\title{
A High Order Finite Element Coupled Multi-Physics Approach To MRI Scanner Design
}

\author{
Bagwell, Scott G.
}

How to cite:

Bagwell, Scott G. (2018) A High Order Finite Element Coupled Multi-Physics Approach To MRI Scanner Design. Doctoral thesis, Swansea University.

http://cronfa.swan.ac.uk/Record/cronfa40797

Use policy:

This item is brought to you by Swansea University. Any person downloading material is agreeing to abide by the terms of the repository licence: copies of full text items may be used or reproduced in any format or medium, without prior permission for personal research or study, educational or non-commercial purposes only. The copyright for any work remains with the original author unless otherwise specified. The full-text must not be sold in any format or medium without the formal permission of the copyright holder. Permission for multiple reproductions should be obtained from the original author.

Authors are personally responsible for adhering to copyright and publisher restrictions when uploading content to the repository.

Please link to the metadata record in the Swansea University repository, Cronfa (link given in the citation reference above.)

http://www.swansea.ac.uk/library/researchsupport/ris-support/ 


\title{
A High Order Finite Element Coupled Multi-Physics Approach To MRI Scanner \\ Design
}
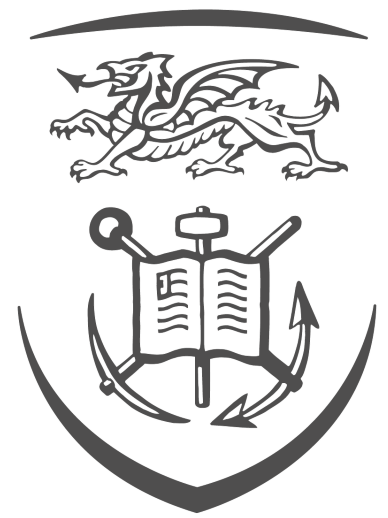

\section{Swansea University Prifysgol Abertawe}

\author{
by \\ Scott Bagwell \\ Submitted to Swansea University \\ in fulfilment of the requirements for the Degree of \\ Doctor of Philosophy \\ at \\ Swansea University
}

May 29, 2018 



\section{Abstract}

Magnetic Resonance Imaging (MRI) scanners are becoming increasingly popular with many clinical experts for use in both medical research and clinical imaging of patients, due to their ability to perform high-resolution non-intrusive imaging examinations. Recently, however, there has been an increasing demand for higherresolution scanners that are capable of performing quicker scans with increased patient comfort. With this demand for more advanced MRI systems, there also follows a number of challenges facing designers. Understanding the physical phenomena behind MRI is crucial in the development of scanners that are capable of producing accurate images of the patient with maximum comfort and minimal noise signatures.

MRI scanners utilise strong static magnetic fields coupled with rapidly time varying gradient magnetic fields to generate images of the patient. In the presence of these time varying fields, the conducting components of MRI scanners generate eddy currents, which give rise to Lorentz forces and cause the conductors to vibrate. These vibrations cause acoustic waves to form that propagate through the air and result in audible noise which can cause significant discomfort for the patient. They also generate Lorentz currents which feedback into the electromagnetic field and this process results in a fully coupled non-linear acousto-magneto-mechanical system. The determination of the coupling mechanisms involved in such a system is a nontrivial task and so, in order to understand the behaviour of MRI systems during operation, advanced computational tools and techniques are required. Moreover, there exists certain small scale physical phenomena that arise in the coupled system which require high resolutions to obtain accurate results.

In this thesis, a new computational framework for the treatment of acoustomagneto-mechanical coupling that arises in low-frequency electro-magneto-mechanical systems, such as MRI scanners, is proposed. The transient Newton-Raphson strategy involves the solution of a monolithic system, obtained from the linearisation of the coupled system of equations and two approaches are considered: (i) the linearised approach and (ii) the non-linear approach.

In (i), physically motivated by the excitation from static and time varying current sources of MRI scanners, the fields may be split into a dominant static component and a much smaller dynamic component. The resulting linearised system is obtained by performing the linearisation of the fields about this dominant static component. This approach permits solutions in the frequency domain, for understanding the response of MRI systems under various excitations, and provides a computationally efficient way to solve this challenging problem, as it allows the tangent stiffness 
matrix to be inverted independently of time or frequency.

In (ii), there is no approximation from a physical standpoint and the linearisation is performed about the current solution. This approach requires that solutions are obtained in the time domain and thus the focus is then put on transient solutions to the coupled system of equations to address the following two important questions: 1) How good is the agreement between the computationally efficient linearised approach compared with the intensive non-linear approach?; and 2) Over what range of MRI operating conditions can the linearised approach be expected to provide acceptable results for MRI scanner design?

Motivated by the need to solve industrial problems rapidly, solutions will be restricted to problems consisting of axisymmetric geometries and current sources. This treatment also discusses, in detail, the computational requirements for the solution of these coupled problems on unbounded domains and the accurate discretisation of the fields using $h p$-finite elements. A set of academic and industrially relevant examples are studied to benchmark and illustrate both approaches, in a $h p$ - finite element context, as well as performing rigorous comparisons between the approaches.

Key Words: Multi-physics; Multifield systems; MRI scanners; Finite element methods; Acousto-Magneto-Mechanical coupling; Newton methods; Frequency domain; Time domain; Transient; Linearisation 


\section{DECLARATION}

This work has not previously been accepted in substance for any degree and is not being concurrently submitted in candidature for any degree

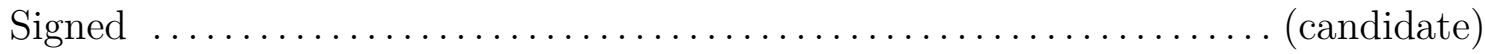

Date

\section{STATEMENT 1}

This thesis is the result of my own investigations, except where otherwise stated. When correction services have been used, the extent and nature of the correction is clearly marked in a footnote(s). Other sources are acknowledged by footnotes giving explicit references. A bibliography is appended.

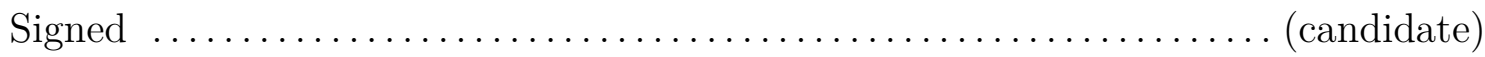

Date

\section{STATEMENT 2}

I herby give consent for my thesis, if accepted, to be available for photocopying and for inter-library loan and for the title and summary to be made available to outside organisations.

Signed

(candidate)

Date 

To Jessica, Willow and my Parents... 



\section{Acknowledgements}

Firstly, I would like to thank Prof. Morgan and Prof. Hassan for convincing me to do a $\mathrm{PhD}$ at Swansea University. It has been a very thrilling and rewarding journey, if not a little relentless! My deepest gratitude goes to my supervisors Dr. Ledger and Prof. Gil for their guidance and wisdom throughout the project. Their meticulous "eye for detail" has helped to improve the presentation of this Thesis. I would like to extend my gratitude to all the folks at Siemens Magnet Technology for accommodating me over the years, whilst carrying out my research. In particular, I would like to thank Mike Mallett, Marcel Kruip and Ian Wilkinson for their continued support as industrial supervisors and helping to setup the project. Thanks must also go to the High Wycombe Radiology department for allowing me to experience an MRI scan first hand, albeit for medical reasons, and the images to add a personal touch to this thesis. This PhD project was funded by the EPSRC as a CASE award studentship in collaboration with Siemens and their financial support is greatly acknowledged.

I have thoroughly enjoyed my time at Swansea University, both through undergraduate study and the more perilous endeavours of studying for a $\mathrm{PhD}$. Were it not for all the friends I have made along the way, I certainly would not have made it through with my sanity in tact. Particular thanks go to Edward Hares, David Naumann and Roman Poya for making academic conferences all the more fun and bearable, with spontaneous trips to the beach and water parks! I would also like to thank all of the people of the Swansea University Rowing Club of whom there are too many to name, you all certainly made studying at Swansea an experience I will never forget. Thanks also go to Caitlin and Dan for allowing me to stay with them and providing welcome distraction during the final stages of writing this beast.

I would like to thank my parents, Greg and Scarlett, for all their love and support throughout my life. I am sure you have both sacrificed more than I know to provide me with the opportunities I have and I am truly grateful for your love and support. Of course, who could forget my siblings Glenn, Alanah and Ross, who have always been there to make my times away from study and family holidays all the more entertaining! And of course thanks also to Willow and Amber, for their endless love, friendship and puppy cuddles! I would also like to thank the Bryant's for being like a second family to me and always being there to provide your support. Thanks must also go to Hans and Nini for being so welcoming during my visits to Canada and the amazing cooking! Thanks also to my grandparents John and Maureen for your love and support. Last but not least, I am incredibly grateful for the endless love and support of my partner-in-crime Jessica Bryant. Were it not for you, I certainly would not have made the finish line! You are my inspiration.

Thank you all so much! 



\section{Contents}

Abstract $\quad$ iii

Acknowledgements $\quad$ ix

$\begin{array}{ll}\text { Nomenclature } & \text { xxvii }\end{array}$

Acronyms and Abbreviations $\quad$ xxxi

I Preliminaries 1

1 Introduction 3

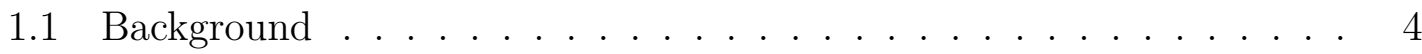

1.2 MRI Scanners . . . . . . . . . . . . . . . . . . . . 4

1.2.1 Physical Issues . . . . . . . . . . . . . . . . . . . 8

1.2.2 Clinical Effects to Patients . . . . . . . . . . . . . 10

1.2 .3 Current Developments . . . . . . . . . . . . . . . . 11

1.3 Computational Methodologies . . . . . . . . . . . . . . . 13

1.3.1 Computational Tools for Single Physics Problems . . . . . . . 13

1.3.2 Coupled Approaches . . . . . . . . . . . . . . . . . . . 17

1.3.3 Applications to MRI Scanners . . . . . . . . . . . . . . 20

1.3.4 Commercial Alternatives . . . . . . . . . . . . . . . . . 21

1.4 Aims and Objectives ... . . . . . . . . . . . . . . . . . 21

1.5 Outline of the Thesis . . . . . . . . . . . . . . . . . 22

1.6 Research Outcomes . . . . . . . . . . . . . . . . . . 25

1.6.1 Journal Publications . . . . . . . . . . . . . . . 26

1.6.2 Conference Presentations . . . . . . . . . . . . . . 26

1.6.3 Research Posters . . . . . . . . . . . . . . . . 27

II General Formulation of the Problem 29

2 Multi-Field Framework $\quad 31$

2.1 Introductory Remarks . . . . . . . . . . . . . . . . . . 32

2.2 Electromagnetics . . . . . . . . . . . . . . . . . 33

2.2.1 Full Maxwell System . . . . . . . . . . . . . . . . . . 33

2.2.2 Isotropic Homogeneous Materials . . . . . . . . . . . 35 
2.2.3 Low Frequency Electromagnetics . . . . . . . . . . . . . 36

2.2.4 Eddy Current Model . . . . . . . . . . . . . . . . . . . . . 38

2.2.5 Interface Conditions . . . . . . . . . . . . . . . . 40

2.3 Mechanics . . . . . . . . . . . . . . . . . . . 41

2.3.1 Transient Linear Elasticity . . . . . . . . . . . . . . . . 41

2.3.2 Magnetic Body Force . . . . . . . . . . . . . . . . . . . . 42

2.3.3 Low Frequency Forcing . . . . . . . . . . . . . . . . . . . . 42

2.3.4 Interface Conditions . . . . . . . . . . . . . . 43

2.4 Acoustics . . . . . . . . . . . . . . . . . . . . . . . . . . . 43

2.4.1 Degenerative Solid Analogy . . . . . . . . . . . . . . . 44

2.4.2 Acoustic Helmholtz Wave Equation . . . . . . . . . . . . . . . 44

2.4.3 Properties of the Source Term . . . . . . . . . . . . . . . 45

2.4 .4 Interface Conditions . . . . . . . . . . . . . . . 46

2.5 Coupled Transmission Problem . . . . . . . . . . . . . . . . 47

2.5.1 Transient Non-Linear Problem . . . . . . . . . . . . . . . 47

2.5.2 MRI Coils . . . . . . . . . . . . . . . . . . . . 48

2.5.3 Static Non-Linear Problem . . . . . . . . . . . . . . . . . 49

2.6 Chapter Summary . . . . . . . . . . . . . . . . . . 50

III Linearised Approach $\quad 51$

3 Linearised Approach

3.1 Introductory Remarks . . . . . . . . . . . . . . . . . . 54

3.2 Iterative Solution Procedures . . . . . . . . . . . . . . . 54

3.2.1 Fixed Point Scheme . . . . . . . . . . . . . . . . 55

3.2 .2 Newton-Raphson Scheme . . . . . . . . . . . . . . . . 57

3.3 Weak Variational Statements . . . . . . . . . . . . . . . 57

3.3.1 Electromagnetics . . . . . . . . . . . . . . . . 59

3.3.2 Mechanics . . . . . . . . . . . . . . . . . 61

3.3 .3 Acoustics . . . . . . . . . . . . . . . . . . 62

3.3.4 Coupled Problem . . . . . . . . . . . . . . . . 63

3.4 Linearisation of the Static Problem . . . . . . . . . . . . . . . . . 64

3.5 Linearisation of the Dynamic Problem . . . . . . . . . . . . . 67

3.5.1 Time Dependent Formulation . . . . . . . . . . . . . 68

3.5.2 Time Harmonic Formulation . . . . . . . . . . . . . . 72

3.6 Chapter Summary . . . . . . . . . . . . . . . . . 75

4 Computational Treatment of the Linearised Approach $\quad 77$

4.1 Introductory Remarks . . . . . . . . . . . . . . . . . . . . . 78

4.2 Axisymmetric Reduction for Rotationally Symmetric Geometries . . . 79

4.2.1 Cylindrical Representation . . . . . . . . . . . . . . . . . 80

4.2 .2 Weighted Spaces . . . . . . . . . . . . . . . . . . 82

4.2.3 Transformation of Variables . . . . . . . . . . . . 86

4.2.4 Axisymmetric Weak Form . . . . . . . . . . . . . . . . . 89

4.3 Far Field Treatment . . . . . . . . . . . . . . . . . . . . 90 
4.3 .1 Electromagnetic Field Decay . . . . . . . . . . . . . . . . . 90

4.3 .2 Acoustic Field Decay . . . . . . . . . . . . . . . . . . . . 91

4.4 Finite Elements . . . . . . . . . . . . . . . . . . . . . . . . 93

4.4.1 Reference Elements . . . . . . . . . . . . . . . . . . 95

4.4.2 Elemental Mapping Functions . . . . . . . . . . . . . . . . . 97

4.4.3 Hierarchic Shape Functions . . . . . . . . . . . . . . . . 100

4.4 .4 Infinite Elements . . . . . . . . . . . . . . . . . . . . 101

4.5 Spatial Discretisation of the Coupled System . . . . . . . . . . . . . . 102

4.5.1 Galerkin Approximation . . . . . . . . . . . . . . . . . 102

4.5.2 Linear System . . . . . . . . . . . . . . . . . . . . . . . . . . . . . . . . . . . . . . . . . . . . .

4.5.3 Numerical Integration . . . . . . . . . . . . . . . . . . . . . . . . . 108

4.6 Numerical Solution Strategy . . . . . . . . . . . . . . . . . . . . . 109

4.7 Chapter Summary . . . . . . . . . . . . . . . . . . . . . . 110

5 Numerical Simulations of the Linearised Approach 113

5.1 Introductory Remarks . . . . . . . . . . . . . . . . . . . . . 114

5.2 Error in the Solution . . . . . . . . . . . . . . . . . . . . 114

5.2 .1 Error Norms . . . . . . . . . . . . . . . . . . . 115

5.3 Single Physics Problems . . . . . . . . . . . . . . . . 115

5.3.1 Conducting Sphere in a Uniform Alternating Magnetic Field . 115

5.3.2 Mechanical Shell Subject to a Pressure Field . . . . . . . . . . 122

5.3.3 Sound-Hard Sphere Subject to an Incident Acoustic Pressure

Field . . . . . . . . . . . . . . . . . . . . 125

5.4 Coupled Multi-Physics Problems . . . . . . . . . . . . . . . . 129

5.4.1 Acoustic Wave Scattering of Thin Elastic Shell . . . . . . . . . 129

5.4 .2 Test Magnet Problem . . . . . . . . . . . . . . . . . . . . . 132

5.5 Chapter Summary . . . . . . . . . . . . . . . . . . . . 141

IV Non Linear Approach 143

6 Non-Linear Approach $\quad 145$

6.1 Introductory Remarks . . . . . . . . . . . . . . . . . . 146

6.2 MRI Operating Conditions . . . . . . . . . . . . . . . . . 147

6.3 Coupled System . . . . . . . . . . . . . . . . . . . . . . 148

6.3.1 Transient Non-Linear System . . . . . . . . . . . . . . . . 148

6.3.2 Static Problem . . . . . . . . . . . . . . . . . . 149

6.3.3 Initial Conditions . . . . . . . . . . . . . . . . . . 149

6.4 Linearisation . . . . . . . . . . . . . . . . . . . . 150

6.4.1 Non-Linear Approach . . . . . . . . . . . . . . . . . . . . 150

6.4.2 Linearised Approach . . . . . . . . . . . . . . . . . . 153

6.5 Non-Linear vs. Linearised Approaches . . . . . . . . . . . . . . . . 154

6.5.1 Comparison of Energies in Non-Linear and Linearised Approaches .................... . . 154

6.5.2 Ratio of the Magnetic Field Strengths . . . . . . . . . . . . . 161

6.5.3 Simple Model Relating Field and Current Strengths . . . . . . 161 
6.6 Chapter Summary . . . . . . . . . . . . . . . . . 163

7 Computational Treatment of the Non Linear Approach 165

7.1 Introductory Remarks . . . . . . . . . . . . . . . . . . 166

7.2 Spatial Discretisation . . . . . . . . . . . . . . . 166

7.3 Temporal Discretisation . . . . . . . . . . . . . . . 167

7.3.1 Time Integration Schemes . . . . . . . . . . . . . . . . 168

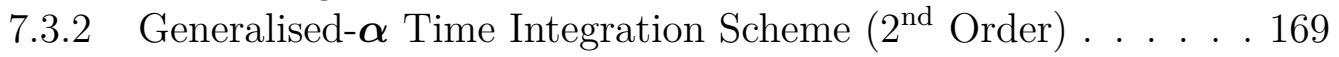

7.3.3 Predictor Multi-Corrector Step . . . . . . . . . . . . . . . 170

7.3.4 Fully Discrete Non-Linear Approach . . . . . . . . . . . . . 170

7.3.5 Fully Discrete Linearised Approach . . . . . . . . . . . . . . . 171

7.4 Solution Strategy . . . . . . . . . . . . . . . . . . . . . 171

7.5 Chapter Summary . . . . . . . . . . . . . . . . . 171

8 Numerical Examples of the Non-Linear Approach 175

8.1 Introductory Remarks . . . . . . . . . . . . . . . . . 176

8.2 Test Magnet Problem . . . . . . . . . . . . . . . . . . 176

8.2.1 Temporal convergence of the physical fields . . . . . . . . . . 179

8.2.2 Spatial Discretisation Study in the Outputs of Interest . . . . 180

8.3 Realistic Magnet Problem . . . . . . . . . . . . . . . . . . . . 190

8.4 Chapter Summary . . . . . . . . . . . . . . . . . . . . . 192

V Conclusions and Further Work 195

9 Conclusions and Future Development 197

9.1 Concluding Remarks . . . . . . . . . . . . . . . . . . . . . . . . 198

9.2 Conclusions . . . . . . . . . . . . . . . . . . . . . . . . . 198

9.3 Recommendations For Further Work . . . . . . . . . . . . . . 201

VI Appendices 205

A Explanation of Key Physical Phenomena 207

A.1 Eddy Currents . . . . . . . . . . . . . . . . . . . . 207

A.2 Skin Depth Effect . . . . . . . . . . . . . . . . . . . . 208

A.3 Lorentz Currents . . . . . . . . . . . . . . . . . . . . . . . . . 209

A.4 Electromotive Force . . . . . . . . . . . . . . . . . . . . . 211

A.5 Electromagnetic (Maxwell) Stress . . . . . . . . . . . . . . . 211

B Interface Conditions

B.1 Electromagnetic Interface Conditions . . . . . . . . . . . . . . . . 213

B.1.1 Electric Field Interface Condition . . . . . . . . . . . . . . 214

B.1.2 Magnetic Field Interface Condition . . . . . . . . . . . . . . . 215

B.2 Mechanical Interface Conditions . . . . . . . . . . . . . . . . 216

B.2.1 Traction Interface Condition . . . . . . . . . . . . . . 217 
B.2.2 Acceleration Interface Condition . . . . . . . . . . . . . . . 218

C Low Frequency Electromagnetics $\quad 221$

C.1 Eddy Current Approximation . . . . . . . . . . . . . . . . . . . . . 221

C.2 Electromagnetic Body Force . . . . . . . . . . . . . . . . . . . . 223

D Acoustic Far Field Treatment 225

D.1 Approximation of the Sommerfeld Condition . . . . . . . . . . . . . 225

D.2 Perfectly Matched Layer Formulation . . . . . . . . . . . . . . . . 226

D.2.1 Axisymmetric (2D Cylindrical) Split Field Form . . . . . . . . 227

D.2.2 Time Harmonic Description . . . . . . . . . . . . . . . . . . . 228

D.2.3 Time Dependent Description . . . . . . . . . . . . . . . . . 229

E Shape Functions on the Triangular Reference Element 233

E.1 Triangular Element . . . . . . . . . . . . . . . . . . . . . . . 233

E.1.1 The $H^{1}(\Omega)$ Vertex Shape Functions . . . . . . . . . . . . . . 233

E.1.2 The $H^{1}(\Omega)$ Edge Shape Functions . . . . . . . . . . . . . . . . 234

E.1.3 The $H^{1}(\Omega)$ Interior Shape Functions . . . . . . . . . . . . . . 235

E.2 Quadrilateral Element . . . . . . . . . . . . . . . . . 236

E.2.1 The $H^{1}(\Omega)$ Vertex Shape Functions . . . . . . . . . . . . . . . 237

E.2.2 The $H^{1}(\Omega)$ Edge Shape Functions . . . . . . . . . . . . . . . . 238

E.2.3 The $H^{1}(\Omega)$ Interior Shape Functions . . . . . . . . . . . . . . 239

F $\quad$ Perturbed Eddy Current Problem $\quad 241$

F.1 Traditional Eddy Current Problem . . . . . . . . . . . . . . . . . 241

F.2 Perturbed Field Problem . . . . . . . . . . . . . . . . . . . . . 242

G Computational Improvements $\quad 243$

G.1 Global System Assembly of Complex Fields . . . . . . . . . . . . . . 243

G.2 Vectorisation . . . . . . . . . . . . . . . . . 245

G.3 Residual Scaling . . . . . . . . . . . . . . . . . . . . . . . 246

$\begin{array}{llr}\text { VII References } & 247\end{array}$ 



\section{List of Figures}

1.1 Comparison between MRI (left) and CT (right) generated images of my brain and eyes! MRI scans typically allow for higher resolution imaging of soft matter, such as the brain and eyes, whilst CT performs better when imaging hard mater, such as the bone structures offering lower resolution of soft matter. These images are courtesy of the High Wycombe Radiology Department to whom I owe thanks for allowing me to add this personal touch to the Thesis. A very useful experience indeed. . . . . . . . . . . . . . . . . 6 6

1.2 Primary components of a simplified clinical MRI scanner. . . . . . . . 7

1.3 Examples of MRI images with imaging artefacts present, known as image ghosting, courtesy of [239]. The imaging artefacts are seen here as a series of images super positioned on top of one-another with an offset. . . . . . . . . . . . . . . . .

1.4 Examples of gradient coil pulse sequences for imaging different parts of the body. . . . . . . . . . . . . . . . . . . .

1.5 Representation of the tangent stiffness matrices of monolithic and partitioned solvers. In the monolithic solver, the pure field and cross derivative blocks (the coupled blocks) are constructed and the tangent stiffness matrix is solved as a whole. In the partitioned solver the tangent stiffness matrix contains only the pure field blocks of the individual physics and can be solved individually as separate systems, which rely on forcing terms to compute coupling contributions. . . . .

1.6 Algorithms for acousto-magneto-mechanical methodologies using both: monolithic (NR) and partitioned (FP) iterative solvers to compute sourcing terms between the physical fields and the system tangent stiffness matrix. . . . . . . . . . . . . . . . . . . . . . 19

2.1 General orthonormal coordinate system. . . . . . . . . . . . . . . 34

2.2 Frequency spectrums of the gradient coil pulse sequences, shown in Figure 1.4, for imaging different parts of the body. . . . . . . . . . . . 37

2.3 Frequency decomposition by Fourier transform of the time signal. . . 37

2.4 Conducting region $\Omega_{c}$ excited by coils contained within the unbounded $\mathbb{R}^{3} \backslash \Omega_{c}$ space. . . . . . . . . . . . . . . . . . 41

2.5 Conducting region $\Omega_{c}$, where the boundary is split into Dirichlet and Neumann parts. . . . . . . . . . . . . . . . . . . 43 
2.6 Interface conditions at the conductor-free space boundary, where $\partial \Omega_{c}^{-}$ corresponds to the contribution of the interface from the conductor and $\partial \Omega_{c}^{+}$the contribution from the non-conducting region. . . . . . . 46

2.7 Physical representation of the coupling effects in an MRI environment. 48

2.8 Current source decomposition, where $\boldsymbol{J}^{s}(t)=\boldsymbol{J}^{D C}+\boldsymbol{J}^{A C}(t) \ldots \ldots . .49$

3.1 Graphical representation of the coupling methodology between the three physical fields; electromagnetic, mechanical and acoustic, coupled through a monolithic linearised scheme. . . . . . . . . . . . 56

3.2 Diagram of the conducting region $\Omega_{c}$ and the magnet coils $\operatorname{supp}\left(\boldsymbol{J}^{s}\right)$, which give rise to the sourcing currents $\boldsymbol{J}^{s}$, contained within an unbounded region of free space $\mathbb{R}^{3}$. The general orthogonal coordinate system $\boldsymbol{x}=x_{1} \boldsymbol{e}_{1}+x_{2} \boldsymbol{e}_{2}+x_{3} \boldsymbol{e}_{3}$ is measured from the centre of $\Omega_{c} . \quad$. 58

3.3 Illustration of the directional derivative of the weighted residual $R_{q}$ about the solution $\boldsymbol{q}$. The weighted residual functions, shown as the green surface, are linearised about the solution by taking the directional derivatives in the direction of the incremental solution $\boldsymbol{\delta}_{q}$ as the scaled distance in the incremental solution $\zeta \rightarrow 0$. . . . . 65

3.4 Linearisation of the full temporal solution about the static field (DC) component, where $\boldsymbol{R}^{D C}(0,0,0)=\left[R_{A}^{D C}(0), R_{u}^{D C}(0), R_{P}^{D C}(0)\right]^{T}$ denotes the static system evaluated at $t=0$ and $\tilde{\boldsymbol{R}}^{A C}\left(\boldsymbol{A}^{D C}, \boldsymbol{u}^{D C}, P^{D C}\right)=$ $\left[\tilde{R}_{A}^{A C}\left(\boldsymbol{A}^{D C}\right), \tilde{R}_{u}^{A C}\left(\boldsymbol{u}^{D C}\right), \tilde{R}_{P}^{A C}\left(P^{D C}\right)\right]^{T}$ denotes the transient system. . 70

4.1 Model order reduction by simplification of the full 3D scanner geometry $\left(\Omega \in \mathbb{R}^{3}\right)$ and an axisymmetric transformation of the simplified geometry to the meridian plane $\left(\Omega^{m} \in \mathbb{R}^{2}\right) \ldots \ldots . . . \ldots$. . . .

4.2 Transformation from a full 3D MRI scanner to the $2 \mathrm{D}$ axisymmetric case. Under simplifications of geometry, and neglection of certain excitation currents, the problem is constant in the azimuthal $\phi$-direction and can thus be axisymmetrically projected onto the meridian domain

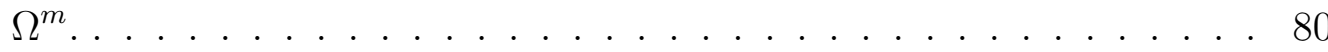

4.3 Typical configurations of the set of $x, y$ and $z$ gradient coils of an MRI scanner. . . . . . . . . . . . . . . . . .

4.4 Differential volume element $\mathrm{d} \Omega$, shown in green, in a cylindrical coordinate system $(r, \phi, z)$. The left most plane illustrates the meridian domain $\Omega^{m}$, with the meridian differential surface element $\mathrm{d} \Omega^{m}$. . .

4.5 Unbounded axisymmetric meridian domain of the problem where the complete domain $\Omega^{m}=\Omega_{n}^{m} \cup \Omega_{c}^{m}$ and the outer boundary of the complete domain $\partial \Omega^{m}=(r=0, z) \cup \partial \Omega_{\infty}^{m}$. The axisymmetric meridian domain $\Omega^{m} \in\left\{\mathbb{R}^{2}: 0 \leq r \leq \infty,-\infty \leq z \leq \infty\right\}$, where the radial coordinates have a lower bound of $(r=0)$ that meets the $z$-axis. . . .

4.6 Artificial truncation of the unbounded domain $\mathbb{R}^{3}$ to create the bounded non-conducting region $\Omega_{n}$, with the outer boundary $\partial \Omega$ located a finite distance from the origin. . . . . . . . . . . . . . . . . . 91

4.7 Representation of the Perfectly Matched Layer (PML) . . . . . . . . . 93 
4.8 The continuous domain $\Omega$ is discretised by a series of points, called nodes. The nodes are connected to form small volumes $\Omega^{(e)}$, known as elements. The discretisation of the domain results in a discrete boundary $\partial \Omega^{(e)}$ that does not, in general, coincide with the domain boundary $\partial \Omega \ldots \ldots \ldots$. . . . . . . . . . . . . . . . . . 94

4.9 Reference triangular element in the referential domain $\Omega_{\xi}=(\xi, \eta)$, with vertices located at $\boldsymbol{\xi}=(1,0),(0, \sqrt{3}),(-1,0)$, in the reference coordinates $\boldsymbol{\xi}=(\xi, \eta)^{T} \ldots \ldots \ldots \ldots \ldots \ldots \ldots \ldots \ldots \ldots \ldots \ldots \ldots \ldots$

4.10 Reference Quadrilateral element in the referential domain $\Omega_{\xi}$, with vertices located at $\boldsymbol{\xi}=(0,0),(1,0),(1,1),(0,1)$, in the reference coordinates $\boldsymbol{\xi}=(\xi, \eta)^{T} \ldots \ldots \ldots \ldots$. . . . . . . . . . . . . . . . .

4.11 Mapping between quadrilateral and triangular finite elements in the reference $\Omega_{\xi}$ and spatial $\Omega$ domains. . . . . . . . . . . . . . . . . . . . 97

4.12 Curved elements in the spatial domain $\Omega \ldots$. . . . . . . . . . . . . 98

4.13 Finite element mesh with a layer of quadrilateral infinite elements located on the outer boundary. In the spatial domain the outer boundary is located at a finite distance from the centre, however through the parametric mapping functions on the reference element, become placed at infinity, where the decay conditions are imposed as Dirichlet conditions. . . . . . . . . . . . . . . . . . . . 101

4.14 Mapping between the reference quadrilateral element and the spatial infinite element. . . . . . . . . . . . . . . . . . . . . . . 102

4.15 Numerical solution strategy flowchart for the linearised approach solver.109

5.1 Conducting sphere in a uniform alternating magnetic field: problem setup. . . . . . . . . . . . . . . . . 116

5.2 Conducting sphere in a uniform alternating magnetic field: convergence of $\left\|\mathcal{A}_{\phi}-\mathcal{A}_{\phi}\right\|_{L^{2}\left(\Omega^{m}\right)} /\left\|\mathcal{A}_{\phi}\right\|_{L^{2}\left(\Omega^{m}\right)}$ under $h$-refinement for elements of $p=[1,2,3]$ for frequencies of $f=[5,50] \mathrm{Hz}$, where the $L^{2}(\Omega)$ norm of the error is plotted against the maximum element edge length $h$. . . . . . . . . . . . . . . . . . . . . . 117

5.3 Conducting sphere in a uniform alternating magnetic field: convergence of $\left\|\mathcal{A}_{\phi}-\mathcal{A}_{\phi}\right\|_{H^{1}\left(\Omega^{m}\right)} /\left\|\mathcal{A}_{\phi}\right\|_{H^{1}\left(\Omega^{m}\right)}$ under $h$-refinement for elements of $p=[1,2,3]$ for frequencies of $f=[5,50] \mathrm{Hz}$, where the $H^{1}(\Omega)$ norm of the error is plotted against the maximum element edge length $h \ldots \ldots \ldots$. . . . . . . . . . . . . . . . 118

5.4 Conducting sphere in a uniform alternating magnetic field: convergence of $\left\|\mathcal{A}_{\phi}-\mathcal{A}_{\phi}\right\|_{L^{2}\left(\Omega^{m}\right)} /\left\|\mathcal{A}_{\phi}\right\|_{L^{2}\left(\Omega^{m}\right)}$ and $\left\|\mathcal{A}_{\phi}-\mathcal{A}_{\phi}\right\|_{H^{1}\left(\Omega^{m}\right)} /\left\|\mathcal{A}_{\phi}\right\|_{H^{1}\left(\Omega^{m}\right)}$ at a frequency $f=5 \mathrm{~Hz}$ for elements of $p=[1,2,3]$ against the number of degrees of freedom NDOF under $h$-refinement. . . . . . . . . . 118

5.5 Conducting sphere in a uniform alternating magnetic field: convergence of $\left\|\mathcal{A}_{\phi}-\mathcal{A}_{\phi}\right\|_{L^{2}\left(\Omega^{m}\right)} /\left\|\mathcal{A}_{\phi}\right\|_{L^{2}\left(\Omega^{m}\right)}$ and $\left\|\mathcal{A}_{\phi}-\mathcal{A}_{\phi}\right\|_{H^{1}\left(\Omega^{m}\right)} /\left\|\mathcal{A}_{\phi}\right\|_{H^{1}\left(\Omega^{m}\right)}$ under $p$-refinement. . . . . . . . . . . . . . . . . . . . . . . 119 
5.6 Conducting sphere in a uniform alternating magnetic field: contours of $|\boldsymbol{B}| \approx\left|\nabla \times\left(\mathcal{A}_{\phi} \boldsymbol{e}_{\phi}\right)\right|$ around the conducting sphere at different frequencies. . . . . . . . . . . . . . . . 120

5.7 Conducting sphere in a uniform alternating magnetic field: convergence of $\left\|\mathcal{A}_{\phi}-\mathcal{A}_{\phi}\right\|_{L^{2}\left(\Omega^{m}\right)} /\left\|\mathcal{A}_{\phi}\right\|_{L^{2}\left(\Omega^{m}\right)}$ and $\left\|\mathcal{A}_{\phi}-\mathcal{A}_{\phi}\right\|_{H^{1}\left(\Omega^{m}\right)} /\left\|\mathcal{A}_{\phi}\right\|_{H^{1}\left(\Omega^{m}\right)}$ for the perturbed eddy current problem using both infinite elements and an approximate boundary condition at $L=[5,10,15,20] m$ under p-refinement. . . . . . . . . . . . . . . . . . 121

5.8 Mechanical shell subject to a pressure field: problem setup. . . . . . . . 122

5.9 Mechanical shell subject to a pressure field: convergence of $\| \boldsymbol{U}-$ $\mathcal{U}\left\|_{L^{2}} /\right\| \boldsymbol{U} \|_{L^{2}}$ and $\|\boldsymbol{U}-\mathcal{U}\|_{S N S} /\|\boldsymbol{U}\|_{S N S}$ under $h$-refinement for elements of $p=[1,2,3]$, where the errors are plotted against the maxi-

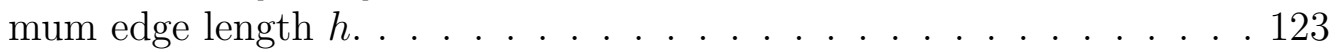

5.10 Mechanical shell subject to a pressure field: convergence of $\| \mathcal{U}-$ $\mathcal{U}\left\|_{L^{2}} /\right\| \boldsymbol{U}\left\|_{L^{2}},\right\| \boldsymbol{U}-\mathcal{U}\left\|_{S N S} /\right\| \boldsymbol{U} \|_{S N S}$ under $p$-refinement. . . . . . . . 124

5.11 Mechanical shell subject to a pressure field: contours of $\mathcal{U}_{r}$ and $\mathcal{U}_{z} \ldots 124$

5.12 Sound-hard sphere subject to an incident acoustic pressure field: problem setup. . . . . . . . . . . . . . . . . . . 125

5.13 Sound-hard sphere subject to an incident acoustic pressure field: convergence of $\left\|\mathcal{P}^{s c}-\mathcal{P}^{s c}\right\|_{L^{2}} /\left\|\mathcal{P}^{s c}\right\|_{L^{2}}$, under $h$-refinement for elements of $p=[1,2,3]$ for wave numbers of $k=[4 \pi / 3,10] \mathrm{m}^{-1}$, where the $L^{2}(\Omega)$ norm of the error is plotted against the maximum element edge length $h \ldots \ldots \ldots$. . . . . . . . . . . . . . 126

5.14 Sound-hard sphere subject to an incident acoustic pressure field: convergence of $\left\|\mathcal{P}^{s c}-\mathcal{P}^{s c}\right\|_{H^{1}} /\left\|\mathcal{P}^{s c}\right\|_{H^{1}}$, under $h$-refinement for elements of $p=[1,2,3]$ for wave numbers of $k=[4 \pi / 3,10] \mathrm{m}^{-1}$, where the $H^{1}(\Omega)$ norm of the error is plotted against the maximum element edge length $h$. . . . . . . . . . . . . . . . . . . . . . . 127

5.15 Sound-hard sphere subject to an incident acoustic pressure field: convergence of $\left\|\mathcal{P}^{s c}-\mathcal{P}^{s c}\right\|_{L^{2}} /\left\|\mathcal{P}^{s c}\right\|_{L^{2}},\left\|\mathcal{P}^{s c}-\mathcal{P}^{s c}\right\|_{H^{1}} /\left\|\mathcal{P}^{s c}\right\|_{H^{1}}$ under $p$-refinement for wave numbers $k=[4 \pi / 3,10,30]$. . . . . . . . . . 128

5.16 Sound-hard sphere subject to an incident acoustic pressure field: contours of $\operatorname{Re}\left(\mathcal{P}^{s c}\right)$ for differing wave numbers $k$. . . . . . . . . . . 128

5.17 Elastic shell subject to an incident acoustic pressure field: problem setup. . . . . . . . . . . . . . . . . . . . 129

5.18 Elastic shell subject to an incident acoustic pressure field: effects of $h$ - and $p$-refinement on the acoustic pressure field for $k=4 \pi / 3 m^{-1}$, plotted along a line along the $z=0$ axis from $r=1$ to $r=5.6$. . . 130

5.19 Elastic shell subject to an incident acoustic pressure field: effects of $p$-refinement for high wave number $k$ on the acoustic pressure field. . 131

5.20 Elastic shell subject to an incident acoustic pressure field: deformed shell interacting with surrounding acoustic pressure field. . . . . . . . 131

5.21 Simplified MRI scanner subject to alternating and static current driven coils: problem setup. . . . . . . . . . . . . . . . . . . . 132 
5.22 Simplified MRI scanner subject to alternating and static current driven coils: ohmic power dissipation as a function of alternating current frequency. . . . . . . . . . . . . . . . . . . . 135

5.23 Simplified MRI scanner subject to alternating and static current driven coils: contours of the eddy currents $\operatorname{Re}\left(\mathcal{J}_{\phi}^{e}\right)$ for $p=1,5$ and $f=$ $[160,4100] \mathrm{Hz} . \ldots \ldots \ldots \ldots$

5.24 Simplified MRI scanner subject to alternating and static current driven coils: effects of $p$ - enrichment on the eddy current resolution $\operatorname{Re}\left(J_{\phi}^{o}\right)$ in $\Omega_{c}^{4 K}$ for $f=[160,4100] \mathrm{Hz} \ldots \ldots \ldots$. . . . . . . . . . . . 136

5.25 Simplified MRI scanner subject to alternating and static current driven coils: $E_{\Omega_{c V} \text { C }}^{k}\left(\omega, \boldsymbol{\delta}_{\mathfrak{U}}\right)$ and resonant mode shapes. . . . . . . . . . 138

5.26 Simplified MRI scanner subject to alternating and static current driven coils: $E_{\Omega_{c}^{77 K}}^{k}\left(\omega, \boldsymbol{\delta}_{\mathfrak{U}}\right)$ and resonant mode shapes. . . . . . . . . . . . 139

5.27 Simplified MRI scanner subject to alternating and static current driven coils: $E_{\Omega^{4 K}}^{k}\left(\omega, \boldsymbol{\delta}_{\mathfrak{U}}\right)$ and resonant mode shapes. . . . . . . . . . . . 140

5.28 Simplified MRI scanner subject to alternating and static current driven coils: magnetic flux lines (red), acoustic contour lines (yellow) and displaced shields $\Omega_{c}$. . . . . . . . . . . . . . . . . . . . . . . . . . . . 142

6.1 Patient on MRI scanner bed with localised torso receiver coils, courtesy of Siemens. . . . . . . . . . . . . . . . . . . . . 148

6.2 Single current loop, representing a lumped mass of coils. . . . . . . . 162

7.1 Summary of steps to solving transient non-linear system. . . . . . . . 172

8.1 Test magnet problem: components of the simplified geometry. . . . . 177

8.2 Test magnet problem: temporal convergence of the generalised $\alpha$ scheme $\left(2^{n d}\right.$ order). The error is taken as the relative error between the computed and reference solutions at some time $t$, which is taken as some time after the steady state response is achieved. . . . . . . 179

8.3 Test magnet problem: output power (left) and kinetic energy (right) of the OVC $\Omega_{c}^{O V C}$ for element order $p=[1,2,3,4,5] \ldots \ldots 180$

8.4 Test magnet problem: output power (left) and kinetic energy (right) of the $77 K$ radiation shield $\Omega_{c}^{77 K}$ for element order $p=[1,2,3,4,5]$.

8.5 Test magnet problem: output power (left) and kinetic energy (right) of the $4 K$ radiation shield $\Omega_{c}^{4 K}$ for element order $p=[1,2,3,4,5] . \quad$. 182

8.6 Test magnet problem: time signals and corresponding FFTs of $\partial \boldsymbol{A} / \partial t$ for both the linearised (red line) and non-linear approaches (black line), for various values of $\left|J_{\phi}^{A C}\right| /\left|J_{\phi}^{D C}\right|$ subject to a $\omega=2 \pi[2000] \mathrm{rad} / \mathrm{s}$ sinusoidal excitation. . . . . . . . . . . . . . . . 183

8.7 Test magnet problem: time signals and corresponding FFTs of $\partial \boldsymbol{A} / \partial t$ for both the linearised and non-linear approaches, for $\left|J_{\phi}^{A C}\right| /\left|J_{\phi}^{D C}\right|=$ $20 \%$ subject to various frequencies of excitation. . . . . . . . . 183

8.8 Test magnet problem: time signals and corresponding FFTs of $\partial u_{r} / \partial t$ for both the linearised and non-linear approaches, for various values of $\left|J_{\phi}^{A C}\right| /\left|J_{\phi}^{D C}\right|$ subject to a $\omega=2 \pi[1000] \mathrm{rad} / \mathrm{s}$ sinusoidal excitation. 
8.9 Test magnet problem: time signals and corresponding FFTs of $\partial u_{r} / \partial t$ for both the linearised and non-linear approaches, for $\left|J_{\phi}^{A C}\right| /\left|J_{\phi}^{D C}\right|=$ $20 \%$ subject to various frequencies of excitation. . . . . . . . . 185

8.10 Test magnet problem: computation of the Output Power (left) and Kinetic Energy (right) of the OVC $\Omega_{c}^{O V C}$ for both the linearised approach in time and frequency domain as well as the non-linear approach.186

8.11 Test magnet problem: computation of the Output Power (left) and Kinetic Energy (right) of the $77 \mathrm{~K}$ radiation shield $\Omega_{c}^{77 K}$ for both the linearised approach in time and frequency domain as well as the nonlinear approach. . . . . . . . . . . . . . . . . . . . . . . 187

8.12 Test magnet problem: computation of the Output Power (left) and Kinetic Energy (right) of the $4 K$ radiation shield $\Omega_{c}^{4 K}$ for both the linearised approach in time and frequency domain as well as the nonlinear approach. . . . . . . . . . . . . . . . . . . . . . . . 187

8.13 Test magnet problem: comparison between the time dependant linearised and non-linear approaches for displacements of the OVC at different times for $\left|J_{\phi}^{A C}\right| /\left|J_{\phi}^{D C}\right|=20 \%$ and $\omega=2 \pi(2000) \mathrm{rad} / \mathrm{s} . \quad$. . 189

8.14 Realistic magnet problem: components of the simplified geometry. . . 191

8.15 Full magnet problem: displacements of the OVC at different times for $\left|J_{\phi}^{A C}\right| /\left|J_{\phi}^{D C}\right|=15 \%$ and $\omega=2 \pi(1500) \mathrm{rad} / \mathrm{s}$ for both the time dependant linearised and non-linear approaches. . . . . . . . . . . . 193

8.16 Full magnet problem: snapshots of the distorted OVC, gradient coils and corresponding gradient magnetic field lines at various time intervals for $\left|J_{\phi}^{A C}\right| /\left|J_{\phi}^{D C}\right|=15 \%$ and $\omega=2 \pi(1500) \mathrm{rad} / \mathrm{s} . \ldots . . . . .194$

A.1 Eddy currents $\boldsymbol{J}^{e}$ arising from time varying magnetic field. . . . . . . 208

A.2 Circulating eddy currents induced by a changing $\boldsymbol{H}$ field inside a conductor. The eddy currents $\boldsymbol{J}^{e}$ reinforce the currents at the surface and oppose the currents towards the centre of the conductor. . . . . . 209

A.3 Current distribution through the cross section of a conductor $\Omega_{c}$ of thickness thick. The current strength decays asymptotically to 0 from the surface. . . . . . . . . . . . . . . . . . . . . . 210

A.4 Lorentz currents $\boldsymbol{J}^{l}$ induced by moving a conductor $\Omega_{c}$ through a magnetic field, with flux density $\boldsymbol{B}$, at a velocity $\boldsymbol{v}$. . . . . . . . . . . 210

A.5 Electromotive (Lorentz) forces $\boldsymbol{f}^{e}$ induced by moving a conductor $\Omega_{c}$ through a magnetic field, with flux density $\boldsymbol{B}$, at a velocity $\boldsymbol{v}$. . . . 211

B.1 A domain $\Omega=\Omega_{1} \cup \Omega_{2}$ consisting of multiple mediums $\Omega_{1}, \Omega_{2}$ with an outer boundary $\partial \Omega$ and an interface $\partial \Omega^{\text {int }}$. A closed loop contour $\partial \Sigma$ is applied at the interface of the two domains, where each side of the interface has an associated unit tangent vector. . . . . . . . . . 214

B.2 A domain $\Omega=\Omega_{1} \cup \Omega_{2}$ consisting of two mediums $\Omega_{1}$ and $\Omega_{2}$ connected at an interface $\partial \Omega^{\text {int }}$, subject to a traction force $\boldsymbol{T}$. . . . . . . . 216

D.1 Computational domain with truncated outer boundary. . . . . . . . 226

D.2 Absorption of outgoing pressure wave through the PML. . . . . . . . 227 
D.3 Truncated computational domain including the PML region. . . . . . 230

E.1 Triangular finite element basis functions associated with the vertex degrees of freedom of the reference triangular element. . . . . . . . . 233

E.2 Triangular finite element basis functions associated with the edge degrees of freedom of the reference triangular element. . . . . . . . . 235

E.3 Triangular finite element basis functions associated with the interior degrees of freedom of the reference triangular element. . . . . . . . . 236

E.4 Quadrilateral finite element basis functions associated with the vertex degrees of freedom of the reference quadrilateral element. . . . . . . . 237

E.5 Quadrilateral finite element basis functions associated with the edge degrees of freedom of the reference quadrilateral element. . . . . . . . 238

E.6 Quadrilateral finite element basis functions associated with the interior degrees of freedom of the reference quadrilateral element. . . . . 239

G.1 Example of a fully coupled sparse system matrix with the definition of the rows and columns. The red, blue and green blocks are associated with the electromagnetic, mechanical and acoustic DOFs, respectively, where the mixed colours indicate coupled two-field DOFs. 244

G.2 Plot of the relative sourcing current density $\boldsymbol{J}^{s}(t) /\left|\boldsymbol{J}^{s}\right|$ as a function of time for a simple single frequency sinusoidal excitation . . . . . . . 246

G.3 Graphical representation of the solution to the system of equations using the scaled residual method. . . . . . . . . . . . . . . 246 



\section{List of Tables}

5.1 Simplified MRI scanner subject to alternating and static current driven coils: Computed modal frequencies and corresponding Eigen-frequencies

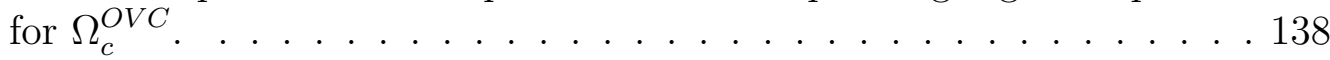

8.1 Test magnet problem: typical values of the current densities in static and gradient coils and ranges of static and gradient field strengths from manufacturers data $[196,229,228] . \ldots \ldots \ldots . \ldots \ldots 177$

8.2 Test magnet problem: average computational times per timestep of the time dependant linearised and non-linear approaches in the time domain for specific element order $p=[1,2,3,4,5] \ldots \ldots$. . . . . 190 



\section{Nomenclature}

Sym

A

H

B

E

$D$

J

$\boldsymbol{j}_{s}$

$u$

$u$

a

$P$

$\boldsymbol{\delta}_{A}$

$\boldsymbol{\delta}_{u}$

$\boldsymbol{\delta}_{P}$

A

u

$\mathrm{P}$

$\delta_{\mathrm{A}}$

$\delta_{\mathrm{u}}$

$\delta_{\mathrm{P}}$
Description

\section{Continuous Time Dependent Fields}

Magnetic vector potential

Magnetic field vector

Magnetic flux density vector

Electric field vector

Electric displacement field vector

Electric current density vector

Surface current density vector

Mechanical displacement vector

Mechanical velocity vector

Mechanical acceleration vector

Acoustic pressure scalar

Magnetic update field

Mechanical update field

Acoustic update field
Webers per metre

Amperes per metre

Teslas

Volts per metre

Coulombs per sq. metre

Amperes per sq. metre

Amperes per metre

Metres

Metres per second

Metres per sq. second

Pascals

Webers per metre

Metres

Pascals

$\left[\mathrm{Wb} \mathrm{m}^{-1}\right]$
$\left[\mathrm{Am}^{-1}\right]$
$[\mathrm{T}]$
$\left[\mathrm{Vm}^{-1}\right]$
$\left[\mathrm{Cm}^{-2}\right]$
$\left[\mathrm{Am}^{-2}\right]$
$\left[\mathrm{Am}^{-1}\right]$
$\left[\mathrm{m}^{-1}\right.$
$\left[\mathrm{m} \mathrm{s}^{-1}\right]$
$\left[\mathrm{ms}^{-2}\right]$
$[\mathrm{Pa}]$
$\left[\mathrm{Wb} \mathrm{m}^{-1}\right]$
$[\mathrm{m}]$
$[\mathrm{Pa}]$

$\left[W^{-1} m^{-1}\right.$

$[m]$

$[P a]$

$\left[W^{-1} m^{-1}\right.$

$[m]$

$[P a]$

\section{Continuous Complex Amplitudes}

$\begin{array}{ll}\mathcal{A} & \text { Magnetic vector potential } \\ \mathcal{H} & \text { Magnetic field vector } \\ \mathcal{B} & \text { Magnetic flux density vector } \\ \mathcal{E} & \text { Electric field vector } \\ \mathcal{D} & \text { Electric displacement field vector } \\ \mathcal{J} & \text { Electric current density vector } \\ \mathcal{U} & \text { Mechanical displacement vector }\end{array}$

Webers per metre

Amperes per metre

Tesla

Volts per metre

Coulombs per sq. metre

Amperes per sq. metre

Metres
$\left[W^{-1} m^{-1}\right.$

$\left[A m^{-1}\right]$

$[T]$

$\left[V m^{-1}\right]$

$\left[\mathrm{Cm}^{-2}\right]$

$\left[A m^{-2}\right]$

$[m]$ 


$\begin{array}{llll}\boldsymbol{P} & \text { Acoustic pressure scalar } & \text { Pascals } & {[\mathrm{Pa}]} \\ \boldsymbol{\delta}_{\mathcal{A}} & \text { Magnetic update field } & \text { Webers per metre } & {\left[\mathrm{Wb} \mathrm{m} \mathrm{m}^{-1}\right]} \\ \boldsymbol{\delta}_{\mathcal{U}} & \text { Mechanical update field } & \text { Metres } & {[\mathrm{m}]} \\ \boldsymbol{\delta}_{\mathcal{P}} & \text { Acoustic update field } & \text { Pascals } & {[\mathrm{Pa}]}\end{array}$

\section{Discrete Complex Amplitudes}

$\begin{array}{llll}\mathcal{A} & \text { Magnetic vector potential } & \text { Webers per metre } & {\left[\mathrm{Wb} \mathrm{m}^{-1}\right]} \\ \mathcal{U} & \text { Mechanical displacement vector } & \text { Metres } & {[\mathrm{m}]} \\ \mathcal{P} & \text { Acoustic pressure scalar } & \text { Pascals } & {[\mathrm{Pa}]} \\ \boldsymbol{\delta}_{\mathcal{A}} & \text { Magnetic update field } & \text { Webers per metre } & {\left[\mathrm{Wb} \mathrm{m} \mathrm{m}^{-1}\right]} \\ \boldsymbol{\delta}_{\mathcal{U}} & \text { Mechanical update field } & \text { Metres } & {[\mathrm{m}]} \\ \boldsymbol{\delta}_{\mathcal{P}} & \text { Acoustic update field } & \text { Pascals } & {[\mathrm{Pa}]}\end{array}$

\section{Continuous Test Functions}

$\boldsymbol{A}^{\delta} \quad$ Magnetic test function

$\boldsymbol{u}^{\delta} \quad$ Mechanical test function

$P^{\delta}$
Dimensionless

Dimensionless

Dimensionless

\section{Discrete Test Functions}

\section{$\mathbf{A}^{\delta}$ \\ $\mathbf{u}^{\delta}$ \\ $\mathrm{P}^{\delta}$}

$\varepsilon$

\section{$\sigma$}

$\boldsymbol{\sigma}^{e}$

$\boldsymbol{\sigma}^{m}$

$\mathcal{S}$

f

$f^{e}$

$T$
Magnetic test function

Mechanical test function

Acoustic test function
Dimensionless

Dimensionless

Dimensionless

\section{Stress, Strain and Force Fields}

Mechanical strain tensor

Total stress tensor

Maxwell stress tensor

Cauchy stress tensor

Linearised Maxwell stress tensor

Mechanical body force vector

Magnetic body force vector

Mechanical traction force vector
Dimensionless

Newtons per sq. metre

Newtons per sq. metre

Newtons per sq. metre

Newtons per sq. metre

Newtons per cbc. metres

Newtons per cbc. metres

Newton metres

$$
\begin{aligned}
& {\left[N m^{-2}\right]} \\
& {\left[N m^{-2}\right]} \\
& {\left[N m^{-2}\right]} \\
& {\left[N m^{-2}\right]} \\
& {\left[N m^{-3}\right]} \\
& {\left[N m^{-3}\right]} \\
& {\left[N m^{-2}\right]}
\end{aligned}
$$

\section{Problem parameters}

$\begin{array}{ll}t & \text { Time } \\ T & \text { Time period } \\ f & \text { Frequency } \\ d_{t} & \text { Thickness } \\ \omega & \text { Angular frequency } \\ k & \text { Wave number } \\ \alpha & \text { Length scale } \\ \text { TOL } & \text { Tolerance in residual for NR }\end{array}$

Seconds

Seconds

Hertz

Metres

Radians per second

Waves per metre

Metres

Dimensionless

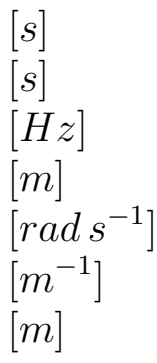




\section{Material parameters}

$\rho \quad$ Material density

$\rho_{V} \quad$ Volume charge density

$\gamma_{0} \quad$ Conductivity of free space

$\mu \quad$ Permeability of medium

$\mu_{0} \quad$ Permeability of free space

$\mu_{r} \quad$ Relative permeability

$\epsilon \quad$ Permittivity of medium

$\epsilon_{0} \quad$ Permittivity of free space

$\epsilon_{r} \quad$ Relative permittivity

$\lambda \quad$ Bulk modulus of solid

$\kappa \quad$ Bulk modulus of fluid

E Young's modulus

$G \quad$ Shear modulus

$\nu \quad$ Poisson's ratio $\gamma \quad$ Material conductivity
Kilograms per cbc. metre Coulombs per cbc. metre Siemens per metre Siemens per metre Henrys per metre Henrys per metre Dimensionless

Farads per metre

Farads per metre

Dimensionless

Newtons per sq. metre

Newtons per sq. metre

Newtons per sq. metre

Newtons per sq. metre

Dimensionless $\left[\mathrm{kg} \mathrm{m}^{-3}\right]$
$\left[\mathrm{Cm}^{-3}\right]$
$\left[\mathrm{S} \mathrm{m}^{-1}\right]$
$\left[\mathrm{S} \mathrm{m}^{-1}\right]$
$\left[\mathrm{H} \mathrm{m}^{-1}\right]$
$\left[\mathrm{H} \mathrm{m}^{-1}\right]$

$\left[F m^{-1}\right]$

$\left[F m^{-1}\right]$

$\left[N m^{-2}\right]$

$\left[N m^{-2}\right]$

$\left[\mathrm{Nm}^{-2}\right]$

$\left[N m^{-2}\right]$

\section{Spatial Discretisation Parameters}

$h \quad$ Maximum element length

Metres

$[m]$

Element order

Dimensionless

\section{Temporal Discretisation Parameters}

$\Delta t \quad$ Timestep size

$\alpha_{f} \quad \alpha$-method parameter

$\alpha_{m} \quad \alpha$-method parameter

$\varsigma \quad \alpha$-method parameter

$\beta \quad \alpha$-method parameter
Seconds

Dimensionless

Dimensionless

Dimensionless

Dimensionless

\section{Discrete System Terms}

$\begin{array}{lll}\mathbf{M} & \text { Mass matrix } & \text { Various } \\ \mathbf{C} & \text { Damping matrix } & \text { Various } \\ \mathbf{K} & \text { Stiffness matrix } & \text { Various } \\ \mathbf{R} & \text { Residual vector } & \text { Various }\end{array}$

\section{Extra Mathematical Terms}
$\boldsymbol{n} \quad$ Unit normal vector
Dimensionless
$\boldsymbol{t} \quad$ Unit traction vector
Dimensionless
$[\cdot]_{\partial \Omega_{c}}$
Boundary interface condition
Dimensionless
$\left.(\cdot)\right|_{\partial \Omega_{c}} ^{+}$ Contribution from outside domain
Dimensionless
$\left.(\cdot)\right|_{\partial \Omega_{c}} ^{-}$
Contribution from inside domain
Dimensionless

\section{Complete Domains and Boundaries}


$\Omega \quad$ Complete domain

$\Omega_{c} \quad$ Conducting domain

$\Omega_{n} \quad$ Non-conducting domain

$\operatorname{supp}\left(\boldsymbol{J}^{s}\right) \quad$ Magnetic coils domain

$\partial \Omega \quad$ Domain boundary

$\partial \Omega^{D} \quad$ Dirichlet boundary

$\partial \Omega^{N} \quad$ Neumann boundary

$\partial \Omega_{c} \quad$ Conductor boundary

$\partial \Omega_{n} \quad$ Non-conductor boundary
Cubic metres

Cubic metres

Cubic metres

Cubic metres

Square metres

Square metres

Square metres

Square metres

Square metres $\left[m^{3}\right]$

$\left[\mathrm{m}^{3}\right]$

$\left[\mathrm{m}^{3}\right]$

$\left[\mathrm{m}^{3}\right]$

$\left[m^{2}\right]$

$\left[\mathrm{m}^{2}\right]$

$\left[\mathrm{m}^{2}\right]$

$\left[m^{2}\right]$

$\left[\mathrm{m}^{2}\right]$

\section{Axisymmetric Domains and Boundaries}

$\Omega^{m} \quad$ Complete meridian domain

$\Omega_{c}^{m} \quad$ Conducting meridian domain

$\Omega_{n}^{m}$

$\partial \Omega^{m}$

$\partial \Omega^{m D}$

$\partial \Omega^{m N}$

$\partial \Omega_{c}^{m}$

$\partial \Omega_{n}^{m}$

$\boldsymbol{x}^{m}$

$\xi$
Non-conducting meridian domain

Meridian domain boundary

Meridian Dirichlet boundary

Meridian Neumann boundary

Conductor meridian boundary

Non-conductor meridian boundary $\boldsymbol{x} \quad$ General orthogonal coordinates

Meridian coordinates

Referential coordinates
Square metres

Square metres

Square metres

Metres

Metres

Metres

Metres

Metres $\left[m^{2}\right]$

$\left[\mathrm{m}^{2}\right]$

$\left[m^{2}\right]$

$[m]$

$[m]$

$[m]$

$[m]$

$[m]$

\section{Coordinate Systems}

Metres

Metres

Metres $[m]$

$[m]$

$[m]$ 


\section{Acronyms and Abbreviations}

$\begin{array}{ll}\text { ABC(s) } & \text { Absorbing Boundary Condition(s) } \\ \text { AC } & \text { Alternating Current } \\ \text { ANSYS } & \text { Analysis System } \\ \text { BC(s) } & \text { Boundary Condition(s) } \\ \text { BEM } & \text { Boundary Element Method } \\ \text { CAD } & \text { Computer Aided Design } \\ \text { CEM } & \text { Computational Electromagnetics } \\ \text { CG } & \text { Continuous Galerkin } \\ \text { COMSOL } & \text { Computer Solution } \\ \text { CT } & \text { Computed Tomography } \\ \text { DC } & \text { Direct Current } \\ \text { DG } & \text { Discontinuous Galerkin } \\ \text { DOF(s) } & \text { Degree(s) of Freedom } \\ \text { DFT } & \text { Discrete Fourier Transform } \\ \text { EM } & \text { Electromagnetics } \\ \text { EMF } & \text { Electro-Motive Force } \\ \text { EOM } & \text { Equation of Motion } \\ \text { EPI } & \text { Echo Planar Imaging } \\ \text { FBV } & \text { Floor Borne Vibrations } \\ \text { fMRI } & \text { functional Magnetic Resonance Imaging } \\ \text { FD } & \text { Finite Difference } \\ \text { FDTD } & \text { Finite Difference Time Domain } \\ \text { FE } & \text { Finite Elements } \\ \text { FEA } & \text { Finite Element Analysis } \\ \text { FEM } & \text { Finite Element Method } \\ \text { FFT } & \text { Fast Fourier Transform } \\ \text { FP } & \text { Fixed Point } \\ \text { FrD } & \text { Frequency Domain } \\ \text { FT } & \text { Fourier Transform } \\ \text { GC } & \text { Gradient Coil } \\ \text { GCI } & \text { Gradient Coil Interactions } \\ \text { HO } & \text { High / Higher Order } \\ \text { HR } & \text { High Resolution } \\ \text { LHS } & \text { Left Hand Side } \\ \text { LO } & \text { Low / Lower Order } \\ & \end{array}$




\begin{tabular}{ll} 
MATLAB & Matrix Laboratory \\
MC & Main Coil \\
MDOF & Multi Degrees of Freedom \\
MoM & Method of Moments \\
MRI & Magnetic Resonance Imaging \\
NACS & Numerical Analysis of Coupled Systems \\
NMR & Nuclear Magnetic Resonance \\
NR & Newton-Raphson \\
NURBS & Non Uniform Rational B-Splines \\
ODE & Ordinary Differential Equation \\
OVC & Outer Vacuum Chamber \\
PDE & Partial Differential Equation \\
PML & Perfectly Matched Layer \\
PUFEM & Partition of Unity Finite Element Method \\
QOI(s) & Quantity(ies) of Interest \\
ROI(s) & Region(s) of Interest \\
R\&D & Research and Development \\
RF & Radio Frequency \\
RHS & Right Hand Side \\
SC & Secondary Coils \\
SDOF & Single Degree of Freedom \\
SMT & Siemens Magnet Technology \\
SNS & Sum of Normal Stresses \\
TD & Time Domain \\
TSM & Tangent Stiffness Matrix \\
1D & One Dimension / Dimensional \\
2D & Two Dimension / Dimensional \\
3D & Three Dimension / Dimensional \\
& \\
\hline
\end{tabular}




\section{Part I}

\section{Preliminaries}





\section{Chapter 1}

\section{Introduction}

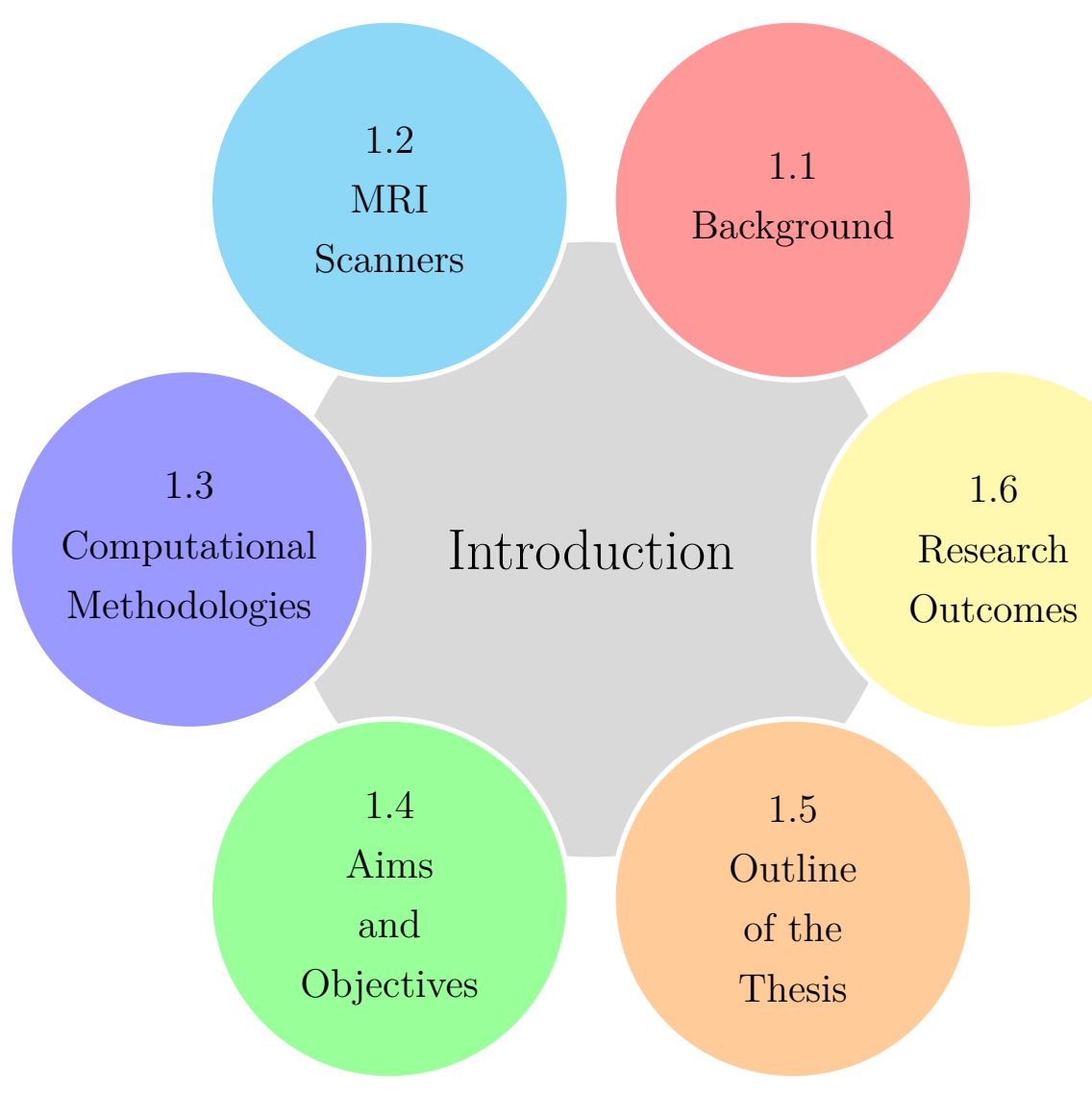

"The journey of a thousand miles begins with one step" 


\subsection{Background}

Since the discovery of Nuclear Magnetic Resonance (NMR) by Rabi of Columbia University in 1937 [200], for which he was awarded the 1944 Nobel Prize in physics, much research into the use of NMR for medical imaging has been accomplished. In 1952, Carr, a Harvard University student, produced a one-dimensional NMR image, reported in his PhD thesis [47]. By the end of the 1950's, Singer had used NMR to study the blood flow in living humans [231] and in the late 1960's reports of the first NMR measurements, of tissue in living humans by Ligon in 1967 [155] and animal subjects by Jackson and Langham in 1968 [117], were published.

In 1971 Physician Damadian published a paper in which he reported that tumours and normal tissue could be distinguished by means of NMR [65] and suggested that these differences could be used to diagnose cancer. In 1972 he applied for a patent, which describes the concept of NMR being used for medical imaging and illustrated major parts of an MRI machine [66]. He went on to build the first MRI scanner and achieved the first MRI scans of a human body [67].

Meanwhile, Lauterbur of the New York State University expanded on Carr's 1D NMR image generation to develop the first 2D and 3D MRI images, which he published in 1973 [149]. In the mid 1970s, Mansfield, a physicist at the University of Nottingham, developed the echo-planar imaging (EPI) technique as an improvement to Lauterbur's initial work, which used the projection-reconstruction method. This new technique would drastically improve the image reconstruction process and lead to scans taking seconds rather than hours and produce clearer images [166]. Both Lauterbur and Mansfield were later awarded the Nobel Prize (2003) in physiology/medicine for their discoveries in MRI.

In 1992, a technique known as functional MRI was introduced [142] which made possible cerebral/brain imaging of humans through analysis of brain activity. By the end of the $20^{\text {th }}$ century MRI scanners were commercially available and clinical MRI units had been introduced into most hospitals and research imaging units in research centres and universities. By 2010, approximately 20,000 MRI scanners were in use worldwide, with those numbers rising to approximately 29,000 in 2014 [9].

\subsection{MRI Scanners}

In recent years, MRI has become an increasingly popular method for medical imaging. Its capability of both neuroimaging [30] and non-invasive diagnosis of a range of 
medical ailments, such as tumours [137], damaged cartilage [170], fractures [45] and even the detection of multiple sclerosis [192] make it a desirable imaging technique for clinical use. MRI scanners differ from other medical imaging techniques, such as X-ray and computed tomography (CT), as they utilise strong magnetic fields for image generation and do not use harmful ionizing radiation. For this reason, they are the preferred imaging technique for children and patients requiring multiple imaging examinations. MRI imaging sequences (15-90 minutes [5]), however, tend to last longer than an X-ray or CT scan (10-20 minutes [4]). Nevertheless, MRI tends to outperform CT scans in terms of image resolution, particularly in soft tissue applications, and as such is often the preferred method when looking to accurately diagnose serious medical conditions such as cancer [29] and strokes [48], as well as in neuroimaging [131]. Figure 1.1 illustrates a comparison between images taken on a CT and MRI scanner. MRI in the imaging of bones has also been carried out [134] and offers positive results in comparison to CT [157].

MRI scanners utilise the principle of NMR to generate images. The human body is largely made up of water molecules (approximately two thirds) which contain hydrogen nuclei, consisting of a single proton. Inside the scanner, the patient is placed in a strong uniform uni-directional magnetic field, known as the $\boldsymbol{H}_{0}$ field $^{1}$, which aligns the hydrogen nuclei of the patient in the direction of $\boldsymbol{H}_{0}$. During an imaging sequence, a series of radio frequency $(\mathrm{RF})$ pulses are generated, perpendicular to the aligning magnetic field, which excite the protons and causes them to flip alignment [60]. When the RF signals are removed, the protons return to their state of alignment with $\boldsymbol{H}_{0}$, known as relaxation, and emit their own radiating RF signal. This signal can then be detected by conductive receiver coils, placed around the object being imaged, and the data encoded. To produce the images the emitted RF signal must be encoded in each Cartesian coordinate direction ( $x, y$ and $z)$. This is accomplished by applying a set of gradient magnetic fields, generated through resistive gradient coils, which result in a total magnetic field $\left(\boldsymbol{H}_{0} \text { plus gradient field }\right)^{2}$ that varies linearly in the direction of each applied gradient [44]. A Fourier transform can then be applied, to the received RF signal, to transform it into the spatial domain and generate an image [166].

Figure 1.2 shows a typical setup of a simplified cylindrical bore MRI scanner, consisting of five main components: the main coils (MC) of the magnet, the secondary

\footnotetext{
${ }^{1}$ The MRI background magnetic field is often referred to as the $\boldsymbol{B}_{0}$ field, measured in Teslas $[T]$. However, it is more correct to refer to the background magnetic field as $\boldsymbol{H}_{0}$ in our formulation.

${ }^{2}$ This total magnetic field is also affected by various effects that result from the magnetomechanical coupling, such as the eddy and Lorentz currents (see Appendix A).
} 


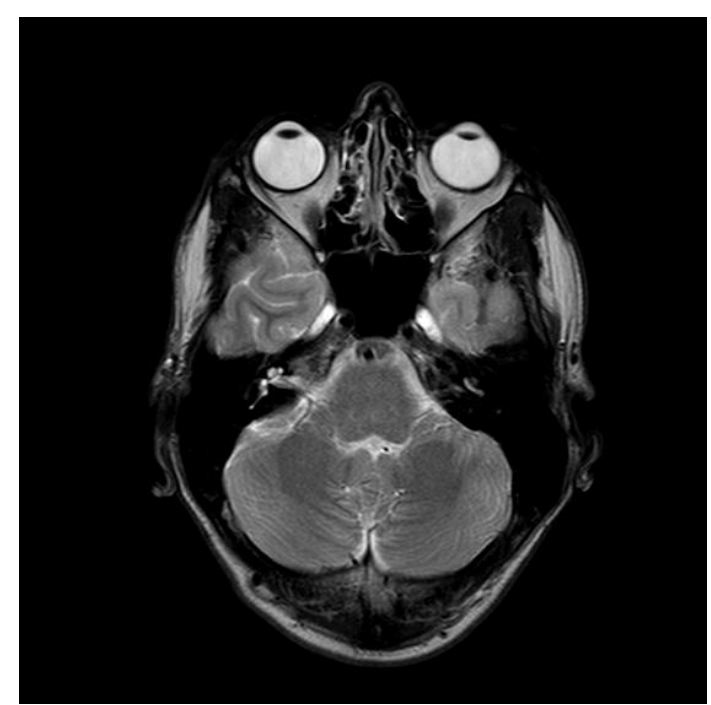

(a) MRI cross-section of eyes

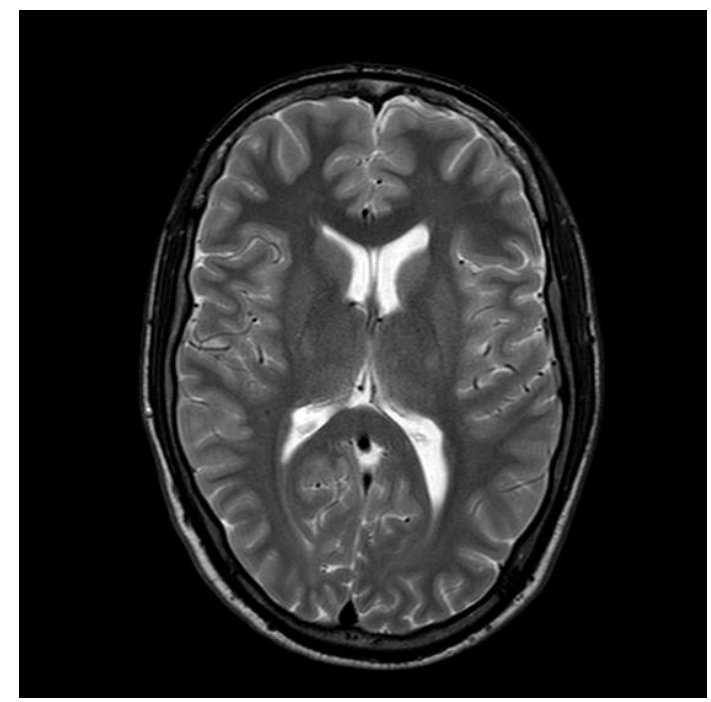

(c) MRI cross-section of brain

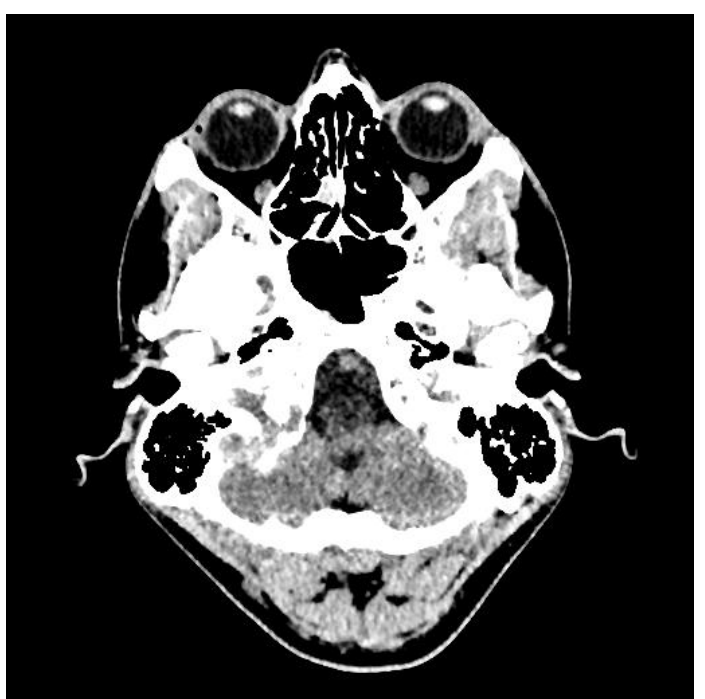

(b) CT cross-section of eyes

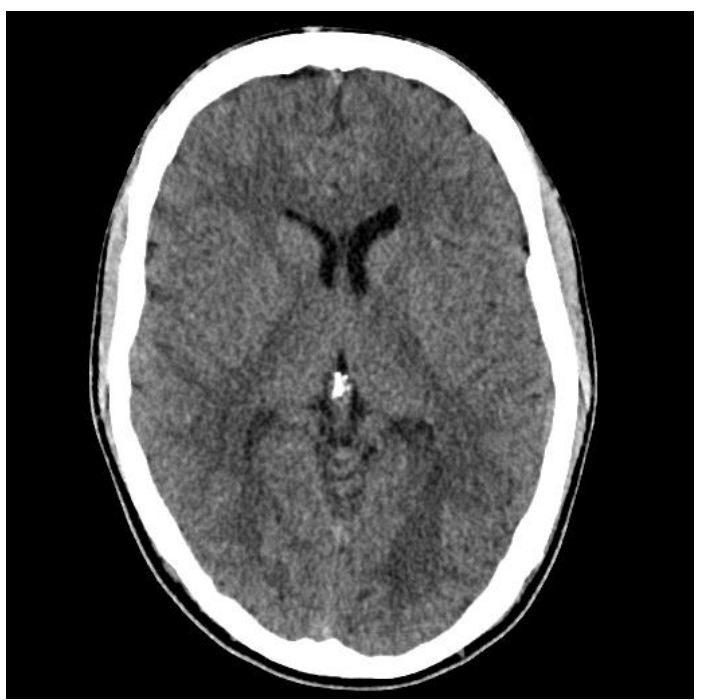

(d) CT cross-section of brain

Figure 1.1: Comparison between MRI (left) and CT (right) generated images of my brain and eyes! MRI scans typically allow for higher resolution imaging of soft matter, such as the brain and eyes, whilst CT performs better when imaging hard mater, such as the bone structures offering lower resolution of soft matter. These images are courtesy of the High Wycombe Radiology Department to whom I owe thanks for allowing me to add this personal touch to the Thesis. A very useful experience indeed. 


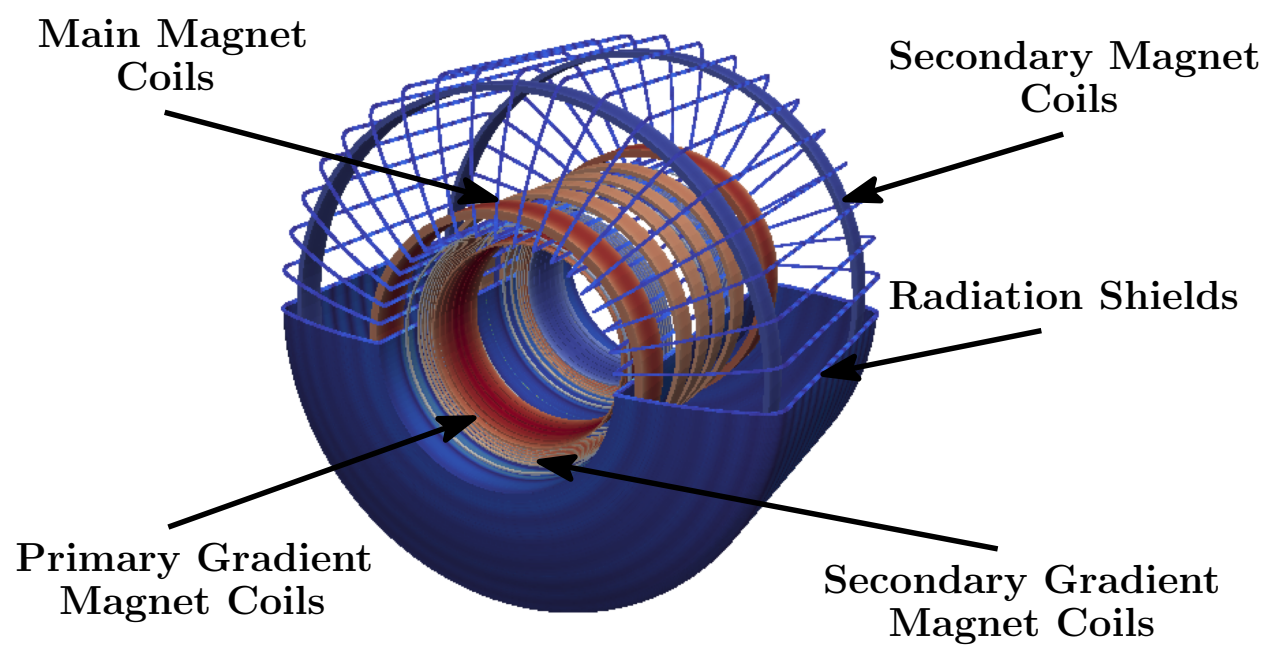

Figure 1.2: Primary components of a simplified clinical MRI scanner.

coils (SC), the cryostat (comprised of a set of radiation shields that encapsulate the liquid helium immersed magnetic coils) and sets of primary and secondary resistive gradient coils (GC). A set of main magnetic coils produce the strong uniform stationary magnetic field across the radial section of the scanner [122], required to align the protons of the hydrogen atoms in the patient in the axial direction. The secondary magnetic coils are used to minimise the large stray magnetic fields arising outside of the scanner. The cryostat consists of a set of metallic vessels in vacuum to maintain the supercooled magnet temperatures and shield the coils from radiation. Scanners typically consist of three shield levels: the Helium vessel, which contains the liquid helium for the superconducting magnet coils, the Inner Radiation shield, and the Outer Vacuum Chamber (OVC). A set of resistive coils inside the imaging volume, known as gradient coils (GC), produce pulsed magnetic field gradients which excite certain regions of the protons to generate an image of the patient. The secondary set of GC are used to shield the radiation shield from the gradient fields and minimise field penetration into the supercooled vessel.

Current commercially available MRI scanners are typically classified into two categories: open (C-shaped) and closed (cylindrical) bore. The most popular type of magnets available in MRI machines are the superconducting solenoidal magnets [14]. These magnets consist of conducting wire contained within a supercooled vessel liquid helium known as the cryostat, and are capable of generating the high field strengths required for high resolution (HR) imaging. The imaging resolution of the scanners is determined by the strength of the static magnetic field produced by the main magnet. The magnetic flux density of such fields is typically in the region 
Chapter 1. Introduction

of 1.5-3T (approximately 30,000-70,000 times the strength of the Earth's magnetic field) for clinical use [196, 229, 228], with some $7 \mathrm{~T}$ units in use for medical research applications $[249,129]$. Some open C-shaped MRI scanners which utilise permanent magnets to generate the static field are still available but are less common in current imaging units due to their relatively low field strengths of approximately $0.2-0.5 \mathrm{~T}$ [193]. However, they do offer the advantage of increased accessibility and comfort for the patient due to the open section around the imaging volume [193]. The gradient strengths of the magnetic fields generated by the gradient coils are much smaller than the strength of the main static field, which is typically in the region of $30-80 \times 10^{-3} \mathrm{~T} / \mathrm{m}[196,229,228]$.

\subsubsection{Physical Issues}

In the presence of conducting materials, such as those used in the manufacture of the metallic radiation shields of an MRI magnet's cryostat, transient magnetic fields give rise to eddy currents (see Appendix A for details) inside these conducting components, which in turn exerts Lorentz forces (see Appendix A for details) on the conductors. These forces generate electro-magneto-mechanical stresses, or Maxwell stresses [80], inside the conducting components which causes them to vibrate and deform. These vibrations then generate Lorentz currents (see Appendix A for details) which causes perturbations in the magnetic field. The vibrations of the conducting components also lead to perturbations of the surrounding air at the conductor surface, which causes acoustic pressure waves to form. In the case of multiple acoustic sources, such as multiple conducting components, the pressure waves can become incident waves to the conductors and reflect off of them, leading to further deformations of the conductors. The acoustic waves result in audible noise that is typically loudest inside the imaging volume, where the patient resides, leading to discomfort [197, 177, 171, 56].

These physical phenomena, present in MRI scanners during operation, lead to a fully coupled acousto-magneto-mechanical problem [26], which is complex in nature and makes the behaviour of the scanners difficult to predict. Some of the major problems facing design engineers today include: imaging artefacts, such as ghosting, decrease in the life span of components due to cyclical loading conditions, and uncomfortable conditions for the patient due to the noise from the mechanical vibrations. Minimising ghosting effects, keeping noise levels to acceptable limits and ensuring patient safety are key criteria for new MRI scanner designs.

Exterior vibrations, known as floor borne vibrations (FBV), caused by nearby 
vehicles and machinery, can also impact on the performance of an MRI scanner. However, these phenomena are considered outside the scope of this Thesis and as such will not be included in the analysis of MRI scanner systems.

\section{Imaging artefacts}

Imaging artefacts in MRI scans are non-physical artefacts that appear on an MRI scan image, during the imaging examination. These artefacts can appear in several different forms for a range of reasons: relatively small movements of the patient during an imaging examination [240, 99, 139], and vibrations caused by imaging sequences $[99,26]$. These artefacts can cause problems for the doctors during examination of the scans and diagnosis of the patient [240] and, in extreme cases, can lead to misdiagnosis of medical conditions [139]. However, the types of artefacts present in images are well documented and experience in recognising and ignoring these artefacts helps minimise this risk [236, 239]. Also, several techniques have recently been developed to remove these artefacts at the processing of images, which helps to improve the image quality $[84,82]$. An example of an imaging artefact present in an MRI scan is shown in Figure 1.3.

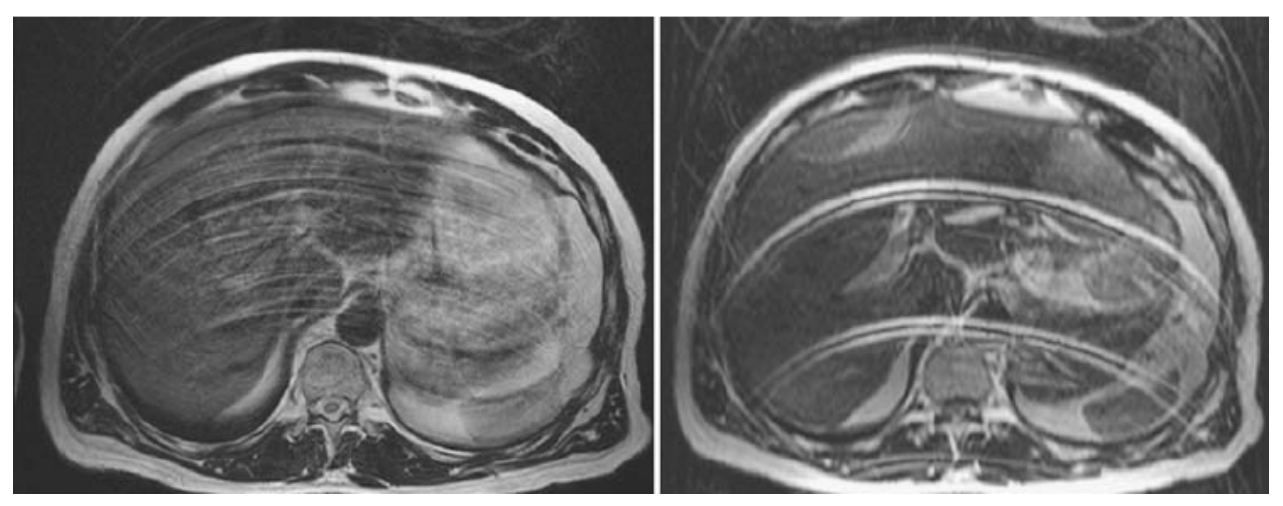

Figure 1.3: Examples of MRI images with imaging artefacts present, known as image ghosting, courtesy of [239]. The imaging artefacts are seen here as a series of images super positioned on top of one-another with an offset.

\section{Mechanical Vibrations}

MRI scanners experience mechanical vibrations during imaging sequences due to the pulsed magnetic fields required for image generation. The magnitude of these vibrations are very small, typically of the order of (microns) $\mu m$, due to the relatively high stiffness of scanner designs and small time varying magnetic fields. However, whilst small in magnitude these vibrations are cyclical, due to the nature of the 
pulsed gradient sequences, and as such can cause residual stresses to develop in the conducting components $[167,147]$ which will eventually cause components to fail.

\subsubsection{Clinical Effects to Patients}

Exposure to the strong magnetic fields, as well as the rapidly time varying magnetic fields from the RF and GC sequences and the corresponding high noise levels of MRI scanners, can have adverse effects on patients as well as the staff handling the scanner $[172,235]$.

\section{Strong Magnetic Fields}

In the presence of strong magnetic fields, such as those generated by the main magnet of an MRI scanner, any person moving around inside these fields, particularly inside or in the proximity of the scanner, must take caution, especially in the case of the higher strength fields with flux densities $>2 \mathrm{~T}$ [11]. The movement inside such strong fields effectively creates a varying field and can lead to inductions of eddy currents inside the person $[172,69]$. With such effects, people moving around scanners with such strong fields have reported feelings of nausea and sickness [212, 11].

\section{Alternating Magnetic Fields}

The imaging sequences used in MRI gradient coils differ depending on what part of body requires imaging, but generally the types of sequences used represent sharp trapezoidal type pulses, shown in Figure 1.4 for the z-gradient coil. These types pulses often involve sharp changes in the magnetic field which result in rapidly time varying magnetic fields [110]. The consistent exposure to time varying magnetic fields during a scan procedure can result in patients experiencing "tingling" sensations [213]. They can also induce eddy currents in regions of the patients, which can lead to potential health risks $[172,60]$.

\section{High noise levels}

During some scans, when patients have been exposed to high sound levels, adverse effects have also been reported [201, 177]. Current guidelines [98] state "there is little risk of a permanent threshold shift in hearing in those exposed to noise associated with MRI procedures on a one-off or occasional basis". It goes on to say that, in current low-frequency MRI scanners, clinically significant effects on hearing are unlikely in most subjects for noise levels below $85 \mathrm{~dB}(\mathrm{~A})$ of scans lasting less 
than an hour. Nevertheless, "IEC recommend that hearing protection should be used if equipment is capable of producing more than $99 \mathrm{~dB}(\mathrm{~A})$ " since such noise levels can still lead to patient discomfort [225], particularly given the tight space of the imaging bore. Many attempts have been made to measure the sound profiles of these harmful noise levels $[56,197]$ as well as proposing methods to reduce them [74], such as active noise cancelling technologies [171].

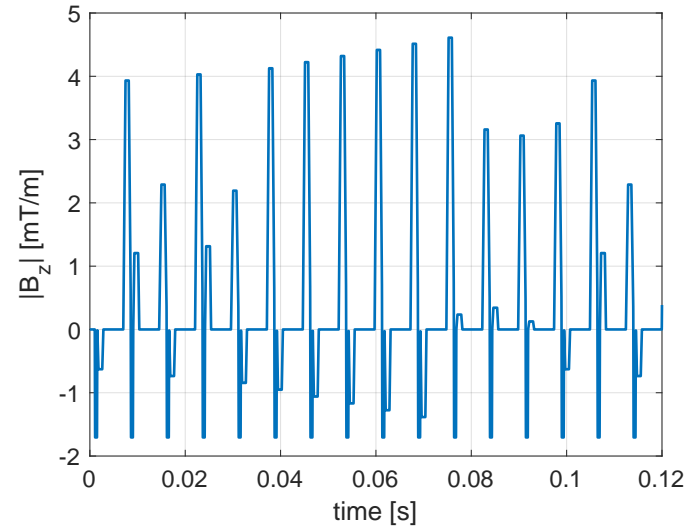

(a) Body

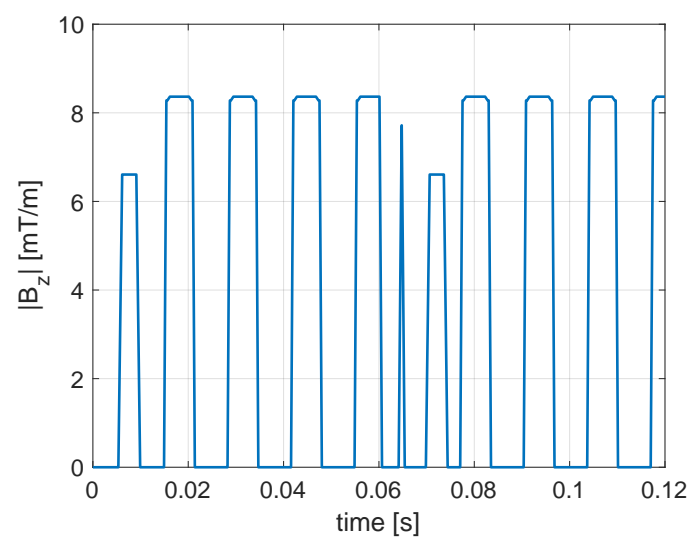

(c) Knee

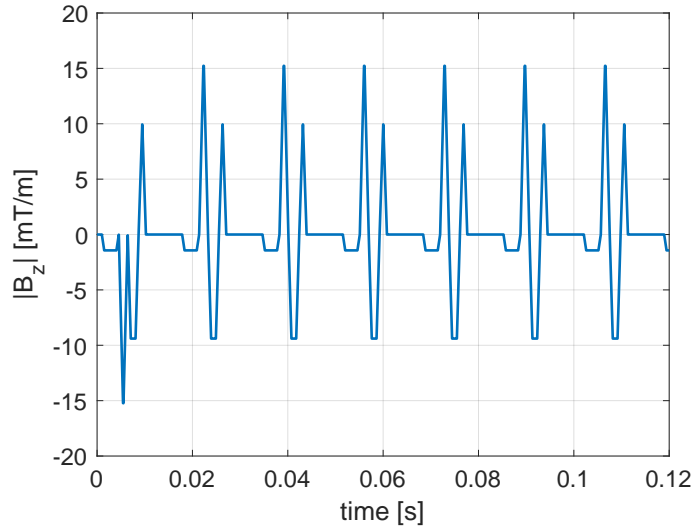

(b) Head

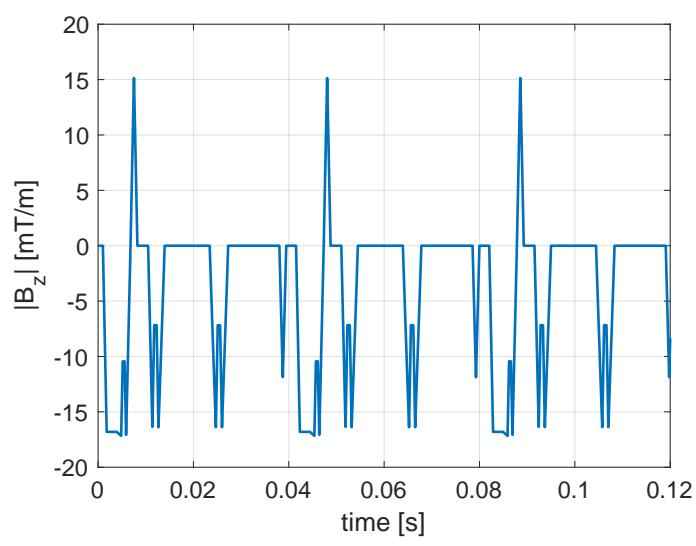

(d) Shoulder

Figure 1.4: Examples of gradient coil pulse sequences for imaging different parts of the body.

\subsubsection{Current Developments}

Due to the ever increasing need for more accurate diagnosis of medical conditions, such as cancer $[132,15]$, recent developments in MRI scanner design have seen increasing interest in: greater magnetic field strengths of the main magnet to improve the quality of MRI images, improvements to the transient gradient coil current signature to speed up scans [110] and minimisation of the stray field (that is the 
magnetic field experienced outside of the scanner) to reduce the impact of scanners in hospitals. Changes in bore geometry and reductions in audible noise have also been areas of interest in terms of patient comfort [62, 235]. An increasing topic of interest has also been in the reduction in cost of scanners, in terms of material usage, cost of materials and power consumption.

\section{Magnetic Field Strength}

Currently, the highest recorded magnetic field strengths used to image human subjects is $9.4 \mathrm{~T}$ [251], whilst in animal subjects field strengths of up to 21.1T [198] have been recorded. Recent advances in MRI design have resulted in clinical magnets for human patients of field strengths up to $12 \mathrm{~T}$ coming into production, which will allow for very high resolution images to be produced, compared with the current systems [211].

\section{Imaging Sequences}

Much research into the development of new imaging sequences to improve the performance of imaging scans has been carried out. GC and RF sequences that have specialist pulse profiles that reduce the rate of time change of the gradient field to reduce the vibrations and noise exerted by MRI scanners [208], with some capable of producing "almost silent" scans [110]. Also, some sequences significantly reduce the time required for imaging examinations [187].

\section{Magnetic Stray Field}

The magnetic stray field of MRI scanners, produced by the superconducting magnet, can cause problems for hospital installation as the level of shielding required in the installation of the room can be significant. Some recent developments have resulted in active shielding of the main magnet where a set of magnetic coils are built on the outside of the primary coils which oppose the main magnetic field to minimise the stray field [108]. Also scanners are now being developed with active gradient coil shielding technology, where a second set of opposing gradient coils are placed between the primary gradient coils and the OVC [226], to minimise eddy currents generated in the radiations shields [75].

However, these developments in MRI also introduce new challenges since image quality must also be balanced with the needs of clinical efficiency and patient safety [98]. 


\subsection{Computational Methodologies}

Due to the recent and increasing interest in the design of cheaper (reduced amount of material, decreased power consumption) and more advanced MRI systems (capable of producing stronger magnetic fields) [62], the prediction of the physical behaviours of such scanners has become a desirable area of research. Experimental prototyping and testing of such devices is expensive and, when requiring multiple designs to be tested, is not practical due to the lead times and cost to build them. As such, there is considerable industrial interest in the development of accurate and efficient computational solvers which can aid in the design process to reduce Research and Development (R\&D) costs and lead times. However, the development of such tools is challenging due to the complexity that results from the coupled physics of electromagnetism, mechanics and acoustics and the non-trivial task of accurately modelling the complex field behaviour.

\subsubsection{Computational Tools for Single Physics Problems}

With complex problems, such as that of MRI scanners, analytical solutions are unobtainable and so alternative methods for computing solutions to these problems are required. In the context of the three fields: electromagnetics, mechanics (or elasticity) and acoustics, a number of alternative computational methods for solving these problems on complex geometries are discussed.

\section{Approaches for Electromagnetics}

In the field of electromagnetics, many real-world applications, for example the prediction of electromagnetic scattering for radar detection and modelling of electromagnetic radiation from devices such as MRI scanners, offer challenges in the calculation of this behaviour. Many attempts to model these applications have been performed using a range of computational techniques, including both integral and differential equation solvers. The boundary element method (BEM) [106, 89], fast multipole method (FMM) [180, 59] and method of moments (MoM) [12] are all surface based methods that require only discretisations of the surface. These types of methods are good for dealing with problems in free space (e.g. scattering in unbounded domains which will be discussed shortly), but not with handling problems with multiple materials.

On the contrary, finite difference (FD) [246], finite volume (FV) [179] and finite 
element (FE) [244] methods are all volume based methods that require the discretisation of the complete volume. These types of methods are good for handling problems with different materials, but less efficient for problems in unbounded domains unless special treatment is used (see later subsection on unbounded domains). All three methods have advantages and disadvantages over the other methods. The FDM has been widely used in many EM applications $[245,162,164]$ due to its simplicity both in understanding and implementing the method. However, the method poses problems when dealing with complex geometries, as the method commonly uses a regular grid. Methods which utilise non-regualr grids have been developed [86], however they require more advanced treatment. The FV and FE methods, on the other hand, are easily applicable to solving problems on irregular grids. The FV method requires only the computation of fluxes across cells and so makes it very computationally efficient for application to electromagnetic problems [13, 104, 223, 105, 165]. Both the Finite Element and Finite Volume allow for higher order approximations to be used to improve the accuracy of the schemes[244, 127], however the application has been more widely associated with the FEM. This application of higher order approximations also permits the use of curved elements to better approximate the geometry for a given polynomial order $[221,195]$ and, through the use of NURBS functions, can represent the exact CAD geometry [219, 220].

In terms of $\mathrm{FE}$ solvers for electromagnetic problems, a number of different formulations for the governing Maxwell equation system exist for a range of applications. For high frequency electromagnetic scattering problems there are possibilities to reduce this system to a Helmholtz wave equation in 2D, in the case of a non-conducting region [151]. In electromagnetic scattering, a major challenge is overcoming problems with pollution (dispersion) error in wave propagation. In terms of low frequency electromagnetics, such as electromagnetic radiation in low frequency devices such as MRI scanners, the eddy current approximation of the full Maxwell system can be adopted [216, 113, 37, 141, 206], which requires special treatment through gauging conditions on the equations [153]. In the eddy current approximation, a major challenge lies in the accurate resolution of the small skin depths in conductors due to higher frequency excitations.

High order finite elements are important in electromagnetics for resolving small skin depths in eddy current problems $[153,217]$. They are needed to avoid problems with pollution (dispersion) error in wave propagation ${ }^{3}$ [16] (This will be revisited in Approaches for Acoustics). Moreover, with the correct combination of $h$ - and

\footnotetext{
${ }^{3}$ Wave-type shape functions, as presented in $[145,146]$, can also be used to reduce dispersion effects in 2D electromagnetic scattering problems.
} 
$p$ - refinement, exponential convergence rates, rather than the algebraic rate of convergence of $h$-refinement, can be achieved. The use of standard nodal-type finite elements in electromagnetic problems can lead to spurious oscillations in the solution $[148,256]$. Also, the electromagnetic field requires tangential continuity across the elements. For these reasons, an alternative set of finite element basis functions have been proposed [183, 184, 39], where the degrees of freedom are associated with the edges of the element, known as (Nédélec) edge elements.

\section{Approaches for Elasticity}

When solving the elasticity equation for the mechanical field a series of possible formulations are available: displacement-based, stress-based and mixed formulations. In a displacement-based formulation, the elasticity equations are expressed purely in terms of the displacements, where the stress and strain tensors are also reformulated $[26,61]$. This formulation allows for the direct application of the displacements in the case of boundary conditions, as well as any traction forces acting externally. The stresses can be obtained a posteriori through the stress-displacement relations. In a stress-based formulation the elasticity equations are reformulated for the stress [230]. In this formulation a series of extra constraints must also be applied in order to obtain a sufficient number of equations for the number of unknowns [174]. The displacements can then be solved a posteriori using the stress-displacement relations. Mixed formulations work by solving for all of the variables in the formulation directly, treating both displacements and stresses as unknowns [189]. A typical approach for this is to solve for both the displacements and pressure ${ }^{4}$ simultaneously, which provides benefits in overcoming volumetric locking [191].

When dealing with the elasticity equations, one problem posed by the displacement based formulation and low order finite elements is a phenomenon known as volumetric locking. This phenomenon occurs as the Poisson's ratio tends to 0.5 (incompressible material) and results in the calculated displacements being (sometimes grossly) under-predicted $[243,52]$. Even worse is that the situation does not improve with an increase in mesh density ( $h$-refinement). This problem can be overcome through the use of mixed formulations [109, 55, 207, 144]. However, this increases the number of degrees of freedom significantly. Another possibility for overcoming this problem is through reduced integration of the stiffness matrix $[115,72]$. However, this results in reductions in accuracy. A final method for overcoming this

\footnotetext{
$(2.25)$.

${ }^{4}$ Here, the pressure is related to the displacements through the divergence, as seen similarly in
} 
phenomenon is through the use of higher order finite elements [243, 52, 255, 109]. Whilst increasing the number of degrees of freedom, $p$-refinement also offers significant increase in accuracy over the low order methods.

\section{Approaches for Acoustics}

In the field of computational acoustics, the primary concern is with the computation of acoustic waves that propagate through domains from some excitation source. Typically, this concerns the solutions to the scalar Helmholtz wave equation, where the wavelengths of the resulting waves depend on the frequency of the excitation source. For significantly high frequency excitations, this results in small wavelengths that can lead to insufficient accuracy in the solution due to an effect known as $n u$ merical dispersion ${ }^{5}$. In some cases, this effect creates not only poor quantitative approximations but also approximations that are not even qualitatively representative [70].

A number of methods exist to overcome the effects of dispersion in solving wave behaviour. Standard techniques would involve refining the mesh, through $h$-refinement, to allow for the wavelengths to be sufficiently resolved. As with the other fields, an alternative approach would be to use high order finite elements, $p$-refinement, to better approximate the underlying wave functions of the problem $[16,218]$. Another approach in overcoming the difficulties of resolving small wavelengths has been to use alternative sets of basis functions that better approximate the wave behaviour of acoustic type problems $[145,146]$ by embedding fundamental solutions to the Helmholtz equation as trial functions. These basis functions represent plane waves of differing wavelengths that propagate in different directions within the element and rely on the partition of unity finite element method (PUFEM) [76, 176].

\section{Unbounded Domains}

Accurate simulation of the electromagnetic and acoustic effects, in unbounded domains, requires special treatment of the fields at the artificial truncated far field boundary. Techniques such as perfectly matched layers (PML), FEM-BEM coupling, infinite elements and absorbing boundary conditions (ABCs) have been developed for this, see e.g. [247, 95] for recent reviews. The boundary element method (BEM) $[87,50]$ is popular for handling acoustic wave propagation and EM field decay prob-

\footnotetext{
${ }^{5}$ Dispersion refers to the effect where the numerical approximation fails to correctly propagate the speed of the wave and results in a non-physical phase-shift in the numerical approximation.
} 
lems, when coupled with FEM, as the discretisation of these schemes is done directly on the boundaries and avoids the need for indirect approximation of an unbounded domain. However, the FEM-BEM approach fails to preserve the sparsity patterns of the matrices available with standard FEM and their storage and inversion becomes prohibitive, particularly in 3D cases $[163,182]$, considerably increasing the computational cost. In terms of an FE discretisation, PMLs are described by an artificial layer of FEs at the boundary of the computational region which absorb outgoing waves and reduce artificial reflections at the boundary [31]. Whilst their ability to minimise artificial reflections depends largely on the computational quality of the mesh, they are capable of reducing artificial reflections on the boundary in the most general of cases. Infinite elements are described by a layer of FEs at the far field which utilise special shape functions to map the outer edge to infinity [34]. Again, their ability to accurately predict the EM field at the truncated boundary depends on the computational quality of the solution ${ }^{6}$. Nevertheless, they provide a general method for handling field decays in truncated domains. Furthermore, in the computation of unbounded domains using PMLs [175] and infinite elements [21, 258, 36], high order FEs have also been shown to offer superior performance compared to low order approaches.

\subsubsection{Coupled Approaches}

When dealing with coupled systems of governing equations, where the coupling is either volumetric or through transmission conditions, iterative solution procedures are often required to resolve the non-linearity of the problem. There exists two primary groups of techniques used to overcome these issues, partitioned and monolithic solution procedures.

As will be seen in later chapters, this is the case for the analysis of MRI scanners, which will combine the need to solve electromagnetics, mechanics and acoustics together on unbounded domains.

\section{Partitioned Solvers}

In order to resolve the non-linearities and coupling of non-linear multifield problems, many approaches utilise partitioned fixed point solution procedures. These methods

\footnotetext{
${ }^{6}$ Depending on the behaviour of the field decay, both static and wave type decay behaviour can be treated by using different types of infinite elements, as seen in [35] and [21] respectively. This is important for distinguishing between the treatment of the full Maxwell system and the Eddy current approximation.
} 


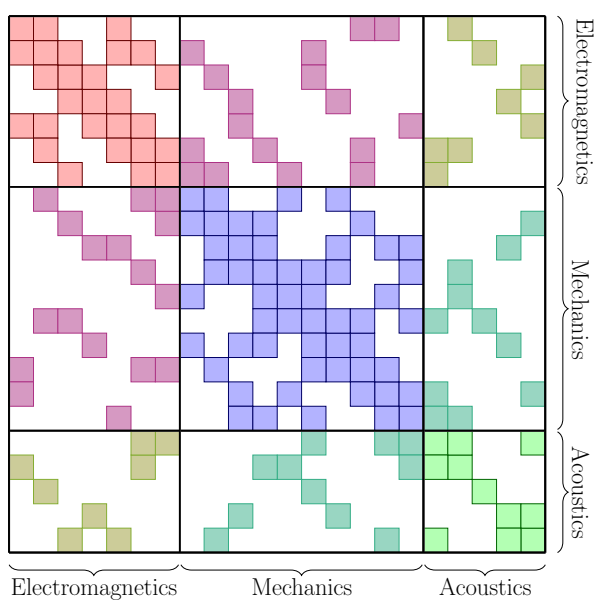

(a) Monolithic Solver

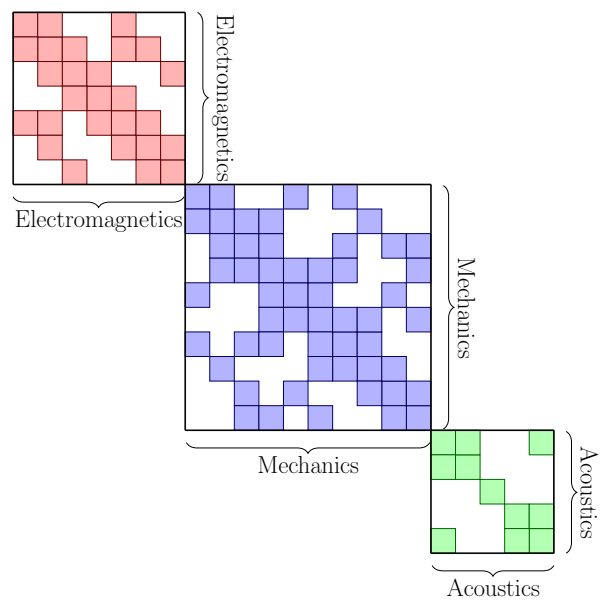

(b) Partitioned Solver

Figure 1.5: Representation of the tangent stiffness matrices of monolithic and partitioned solvers. In the monolithic solver, the pure field and cross derivative blocks (the coupled blocks) are constructed and the tangent stiffness matrix is solved as a whole. In the partitioned solver the tangent stiffness matrix contains only the pure field blocks of the individual physics and can be solved individually as separate systems, which rely on forcing terms to compute coupling contributions.

work by solving each field individually and then compute the coupling mechanisms via source terms that are passed between the fields. The solution is then recomputed and this process continues until a convergence criteria is met. Applications of this type of scheme to multifield problems have been considered [123, 125, 85, 215], with more specific applications in the magneto-mechanical coupling of MRI scanners [202, 150] and acoustic magneto-mechanical coupling as seen in [203].

Partitioned fixed point type schemes are relatively easy to implement when coupling individual black box solvers together as only the calculation of the coupling forces/sources are required between the physics. This makes them very customisable solution procedures in terms of solving coupled problems and are therefore used by many commercial multiphysics codes, such as COMSOL [7]. However, these approaches are typically less robust than the Newton-Raphson (NR) method (see monolithic solvers below) and are very sensitive to guesses in the fields [209, 128, 33]. Furthermore, they typically require a large number of iterations to solve, which can be significantly decreased by the quadratically converging Newton-Raphson scheme, particularly in the case of non-linear multifield problems.

An example of a typical iterative fixed point procedure for an acousto-magnetomechanical system is summarised in the flowchart illustrated in Figure 1.6a. 


\section{Monolithic Solvers}

Monolithic Newton-Raphson solution procedures are often used when dealing with non-linear coupled systems of equations. NR procedures are gradient based methods and, as such, require a linearisation of the system of non-linear equations to obtain the tangent stiffness operator [38]. Complete linearisation of the system results in quadratic convergence of the solution, reducing the total number of iterations required. Applications of this type of scheme to multifield problems have been extensively considered [93, 26, 121, 194, 209, 199].

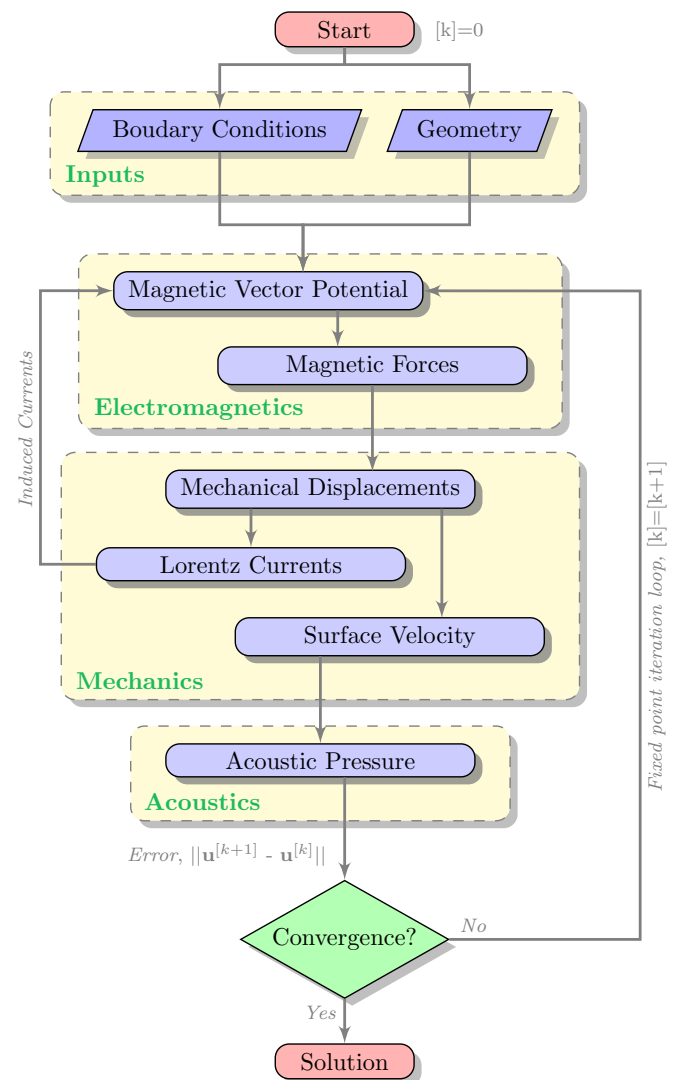

(a) Fixed Point Scheme

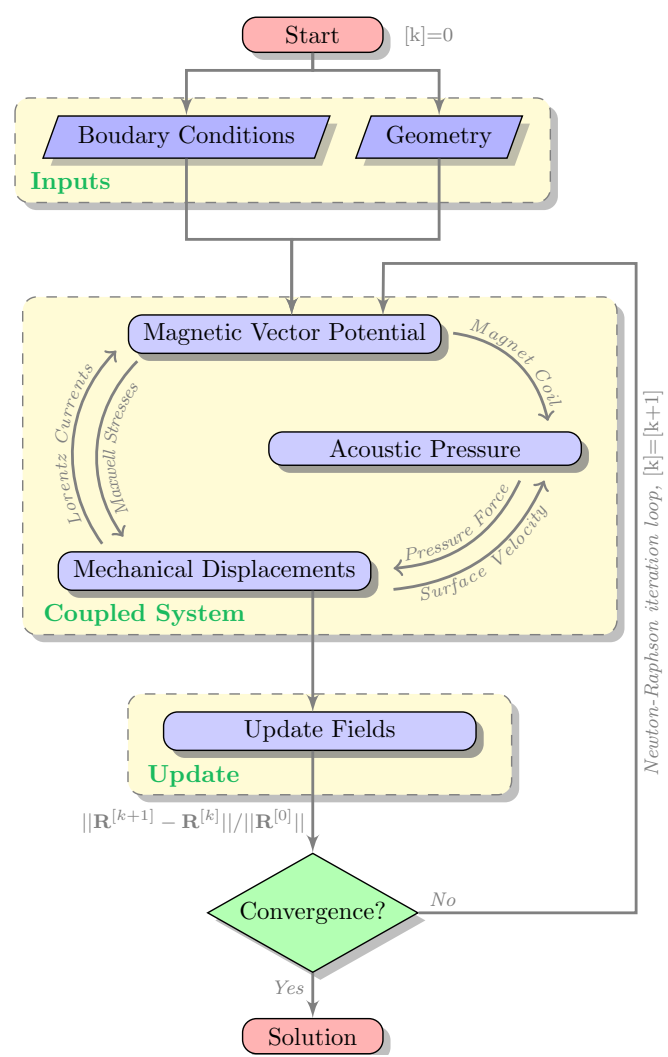

(b) Newton-Raphson Scheme

Figure 1.6: Algorithms for acousto-magneto-mechanical methodologies using both: monolithic (NR) and partitioned (FP) iterative solvers to compute sourcing terms between the physical fields and the system tangent stiffness matrix.

NR procedures offer a more robust solution procedure as they more accurately calculate the coupling between the schemes and are also less sensitive to initial guesses, although still require for the guess to be sufficiently close to the root. However, in terms of dealing with black box solvers, computing the cross derivative terms in the linearisation becomes far more challenging due to the need for specific 
information within the code. For this reason, NR procedures are far more commonly used in dedicated design tools, where the types of problems considered are known $a$ priori to its design.

An example of a typical iterative Newton-Rapshon procedure for an acoustomagneto-mechanical system is summarised in the flowchart illustrated in Figure $1.6 \mathrm{~b}$.

\subsubsection{Applications to MRI Scanners}

Many single field attempts to analyse the magnetic field of MRI scanners have been published; finite difference time domain methods for the calculation of eddy currents arising from transient gradient coils in MRI scanners [94, 248, 158], with analysis also of the effects on the human body [257, 159, 160], and methods for 'fast' analysis and design of the MRI coils [136, 138]. A number of works have also been published which focus on the analysis of superconducting solenoids [168], as well as on full MRI scanners $[154,51]$ and also consider the structural design of higher field scanners [64]. Acoustic effects in MRI scanners have also been investigated $[224,173]$, with attempts to design noise reduction systems [252, 253, 208] and even analyse the acoustic effects in the human head [156]. Others have attempted to model these complex physical effects to aid the design of the MRI magnetic coils $[101,210]$, to analyse the plane strain effects on superconducting solenoids [168] and the effects of the magnetic field exposure on the patient [63].

The simulation of MRI scanners also builds on the expanding literature devoted to magneto-mechanics and coupled problems including FEM-BEM coupling [125, 141], magneto-mechanical damping machines [40], magneto-mechanical effects on material parameters [133], enhanced basis functions for magneto-mechanical coupling [107] and strongly coupled systems [204]. More specifically, attempts to analyse the magneto-mechanical coupling in MRI systems have been considered; with the modelling of axisymmetric superconducting solenoids in self magnetic fields [101] and efficient low order FE solvers for magneto-mechanical coupling in [202], based on the FEM-BEM program CAPA [126], with extensions also to include acoustic effects [203]. This work utilises the approach by Kaltenbacher [124], which employs a layer of elements adjacent to the conductor, for the direct calculation of electromagnetic body forces to generate a weakly coupled algorithm. More recent works $[26,150,93,120,121]$ have utilised an alternative approach, which avoids the direct computation of electromotive forces and instead works directly with a (physically motivated) Maxwell stress tensor. This work permits a more rigorous treatment of 
the coupling through a complete linearisation of the coupled system [26, 25].

\subsubsection{Commercial Alternatives}

Previously, there has also been great interest in applying commercial multi-physics FE(M) packages, such as COMSOL [7], NACS [8] and even the coupling of software packages like Ansys [6] coupled with Opera [10], to simulate the coupled nature of MRI scanners [26]. Although commercial codes provide an efficient environment for many problems, significant interest lies in the development of dedicated low-cost industrial design tools. Such a tool should not only produce accurate predictions of the acousto-magneto-mechanical coupling mechanisms in MRI scanners, but also be able to accurately resolve the potentially small skin depths (see Appendix A) in conducting components, as well as the propagation of acoustic waves in free space [26]. The aforementioned commercial codes have historically been designed with low order FE discretisations in mind which require denser meshes ( $h$-refinement) to converge to accurate solutions [259], particularly at higher frequency excitations. These packages have, however, recently seen an introduction of higher order methods to solve these issues. Nevertheless, the high order technologies applied to these solvers is not always optimal and can lead to ill conditioned matrices with a large number of iterations. Hence using these tools is not always practical for specific multi-scale coupled problems.

\subsection{Aims and Objectives}

As described above, there is an ever increasing need for the more accurate prediction of MRI scanner behaviour under the current operational limits of the magnetic field strengths of both the static and gradient field. With this in mind, the main aim of this Thesis is to develop a new computational framework for the rapid, accurate and robust solution to non-linear coupled acousto-magneto-mechanical problems that arise in MRI scanners, named the linearised approach. In particular, the desired outcome of this work is the development of a computational analysis tool for the fast and accurate simulation of a number of design configurations of MRI scanners that can be used to enhance the analysis within the conceptual design cycle of MRI scanners. By presenting also the fully non-linear approach to solving the coupled system, the two approaches are to be compared to determine the accuracy of the model in providing useful design insight into challenging problems that can be clinically applicable. 
The aim of this Thesis will be achieved through the following objectives:

1. "To accurately describe the individual physical fields and the coupling mechanisms associated with MRI scanners."

2. "To develop an efficient novel solution procedure, named the linearised approach, through a rigorous linearisation of the non-linear system of equations."

3. "To develop the complete non-linear approach to solving the coupled system of equations."

4. "To provide an efficient computational methodology for handling the solution to the coupled problem of MRI scanners using both approaches."

5. "To determine a series of theoretical measures that can be used to measure the non-linearity of the coupled problem in MRI scanners."

6. "To determine the accuracy of the linearised approach across the full operating range of MRI scanners."

In order to achieve these aims and objectives, the research is split into a number of stages, which are described in the outline of this Thesis.

\subsection{Outline of the Thesis}

This Thesis comprises of 9 chapters split into 7 parts and is supported by 7 appendices, as follows:

\section{- PART II - \\ General Formulation of the problem}

- Chapter 2 presents the governing equations of the electromagnetic, mechanical and acoustic fields present in an MRI scanner during an imaging sequence. The corresponding coupling mechanisms between the fields are derived through both body and transmission terms and the farfield boundary conditions of the fields are also presented. The governing equations are reformulated, through a series of physically motivated assumptions, to obtain the fully coupled transient and static acousto-magneto-mechanical transmission problem.

- PART III -

Linearised Approach 
- Chapter 3 presents the rigorous linearisation of the fully coupled acoustomagneto-mechanical system through the use of a Newton-Raphson methodology. The weighted residual form of the coupled transmission problems, in Chapter 2, is first presented and the corresponding directional derivatives with respect to the solution fields are then taken to permit a monolithic description of the equation system. A novel technique for the linearisation of the system is then posed, where the linearisation of the fields is performed about the static fields, to obtain a new approach named the linearised approach. This linearisation is motivated by the fact that the static excitation of MRI scanners is orders of magnitude larger than the transient. Based on this linearisation the linearised approach can be recast in terms of a time harmonic (frequency domain) formulation to avoid the need for temporal integration techniques and improve the efficiency.

- Chapter 4 presents the computational treatment of the newly proposed linearised approach. An axisymmetric description of the system is first presented, where the solution fields are suitably scaled in order to permit the use of standard finite element basis functions. The computational treatment of the artificially truncated outer boundary is considered, where the decay conditions in Chapter 2 must be well approximated, to allow for accurate simulations of unbounded problems. Finally, the spatial discretisation through the use of high order hierarchic (modal) hp-finite elements is presented for both the static and transient coupled transmission problems, where the technical details of the FEM are recalled.

- Chapter 5 presents a series of academic and industrial benchmark numerical examples to test the accuracy of the linearised approach. First, three simple single field problems are presented in order to determine whether the $h p$-finite elements converge with the expected rates as predicted in the theory [127, 244]. The new approach is then applied to a series of coupled multi-physics problems to determine the accuracy of the newly proposed scheme compared with other coupled solvers.

\section{- PART IV - Non-Linear Approach}

- Chapter $\boldsymbol{6}$ briefly discusses the typical operating ranges of MRI scanners during clinical use. This Chapter then revisits the linearisation, performed 
in Chapter 3, in order to treat the non-linear system and provides a monolithic NR solution procedure for this, named the non-linear approach. Both approaches are then recast in terms of the stiffness, damping and mass contributions which will be used to form the corresponding linearised system matrices, once discretised. A comparison of the energy associated with the two approaches is also performed in order to derive a set of rigorous bounds that are used to define a series of theoretical measures that determine the non-linearity of the problem. Finally a simplified model, which relates these measures to industrial data, is presented based on the Biot-Savart law [100].

- Chapter 7 presents the generalised $\alpha$ temporal discretisation scheme [57], chosen for the numerical solution of both the linearised and non-linear approaches in the time domain. The linearised approach results in a system where the stiffness, mass and damping matrices do not depend on NR iteration or time. Thus they do not require recomputing at each NR step, resulting in a more efficient system. A generalised solution procedure for the solution of the coupled system using both approaches is summarised.

- Chapter 8 presents two MRI-type numerical examples: the first a simplified scanner geometry and the second a realistic MRI scanner resembling current scanners in operation. The possible range of operating conditions of the theoretical measures is derived for each problem using the simplified model presented in Chapter 6. A series of numerical tests are first run to determine suitable discretisation parameters to use for the complete problems. Then, a series of computational studies are performed to analyse the non-linearity across a range of different operating conditions to determine: 1) How good is the agreement between the computationally efficient linearised approach compared with the intensive non-linear approach?; and 2) Over what range of MRI operating conditions can the linearised approach be expected to provide acceptable results for MRI scanner design?

\section{- PART V - \\ Conclusions and Further Work}

- Chapter 9 presents a brief overview of the work carried out in this Thesis and presents the findings of each element involved. A series of recommendations of work to be carried out in the future are also included that will build on the underlying concepts provided within this Thesis. 


\section{- PART VI -}

\section{Appendices}

- Appendix $\boldsymbol{A}$ explains the key physical phenomena in the magnetic and mechanical fields that lead to the magneto-mechanical coupling in MRI scanners.

- Appendix $\boldsymbol{B}$ derives the continuity of the magnetic, mechanical and acoustic fields for problems considering domains consisting of multiple subdomains of different materials across the interfaces. These conditions are known as the interface (or jump) conditions.

- Appendix $\boldsymbol{C}$ derives conditions that must be satisfied in order for the eddy current model of the electromagnetic field to be valid.

- Appendix $\boldsymbol{D}$ derives the farfield conditions of the electromagnetic and acoustic fields that arise from solving problems in unbounded domains.

- Appendix $\boldsymbol{E}$ presents the high order hierarchic $H^{1}(\Omega)$ conforming finite element shape functions on both the triangular and quadrilateral elements used in this Thesis.

- Appendix $\boldsymbol{F}$ presents the perturbed magnetic eddy current problem for the application of infinite elements, where the field on the outer boundary decays to zero.

- Appendix $\boldsymbol{G}$ describes a range of algorithmic procedures that may be used to improve the computational efficiency of the implementation of the linearised approach in both a time harmonic and time dependant description.

\section{- PART VII -}

\section{References}

\subsection{Research Outcomes}

The following lists the research outputs of the work carried out as part of this Thesis in terms of the journal publications, conference presentations and research posters: 


\subsubsection{Journal Publications}

- S. Bagwell, P.D. Ledger, A.J. Gil and M. Mallett, "Transient solutions to nonlinear acousto-magneto-mechanical coupling for axisymmetric MRI scanner design", International Journal for Numerical Methods in Engineering, DOI: 10.1002/nme.5802, 2018.

- S. Bagwell, P.D. Ledger, A.J. Gil, M. Mallett and M. Kruip, "A linearised $h p$-finite element framework for acousto-magneto-mechanical coupling in axisymmetric MRI scanners", International Journal for Numerical Methods in Engineering, 112(10):1323-1352, 2017.

- P.D. Ledger, A.J. Gil, R. Poya, M. Kruip, I. Wilkinson and S. Bagwell, "Solution of an industrially relevant coupled magneto-mechanical problem set on an axisymmetric domain", Applied Mathematical Modelling, 40:1959-1971, 2016 .

\subsubsection{Conference Presentations}

- S. Bagwell, P.D. Ledger, A.J. Gil, "Non-linear Transient Simulations of Coupled Acousto-Magneto-Mechanical Systems in MRI Scanners", VII International Conference on Coupled Problems in Science and Engineering, Rhodes, Greece, Jun. 2017

- S. Bagwell, P.D. Ledger, A.J. Gil, "Finite elements for the simulation of coupled acousto-magneto-mechanical systems with application to MRI scanner design", $11^{\text {th }}$ International Conference on Scientific Computing in Electrical Engineering, St. Wolfgang, Austria, Oct. 2016 URL: https://www.ricam.oeaw.ac.at/events/conferences/scee2016/links/ ...

... SCEE2016_Book_of_Abstracts.pdf

- S. Bagwell, P.D. Ledger, A.J. Gil, "Accurate Simulation of Acousto- Magnetomechanical Systems using $h p$ Finite Elements with Application to MRI Coil Design", VII European Congress on Computational Methods in Applied Sciences and Engineering, Crete, Greece, Jun. 2016

- S. Bagwell, P.D. Ledger, A.J. Gil, " $h p$ finite elements for the simulation of coupled acousto-magneto-mechanical systems with application to MRI scanner design", 5 $5^{\text {th }}$ National SIAM Student Chapter Conference, Cardiff, May. 2016 
- S. Bagwell, P.D. Ledger, A.J. Gil, " $h p$ finite elements for the simulation of coupled acousto-magneto-mechanical systems with application to MRI scanner design", 25 $5^{\text {th }}$ UK conference of the Association for Computational Mechanics in Engineering, Cardiff, Apr. 2016

https://acme2016.sciencesconf.org/conference/acme2016/pages/ ...

... proceedings.pdf

- S. Bagwell, P.D. Ledger, A.J. Gil, "A Newton-Raphson scheme for coupled magneto-mechanical problems applied to MRI scanners", 24th $U K$ conference of the Association for Computational Mechanics in Engineering, Swansea, Apr. 2015

URL: http://eng-intranet-web.swan.ac.uk/acme2015/ACMEUK2015.pdf

\subsubsection{Research Posters}

All three posters were awarded prizes for best research poster at the respective conferences:

- S. Bagwell, P.D. Ledger, A.J. Gil, M. Mallett and M. Kruip, "AcoustoMagneto-Mechanical simulation using $h p$-finite elements for MRI scanner design", ZCCE Student Workshop, Swansea, 2017, URL: http://www.swansea.ac.uk/media/638988\%20-\%20Bagwell.pdf

- S. Bagwell, P.D. Ledger, A.J. Gil, G. Barroso Gassiot, M. Seoane Chouciño, M. Kruip, M. Mallett, "Acousto-Magneto-Mechanical simulation using $h p$ finite elements for MRI scanner design", SIAM/UKIE Annual Conference, Glasgow, 2017,

URL: http://maths.manchester.ac.uk/siam-ukie/annual2017/posters/ ...

... bagwell.pdf

- S. Bagwell, P.D. Ledger, A.J. Gil, M. Kruip and M. Mallett, "Coupled acoustic magneto-mechanical systems using $h p$-finite elements for MRI coil design", ZCCE Student Workshop, Swansea, 2016,

URL: https://www.swansea.ac.uk/media/Scott\%20Bagwell.pdf 



\section{Part II}

\section{General Formulation of the Problem}





\section{Chapter 2}

\section{Multi-Field Framework}

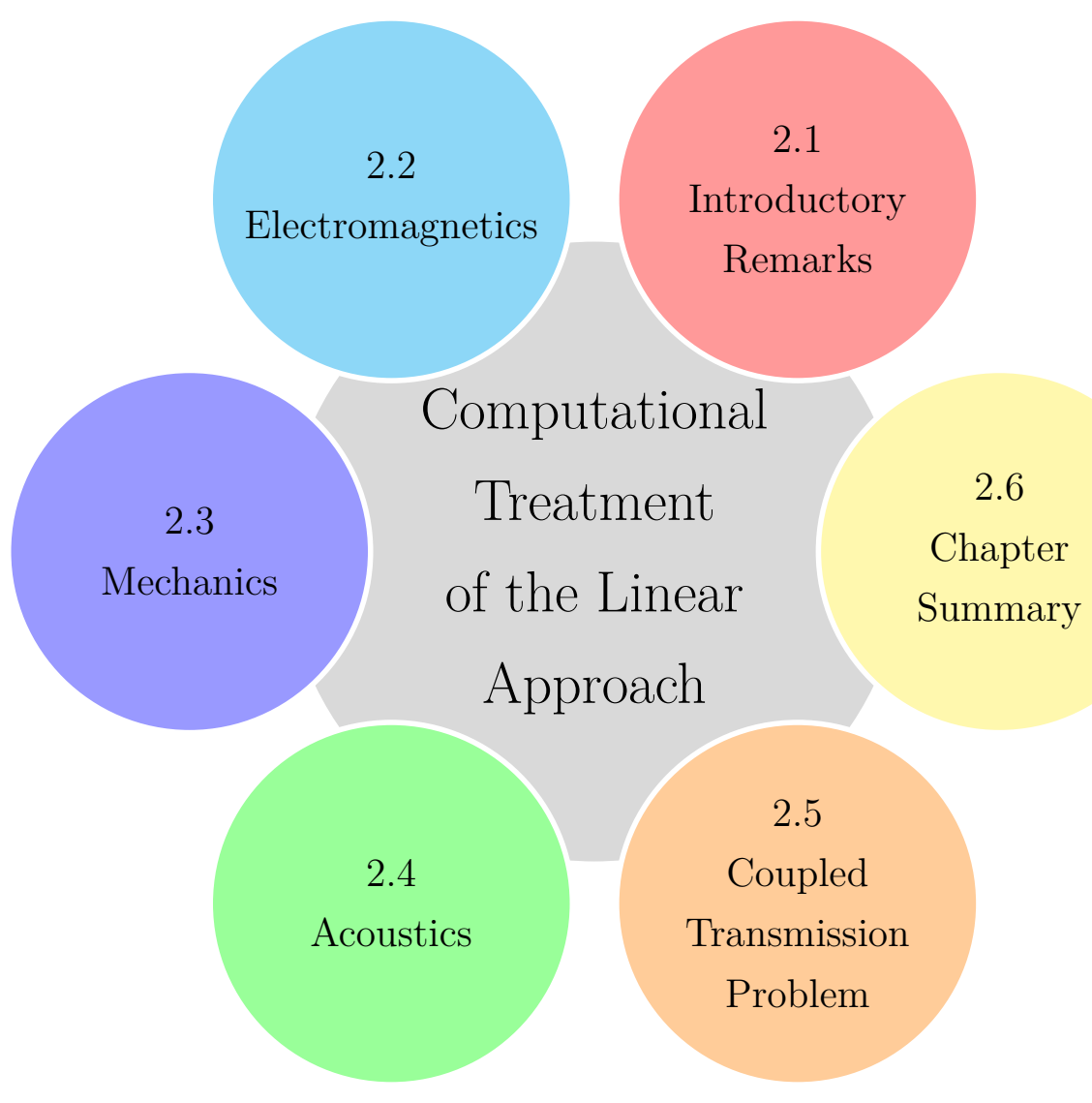

"Thoroughly conscious ignorance is the prelude to every real advance in science." 


\section{$2.1 \quad$ Introductory Remarks}

This Chapter presents a novel coupled acousto-magneto-mechanical system describing the physical behaviour of superconducting solenoidal MRI scanners in clinical operation. This system is described through the governing partial differential equations of the magnetic, mechanical and acoustic fields, set in unbounded regions, where the behaviour of the fields at the farfield boundary is required. The coupling mechanisms are described via a series of volumetric terms, introduced through induced currents and magnetic stresses (see Appendix A for details) and boundary terms at the conductor-free space interface. These boundary terms, also referred to as interface conditions, describe the behaviour of the fields between multiple domains of different materials (see Appendix B for details).

A magneto-mechanical formulation of the MRI problem, more recently including acoustic effects, was previously considered in [203, 203]. The coupling of this formulation is described through body and surface forces in the conductors, where the coupled system is calculated by means of a staggered approach through direct calculation of the electromagnetic forces. This method utilises the approach by Kaltenbacher [124] of applying a layer of elements adjacent to the conductor for the calculation of the electromagnetic forces. In contrast, the method presented in this thesis describes the mechanical body forces through magnetic stresses (see [93]), which provides a more natural treatment of the coupling. The coupled system is described through a rigorous linearisation procedure and provides the basis for a strongly coupled monolithic algorithm, where the system is described naturally in the frequency domain. This provides a novel description of the fully coupled acoustomagneto-mechanical system and also gives rise-under a Biot-Savart assumption of the coils [100]-to a novel magnetic source term in the acoustic equation.

From the objectives of this Thesis, discussed in Section 1.4, the description of the novel acousto-magneto-mechanical system aligns with the objective; "To accurately describe the individual fields and the coupling mechanisms of current clinical MRI scanners.". The contents of this Chapter builds on the work carried out in the published papers $[150,26]$, through an extension of the discussion of the technical details.

Section 2.2 begins with a discussion of the Maxwell system, which describes the electromagnetic response of a conducting region when illuminated by a transient magnetic field. Section 2.3 then describes the corresponding transient mechanical response of conductors resulting from electromagnetic stresses generated in this 
region. Finally, in Section 2.4, the transient acoustic response resulting from the vibration of conductors in free space and a novel magnetic source term is presented. The equations of each of the fields are individually reduced, by ignoring effects that are negligible within the operating conditions of MRI scanners. These systems of equations are combined to form the complete coupled transmission problem, in 2.5, describing the acousto-magneto-mechanical coupled behaviour of MRI scanners. Finally, the contributions of this Chapter are then summarised in Section 2.6.

\section{$2.2 \quad$ Electromagnetics}

MRI scanners generate high strength static magnetic fields, as well as transient magnetic fields, to perform scans of a patient and acquire data for imaging. Understanding these magnetic fields generated in an MRI scanner is crucial in the design phase to ensure the scanner is capable of supporting such fields. The electromagnetic field is described by Maxwell's system of equations.

\subsubsection{Full Maxwell System}

Defining $\boldsymbol{E}, \boldsymbol{H}, \boldsymbol{D}, \boldsymbol{B}$ as the electric, magnetic, electric flux and magnetic flux intensity field vectors respectively, the differential form of the Maxwell system, is described by

$$
\begin{aligned}
\nabla \times \boldsymbol{H} & =\boldsymbol{J}+\frac{\partial \boldsymbol{D}}{\partial t}, \\
\nabla \times \boldsymbol{E} & =-\frac{\partial \boldsymbol{B}}{\partial t}, \\
\nabla \cdot \boldsymbol{B} & =0 \\
\nabla \cdot \boldsymbol{D} & =\rho_{V},
\end{aligned}
$$

where $\boldsymbol{J}$ and $\rho_{V}$ represent the electric current density vector and the electric volume charge density respectively. The total electric current density vector comprises of the addition of several current density terms $\boldsymbol{J}=\boldsymbol{J}^{e}+\boldsymbol{J}^{l}+\boldsymbol{J}^{s}$; the Eddy current density $\boldsymbol{J}^{e}$, the Lorentz current density $\boldsymbol{J}^{l}$ and the source current density $\boldsymbol{J}^{s}$, which arises from electrical currents being passed through a set of coils.

The relationships between the magnetic and magnetic flux density fields, the electric and electric displacement fields and the current density terms are expressed 


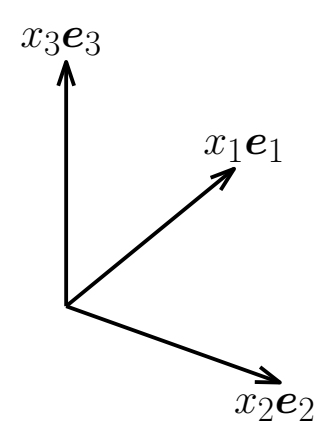

Figure 2.1: General orthonormal coordinate system.

through the constitutive laws

$$
\begin{aligned}
\boldsymbol{B} & =\boldsymbol{\mu} \boldsymbol{H}, \\
\boldsymbol{D} & =\boldsymbol{\epsilon} \boldsymbol{E}, \\
\boldsymbol{J}^{e} & =\gamma \boldsymbol{E}, \\
\boldsymbol{J}^{l} & =\gamma \boldsymbol{v} \times \boldsymbol{B},
\end{aligned}
$$

where $\boldsymbol{v}$ defines the velocity of the medium and the material parameters $\boldsymbol{\gamma}, \boldsymbol{\mu}$ and $\boldsymbol{\epsilon}$ represent the conductivity, permeability and permittivity respectively. In general, these parameters can describe inhomogeneous and anisotropic materials and, as such, can be functions of space with different properties in different directions. For this reason they are represented as spatially varying rank 2 tensors, where $\boldsymbol{\mu}=\boldsymbol{\mu}(\boldsymbol{x})$, $\boldsymbol{\gamma}=\boldsymbol{\gamma}(\boldsymbol{x})$ and $\boldsymbol{\epsilon}=\boldsymbol{\epsilon}(\boldsymbol{x})$. The vector $\boldsymbol{x}$ represents position, illustrated in Figure 2.1, defined as

$$
\boldsymbol{x}=\sum_{i=1}^{3} x_{i} \boldsymbol{e}_{i},
$$

where for a Cartesian coordinate system $x_{1}=x, x_{2}=y, x_{3}=z$ and for a cylindrical coordinate system $x_{1}=r, x_{2}=\phi, x_{3}=z$.

The $\boldsymbol{A}$-based formulation ${ }^{1}$ of the Maxwell system may be obtained by introducing the magnetic vector potential representation of the $\boldsymbol{B}$ field as

$$
\boldsymbol{B}=\nabla \times \boldsymbol{A}
$$

such that $(2.1 \mathrm{c})$ is automatically satisfied. Substituting this vector potential repre-

\footnotetext{
${ }^{1}$ Two possible formulations of the Maxwell system are the $\boldsymbol{A}$ based and $\boldsymbol{H}$ based formulations, see [153] and [113]. This Thesis focusses on the $\boldsymbol{A}$ based formulation and its reduction to the eddy current model.
} 
sentation directly into equation (2.1b), the electric field may be represented as

$$
\boldsymbol{E}=-\nabla \varphi-\frac{\partial \boldsymbol{A}}{\partial t}
$$

where the scalar potential $\varphi$ arises due to the freedom on the choice of $\boldsymbol{A}$. This results in a non-uniqueness of the vector potential as can be seen by taking $\boldsymbol{A} \rightarrow \boldsymbol{A}+\nabla \psi$, where $\varphi=\partial \psi / \partial t$, and noting that $\nabla \times \nabla \psi=\mathbf{0}$, without affecting the result of (2.4).

Thus the $\boldsymbol{A}-\varphi$ formulation of the Maxwell system in (2.1) becomes

$$
\begin{aligned}
\nabla \times \boldsymbol{\mu}^{-1} \nabla \times \boldsymbol{A}=\boldsymbol{J}^{s}-\gamma\left(\nabla \varphi+\frac{\partial \boldsymbol{A}}{\partial t}\right)+\gamma \boldsymbol{v} \times(\nabla \times \boldsymbol{A}) & \\
-\frac{\partial}{\partial t}\left(\boldsymbol{\epsilon} \nabla \varphi+\boldsymbol{\epsilon} \frac{\partial \boldsymbol{A}}{\partial t}\right) & \text { in } \mathbb{R}^{3}, \\
\nabla \cdot\left(\boldsymbol{\epsilon} \nabla \varphi+\boldsymbol{\epsilon} \frac{\partial \boldsymbol{A}}{\partial t}\right)=\rho_{V} & \text { in } \mathbb{R}^{3},
\end{aligned}
$$

The treatment of this formulation and any gauges required is discussed below in Section 2.2.4

\subsubsection{Isotropic Homogeneous Materials}

Given that a large number of the components of MRI scanners are currently made of metals; specifically aluminium and stainless steel, which do not exhibit hysteresis effects $[3,2]$, they can be described as isotropic and homogeneous. This means that, for each individual component within an MRI scanner, a constant material parameter that has the same behaviour in all the principle directions of the coordinate system can be employed. This results in the material tensors becoming

$$
\boldsymbol{\mu}=\left[\begin{array}{ccc}
\mu & 0 & 0 \\
0 & \mu & 0 \\
0 & 0 & \mu
\end{array}\right]=\mu \boldsymbol{I} ; \quad \boldsymbol{\gamma}=\left[\begin{array}{ccc}
\gamma & 0 & 0 \\
0 & \gamma & 0 \\
0 & 0 & \gamma
\end{array}\right]=\gamma \boldsymbol{I} ; \quad \boldsymbol{\epsilon}=\left[\begin{array}{ccc}
\epsilon & 0 & 0 \\
0 & \epsilon & 0 \\
0 & 0 & \epsilon
\end{array}\right]=\epsilon \boldsymbol{I}
$$

where $\boldsymbol{I}$ is the rank 2 identity tensor. In the free space region $\mathbb{R}^{3} \backslash \Omega_{c}$ of the problem the material parameters are that of a vacuum, where the permeability $\mu=\mu_{0}=4 \pi \times 10^{-7} \mathrm{Hm}^{-1}$, the permittivity $\epsilon=\epsilon_{0}=8.854 \times 10^{-12} \mathrm{Fm}^{-1}$ and the conductivity $\gamma=\gamma_{0}=0 \mathrm{Sm}^{-1}$.

This model can be used to describe all material parameters of isotropic, homogeneous materials. Therefore, given this is typically the case in MRI scanners, all 
equations from this point will be expressed with constant scalar material parameters as opposed to spatially dependant tensors.

\subsubsection{Low Frequency Electromagnetics}

The gradient coils are an important component of an MRI scanner, as they are used to generate an image of the patient. They are subject to pulsed electrical current sources that pass through the set of resistive coils and generate a transient magnetic field, based on Ampere's law (2.1a). For typical MRI scanner applications the frequency range- at which the gradient coils are excited by pulsed current sourcesis in the region of $0-5 \mathrm{kHz}$ as can be seen in Figure 1.4 [83]. Figure 1.4 illustrates the typical time varying profiles of a series of generic gradient field sequences in an MRI scanner for imaging different parts of the body. The plots show the gradient magnetic flux density across the imaging bore axis against time. The reader is referred to Section 6.5.3 for how this relates to the current density in the coils.

In order to determine the fundamental frequencies associated with the pulsed transient field signals, a Fourier transform (FT) can be applied. Figure 2.2 illustrates the amplitudes of the fundamental frequencies of the gradient field time signals in Figure 1.4. The signals appear to be dominated by frequencies below $f=2 \mathrm{kHz}^{2}$. However, the imaging sequence of the body, illustrated in Figure 2.3, suggests that there is a further region of increasing dominance at around $3 k H z$ for this signal. Nonetheless, for frequencies above $4 k \mathrm{~Hz}$, shown in the zoom section of Figure 2.3, the amplitudes of the gradient field are $<3 \%$ of the lower frequencies. In general, therefore, frequencies above $5 \mathrm{kHz}$ in MRI scanners can be ignored.

The electromagnetic fields generated in MRI scanners by these pulsed gradient fields are therefore considered to be relatively low frequency, compared with frequencies associated with electromagnetic scattering applications, such as antennas etc. [152]. For such problems, the system of Maxwell's equations may be further reduced by considering the magnitude of the displacement currents ${ }^{3} \partial \boldsymbol{D} / \partial t$, compared with those of the other terms. Two conditions arise from the Maxwell system that must be satisfied in order to ensure the neglection of the displacement currents is valid.

$$
\epsilon \mu \alpha^{2} \omega^{2}<<1, \quad \frac{\epsilon \omega}{\gamma}<<1
$$

\footnotetext{
${ }^{2}$ The frequency $f$ of a wave, measured in $H z$, is the reciprocal of its time period $T=1 / f$ and can be related to the angular frequency $\omega=2 \pi f$ measured in $\mathrm{rad} / \mathrm{s}$.

${ }^{3}$ The displacement currents $\partial \boldsymbol{D} / \partial t$ are associated with magnetic wave propagation, since they are defined by a second time derivative of the magnetic vector potential.
} 


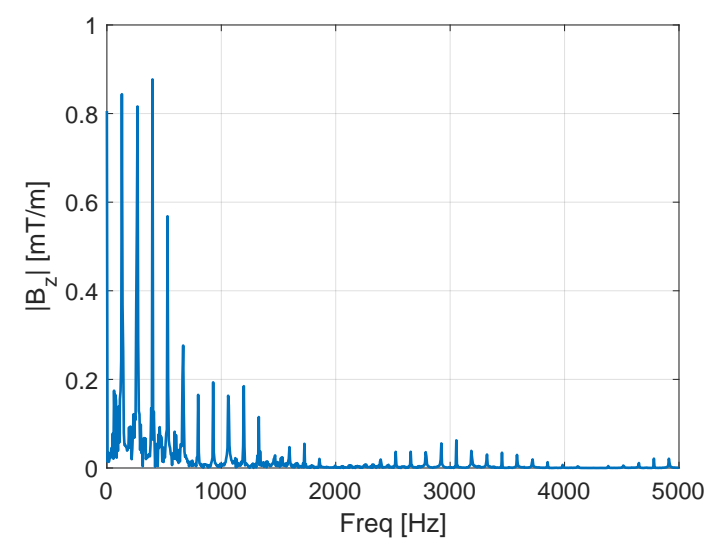

(a) Body

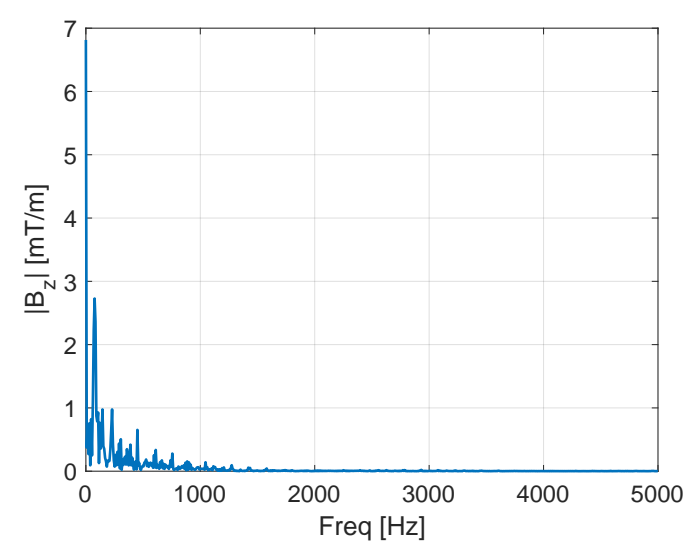

(c) Knee

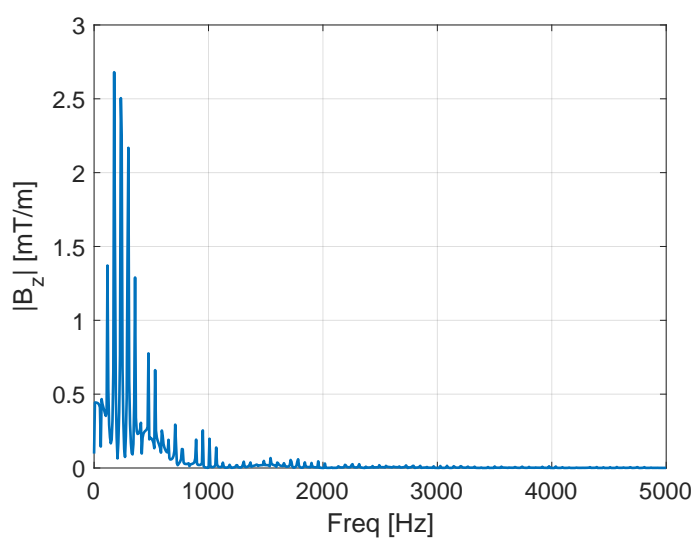

(b) Head

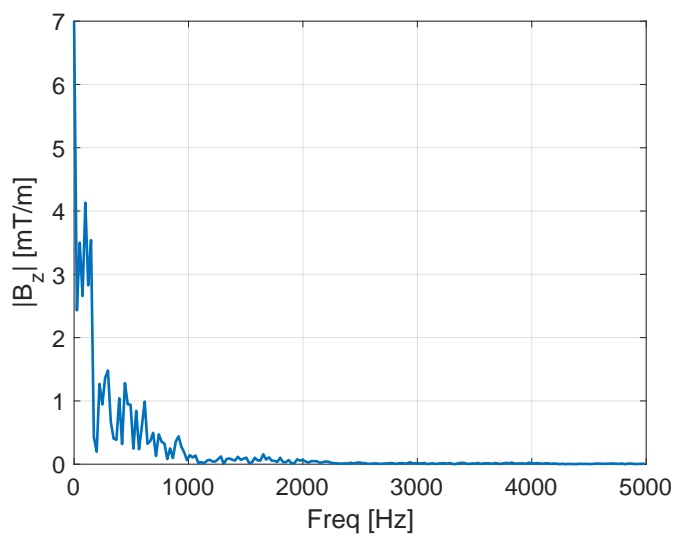

(d) Shoulder

Figure 2.2: Frequency spectrums of the gradient coil pulse sequences, shown in Figure 1.4, for imaging different parts of the body.

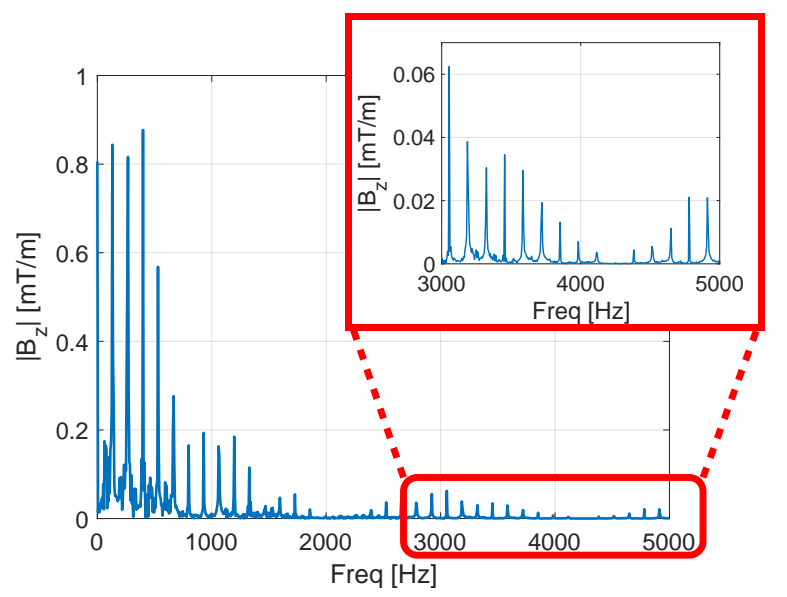

Figure 2.3: Frequency decomposition by Fourier transform of the time signal. 
where $\alpha$ is the length scale of the problem, as a result of taking the gradient of the fields, and $\omega$ is the frequency of the excitation current source, in rad/s. Appendix C.1 summarises the steps required to derive these conditions for ensuring this approximation remains valid. A rigorous justification involves the topology of the conducting region [18].

For MRI scanners in operation the typical ranges of material parameters are: $10^{6} \leq \gamma \leq 10^{8} \mathrm{Sm}^{-1}, \mu \approx \mu_{0}$ and $\epsilon \approx \epsilon_{0}$, subject to angular frequencies typically of $\omega \leq 10000 \pi$. Substituting the worst case parameters into the above conditions, in (2.8), results in

$$
\mu_{0} \epsilon_{0} \omega^{2} \alpha^{2} \approx 10^{-8} \alpha^{2}<<1, \quad \frac{\epsilon_{0} \omega}{\gamma} \approx 3 \times 10^{-13}<<1,
$$

which still hold ${ }^{4}$, provided that the length scale $\alpha<<10^{4} m$. Thus, suggesting that the eddy current approximation is valid for the study of MRI scanners, as the scanners are typically only a few metres in size, resulting in much smaller length scales of $\alpha \sim O\left(10^{1}\right)$.

\subsubsection{Eddy Current Model}

Given the problems considered for MRI application involve low frequency fields and materials of high conductivity, low permittivity and permeability, the transient eddy current approximation of Maxwell's equations can be used, as described above. With this, the displacement currents $\partial \boldsymbol{D} / \partial t$ in (2.6) can be neglected and thus the system may be described through the following parabolic PDEs

$$
\begin{aligned}
\nabla \times \mu^{-1} \nabla \times \boldsymbol{A} & =\boldsymbol{J}^{s}-\gamma\left(\nabla \varphi+\frac{\partial \boldsymbol{A}}{\partial t}\right)+\gamma \boldsymbol{v} \times(\nabla \times \boldsymbol{A}) & & \text { in } \mathbb{R}^{3}, \\
\epsilon \nabla \cdot\left(\nabla \varphi+\frac{\partial \boldsymbol{A}}{\partial t}\right) & =0 & & \text { in } \mathbb{R}^{3},
\end{aligned}
$$

where the problem is charge free $\rho_{V}=0$. In the above the previous assumptions of homogeneous and isotropic materials have been imposed to render the material parameters as scalar constants. The term $\boldsymbol{J}^{l}=\gamma \partial \boldsymbol{u} / \partial t \times \nabla \times \boldsymbol{A}$ denotes the Lorentz currents where $\partial \boldsymbol{u} / \partial t=\boldsymbol{v}$ is the mechanical velocity in the conducting region $\Omega_{c}$.

Under a Biot-Savart conductor assumption of the main and gradient coils [100], the coils are assumed to be non-conductors and thus the solenoidal external current

\footnotetext{
[216].

${ }^{4}$ For an estimation on the error of the eddy current model using geometrical definitions see
} 
sources $\boldsymbol{J}^{s}$ are assumed to lie in free space, $\mathbb{R}^{3} \backslash \Omega_{c}$.

Equation (2.10) describes a transient first order system for the magnetic vector potential. The radiation condition of the field is, therefore, given by ${ }^{5}$

$$
\boldsymbol{A}=O\left(|\boldsymbol{x}|^{-1}\right) \quad \text { as }|\boldsymbol{x}| \rightarrow \infty
$$

where $\boldsymbol{x}$ is assumed to be measured from the centre of the conducting region $\Omega_{c}$. The above describes the static decay of the magnetic vector potential $\boldsymbol{A}$ away from the current sources $\operatorname{supp}\left(\boldsymbol{J}^{s}\right)$ and conductors $\Omega_{c}$.

In order to ensure uniqueness in the solution of (2.10) a series of gauge conditions must be applied in both the conducting $\Omega_{c}$ and non-conducting $\mathbb{R}^{3} \backslash \Omega_{c}$ regions.

\section{Gauging in the Conducting Region}

To satisfy (2.10) in the conducting region, some gauging on the scalar potential can be applied to ensure the uniqueness of $\boldsymbol{A}$. Choosing to employ the temporal gauge, also known as the Weyl gauge,

$$
\varphi=0
$$

allows for the system in (2.10) to become

$$
\begin{aligned}
\nabla \times \mu^{-1} \nabla \times \boldsymbol{A} & =\boldsymbol{J}^{s}-\gamma \frac{\partial \boldsymbol{A}}{\partial t}+\gamma \boldsymbol{v} \times(\nabla \times \boldsymbol{A}) & & \text { in } \Omega_{c} \\
\epsilon \nabla \cdot \frac{\partial \boldsymbol{A}}{\partial t} & =0 & & \text { in } \Omega_{c} .
\end{aligned}
$$

In the above the result of $\boldsymbol{A}$, from (2.13a), is uniquely defined and thus (2.13b) is not required ${ }^{6}$.

\section{Gauging in the Non-Conducting Region}

In the non-conducting region $\mathbb{R}^{3} \backslash \Omega_{c}$ the eddy current system, in (2.10), reduces to

$$
\begin{aligned}
\nabla \times \mu_{0}^{-1} \nabla \times \boldsymbol{A} & =\boldsymbol{J}^{s} & & \text { in } \mathbb{R}^{3} \backslash \Omega_{c}, \\
\nabla \cdot\left(\epsilon_{0} \nabla \varphi+\epsilon_{0} \frac{\partial \boldsymbol{A}}{\partial t}\right) & =0 & & \text { in } \mathbb{R}^{3} \backslash \Omega_{c} .
\end{aligned}
$$

\footnotetext{
${ }^{5}$ Note that $\boldsymbol{A}=O\left(|\boldsymbol{x}|^{-1}\right)$ as $\boldsymbol{x} \rightarrow \infty$ is according to the mathematical model described by Ammari, Buffa and Nédélec [18]. Here, the big O notation implies that the rate is at least as fast as $|\boldsymbol{x}|^{-1}$ and can be faster in practice, for details see the aforementioned paper.

${ }^{6}$ Note that the result of this gauging is often called the $\boldsymbol{A}^{*}-\boldsymbol{A}$ formulation, eg. [37], but in this Thesis no distinction between $\boldsymbol{A}^{*}$ and $\boldsymbol{A}$ is made.
} 
In this case one can choose to employ the Coulomb gauge, where

$$
\nabla \cdot \boldsymbol{A}=0
$$

in order to ensure uniqueness on the gradients of $\boldsymbol{A}$ and satisfy the set of equations in (2.14). If $\boldsymbol{A}$ is divergence free, then so too must $\nabla \varphi$ in order for (2.14b) to hold. Thus, in this case $(2.14 \mathrm{~b})$ can be replaced by the Coulomb gauge (2.15) and the system now becomes

$$
\begin{aligned}
\nabla \times \mu_{0}^{-1} \nabla \times \boldsymbol{A} & =\boldsymbol{J}^{s} & & \text { in } \mathbb{R}^{3} \backslash \Omega_{c} \\
\nabla \cdot \boldsymbol{A} & =0 & & \text { in } \mathbb{R}^{3} \backslash \Omega_{c} .
\end{aligned}
$$

\section{Complete Gauged System}

Given the gauge conditions in the conducting and non-conducting regions the complete eddy current system now becomes ${ }^{7}$

$$
\begin{aligned}
\nabla \times \mu^{-1} \nabla \times \boldsymbol{A} & =\boldsymbol{J}^{s}-\gamma \frac{\partial \boldsymbol{A}}{\partial t}+\gamma \boldsymbol{v} \times(\nabla \times \boldsymbol{A}) & & \text { in } \Omega_{c}, \\
\nabla \times \mu_{0}^{-1} \nabla \times \boldsymbol{A} & =\boldsymbol{J}^{s} & & \text { in } \mathbb{R}^{3} \backslash \Omega_{c} \\
\nabla \cdot \boldsymbol{A} & =0 & & \text { in } \mathbb{R}^{3} \backslash \Omega_{c} .
\end{aligned}
$$

\subsubsection{Interface Conditions}

To fully describe the interaction of the magnetic field across the interfaces of multiple domains, the vector potential $\boldsymbol{A}$ satisfies the following transmission conditions (see Appendix B.1.1 and B.1.2) on the conductor boundary $\partial \Omega_{c}$

$$
\boldsymbol{n} \times[\boldsymbol{A}]_{\partial \Omega_{c}}=\mathbf{0}, \quad \boldsymbol{n} \times\left[\mu^{-1} \nabla \times \boldsymbol{A}\right]_{\partial \Omega_{c}}=\boldsymbol{j}_{s},
$$

which describe the tangential continuity in the electric and magnetic fields, respectively. In the absence of the surface current density $\boldsymbol{j}_{s}$ the transmission conditions become

$$
\boldsymbol{n} \times[\boldsymbol{A}]_{\partial \Omega_{c}}=\mathbf{0}, \quad \boldsymbol{n} \times\left[\mu^{-1} \nabla \times \boldsymbol{A}\right]_{\partial \Omega_{c}}=\mathbf{0},
$$

where $[\cdot]_{\partial \Omega_{c}}$ denotes the jump ${ }^{8}$ on the conductor-non-conductor interface and $\boldsymbol{n}$ is a unit outward normal vector to $\partial \Omega_{c}$.

\footnotetext{
${ }^{7}$ The temporal gauge has been applied in $\Omega_{c}$ and the Coulomb gauge in $\mathbb{R}^{3} \backslash \Omega_{c}[153]$.

${ }^{8}$ The jump describes the continuity of a field across an interface between two mediums $[w]_{\partial \Omega_{c}}=$ $\left.w\right|_{+}-\left.w\right|_{-}$, see Appendix B for more details.
} 


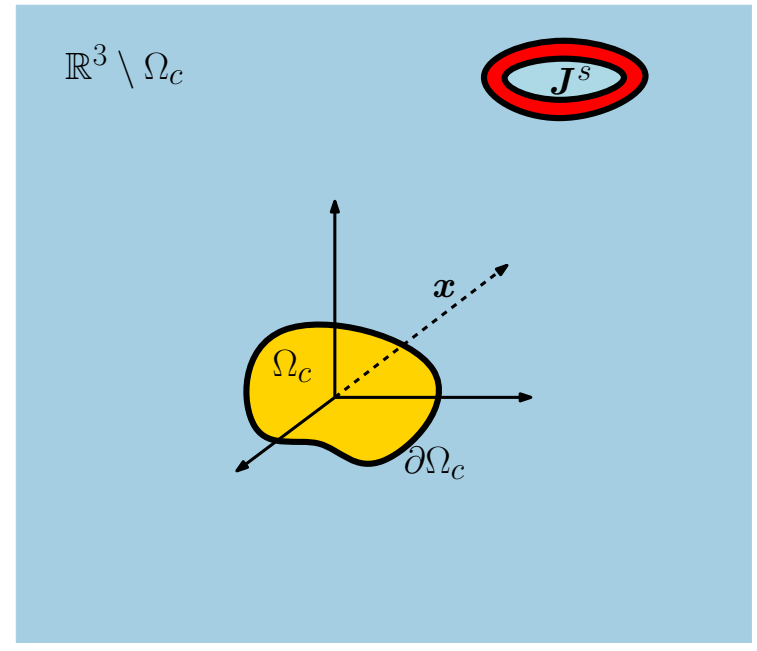

Figure 2.4: Conducting region $\Omega_{c}$ excited by coils contained within the unbounded $\mathbb{R}^{3} \backslash \Omega_{c}$ space.

\subsection{Mechanics}

In the presence of time varying magnetic fields the conducting components of an MRI scanner undergo deformations due to magneto-ponderomotive forces exerted by the magnetic field. These displacements are typically of the order of microns [25] and the conductors can therefore be assumed to behave elastically, given the small strains developed.

\subsubsection{Transient Linear Elasticity}

The mechanical displacements $\boldsymbol{u}$ for the conducting region $\Omega_{c}$ therefore satisfy the transient equilibrium equations

$$
\nabla \cdot \boldsymbol{\sigma}^{m}(\boldsymbol{u})+\boldsymbol{f}=\rho \frac{\partial^{2} \boldsymbol{u}}{\partial t^{2}} \quad \text { in } \Omega_{c}
$$

where $\boldsymbol{\sigma}^{m}$ is the mechanical stress tensor due to the mechanical displacements $\boldsymbol{u}$, defined as

$$
\boldsymbol{\sigma}^{m}(\boldsymbol{u})=\lambda \operatorname{tr}(\boldsymbol{\varepsilon}(\boldsymbol{u})) \boldsymbol{I}+2 G \boldsymbol{\varepsilon}(\boldsymbol{u}),
$$

is the mechanical contribution to the Cauchy stress tensor, $\lambda, G$ denote the Lamé parameters, $\boldsymbol{\varepsilon}=\left(\nabla \boldsymbol{u}+\nabla \boldsymbol{u}^{T}\right) / 2$ the linear strain tensor, ${ }^{T}$ the transpose and $\boldsymbol{f}$ the body force exerted on the mechanical region. 


\subsubsection{Magnetic Body Force}

The volumetric magneto-mechanical coupling arises due to a magneto-ponderomotive body force exerted by the magnetic field on the conductor. This electromagnetic body force may be expressed through the Lorentz force law, per unit volume, as

$$
\boldsymbol{f}^{e}=\rho_{V} \boldsymbol{E}+\boldsymbol{J} \times \boldsymbol{B}
$$

where $\boldsymbol{J}$ denotes the complete current density of the system.

It has been shown in [80] (see also Appendix A.5 for details) that this force can be further described in terms of the divergence of a stress tensor plus a momentum term as

$$
\boldsymbol{f}^{e}=\nabla \cdot \boldsymbol{\sigma}^{e}(\boldsymbol{H}, \boldsymbol{E})+\frac{\partial}{\partial t}(\boldsymbol{B} \times \boldsymbol{D}),
$$

where $\boldsymbol{\sigma}^{e}$ represents the maxwell stress tensor. This stress tensor can be split into a magnetic and an electric component as $\boldsymbol{\sigma}^{e}(\boldsymbol{H}, \boldsymbol{E})=\boldsymbol{\sigma}_{M}^{e}(\boldsymbol{H})+\boldsymbol{\sigma}_{E}^{e}(\boldsymbol{E})$, where

$$
\begin{array}{r}
\boldsymbol{\sigma}_{M}^{e}(\boldsymbol{H})=\mu\left(\boldsymbol{H} \otimes \boldsymbol{H}-\frac{1}{2}(\boldsymbol{H} \cdot \boldsymbol{H}) \boldsymbol{I}\right) \\
\boldsymbol{\sigma}_{E}^{e}(\boldsymbol{E})=\epsilon\left(\boldsymbol{E} \otimes \boldsymbol{E}-\frac{1}{2}(\boldsymbol{E} \cdot \boldsymbol{E}) \boldsymbol{I}\right)
\end{array}
$$

for homogeneous, isotropic materials in the absence of electro- and magneto-strictive effects. The Maxwell stress tensor in materials that exhibit electro- and magnetostrictive effects have been studied in $[93,120,121]$.

\subsubsection{Low Frequency Forcing}

For problems involving the same conditions in (2.8) required to invoke the transient eddy current approximation, made in Section 2.2.3, the momentum term $\frac{\partial}{\partial t}(\boldsymbol{B} \times \boldsymbol{D})$ and the electric part of the stress tensor $\boldsymbol{\sigma}_{E}^{e}$ may be neglected (see Appendix C.2) and so (2.20) simplifies to

$$
\nabla \cdot\left(\boldsymbol{\sigma}^{m}(\boldsymbol{u})+\boldsymbol{\sigma}^{e}(\boldsymbol{A})\right)=\rho \frac{\partial^{2} \boldsymbol{u}}{\partial t^{2}} \quad \text { in } \Omega_{c}
$$

In this case, the magneto-ponderomotive force exerted on $\Omega_{c}$ is described purely by the divergence of the magnetic component of the Maxwell stress tensor $\boldsymbol{f}^{e}=$ $\nabla \cdot \boldsymbol{\sigma}^{e}(\boldsymbol{A})$. The magnetic component of the Maxwell stress tensor in a homogeneous 
isotropic medium [150] is given by

$$
\begin{aligned}
\boldsymbol{\sigma}^{e}(\boldsymbol{A}) & =\mu\left(\boldsymbol{H} \otimes \boldsymbol{H}-\frac{1}{2}|\boldsymbol{H}|^{2} \boldsymbol{I}\right) \\
& =\mu^{-1}\left(\nabla \times \boldsymbol{A} \otimes \nabla \times \boldsymbol{A}-\frac{1}{2}|\nabla \times \boldsymbol{A}|^{2} \boldsymbol{I}\right),
\end{aligned}
$$

The boundary of the conducting region may be split into its Dirichlet and Neumann parts as $\partial \Omega_{c}=\partial \Omega_{c}^{D} \cup \partial \Omega_{c}^{N}$, which do not intersect as $\partial \Omega_{c}^{D} \cap \partial \Omega_{c}^{N}=\emptyset$, illustrated in Figure 2.5. The displacements are fixed $\boldsymbol{u}=\boldsymbol{u}^{D}$ on $\partial \Omega_{c}^{D}$ in order to stop the conducting components from floating away.

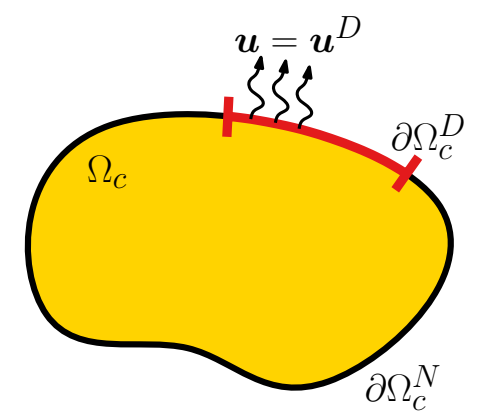

Figure 2.5: Conducting region $\Omega_{c}$, where the boundary is split into Dirichlet and Neumann parts.

\subsubsection{Interface Conditions}

The discussion of the interface conditions for the mechanical problem at the conductorfree space interface is delayed until Section 2.4.4. This is because the description of the acoustic fields will be derived by assuming the analogy of a degenerative solid, where the mechanical equations are used to describe the behaviour of free space and the acoustic pressure $P$ is related to the mechanical displacements $\boldsymbol{u}$. However, for derivation of the interface conditions of the traction forces across interfaces between multiple domains in equilibrium see Appendix B.2.1.

\subsection{Acoustics}

Given that the conducting components in MRI scanners deform and vibrate, throughout an imaging cycle, sound waves will radiate off the conductor surfaces [46]. These sounds can become high in amplitude and lead to uncomfortable conditions for the patient, during a scan. Therefore the analysis of these acoustic sound waves is of great importance in the field of MRI scanner design. 


\subsubsection{Degenerative Solid Analogy}

Based on the previously described coupling phenomena the acoustic field will also depend on sourcing terms from both the magnetic (under certain assumptions) and mechanical fields. Thus, to describe the acoustic behaviour of an MRI scanner the acoustic wave equation under sourcing must be developed to form part of the fully coupled system.

The equation describing the acoustic behaviour is obtained by first considering the relationship between the pressure $P$ and displacement field in the non-conducting medium by

$$
P=\kappa \operatorname{tr}(\varepsilon(\boldsymbol{u}))=\kappa \nabla \cdot \boldsymbol{u},
$$

where $\kappa$ defines the bulk modulus of the acoustic (free space) medium. This definition corresponds to the hydrostatic mechanical part of the Cauchy stress tensor

$$
\boldsymbol{\sigma}^{m}=\kappa(\nabla \cdot \boldsymbol{u}) \boldsymbol{I}+\boldsymbol{\sigma}_{d e v}^{m}
$$

where $\boldsymbol{\sigma}_{d e v}^{m}$ defines the deviatoric component of the Cauchy stress tensor.

Given that the medium of free space surrounding MRI scanners- in which acoustic effects are propagated- consists of air, the presence of shear waves is negligible due to the very small values of shear modulus. This means that $\boldsymbol{\sigma}_{\text {dev }}^{m}$ can be neglected and (2.26) reduces to

$$
\boldsymbol{\sigma}^{m}=\kappa(\nabla \cdot \boldsymbol{u}) \boldsymbol{I}=P \boldsymbol{I}
$$

From this relationship the free space region surrounding the scanner can now be modelled as a solid, governed by the elasticity equation

$$
\nabla \cdot \boldsymbol{\sigma}=\rho \frac{\partial^{2} \boldsymbol{u}}{\partial t^{2}}
$$

where the total stress tensor can be expressed as a linear combination of the Cauchy $\boldsymbol{\sigma}^{m}$ and Maxwell $\boldsymbol{\sigma}^{e}$ stresses, as seen previously in Section 2.3.3. Combining (2.27) with (2.28) yields

$$
\nabla \cdot\left(P \boldsymbol{I}+\boldsymbol{\sigma}^{e}\right)=\rho \frac{\partial^{2} \boldsymbol{u}}{\partial t^{2}}
$$

\subsubsection{Acoustic Helmholtz Wave Equation}

Combining (2.29) and the temporal derivative of (2.25) and realising the relation between the first temporal derivative of the displacement and the velocity $\partial \boldsymbol{u} / \partial t=$ 
$\boldsymbol{v}$, the first order system governing the acoustic behaviour in free space is described by the system

$$
\begin{aligned}
\frac{\partial P}{\partial t} & =\kappa \nabla \cdot \boldsymbol{v} \\
\nabla P+\nabla \cdot \boldsymbol{\sigma}^{e} & =\rho \frac{\partial \boldsymbol{v}}{\partial t} .
\end{aligned}
$$

By taking the divergence of (2.30b) and combining with (2.30a), the behaviour of the acoustic pressure in the free space region is described by the acoustic scalar Helmholtz wave equation

$$
\nabla^{2} P-\frac{1}{c^{2}} \frac{\partial^{2} P}{\partial t^{2}}=-\nabla \cdot\left(\nabla \cdot \boldsymbol{\sigma}^{e}\right) \quad \text { in } \mathbb{R}^{3} \backslash \Omega_{c},
$$

where $c=\sqrt{\kappa / \rho}$ is the speed of sound through the medium. This must be accompanied by the associated (Sommerfeld) radiation condition

$$
\lim _{|\boldsymbol{x}| \rightarrow \infty}\left(\frac{\partial P}{\partial|\boldsymbol{x}|}+\frac{\partial P}{\partial t}\right)=O\left(|\boldsymbol{x}|^{-1}\right),
$$

and can be rewritten in terms of the $\nabla$ operator as

$$
\lim _{|\boldsymbol{x}| \rightarrow \infty}\left(\nabla P \cdot \boldsymbol{n}+\frac{\partial P}{\partial t}\right)=O\left(|\boldsymbol{x}|^{-1}\right) .
$$

which describes the decay behaviour of the field away from the radiation source.

\subsubsection{Properties of the Source Term}

The source term on the RHS of (2.31) is only non-zero inside the magnetic coils, defined as the region $\operatorname{supp}\left(\boldsymbol{J}^{s}\right)^{9}{ }^{10}$, this can be seen by considering the alternative form of $\boldsymbol{f}^{e}$ in free space below ${ }^{11}$

$$
\boldsymbol{f}^{e}=\nabla \cdot \boldsymbol{\sigma}^{e}=-\mu_{0} \boldsymbol{H} \times \nabla \times \boldsymbol{H}=-\nabla \times \boldsymbol{A} \times \boldsymbol{J}^{s}=-\nabla \times \boldsymbol{A} \times\left(\nabla \times \mu_{0}^{-1} \nabla \times \boldsymbol{A}\right),
$$

\footnotetext{
${ }^{9}$ The region $\operatorname{supp}\left(\boldsymbol{J}^{s}\right)$, which means the support of $\boldsymbol{J}^{s}$ defines a volume in which the sourcing current is located, inside the coils in this case

${ }^{10}$ Here the coils are assumed as Biot-Savart conductors, where they remain in $\mathbb{R}^{3} \backslash \Omega_{c}$ and are assumed to have $\gamma=0$ [100]. If the coils are instead treated as rigid or deformable conducting bodies then their support instead forms part of $\Omega_{c}$.

${ }^{11}$ Recall here the relationship between the curl and gradient operators $(\nabla \boldsymbol{H}) \boldsymbol{H}-1 / 2 \nabla(\boldsymbol{H} \cdot \boldsymbol{H})=$ $-\boldsymbol{H} \times(\nabla \times \boldsymbol{H})$.
} 
and thus it follows that in the same region

$$
\nabla \cdot \boldsymbol{f}^{e}=\nabla \cdot\left(\nabla \cdot \boldsymbol{\sigma}^{e}\right)=\mu_{0}\left(\boldsymbol{H} \cdot \nabla \times \boldsymbol{J}^{s}-\left|\boldsymbol{J}^{s}\right|^{2}\right)
$$

\subsubsection{Interface Conditions}

Taking this into account, on the interface $\partial \Omega_{c}$ shown in Figure 2.6, the pressure field, mechanical displacements and stresses are coupled by the transmission conditions (see Appendix B.2.1 and B.2.2 for details)

$$
\begin{aligned}
\left.\left(\boldsymbol{\sigma}^{m}+\boldsymbol{\sigma}^{e}\right)\right|_{\partial \Omega_{c}} ^{-} \boldsymbol{n} & =\left.\left(P \boldsymbol{I}+\boldsymbol{\sigma}^{e}\right)\right|_{\partial \Omega_{c}} ^{+} \boldsymbol{n}, \\
\left.\rho^{+} \frac{\partial^{2} \boldsymbol{u}}{\partial t^{2}}\right|_{\partial \Omega_{c}} ^{-} \cdot \boldsymbol{n} & =\left.\left(\nabla P+\nabla \cdot \boldsymbol{\sigma}^{e}\right)\right|_{\partial \Omega_{c}} ^{+} \cdot \boldsymbol{n} .
\end{aligned}
$$

The above describes the normal continuity of the stresses and accelerations of two different mediums across an interface. Note that the latter condition also further reduces to

$$
\left.\left(\nabla P+\nabla \cdot \boldsymbol{\sigma}^{e}\right)\right|_{\partial \Omega_{c}^{D}} ^{+} \cdot \boldsymbol{n}=0,\left.\quad \rho^{+} \frac{\partial^{2} \boldsymbol{u}}{\partial t^{2}}\right|_{\partial \Omega_{c}^{N}} ^{-} \cdot \boldsymbol{n}=\left.\left(\nabla P+\nabla \cdot \boldsymbol{\sigma}^{e}\right)\right|_{\partial \Omega_{c}^{N}} ^{+} \cdot \boldsymbol{n}
$$

in light of the known Dirichlet displacement condition on $\partial \Omega_{c}^{D}$ for the mechanical problem.

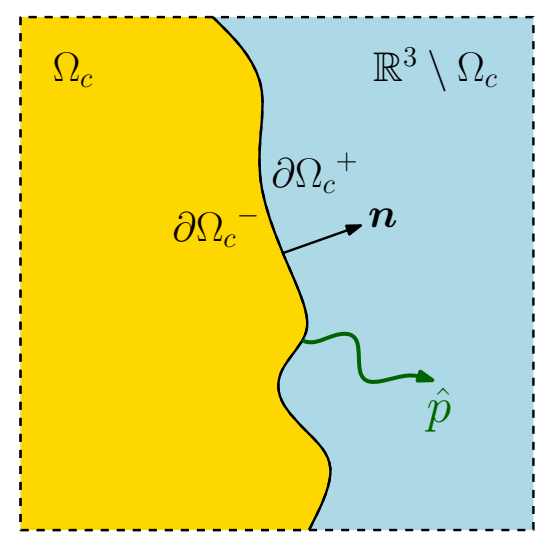

Figure 2.6: Interface conditions at the conductor-free space boundary, where $\partial \Omega_{c}^{-}$corresponds to the contribution of the interface from the conductor and $\partial \Omega_{c}^{+}$the contribution from the nonconducting region. 


\subsection{Coupled Transmission Problem}

In order to describe the fully coupled acousto-magneto-mechanical system a coupled transmission problem describing the three physical fields throughout the domains, the constitutive laws governing the material relations, the conditions at the interface between the domains and the boundary conditions must be developed.

\subsubsection{Transient Non-Linear Problem}

Combining the statements from the previous Sections 2.2, 2.3 and 2.4 the transmission problem for describing the coupled acousto-magneto-mechanical system in a time period $[0, T]$ is given by: Find $(\boldsymbol{A}, \boldsymbol{u}, P)(t) \in\left(\mathbb{R}^{3} \times \mathbb{R}^{3} \times \mathbb{R}\right)[0, T]$ such that

$$
\begin{aligned}
\nabla \times\left(\mu^{-1} \nabla \times \boldsymbol{A}\right)+\gamma \frac{\partial \boldsymbol{A}}{\partial t} & =\boldsymbol{J}^{s}+\gamma \frac{\partial \boldsymbol{u}}{\partial t} \times(\nabla \times \boldsymbol{A}) & & \text { in } \mathbb{R}^{3}, \\
\nabla \cdot \boldsymbol{A} & =0 & & \text { in } \mathbb{R}^{3} \backslash \Omega_{c} \\
\nabla \cdot\left(\boldsymbol{\sigma}^{m}(\boldsymbol{u})+\boldsymbol{\sigma}^{e}(\boldsymbol{A})\right) & =\rho \frac{\partial^{2} \boldsymbol{u}}{\partial t^{2}} & & \text { in } \Omega_{c}, \\
\nabla^{2} P-\frac{1}{c^{2}} \frac{\partial^{2} P}{\partial t^{2}} & =-\nabla \cdot\left(\nabla \cdot \boldsymbol{\sigma}^{e}(\boldsymbol{A})\right) & & \text { in } \mathbb{R}^{3} \backslash \Omega_{c} \\
\boldsymbol{A} & =O\left(|\boldsymbol{x}|^{-1}\right), & & \\
\lim _{|\boldsymbol{x}| \rightarrow \infty}\left(\nabla P \cdot \boldsymbol{n}+\frac{\partial P}{\partial t}\right) & =O\left(|\boldsymbol{x}|^{-1}\right) & & \text { as }|\boldsymbol{x}| \rightarrow \infty \\
\left.\left(\nabla P+\nabla \cdot \boldsymbol{\sigma}^{e}(\boldsymbol{A})\right)\right|_{\partial \Omega_{c}^{D}} ^{+} \cdot \boldsymbol{n} & =0 & & \text { on } \partial \Omega_{c}^{D} \\
\left.\rho^{+} \frac{\partial^{2} \boldsymbol{u}}{\partial t^{2}}\right|_{\partial \Omega_{c}^{N}} ^{-} \cdot \boldsymbol{n} & =\left.\left(\nabla P+\nabla \cdot \boldsymbol{\sigma}^{e}(\boldsymbol{A})\right)\right|_{\partial \Omega_{c}^{+}} ^{+} \cdot \boldsymbol{n}, & & \\
\left.\left(\boldsymbol{\sigma}^{e}(\boldsymbol{A})+\boldsymbol{\sigma}^{m}(\boldsymbol{u})\right)\right|_{\partial \Omega_{c}} ^{-} \boldsymbol{n} & =\left.\left(P \boldsymbol{I}+\boldsymbol{\sigma}^{e}(\boldsymbol{A})\right)\right|_{\partial \Omega_{c}} ^{+} \boldsymbol{n} & & \text { on } \partial \Omega_{c}^{N} \\
\boldsymbol{n} \times[\boldsymbol{A}]_{\partial \Omega_{c}} & =\mathbf{0}, & & \\
\boldsymbol{n} \times\left[\mu^{-1} \nabla \times \boldsymbol{A}\right]_{\partial \Omega_{c}} & =\mathbf{0} & & \text { on } \partial \Omega_{c},
\end{aligned}
$$

subject to the following general initial conditions

$$
\begin{aligned}
\boldsymbol{A}(t=0) & =\boldsymbol{A}_{0} & & \text { in } \mathbb{R}^{3}, \\
\boldsymbol{u}(t=0)=\boldsymbol{u}_{0}, \quad \frac{\partial \boldsymbol{u}}{\partial t}(t=0) & =\boldsymbol{v}_{0} & & \text { in } \Omega_{c}, \\
P(t=0)=P_{0}, & \frac{\partial P}{\partial t}(t=0)=\left.\frac{\partial P}{\partial t}\right|_{0} & & \text { in } \mathbb{R}^{3} \backslash \Omega_{c}
\end{aligned}
$$


In the coupled system, the magnetic vector potential depends on the mechanical displacements through generated Lorentz currents in (2.36a). The mechanical displacements depend on the magnetic vector potential through the generated electromagnetic stresses in (2.36c). This two-way coupling describes the magnetomechanical system obtained in [150]. In consideration of the acoustic behaviour, the displacements are transmitted into the acoustic pressure field through $(2.36 \mathrm{~h}, 2.36 \mathrm{i})$ and the magnetic vector potential is transmitted to the acoustic field through electromagnetic stresses in (2.36d). This system describes a fully coupled system, where two-way coupling mechanisms between the magnetic and mechanical and acoustic and mechanical fields and a one-way coupling between the magnetic and acoustic fields are generated. An illustration of the fully coupled system is shown in Figure 2.7 .

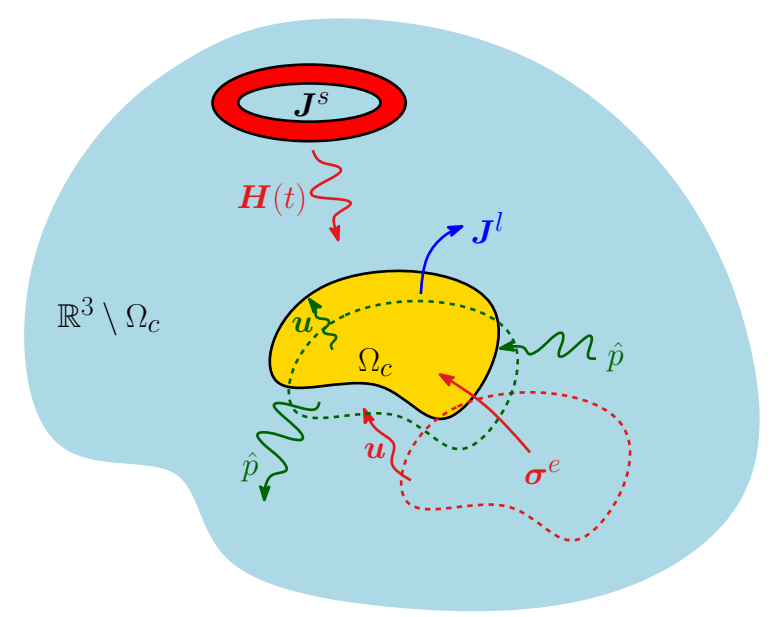

Figure 2.7: Physical representation of the coupling effects in an MRI environment.

\subsubsection{MRI Coils}

There are typically two types of coils in MRI scanners: the main coils, which are superconducting coils supercooled to temperature around $4 K$ by being submersed in liquid helium to achieve high strength static magnetic fields and the gradient coils, which are a set of resistive coils that generate low strength transient magnetic fields.

For MRI application, the system (2.36) is excited through the current source $\boldsymbol{J}^{s}(t)$. In practice, however, MRI scanners are typically maintained at full static field strength and apply the gradient fields only during imaging examinations of the patient. As such, the application allows for the decomposition of the current source into $\boldsymbol{J}^{s}(t)=\boldsymbol{J}^{D C}+\boldsymbol{J}^{A C}(t)$, illustrated in Figure 2.8. Here, $\boldsymbol{J}^{D C}$ corresponds to the 


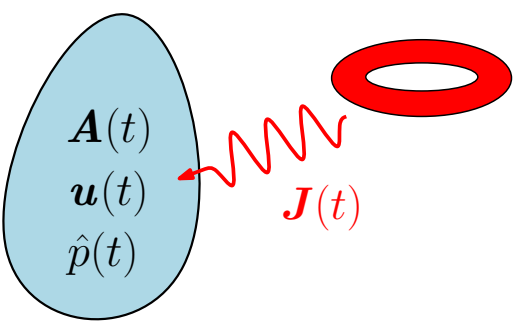

(a) Time dependant current source

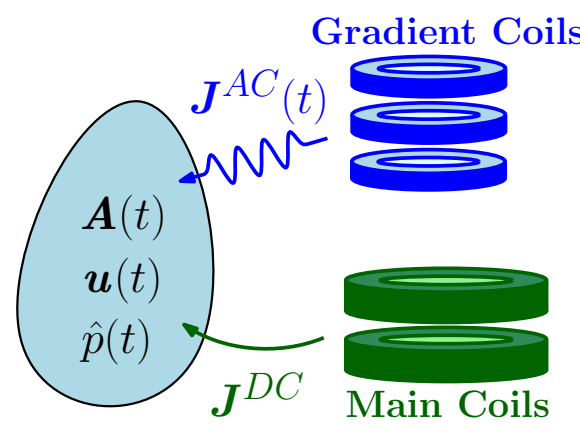

(b) Decomposition of the current source

Figure 2.8: Current source decomposition, where $\boldsymbol{J}^{s}(t)=\boldsymbol{J}^{D C}+\boldsymbol{J}^{A C}(t)$.

static current source of the main magnet and $\boldsymbol{J}^{A C}(t)$ the transient current source of the gradient coils [26].

Under a Biot-Savart assumption of the coils [100], they may be considered as nonconductors and as a result the coils form part of the non-conducting region $\mathbb{R}^{3} \backslash \Omega_{c}$, where the currents arise in $\operatorname{supp}\left(\boldsymbol{J}^{s}\right)$. In the case that the coils are modelled as complete conductors, the supp $\left(\boldsymbol{J}^{s}\right)$ no longer lies in $\mathbb{R}^{3} \backslash \Omega_{c}$ and instead forms part of $\Omega_{c}$. In this case, certain terms in the acoustic system and interface conditions vanish, as previously discussed.

\subsubsection{Static Non-Linear Problem}

The decomposition, illustrated in Figure 2.8, allows for the introduction of the following static problem: Find $\boldsymbol{A}^{D C}, \boldsymbol{u}^{D C}, P^{D C} \in \mathbb{R}^{3} \times \mathbb{R}^{3} \times \mathbb{R}$ such that

$$
\begin{aligned}
\nabla \times\left(\mu^{-1} \nabla \times \boldsymbol{A}^{D C}\right) & =\boldsymbol{J}^{D C} & & \text { in } \mathbb{R}^{3}, \\
\nabla \cdot \boldsymbol{A}^{D C} & =0 & & \text { in } \mathbb{R}^{3}, \\
\nabla \cdot\left(\boldsymbol{\sigma}^{m}\left(\boldsymbol{u}^{D C}\right)+\boldsymbol{\sigma}^{e}\left(\boldsymbol{A}^{D C}\right)\right) & =0 & & \text { in } \Omega_{c}, \\
\nabla^{2} P^{D C} & =-\nabla \cdot\left(\nabla \cdot \boldsymbol{\sigma}^{e}\left(\boldsymbol{A}^{D C}\right)\right) & & \text { in } \mathbb{R}^{3} \backslash \Omega_{c} \\
\boldsymbol{A}^{D C} & =O\left(|\boldsymbol{x}|^{-1}\right), & & \\
P^{D C} & =O\left(|\boldsymbol{x}|^{-1}\right) & & \text { as }|\boldsymbol{x}| \rightarrow \infty \\
\boldsymbol{u}^{D C} & =\boldsymbol{u}_{D}^{D C} & & \text { on } \partial \Omega_{c}^{D}, \\
\boldsymbol{n} \times\left[\boldsymbol{A}^{D C}\right]_{\partial \Omega_{c}} & =\mathbf{0}, & &
\end{aligned}
$$




$$
\begin{aligned}
\boldsymbol{n} \times\left[\mu^{-1} \nabla \times \boldsymbol{A}^{D C}\right]_{\partial \Omega_{c}} & =\mathbf{0} \\
\left.\left(\nabla P^{D C}+\nabla \cdot \boldsymbol{\sigma}^{e}\left(\boldsymbol{A}^{D C}\right)\right)\right|_{\partial \Omega_{c}} ^{+} \cdot \boldsymbol{n} & =0 \\
\left.\left(\boldsymbol{\sigma}^{m}\left(\boldsymbol{u}^{D C}\right)+\boldsymbol{\sigma}^{e}\left(\boldsymbol{A}^{D C}\right)\right)\right|_{\partial \Omega_{c}} ^{-} \boldsymbol{n} & =\left.\left(P^{D C} \boldsymbol{I}+\boldsymbol{\sigma}^{e}\left(\boldsymbol{A}^{D C}\right)\right)\right|_{\partial \Omega_{c}} ^{+} \boldsymbol{n} \text { on } \partial \Omega_{c},
\end{aligned}
$$

where the time variance of the DC fields vanish and a similar decomposition of the Dirichlet displacement condition $\boldsymbol{u}^{D}=\boldsymbol{u}_{D C}^{D}+\boldsymbol{u}_{A C}^{D}(t)$ has been assumed.

The static system in (2.38) results in a coupled acousto-magneto-mechanical system, where both the magneto-mechanical and acousto-mechanical coupling mechanisms are one way coupling due to the absence of any Lorentz currents or acceleration continuity conditions, where these vanish in the case of time independent fields. The solution to this static problem can be used as a starting point for the transient system and provides a useful step in the treatment of the linearisation of the coupled system of equations, discussed later in Chapter 3.

The transient and static coupled systems in (2.36) and (2.38), respectively, will form the transmission problems that will be the focus in the remaining Chapters of this Thesis.

\subsection{Chapter Summary}

This Chapter has derived the novel acousto-magneto-mechanical coupled system describing MRI scanner behaviour. The coupling mechanisms between the three fields have been discussed in detail, where the expression of the magnetic forces in terms of a stress tensor result in a novel set of interface conditions and sourcing terms. The fully coupled systems, which will form the transmission problems for the remainder of this Thesis, have been derived for both the static and transient cases by utilising a physically motivated decomposition of the magnetic source currents.

From the objectives of this Thesis, discussed in section 1.4, the topics discussed in this Chapter align with the objective: "To accurately describe the individual fields and the coupling mechanisms of current clinical MRI scanners.".

In the next Chapter, possible solution procedures to solve the fully coupled transmission problems will be discussed: a fixed point iterative scheme, as in [150] and a Newton-Raphson procedure, as in [26]. The linearisation of the coupled system is performed, using a physically motivated approach, in order to obtain a novel single-step solution strategy to the fully coupled non-linear transmission problems. 


\section{Part III}

\section{Linearised Approach}





\section{Chapter 3}

\section{Linearised Approach}

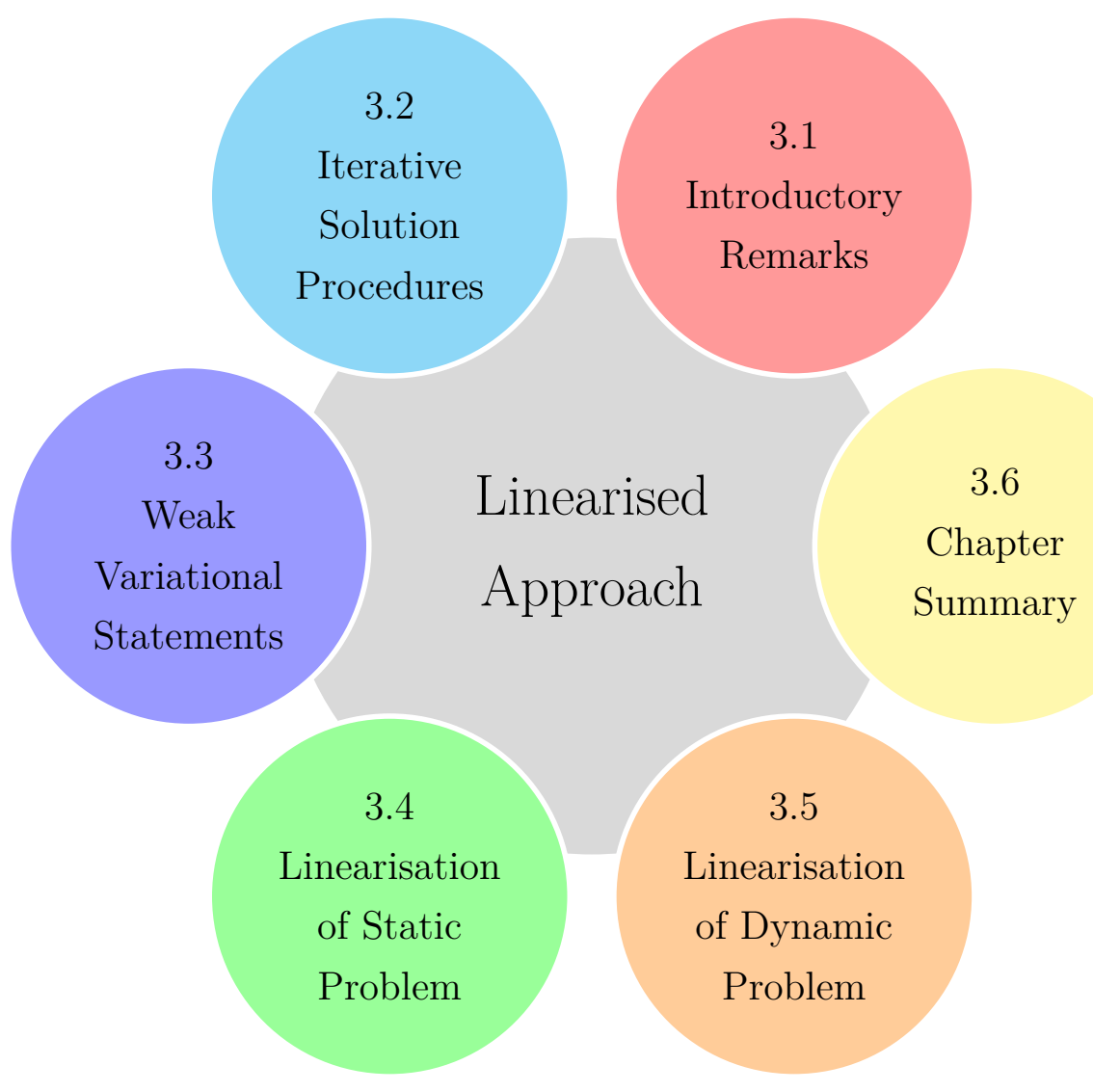

"Hard work beats talent when talent doesn't work hard."

Kevin Durant 


\subsection{Introductory Remarks}

This Chapter describes the novel coupling methodology for the solution of coupled acousto-magneto-mechanical problems, taking into consideration interface conditions between different domains. The coupling scheme is presented in Figure 3.1. A rigorous linearisation of the coupled equation set, motivated by the physics of an MRI scanner during operation is presented in order to achieve an efficient computational procedure. This solution strategy is dubbed the linearised approach, due to the structure of the resulting equation system.

From the objectives of this Thesis, discussed in section 1.4, this new solution strategy aligns with the objective; "To develop an efficient novel solution procedure, named the linearised approach, through a rigorous linearisation of the non-linear system of equations." The contents of this Chapter builds on the work carried out in the published paper [26], through an extension of the discussion of the technical details.

Starting with a discussion of iterative solution procedures for handling coupled sets of non-linear equations in Section 3.2, this Chapter then goes on to describe the weak variational statements of the individual fields: magnetics, mechanics and acoustics and the coupled problem, in Section 3.3. The linearisation of the static (2.38) and transient (2.36) transmission problems, described in Chapter 2, are then discussed in Section 3.4 and 3.5 respectively. The contents of this Chapter are then summarised in Section 3.6.

\subsection{Iterative Solution Procedures}

The numerical simulation of coupled sets of non-linear equations, such as those describing acousto-magneto-mechanical coupling in MRI scanners, presented in Chapter 2, requires approximations of the non-linearities in the equations in order to compute solutions. There exists a range of iterative methods for the solution to such systems of equations, these include fixed point (FP) schemes [202, 203, 150], gradient free methods, such as the Secant, Gauss-Seidel and classic-chord methods [128] and the gradient based Newton-Raphson (NR) method [38].

$\mathrm{FP}$ and NR schemes involve solving iterations of the equation system to obtain converged solutions. The FP scheme requires no knowledge of the gradient of the equation system and so can be easily applied to a range of applications [128]. This, typically, makes it a popular scheme for use in commercial codes [6, 7], where a range 
of applications may be required. NR schemes, on the other hand, provide a more robust method for solving systems of non-linear equations, as they directly compute the gradients, known as the tangent stiffness (or Jacobian) operators. For purposebuilt solvers, with designated applications, the NR scheme provides a more robust technique for solving non-linear coupled equation systems than the FP scheme. They also require fewer iterations, as they converge quadratically and are less prone to poor initial guesses [19].

In terms of defining iterative solution procedures, consider, first, a general nonlinear vector field equation of the form

$$
\boldsymbol{F}(\boldsymbol{x})=\mathbf{0}
$$

which defines the system in terms of a residual function. In general, the form of (3.1) cannot always be solved analytically, due to the non-linearities in the field $\boldsymbol{x}$ and so require numerical techniques in order to solve.

\subsubsection{Fixed Point Scheme}

Iterative fixed point (FP) methods [125, 123] typically work by reformulating a general non-linear equation, in (3.1), to form the following fixed point problem,

$$
\boldsymbol{x}=\boldsymbol{G}(\boldsymbol{x})
$$

Iterations on (3.2), in the form of

$$
\boldsymbol{x}^{[\mathrm{k}+1]}=\boldsymbol{G}\left(\boldsymbol{x}^{[\mathrm{k}]}\right), \quad \mathrm{k}=0,1,2, \ldots, N_{\max },
$$

are performed, where an initial guess of the solution $\boldsymbol{x}^{[0]}$ is provided, until the convergence criterion in the residual $\left|\boldsymbol{x}^{[\mathrm{k}+1]}-\boldsymbol{G}\left(\boldsymbol{x}^{[\mathrm{k}]}\right)\right|<T O L$ and the solution $\left|\boldsymbol{x}^{[\mathrm{k}+1]}-\boldsymbol{x}^{[\mathrm{k}]}\right|<T O L$ are achieved or the specified maximum number of iterations $N_{\max }$ have been performed. TOL is a user specified tolerance of the error. After a number of iterations the update in the solution $\boldsymbol{x}^{[\mathrm{k}+1]}$ will converge to correct solution $\boldsymbol{x}$.

There are often multiple ways of expressing the non-linear equation in this form and conditions need to be fulfilled in order for $\boldsymbol{G}(\boldsymbol{x})$ to be a valid fixed point iteration [128]. Even if these conditions are fulfilled, the convergence in the iterations is at most linear and the scheme is quite sensitive to the choice of the initial guess. For applications of the Fixed point scheme to magneto-mechanical problems within the 


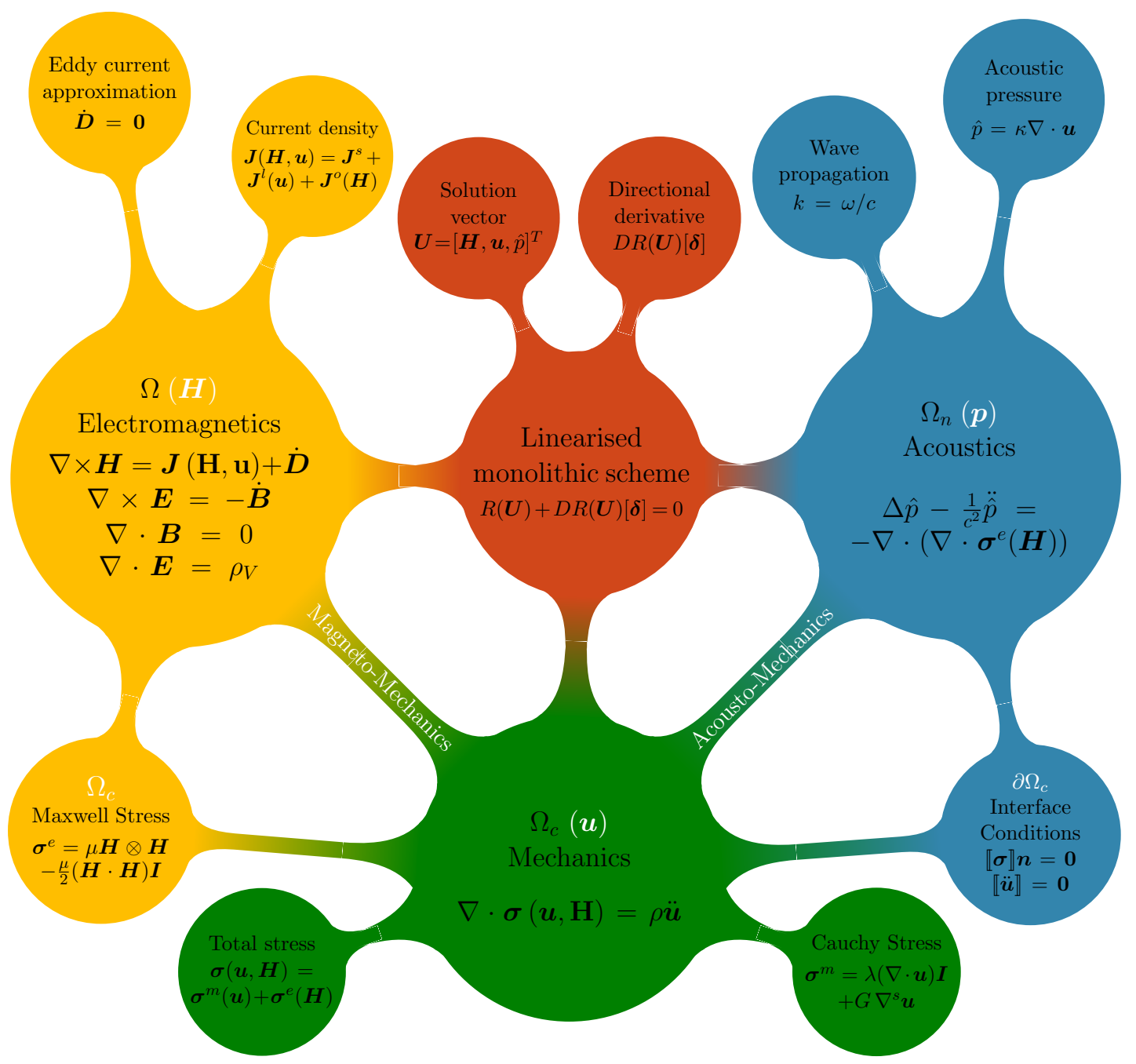

Figure 3.1: Graphical representation of the coupling methodology between the three physical fields; electromagnetic, mechanical and acoustic, coupled through a monolithic linearised scheme. 
field of MRI scanners see [202, 150].

\subsubsection{Newton-Raphson Scheme}

Newton-Raphson schemes are derived from Taylor series approximations to nonlinear equations, in (3.1). They give an iteration of the form

$$
\boldsymbol{x}^{[\mathrm{k}+1]}=\boldsymbol{x}^{[\mathrm{k}]}-\left[\nabla_{x} \boldsymbol{F}\left(\boldsymbol{x}^{[\mathrm{k}]}\right)\right]^{-1} \boldsymbol{F}\left(\boldsymbol{x}^{[\mathrm{k}]}\right),
$$

where the initial guess $\boldsymbol{x}^{[0]}$ is required a-priori. The Jacobian operator, given by $\nabla_{x} \boldsymbol{F}(\boldsymbol{x})$, refers to the derivative of each component of the Residual vector with respect to the components of the solution vector $\boldsymbol{x}$.

Notice that, if $\boldsymbol{G}(\boldsymbol{x})=\boldsymbol{x}-\left[\nabla_{x} \boldsymbol{F}(\boldsymbol{x})\right]^{-1} \boldsymbol{F}(\boldsymbol{x})$, then the Newton-Raphson scheme in (3.4) may be rewritten as the iterative fixed point scheme in (3.3). The fixed point theory can be applied to this choice to establish valid initial guesses for the Newton-Raphson scheme. Generally, however, they need to be close to the root. This iterative scheme converges to the correct root quadratically, which is superior to the linear convergence achieved by the FP scheme.

Gradient free methods (e.g. Secant method) do not require the explicit knowledge of the exact derivative, used in NR methods, and make approximations. However, as a result, they do not experience quadratic convergence in the solution.

\subsection{Weak Variational Statements}

With the development of a finite element analysis tool, the weak variational statements of the coupled set of non-linear partial differential equations will first be established. Building on this, the resulting non-linear weighted residual system will then be linearised, where two approaches for solving the equation system (the linearised and non-linear approaches) will be presented. In obtaining the weak form of the equations, it is convenient to introduce the following definitions

$$
\begin{aligned}
X & :=\left\{\boldsymbol{A} \in \boldsymbol{H}\left(\operatorname{curl}, \mathbb{R}^{3}\right): \nabla \cdot \boldsymbol{A}=0 \text { in } \mathbb{R}^{3} \backslash \Omega_{c}\right\}, \\
Y(\boldsymbol{g}) & :=\left\{\boldsymbol{u} \in\left(H^{1}\left(\Omega_{c}\right)\right)^{3}: \boldsymbol{u}=\boldsymbol{g} \text { on } \partial \Omega_{c}^{D}\right\}, \\
Z & :=\left\{P \in H^{1}\left(\mathbb{R}^{3} \backslash \Omega_{c}\right)\right\},
\end{aligned}
$$

which will be used to describe the weak solutions to the dynamic and static transmission problems, in (2.36) and (2.38) respectively. The domains of the problem 
in which these definitions apply are illustrated in Figure 3.2. The respective spaces $\boldsymbol{H}\left(\operatorname{curl}, \mathbb{R}^{3}\right)$ and $H^{1}\left(\mathbb{R}^{3}\right)$ have their usual definitions, e.g. [178], of

$$
\begin{aligned}
L^{2}(\Omega) & :=\left\{w: \int_{\Omega}|w|^{2} \mathrm{~d} \Omega<\infty\right\}, \\
H^{1}(\Omega) & :=\left\{w \in L^{2}(\Omega): \nabla w \in\left(L^{2}(\Omega)\right)^{3}\right\}, \\
\boldsymbol{H}(\operatorname{curl}, \Omega) & :=\left\{\boldsymbol{w} \in\left(L^{2}(\Omega)\right)^{3}: \nabla \times \boldsymbol{w} \in\left(L^{2}(\Omega)\right)^{3}\right\} .
\end{aligned}
$$

The weighted space $X$ has been chosen such that it satisfies the Coulomb gauge condition in (2.36b). For the full 3D problem this constraint requires extra treatment by either regularisation or introducing Lagrange multipliers, described in [217]. However, the scope of this Thesis is concerned with only axisymmetric problems, where this condition is automatically satisfied.

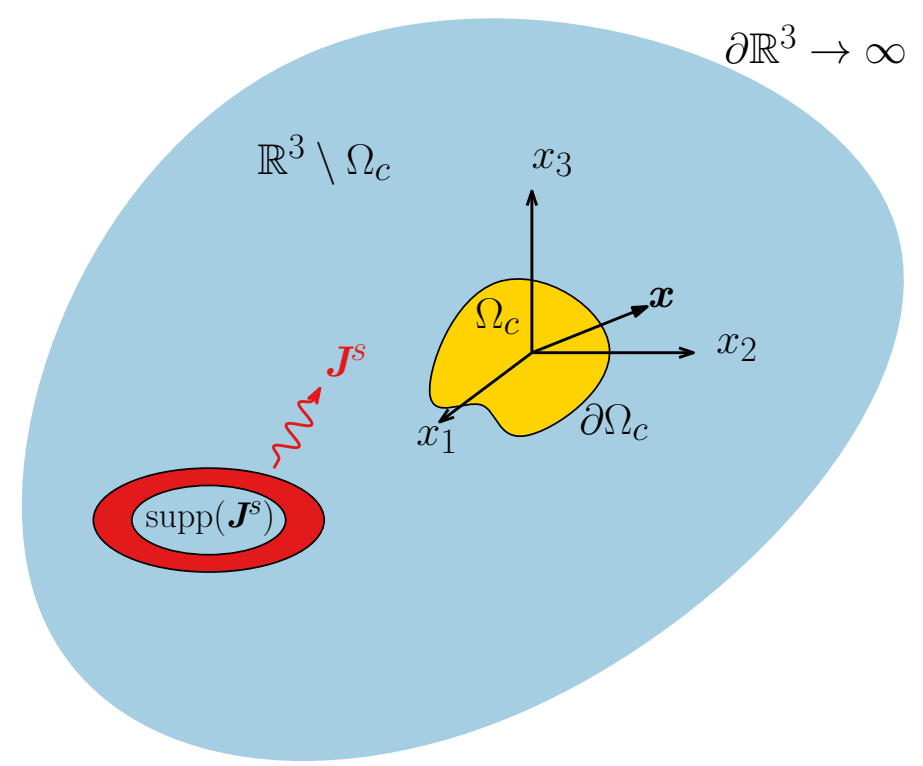

Figure 3.2: Diagram of the conducting region $\Omega_{c}$ and the magnet coils $\operatorname{supp}\left(\boldsymbol{J}^{s}\right)$, which give rise to the sourcing currents $\boldsymbol{J}^{s}$, contained within an unbounded region of free space $\mathbb{R}^{3}$. The general orthogonal coordinate system $\boldsymbol{x}=x_{1} \boldsymbol{e}_{1}+x_{2} \boldsymbol{e}_{2}+x_{3} \boldsymbol{e}_{3}$ is measured from the centre of $\Omega_{c}$.

First, the weak statements of the individual physics will be presented, before the coupled system of weighted residuals is obtained. The weak statement of the nonlinear static problem will then be presented, followed by the full non-linear transient problem. 


\subsubsection{Electromagnetics}

The strong form of the eddy current problem is given in Equation (2.36a), where an extra constraint on the magnetic vector potential, the Coulomb gauge, is given in (2.36b). The weighted residual form of the eddy current problem is obtained by equating (2.36a) to zero, pre-multiplication by real valued test or weighting functions and integration over $\mathbb{R}^{3}$, which results in

$$
R_{A}=\int_{\mathbb{R}^{3}} \boldsymbol{A}^{\delta} \cdot(\nabla \times\left(\mu^{-1} \nabla \times \boldsymbol{A}\right)+\underbrace{\gamma \frac{\partial \boldsymbol{A}}{\partial t}}_{\boldsymbol{J}^{o}}-\underbrace{\gamma \frac{\partial \boldsymbol{u}}{\partial t} \times(\nabla \times \boldsymbol{A})}_{\boldsymbol{J}^{l}}-\boldsymbol{J}^{s}) \mathrm{d} \Omega,
$$

where $\boldsymbol{A}^{\delta} \in X$ is a vector weighting function for the electromagnetic problem, the eddy and Lorentz current terms are only non-zero in the conducting region $\Omega_{c}$ and the source currents are only non-zero in the coils, $\operatorname{supp}\left(\boldsymbol{J}^{s}\right)$. Performing integration by parts on the curl-curl term ${ }^{1}$ over the complete domain $\mathbb{R}^{3}=\mathbb{R}^{3} \backslash \Omega_{c} \cup \Omega_{c}$ and separating the integral terms gives

$$
\begin{aligned}
R_{A}= & \int_{\mathbb{R}^{3}} \mu^{-1} \nabla \times \boldsymbol{A}^{\delta} \cdot \nabla \times \boldsymbol{A} \mathrm{d} \Omega+\int_{\Omega_{c}} \gamma \frac{\partial \boldsymbol{A}}{\partial t} \cdot \boldsymbol{A}^{\delta} \mathrm{d} \Omega-\int_{\Omega_{c}} \gamma \frac{\partial \boldsymbol{u}}{\partial t} \times(\nabla \times \boldsymbol{A}) \cdot \boldsymbol{A}^{\delta} \mathrm{d} \Omega \\
& -\int_{\operatorname{supp}\left(\boldsymbol{J}^{s}\right)} \boldsymbol{J}^{s} \cdot \boldsymbol{A}^{\delta} \mathrm{d} \Omega-\int_{\mathbb{R}^{3} \backslash \Omega_{c}} \nabla \cdot\left(\boldsymbol{A}^{\delta} \times \mu^{-1} \nabla \times \boldsymbol{A}\right) \mathrm{d} \Omega \\
& -\int_{\Omega_{c}} \nabla \cdot\left(\boldsymbol{A}^{\delta} \times \mu^{-1} \nabla \times \boldsymbol{A}\right) \mathrm{d} \Omega .
\end{aligned}
$$

Applying the divergence theorem and manipulating the resulting boundary terms ${ }^{2}$ 3 , see Remark 3.1, results in

$$
\begin{aligned}
R_{A}= & \int_{\mathbb{R}^{3}} \mu^{-1} \nabla \times \boldsymbol{A}^{\delta} \cdot \nabla \times \boldsymbol{A} \mathrm{d} \Omega+\int_{\Omega_{c}} \gamma \frac{\partial \boldsymbol{A}}{\partial t} \cdot \boldsymbol{A}^{\delta} \mathrm{d} \Omega-\int_{\Omega_{c}} \gamma \frac{\partial \boldsymbol{u}}{\partial t} \times(\nabla \times \boldsymbol{A}) \cdot \boldsymbol{A}^{\delta} \mathrm{d} \Omega \\
& -\int_{\operatorname{supp}\left(\boldsymbol{J}^{s}\right)} \boldsymbol{J}^{s} \cdot \boldsymbol{A}^{\delta} \mathrm{d} \Omega+\int_{\partial \Omega_{c}}\left[\boldsymbol{n} \times \mu^{-1} \nabla \times \boldsymbol{A}\right]_{\partial \Omega_{c}} \cdot \boldsymbol{A}^{\delta} \mathrm{d} S \\
& +\int_{\partial \mathbb{R}^{3}}\left(\boldsymbol{n} \times \mu^{-1} \nabla \times \boldsymbol{A}\right) \cdot \boldsymbol{A}^{\delta} \mathrm{d} S .
\end{aligned}
$$

Remark 3.1 Applying the divergence theorem to the two divergence terms in (3.8)

\footnotetext{
${ }^{1}$ Recall here the product rule of differentiation $\nabla \cdot\left(\boldsymbol{A}^{\delta} \times \mu^{-1} \nabla \times \boldsymbol{A}\right)=\mu^{-1} \nabla \times \boldsymbol{A} \cdot \nabla \times \boldsymbol{A}^{\delta}$ $\boldsymbol{A}^{\delta} \cdot\left(\nabla \times \mu^{-1} \nabla \times \boldsymbol{A}\right)$.

${ }^{2}$ Recall here the relationship $(\boldsymbol{A} \times \boldsymbol{B}) \cdot \boldsymbol{C}=\boldsymbol{A} \cdot(\boldsymbol{B} \times \boldsymbol{C})$.

${ }^{3}$ Recall here the property of the cross product $\boldsymbol{A} \times \boldsymbol{B}=-\boldsymbol{B} \times \boldsymbol{A}$.
} 
results $i^{2,3}$

$$
\begin{aligned}
\int_{\mathbb{R}^{3} \backslash \Omega_{c}} \nabla & \cdot\left(\boldsymbol{A}^{\delta} \times \mu^{-1} \nabla \times \boldsymbol{A}\right) \mathrm{d} \Omega+\int_{\Omega_{c}} \nabla \cdot\left(\boldsymbol{A}^{\delta} \times \mu^{-1} \nabla \times \boldsymbol{A}\right) \mathrm{d} \Omega= \\
& -\int_{\partial \mathbb{R}^{3}}\left(\boldsymbol{n} \times \mu^{-1} \nabla \times \boldsymbol{A}\right) \cdot \boldsymbol{A}^{\delta} \mathrm{d} S-\left.\int_{\partial \Omega_{c}^{+}}\left(\boldsymbol{n} \times \mu^{-1} \nabla \times \boldsymbol{A}\right)\right|_{+} \cdot \boldsymbol{A}^{\delta} \mathrm{d} S \\
& -\left.\int_{\partial \Omega_{c}^{-}}\left(\boldsymbol{n} \times \mu^{-1} \nabla \times \boldsymbol{A}\right)\right|_{-} \cdot \boldsymbol{A}^{\delta} \mathrm{d} S,
\end{aligned}
$$

where two boundary terms on the conductor boundary $\partial \Omega_{c}$ have emerged, due to contributions from the free space side, denoted by the subscript + on $\partial \Omega_{c}^{+}$, and the conductor side, denoted the subscript - on $\partial \Omega_{c}^{-}$.

Given that the normals on both sides of the conductor boundary are related by $\boldsymbol{n}_{-}=-\boldsymbol{n}_{+}=\boldsymbol{n}$, the divergence terms thus become

$$
\begin{aligned}
\int_{\mathbb{R}^{3} \backslash \Omega_{c}} & \nabla \cdot\left(\boldsymbol{A}^{\delta} \times \mu^{-1} \nabla \times \boldsymbol{A}\right) \mathrm{d} \Omega+\int_{\Omega_{c}} \nabla \cdot\left(\boldsymbol{A}^{\delta} \times \mu^{-1} \nabla \times \boldsymbol{A}\right) \mathrm{d} \Omega \\
= & -\int_{\partial \mathbb{R}^{3}}\left(\boldsymbol{n} \times \mu^{-1} \nabla \times \boldsymbol{A}\right) \cdot \boldsymbol{A}^{\delta} \mathrm{d} S-\int_{\partial \Omega_{c}} \boldsymbol{n} \times\left[\left.\left(\mu^{-1} \nabla \times \boldsymbol{A}\right)\right|_{-}-\left.\left(\mu^{-1} \nabla \times \boldsymbol{A}\right)\right|_{+}\right] \cdot \boldsymbol{A}^{\delta} \mathrm{d} S \\
= & -\int_{\partial \mathbb{R}^{3}}\left(\boldsymbol{n} \times \mu^{-1} \nabla \times \boldsymbol{A}\right) \cdot \boldsymbol{A}^{\delta} \mathrm{d} S-\int_{\partial \Omega_{c}}\left(\boldsymbol{n} \times\left[\mu^{-1} \nabla \times \boldsymbol{A}\right]_{\partial \Omega_{c}}\right) \cdot \boldsymbol{A}^{\delta} \mathrm{d} S,
\end{aligned}
$$

which is thus defined in terms of the interface condition in (2.36l).

Given that the problem lies in an unbounded domain $\mathbb{R}^{3}$, see Figure 3.2 , the boundary $\partial \mathbb{R}^{3}$ lies at $\infty$. In this case the decay condition of the eddy current problem, in $(2.36 \mathrm{e})$, can be applied directly ${ }^{4}$ and thus the terms associated with $\partial \mathbb{R}^{3}$ disappear. Substituting, also, the interface condition for the electromagnetic problem (2.361) results in the boundary terms vanishing and thus the weighted residual form of the electromagnetic problem becomes

$$
\begin{aligned}
R_{A}= & \int_{\mathbb{R}^{3}} \mu^{-1} \nabla \times \boldsymbol{A}^{\delta} \cdot \nabla \times \boldsymbol{A} \mathrm{d} \Omega+\int_{\Omega_{c}} \gamma \frac{\partial \boldsymbol{A}}{\partial t} \cdot \boldsymbol{A}^{\delta} \mathrm{d} \Omega \\
& -\int_{\Omega_{c}} \gamma \frac{\partial \boldsymbol{u}}{\partial t} \times(\nabla \times \boldsymbol{A}) \cdot \boldsymbol{A}^{\delta} \mathrm{d} \Omega-\int_{\operatorname{supp}\left(\boldsymbol{J}^{s}\right)} \boldsymbol{J}^{s} \cdot \boldsymbol{A}^{\delta} \mathrm{d} \Omega
\end{aligned}
$$

where the magnet coils, contained within $\operatorname{supp}\left(\boldsymbol{J}^{s}\right)$, are located in free space $\mathbb{R}^{3} \backslash \Omega_{c}$ at a finite distance from the conductors.

\footnotetext{
${ }^{4}$ The decay condition of the magnetic vector potential for the eddy current problem, when applied at $\infty$ yields $\boldsymbol{A}=\mathbf{0}$.
} 


\subsubsection{Mechanics}

Pre-multiplication of the linear elastic problem, in (2.36c), by real valued test or weighting functions and integration over the conducting region $\Omega_{c}$, results in

$$
R_{u}=\int_{\Omega_{c}} \boldsymbol{u}^{\delta} \cdot\left(\nabla \cdot\left(\boldsymbol{\sigma}^{m}(\boldsymbol{u})+\boldsymbol{\sigma}^{e}(\boldsymbol{A})\right)-\rho \frac{\partial^{2} \boldsymbol{u}}{\partial t^{2}}\right) \mathrm{d} \Omega,
$$

where $\boldsymbol{u}^{\delta} \in Y(\mathbf{0})$ is a vector weighting function for the mechanical problem. Performing integration by parts on the term involving the stress tensors ${ }^{5}$ gives

$$
\begin{aligned}
R_{u}=\int_{\Omega_{c}} \nabla \boldsymbol{u}^{\delta}:\left(\boldsymbol{\sigma}^{m}(\boldsymbol{u})+\boldsymbol{\sigma}^{e}(\boldsymbol{A})\right) \mathrm{d} \Omega & +\int_{\Omega_{c}} \rho \frac{\partial^{2} \boldsymbol{u}}{\partial t^{2}} \cdot \boldsymbol{u}^{\delta} \mathrm{d} \Omega \\
& -\int_{\Omega_{c}} \nabla \cdot\left(\left(\boldsymbol{\sigma}^{m}(\boldsymbol{u})+\boldsymbol{\sigma}^{e}(\boldsymbol{A})\right)^{T} \boldsymbol{u}^{\delta}\right) \mathrm{d} \Omega .
\end{aligned}
$$

Applying the divergence theorem and manipulating the resulting boundary term results in

$$
\begin{aligned}
R_{u}=\int_{\Omega_{c}} \nabla \boldsymbol{u}^{\delta}:\left(\boldsymbol{\sigma}^{m}(\boldsymbol{u})+\boldsymbol{\sigma}^{e}(\boldsymbol{A})\right) \mathrm{d} \Omega & +\int_{\Omega_{c}} \rho \frac{\partial^{2} \boldsymbol{u}}{\partial t^{2}} \cdot \boldsymbol{u}^{\delta} \mathrm{d} \Omega \\
& -\int_{\partial \Omega_{c}} \boldsymbol{u}^{\delta} \cdot\left(\boldsymbol{\sigma}^{m}(\boldsymbol{u})+\boldsymbol{\sigma}^{e}(\boldsymbol{A})\right) \boldsymbol{n} \mathrm{d} S
\end{aligned}
$$

The interface condition for the mechanical problem from $(2.36 \mathrm{j})$ can be applied to yield

$$
\begin{aligned}
R_{u}=\int_{\Omega_{c}} \nabla \boldsymbol{u}^{\delta}:\left(\boldsymbol{\sigma}^{m}(\boldsymbol{u})+\boldsymbol{\sigma}^{e}(\boldsymbol{A})\right) \mathrm{d} \Omega & +\int_{\Omega_{c}} \rho \frac{\partial^{2} \boldsymbol{u}}{\partial t^{2}} \cdot \boldsymbol{u}^{\delta} \mathrm{d} \Omega \\
& -\left.\int_{\partial \Omega_{c}} \boldsymbol{u}^{\delta} \cdot\left(P \boldsymbol{I}+\boldsymbol{\sigma}^{e}(\boldsymbol{A})\right)\right|_{\partial \Omega_{c}} ^{+} \boldsymbol{n} \mathrm{d} S .
\end{aligned}
$$

The conductor boundary can be separated into Dirichlet and Neumann portions $\partial \Omega=\partial \Omega_{c}^{D} \cup \partial \Omega_{c}^{N}$, illustrated in Figure 2.5. Thus the weighted residual form of the elasticity equation becomes

$$
\begin{aligned}
R_{u}=\int_{\Omega_{c}} \nabla \boldsymbol{u}^{\delta}:\left(\boldsymbol{\sigma}^{m}(\boldsymbol{u})+\boldsymbol{\sigma}^{e}(\boldsymbol{A})\right) \mathrm{d} \Omega & +\int_{\Omega_{c}} \rho \frac{\partial^{2} \boldsymbol{u}}{\partial t^{2}} \cdot \boldsymbol{u}^{\delta} \mathrm{d} \Omega \\
& -\left.\int_{\partial \Omega_{c}^{N}} \boldsymbol{u}^{\delta} \cdot\left(P \boldsymbol{I}+\boldsymbol{\sigma}^{e}(\boldsymbol{A})\right)\right|_{\partial \Omega_{c}^{N}} ^{+} \boldsymbol{n} \mathrm{d} S
\end{aligned}
$$

\footnotetext{
${ }^{5}$ Recall here the product rule of differentiation of the product between a tensor $\sigma$ and a vector $\boldsymbol{v}$, where from index notation $\nabla \cdot\left(\boldsymbol{\sigma}^{T} \boldsymbol{v}\right)=\frac{\partial}{\partial x_{j}}\left(\sigma_{i j} v_{i}\right)=\sigma_{i j} \frac{\partial v_{i}}{\partial x_{j}}+v_{i} \frac{\partial \sigma_{i j}}{\partial x_{j}}=\boldsymbol{\sigma}: \nabla \boldsymbol{v}+\boldsymbol{v} \cdot(\nabla \cdot \boldsymbol{\sigma})$.
} 
where the integral over the Dirichlet portion of the boundary vanishes, due to $\boldsymbol{u}^{\delta} \in$ $Y(\mathbf{0})$, which vanishes on this portion of the boundary.

\subsubsection{Acoustics}

Pre-multiplication of the acoustic Helmholtz problem, in (2.36d), by test or weighting functions and integration over the free space region $\mathbb{R}^{3} \backslash \Omega_{c}$, results in

$$
R_{P}=\int_{\mathbb{R}^{3} \backslash \Omega_{c}} P^{\delta}\left(\nabla^{2} P-\frac{1}{c^{2}} \frac{\partial^{2} P}{\partial t^{2}}+\nabla \cdot\left(\nabla \cdot \boldsymbol{\sigma}^{e}(\boldsymbol{A})\right)\right) \mathrm{d} \Omega
$$

where $P^{\delta} \in Z$ is a scalar weighting function for the acoustic problem. Performing integration by parts on the Laplacian term ${ }^{6}$ and the divergence-divergence term ${ }^{6}$ gives

$$
\begin{aligned}
R_{P}= & \int_{\mathbb{R}^{3} \backslash \Omega_{c}} \nabla P \cdot \nabla P^{\delta} \mathrm{d} \Omega+\int_{\mathbb{R}^{3} \backslash \Omega_{c}} \frac{1}{c^{2}} \frac{\partial^{2} P}{\partial t^{2}} P^{\delta} \mathrm{d} \Omega+\int_{\mathbb{R}^{3} \backslash \Omega_{c}}\left(\nabla \cdot \boldsymbol{\sigma}^{e}(\boldsymbol{A})\right) \cdot \nabla P^{\delta} \mathrm{d} \Omega \\
& -\int_{\mathbb{R}^{3} \backslash \Omega_{c}} \nabla \cdot\left(P^{\delta} \nabla P\right) \mathrm{d} \Omega-\int_{\mathbb{R}^{3} \backslash \Omega_{c}} \nabla \cdot\left(P^{\delta} \nabla \cdot \boldsymbol{\sigma}^{e}(\boldsymbol{A})\right) \mathrm{d} \Omega
\end{aligned}
$$

Applying the divergence theorem and manipulating the resulting boundary term results in

$$
\begin{aligned}
R_{P}= & \int_{\mathbb{R}^{3} \backslash \Omega_{c}}\left(\nabla P+\nabla \cdot \boldsymbol{\sigma}^{e}(\boldsymbol{A})\right) \cdot \nabla P^{\delta} \mathrm{d} \Omega+\int_{\mathbb{R}^{3} \backslash \Omega_{c}} \frac{1}{c^{2}} \frac{\partial^{2} P}{\partial t^{2}} P^{\delta} \mathrm{d} \Omega \\
& -\int_{\partial \Omega_{c}} \boldsymbol{n} \cdot\left(\nabla P+\nabla \cdot \boldsymbol{\sigma}^{e}(\boldsymbol{A})\right) P^{\delta} \mathrm{d} S-\int_{\partial \mathbb{R}^{3}} \boldsymbol{n} \cdot\left(\nabla P+\nabla \cdot \boldsymbol{\sigma}^{e}(\boldsymbol{A})\right) P^{\delta} \mathrm{d} S .
\end{aligned}
$$

Like in the mechanical problem, the conductor boundary can again be split into the Dirichlet and Neumann part. The interface conditions for the acoustic problem from $(2.36 \mathrm{~h})$ and $(2.36 \mathrm{i})$ can be applied to yield

$$
\begin{aligned}
R_{P}=\int_{\mathbb{R}^{3} \backslash \Omega_{c}} & \left(\nabla P+\nabla \cdot \boldsymbol{\sigma}^{e}(\boldsymbol{A})\right) \cdot \nabla P^{\delta} \mathrm{d} \Omega+\int_{\mathbb{R}^{3} \backslash \Omega_{c}} \frac{1}{c^{2}} \frac{\partial^{2} P}{\partial t^{2}} P^{\delta} \mathrm{d} \Omega \\
& -\left.\int_{\partial \Omega_{c}^{N}} \rho^{+} \boldsymbol{n} \cdot \frac{\partial^{2} \boldsymbol{u}}{\partial t^{2}}\right|_{\partial \Omega_{c}^{N}} P^{\delta} \mathrm{d} S-\int_{\partial \mathbb{R}^{3}}(\boldsymbol{n} \cdot \nabla P) P^{\delta} \mathrm{d} S
\end{aligned}
$$

\footnotetext{
${ }^{6}$ Recall here the product rule of differentiation of the product between a scalar $f$ and a vector $\boldsymbol{v}$, where from index notation $\nabla \cdot(f \boldsymbol{v})=\frac{\partial}{\partial x_{j}}\left(f v_{i}\right)=v_{i} \frac{\partial f}{\partial x_{j}}+f \frac{\partial \boldsymbol{v}_{i}}{\partial x_{j}}=\boldsymbol{v} \cdot \nabla f+f \nabla \boldsymbol{v}$.
} 
where the $\nabla \cdot \boldsymbol{\sigma}^{e}(\boldsymbol{A})$ term vanishes on the outer boundary, due to the fact that the coils are located a finite distance from $\Omega_{c}$ and hence well away from $\partial \mathbb{R}^{3}$.

Given that the problem lies in an unbounded domain $\mathbb{R}^{3}$, the boundary $\partial \mathbb{R}^{3}$ lies at $\infty$. In this case the Sommerfeld radiation condition, in (2.36f), can be applied exactly to yield

$$
\begin{aligned}
R_{P}=\int_{\mathbb{R}^{3} \backslash \Omega_{c}}\left(\nabla P+\nabla \cdot \boldsymbol{\sigma}^{e}(\boldsymbol{A})\right) & \cdot \nabla P^{\delta} \mathrm{d} \Omega+\int_{\mathbb{R}^{3} \backslash \Omega_{c}} \frac{1}{c^{2}} \frac{\partial^{2} P}{\partial t^{2}} P^{\delta} \mathrm{d} \Omega \\
& -\left.\int_{\partial \Omega_{c}^{N}} \rho^{+} \boldsymbol{n} \cdot \frac{\partial^{2} \boldsymbol{u}}{\partial t^{2}}\right|_{\partial \Omega_{c}^{N}} P^{\delta} \mathrm{d} S+\int_{\partial \mathbb{R}^{3}} \frac{\partial P}{\partial t} P^{\delta} \mathrm{d} S
\end{aligned}
$$

The treatment of this term for computational problems set on truncated domains will be discussed in Chapter 4 .

\subsubsection{Coupled Problem}

Combining the weighted residual statements of all three fields, in (3.10), (3.15) and (3.20), the fully coupled weighted residual statement of the transient problem is: Find weak solutions $(\boldsymbol{A}, \boldsymbol{u}, P)(t) \in\left(X \times Y\left(\boldsymbol{u}_{D}\right) \times Z\right)[0, T]$ such that

$$
\begin{aligned}
R_{A}\left(\boldsymbol{A}^{\delta} ; \boldsymbol{A}, \boldsymbol{u}\right)= & \int_{\mathbb{R}^{3}} \mu^{-1} \nabla \times \boldsymbol{A}^{\delta} \cdot \nabla \times \boldsymbol{A} \mathrm{d} \Omega+\int_{\Omega_{c}} \gamma \frac{\partial \boldsymbol{A}}{\partial t} \cdot \boldsymbol{A}^{\delta} \mathrm{d} \Omega \\
& -\int_{\Omega_{c}} \gamma \frac{\partial \boldsymbol{u}}{\partial t} \times(\nabla \times \boldsymbol{A}) \cdot \boldsymbol{A}^{\delta} \mathrm{d} \Omega-\int_{\operatorname{supp}\left(\boldsymbol{J}^{s}\right)} \boldsymbol{J}^{s} \cdot \boldsymbol{A}^{\delta} \mathrm{d} \Omega, \\
R_{u}\left(\boldsymbol{u}^{\delta} ; \boldsymbol{A}, \boldsymbol{u}, P\right)= & \int_{\Omega_{c}}\left(\boldsymbol{\sigma}^{m}(\boldsymbol{u})+\boldsymbol{\sigma}^{e}(\boldsymbol{A})\right): \nabla \boldsymbol{u}^{\delta} \mathrm{d} \Omega+\int_{\Omega_{c}} \rho \frac{\partial^{2} \boldsymbol{u}}{\partial t^{2}} \cdot \boldsymbol{u}^{\delta} \mathrm{d} \Omega \\
& -\left.\int_{\partial \Omega_{c}^{N}} \boldsymbol{u}^{\delta} \cdot\left(P \boldsymbol{I}+\boldsymbol{\sigma}^{e}(\boldsymbol{A})\right)\right|_{\partial \Omega_{c}} ^{+} \boldsymbol{n} \mathrm{d} S, \\
R_{P}\left(P^{\delta} ; \boldsymbol{A}, \boldsymbol{u}, P\right)= & \int_{\mathbb{R}^{3} \backslash \Omega_{c}}\left(\nabla P+\nabla \cdot \boldsymbol{\sigma}^{e}(\boldsymbol{A})\right) \cdot \nabla P^{\delta} \mathrm{d} \Omega+\int_{\mathbb{R}^{3} \backslash \Omega_{c}} \frac{1}{c^{2}} \frac{\partial^{2} P}{\partial t^{2}} P^{\delta} \mathrm{d} \Omega \\
& -\left.\int_{\partial \Omega_{c}^{N}} \rho^{+} \boldsymbol{n} \cdot \frac{\partial^{2} \boldsymbol{u}}{\partial t^{2}}\right|_{\partial \Omega_{c}^{N}} ^{-} P^{\delta} \mathrm{d} S,
\end{aligned}
$$

for all $\left(\boldsymbol{A}^{\delta}, \boldsymbol{u}^{\delta}, P^{\delta}\right) \in X \times Y(\mathbf{0}) \times Z$.

The weighted residual form of the static problem is obtained analogously to (3.21) by obtaining the weak form of Equation (2.38). From the transient weak form in (3.21), using the same additive splitting of the current source as in Section 2.5.2, expressing the fields purely in terms of their static component only- hence time independent- the static weak form of the coupled system becomes: Find weak 
solutions $\left(\boldsymbol{A}^{D C}, \boldsymbol{u}^{D C}, P^{D C}\right)(t) \in\left(X \times Y\left(\boldsymbol{u}_{D}^{D C}\right) \times Z\right)[0, T]$ such that

$$
\begin{aligned}
R_{A}\left(\boldsymbol{A}^{\delta} ; \boldsymbol{A}^{D C}, \boldsymbol{u}^{D C}\right)= & \int_{\mathbb{R}^{3}} \mu^{-1} \nabla \times \boldsymbol{A}^{\delta} \cdot \nabla \times \boldsymbol{A}^{D C} \mathrm{~d} \Omega-\int_{\operatorname{supp}\left(\boldsymbol{J}^{D C}\right)} \boldsymbol{J}^{D C} \cdot \boldsymbol{A}^{\delta} \mathrm{d} \Omega, \\
R_{u}\left(\boldsymbol{u}^{\delta} ; \boldsymbol{A}^{D C}, \boldsymbol{u}^{D C}, P^{D C}\right)= & \int_{\Omega_{c}}\left(\boldsymbol{\sigma}^{m}\left(\boldsymbol{u}^{D C}\right)+\boldsymbol{\sigma}^{e}\left(\boldsymbol{A}^{D C}\right)\right): \nabla \boldsymbol{u}^{\delta} \mathrm{d} \Omega \\
& -\left.\int_{\partial \Omega_{c}^{N}} \boldsymbol{u}^{\delta} \cdot\left(P^{D C} \boldsymbol{I}+\boldsymbol{\sigma}^{e}\left(\boldsymbol{A}^{D C}\right)\right)\right|_{\partial \Omega_{c}} ^{+} \boldsymbol{n} \mathrm{d} S, \\
R_{P}\left(P^{\delta} ; \boldsymbol{A}^{D C}, \boldsymbol{u}^{D C}, P^{D C}\right)= & \int_{\mathbb{R}^{3} \backslash \Omega_{c}}\left(\nabla P^{D C}+\nabla \cdot \boldsymbol{\sigma}^{e}\left(\boldsymbol{A}^{D C}\right)\right) \cdot \nabla P^{\delta} \mathrm{d} \Omega
\end{aligned}
$$

for all $\left(\boldsymbol{A}^{\delta}, \boldsymbol{u}^{\delta}, P^{\delta}\right) \in X \times Y(\mathbf{0}) \times Z$.

\subsection{Linearisation of the Static Problem}

In order to apply the superior NR strategy to the non-linear static transmission problem, described in (2.38) Chapter 2, the weak form of the static equation system must be linearised with respect to the solution fields. This is achieved by taking the directional derivatives of the weak variational statement of the static problem, in order to obtain the tangent stiffness (or Jacobian) operator.

First, by following a similar procedure to that carried out in Section 3.3, the static weighted residual form of the static problem in (2.38) is: Find weak solutions $\left(\boldsymbol{A}^{D C}, \boldsymbol{u}^{D C}, P^{D C}\right) \in X \times Y\left(\boldsymbol{u}_{D}^{D C}\right) \times Z$ such that

$$
\begin{aligned}
R_{A}^{D C}\left(\boldsymbol{A}^{\delta} ; \boldsymbol{A}^{D C}\right):= & \int_{\mathbb{R}^{3}}\left(\mu^{-1} \nabla \times \boldsymbol{A}^{D C} \cdot \nabla \times \boldsymbol{A}^{\delta}\right) \mathrm{d} \Omega \\
& -\int_{\operatorname{supp}\left(\boldsymbol{J}^{D C}\right)} \boldsymbol{J}^{D C} \cdot \boldsymbol{A}^{\delta} \mathrm{d} \Omega \\
R_{u}^{D C}\left(\boldsymbol{u}^{\delta} ; \boldsymbol{A}^{D C}, \boldsymbol{u}^{D C}, P^{D C}\right):= & \int_{\Omega_{c}}\left(\boldsymbol{\sigma}^{m}\left(\boldsymbol{u}^{D C}\right)+\boldsymbol{\sigma}^{e}\left(\boldsymbol{A}^{D C}\right)\right): \nabla \boldsymbol{u}^{\delta} \mathrm{d} \Omega \\
& -\left.\int_{\partial \Omega_{c}^{N}} \boldsymbol{u}^{\delta} \cdot\left(P^{D C} \boldsymbol{I}+\boldsymbol{\sigma}^{e}\left(\boldsymbol{A}^{D C}\right)\right)\right|_{\partial \Omega_{c}} ^{+} \boldsymbol{n} \mathrm{d} S, \\
R_{P}^{D C}\left(P^{\delta} ; \boldsymbol{A}^{D C}, P^{D C}\right):= & \int_{\mathbb{R}^{3} \backslash \Omega_{c}}\left(\nabla P^{D C}+\nabla \cdot \boldsymbol{\sigma}^{e}\left(\boldsymbol{A}^{D C}\right)\right) \cdot \nabla P^{\delta} \mathrm{d} \Omega,
\end{aligned}
$$

for all $\left(\boldsymbol{A}^{\delta}, \boldsymbol{u}^{\delta}, P^{\delta}\right) \in X \times Y(\mathbf{0}) \times Z$. From the definition of the Maxwell stress tensor $\boldsymbol{\sigma}^{e}$ in (2.1) it is clear that this term is quadratic in the magnetic vector potential and that taking directional derivatives of this term will result in a linear approximation.

Remark 3.2 The directional derivative of weighted residuals $R_{q}$ in the direction of 


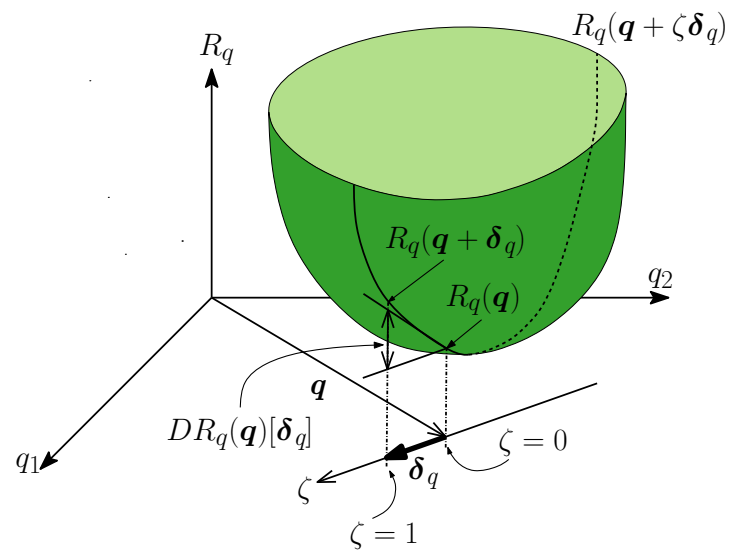

Figure 3.3: Illustration of the directional derivative of the weighted residual $R_{q}$ about the solution $\boldsymbol{q}$. The weighted residual functions, shown as the green surface, are linearised about the solution by taking the directional derivatives in the direction of the incremental solution $\boldsymbol{\delta}_{q}$ as the scaled distance in the incremental solution $\zeta \rightarrow 0$.

an increment in the solution $\boldsymbol{\delta}_{q}$ may be evaluated, as described in [38], by

$$
D R_{q}(\boldsymbol{q})\left[\boldsymbol{\delta}_{q}\right]=\left.\frac{\mathrm{d}}{\mathrm{d} \zeta}\right|_{\zeta=0} R\left(\boldsymbol{q}+\zeta \boldsymbol{\delta}_{q}\right)
$$

where $\boldsymbol{q}$ is the solution vector and $\zeta$ is a scaling parameter in the incremental $d i$ rection of the solution, illustrated in Figure 3.3. This definition arises by taking a first-order Taylor series expansion ${ }^{7}$ of $R_{q}\left(\boldsymbol{q}+\zeta \boldsymbol{\delta}_{q}\right)$ about $\zeta=0$ and setting $\zeta=1$.

Thus taking directional derivatives of the weighted residuals $\left(R_{A}^{D C}, R_{u}^{D C}, R_{P}^{D C}\right)$ in the direction of the incremental solution variables $\left(\boldsymbol{\delta}_{A}^{D C}, \boldsymbol{\delta}_{u}^{D C}, \delta_{P}^{D C}\right)$ yields

$$
\begin{aligned}
D R_{A}^{D C}\left(\boldsymbol{A}^{\delta} ; \boldsymbol{A}^{D C}\right)\left[\boldsymbol{\delta}_{A}^{D C}\right]= & \int_{\mathbb{R}^{3}}\left(\mu^{-1} \nabla \times \boldsymbol{\delta}_{A}^{D C} \cdot \nabla \times \boldsymbol{A}^{\delta}\right) \mathrm{d} \Omega, \\
D R_{u}^{D C}\left(\boldsymbol{u}^{\delta} ; \boldsymbol{A}^{D C}, \boldsymbol{u}^{D C}, P^{D C}\right)\left[\boldsymbol{\delta}_{A}^{D C}\right]= & \int_{\Omega_{c}} \mu^{-1} \mathcal{S}\left(\boldsymbol{A}^{D C}, \boldsymbol{\delta}_{A}^{D C}\right): \nabla \boldsymbol{u}^{\delta} \mathrm{d} \Omega \\
& -\left.\int_{\partial \Omega_{c}^{N}} \mu_{0}^{-1} \mathcal{S}\left(\boldsymbol{A}^{D C}, \boldsymbol{\delta}_{A}^{D C}\right)\right|^{+} \boldsymbol{n} \cdot \boldsymbol{u}^{\delta} \mathrm{d} S, \\
D R_{u}^{D C}\left(\boldsymbol{u}^{\delta} ; \boldsymbol{A}^{D C}, \boldsymbol{u}^{D C}, P^{D C}\right)\left[\boldsymbol{\delta}_{u}^{D C}\right]= & \int_{\Omega_{c}} \boldsymbol{\sigma}^{m}\left(\boldsymbol{\delta}_{u}^{D C}\right): \nabla \boldsymbol{u}^{\delta} \mathrm{d} \Omega, \\
D R_{u}^{D C}\left(\boldsymbol{u}^{\delta} ; \boldsymbol{A}^{D C}, \boldsymbol{u}^{D C}, P^{D C}\right)\left[\delta_{P}^{D C}\right]= & -\left.\int_{\partial \Omega_{c}^{N}} \delta_{P}^{D C}\right|^{+} \boldsymbol{n} \cdot \boldsymbol{u}^{\delta} \mathrm{d} S,
\end{aligned}
$$

${ }^{7}$ The Taylor series expansion of a function $f(x)$ around a fixed point $x=a$ results in $f(x)=$ $f(a)+\left.(x-a) \frac{\mathrm{d} f(x)}{\mathrm{d} x}\right|_{x=a}+\ldots+\left.\frac{1}{n !}(x-a)^{n} \frac{\mathrm{d}^{n} f(x)}{\mathrm{d} x^{n}}\right|_{x=a}+\ldots$ 


$$
\begin{aligned}
D R_{P}^{D C}\left(P^{\delta} ; \boldsymbol{A}^{D C}, P^{D C},\right)\left[\boldsymbol{\delta}_{A}^{D C}\right] & =-\int_{\operatorname{supp}\left(\boldsymbol{J}^{D C}\right)}\left(\left(\nabla \times \boldsymbol{\delta}_{A}^{D C}\right) \times \boldsymbol{J}^{D C}\right) \cdot \nabla P^{\delta} \mathrm{d} \Omega \\
D R_{P}^{D C}\left(P^{\delta} ; \boldsymbol{A}^{D C} P^{D C}\right)\left[\delta_{P}^{D C}\right] & =\int_{\mathbb{R}^{3} \backslash \Omega_{c}} \nabla \delta_{P}^{D C} \cdot \nabla P^{\delta} \mathrm{d} \Omega
\end{aligned}
$$

where the linearised electromagnetic stress tensor, defined as

$\mathcal{S}\left(\boldsymbol{A}^{D C}, \boldsymbol{\delta}_{A}^{D C}\right):=\nabla \times \boldsymbol{A}^{D C} \otimes \nabla \times \boldsymbol{\delta}_{A}^{D C}+\nabla \times \boldsymbol{\delta}_{A}^{D C} \otimes \nabla \times \boldsymbol{A}^{D C}-\left(\nabla \times \boldsymbol{A}^{D C} \cdot \nabla \times \boldsymbol{\delta}_{A}^{D C}\right) \boldsymbol{I}$,

has been introduced to simplify the notation of the linearised system. The system of equations in (3.24) is linear in the incremental solution variables and thus it becomes clear that the weighted residual system has been linearised.

In order to solve the linear system, at a continuous level, the NR iterative solution procedure is applied. Equations $(3.23,3.24)$ can be used to introduce the NR scheme for a particular iteration $[\mathrm{k}]$ as: Find $\left(\boldsymbol{\delta}_{A}^{D C[\mathrm{k}]}, \boldsymbol{\delta}_{u}^{D C[\mathrm{k}]}, \delta_{P}^{D C[\mathrm{k}]}\right) \in X \times Y(\mathbf{0}) \times Z$ such that

$$
\begin{gathered}
D R_{A}^{D C}\left(\boldsymbol{A}^{\delta} ; \boldsymbol{A}^{D C[\mathrm{k}]}\right)\left[\boldsymbol{\delta}_{A}^{D C[\mathrm{k}]}\right]=-R_{A}^{D C}\left(\boldsymbol{A}^{\delta} ; \boldsymbol{A}^{D C[\mathrm{k}]}\right) \\
D R_{u}^{D C}\left(\boldsymbol{u}^{\delta} ; \boldsymbol{A}^{D C[\mathrm{k}]}, \boldsymbol{u}^{D C[\mathrm{k}]}, P^{D C[\mathrm{k}]}\right)\left[\boldsymbol{\delta}_{A}^{D C[\mathrm{k}]}\right]+ \\
D R_{u}^{D C}\left(\boldsymbol{u}^{\delta} ; \boldsymbol{A}^{D C[\mathrm{k}]}, \boldsymbol{u}^{D C[\mathrm{k}]}, P^{D C[\mathrm{k}]}\right)\left[\boldsymbol{\delta}_{u}^{D C[\mathrm{k}]}\right]+ \\
D R_{u}^{D C}\left(\boldsymbol{u}^{\delta} ; \boldsymbol{A}^{D C[\mathrm{k}]}, \boldsymbol{u}^{D C[\mathrm{k}]}, P^{D C[\mathrm{k}]}\right)\left[\delta_{P}^{D C[\mathrm{k}]}\right]=-R_{u}^{D C}\left(\boldsymbol{u}^{\delta} ; \boldsymbol{A}^{D C[\mathrm{k}]}, \boldsymbol{u}^{D C[\mathrm{k}]}, P^{D C[\mathrm{k}]}\right),
\end{gathered}
$$

$$
\begin{aligned}
& D R_{P}^{D C}\left(P^{\delta} ; \boldsymbol{A}^{D C[\mathrm{k}]}, P^{D C[\mathrm{k}]}\right)\left[\boldsymbol{\delta}_{A}^{D C[\mathrm{k}]}\right]+ \\
& \quad D R_{P}^{D C}\left(P^{\delta} ; \boldsymbol{A}^{D C}, P^{D C[\mathrm{k}]}\right)\left[\delta_{P}^{D C[\mathrm{k}]}\right]=-R_{P}^{D C}\left(P^{\delta} ; \boldsymbol{A}^{D C[\mathrm{k}]}, P^{D C[\mathrm{k}]}\right),
\end{aligned}
$$

for all $\left(\boldsymbol{A}^{\delta}, \boldsymbol{u}^{\delta}, P^{\delta}\right) \in X \times Y(\mathbf{0}) \times Z$ where the updated solutions at the current iteration are given by

$$
\begin{aligned}
& \boldsymbol{A}^{D C[\mathrm{k}+1]}=\boldsymbol{A}^{D C[\mathrm{k}]}+\boldsymbol{\delta}_{A}^{D C[\mathrm{k}]}, \\
& \boldsymbol{u}^{D C[\mathrm{k}+1]}=\boldsymbol{u}^{D C[\mathrm{k}]}+\boldsymbol{\delta}_{u}^{D C[\mathrm{k}]} \\
& P^{D C[\mathrm{k}+1]}=P^{D C[\mathrm{k}]}+\delta_{P}^{D C[\mathrm{k}]}
\end{aligned}
$$

The NR scheme starts by defining the initial guess of the solution vector such that $\left(\boldsymbol{A}^{D C[0]}, \boldsymbol{u}^{D C[0]}, P^{D C[0]}\right) \in\left(X \times Y\left(\boldsymbol{u}_{D}^{D C}\right) \times Z\right)$. The residuals and directional derivatives must then evaluated using the initial guess of the solution vector. The system in (3.26) is then solved to obtain the incremental solution variables which are 
used in (3.27) to obtain the updated solution variables. Traditionally, the scheme would then require a number of iterations to be performed until the solution converges. The convergence of the scheme is determined by calculating the norm of the weighted residual vector and comparing it against a user defined tolerance. However, at this stage, it is already useful to note that, due to the specific nature of the equations, (3.26a) can be solved independently, followed by (3.26c) and then (3.26b) without iteration. Moreover, if the system is solved monolithically, then the solution will converge to $\left(\boldsymbol{A}^{D C}, \boldsymbol{u}^{D C}, P^{D C}\right) \in\left(X \times Y\left(\boldsymbol{u}_{D}^{D C}\right) \times Z\right)$ in a single iteration.

To permit the computational solution of (3.26) a spatial FE discretisation is required, which is considered in Chapter 4.

\subsection{Linearisation of the Dynamic Problem}

The weighted residual system of the dynamic problem in (3.21), similarly to that of the static problem, is non-linear in the solution variables due to the quadratic dependence of the Maxwell stress on the magnetic vector potential. The transient system, however, also contains other terms which involve the product of two of the solution fields. Thus, in order to solve this system the equations must first be linearised.

In this Section, two linearisation methods will be considered, the linearised and non-linear approaches. Firstly, the linearisation of the coupled non-linear system of weighted residuals, in (3.21), with respect to the complete solution fields (static plus transient components) is considered by taking their directional derivatives directly, denoted the non-linear approach. With the knowledge of the static solution, from the linearised static system in (3.26), an alternative linearisation method, where the residual system is linearised about the static solution, is considered. The resulting linearised system, when cast in a monolithic NR iterative procedure, permits a simplified single-step monolithic solution strategy where the resulting system is linear in the transient solution variables, therefore denoted as the linearised approach. This approach is physically motivated by the difference in the strengths between the static and transient fields in MRI scanners. 


\subsubsection{Time Dependent Formulation}

\section{Non-linear Approach}

In order to perform the linearisation of the full transient problem the weighted residuals of the dynamic system are recalled as: Find weak solutions $(\boldsymbol{A}, \boldsymbol{u}, P)(t) \in$ $\left(X \times Y\left(\boldsymbol{u}_{D}\right) \times Z\right)[0, T]$ such that

$$
\begin{aligned}
R_{A}\left(\boldsymbol{A}^{\delta} ; \boldsymbol{A}, \boldsymbol{u}\right)= & \int_{\mathbb{R}^{3}} \mu^{-1} \nabla \times \boldsymbol{A}^{\delta} \cdot \nabla \times \boldsymbol{A} \mathrm{d} \Omega+\int_{\Omega_{c}} \gamma \frac{\partial \boldsymbol{A}}{\partial t} \cdot \boldsymbol{A}^{\delta} \mathrm{d} \Omega \\
& -\int_{\Omega_{c}} \gamma \frac{\partial \boldsymbol{u}}{\partial t} \times(\nabla \times \boldsymbol{A}) \cdot \boldsymbol{A}^{\delta} \mathrm{d} \Omega-\int_{\operatorname{supp}\left(\boldsymbol{J}^{s}\right)} \boldsymbol{J}^{s} \cdot \boldsymbol{A}^{\delta} \mathrm{d} \Omega, \\
R_{u}\left(\boldsymbol{u}^{\delta} ; \boldsymbol{A}, \boldsymbol{u}, P\right)= & \int_{\Omega_{c}}\left(\boldsymbol{\sigma}^{m}(\boldsymbol{u})+\boldsymbol{\sigma}^{e}(\boldsymbol{A})\right): \nabla \boldsymbol{u}^{\delta} \mathrm{d} \Omega+\int_{\Omega_{c}} \rho \frac{\partial^{2} \boldsymbol{u}}{\partial t^{2}} \cdot \boldsymbol{u}^{\delta} \mathrm{d} \Omega \\
& -\left.\int_{\partial \Omega_{c}^{N}} \boldsymbol{u}^{\delta} \cdot\left(P \boldsymbol{I}+\boldsymbol{\sigma}^{e}(\boldsymbol{A})\right)\right|_{\partial \Omega_{c}} ^{+} \boldsymbol{n} \mathrm{d} S \\
R_{P}\left(P^{\delta} ; \boldsymbol{A}, \boldsymbol{u}, P\right)= & \int_{\mathbb{R}^{3} \backslash \Omega_{c}}\left(\nabla P+\nabla \cdot \boldsymbol{\sigma}^{e}(\boldsymbol{A})\right) \cdot \nabla P^{\delta} \mathrm{d} \Omega+\int_{\mathbb{R}^{3} \backslash \Omega_{c}} \frac{1}{c^{2}} \frac{\partial^{2} P}{\partial t^{2}} P^{\delta} \mathrm{d} \Omega \\
& -\left.\int_{\partial \Omega_{c}^{N}} \rho^{+} \boldsymbol{n} \cdot \frac{\partial^{2} \boldsymbol{u}}{\partial t^{2}}\right|_{\partial \Omega_{c}^{N}} ^{-} P^{\delta} \mathrm{d} S
\end{aligned}
$$

for all $\left(\boldsymbol{A}^{\delta}, \boldsymbol{u}^{\delta}, P^{\delta}\right) \in X \times Y(\mathbf{0}) \times Z$. Again, taking the directional derivatives of these weighted residuals in the direction of the incremental solution variables $\left(\boldsymbol{\delta}_{A}, \boldsymbol{\delta}_{u}, \delta_{P}\right)$ yields

$$
\begin{aligned}
D R_{A}\left(\boldsymbol{A}^{\delta} ; \boldsymbol{A}, \boldsymbol{u}\right)\left[\boldsymbol{\delta}_{A}\right]= & \int_{\mathbb{R}^{3}}\left(\mu^{-1} \nabla \times \boldsymbol{\delta}_{A} \cdot \nabla \times \boldsymbol{A}^{\delta}+\gamma \frac{\partial \boldsymbol{\delta}_{A}}{\partial t} \cdot \boldsymbol{A}^{\delta}\right) \mathrm{d} \Omega \\
& -\int_{\Omega_{c}}\left(\gamma \frac{\partial \boldsymbol{u}}{\partial t} \times\left(\nabla \times \boldsymbol{\delta}_{A}\right)\right) \cdot \boldsymbol{A}^{\delta} \mathrm{d} \Omega \\
D R_{A}\left(\boldsymbol{A}^{\delta} ; \boldsymbol{A}, \boldsymbol{u}\right)\left[\boldsymbol{\delta}_{u}\right]= & -\int_{\Omega_{c}}\left(\gamma \frac{\partial \boldsymbol{\delta}_{u}}{\partial t} \times(\nabla \times \boldsymbol{A}) \cdot \boldsymbol{A}^{\delta}\right) \mathrm{d} \Omega \\
D R_{u}\left(\boldsymbol{u}^{\delta} ; \boldsymbol{A}, \boldsymbol{u}, P\right)\left[\boldsymbol{\delta}_{A}\right]= & \int_{\Omega_{c}} \mu^{-1} \mathcal{S}\left(\boldsymbol{A}, \boldsymbol{\delta}_{A}\right): \nabla \boldsymbol{u}^{\delta} \mathrm{d} \Omega \\
& -\left.\int_{\partial \Omega_{c}^{N}} \mu_{0}^{-1} \mathcal{S}\left(\boldsymbol{A}, \boldsymbol{\delta}_{A}\right)\right|_{\partial \Omega_{c}} ^{+} \boldsymbol{n} \cdot \boldsymbol{u}^{\delta} \mathrm{d} S \\
D R_{u}\left(\boldsymbol{u}^{\delta} ; \boldsymbol{A}, \boldsymbol{u}, P\right)\left[\boldsymbol{\delta}_{u}\right]= & \int_{\Omega_{c}}\left(\boldsymbol{\sigma}^{m}\left(\boldsymbol{\delta}_{u}\right): \nabla \boldsymbol{u}^{\delta}+\rho \frac{\partial^{2} \boldsymbol{\delta}_{u}}{\partial t^{2}} \cdot \boldsymbol{u}^{\delta}\right) \mathrm{d} \Omega \\
D R_{u}\left(\boldsymbol{u}^{\delta} ; \boldsymbol{A}, \boldsymbol{u}, P\right)\left[\delta_{P}\right]= & -\left.\int_{\partial \Omega_{c}^{N}} \delta_{P}\right|_{\partial \Omega_{c}} ^{+} \boldsymbol{n} \cdot \boldsymbol{u}^{\delta} \mathrm{d} S, \\
D R_{P}\left(P^{\delta} ; \boldsymbol{A}, \boldsymbol{u}, P\right)\left[\boldsymbol{\delta}_{A}\right]= & -\int_{\operatorname{supp}\left(\boldsymbol{J}^{s}\right)}\left(\nabla \times \boldsymbol{\delta}_{A} \times\left(\nabla \times\left(\mu_{0}^{-1} \nabla \times \boldsymbol{A}\right)\right)\right.
\end{aligned}
$$




$$
\begin{aligned}
& \left.+\nabla \times \boldsymbol{A} \times\left(\nabla \times\left(\mu_{0}^{-1} \nabla \times \boldsymbol{\delta}_{A}\right)\right)\right) \cdot \nabla P^{\delta} \mathrm{d} \Omega, \\
D R_{P}\left(P^{\delta} ; \boldsymbol{A}, \boldsymbol{u}, P\right)\left[\boldsymbol{\delta}_{u}\right]= & -\left.\int_{\partial \Omega_{c}^{N}} \rho^{+} \frac{\partial^{2} \boldsymbol{\delta}_{u}}{\partial t^{2}}\right|_{\partial \Omega_{c}^{N}} ^{-} \cdot \boldsymbol{n} P^{\delta} \mathrm{d} S \\
D R_{P}\left(P^{\delta} ; \boldsymbol{A}, \boldsymbol{u}, P\right)\left[\delta_{P}\right]= & \int_{\mathbb{R}^{3} \backslash \Omega_{c}}\left(\nabla \delta_{P} \cdot \nabla P^{\delta}+\frac{1}{c^{2}} \frac{\partial^{2} \delta_{P}}{\partial t^{2}} \cdot P^{\delta}\right) \mathrm{d} \Omega .
\end{aligned}
$$

In the above, the alternative form of $\nabla \cdot \boldsymbol{\sigma}^{e}$, introduced in Equation (2.34), has been used when linearising $R_{P}$ in order to simplify the notation. This alternative form defines clearly that this term is present only in the coils $\operatorname{supp}\left(\boldsymbol{J}^{s}\right)$.

The typical strategy for solving the temporal system in (3.28) and (3.29), after spatial discretisation, would be to adopt a discrete time integration scheme for solving the temporal component of the solution fields. The Newton-Raphson scheme could then be applied at each time-step to solve the non-linear system using the directional derivatives computed above. The discussion and treatment of such a solution method is delayed until Chapter 6 .

To fully resolve the non-linearities of the system, the non-linear approach is required. However, despite the accuracy of such approaches they are often computationally intensive. In the interest of developing a fast computational technique a new strategy for linearising the system of equations is adopted. This approach is justified on the basis that the static fields are orders of magnitude stronger than the corresponding transient fields, due to the forms of the excitation in MRI scanners. This scheme is particularly beneficial for the case when $\boldsymbol{J}^{A C}$ is a harmonic current excitation.

\section{Linearised Approach}

Rather than solving the full non-linear equations at each time-step, the full transient system in (3.28) can instead be linearised about the static solution, illustrated in Figure 3.4. This linearisation technique, known as the linearised approach, in the context of MRI scanners, is motivated by the knowledge that the static DC current source $\boldsymbol{J}^{D C}$ is several orders of magnitude stronger than the weaker AC time varying source $\boldsymbol{J}^{A C}$, leading to a strong DC field and a weaker time varying AC field [25]. Similar techniques, involving the additive split of a non-linear problem to a series of linear problems, have been successfully applied to the field of computational mechanics such as; analysis of structural membranes [91, 90, 92], high order mesh generation [195] and in biomedical applications [24].

A rigorous comparison between the linearised and non-linear approaches will be 
presented later in Chapter 6. In this case the residuals of the dynamic problem are

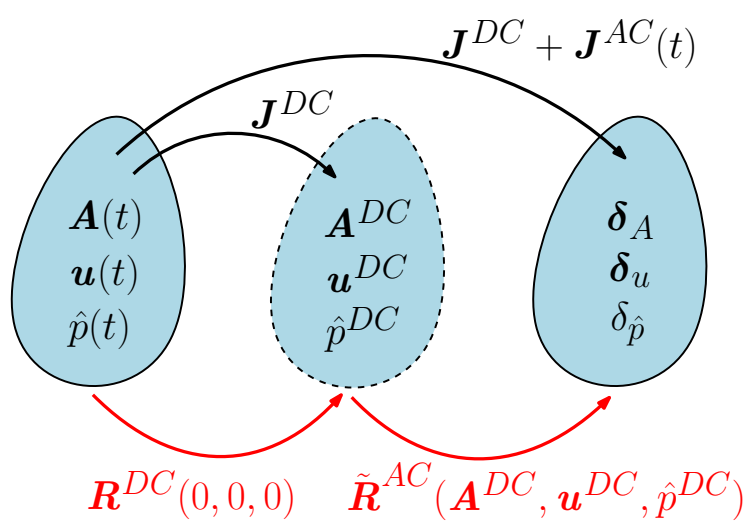

Figure 3.4: Linearisation of the full temporal solution about the static field (DC) component, where $\boldsymbol{R}^{D C}(0,0,0)=\left[R_{A}^{D C}(0), R_{u}^{D C}(0), R_{P}^{D C}(0)\right]^{T}$ denotes the static system evaluated at $t=0$ and $\tilde{\boldsymbol{R}}^{A C}\left(\boldsymbol{A}^{D C}, \boldsymbol{u}^{D C}, P^{D C}\right)=\left[\tilde{R}_{A}^{A C}\left(\boldsymbol{A}^{D C}\right), \tilde{R}_{u}^{A C}\left(\boldsymbol{u}^{D C}\right), \tilde{R}_{P}^{A C}\left(P^{D C}\right)\right]^{T}$ denotes the transient system.

evaluated at the static solution $\left(\boldsymbol{A}^{D C}, \boldsymbol{u}^{D C}, P^{D C}\right)$ and the source current is split into its DC and AC components which results in

$$
\begin{aligned}
\tilde{R}_{A}^{A C}\left(\boldsymbol{A}^{\delta}\right):=R_{A}\left(\boldsymbol{A}^{\delta} ; \boldsymbol{A}^{D C}, \boldsymbol{u}^{D C}\right)= & \int_{\mathbb{R}^{3}} \mu^{-1} \nabla \times \boldsymbol{A}^{\delta} \cdot \nabla \times \boldsymbol{A}^{D C} \mathrm{~d} \Omega \\
& -\int_{\operatorname{supp}\left(\boldsymbol{J}^{s}\right)}\left(\boldsymbol{J}^{D C}+\boldsymbol{J}^{A C}\right) \cdot \boldsymbol{A}^{\delta} \mathrm{d} \Omega, \quad(3.30 \mathrm{a}) \\
\tilde{R}_{u}^{A C}\left(\boldsymbol{u}^{\delta}\right):=R_{u}\left(\boldsymbol{u}^{\delta} ; \boldsymbol{A}^{D C}, \boldsymbol{u}^{D C}, P^{D C}\right)= & \int_{\Omega_{c}}\left(\boldsymbol{\sigma}^{m}\left(\boldsymbol{u}^{D C}\right)+\boldsymbol{\sigma}^{e}\left(\boldsymbol{A}^{D C}\right)\right): \nabla \boldsymbol{u}^{\delta} \mathrm{d} \Omega \\
& -\left.\int_{\partial \Omega_{c}^{N}} \boldsymbol{u}^{\delta} \cdot\left(P^{D C} \boldsymbol{I}+\boldsymbol{\sigma}^{e}\left(\boldsymbol{A}^{D C}\right)\right)\right|_{\partial \Omega_{c}} ^{+} \boldsymbol{n} \mathrm{d} S, \\
\tilde{R}_{P}^{A C}\left(P^{\delta}\right):=R_{P}\left(P^{\delta} ; \boldsymbol{A}^{D C}, \boldsymbol{u}^{D C}, P^{D C}\right)= & \int_{\mathbb{R}^{3} \backslash \Omega_{c}} \nabla P^{D C} \cdot \nabla P^{\delta} \mathrm{d} \Omega, \\
- & \int_{\operatorname{supp}\left(\boldsymbol{J}^{s}\right)}\left(\nabla \times \boldsymbol{A}^{D C} \times\left(\boldsymbol{J}^{D C}+\boldsymbol{J}^{A C}\right)\right) \cdot \nabla P^{\delta} \mathrm{d} \Omega,
\end{aligned}
$$

where the time derivative terms have vanished, due to that fact that the DC fields are independent of time and the alternative form of $\nabla \cdot \boldsymbol{\sigma}^{e}\left(\boldsymbol{A}^{D C}\right)$, from $(2.34)$, has been used in $(3.30 \mathrm{c})$. From the definitions of the residuals of the static problem, obtained in (3.23), some of the terms in (3.30) can be eliminated to yield

$$
\begin{aligned}
\tilde{R}_{A}^{A C}\left(\boldsymbol{A}^{\delta} ; \boldsymbol{A}^{D C}, \boldsymbol{u}^{D C}\right) & =-\int_{\operatorname{supp}\left(\boldsymbol{J}^{A C}\right)} \boldsymbol{J}^{A C} \cdot \boldsymbol{A}^{\delta} \mathrm{d} \Omega, \\
\tilde{R}_{A}^{A C}\left(\boldsymbol{u}^{\delta} ; \boldsymbol{A}^{D C}, \boldsymbol{u}^{D C}, P^{D C}\right) & =0
\end{aligned}
$$




$$
\tilde{R}_{P}^{A C}\left(P^{\delta} ; \boldsymbol{A}^{D C}, \boldsymbol{u}^{D C}, P^{D C}\right)=-\int_{\operatorname{supp}\left(J^{A C}\right)}\left(\left(\nabla \times \boldsymbol{A}^{D C}\right) \times \boldsymbol{J}^{A C}\right) \cdot \nabla P^{\delta} \mathrm{d} \Omega .
$$

The directional derivatives must also be evaluated about the static solution. Performing this evaluation, by substituting the static solution $\left(\boldsymbol{A}^{D C}, \boldsymbol{u}^{D C}, P^{D C}\right)$ into the directional derivative definitions in (3.29), analogously to the evaluation of the residuals above, yields

$$
\begin{aligned}
& D \tilde{R}_{A}^{A C}\left(\boldsymbol{A}^{\delta}\right)\left[\boldsymbol{\delta}_{A}\right]:=D R_{A}\left(\boldsymbol{A}^{\delta} ; \boldsymbol{A}^{D C}, \boldsymbol{u}^{D C}\right)\left[\boldsymbol{\delta}_{A}\right] \\
& =\int_{\mathbb{R}^{3}}\left(\mu^{-1} \nabla \times \boldsymbol{\delta}_{A} \cdot \nabla \times \boldsymbol{A}^{\delta}+\gamma \frac{\partial \boldsymbol{\delta}_{A}}{\partial t} \cdot \boldsymbol{A}^{\delta}\right) \mathrm{d} \Omega, \\
& D \tilde{R}_{A}^{A C}\left(\boldsymbol{A}^{\delta} ; \boldsymbol{A}^{D C}\right)\left[\boldsymbol{\delta}_{u}\right]:=D R_{A}\left(\boldsymbol{A}^{\delta} ; \boldsymbol{A}^{D C}, \boldsymbol{u}^{D C}\right)\left[\boldsymbol{\delta}_{u}\right] \\
& =-\int_{\Omega_{c}} \gamma \frac{\partial \boldsymbol{\delta}_{u}}{\partial t} \times\left(\nabla \times \boldsymbol{A}^{D C}\right) \cdot \boldsymbol{A}^{\delta} \mathrm{d} \Omega, \\
& D \tilde{R}_{u}^{A C}\left(\boldsymbol{u}^{\delta} ; \boldsymbol{A}^{D C}\right)\left[\boldsymbol{\delta}_{A}\right]:=D R_{u}\left(\boldsymbol{u}^{\delta} ; \boldsymbol{A}^{D C}, \boldsymbol{u}^{D C}, P^{D C}\right)\left[\boldsymbol{\delta}_{A}\right] \\
& =\int_{\Omega_{c}} \mu^{-1} \mathcal{S}\left(\boldsymbol{A}^{D C}, \boldsymbol{\delta}_{A}\right): \nabla \boldsymbol{u}^{\delta} \mathrm{d} \Omega \\
& -\left.\int_{\partial \Omega_{c}^{N}} \mu_{0}^{-1} \mathcal{S}\left(\boldsymbol{A}^{D C}, \boldsymbol{\delta}_{A}\right)\right|_{\partial \Omega_{c}} ^{+} \boldsymbol{n} \cdot \boldsymbol{u}^{\delta} \mathrm{d} S, \\
& D \tilde{R}_{u}^{A C}\left(\boldsymbol{u}^{\delta}\right)\left[\boldsymbol{\delta}_{u}\right]:=D R_{u}\left(\boldsymbol{u}^{\delta} ; \boldsymbol{A}^{D C}, \boldsymbol{u}^{D C}, P^{D C}\right)\left[\boldsymbol{\delta}_{u}\right] \\
& =\int_{\Omega_{c}}\left(\boldsymbol{\sigma}^{m}\left(\boldsymbol{\delta}_{u}\right): \nabla \boldsymbol{u}^{\delta}+\rho \frac{\partial \boldsymbol{\delta}_{u}}{\partial t} \cdot \boldsymbol{u}^{\delta}\right) \mathrm{d} \Omega, \\
& D \tilde{R}_{u}^{A C}\left(\boldsymbol{u}^{\delta}\right)\left[\delta_{P}\right]:=D R_{u}\left(\boldsymbol{u}^{\delta} ; \boldsymbol{A}^{D C}, \boldsymbol{u}^{D C}, P^{D C}\right)\left[\delta_{P}\right] \\
& =-\left.\int_{\partial \Omega_{c}^{N}} \delta_{P}\right|^{+} \boldsymbol{n} \cdot \boldsymbol{u}^{\delta} \mathrm{d} S, \\
& D \tilde{R}_{P}^{A C}\left(P^{\delta} ; \boldsymbol{A}^{D C}\right)\left[\boldsymbol{\delta}_{A}\right]:=D R_{P}\left(P^{\delta} ; \boldsymbol{A}^{D C}, P^{D C}\right)\left[\boldsymbol{\delta}_{A}\right] \\
& =-\int_{\operatorname{supp}\left(\boldsymbol{J}^{D C}\right)}\left(\left(\nabla \times \boldsymbol{\delta}_{A}\right) \times \boldsymbol{J}^{D C}\right) \cdot \nabla P^{\delta} \mathrm{d} \Omega \\
& -\int_{\operatorname{supp}\left(\boldsymbol{J}^{D C}\right) \cup \operatorname{supp}\left(\boldsymbol{J}^{A C}\right)}\left(\nabla \times \boldsymbol{A}^{D C} \times\left(\nabla \times\left(\mu_{0}^{-1} \nabla \times \boldsymbol{\delta}_{A}\right)\right)\right) \cdot \nabla P^{\delta} \mathrm{d} \Omega, \\
& D \tilde{R}_{P}^{A C}\left(P^{\delta}\right)\left[\boldsymbol{\delta}_{u}\right]:=D R_{P}\left(P^{\delta} ; \boldsymbol{A}^{D C}, \boldsymbol{u}^{D C}, P^{D C}\right)\left[\boldsymbol{\delta}_{u}\right] \\
& =-\left.\int_{\partial \Omega_{c}^{N}} \rho^{+} \frac{\partial \boldsymbol{\delta}_{u}}{\partial t}\right|^{-} \cdot \boldsymbol{n} P^{\delta} \mathrm{d} S, \\
& D \tilde{R}_{P}^{A C}\left(P^{\delta}\right)\left[\delta_{P}\right]:=D R_{P}\left(P^{\delta} ; \boldsymbol{A}^{D C}, P^{D C}\right)\left[\delta_{P}\right] \\
& =\int_{\mathbb{R}^{3} \backslash \Omega_{c}}\left(\nabla \delta_{P} \cdot \nabla P^{\delta}+\frac{1}{c^{2}} \frac{\partial^{2} \delta_{P}}{\partial t^{2}} \cdot P^{\delta}\right) \mathrm{d} \Omega,
\end{aligned}
$$

where the temporal derivative terms of the static fields vanish, due to the independence of the static fields on time. The equation system, in (3.31) and (3.32), can be 
solved monolithically using a Newton-Raphson procedure. However, given that the system is linear in the solution variables $\left(\boldsymbol{\delta}_{A}, \boldsymbol{\delta}_{u}, \delta_{P}\right)$ it can be solved in a single iteration as the directional derivative and residual terms are all independent of iteration. Thus the linear system can be st up as: Find $\left(\boldsymbol{\delta}_{A}, \boldsymbol{\delta}_{u}, \delta_{P}\right)(t) \in(X \times Y(\mathbf{0}) \times Z)[0, T]$ such that

$$
\begin{aligned}
& D \tilde{R}_{A}^{A C}\left(\boldsymbol{A}^{\delta} ; \boldsymbol{A}^{D C}, \boldsymbol{u}^{D C}\right)\left[\boldsymbol{\delta}_{A}\right]+ \\
& D \tilde{R}_{A}^{A C}\left(\boldsymbol{A}^{\delta} ; \boldsymbol{A}^{D C}, \boldsymbol{u}^{D C}\right)\left[\boldsymbol{\delta}_{u}\right]=-\tilde{R}_{A}^{D C}\left(\boldsymbol{A}^{\delta} ; \boldsymbol{A}^{D C}, \boldsymbol{u}^{D C},\right), \\
& D \tilde{R}_{u}^{A C}\left(\boldsymbol{u}^{\delta} ; \boldsymbol{A}^{D C}, \boldsymbol{u}^{D C}, P^{D C}\right)\left[\boldsymbol{\delta}_{A}\right]+ \\
& D \tilde{R}_{u}^{A C}\left(\boldsymbol{u}^{\delta} ; \boldsymbol{A}^{D C}, \boldsymbol{u}^{D C}, P^{D C}\right)\left[\boldsymbol{\delta}_{u}\right]+ \\
& D \tilde{R}_{u}^{A C}\left(\boldsymbol{u}^{\delta} ; \boldsymbol{A}^{D C}, \boldsymbol{u}^{D C}, P^{D C}\right)\left[\delta_{P}\right]=-\tilde{R}_{u}^{A C}\left(\boldsymbol{u}^{\delta} ; \boldsymbol{A}^{D C}, \boldsymbol{u}^{D C}, P^{D C}\right), \\
& D \tilde{R}_{P}^{A C}\left(P^{\delta} ; \boldsymbol{A}^{D C}, \boldsymbol{u}^{D C}, P^{D C}\right)\left[\boldsymbol{\delta}_{A}\right]+ \\
& D \tilde{R}_{P}^{A C}\left(P^{\delta} ; \boldsymbol{A}^{D C}, \boldsymbol{u}^{D C}, P^{D C}\right)\left[\boldsymbol{\delta}_{u}\right]+ \\
& D \tilde{R}_{P}^{A C}\left(P^{\delta} ; \boldsymbol{A}^{D C}, \boldsymbol{u}^{D C}, P^{D C}\right)\left[\delta_{P}\right]=-\tilde{R}_{P}^{A C}\left(P^{\delta} ; \boldsymbol{A}^{D C}, \boldsymbol{u}^{D C}, P^{D C}\right),
\end{aligned}
$$

for all $\left(\boldsymbol{A}^{\delta}, \boldsymbol{u}^{\delta}, P^{\delta}\right) \in X \times Y(\mathbf{0}) \times Z$. The complete transient solutions of the fields are given by

$$
\begin{aligned}
\boldsymbol{A}(t) & =\boldsymbol{A}^{D C}+\boldsymbol{\delta}_{A}(t), \\
\boldsymbol{u}(t) & =\boldsymbol{u}^{D C}+\boldsymbol{\delta}_{u}(t), \\
P(t) & =P^{D C}+\delta_{P}(t) .
\end{aligned}
$$

This system, after discretisation, can be solved using a temporal integration scheme to obtain the transient response of the system. The benefit of this system is that it can be solved monolithically in a single iteration and for this reason the approach is called the linearised approach. Moreover, after applying suitable temporal integration schemes, the directional derivative terms in (3.32) will also be independent of time, and thus need to be computed only once for a given problem.

\subsubsection{Time Harmonic Formulation}

By noting that $\boldsymbol{A}^{D C}, \boldsymbol{u}^{D C}, P^{D C}$ are all time invariant, it is clear that the residuals and the directional derivatives in (3.31) and (3.32), respectively, are linear in the time dependent terms $\boldsymbol{\delta}_{A}, \boldsymbol{\delta}_{u}, \delta_{P}$ and $\boldsymbol{J}^{A C}$. Rather than integrating the fields in time, this linearity of the system, in time, allows for a time harmonic representation 
of the fields to be used, where the solution fields may be described as

$$
\begin{aligned}
\boldsymbol{\delta}_{A} & \rightarrow \boldsymbol{\delta}_{\mathcal{A}} e^{\mathrm{i} \omega t}, \\
\boldsymbol{\delta}_{u} & \rightarrow \boldsymbol{\delta}_{\mathcal{U}} e^{\mathrm{i} \omega t}, \\
\delta_{P} & \rightarrow \delta_{\mathcal{P}} e^{\mathrm{i} \omega t}, \\
\boldsymbol{J}^{A C} & \rightarrow \boldsymbol{J}^{A C} e^{\mathrm{i} \omega t},
\end{aligned}
$$

where, in this case, the total time dependent fields and source current density can be recovered, similar to (3.34), by

$$
\begin{aligned}
\boldsymbol{A}(t) & =\boldsymbol{A}^{D C}+\operatorname{Re}\left(\boldsymbol{\delta}_{\mathcal{A}} e^{\mathrm{i} \omega t}\right), \\
\boldsymbol{u}(t) & =\boldsymbol{u}^{D C}+\operatorname{Re}\left(\boldsymbol{\delta}_{\mathcal{U}} e^{\mathrm{i} \omega t}\right), \\
P(t) & =P^{D C}+\operatorname{Re}\left(\delta_{\mathcal{P}} e^{\mathrm{i} \omega t}\right), \\
\boldsymbol{J}^{s}(t) & =\boldsymbol{J}^{D C}+\operatorname{Re}\left(\boldsymbol{J}^{A C} e^{\mathrm{i} \omega t}\right),
\end{aligned}
$$

This time harmonic representation of the fields eliminates the time dependence so that the resulting transient equations depend only on space.

In the above definitions in $(3.35) \mathcal{A}, \boldsymbol{U}, \mathcal{P}$ and $\boldsymbol{g}$ represent the complex amplitudes of the corresponding time varying fields $\boldsymbol{A}, \boldsymbol{u}, P$ and $\boldsymbol{J}$, respectively, and $\omega$ denotes the angular frequency of the driving current in the gradient coils, in the case of a harmonic excitation. In reality, the gradient coils are driven using nonharmonic pulse type excitation sequences, illustrated in Figure 1.4. However, the time signals of the sequences can be decomposed into the different frequency modes, using a FFT as shown in Figure 2.2, and the same approach can still be applied to each frequency to analyse the behaviour of the system for the complete range of frequencies in the signal. Thus, the solution to the linear harmonic problem becomes: Find $\left(\boldsymbol{\delta}_{\mathcal{A}}, \boldsymbol{\delta}_{\mathfrak{U}}, \delta_{\mathcal{P}}\right) \in X \times Y\left(\boldsymbol{U}_{D}^{A C}\right) \times Z$ such that

$$
\begin{aligned}
D \tilde{R}_{\mathcal{A}}^{A C}\left(\boldsymbol{A}^{\delta}\right)\left[\boldsymbol{\delta}_{\mathcal{A}}\right]+D \tilde{R}_{\mathcal{A}}^{A C}\left(\boldsymbol{A}^{\delta} ; \boldsymbol{A}^{D C}\right)\left[\boldsymbol{\delta}_{\mathcal{U}}\right] & =-\tilde{R}_{\mathcal{A}}^{A C}\left(\boldsymbol{A}^{\delta}\right) \\
& =\int_{\operatorname{supp}\left(\boldsymbol{g}^{A C}\right)} \boldsymbol{g}^{A C} \cdot \boldsymbol{A}^{\delta} \mathrm{d} \Omega,
\end{aligned}
$$

$$
\begin{aligned}
D \tilde{R}_{\mathcal{U}}^{A C}\left(\boldsymbol{u}^{\delta} ; \boldsymbol{A}^{D C}\right)\left[\boldsymbol{\delta}_{\mathcal{A}}\right]+D \tilde{R}_{\mathcal{U}}^{A C}\left(\boldsymbol{u}^{\delta}\right)\left[\boldsymbol{\delta}_{\mathcal{U}}\right]+D \tilde{R}_{\mathcal{U}}^{A C}\left(\boldsymbol{u}^{\delta}\right)\left[\delta_{\mathcal{P}}\right] & =-\tilde{R}_{\mathcal{U}}^{A C}\left(\boldsymbol{u}^{\delta}\right) \\
& =0 \\
D \tilde{R}_{\mathcal{P}}^{A C}\left(P^{\delta} ; \boldsymbol{A}^{D C}\right)\left[\boldsymbol{\delta}_{\mathcal{A}}\right]+D \tilde{R}_{\mathcal{P}}^{A C}\left(P^{\delta}\right)\left[\boldsymbol{\delta}_{\mathcal{U}}\right]+D \tilde{R}_{\mathcal{P}}^{A C}\left(P^{\delta}\right)\left[\delta_{\mathcal{P}}\right] & =-\tilde{R}_{\mathcal{P}}^{A C}\left(P^{\delta}\right)
\end{aligned}
$$




$$
=\int_{\operatorname{supp}\left(\boldsymbol{g}^{A C}\right)}\left(\nabla \times \boldsymbol{A}^{D C}\right) \times \boldsymbol{g}^{A C} \cdot \nabla P^{\delta} \mathrm{d} \Omega
$$

for all $\left(\boldsymbol{A}^{\delta}, \boldsymbol{u}^{\delta}, P^{\delta}\right) \in X \times Y(\mathbf{0}) \times Z$ where it is assumed that the Dirichlet boundary value of the displacements are transformed similarly to the fields in (3.35) as $\boldsymbol{u}_{D}^{A C} \rightarrow$ $\boldsymbol{U}_{D}^{A C} e^{\mathrm{i} \omega t}$ and the definitions of $X, Y$ and $Z$ are now contained in $\mathbb{C}^{3}$ and valid for complex variables.

The full temporal solution is then given by the sum of its static and time varying components, which in the case of a single frequency excitation are given by

$$
\begin{aligned}
\boldsymbol{A}(t) & =\boldsymbol{A}^{D C}+\operatorname{Re}\left(\boldsymbol{\delta}_{\mathcal{A}} e^{\mathrm{i} \omega t}\right), \\
\boldsymbol{u}(t) & =\boldsymbol{u}^{D C}+\operatorname{Re}\left(\boldsymbol{\delta}_{\mathfrak{U}} e^{\mathrm{i} \omega t}\right), \\
P(t) & =P^{D C}+\operatorname{Re}\left(\delta_{\mathcal{P}} e^{\mathrm{i} \omega t}\right) .
\end{aligned}
$$

From the definitions in (3.35), the first and second order temporal derivatives of the fields can be expressed in terms of the imaginary unit " $\mathrm{i}$ " and the angular frequency $\omega^{89}$. Thus, the directional derivatives in equation (3.37) explicitly become

$$
\begin{aligned}
& D \tilde{R}_{\mathcal{A}}^{A C}\left(\boldsymbol{A}^{\delta}\right)\left[\boldsymbol{\delta}_{\mathcal{A}}\right]= \int_{\mathbb{R}^{3}}\left(\mu^{-1} \nabla \times \boldsymbol{\delta}_{\mathcal{A}} \cdot \nabla \times \boldsymbol{A}^{\delta}+\mathrm{i} \omega \gamma \boldsymbol{\delta}_{\mathcal{A}} \cdot \boldsymbol{A}^{\delta}\right) \mathrm{d} \Omega, \\
& D \tilde{R}_{\mathcal{A}}^{A C}\left(\boldsymbol{A}^{\delta} ; \boldsymbol{A}^{D C}\right)\left[\boldsymbol{\delta}_{\mathcal{U}}\right]=-\int_{\Omega_{c}} \mathrm{i} \omega \gamma \boldsymbol{\delta}_{\mathcal{U}} \times\left(\nabla \times \boldsymbol{A}^{D C}\right) \cdot \boldsymbol{A}^{\delta} \mathrm{d} \Omega, \\
& D \tilde{R}_{\mathcal{U}}^{A C}\left(\boldsymbol{u}^{\delta} ; \boldsymbol{A}^{D C}\right)\left[\boldsymbol{\delta}_{\mathcal{A}}\right]= \int_{\Omega_{c}} \mu^{-1} \mathcal{S}\left(\boldsymbol{A}^{D C}, \boldsymbol{\delta}_{\mathcal{A}}\right): \nabla \boldsymbol{u}^{\delta} \mathrm{d} \Omega \\
&-\left.\int_{\partial \Omega_{c}^{N}} \mu_{0}^{-1} \mathcal{S}\left(\boldsymbol{A}^{D C}, \boldsymbol{\delta}_{\mathcal{A}}\right)\right|^{+} \boldsymbol{n} \cdot \boldsymbol{u}^{\delta} \mathrm{d} S, \\
& D \tilde{R}_{\mathcal{U}}^{A C}\left(\boldsymbol{u}^{\delta}\right)\left[\boldsymbol{\delta}_{\mathcal{U}}\right]= \int_{\Omega_{c}}\left(\boldsymbol{\sigma}^{m}\left(\boldsymbol{\delta}_{\mathfrak{U}}\right): \nabla \boldsymbol{u}^{\delta}-\rho \omega^{2} \boldsymbol{\delta}_{\mathfrak{U}} \cdot \boldsymbol{u}^{\delta}\right) \mathrm{d} \Omega, \\
& D \tilde{R}_{\mathcal{U}}^{A C}\left(\boldsymbol{u}^{\delta}\right)\left[\delta_{\mathcal{P}}\right]=-\left.\int_{\partial \Omega_{c}^{N}} \delta_{\mathcal{P}}\right|^{+} \boldsymbol{n} \cdot \boldsymbol{u}^{\delta} \mathrm{d} S, \\
& D \tilde{R}_{\mathcal{P}}^{A C}\left(P^{\delta} ; \boldsymbol{A}^{D C}\right)\left[\boldsymbol{\delta}_{\mathcal{A}}\right]=-\int_{\operatorname{supp}\left(\boldsymbol{J}^{D C}\right)}\left(\left(\nabla \times \boldsymbol{\delta}_{\mathscr{A}}\right) \times \boldsymbol{J}^{D C}\right) \cdot \nabla P^{\delta} \mathrm{d} \Omega \\
&-\int_{\operatorname{supp}\left(J^{D C}\right) \cup \operatorname{supp}\left(\boldsymbol{g}^{A C}\right)}\left(\nabla \times \boldsymbol{A}^{D C} \times\left(\nabla \times\left(\mu_{0}^{-1} \nabla \times \boldsymbol{\delta}_{\mathcal{A}}\right)\right)\right) \cdot \nabla P^{\delta} \mathrm{d} \Omega,
\end{aligned}
$$

\footnotetext{
${ }^{8}$ The first order time derivative of a transient field, using a time harmonic representation, gives $\frac{\partial}{\partial t}\left(\boldsymbol{Q} e^{i \omega t}\right)=i \omega \boldsymbol{Q} e^{i \omega t}$

${ }^{9}$ The second order time derivative of a transient field, using a time harmonic representation, gives $\frac{\partial^{2}}{\partial t^{2}}\left(\boldsymbol{Q} e^{\mathrm{i} \omega t}\right)=\mathrm{i}^{2} \omega^{2} \boldsymbol{Q} e^{\mathrm{i} \omega t}=-\omega^{2} \boldsymbol{Q} e^{\mathrm{i} \omega t}$.
} 


$$
\begin{aligned}
D \tilde{R}_{\mathcal{P}}^{A C}\left(P^{\delta}\right)\left[\boldsymbol{\delta}_{\mathcal{U}}\right] & =\left.\int_{\partial \Omega_{c}^{N}} \omega^{2} \rho^{+} \boldsymbol{\delta}_{\mathcal{U}}\right|^{-} \cdot \boldsymbol{n} P^{\delta} \mathrm{d} S \\
D \tilde{R}_{\mathcal{P}}^{A C}\left(P^{\delta}\right)\left[\delta_{\mathcal{P}}\right] & =\int_{\mathbb{R}^{3} \backslash \Omega_{c}}\left(\nabla \delta_{\mathcal{P}} \cdot \nabla P^{\delta}-\frac{\omega^{2}}{c^{2}} \delta_{\mathcal{P}} \cdot P^{\delta}\right) \mathrm{d} \Omega
\end{aligned}
$$

The linear system, in (3.37), is linear in each of the unknown update fields $\left(\boldsymbol{\delta}_{\mathcal{A}}, \boldsymbol{\delta}_{\mathfrak{U}}, \delta_{\mathcal{P}}\right)$ and as a result, the system, when solved monolithically, can be obtained in a single solution step. In the absence of pressure, this formulation of the linearised approach becomes identical to the fixed point magneto-mechanical solution strategy proposed in [150] for the dynamic case. However, in this approach, the assumptions which were made in deriving the fixed point scheme are no longer made.

\subsection{Chapter Summary}

This Chapter has presented a novel coupling methodology for the solution to coupled acousto-magneto-mechanical problems in MRI scanners, which results in a monolithic single step solution strategy at a continuous level, named the linearised approach. This strategy greatly reduces the computational cost of solving the system. Furthermore, the extension to a time harmonic (Fourier domain) representation has resulted in a coupled system that is independent of time and thus provides a computationally efficient algorithm for rapid solutions of the fundamental characteristics of MRI scanner designs.

The weak variational statements of the electromagnetic, mechanical and acoustic problems have been derived and the relevant boundary and interface conditions, discussed in Chapter 2, are applied to obtain the weighted residual form of the static and transient coupled transmission problem. The corresponding directional derivatives of the static and transient problems have been computed to define the monolithic linear systems which can be solved in a single iteration, using a NR solution procedure, due to the nature of the linearisation technique.

From the objectives of this Thesis, discussed in section 1.4, the topics discussed in this Chapter align with the objective: "To develop an efficient novel solution procedure, named the linearised approach, through a rigorous linearisation of the non-linear system of equations."

In the next Chapter, the numerical treatment of the monolithic linearised approach will be considered through: the reduction to axisymmetric domains, the application of the far field boundary conditions located at $\infty$, the discussion of $h p$ finite elements and the finite element spatial discretisation of the coupled linearised 
weighted residual system. 


\section{Chapter 4}

\section{Computational Treatment of the Linearised Approach}

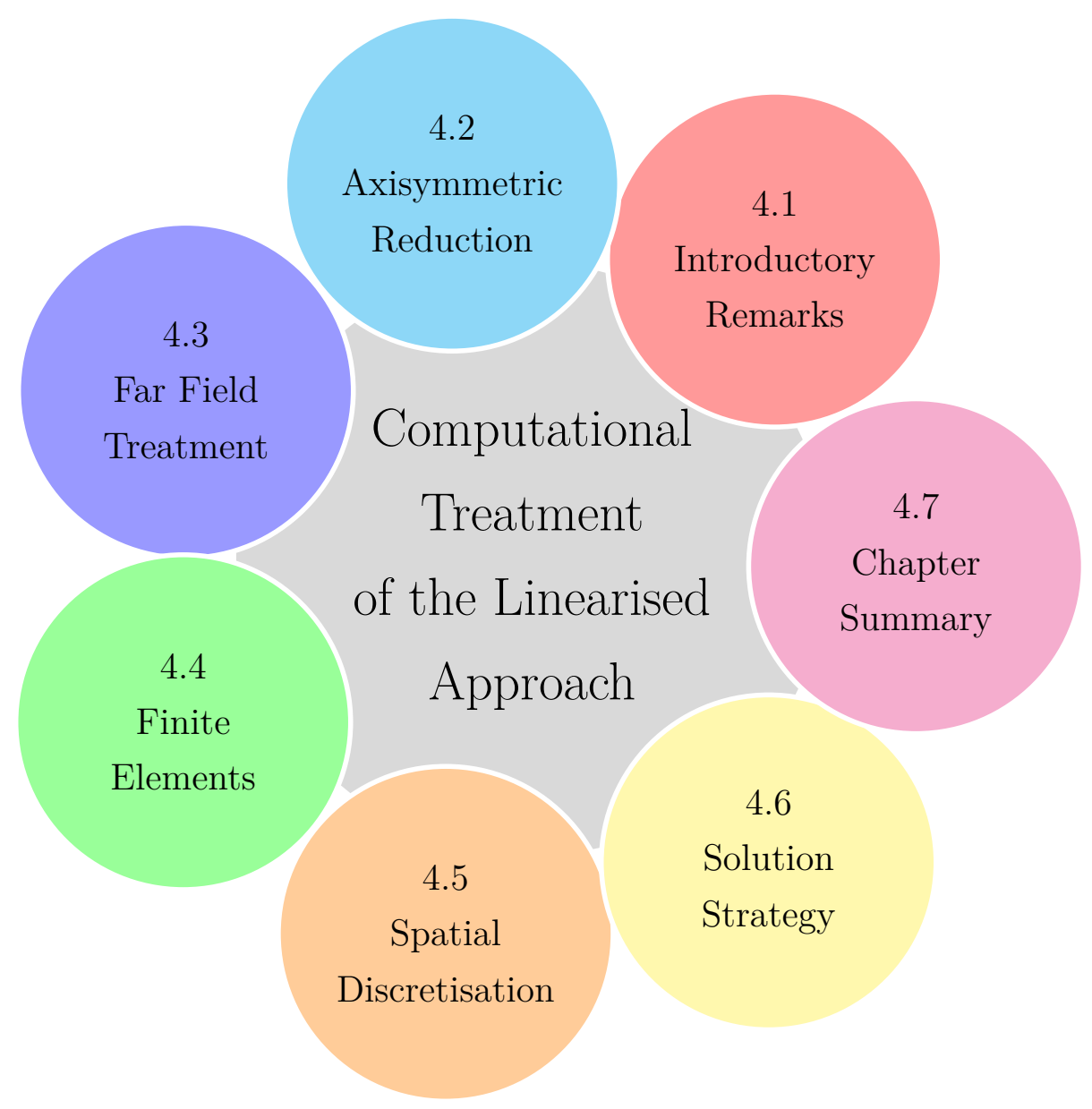

"Don't waste your time looking back, you're not going that way."

Ragnar Lothbrok 


\subsection{Introductory Remarks}

In this Chapter, the efficient computational treatment of the novel linearised coupled equation system presented in Chapter 3 is discussed, where accurate spatial solutions are required. In the interest of providing an efficient solver, the novel treatment of the equation system in an axisymmetric representation is presented, by considering suitably scaled transformations of the solution variables. The computational treatment of such axisymmetric problems in unbounded domains are considered, and extra consideration for the approximation of the far field conditions is given.

From the objectives of this Thesis, discussed in Section 1.4, the computational treatment of the novel coupled acousto-magneto-mechanical system, through an axisymmetric reduction and far field treatment, aligns with the objective: "To provide an efficient computational methodology for handling the solution to the coupled problem of MRI scanners using both approaches." The contents of this Chapter builds on the work presented in the published papers $[150,26]$, by an extension of the discussion of the technical details.

This Chapter starts with a reduction of the three dimensional problem to an axisymmetric treatment in Section 4.2. The computational treatment of the farfield conditions in unbounded domains is then considered in Section 4.3. The description of high order $h p$ finite elements as well as the mapping between the referential and physical element, for both triangular and quadrilateral elements, are then discussed in Section 4.4. The spatial discretisation of the linearised coupled weighted residual system in the context of using high order $h p$ finite elements is discussed in Section 4.5 , with a description of the solution strategy for computing the discrete solutions of the fields described in Section 4.6. The main contributions of this Chapter are then summarised in Section 4.7.

Remark 4.1 Given that this Chapter is concerned with the spatial treatment of the linearised approach, in a time harmonic description, the solution variables are referred to by their complex amplitudes, where the time dependent fields $(\boldsymbol{A}, \boldsymbol{u}, P)$ become complex amplitudes $(\boldsymbol{A}, \boldsymbol{U}, \mathcal{P})$. However, the numerical treatment in this chapter is still valid for the spatial discretisation of both the linearised and nonlinear approaches in the time domain also, which is discussed later in Chapter $\%$. 


\subsection{Axisymmetric Reduction for Rotationally Symmetric Geometries}

In the interest of developing an efficient computational analysis tool for the simulation of acousto-magneto-mechanical coupling, as has been set out in the objectives, some properties of MRI scanners can be exploited to reduce the computational complexity of the model. The geometry of MRI scanners, when simplified, lends itself to a cylindrical coordinate representation $(r, \phi, z)$ and when considering only certain sets of exciting coils, can be reduced to the axisymmetric case, see Figure 4.1 for details.

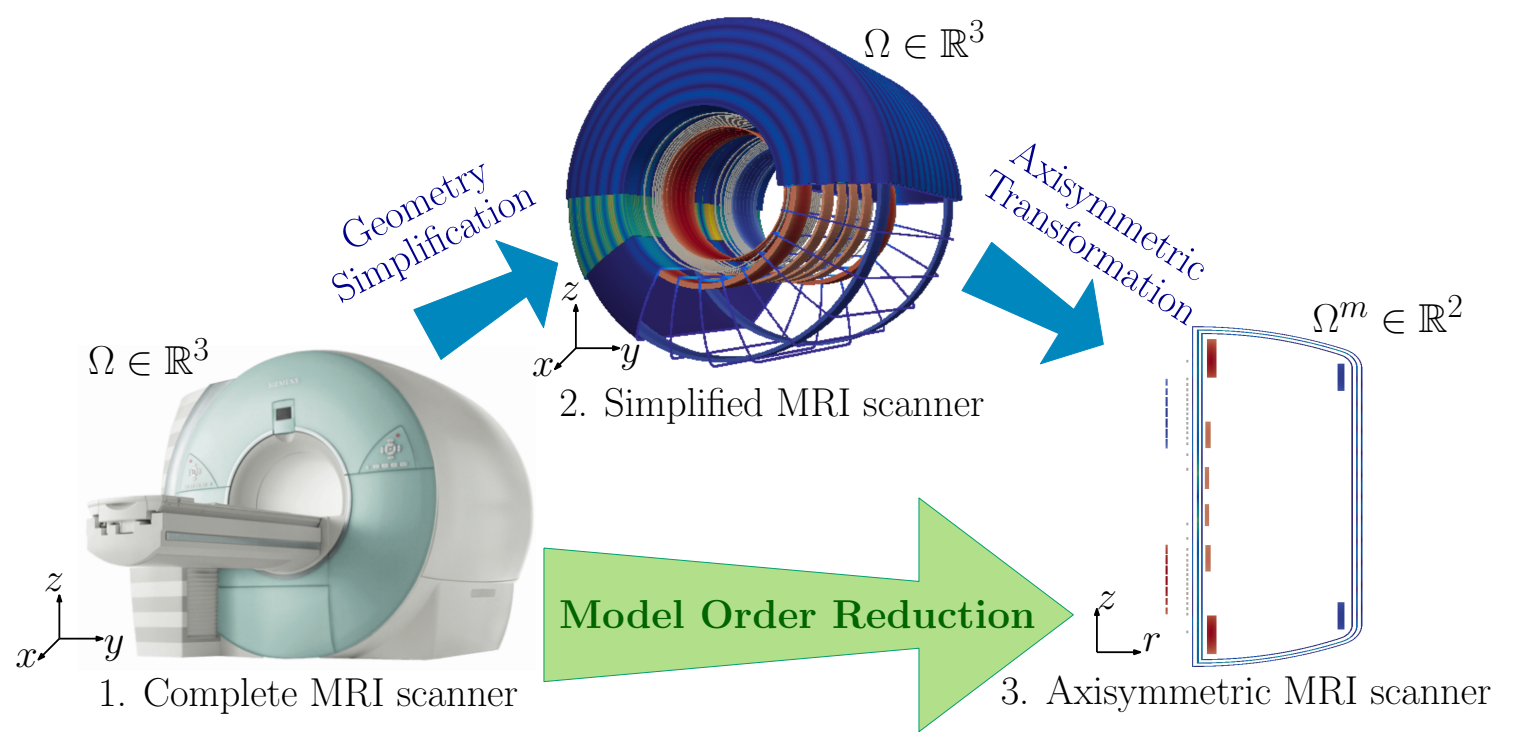

Figure 4.1: Model order reduction by simplification of the full $3 \mathrm{D}$ scanner geometry $\left(\Omega \in \mathbb{R}^{3}\right)$ and an axisymmetric transformation of the simplified geometry to the meridian plane $\left(\Omega^{m} \in \mathbb{R}^{2}\right)$.

In order to perform this reduction in the order of the problem, the solution fields are first recast in terms of their cylindrical descriptions in Section 4.2.1, where a reduction in the number of variables can be performed, based on assumptions of MRI scanners. The transformation of the solution spaces under the axisymmetric treatment are then described in Section 4.2.2, where suitable scaling of the variables to overcome this transformation are presented in Section 4.2.3. The axisymmetric weak form of the coupled linearised approach is then presented in Section 4.2.4. 


\subsubsection{Cylindrical Representation}

The formulation proposed in Chapter 3 is valid for general three dimensional domains involving a conducting region surrounded by an unbounded region of free space, containing the current sources.

To a first approximation, the geometry of a solenoidal (superconducting) MRI scanner is close to cylindrical and is therefore much better described in terms of cylindrical coordinates $(r, \phi, z)$. The unit vectors $\left(\boldsymbol{e}_{r}, \boldsymbol{e}_{\phi}, \boldsymbol{e}_{z}\right)$ denote the standard bases of the cylindrical coordinate system. The strong DC current source in the main coils has only an angular $\phi$-component and thus can be described in cylindrical coordinates as $\boldsymbol{J}^{D C}=J_{\phi}^{D C} \boldsymbol{e}_{\phi}$. Given these properties of MRI scanners, the problem can be described as being symmetric about the $z$ axis, with respect to the angular (azimuthal) $\phi$-component. Through this symmetry (known as axisymmetry) the problem can be reduced to solving in a 2D-planar domain (known as the meridian plane), where the full 3D solutions are achieved by projection of the meridian plane [150]. The reduction of the full 3D problem to the axisymmetric meridian $(r, z)$ plane $\Omega^{m}$ is shown in Figure 4.2, where the reduction of the domain $\Omega$ to $\Omega^{m}$ is illustrated in Figure 4.4 .
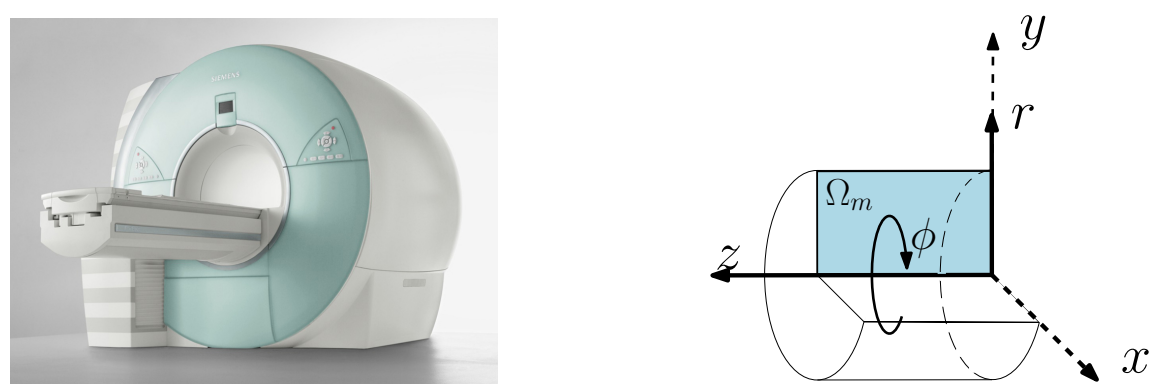

Figure 4.2: Transformation from a full 3D MRI scanner to the 2D axisymmetric case. Under simplifications of geometry, and neglection of certain excitation currents, the problem is constant in the azimuthal $\phi$-direction and can thus be axisymmetrically projected onto the meridian domain $\Omega^{m}$.

However, of the three sets of AC gradient coils, the $x, y$ and $z$ gradient coils, it is only the $z$ gradient coils that exhibit the same properties as the main coils, having only an angular component of the current source $\boldsymbol{g}^{A C}=\mathcal{J}_{\phi}^{A C} \boldsymbol{e}_{\phi}$. Therefore, in order to provide a reduced order computational analysis tool that can rapidly simulate the physical effects of an MRI scanner under operation, then the $x$ and $y$ gradient coils must be neglected. Typical $x, y$ and $z$ gradient coil configurations are illustrated in Figure 4.3, where the non-axisymmetry of the $x$ and $y$ coils is visualised. 


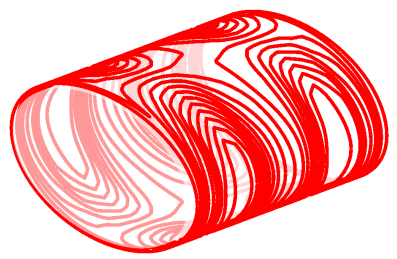

(a) $x$ gradient coil

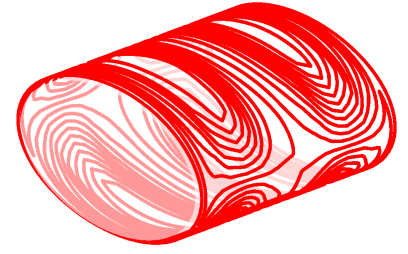

(b) y gradient coil

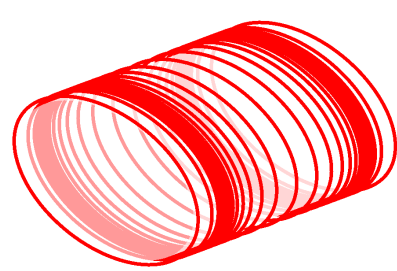

(c) $z$ gradient coil

Figure 4.3: Typical configurations of the set of $x, y$ and $z$ gradient coils of an MRI scanner.

Further to this neglection of the coils, a number of geometric simplifications, which involve neglecting non-axisymmetric components such as the patient test bed etc., must also be considered. Under these assumptions, the simplified MRI scanner, illustrated in Figure 4.1, can be described as being rotationally symmetric with respect to the azimuthal component. Thus, the problem reduces to solving for the reduced axisymmetric variables

$$
\begin{aligned}
& \mathcal{A}(r, \phi, z)=\mathcal{A}_{\phi}(r, z) \boldsymbol{e}_{\phi}, \\
& \mathcal{U}(r, \phi, z)=\mathcal{U}_{r}(r, z) \boldsymbol{e}_{r}+\mathcal{U}_{z}(r, z) \boldsymbol{e}_{z} \\
& \mathcal{P}(r, \phi, z)=\mathcal{P}(r, z)
\end{aligned}
$$

where the fields are functions of $r$ and $z$ only and are expressed in terms of an axisymmetric meridian coordinate system

$$
\boldsymbol{x}^{m}=\sum_{i=1}^{2} x_{i}^{m} \boldsymbol{e}_{i}=r \boldsymbol{e}_{r}+z \boldsymbol{e}_{z},
$$

where the superscript ${ }^{m}$ denotes a variable that lies on the meridian plane, illustrated in Figure 4.2.

This treatment of the solution fields renders the eddy and Lorentz currents to being angular and the full set of current densities are described similarly as

$$
\begin{aligned}
\boldsymbol{g}^{o}(r, \phi, z) & =\mathcal{J}_{\phi}^{o}(r, z) \boldsymbol{e}_{\phi}, \\
\boldsymbol{J}^{l}(r, \phi, z) & =\mathcal{J}_{\phi}^{l}(r, z) \boldsymbol{e}_{\phi}, \\
\boldsymbol{J}^{s}(r, \phi, z) & =\mathcal{J}_{\phi}^{s}(r, z) \boldsymbol{e}_{\phi} .
\end{aligned}
$$

The derived field variables for the magnetic and mechanical fields are similarly 
given by

$$
\begin{aligned}
\mathcal{B}(r, \phi, z)= & \mathcal{B}_{r}(r, z) \boldsymbol{e}_{r}+\mathcal{B}_{z}(r, z) \boldsymbol{e}_{z}, \\
\mathcal{H}(r, \phi, z)= & \mathcal{H}_{r}(r, z) \boldsymbol{e}_{r}+\mathcal{H}_{z}(r, z) \boldsymbol{e}_{z}, \\
\mathcal{E}(r, \phi, z)= & \mathcal{E}_{\phi}(r, z) \boldsymbol{e}_{\phi}, \\
\boldsymbol{\varepsilon}(r, \phi, z)= & \varepsilon_{r r}(r, z) \boldsymbol{e}_{r} \otimes \boldsymbol{e}_{r}+\varepsilon_{\phi \phi}(r, z) \boldsymbol{e}_{\phi} \otimes \boldsymbol{e}_{\phi}+\varepsilon_{z z}(r, z) \boldsymbol{e}_{z} \otimes \boldsymbol{e}_{z} \\
& +\varepsilon_{r z}(r, z) \boldsymbol{e}_{r} \otimes \boldsymbol{e}_{z}+\varepsilon_{z r}(r, z) \boldsymbol{e}_{z} \otimes \boldsymbol{e}_{r}, \\
\boldsymbol{\sigma}(r, \phi, z)= & \sigma_{r r}(r, z) \boldsymbol{e}_{r} \otimes \boldsymbol{e}_{r}+\sigma_{\phi \phi}(r, z) \boldsymbol{e}_{\phi} \otimes \boldsymbol{e}_{\phi}+\sigma_{z z}(r, z) \boldsymbol{e}_{z} \otimes \boldsymbol{e}_{z} \\
& +\sigma_{r z}(r, z) \boldsymbol{e}_{r} \otimes \boldsymbol{e}_{z}+\sigma_{z r}(r, z) \boldsymbol{e}_{z} \otimes \boldsymbol{e}_{r} .
\end{aligned}
$$

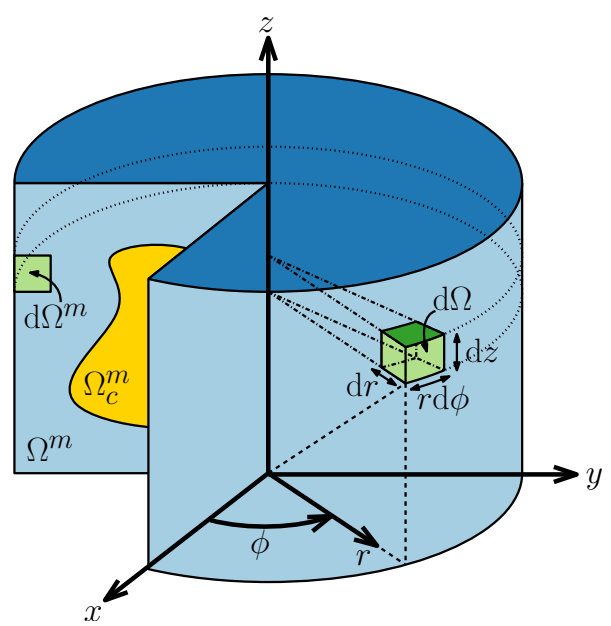

Figure 4.4: Differential volume element $\mathrm{d} \Omega$, shown in green, in a cylindrical coordinate system $(r, \phi, z)$. The left most plane illustrates the meridian domain $\Omega^{m}$, with the meridian differential surface element $\mathrm{d} \Omega^{m}$.

\subsubsection{Weighted Spaces}

When the problem is projected onto the axisymmetric meridian plane, the spaces in which the weak solutions are sought in the variational statements (3.37) and (3.26) must also be adapted.

A transformation of the divergence ${ }^{1}$ and $\operatorname{curl}^{2}$ operators into cylindrical coordinates contain terms involving $1 / r$ (see $[100,169,241]$ for details on the transformation of these operators). This causes problems to occur on the $z$-axis of the meridian

\footnotetext{
${ }^{1}$ The divergence of a vector field in cylindrical coordinates is $\nabla \cdot \boldsymbol{w}=\frac{1}{r} \frac{\partial\left(r w_{r}\right)}{\partial r}+\frac{1}{r} \frac{\partial w_{\phi}}{\partial \phi}+\frac{\partial w_{z}}{\partial z}$.

${ }^{2}$ The curl operator on a vector field in cylindrical coordinates becomes $\nabla \times \boldsymbol{w}=$ $\boldsymbol{e}_{r}\left(\frac{1}{r} \frac{\partial w_{z}}{\partial \phi}-\frac{\partial w_{\phi}}{\partial z}\right)+\boldsymbol{e}_{\phi}\left(\frac{\partial w_{r}}{\partial z}-\frac{\partial w_{z}}{\partial r}\right)+\boldsymbol{e}_{z} \frac{1}{r}\left(\frac{\partial\left(r w_{\phi}\right)}{\partial r}-\frac{\partial w_{r}}{\partial \phi}\right)$.
} 
plane, where $(r=0)$, as $1 / r \rightarrow \infty$ when $r \rightarrow 0$. In general, this leads to a necessity to seek for solutions in weighted spaces to ensure the fields are well behaved at the $z$ axis [143]. Based on the axisymmetric transformation of the domains, variables and differential operators, the definitions in (3.5), when combined with (3.6), become

$$
\begin{aligned}
X^{m} & :=\left\{\mathcal{A}_{\phi}: \mathcal{A}_{\phi} \in L_{1}^{2}\left(\Omega^{m}\right),\left(\begin{array}{c}
-\frac{\partial \mathcal{A}_{\phi}}{\partial z} \\
\frac{1}{r} \frac{\partial\left(r \mathcal{A}_{\phi}\right)}{\partial r}
\end{array}\right) \in\left(L_{1}^{2}\left(\Omega^{m}\right)\right)^{2}\right\} \\
Y^{m}(\boldsymbol{g}) & :=\left\{\boldsymbol{U}: \boldsymbol{U} \in\left(L_{1}^{2}\left(\Omega_{c}^{m}\right)\right)^{2}, \nabla^{s} \boldsymbol{U} \in\left(L_{1}^{2}\left(\Omega_{c}^{m}\right)\right)^{3 \times 3}, \boldsymbol{U}=\boldsymbol{g} \text { on } \partial \Omega_{c}^{m D}\right\}, \\
Z^{m} & :=\left\{\boldsymbol{P}: \mathcal{P} \in L_{1}^{2}\left(\Omega_{n}^{m}\right), \nabla \mathcal{P} \in\left(L_{1}^{2}\left(\Omega_{c}^{m}\right)\right)^{2}\right\} .
\end{aligned}
$$

Both $\nabla \times \mathcal{A} \in\left(L_{1}^{2}\left(\Omega^{m}\right)\right)^{3}$ and $\boldsymbol{\varepsilon}(\boldsymbol{U}) \in\left(L_{1}^{2}\left(\Omega^{m}\right)\right)^{3 \times 3}$ need to be bounded, but due to the switch to cylindrical coordinates the requirements on the derivatives of $\mathcal{A}_{\phi}$ in (4.7a) and symmetric gradient of $\boldsymbol{U}$ in (4.7b) arise. In the above, the domain $\Omega^{m}=\Omega_{n}^{m} \cup \Omega_{c}^{m}$, the superscript ${ }^{m}$ denotes a domain or boundary that lies on the meridian plane, the fields must satisfy the decay and radiation conditions in (2.11) and (2.33) and the Coulomb gauge on $\mathcal{A}$ is no longer required, see Remark 4.2.

Remark 4.2 Note here, that the Coulomb gauge is no longer required in (4.7a) since it is automatically satisfied for the axisymmetric case, where ${ }^{1}$

$$
\nabla \cdot \mathcal{A}=\nabla \cdot \mathcal{A}_{\phi}(r, z) \boldsymbol{e}_{\phi}=\frac{1}{r} \frac{\partial \mathcal{A}_{\phi}(r, z)}{\partial \phi}=0
$$

because of the independence of $\mathcal{A}_{\phi}(r, z)$ on $\phi$.

The "unbounded" meridian domain $\Omega^{m} \in\left\{\mathbb{R}^{2}: 0 \leq r \leq \infty,-\infty \leq z \leq \infty\right\}$, illustrated in Figure 4.5, still requires that the radial coordinate have a lower bound of $(r=0)$, in order to maintain axisymmetry, due to the construction of the meridian plane from $\mathbb{R}^{3}$ (see Figure 4.2). The $L_{1}^{2}\left(\Omega^{m}\right)$ space is defined as

$$
L_{1}^{2}\left(\Omega^{m}\right):=\left\{w: w \in L^{2}\left(\Omega^{m}\right), \sqrt{r} w \in L^{2}\left(\Omega^{m}\right)\right\}
$$

The definitions in (4.7) are based on suitable weighted spaces, as described in 
[143]. In order to understand the requirements on $\mathcal{A}_{\phi}, \boldsymbol{U}$ and $\mathcal{P}$, defined in (4.7), the requirements for $\int_{\Omega}|\nabla \times \mathcal{A}|^{2} \mathrm{~d} \Omega<\infty, \int_{\Omega}|\boldsymbol{\varepsilon}(\boldsymbol{U})|^{2} \mathrm{~d} \Omega<\infty$ and $\int_{\Omega}|\nabla \mathcal{P}|^{2} \mathrm{~d} \Omega<\infty$ under an axisymmetric treatment of the problem will be considered.

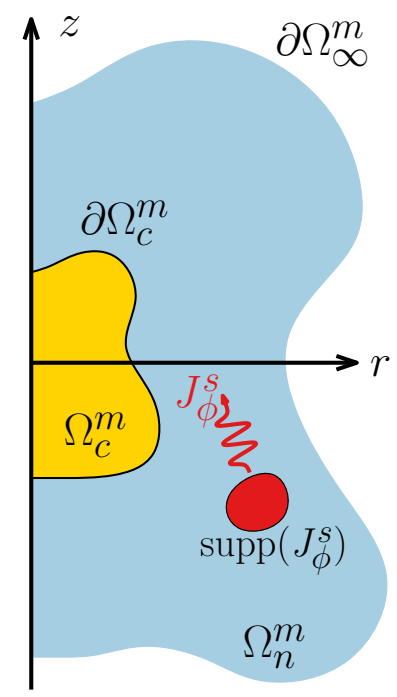

Figure 4.5: Unbounded axisymmetric meridian domain of the problem where the complete domain $\Omega^{m}=\Omega_{n}^{m} \cup \Omega_{c}^{m}$ and the outer boundary of the complete domain $\partial \Omega^{m}=(r=0, z) \cup \partial \Omega_{\infty}^{m}$. The axisymmetric meridian domain $\Omega^{m} \in\left\{\mathbb{R}^{2}: 0 \leq r \leq \infty,-\infty \leq z \leq \infty\right\}$, where the radial coordinates have a lower bound of $(r=0)$ that meets the $z$-axis.

In terms of the electromagnetic problem, from the definition in (4.7a) it is important to note that $\left(\begin{array}{c}-\frac{\partial \mathcal{A}_{\phi}}{\partial z} \\ \frac{\mathcal{A}_{\phi}}{r}+\frac{\partial \mathcal{A}_{\phi}}{\partial r}\end{array}\right) \in\left(L_{1}^{2}\left(\Omega^{m}\right)\right)^{2}$, which implies that

$$
\begin{gathered}
\int_{\Omega^{m}}\left|\left(\frac{\partial \mathcal{A}_{\phi}}{\partial z}\right)^{2}+\frac{\mathcal{A}_{\phi}{ }^{2}}{r^{2}}+\frac{2}{r} \mathcal{A}_{\phi} \frac{\partial \mathcal{A}_{\phi}}{\partial r}+\left(\frac{\partial \mathcal{A}_{\phi}}{\partial r}\right)^{2}\right| r \mathrm{~d} \Omega^{m}<\infty, \\
\Rightarrow \int_{\Omega^{m}}\left|r\left(\frac{\partial \mathcal{A}_{\phi}}{\partial z}\right)^{2}+\frac{\mathcal{A}_{\phi}{ }^{2}}{r}+2 \mathcal{A}_{\phi} \frac{\partial \mathcal{A}_{\phi}}{\partial r}+r\left(\frac{\partial \mathcal{A}_{\phi}}{\partial r}\right)^{2}\right| \mathrm{d} \Omega^{m}<\infty,
\end{gathered}
$$

where the $1 / r$ term arises. The volume integral in $3 \mathrm{D}$ is transformed to the axisymmetric case as per Remark 4.3.

Similarly, in terms of the mechanical problem, from the definition in $(4.7 \mathrm{~b})$ it is important to note that, due to $\boldsymbol{U} \in\left(H^{1}\left(\Omega_{c}^{m}\right)\right)^{2}$, the symmetric gradient tensor of the displacements $\nabla^{s} \boldsymbol{U} \in\left(L_{1}^{2}\left(\Omega^{m}\right)\right)^{3 \times 3}$ which implies that

$$
\int_{\Omega_{c}^{m}}\left|\nabla^{s} \boldsymbol{U}: \nabla^{s} \boldsymbol{U}\right| r \mathrm{~d} \Omega^{m}<\infty
$$


where

$$
\nabla^{s} \boldsymbol{U}=\left[\begin{array}{ccc}
\frac{\partial \mathcal{U}_{r}}{\partial r} & 0 & \frac{1}{2}\left(\frac{\partial \mathcal{U}_{r}}{\partial z}+\frac{\partial \mathcal{U}_{r}}{\partial z}\right) \\
0 & \frac{\mathcal{U}_{r}}{r} & 0 \\
\text { sym. } & 0 & \frac{\partial \mathcal{U}_{z}}{\partial z}
\end{array}\right]
$$

which ensures that $\nabla \cdot \mathcal{U} \in L_{1}^{2}\left(\Omega_{c}^{m}\right)$ so that

$$
\int_{\Omega_{c}^{m}}|\nabla \cdot \boldsymbol{U}|^{2} r \mathrm{~d} \Omega^{m}=\int_{\Omega_{c}^{m}}\left|\frac{\partial \mathcal{U}_{r}}{\partial r}+\frac{\mathcal{U}_{r}}{r}+\frac{\partial \mathcal{U}_{z}}{\partial z}\right|^{2} r \mathrm{~d} \Omega^{m}<\infty
$$

where the $1 / r$ term also arises.

With regards to the acoustic pressure, from the definition in $(4.7 \mathrm{c})$ it is important to note that, $\left(\begin{array}{c}\frac{\partial \mathcal{P}}{\partial r} \\ \frac{\partial \mathcal{P}}{\partial z}\end{array}\right) \in\left(L_{1}^{2}\left(\Omega^{m}\right)\right)^{2}$, which implies that

$$
\int_{\Omega^{m}}\left|\left(\frac{\partial \mathcal{P}}{\partial r}\right)^{2}+\left(\frac{\partial \mathcal{P}}{\partial z}\right)^{2}\right| r \mathrm{~d} \Omega^{m}<\infty
$$

where the $1 / r$ term is absent and so no complications in the treatment of $\mathcal{P}$ result.

Remark 4.3 The differential volume element of the full 3D problem can be expressed in terms of both Cartesian and cylindrical coordinates as

$$
\mathrm{d} \Omega=\mathrm{d} x \mathrm{~d} y \mathrm{~d} z=r \mathrm{~d} r \mathrm{~d} \phi \mathrm{d} z,
$$

where Figure 4.4 illustrates the relationship between the differential volume element $\mathrm{d} \Omega$ in Cartesian and cylindrical coordinates.

The $3 D$ volume integral in cylindrical coordinates, given that all fields are independent of $\phi$, by integration over the azimuthal angle for $0 \leq \phi \leq 2 \pi$, reduces to

$$
\begin{aligned}
\int_{\Omega} f(r, z) \mathrm{d} \Omega & =\int_{0}^{2 \pi} \int_{\Omega^{m}} f(r, z) r \mathrm{~d} r \mathrm{~d} z \mathrm{~d} \phi \\
& =2 \pi \int_{\Omega^{m}} f(r, z) r \mathrm{~d} \Omega^{m},
\end{aligned}
$$


where the differential element of the meridian plane is given by

$$
\mathrm{d} \Omega^{m}=\mathrm{d} r \mathrm{~d} z,
$$

purely in terms of the meridian coordinates.

In order to ensure that the solution remains bounded across the entire domain, including at the $(r=0)$ axis, a suitably scaled transformation of the solution variables can be performed.

\subsubsection{Transformation of Variables}

In terms of the development of a finite element approach for the spatial discretisation of the fields, one could consider deriving suitable finite element basis functions for the definitions in (4.7), in a similar manner to [143]. Or, alternatively choose to treat the unbounded integrations by applying regularisation through the numerical integration of the weighted residual statements, similar to [112]. However, an alternative approach to overcome singularities at the $(r=0)$ axis, is to use standard basis functions and apply a transformation of the solution variables.

Difficulties occur due to terms involving $1 / r$ and the transformation will be performed both on the solution variables $\mathcal{A}_{\phi}, \mathcal{U}_{r}$ and trial functions $A_{\phi}^{\delta}, u_{r}^{\delta}$. Scaling the field components by a variable exponent of the radial coordinate $r^{a}$ results in the solution variables taking the form

$$
\begin{aligned}
& \mathcal{A}_{\phi}(r, z)=r^{a} \hat{\mathcal{A}}_{\phi}(r, z) \\
& \boldsymbol{U}(r, z)=r^{a} \hat{\mathcal{U}}(r, z) \boldsymbol{e}_{r}+\mathcal{U}_{z}(r, z) \boldsymbol{e}_{z}
\end{aligned}
$$

where the problem would then be adjusted to solving for $\hat{\mathcal{A}}_{\phi}, \hat{\mathcal{U}}{ }_{r}, \mathcal{U}_{z}, \mathcal{P}$. Scaling of the axial displacements $\mathcal{U}_{z}$ and acoustic pressure $\mathcal{P}$ is not required, as there are no complications in the $\nabla$ operator terms for these variables. The definitions of $X^{m}$ and $Y^{m}$ in (4.7a) and (4.7b) become

$$
X^{m}:=\left\{r^{a} \hat{\mathcal{A}}_{\phi}: r^{a} \hat{\mathcal{A}}_{\phi} \in L_{1}^{2}\left(\Omega^{m}\right),\left(\begin{array}{c}
-r^{a} \frac{\partial \hat{\mathcal{A}}_{\phi}}{\partial z} \\
\frac{1}{r} \frac{\partial\left(r^{a+1} \hat{\mathcal{A}}_{\phi}\right)}{\partial r}
\end{array}\right) \in\left(L_{1}^{2}\left(\Omega^{m}\right)\right)^{2}\right\},
$$




$$
Y^{m}(\boldsymbol{g}):=\left\{\mathfrak{R}^{a} \tilde{\boldsymbol{U}}: \mathfrak{R}^{a} \tilde{\boldsymbol{U}} \in\left(H^{1}\left(\Omega^{m}\right)\right)^{2}, \mathfrak{R}^{1 / 2} \tilde{\boldsymbol{U}}=\boldsymbol{g} \text { on } \partial \Omega_{c}^{m D}\right\}
$$

where $\tilde{\boldsymbol{U}}=\hat{\mathcal{U}} \boldsymbol{e}_{r}+\mathcal{U}_{z} \boldsymbol{e}_{z}$ and $\mathfrak{R}=\left[\begin{array}{ll}r & 0 \\ 0 & 1\end{array}\right]$ denotes the axisymmetric transformation matrix of the displacements, thus $\mathfrak{R}^{a}=\left[\begin{array}{cc}r^{a} & 0 \\ 0 & 1\end{array}\right]$.

With regards to the electromagnetic problem, based on the definition $X^{m}$ in

(4.15a), then the definition of the curl on the meridian plane $\left(\begin{array}{c}-r^{a} \frac{\partial \hat{\mathcal{A}}_{\phi}}{\partial z} \\ (a+1) r^{a-1} \hat{\mathcal{A}}_{\phi}+r^{a} \frac{\partial \hat{\mathcal{A}}_{\phi}}{\partial r}\end{array}\right) \in$

$\left(L_{1}^{2}\left(\Omega^{m}\right)\right)^{2}$ implies that

$$
\begin{aligned}
& \int_{\Omega^{m}} \mid\left(r^{a} \frac{\partial \hat{\mathcal{A}}_{\phi}}{\partial z}\right)^{2}+\left((a+1) r^{a-1} \hat{\mathcal{A}}_{\phi}+r^{a} \frac{\partial \hat{\mathcal{A}}_{\phi}}{\partial r}\right)^{2} \mid r \mathrm{~d} \Omega^{m}<\infty, \\
& \Rightarrow \int_{\Omega^{m}} \mid r^{2 a+1}\left(\frac{\partial \hat{\mathcal{A}}_{\phi}}{\partial z}\right)^{2}+(a+1)^{2} r^{2 a-1} \hat{\mathcal{A}}_{\phi}^{2}+2(a+1) r^{2 a} \hat{\mathcal{A}}_{\phi} \frac{\partial \hat{\mathcal{A}}_{\phi}}{\partial r} \\
&+r^{2 a+1}\left(\frac{\partial \hat{\mathcal{A}}_{\phi}}{\partial r}\right)^{2} \mid \mathrm{d} \Omega^{m}<\infty .
\end{aligned}
$$

In this case, identifying the term previously associated with the $1 / r$ coefficient in (4.9) as $(a+1)^{2} r^{2 a-1} \hat{\mathcal{A}}_{\phi}^{2}$, then it is clear that to avoid any singularities at the $r=0$ axis (induced through any negative exponent of $r$ ) then $2 a-1 \geq 0 \rightarrow a \geq 1 / 2$.

With regards to the mechanical problem, the definition in $(4.15 \mathrm{~b})$ suggests that $\nabla \cdot\left(\Re^{a} \tilde{\boldsymbol{U}}\right) \in L_{1}^{2}\left(\Omega_{c}^{m}\right)$, from (4.10) and (4.12), which implies that

$$
\begin{aligned}
& \int_{\Omega^{m}} \mid r^{a} \frac{\partial \hat{\mathcal{U}}}{\partial r}+(a+1) r^{a-1} \hat{\mathcal{U}}+\left.\frac{\partial \mathcal{U}_{z}}{\partial z}\right|^{2} r \mathrm{~d} \Omega^{m}<\infty, \\
& \Rightarrow \int_{\Omega^{m}} \mid r^{2 a+1}\left(\frac{\partial \hat{\mathcal{U}}}{\partial r}\right)^{2}+(a+1)^{2} r^{2 a-1} \hat{\mathcal{U}}{ }_{r}^{2}+r\left(\frac{\partial \mathcal{U}_{z}}{\partial z}\right)^{2}+2(a+1) r^{2 a} \hat{\mathcal{U}}{ }_{r} \frac{\partial \hat{\mathcal{U}}}{\partial r} \\
&+2(a+1) r^{a} \hat{\mathfrak{U}_{r}} \frac{\partial \mathcal{U}_{z}}{\partial z}+2 r^{a+1} \frac{\partial \hat{\mathcal{U}}}{\partial r} \frac{\partial \mathcal{U}_{z}}{\partial z} \mid \mathrm{d} \Omega^{m}<\infty,
\end{aligned}
$$

In this case, identifying the term previously associated with the $1 / r$ coefficient in 
(4.12) as $(a+1)^{2} r^{2 a-1} \hat{\mathcal{U}}{ }_{r}^{2}$, then it is clear that to avoid any singularities at the $r=0$ axis (induced through any negative exponent of $r$ ) then $2 a-1 \geq 0 \rightarrow a \geq \frac{1}{2}$. For this case of variable transformation, the solution variables are now suitably scaled such that they satisfy the definitions in (4.7) provided that $a \geq 1 / 2$.

Given this bound on the exponent of $r$, one could choose to set this to any number of possibilities. One suitable choice would be to set $a=1 / 2$, which is the lowest possible value of $a$ to satisfy the conditions. However, arguably a more suitable choice is to set the exponent $a=1$. Computationally speaking, this choice of scaling is more efficient than $a=1 / 2$ because $\sqrt{r}$ isn't a polynomial, whereas $r$ is. Hence, the expressions involved in the weak form can be exactly integrated by numerical quadrature rules [68]. For further details on this topic see [143] and [112]. For this reason the scaling adopted in the following formulations is for the case of $a=1$ and thus the scaled variables in 4.14 explicitly become

$$
\begin{aligned}
\mathcal{A}_{\phi}(r, z) & =r \hat{\mathcal{A}}_{\phi}(r, z), \\
\mathcal{U}(r, z) & =r \hat{\mathcal{U}}{ }_{r}(r, z) \boldsymbol{e}_{r}+\mathcal{U}_{z}(r, z) \boldsymbol{e}_{z},
\end{aligned}
$$

From the scaled variables in both cases, it is clear that the problem is satisfied for $\hat{\mathcal{A}}_{\phi} \in L^{2}\left(\Omega^{m}\right)$ and $\nabla^{m} \hat{\mathcal{A}}_{\phi} \in\left(L^{2}\left(\Omega^{m}\right)\right)^{2}$, and $\nabla \cdot \boldsymbol{U} \in L^{2}\left(\Omega_{c}^{m}\right)$ and $\nabla^{s} \boldsymbol{U} \in$ $\left(L^{2}\left(\Omega_{c}^{m}\right)\right)^{3 \times 3}$. This implies that $\hat{\mathcal{A}}_{\phi} \in H^{1}\left(\Omega^{m}\right)$ and $\tilde{\boldsymbol{U}} \in\left(H^{1}\left(\Omega_{c}^{m}\right)\right)^{2}$ and thus, the definitions of (4.7) reduce to

$$
\begin{aligned}
\hat{X}^{m} & :=\left\{\hat{\mathcal{A}}_{\phi}: \hat{\mathcal{A}}_{\phi} \in H^{1}\left(\Omega^{m}\right), \hat{\mathcal{A}}_{\phi}=0 \text { on } \partial \Omega_{\infty}^{m}\right\} \\
\hat{Y}^{m}(\boldsymbol{g}) & :=\left\{\tilde{\boldsymbol{U}}: \tilde{\boldsymbol{U}} \in\left(H^{1}\left(\Omega_{c}^{m}\right)\right)^{2}, \tilde{\boldsymbol{U}}=\mathfrak{R}^{-1} \boldsymbol{g} \text { on } \partial \Omega_{c}^{m}\right\}, \\
Z^{m} & :=\left\{\boldsymbol{P}: \boldsymbol{P} \in\left(H^{1}\left(\Omega^{m}\right)\right)^{2}, \nabla^{m} \boldsymbol{P} \cdot \boldsymbol{n}^{m}+\mathrm{i} \omega \boldsymbol{P}=0 \text { on } \partial \Omega_{\infty}^{m}\right\},
\end{aligned}
$$

where $\boldsymbol{n}^{m}$ is the meridian normal ${ }^{3}, \nabla^{m}$ is the meridian gradient operator ${ }^{4}$ and the decay and radiation conditions in (2.11) and (2.33) have been included. The transformation of the variables for both cases, such that the scaled solution variables lie in the $H^{1}\left(\Omega^{m}\right)$ space, permit the use of standard $H^{1}(\Omega)$ conforming finite element basis functions, which will be discussed in Section 4.4 .

\footnotetext{
${ }^{3}$ The meridian normal vector is given as $\boldsymbol{n}^{m}=n_{r} \boldsymbol{e}_{r}+n_{z} \boldsymbol{e}_{z}$.

${ }^{4}$ The meridian gradient of a scalar field $w$ is defined as $\nabla^{m} w=\frac{\partial w}{\partial r} \boldsymbol{e}_{r}+\frac{\partial w}{\partial z} \boldsymbol{e}_{z}$.
} 


\subsubsection{Axisymmetric Weak Form}

Applying the cylindrical representation of the fields, discussed in Section 4.2.1, and the transformation of variables for case 2, from Section 4.2.3, the time harmonic linear system in $(3.37)$ becomes: Find $\left(\delta_{\hat{\mathcal{A}}_{\phi}}, \boldsymbol{\delta}_{\tilde{\mathfrak{u}}}, \delta_{\mathcal{P}}\right) \in \hat{X}^{m} \times \hat{Y}^{m}(\mathbf{0}) \times Z^{m}$ such that

$$
\begin{array}{r}
D \tilde{R}_{\mathcal{A}}^{A C}\left(\hat{A}_{\phi}^{\delta}\right)\left[\delta_{\hat{\mathcal{A}}_{\phi}}\right]+D \tilde{R}_{\mathcal{A}}^{A C}\left(\hat{A}_{\phi}^{\delta} ; A_{\phi}^{D C}\right)\left[\boldsymbol{\delta}_{\mathcal{U}}\right]=-\tilde{R}_{\mathcal{A}}^{A C}\left(\hat{A}_{\phi}^{\delta}\right) \\
=2 \pi \int_{\operatorname{supp}\left(\mathcal{g}_{\phi}^{A C}\right)} \mathcal{g}_{\phi}^{A C} \hat{A}_{\phi}^{\delta} r^{2} \mathrm{~d} \Omega^{m} \\
D \tilde{R}_{\mathcal{U}}^{A C}\left(\boldsymbol{u}^{\delta} ; A_{\phi}^{D C}\right)\left[\delta_{\hat{\mathcal{A}}_{\phi}}\right]+D \tilde{R}_{\mathcal{U}}^{A C}\left(\boldsymbol{u}^{\delta}\right)\left[\boldsymbol{\delta}_{\mathcal{U}}\right]+D \tilde{R}_{\mathcal{U}}^{A C}\left(\boldsymbol{u}^{\delta}\right)\left[\delta_{\mathcal{P}}\right]=-\tilde{R}_{\mathcal{U}}^{A C}\left(\boldsymbol{u}^{\delta}\right) \\
=0 \\
D \tilde{R}_{\mathcal{P}}^{A C}\left(P^{\delta} ; A_{\phi}^{D C}\right)\left[\delta_{\hat{\mathcal{A}}_{\phi}}\right]+D \tilde{R}_{\mathcal{P}}^{A C}\left(P^{\delta}\right)\left[\boldsymbol{\delta}_{\mathcal{U}}\right]+D \tilde{R}_{\mathcal{P}}^{A C}\left(P^{\delta}\right)\left[\delta_{\mathcal{P}}\right]=-\tilde{R}_{\mathcal{P}}^{A C}\left(P^{\delta}\right) \\
=-2 \pi \int_{\operatorname{supp}\left(\mathcal{g}_{\phi}^{A C}\right)} \boldsymbol{g}_{\phi}^{A C} \nabla^{m}\left(r A_{\phi}^{D C}\right) \cdot \nabla^{m} P^{\delta} \mathrm{d} \Omega^{m}
\end{array}
$$

for all $\left(\hat{A}_{\phi}^{\delta}, \tilde{\boldsymbol{u}}^{\delta}, P^{\delta}\right) \in \hat{X} \times \hat{Y}(\mathbf{0}) \times Z$, where the directional derivatives are given by

$$
\begin{aligned}
& D \tilde{R}_{\mathcal{A}}^{A C}\left(\hat{A}_{\phi}^{\delta}\right)\left[\delta_{\hat{\mathcal{A}}_{\phi}}\right]=2 \pi \int_{\mathbb{R}^{2}} \frac{\mu^{-1}}{r} \nabla^{m}\left(r^{2} \delta_{\hat{\mathcal{A}}_{\phi}}\right) \cdot \nabla^{m}\left(r^{2} \hat{A}_{\phi}^{\delta}\right) \mathrm{d} \Omega^{m} \\
& +2 \pi \int_{\mathbb{R}^{2}} \mathrm{i} \omega \gamma \delta_{\hat{\mathcal{A}}_{\phi}} \hat{A}_{\phi}^{\delta} r^{3} \mathrm{~d} \Omega^{m}, \\
& D \tilde{R}_{\mathcal{A}}^{A C}\left(\hat{A}_{\phi}^{\delta} ; A_{\phi}^{D C}\right)\left[\boldsymbol{\delta}_{\mathcal{U}}\right]=-2 \pi \int_{\Omega_{c}^{m}} \mathrm{i} \omega \gamma \boldsymbol{\delta}_{\mathcal{U}} \cdot \nabla^{m}\left(r A_{\phi}^{D C}\right) \hat{A}_{\phi}^{\delta} r \mathrm{~d} \Omega^{m}, \\
& D \tilde{R}_{\mathcal{U}}^{A C}\left(\boldsymbol{u}^{\delta} ; A_{\phi}^{D C}\right)\left[\delta_{\hat{\mathcal{A}}_{\phi}}\right]=2 \pi \int_{\Omega_{c}^{m}} \mu^{-1} \mathcal{S}\left(A_{\phi}^{D C}, r \delta_{\hat{\mathcal{A}}_{\phi}}\right): \nabla \boldsymbol{u}^{\delta} r \mathrm{~d} \Omega^{m} \\
& -\left.2 \pi \int_{\partial \Omega_{c}^{m N}} \mu_{0}^{-1} \mathcal{S}\left(A_{\phi}^{D C}, r \delta_{\hat{\mathcal{A}}_{\phi}}\right)\right|^{+} \boldsymbol{n}^{m} \cdot \boldsymbol{u}^{\delta} r \mathrm{~d} S^{m}, \\
& D \tilde{R}_{\mathcal{U}}^{A C}\left(\boldsymbol{u}^{\delta}\right)\left[\boldsymbol{\delta}_{\mathcal{U}}\right]=2 \pi \int_{\Omega_{c}^{m}}\left(\boldsymbol{\sigma}^{m}\left(\boldsymbol{\delta}_{\mathcal{U}}\right): \nabla\left(\boldsymbol{u}^{\delta}\right)-\rho \omega^{2} \boldsymbol{\delta}_{\mathcal{U}} \cdot \boldsymbol{u}^{\delta}\right) r \mathrm{~d} \Omega^{m}, \\
& D \tilde{R}_{\mathcal{U}}^{A C}\left(\boldsymbol{u}^{\delta}\right)\left[\delta_{\mathcal{P}}\right]=-\left.2 \pi \int_{\partial \Omega_{c}^{m N}} \delta_{\mathcal{P}}\right|^{+} \boldsymbol{n}^{m} \cdot \boldsymbol{u}^{\delta} r \mathrm{~d} S^{m}, \\
& D \tilde{R}_{\mathcal{P}}^{A C}\left(P^{\delta} ; A_{\phi}^{D C}\right)\left[\delta_{\hat{\mathcal{A}}_{\phi}}\right]=2 \pi \int_{\operatorname{supp}\left(J_{\phi}^{D C}\right)}\left(J_{\phi}^{D C} \nabla^{m} \delta_{\hat{\mathcal{A}}_{\phi}}\right) \cdot \nabla^{m} P^{\delta} \mathrm{d} \Omega^{m} \\
& -2 \pi \int_{\operatorname{supp}\left(\mathcal{g}_{\phi}^{s}\right)} \mu_{0}^{-1}\left(r \nabla^{m 2} \delta_{\hat{\mathcal{A}}_{\phi}}+3 \frac{\partial \delta_{\hat{\mathcal{A}}_{\phi}}}{\partial r}\right) \nabla^{m}\left(r A_{\phi}^{D C}\right) \cdot \nabla^{m} P^{\delta} \mathrm{d} \Omega^{m} \\
& D \tilde{R}_{\mathcal{P}}^{A C}\left(P^{\delta}\right)\left[\boldsymbol{\delta}_{\mathcal{U}}\right]=\left.2 \pi \int_{\partial \Omega_{c}^{m N}} \omega^{2} \rho^{+} \boldsymbol{\delta}_{\mathcal{U}}\right|^{-} \cdot \boldsymbol{n}^{m} P^{\delta} r \mathrm{~d} S^{m}, \\
& D \tilde{R}_{\mathcal{P}}^{A C}\left(P^{\delta}\right)\left[\delta_{\mathcal{P}}\right]=2 \pi \int_{\mathbb{R}^{2} \backslash \Omega_{c}^{m}}\left(\nabla^{m} \delta_{\mathcal{P}} \cdot \nabla^{m} P^{\delta}-\frac{\omega^{2}}{c^{2}} \delta_{\mathcal{P}} \cdot P^{\delta}\right) r \mathrm{~d} \Omega^{m} .
\end{aligned}
$$


In the above, $\boldsymbol{A}^{D C}=A_{\phi}^{D C} \boldsymbol{e}_{\phi}, \boldsymbol{\delta}_{\mathcal{A}}=r \delta_{\hat{\mathcal{A}}_{\phi}} \boldsymbol{e}_{\phi}, \boldsymbol{\delta}_{\mathfrak{U}}=\mathfrak{R} \boldsymbol{\delta}_{\tilde{\mathcal{U}}}=\left[\begin{array}{cc}r & 0 \\ 0 & 1\end{array}\right]\left(\begin{array}{c}\delta_{\hat{\mathfrak{u}}_{r}} \\ \delta_{\mathfrak{U}_{z}}\end{array}\right)=\left(\begin{array}{c}r \delta_{\hat{\mathfrak{u}}_{r}} \\ \delta_{\mathfrak{U}_{z}}\end{array}\right)$, $\boldsymbol{A}^{\delta}=r \hat{A}_{\phi}^{\delta} \boldsymbol{e}_{\phi}$ and $\boldsymbol{u}^{\delta}=\mathfrak{R} \tilde{\boldsymbol{u}}^{\delta}=\left[\begin{array}{cc}r & 0 \\ 0 & 1\end{array}\right]\left(\begin{array}{c}\hat{u}_{r}^{\delta} \\ u_{z}^{\delta}\end{array}\right)=\left(\begin{array}{c}r \hat{u}_{r}^{\delta} \\ u_{z}^{\delta}\end{array}\right)$.

For a description of the axisymmetric transformation of the individual terms see [150], where the axisymmetric magneto-mechanical terms are derived. The $2 \pi$ coefficient appears in all terms of each equation in (4.20) and as a result can be dropped from all the residual and the directional derivative terms in (4.20) and (4.21) respectively, when solving the system.

\subsection{Far Field Treatment}

The axisymmetric linearised system, presented in (4.20) and (4.21), permits finite element discretisation, which allows for the computational solutions to the coupled acousto-magneto-mechanical problem.

Given, however, that the current state of the problem resides in an unbounded region of free space, an artificial truncation of the unbounded region must be applied to create a bounded domain on which computations can be performed. The unbounded free space region $\mathbb{R}^{3} \backslash \Omega_{c}$ is truncated at a finite distance from $\Omega_{c}$ and the non-conducting region $\Omega_{n}$ is created, which contains all the current sources, illustrated in Figure 4.6. For the axisymmetric case discussed above, the 3D computational domain $\Omega:=\Omega_{n} \cup \Omega_{c} \subset \mathbb{R}^{3}$ becomes $\Omega^{m}:=\Omega_{n}^{m} \cup \Omega_{c}^{m} \subset \mathbb{R}^{2}$. This means that integrals over $\mathbb{R}^{2}$ and $\mathbb{R}^{2} \backslash \Omega_{c}$, in (4.20) and (4.21), become integrals over $\Omega^{m}$ and $\Omega_{n}^{m}$ respectively.

The boundary conditions of the problems on the unbounded region, where the fields rely on decay conditions at $\infty$, must therefore be approximated to provide boundary conditions for the finite element solutions. An ideal scenario would be that the analytical solution at the boundary is known and then the analytical solution of the fields could be applied directly. However, analytical solutions are only available for simple geometries and certainly are not available for the coupled multi-physics problems attempted in this Thesis.

\subsubsection{Electromagnetic Field Decay}

The strong form of the coupled transmission conditions for the static and transient problems in (2.38) and (2.36), respectively, include radiation and decay conditions, which describe the behaviour of $\boldsymbol{A}, \boldsymbol{A}^{D C}, \mathcal{P}$ and $P^{D C}$ as $|\boldsymbol{x}| \rightarrow \infty$. 


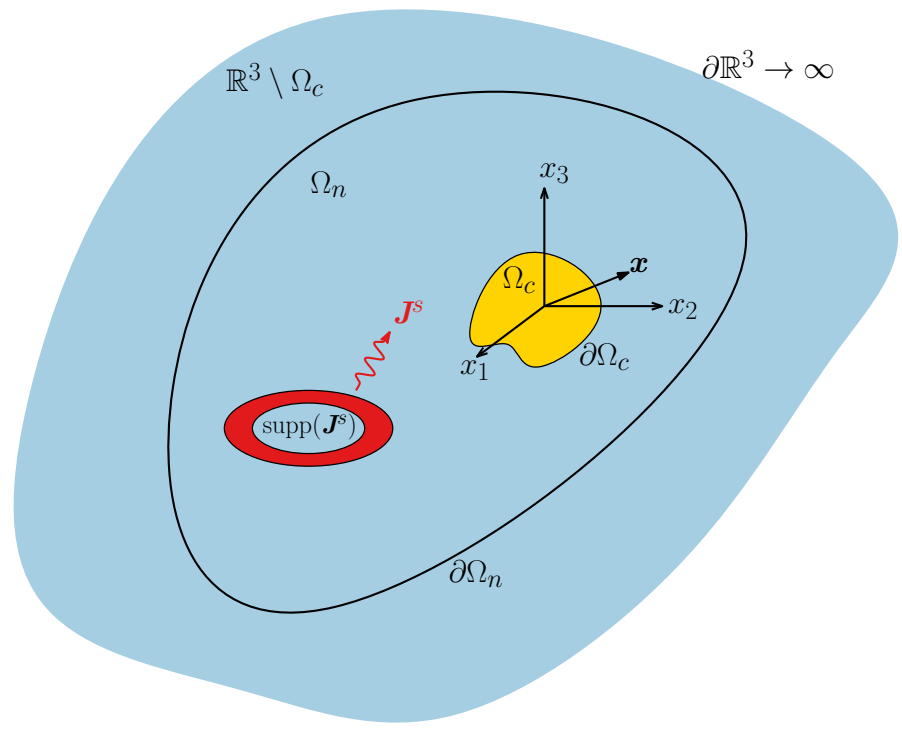

Figure 4.6: Artificial truncation of the unbounded domain $\mathbb{R}^{3}$ to create the bounded nonconducting region $\Omega_{n}$, with the outer boundary $\partial \Omega$ located a finite distance from the origin.

On the outer boundary of the non-conducting meridian domain $\partial \Omega^{m}$, the static decay of $\boldsymbol{A}, \boldsymbol{A}^{D C}, P^{D C}$ is approximated by fixing $\partial \Omega^{m}$ to be located sufficiently far from the region of interest and setting

$$
\begin{aligned}
\mathcal{A}=\boldsymbol{A}^{D C}=\mathcal{A}_{\phi} \boldsymbol{e}_{\phi}=A_{\phi}^{D C} \boldsymbol{e}_{\phi} & =\mathbf{0}, \\
P^{D C} & =0
\end{aligned}
$$

Naturally, the quality of the approximation improves as the size of $\Omega_{n}\left(\Omega_{n}^{m}\right)$ is increased. By increasing the size of the domain, thus pushing the boundary further from the origin, the computational requirement of the model is increased. In the interest of providing an efficient computational analysis tool, the size of the domain must be kept to a minimum, thus another method for approximating field decay conditions will be considered. This approximate condition requires knowledge of $h p$ finite elements and thus its discussion will be delayed until Section 4.4.4.

\subsubsection{Acoustic Field Decay}

The harmonic pressure field, and in particular the treatment of $D \tilde{R}_{\mathcal{P}}^{A C}\left(P^{\delta}\right)\left[\delta_{\mathcal{P}}\right]$ can not merely be approximated by truncating the domain and fixing $\mathcal{P}=0$ at $\partial \Omega^{m}$, since this would result in reflections which would pollute the computational domain, due to the wave behaviour of the acoustic pressure. The radiation condition (2.33) in the continuous problem describes the correct decay of this field, which must also be approximated computationally. 
One method for approximating this condition, as in the magnetic case, is to apply the radiation condition of the field in (2.33) at the truncated boundary. For a time harmonic description the condition approximates to

$$
\nabla^{m} \mathcal{P} \cdot \boldsymbol{n}^{m}+\mathrm{i} \omega \mathcal{P}=0 \quad \text { on } \partial \Omega_{n}^{m}
$$

As with the magnetic decay condition this approximation improves with the increase in $\Omega_{n}\left(\Omega_{n}^{m}\right)$. For further details of this condition see Appendix D.

Another method for handling the radiation condition of the acoustic pressure field is to employ a perfectly matched layer (PML) [175]. The PML $\Omega_{p m l}\left(\Omega_{p m l}^{m}\right)$ is applied to the exterior of $\Omega_{n}\left(\Omega_{n}^{m}\right)$ so that the computational domain now becomes $\Omega=\Omega_{c} \cup \Omega_{n} \cup \Omega_{p m l}\left(\Omega^{m}=\Omega_{c}^{m} \cup \Omega_{n}^{m} \cup \Omega_{p m l}^{m}\right)$. For terms other than $D \tilde{R}_{\mathcal{P}}^{A C}\left(P^{\delta}\right)\left[\delta_{\mathcal{P}}\right]$ in (3.37) $\Omega_{p m l}$ can be merely thought of as a free space extension of $\Omega_{n}$. However, the aforementioned term $(4.21 \mathrm{~h})$ is treated differently as

$$
\begin{aligned}
D \tilde{R}_{\mathcal{P}}^{A C}\left(P^{\delta}\right)\left[\delta_{\mathcal{P}}\right] & =\int_{\Omega_{n}^{m}}\left(\nabla^{m} \delta_{\mathcal{P}} \cdot \nabla^{m} P^{\delta}-\frac{\omega^{2}}{c^{2}} \delta_{\mathcal{P}} P^{\delta}\right) r \mathrm{~d} \Omega^{m} \\
& +\int_{\Omega_{p m l}^{m}}\left(\left(\boldsymbol{\Lambda}_{1} \nabla^{m} \delta_{\mathcal{P}}\right) \cdot \nabla^{m} P^{\delta}-\frac{\omega^{2}}{c^{2}} \Lambda_{2} \delta_{\mathcal{P}} P^{\delta}\right) r \mathrm{~d} \Omega^{m}
\end{aligned}
$$

where $\Lambda_{1}, \Lambda_{2}$ are both complex functions of position in the layer and reduce to identity on $\partial \Omega_{n}^{m} \cap \partial \Omega_{p m l}^{m}$. For details in deriving this system for the time harmonic case see Appendix D. The coefficients of these functions can be established through a complex coordinate stretching of the domain $\Omega_{n}^{m}$ following the approach in [175]. For the axisymmetric case this complex stretching is equivalent to introducing the complex position dependent functions

$$
\Lambda_{1}(r, z)=\frac{1}{r}\left[\begin{array}{cc}
\frac{z_{z}^{\prime} z_{r}}{z_{r}^{\prime}} & 0 \\
0 & \frac{z_{r}^{\prime} z_{r}}{z_{z}^{\prime}}
\end{array}\right], \quad \Lambda_{2}(r, z)=\frac{z_{r}^{\prime} z_{z}^{\prime} z_{r}}{r}
$$

In the above, the complex coordinate transform $z_{s}$ is described as a power law in terms of the distance to the layer $d_{s}$ and thickness of the layer $t_{s}$, as illustrated in Figure $4.7 \mathrm{~b}$, where the prime indicates differentiation with respect to the argument. The complex coordinate transform function may be given as

$$
z_{s}(s)=\left\{\begin{array}{ll}
s & 0 \leq|s|<d_{k} \\
s-\mathrm{i}\left(\frac{|s|-d_{s}}{t_{s}}\right)^{5} s & |s| \geq d_{k}
\end{array},\right.
$$


where $s=(r, z)^{T}$. The choice of a power law of degree 5 and user defined thickness $t_{s}$ is somewhat arbitrary, provided that the resulting complex field behaviour in $\Omega_{p m l}^{m}$ is properly resolved. It has been shown in [175] that this choice provides an accurate description when combined with a $h p$-FEM description. If this is accomplished, the acoustic pressure field is absorbed without reflection and the outer boundary condition can be set as $\boldsymbol{P}=0$ on $\partial \Omega^{m}$.

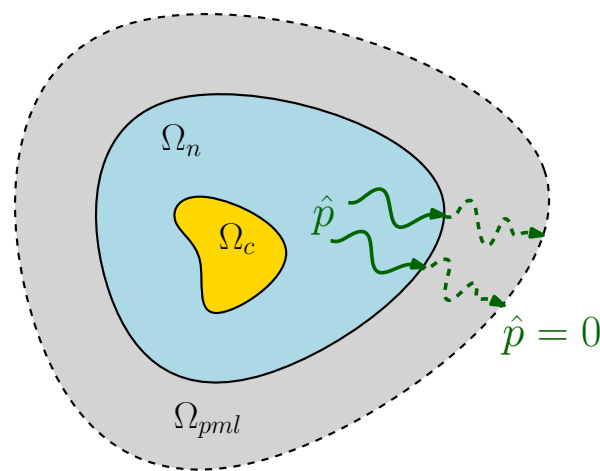

(a) 3D representation, with absorption of the outgoing waves

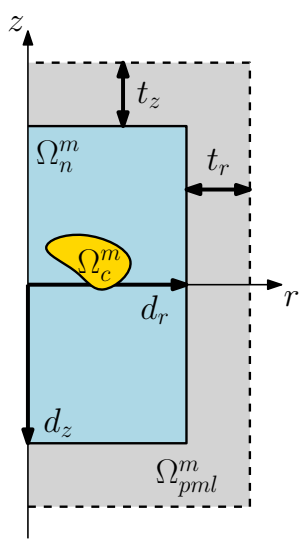

(b) Axisymmetric representation

Figure 4.7: Representation of the Perfectly Matched Layer (PML).

To account for this treatment of the farfield conditions the definitions of $\hat{X}^{m}$, $\hat{Y}^{m}$ and $Z^{m}$ in (4.19) are redefined, for the discrete time harmonic approximation, as

$$
\begin{aligned}
\hat{X}^{m} & :=\left\{\hat{\mathcal{A}}_{\phi}: \hat{\mathcal{A}}_{\phi} \in H^{1}\left(\Omega^{m}\right), \hat{\mathcal{A}}_{\phi}=0 \text { on } \partial \Omega^{m}\right\}, \\
\hat{Y}^{m}(\boldsymbol{g}) & :=\left\{\tilde{\boldsymbol{U}}: \tilde{\boldsymbol{U}} \in\left(H^{1}\left(\Omega_{c}^{m}\right)\right)^{2}, \tilde{\boldsymbol{U}}=\mathfrak{R}^{-1} \boldsymbol{g} \text { on } \partial \Omega_{c}^{m}\right\}, \\
\hat{Z}^{m} & :=\left\{\boldsymbol{P}: \mathcal{P} \in H^{1}\left(\Omega_{n}^{m} \cup \Omega_{p m l}^{m}\right), \mathcal{P}=0 \text { on } \partial \Omega^{m}\right\} .
\end{aligned}
$$

\subsection{Finite Elements}

In this Thesis the use of high order $h p$ finite elements for the spatially accurate numerical solution of acousto-magneto-mechanical coupling problems in MRI scanner is proposed. This method allows for sufficient resolution of the small scale physical phenomena described in Chapter 2. This section recalls the fundamentals of high 
order finite elements and advocates the use of a hierarchic set of shape functions, in providing functional approximations to the solution.

Given a continuous bounded (spatial) domain $\Omega$, in which the problem is defined, a finite element discretisation involves the representation of the domain by a series of points, called nodes, where a partition of the domain into elements $\Omega^{(e)}$ is performed, see Figure 4.8 for a hybrid mesh of both triangular and quadrilateral elements. For 2D problems, finite element discretisations typically utilise two types of element: the triangular and quadrilateral element. Each element provides advantages over the other, and can be used together to form hybrid meshes, illustrated in Figure 4.8.

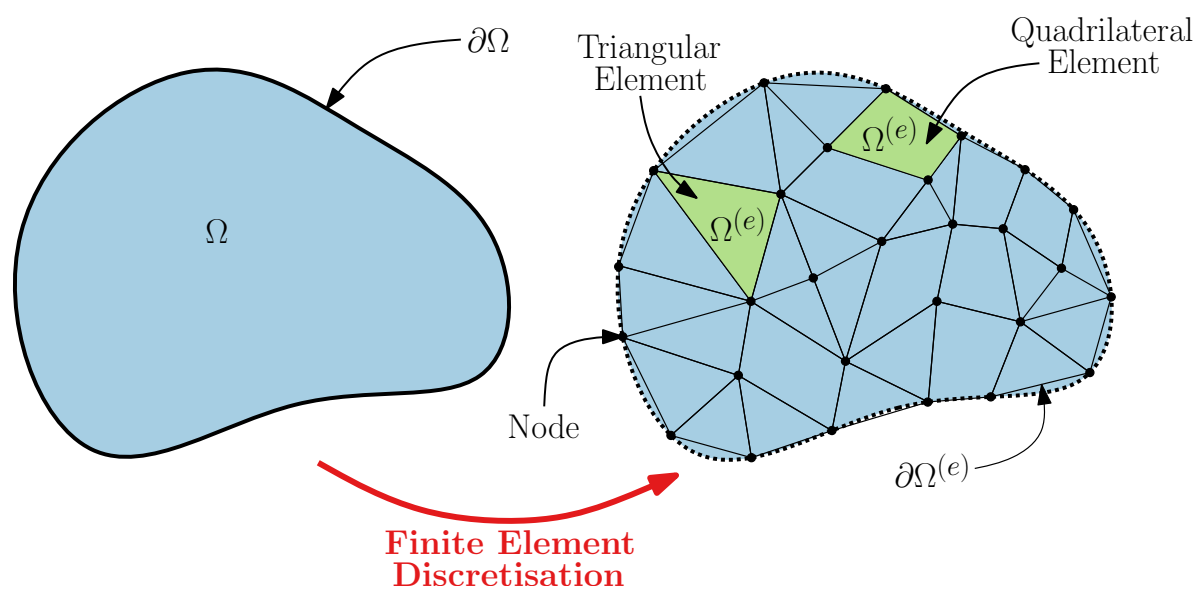

Figure 4.8: The continuous domain $\Omega$ is discretised by a series of points, called nodes. The nodes are connected to form small volumes $\Omega^{(e)}$, known as elements. The discretisation of the domain results in a discrete boundary $\partial \Omega^{(e)}$ that does not, in general, coincide with the domain boundary $\partial \Omega$.

In standard $h$ based finite element procedures, seen typically in commercial codes, the resolution and accuracy of the solution is improved by refining the mesh, either globally or in some cases locally around specific features ${ }^{5}$. Whereas, in high order $p$ finite elements the accuracy of the solution is improved through increasing the polynomial degree of the basis functions of the elements ${ }^{6}$. A combination of both methods results in a $h p$ finite element procedure and is adopted in this Thesis. The advantages of the $h p$ finite element method are discussed in [127, 244, 119, 254] and are therefore not repeated here.

With a $p$-version finite element approach in mind, hierarchic (modal-type) basis functions are a better choice than standard Lagrange (nodal-type) basis function. They make for a more efficient implementation- the set of functions for an order

\footnotetext{
${ }^{5}$ The lower case $h$ denotes the maximum characteristic length of the elements.

${ }^{6}$ The lower case $p$ denotes the order of the elemental basis functions.
} 
$p$ are contained in the set of functions for order $p+1$ and so do not need to be regenerated for different $p$ - and lead to better conditioned matrices [244, 127, 217], crucial for multi-physics problems of varying scale.

In order to determine the solution to the equations in (4.20), integration in space will have to be performed. This poses a significant problem from a numerical point of view. When integrating in the spatial domain $\Omega$, not only do the limits of integration change between elements $\Omega^{(e)}$, but also determining the finite element basis functions for each element can be time consuming for large meshes and high $p$. This task becomes significantly more simple when considering a regular element in the reference domain $\Omega_{\xi}$, with fixed and constant limits of integration. The shape functions of this element can be defined a priori and then mapped to the continuous basis functions of the elements in the spatial domain.

\subsubsection{Reference Elements}

In order to determine the hierarchic basis functions, the reference element must first be defined. In practice, any regular triangle and quadrilateral will provide a practical reference element. The reference triangular and quadrilateral elements used in this Thesis are introduced below.

\section{Triangular Element}

The reference triangular element, also used in [244, 119], is taken to be an equilateral, shown in Figure 4.9, where the vertex degrees of freedom (DOF) are denoted by the hollow circles, the edge DOF are highlighted with the double arrows and the interior DOF are denoted by the cross. The triangular reference element has vertices $V_{1}=(1,0), V_{2}=(0, \sqrt{3})$ and $V_{3}=(-1,0)$ defined in the referential coordinate system, given as

$$
\boldsymbol{\xi}=\sum_{i=1}^{2} \xi_{i} \boldsymbol{e}_{i}=\xi \boldsymbol{e}_{\xi}+\eta \boldsymbol{e}_{\eta}
$$

in the reference domain $\Omega_{\xi}$.

The hierarchic set of low (vertex) and high order (edge, interior) modal shape functions, generated on the reference triangular element, are defined in [217, 256, 119] and Appendix E. 


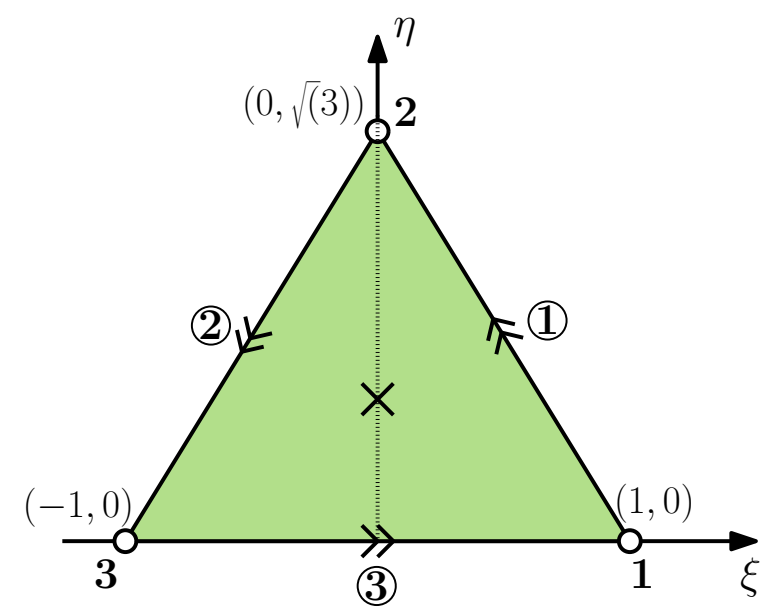

Figure 4.9: Reference triangular element in the referential domain $\Omega_{\xi}=(\xi, \eta)$, with vertices located at $\boldsymbol{\xi}=(1,0),(0, \sqrt{3}),(-1,0)$, in the reference coordinates $\boldsymbol{\xi}=(\xi, \eta)^{T}$.

\section{Quadrilateral Element}

The reference quadrilateral element is taken to be the regular equal sided quadrilateral, shown in Figure 4.10, where the vertex DOF are denoted by the hollow circles, the edge DOF are highlighted with the double arrows and the interior DOF are denoted by the cross. The quadrilateral reference element has vertices $V_{1}=(0,0)$, $V_{2}=(1,0), V_{3}=(1,1)$ and $V_{4}=(0,1)$ in referential coordinates $\boldsymbol{\xi}=(\xi, \eta)^{T}$ in the reference domain $\Omega_{\xi}$.

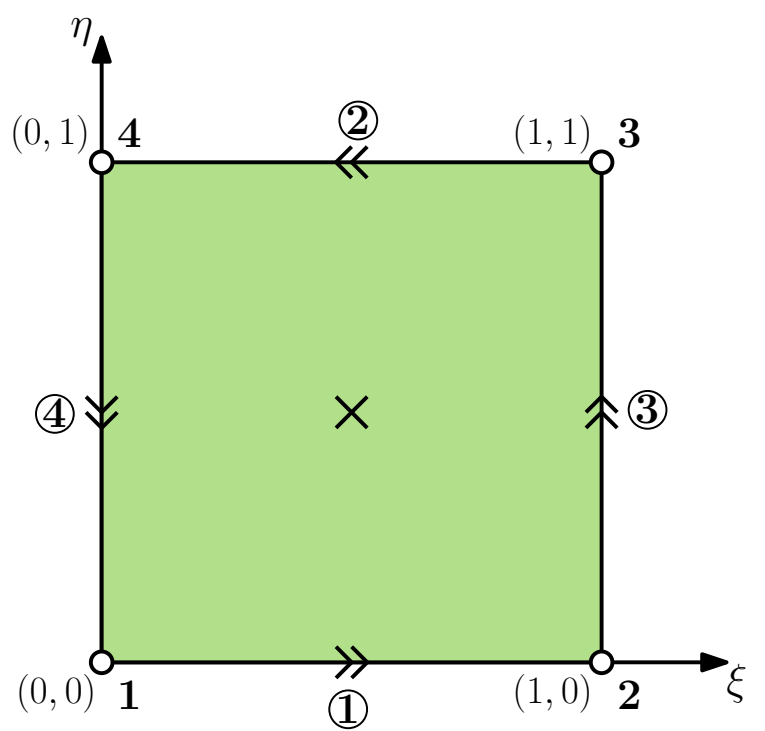

Figure 4.10: Reference Quadrilateral element in the referential domain $\Omega_{\xi}$, with vertices located at $\boldsymbol{\xi}=(0,0),(1,0),(1,1),(0,1)$, in the reference coordinates $\boldsymbol{\xi}=(\xi, \eta)^{T}$.

The hierarchic set of low (vertex) and high order (edge, interior) modal shape functions, generated on the reference quadrilateral element, are defined below. 


\subsubsection{Elemental Mapping Functions}

In order to generate the basis functions for the elements in the spatial domain, where the problem is defined, mapping functions to map the shape functions in the reference domain to the basis functions in the spatial domain are required. Figure 4.11 shows the mapping function $\varphi$ that maps points $(\xi, \eta)$ from the reference $\Omega_{\xi}$ to the spatial $\Omega$ domains at $(r, z)$. If integration was to be performed in the spatial domain one would require the inverse mapping $\varphi^{-1}$, which is not trivial to obtain in all cases. Note that, given the system of equations was reduced to the axisymmetric meridian domain in Section 4.2, the spatial domain (which is the meridian domain) is referred to in terms of the $2 \mathrm{D}$ cylindrical coordinates $(r, z)$.

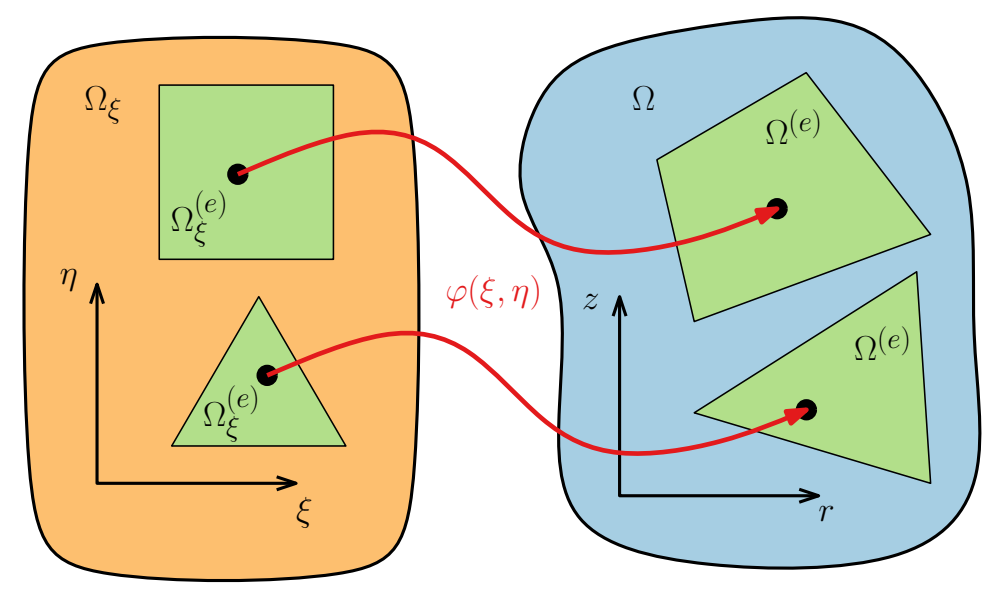

Figure 4.11: Mapping between quadrilateral and triangular finite elements in the reference $\Omega_{\xi}$ and spatial $\Omega$ domains.

The mapping from the reference domain to the spatial domain may be given by

$$
\left(\begin{array}{l}
r \\
z
\end{array}\right)=\varphi^{(e)}(\xi, \eta)
$$

where $\varphi$ denotes the mapping function for the $e^{\text {th }}$ element. Low order finite element methods use straight edged elements to discretise the spatial domain. In this case, linear mapping functions are required for triangular elements and bilinear mapping functions for quadrilaterals.

\section{Linear Mapping}

The linear mapping between the reference $\Omega_{\xi}^{(e)}$ and spatial element $\Omega^{(e)}$ may be given in terms of the barycentric coordinates, which coincide with the lowest order finite 
element hat functions on the reference element, $L_{i}$ as $^{7}$

$$
\left(\begin{array}{l}
r \\
z
\end{array}\right)=\sum_{i=1}^{n} L_{i}(\xi, \eta)\left(\begin{array}{c}
R_{i} \\
Z_{i}
\end{array}\right)
$$

where $\boldsymbol{X}_{i}^{m}=\left(R_{i}, Z_{i}\right)^{T}$ are the nodal coordinates of the spatial element and $n$ denotes the number of vertices of the element. In terms of computing the inverse mapping of the linear element, one simply has to compute the inverse mapping function $\varphi^{-1}$ to determine $(\xi, \eta)$ in terms of $(r, z)$, which is trivial for the linear case.

\section{Non-Linear Mapping through Blending Functions}

In some cases, where complex curved edge geometry is considered, the user may wish to define, more accurately, the discretisation of the geometry. In the case of high order finite elements, the linear mapping function can be complimented to better approximate the realistic geometry in the spatial domain. Thus, a non-linear mapping between the straight edged reference element and a curved element in the spatial domain, illustrated in Figure 4.12, can be applied.

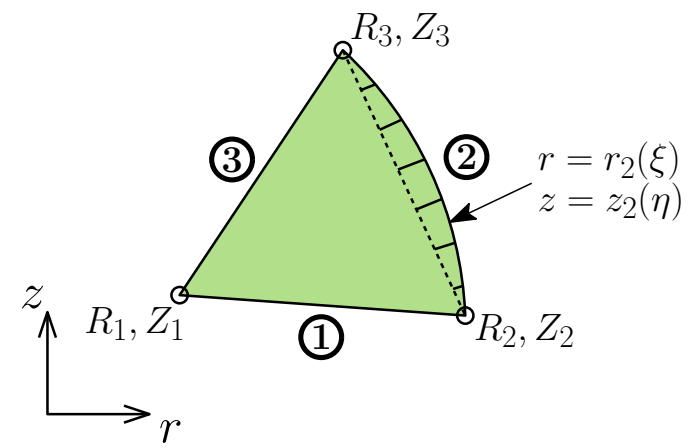

(a) Triangular

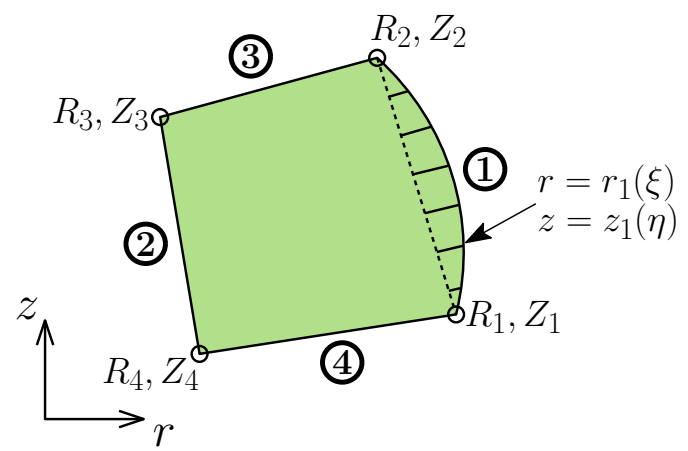

(b) Quadrilateral

Figure 4.12: Curved elements in the spatial domain $\Omega$.

In the context of hierarchic $h p$ finite elements a method, known as the method of blending functions, can be used to represent curved geometries by applying elements with curved edges in the spatial domain ${ }^{8}$. The geometry of curved edges of elements are then parametrised through the functions $r=r_{e}(\xi)$ and $z=z_{e}(\eta)$, where the subscript $e$ refers to specific curved edge(s). The geometric mapping function in

\footnotetext{
${ }^{7}$ The barycentric coordinates $L_{i}$ depend on the vertices of the reference element for which they are defined. These are shown for the reference element in Section 4.4.3.

${ }^{8}$ The exact representation of the geometry can be achieved if that exact parametrisation of the edges is known.
} 
(4.27) then becomes

$$
\left(\begin{array}{l}
r \\
z
\end{array}\right)=\sum_{i=1}^{n} L_{i}(\xi, \eta)\left(\begin{array}{c}
R_{i} \\
Z_{i}
\end{array}\right)+\sum_{e=n c} L_{n_{1}}(\xi, \eta) L_{n_{2}}(\xi, \eta)\left(\begin{array}{c}
r_{e}(\xi) \\
z_{e}(\eta)
\end{array}\right)
$$

where $n c$ is a list containing the curved edges for a given element and $n_{1}$ and $n_{2}$ are the first and second vertices of the specific edge. For further details on the method of blending functions see [244]. In this case the mapping functions are non-linear and so obtaining the inverse mapping function $\varphi^{-1}$ would require a Newton-Raphson, or other similar procedure to obtain the referential coordinates $(\xi, \eta)$ from the spatial coordinates $(r, z)$. But this is only required for post processing and not used in the calculation.

\section{Jacobian}

The final ingredient in performing the integration over the reference elements, shown in Figures 4.9 and 4.10, is to map the geometric integration to the reference domain. Consider, first, the differential meridian "volume" element $\mathrm{d} \Omega^{m}$ which when mapped to the reference domain becomes

$$
\begin{aligned}
\mathrm{d} \Omega^{m}=\mathrm{d} r \mathrm{~d} z & =\left(\frac{\partial r}{\partial \xi} \boldsymbol{e}_{r}+\frac{\partial z}{\partial \xi} \boldsymbol{e}_{z}\right) \mathrm{d} \xi \times\left(\frac{\partial r}{\partial \eta} \boldsymbol{e}_{r}+\frac{\partial z}{\partial \eta} \boldsymbol{e}_{z}\right) \mathrm{d} \eta \\
& =\left(\frac{\partial r}{\partial \xi} \frac{\partial z}{\partial \eta}-\frac{\partial r}{\partial \eta} \frac{\partial z}{\partial \xi}\right) \mathrm{d} \xi \mathrm{d} \eta
\end{aligned}
$$

The integration of a function over an element in the spatial domain can therefore be transformed into an integration in the reference element as

$$
\int_{\Omega^{(e)}} f(r, z) r \mathrm{~d} r \mathrm{~d} z \rightarrow \int_{\Omega_{\xi}^{(e)}} f(\xi, \eta) r|\mathbf{J}| \mathrm{d} \xi \mathrm{d} \eta
$$

where the limits of integration are constant and known a priori and $|\mathbf{J}|$ denotes the determinant of the Jacobian matrix. In terms of computing the gradients in the function, which is the case for some terms in the coupled system of equations, then

$$
\frac{\partial f}{\partial \boldsymbol{x}^{m}}(\xi, \eta)=\sum_{i}^{M} \mathbf{J}^{-T} \frac{\partial N_{i}}{\partial \boldsymbol{\xi}}(\xi, \eta) F_{i}
$$

where the modal values of the function are constant and thus do not depend on space. The integration in the reference domain depends on the determinant of the 
Jacobian matrix, where the Jacobian matrix is given by

$$
\mathbf{J}=\frac{\partial \boldsymbol{x}^{m}}{\partial \boldsymbol{\xi}}=\left[\begin{array}{ll}
\frac{\partial r}{\partial \xi} & \frac{\partial z}{\partial \xi} \\
\frac{\partial r}{\partial \eta} & \frac{\partial z}{\partial \eta}
\end{array}\right]
$$

Based on the mapping between the spatial and referential domain, defined previously, the Jacobian matrix can be readily determined, for 2D geometries, by

$$
\frac{\partial \boldsymbol{x}^{m}}{\partial \boldsymbol{\xi}}=\sum_{i=1}^{M} \frac{\partial \boldsymbol{x}^{m}}{\partial L_{i}} \frac{\partial L_{i}}{\partial \boldsymbol{\xi}} .
$$

With the mapping between the referential and spatial domains now defined the reference triangular and quadrilateral elements will now be introduced and the shape functions determined.

\subsubsection{Hierarchic Shape Functions}

The hierarchic shape functions must be defined on the reference elements, defined above in Section 4.4.1. This Thesis considers a $h p$-finite element discretisation using the $H^{1}(\Omega)$ conforming hierarchic shape function set proposed by Schöberl and Zaglmayr [217, 256]. For this, a discretisation of the domain $\Omega$ into a hybrid mesh consisting of triangular and quadrilateral elements is considered, as illustrated in Figure 4.8. The global finite element space can be decomposed into vertex, edge and interior (or cell) based spaces as

$$
\mathrm{X}_{h p}=\mathrm{X}_{h, 1} \oplus \sum_{\text {edges }}^{M_{E}} \mathrm{X}_{p}^{E} \oplus \sum_{\text {cells }}^{M_{I}} \mathrm{X}_{p}^{I} \subset H^{1}(\Omega),
$$

where $\mathrm{X}_{h, 1}$ denotes the standard vertex based finite element space, $\mathrm{X}_{p}^{E}$ the edge and $\mathrm{X}_{p}^{I}$ the interior (or cell) based spaces $[217,256]$. The shape functions for the triangular elements have already been treated in $[256,119]$ and quadrilateral elements in $[244,127]$ and so are omitted from this Chapter. For details on the hierarchic $H^{1}(\Omega)$ conforming shape functions generated on the reference elements used in this Thesis see Appendix E. 


\subsubsection{Infinite Elements}

In the case of the far field conditions for the static magnetic vector potential and acoustic fields $\left(\boldsymbol{A}^{D C}, P^{D C}\right)$ and the transient magnetic vector potential $\mathcal{A}$, the decay conditions have been approximated at a finite distance from the radiation source, discussed in Section 4.3.1. However, an alternative method, where the parametric mapping function of the quadrilateral element is adjusted such that one boundary is mapped to infinity can be used to better approximate the decay conditions at infinity [34]. A layer of elements can be placed on the outer boundary of a given mesh, where the outer edge is mapped through the parametric mapping to infinity, shown in Figure 4.13.

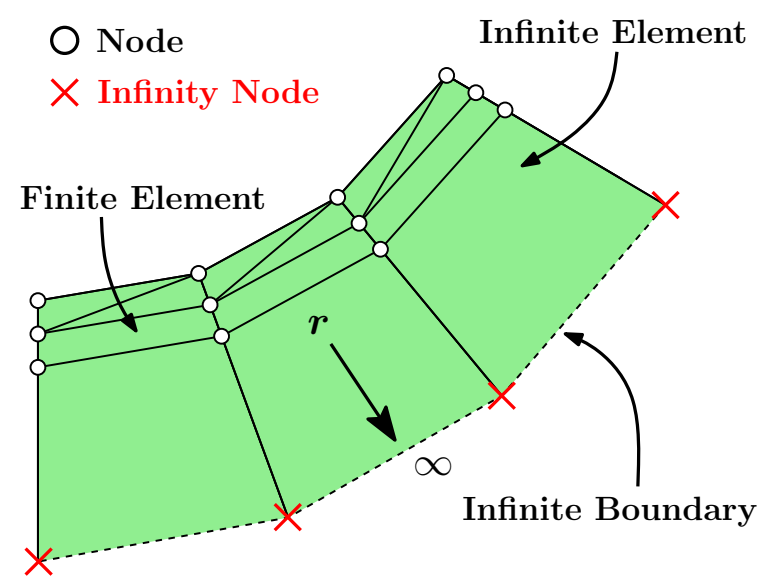

Figure 4.13: Finite element mesh with a layer of quadrilateral infinite elements located on the outer boundary. In the spatial domain the outer boundary is located at a finite distance from the centre, however through the parametric mapping functions on the reference element, become placed at infinity, where the decay conditions are imposed as Dirichlet conditions.

The outer layer of elements must always be convex, such that their outer most nodes never cross or collapse to a point, creating an invalid element (with a negative determinant of the Jacobian matrix). For this to be the case the use of quadrilateral elements, discussed previously, are required. The parametric mapping functions of the reference quadrilateral element must be altered such that the nodes of a particular boundary map to infinity. For this to be the case the nodal coordinates of the quadrilateral element in (E.6) become

$$
\begin{aligned}
L_{1} & =\frac{1-\eta}{1-\xi}, \\
L_{4} & =\frac{\eta}{1-\xi},
\end{aligned}
$$

where only the coordinate functions of vertex 1 and 4 are used as the integration of 
the system is never performed on the infinite boundary ${ }^{9}$. The value of the field at infinity, according to (2.11), is simply applied on the outer boundary as a Dirichlet condition instead. The adjusted definitions of the nodal coordinate functions do not change the shape functions, as these remain the same as previously discussed to maintain bounded solutions. However, they do affect the geometric mapping between the reference and spatial domains.

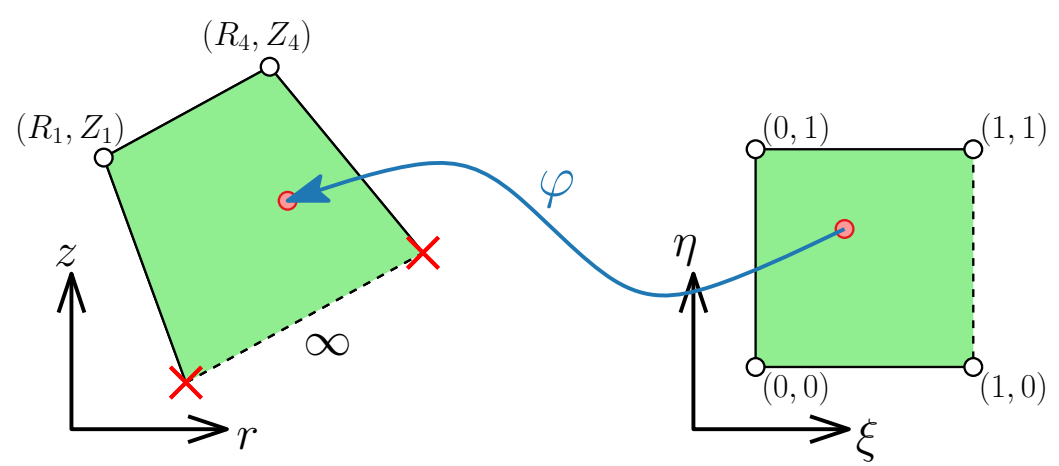

Figure 4.14: Mapping between the reference quadrilateral element and the spatial infinite element.

With knowledge of the required parametric mapping functions and element shape functions the discretised linear system of coupled equations will now be presented.

\subsection{Spatial Discretisation of the Coupled System}

With knowledge of the hierarchic $h p$ finite element basis, discussed in the previous section, the spatial discretisation of the linearised monolithic coupled axisymmetric system of equations in (4.20) is required in order to obtain solutions to the problem.

\subsubsection{Galerkin Approximation}

Based on the finite element functional approximations and parametric mapping, discussed in the previous section, the Galerkin approximation of the solution fields and corresponding test functions can be applied such that:

$$
\mathcal{A}_{\phi}=\sum_{a=1}^{M} \mathcal{A}_{\phi}^{a} N^{a}, \quad \delta_{\mathcal{A}_{\phi}}=\sum_{a=1}^{M} \delta_{\mathcal{A}_{\phi}}^{a} N^{a}, \quad A_{\phi}^{\delta}=\sum_{b=1}^{M} \mathrm{~A}_{\phi}^{\delta b} N^{b},
$$

\footnotetext{
${ }^{9}$ From the reference quadrilateral it is trivial to show that for the infinite boundary (where $\xi=1$ the mapping functions $L_{1}$ and $L_{4}$ become infinity, and remain unit valued at the node and zero at the other node.
} 


$$
\begin{aligned}
& \boldsymbol{U}=\sum_{a=1}^{M} \mathcal{U}^{a} N^{a}, \quad \boldsymbol{\delta}_{\mathfrak{U}}=\sum_{a=1}^{M} \boldsymbol{\delta}_{\mathcal{U}}^{a} N^{a}, \quad \boldsymbol{u}^{\delta}=\sum_{b=1}^{M} \mathbf{u}^{\delta b} N^{b}, \\
& \mathcal{P}=\sum_{a=1}^{M} \mathcal{P}^{a} N^{a}, \quad \delta_{\mathcal{P}}=\sum_{a=1}^{M} \delta_{\mathcal{P}}^{a} N^{a}, \quad P^{\delta}=\sum_{b=1}^{M} \mathrm{P}^{\delta b} N^{b},
\end{aligned}
$$

where $a, b$ indicate the indices of the basis functions, the non-italics versions of the variables $\mathcal{A}_{\phi}, \mathcal{U}, \mathcal{P}, \delta_{\mathcal{A}_{\phi}}, \boldsymbol{\delta}_{\mathcal{U}}, \delta_{\mathcal{P}}, \mathrm{A}_{\phi}^{\delta}, \mathbf{u}^{\delta}, \mathrm{P}^{\delta}$ have been introduced to denote the discrete variables and $M$ denotes the number of shape functions for the $H^{1}(\Omega)$ conforming finite elements for a given element order. The shape functions $N=$ $\left\{N^{V}, N_{i}^{E_{m}}, i=p-1, m=1,2,3, N_{i j}^{I}, i, j=p-1\right\}$ are basis functions that vary in space and the discrete solution variables are constants associated with a given shape function and are not functions of space.

\subsubsection{Linear System}

With knowledge of the Galerkin approximation of the variables and test functions, in (4.35), the coupled system of equations in (4.20) can now be discretised to form the linear system

$$
\mathbf{K} \delta=\mathbf{R},
$$

where $\mathbf{K}$ is the discrete tangent stiffness matrix, $\boldsymbol{\delta}$ is vector of discrete modal solution variables and $\mathbf{R}$ is the vector of discrete residuals.

\section{Static System}

The solution to the static coupled transmission problem becomes: Find $\delta^{D C[\mathrm{k}]}=$ $\left(\delta_{\mathrm{A}_{\phi}}^{D C[\mathrm{k}]} \boldsymbol{e}_{\phi}, \boldsymbol{\delta}_{\tilde{\mathrm{u}}}^{D C[\mathrm{k}]}, \delta_{\mathrm{P}}^{D C[\mathrm{k}]}\right)$ such that

$$
\begin{aligned}
& \text { Magneto-Mechanical One-way Coupling } \\
& \underbrace{\left[\begin{array}{c:c:c}
\mathrm{K}_{\mathrm{AA}}^{D C} & \mathbf{0} & 0 \\
\hdashline \mathbf{K}_{\mathrm{uA}}^{D C} & \mathbf{K}_{\mathrm{uu}}^{D C} & \mathbf{K}_{\mathrm{uP}}^{D C} \\
\mathbf{K}_{\mathrm{PA}}^{D C} & \mathbf{0} & \mathrm{K}_{\mathrm{PP}}^{D C}
\end{array}\right]}_{\mathbf{K}} \underbrace{\left(\begin{array}{c}
\delta_{\hat{A}_{\phi}}^{D C} \\
\boldsymbol{\delta}_{\tilde{\mathrm{u}}}^{D C} \\
\delta_{\mathrm{P}}^{D C}
\end{array}\right)}_{\boldsymbol{\delta}}=-\underbrace{\left(\begin{array}{c}
\mathrm{R}_{\mathrm{A}}^{D C} \\
\mathbf{R}_{\mathrm{u}}^{D C} \\
\mathbf{R}_{\mathrm{P}}^{D C}
\end{array}\right)}_{\mathbf{R}} . \\
& \text { Magneto-Acoustic Acousto-Mechanical }
\end{aligned}
$$

The magnetic and acoustic fields force the mechanical, but the mechanical field does not force the magnetic and acoustic fields, generating both one-way magnetomechanical and acousto-mechanical coupling. 
The discrete residual vector terms in the linear system (4.37) are described, in index notation ${ }^{10}$, by

$$
\begin{aligned}
\left(\mathrm{R}_{\mathrm{A}}^{D C}\right)^{b} & :=-\int_{\operatorname{supp}\left(g_{\phi}^{D C}\right)} J_{\phi}^{D C} N^{b} r^{2} \mathrm{~d} \Omega^{m}, \\
\left(\mathbf{R}_{\mathrm{u}}^{D C}\right)^{b} & :=\mathbf{0}, \\
\left(\mathrm{R}_{\mathrm{P}}^{D C}\right)^{b} & :=\int_{\operatorname{supp}\left(g_{\phi}^{D C}\right)} J_{\phi}^{D C} \frac{\partial r A_{\phi}^{D C}[\mathrm{k}]}{\partial x_{k}^{m}} \frac{\partial N^{b}}{\partial x_{k}^{m}} \mathrm{~d} \Omega^{m} .
\end{aligned}
$$

Remark 4.4 Typical finite element discretisations utilise the third order permutation tensor $\vartheta$ to describe the curl operator in index form, which for the full $3 D$ problem is given as

$$
\nabla \times \boldsymbol{A}=\vartheta_{i j k} \frac{\partial A_{k}}{\partial x_{j}^{m}}
$$

where the third order permutation tensor is given as

$$
\vartheta_{i j k}= \begin{cases}0, & \text { if } i=j, i=k \text { or } j=k \\ -1, & \text { if }(i, j, k)=(1,2,3),(2,3,1) \text { or }(3,1,2) \\ 1, & \text { if }(i, j, k)=(1,3,2),(3,2,1) \text { or }(2,1,3) .\end{cases}
$$

In the axisymmetric case, however, the curl reduces to a two component column vector, which allows for the following expression

$$
\nabla \times\left(A_{\phi} \boldsymbol{e}_{\phi}\right)=\frac{1}{r}\left(-\frac{\partial r A_{\phi}}{\partial z} \boldsymbol{e}_{r}+\frac{\partial r A_{\phi}}{\partial r} \boldsymbol{e}_{z}\right)=\frac{1}{r} \vartheta_{i j}^{m} \frac{\partial r A_{\phi}}{\partial x_{j}^{m}},
$$

where the axisymmetric permutation operator $\vartheta^{m}$ is given as

$$
\vartheta^{m}=\left(\begin{array}{cc}
0 & -1 \\
1 & 0
\end{array}\right)
$$

The tangent stiffness matrix terms, in (4.45), are obtained by applying the Galerkin approximation and finite element discretisation to the directional deriva-

\footnotetext{
${ }^{10}$ Einstein notation is implied here. Einstein notation is a shorthand method for writing a sum in index notation and can be expressed, for the dot product between two vectors $\boldsymbol{a}$ and $\boldsymbol{b}$, as $\boldsymbol{a} \cdot \boldsymbol{b}=\sum_{i=1}^{n} a_{i} b_{i} \underset{\text { notation }}{\stackrel{\text { Einstein }}{\longrightarrow}} a_{i} b_{i}$.
} 
tives, defined in (4.21), which in index notation (see remark 4.4), gives

$$
\begin{aligned}
& {\left[\mathrm{K}_{\mathrm{AA}}^{D C}\right]^{a b}:=\int_{\Omega^{m}} \frac{\mu^{-1}}{r} \frac{\partial r^{2} N^{a}}{\partial x_{k}^{m}} \frac{\partial r^{2} N^{b}}{\partial x_{k}^{m}} \mathrm{~d} \Omega^{m},} \\
& {\left[\mathbf{K}_{\mathrm{uA}}^{D C}\right]_{i}^{a b}:=\int_{\Omega_{c}^{m}} \frac{\mu^{-1}}{r} \vartheta_{n k}^{m} \vartheta_{j l}^{m} \frac{\partial r A_{\phi}^{D C[\mathrm{k}]}}{\partial x_{k}^{m}} \frac{\partial r^{2} N^{a}}{\partial x_{l}^{m}}\left(\frac{\partial \mathfrak{R}_{n i}}{\partial x_{j}^{m}} N^{b}+\mathfrak{R}_{n i} \frac{\partial N^{b}}{\partial x_{j}^{m}}\right) \mathrm{d} \Omega^{m}} \\
& +\int_{\Omega_{c}^{m}} \frac{\mu^{-1}}{r} \vartheta_{n k}^{m} \vartheta_{j l}^{m} \frac{\partial r^{2} N^{a}}{\partial x_{k}^{m}} \frac{\partial r A_{\phi}^{D C[\mathrm{k}]}}{\partial x_{l}^{m}}\left(\frac{\partial \Re_{n i}}{\partial x_{j}^{m}} N^{b}+\mathfrak{R}_{n i} \frac{\partial N^{b}}{\partial x_{j}^{m}}\right) \mathrm{d} \Omega^{m} \\
& -\int_{\Omega_{c}^{m}} \frac{\mu^{-1}}{r} \frac{\partial r A_{\phi}^{D C[\mathrm{k}]}}{\partial x_{k}^{m}} \frac{\partial r^{2} N^{a}}{\partial x_{k}^{m}}\left(\frac{\partial \Re_{j i}}{\partial x_{j}^{m}} N^{b}+\mathfrak{R}_{j i} \frac{\partial N^{b}}{\partial x_{j}^{m}}\right) \mathrm{d} \Omega^{m} \\
& -\int_{\partial \Omega_{c}^{m N}} \frac{\mu^{-1}}{r} \vartheta_{n k}^{m} \vartheta_{j l}^{m}\left(\frac{\partial r A_{\phi}^{D C[\mathrm{k}]}}{\partial x_{k}^{m}} \frac{\partial r^{2} N^{a}}{\partial x_{l}^{m}}+\frac{\partial r^{2} N^{a}}{\partial x_{k}^{m}} \frac{\partial r A_{\phi}^{D C[\mathrm{k}]}}{\partial x_{l}^{m}}\right) n_{j}^{m} \Re_{n i} N^{b} \mathrm{~d} S^{m} \\
& +\int_{\partial \Omega_{c}^{m N}} \frac{\mu^{-1}}{r} \frac{\partial r A_{\phi}^{D C[\mathrm{k}]}}{\partial x_{k}^{m}} \frac{\partial r^{2} N^{a}}{\partial x_{k}^{m}} n_{j}^{m} \Re_{j i} N^{b} \mathrm{~d} S^{m}, \\
& {\left[\mathbf{K}_{\mathrm{uu}}^{D C}\right]_{i j}^{a b}:=\int_{\Omega_{c}^{m}} \frac{\lambda}{r^{3}} \mathfrak{R}_{k j} \frac{\partial r^{2} N^{a}}{\partial x_{k}^{m}} \mathfrak{R}_{l i} \frac{\partial r^{2} N^{b}}{\partial x_{l}^{m}} \mathrm{~d} \Omega^{m}} \\
& +\int_{\Omega_{c}^{m}} G\left(\frac{\partial \Re_{l j}}{\partial x_{k}^{m}} N^{a}+\mathfrak{R}_{l j} \frac{\partial N^{a}}{\partial x_{k}^{m}}\right)\left(\frac{\partial \Re_{l i}}{\partial x_{k}^{m}} N^{b}+\mathfrak{R}_{l i} \frac{\partial N^{b}}{\partial x_{k}^{m}}\right) r \mathrm{~d} \Omega^{m} \\
& +\int_{\Omega_{c}^{m}} G\left(\frac{\partial \mathfrak{R}_{k j}}{\partial x_{l}^{m}} N^{a}+\mathfrak{R}_{k j} \frac{\partial N^{a}}{\partial x_{l}^{m}}\right)\left(\frac{\partial \mathfrak{R}_{l i}}{\partial x_{k}^{m}} N^{b}+\mathfrak{R}_{l i} \frac{\partial N^{b}}{\partial x_{k}^{m}}\right) r \mathrm{~d} \Omega^{m}, \\
& {\left[\mathbf{K}_{\mathrm{uP}}^{D C}\right]_{i}^{a b}:=-\int_{\partial \Omega_{c}^{m N}} N^{a} N^{b} \Re_{i k} n_{k}^{m} r \mathrm{~d} S^{m},} \\
& {\left[\mathrm{~K}_{\mathrm{PA}}^{D C}\right]^{a b}:=\int_{\operatorname{supp}\left(J_{\phi}^{D C}\right)} J_{\phi}^{D C} \frac{\partial N^{a}}{\partial x_{k}^{m}} \frac{\partial N^{b}}{\partial x_{k}^{m}} \mathrm{~d} \Omega^{m}} \\
& -\int_{\operatorname{supp}\left(g_{\phi}^{D C}\right)} \mu_{0}^{-1} \frac{\partial r A_{\phi}^{D C[\mathrm{k}]}}{\partial x_{k}^{m}} \frac{\partial N^{b}}{\partial x_{k}^{m}}\left(r \nabla^{m 2} N^{a}+3 \frac{\partial N^{a}}{\partial r}\right) \mathrm{d} \Omega^{m}, \\
& {\left[\mathrm{~K}_{\mathrm{PP}}^{D C}\right]^{a b}:=\int_{\Omega_{n}^{m}} \frac{\partial N^{a}}{\partial x_{k}^{m}} \frac{\partial N^{b}}{\partial x_{k}^{m}} r \mathrm{~d} \Omega^{m} .}
\end{aligned}
$$

Here, the nodal test functions $\mathrm{A}_{\phi}^{\delta b}, \mathbf{u}^{\delta b}, \mathrm{P}^{\delta b}$ appear on both the left and right hand side of the equation system, and so cancel from the system, thus are not included in the stiffness and forcing terms, see [38].

In the above system the solution to the DC field is obtained ${ }^{11}$ by performing a NR procedure until the norm of the residual is less than a user defined tolerance

\footnotetext{
${ }^{11}$ The meridian Laplacian operator of a scalar field $w$ is denoted by $\nabla^{m 2}(w)=\frac{\partial}{\partial x_{k}^{m}}\left(\frac{\partial w}{\partial x_{k}^{m}}\right)$ in index notation.
} 
$|\mathbf{R}|<T O L$. The updated solution field for a given iteration is obtained by

$$
\left(\begin{array}{c}
\hat{\mathrm{A}}_{\phi}^{D C} \\
\tilde{\mathbf{u}}^{D C} \\
\mathrm{P}^{D C}
\end{array}\right)^{[\mathrm{k}+1]}=\left(\begin{array}{c}
\hat{\mathrm{A}}_{\phi}^{D C} \\
\tilde{\mathbf{u}}^{D C} \\
\mathrm{P}^{D C}
\end{array}\right)^{[\mathrm{k}]}+\left(\begin{array}{c}
\delta_{\hat{\mathrm{A}}_{\phi}}^{D C} \\
\boldsymbol{\delta}_{\tilde{\mathrm{u}}}^{D C} \\
\delta_{\mathrm{P}}^{D C}
\end{array}\right)^{[\mathrm{k}]}
$$

\section{Transient System}

The general linear system of equations results from the acousto-magneto-mechanical problem and is thus interpreted as: Find $\boldsymbol{\delta}_{=}\left(\boldsymbol{\delta}_{\mathcal{A}_{\phi}} \boldsymbol{e}_{\phi}, \boldsymbol{\delta}_{\tilde{\mathcal{U}}}, \boldsymbol{\delta}_{\mathcal{P}}\right)$ such that

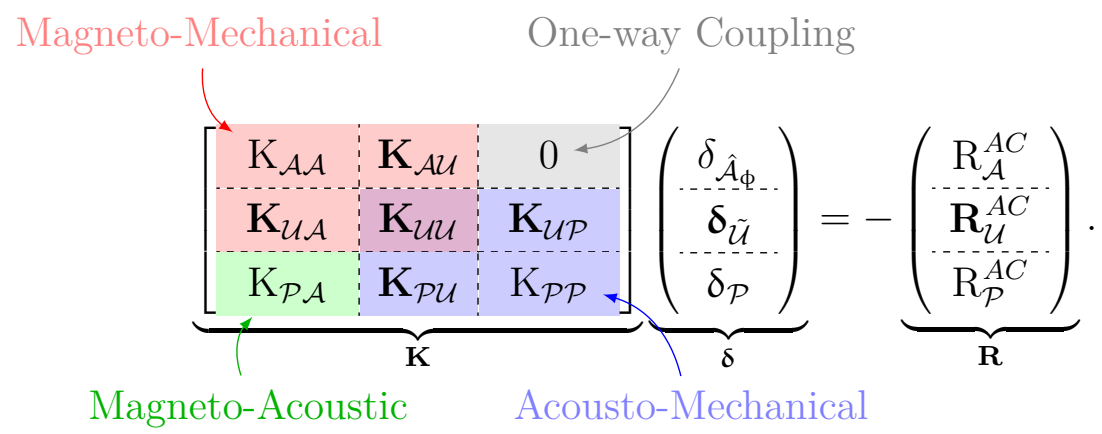

In the linear system, the subscripts on the tangent stiffness matrix entries are used to denote the corresponding discretised linearised terms, or directional derivatives as in (4.21), where $\mathrm{K}_{\mathcal{A A}}=D \tilde{R}_{\mathcal{A}}^{A C}\left(N^{b} \hat{\mathcal{A}}_{\phi}^{\delta b}\right)\left[N^{a} \delta_{\hat{\mathcal{A}}_{\phi}}^{a}\right]$ denotes the pure magnetic linearised term, $\mathrm{K}_{\mathcal{A U}}=D \tilde{R}_{\mathcal{A}}^{A C}\left(N^{b} \hat{\mathcal{A}}_{\phi}^{\delta b}\right)\left[N^{a} \boldsymbol{\delta}_{\tilde{\mathcal{U}}}^{a}\right]$ denotes the magnetic system linearised with respect to displacements (the Lorentz current term) etc.

The discrete residual vector terms in the linear system (4.45) are described by

$$
\begin{aligned}
\left(\mathrm{R}_{\mathcal{A}}^{A C}\right)^{b} & :=-\int_{\operatorname{supp}\left(\mathcal{I}_{\phi}^{A C}\right)} \mathcal{I}_{\phi}^{A C} N^{b} r^{2} \mathrm{~d} \Omega^{m}, \\
\left(\mathbf{R}_{\mathcal{U}}^{A C}\right)^{b} & :=\mathbf{0}, \\
\left(\mathrm{R}_{\mathcal{P}}^{A C}\right)^{b} & :=\int_{\operatorname{supp}\left(g_{\phi}^{A C}\right)} \mathcal{I}_{\phi}^{A C} \frac{\partial r A_{\phi}^{D C}}{\partial x_{k}^{m}} \frac{\partial N^{b}}{\partial x_{k}^{m}} \mathrm{~d} \Omega^{m} .
\end{aligned}
$$

The tangent stiffness matrix terms, in (4.45), are obtained by applying the Galerkin approximation and finite element discretisation to the directional derivatives, defined in (4.21), which gives

$$
\begin{aligned}
{\left[\mathrm{K}_{\mathcal{A} \mathcal{A}}\right]^{a b} } & :=\int_{\Omega^{m}} \frac{\mu^{-1}}{r} \frac{\partial r^{2} N^{a}}{\partial x_{k}^{m}} \frac{\partial r^{2} N^{b}}{\partial x_{k}^{m}} \mathrm{~d} \Omega^{m}+\mathrm{i} \omega \int_{\Omega^{m}} \gamma N^{a} N^{b} r^{3} \mathrm{~d} \Omega^{m} \\
{\left[\mathbf{K}_{\mathcal{A U}}\right]_{j}^{a b} } & :=-\mathrm{i} \omega \int_{\Omega_{c}^{m}} \gamma \frac{\partial r A_{\phi}^{D C}}{\partial x_{k}^{m}} \mathfrak{R}_{k j} N^{a} N^{b} r \mathrm{~d} \Omega^{m}
\end{aligned}
$$




$$
\begin{aligned}
& {\left[\mathbf{K}_{\mathcal{U A} \mathcal{A}}\right]_{i}^{a b}:=\int_{\Omega_{c}^{m}} \frac{\mu^{-1}}{r} \vartheta_{n k}^{m} \vartheta_{j l}^{m} \frac{\partial r A_{\phi}^{D C}}{\partial x_{k}^{m}} \frac{\partial r^{2} N^{a}}{\partial x_{l}^{m}}\left(\frac{\partial \Re_{n i}}{\partial x_{j}^{m}} N^{b}+\Re_{n i} \frac{\partial N^{b}}{\partial x_{j}^{m}}\right) \mathrm{d} \Omega^{m}} \\
& +\int_{\Omega_{c}^{m}} \frac{\mu^{-1}}{r} \vartheta_{n k}^{m} \vartheta_{j l}^{m} \frac{\partial r^{2} N^{a}}{\partial x_{k}^{m}} \frac{\partial r A_{\phi}^{D C}}{\partial x_{l}^{m}}\left(\frac{\partial \Re_{n i}}{\partial x_{j}^{m}} N^{b}+\Re_{n i} \frac{\partial N^{b}}{\partial x_{j}^{m}}\right) \mathrm{d} \Omega^{m} \\
& -\int_{\Omega_{c}^{m}} \frac{\mu^{-1}}{r} \frac{\partial r A_{\phi}^{D C}}{\partial x_{k}^{m}} \frac{\partial r^{2} N^{a}}{\partial x_{k}^{m}}\left(\frac{\partial \Re_{j i}}{\partial x_{j}^{m}} N^{b}+\mathfrak{R}_{j i} \frac{\partial N^{b}}{\partial x_{j}^{m}}\right) \mathrm{d} \Omega^{m} \\
& -\int_{\partial \Omega_{c}^{m} N} \frac{\mu^{-1}}{r} \vartheta_{n k}^{m} \vartheta_{j l}^{m}\left(\frac{\partial r A_{\phi}^{D C}}{\partial x_{k}^{m}} \frac{\partial r^{2} N^{a}}{\partial x_{l}^{m}}+\frac{\partial r^{2} N^{a}}{\partial x_{k}^{m}} \frac{\partial r A_{\phi}^{D C}}{\partial x_{l}^{m}}\right) n_{j}^{m} \mathfrak{R}_{n i} N^{b} \mathrm{~d} S^{m} \\
& +\int_{\partial \Omega_{c}^{m N}} \frac{\mu^{-1}}{r} \frac{\partial r A_{\phi}^{D C}}{\partial x_{k}^{m}} \frac{\partial r^{2} N^{a}}{\partial x_{k}^{m}} n_{j}^{m} \Re_{j i} N^{b} \mathrm{~d} S^{m}, \\
& {\left[\mathbf{K}_{\mathcal{U} \mathcal{U}}\right]_{i j}^{a b}:=\int_{\Omega_{c}^{m}} \frac{\lambda}{r^{3}} \mathfrak{R}_{k j} \frac{\partial r^{2} N^{a}}{\partial x_{k}^{m}} \Re_{l i} \frac{\partial r^{2} N^{b}}{\partial x_{l}^{m}} \mathrm{~d} \Omega^{m}} \\
& +\int_{\Omega_{c}^{m}} G\left(\frac{\partial \mathfrak{R}_{l j}}{\partial x_{k}^{m}} N^{a}+\mathfrak{R}_{l j} \frac{\partial N^{a}}{\partial x_{k}^{m}}\right)\left(\frac{\partial \mathfrak{R}_{l i}}{\partial x_{k}^{m}} N^{b}+\mathfrak{R}_{l i} \frac{\partial N^{b}}{\partial x_{k}^{m}}\right) r \mathrm{~d} \Omega^{m} \\
& +\int_{\Omega_{c}^{m}} G\left(\frac{\partial \Re_{k j}}{\partial x_{l}^{m}} N^{a}+\mathfrak{R}_{k j} \frac{\partial N^{a}}{\partial x_{l}^{m}}\right)\left(\frac{\partial \Re_{l i}}{\partial x_{k}^{m}} N^{b}+\mathfrak{R}_{l i} \frac{\partial N^{b}}{\partial x_{k}^{m}}\right) r \mathrm{~d} \Omega^{m} \\
& -\omega^{2} \int_{\Omega_{c}^{m}} \rho \Re_{k i} \Re_{k j} N^{a} N^{b} r \mathrm{~d} \Omega^{m}, \\
& {\left[\mathbf{K}_{\mathcal{U P}}\right]_{i}^{a b}:=-\int_{\partial \Omega_{c}^{m} N} N^{a} N^{b} \Re_{k i} n_{k}^{m} r \mathrm{~d} S^{m},} \\
& {\left[\mathrm{~K}_{\mathcal{P} \mathcal{A}}\right]^{a b}:=\int_{\operatorname{supp}\left(J_{\phi}^{D C}\right)} J_{\phi}^{D C} \frac{\partial N^{a}}{\partial x_{k}^{m}} \frac{\partial N^{b}}{\partial x_{k}^{m}} \mathrm{~d} \Omega^{m}} \\
& -\int_{\operatorname{supp}\left(\mathcal{I}_{\phi}^{s}\right)} \mu_{0}^{-1} \frac{\partial r A_{\phi}^{D C[\mathrm{k}]}}{\partial x_{k}^{m}} \frac{\partial N^{b}}{\partial x_{k}^{m}}\left(r \nabla^{m 2} N^{a}+3 \frac{\partial N^{a}}{\partial r}\right) \mathrm{d} \Omega^{m}, \\
& {\left[\mathbf{K}_{\mathcal{P U}}\right]_{j}^{a b}:=\omega^{2} \int_{\partial \Omega_{c}^{m N}} \rho^{+} n_{k}^{m} \mathfrak{R}_{k j} N^{a} N^{b} r \mathrm{~d} S^{m}} \\
& {\left[\mathrm{~K}_{\mathcal{P} \mathcal{P}}\right]^{a b}:=\int_{\Omega_{n}^{m}} \frac{\partial N^{a}}{\partial x_{k}^{m}} \frac{\partial N^{b}}{\partial x_{k}^{m}} r \mathrm{~d} \Omega^{m}-\omega^{2} \int_{\Omega_{c}^{n}} \frac{1}{c^{2}} N^{a} N^{b} r \mathrm{~d} \Omega^{m}} \\
& +\int_{\Omega_{p m l}^{m}} \Lambda_{1 k l} \frac{\partial N^{a}}{\partial x_{l}^{m}} \frac{\partial N^{b}}{\partial x_{k}^{m}} r \mathrm{~d} \Omega^{m}-\omega^{2} \int_{\Omega_{p m l}^{n}} \frac{1}{c^{2}} \Lambda_{2} N^{a} N^{b} r \mathrm{~d} \Omega^{m} .
\end{aligned}
$$

The linear system of the static problem can be obtained analogously to the linear system in (4.45), which describes the discrete form of the linearised approach of the fully coupled transient acousto-magneto-mechanical problem. This linear system can be further split into individual coupling mechanisms. The magnetomechanical coupling can thus be defined by the block monolithic scheme between the magnetic and mechanical equations, highlighted in red, and is exactly the same as that reported in [150]. The acousto-mechanical coupling through the acoustic and magnetic equations is highlighted in the blue block. The green blocks denote 
the magneto-acoustic terms, where the term arising from the assumption of the Biot-Savart coils, which results in $\operatorname{supp}\left(\mathcal{J}_{\phi}^{s}\right)$ being located in the free space region $\Omega_{n}$ is present. The magneto-acoustic coupling is purely one-way coupling, where the magnetic field excites the acoustic field, but the acoustic field does not force the magnetic field.

Note that, if the coils are considered as conductors where the Biot-Savart assumption is dropped, then the term $\left[\mathbf{K}_{\mathcal{P} \mathcal{A}}\right]_{a b}$ will become a block of zeros and $\left(\mathbf{R}_{\mathcal{P}}\right)_{b}$ a vector of zeros. In this case, the coils will form part of the conducting region $\Omega_{c}$ and thus supp $\left(\mathcal{J}_{\phi}^{s}\right)$ will not be located in free space $\mathbb{R}^{2} \backslash \Omega_{c}^{m}$. This will remove any magneto-acoustic coupling (and hence the green block in (4.45)) and the three fields will interact through the mutual coupling between the mechanical field.

For a full description of the assembly procedures involved in the construction of the linear system and the numbering of the degrees of freedom, the reader is referred to the approach discussed for hierarchical shape functions in [119] .

\subsubsection{Numerical Integration}

The terms in the linear system, whilst expressed in terms of the shape functions and thus polynomials, are still very difficult to integrate. Consider however, that a quadrature rule can be used to approximate definite integrals and avoid the complexities of exact integration. The Gaussian quadrature rule is a rule constructed to yield exact integration of polynomials of degree $2 n-1$, where $n$ is the number of quadrature points, in 1D. For 2D quadrilaterals the integration is trivial due to its tensor product structure of 1D Gauss-Legendre rules. For 2D triangles it is more complicated. The approach used is described in [242] and combines Gauss-Legendre and Gauss-Jacobi rules. But more efficient schemes is a current hot topic of research. The integration of the function of the reference element can thus be obtained by a product of weights $w_{i}$ and points $\boldsymbol{\xi}_{i}$ as

$$
\int_{\Omega_{\xi}^{(e)}} f(\boldsymbol{\xi}) \mathrm{d} \Omega^{m} \approx \sum_{i=1}^{n} w_{i} f\left(\boldsymbol{\xi}_{i}\right)
$$

The integration weights and points can therefore be determined for arbitrary orders of degree [135]. Using this rule the integration of the linear system can thus be redefined in terms of the Gaussian quadrature rule.

Whilst the infinite element mapping functions in (4.34) and blending functions in (4.28) do not, in general, resemble polynomials, the integration of such functions is still approximated to an excellent degree of accuracy using Gaussian quadrature 
$[28,97]$.

\subsection{Numerical Solution Strategy}

The computational strategy for the implementation of the linearised approach to solving the coupled axisymmetric acousto-magneto-mechanical system, in (4.20), is defined as a flowchart in Figure 4.15.

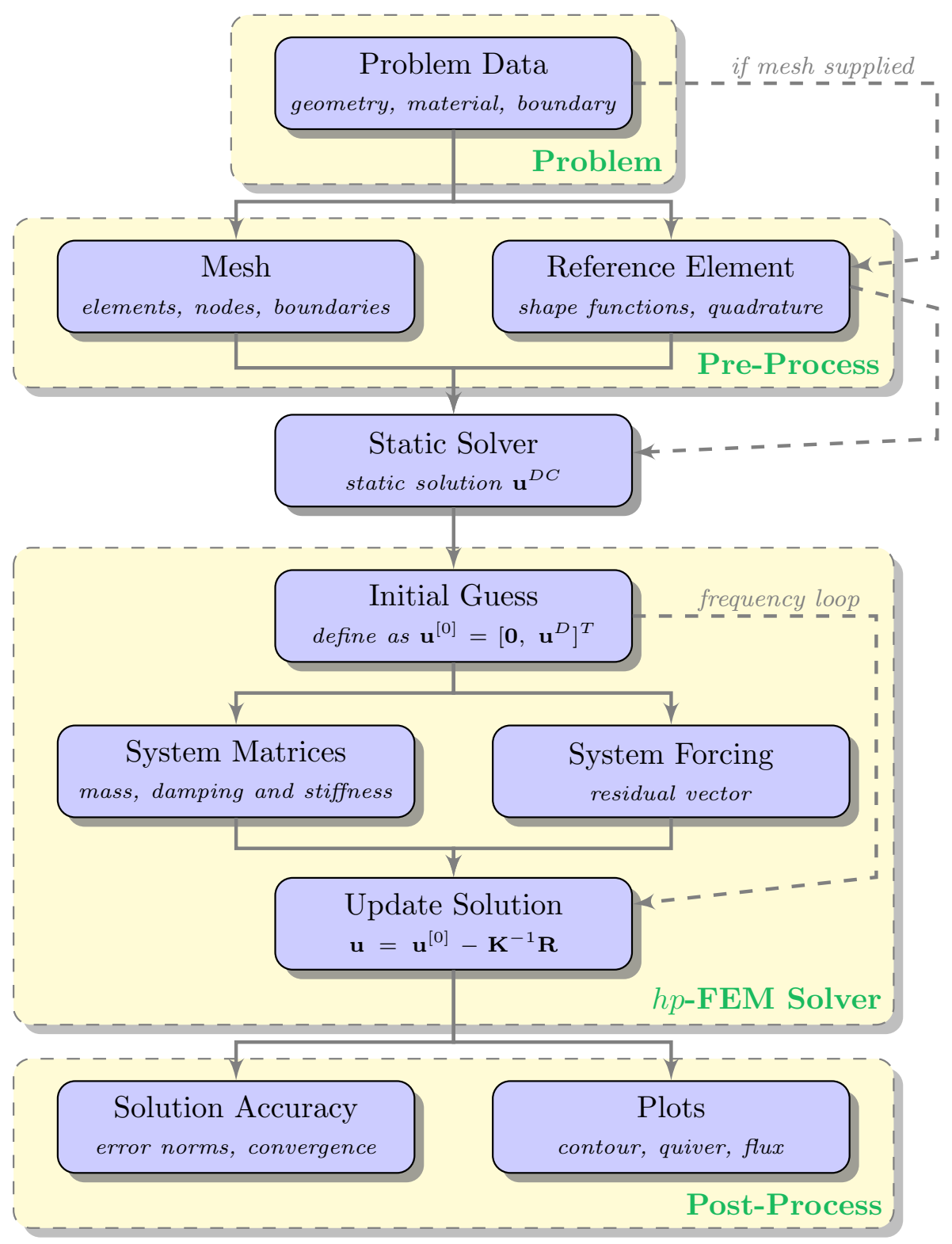

Figure 4.15: Numerical solution strategy flowchart for the linearised approach solver.

The idea for the numerical implementation is a modularised code, where the problem definition, pre-processing, static and dynamic $h p$-FEM solvers and the post- 
processing are all contained in modules. The problem data module incorporates all of the problem definitions; including the geometry, materials and boundary information etc. of the problem. The problem data feeds into the pre-processing where, based on the defined $h$ and $p$, a mesh is generated and the shape functions and quadrature information of the reference element are obtained. Note that in the case a mesh is pre-defined, the code skips the mesh generation step. The static solver is then run to obtain the static solution, which uses the same $h p$-FEM solver as the dynamic problem, just with different system matrix terms. The dynamic solver is then run whereby the initial guess of the field is defined before constructing the system matrices and residual vector. The matrices are obtained first at an element level, where numerical integration on the reference element is performed, and then the elemental contributions are summed into the system matrices. The complete linear system is solved using a direct solver ${ }^{12}$ to obtain the update variable which is added to the initial guess to obtain the complete fields. This data can then be post-processed where error performance and plots are defined. For a series of techniques required to improve the efficiency of the implementation in MATLAB [1], see Appendix G.

The complete solution algorithm is summarised in Algorithm 2, at the end of Chapter 7 .

\subsection{Chapter Summary}

This Chapter has provided a computational implementation of the novel linearised approach for an axisymmetric system. A suitable transformation of variables has been performed to allow for standard $H^{1}(\Omega)$ conforming hierarchic finite element shape functions to be used. The treatment of the far field conditions (located at $\infty$ ) of the fields in truncated domains has also been considered to allow for the accurate simulation of the fields with minimal increase in the number of unknowns required in the system. The discrete linear system is defined first for the non-linear static problem, where a series of one-way coupling phenomena arise. Based on the physically motivated linearised approach, the solution to the static system is then used in the discrete transient system to compute the transient fields, in a time harmonic description, of the fully coupled problem.

\footnotetext{
${ }^{12}$ Given the reduction in order to axisymmetric domains, the reduction in NDOF from 3D permits the use of direct matrix solvers. For 3D problems this will, however, require the use of iterative solvers and an efficient pre-conditioner to compute the matrix system, for examples of such solvers see $[77,153]$.
} 
The discrete system of the novel linearised approach, obtained in this Chapter, permits an efficient monolithic single step solution strategy as the system matrices are independent of NR iteration. Thus, this system offers an efficient solution strategy and aligns with the objective of "To provide an efficient computational methodology for handling the solution to the coupled problem of MRI scanners using both approaches."

In the next Chapter a series of numerical experiments will be performed on a variety of academic and industrially relevant benchmark examples. These experiments will test the algorithms and computational implementation of the linearised approach provided in the preceding Chapters 3 and 4 .

$\overline{\text { Algorithm } 1 \text { Algorithm for the discrete } h p \text {-FE solution to linearised acousto- }}$ magneto-mechanical coupling in MRI scanners.

1: Prescribe an initial guess for the static $\left(\mathbf{A}^{D C[0]}, \mathbf{u}^{D C[0]}, \mathrm{P}^{D C[0]}\right)=$ $\left(r \hat{\mathrm{A}}_{\phi}^{D C[0]} \boldsymbol{e}_{\phi}, r \hat{\mathrm{u}}_{r}^{D C[0]} \boldsymbol{e}_{r}+\mathrm{u}_{z}^{D C[0]} \boldsymbol{e}_{z}, \mathrm{P}^{D C[0]}\right) \in\left(X \times Y\left(\boldsymbol{u}_{D}^{D C}\right) \times Z\right)$ and dynamic $\left(r \hat{\mathcal{A}}_{\phi}^{[0]} \boldsymbol{e}_{\phi}, r \hat{\mathcal{U}}_{r}^{[0]} \boldsymbol{e}_{r}+\mathcal{U}_{z}^{[0]} \boldsymbol{e}_{z}, \mathcal{P}^{[0]}\right) \in\left(X \times Y\left(\boldsymbol{u}_{D}^{D C}\right) \times Z\right)$ fields

2: Obtain the discrete static DC solution updates $\left(\delta_{\hat{\mathrm{A}}_{\phi} C}^{D C} \boldsymbol{e}_{\phi}, \boldsymbol{\delta}_{\tilde{\mathrm{u}}}^{D C}, \delta_{\mathrm{P}}^{D C}\right)$ by solving the linear monolithic system of the static problem, which is obtained analogous to (4.45).

3: Set the DC solutions to be

$$
\begin{aligned}
\mathbf{A}^{D C} & =r\left(\hat{\mathrm{A}}_{\phi}^{D C[0]}+\delta_{\hat{\mathrm{A}}_{\phi}}^{D C[0]}\right) \boldsymbol{e}_{\phi}, \\
\mathbf{u}^{D C} & =\mathfrak{R}\left(\tilde{\mathbf{u}}^{D C[0]}+\boldsymbol{\delta}_{\tilde{\mathrm{u}}}^{D C[0]}\right), \\
\mathrm{P}^{D C} & =\mathrm{P}^{D C[0]}+\delta_{\mathrm{P}}^{D C[0]} .
\end{aligned}
$$

4: Obtain the discrete harmonic $\mathrm{AC}$ solution updates $\left(\delta_{\hat{\mathcal{A}}_{\phi}} \boldsymbol{e}_{\phi}, \boldsymbol{\delta}_{\tilde{\mathcal{U}}}, \boldsymbol{\delta}_{\mathcal{P}}\right)$ by solving the monolithic system (4.45).

5: Set the AC solutions to be

$$
\begin{aligned}
& \mathcal{A}^{A C}=r\left(\hat{\mathcal{A}}_{\phi}^{[0]}+\delta_{\hat{\mathcal{A}}_{\phi}}\right) \boldsymbol{e}_{\phi}, \\
& \mathcal{U}^{A C}=\mathfrak{R}\left(\tilde{\mathcal{U}}^{[0]}+\boldsymbol{\delta}_{\tilde{\mathcal{U}}}\right), \\
& \mathcal{P}^{A C}=\mathcal{P}^{[0]}+\delta_{\mathcal{P}} .
\end{aligned}
$$

6: The complete linearised transient solutions are then

$$
\begin{aligned}
\mathbf{A}(t) & =\mathbf{A}^{D C}+\operatorname{Re}\left(\mathcal{A}^{A C} e^{\mathrm{i} \omega t}\right) \\
\mathbf{u}(t) & =\mathbf{u}^{D C}+\operatorname{Re}\left(\mathcal{U}^{A C} e^{\mathrm{i} \omega t}\right) \\
\mathrm{P}(t) & =\mathrm{P}^{D C}+\operatorname{Re}\left(\mathcal{P}^{A C} e^{\mathrm{i} \omega t}\right)
\end{aligned}
$$





\section{Chapter 5}

\section{Numerical Simulations of the Linearised Approach}

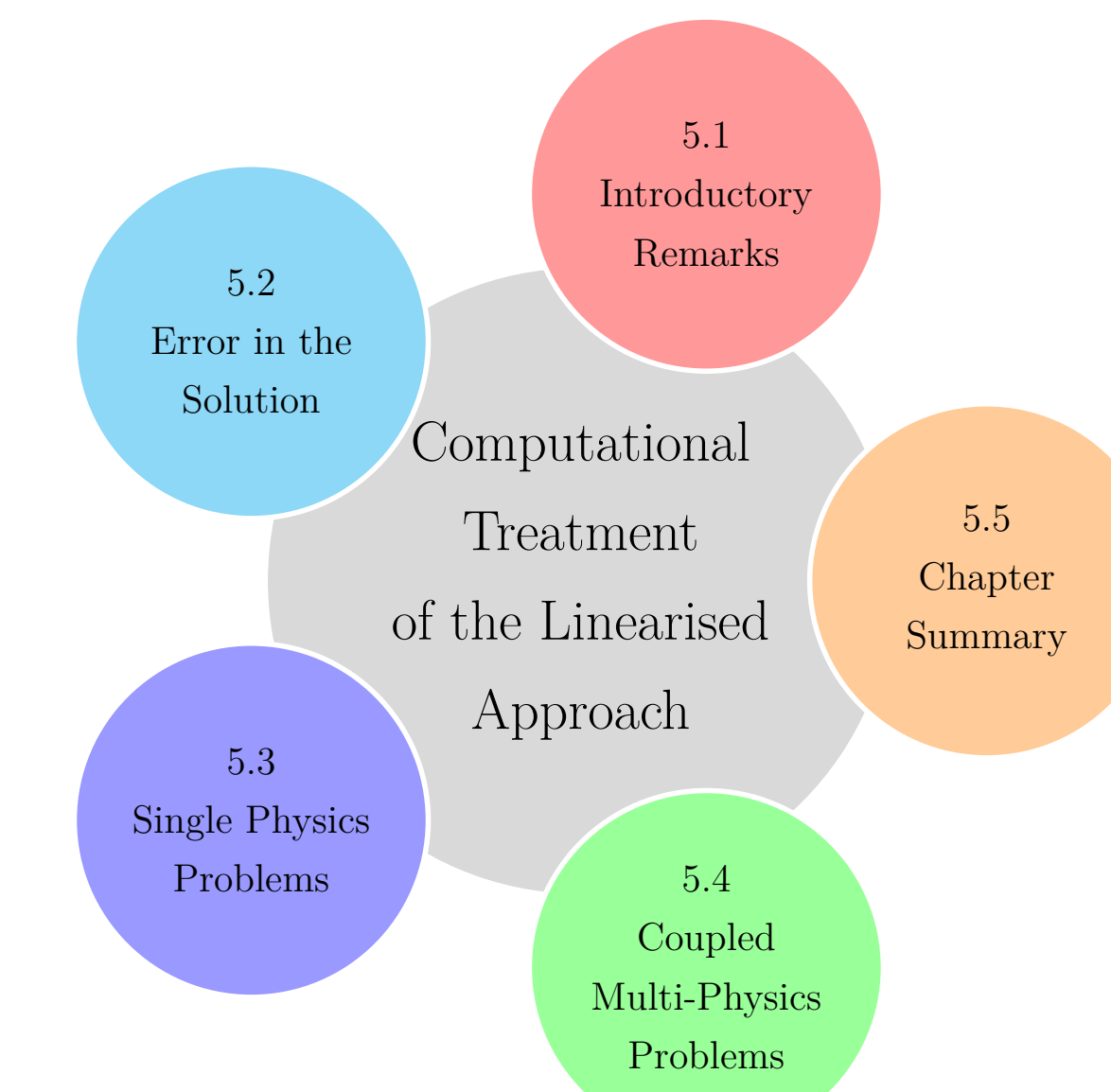

"Logic will get you from A to Z; imagination will take you everywhere."

Albert Einstein 


\subsection{Introductory Remarks}

This Chapter presents a series of academic and industrial numerical examples used to benchmark the newly proposed linearised approach, as presented in Chapter 3, and the discrete approach, as described in Chapter 4. First, the computational solution to a series of single physics problems, where the analytical solutions are known, are considered. Each of the three problems considered are concerned with a single physical field: the magnetic, mechanical and acoustic fields. The computational solution to a series of coupled physics problems are then considered.

From the objectives of this Thesis, discussed in section 1.4, this Chapter addresses the objective: "To determine the accuracy of the linearised approach across the full operating range of MRI scanners" by benchmarking the computational solutions to single and coupled physics problems against known analytical solutions as well as commercial codes to test the accuracy. The contents of this Chapter builds on the work carried out in the published papers $[150,26]$, where the discussion of the technical details has been extended.

The Chapter presents a series of single physics problems in Section 5.3 to demonstrate the independent validation of the electromagnetic, mechanical and acoustic fields for uncoupled problems in Sections 5.3.1, 5.3.2 and 5.3.3 respectively. The Chapter then goes on to present a series of coupled physics problems in Section 5.4. A coupled acousto-mechanical problem is presented in Section 5.4.1, followed by a simple MRI magnet type problem, for both magneto-mechanical and acoustomagneto-mechanical coupling in Section 5.4.2.

\subsection{Error in the Solution}

In order to confirm that the finite element implementation of the linear system presented in Chapter 4 produces the correct solutions to a given problem, the accuracy of the solution must be compared to known solutions. Certain simple academic problems provide analytical solutions that can be used to benchmark the $h p$-finite element implementation. To confirm the solutions are correct a series of error measures can be used to determine the accuracy of the solution across the complete domain by integration of the difference in the solutions. 


\subsubsection{Error Norms}

In order to measure the quality of the computed solution, compared with the analytical, the error in the solution can be measured through the following norms

$$
\begin{aligned}
\|\boldsymbol{e}\|_{L^{2}(\Omega)} & :=(\boldsymbol{e}, \overline{\boldsymbol{e}})^{1 / 2}, \\
\|e\|_{H^{1}(\Omega)} & :=\left(\|e\|_{L^{2}(\Omega)}^{2}+\|\nabla e\|_{L^{2}(\Omega)}^{2}\right)^{1 / 2}, \\
\|\boldsymbol{\sigma}(\boldsymbol{e})\|_{S N S(\Omega)} & :=\|\operatorname{tr}(\boldsymbol{\sigma}(\boldsymbol{e}))\|_{L^{2}(\Omega)},
\end{aligned}
$$

where $(5.1 \mathrm{c})$ is only computed for mechanical problems. In the above, $\boldsymbol{e}$ denotes the difference between the computed and analytical solutions. In the above $(\boldsymbol{e}, \overline{\boldsymbol{e}})=$ $\int_{\Omega} \boldsymbol{e} \cdot \overline{\boldsymbol{e}} \mathrm{d} \Omega$ is the standard $L^{2}$ inner product and $\overline{\boldsymbol{e}}$ denotes the complex conjugate of $\boldsymbol{e}$. The three norms are used to measure the performance of the solution and its spatial gradients as well as the error associated with the sum of normal stresses to examine the extent to which the developed formulation can overcome mechanical locking [93, 52, 243].

Equipped with these error norms and knowledge of the theoretical a priori convergence rates of the $h p$-finite element scheme [244, 127], the academic single physics problems can be analysed, starting the electromagnetic problem.

\subsection{Single Physics Problems}

A series of single field problems are first presented, in order to determine the accuracy of the implementation for each of the three fields: magnetic, mechanical and acoustics. The three problems all have known analytical solutions and as such can be used to test the accuracy of the finite element implementation.

\subsubsection{Conducting Sphere in a Uniform Alternating Magnetic Field}

A closely related problem to the solution of (2.10), with $\boldsymbol{g}^{s}=\boldsymbol{g}^{l}=\mathbf{0}$, is that of a rigid conducting object located in free space excited by a uniform harmonic background magnetic field of amplitude $\mathcal{H}_{0}$ and frequency $\omega$. In this case $\mu_{0}^{-1} \nabla \times \boldsymbol{A} \rightarrow \boldsymbol{\mathcal { H }}_{0}$ as $|\boldsymbol{x}| \rightarrow \infty$. For a spherical conductor $\Omega_{c}=\left\{\boldsymbol{x}:|\boldsymbol{x}|^{2} \leq R^{2}\right\}$ of radius $R$, permeability $\mu_{s}$ and conductivity $\gamma_{s}$, as illustrated in Figure 5.1, an analytical axisymmetric solution is presented in [234] for $A_{\phi}$. 


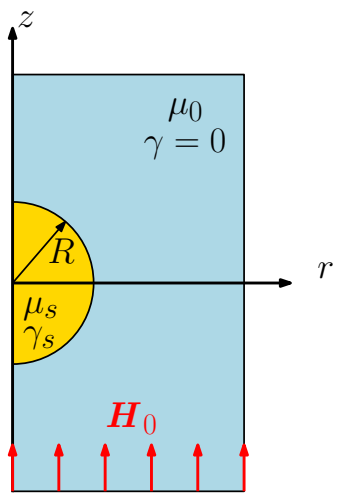

Figure 5.1: Conducting sphere in a uniform alternating magnetic field: problem setup.

Here, the case of $R=1 \mathrm{~m}, \gamma_{s}=10^{7} \mathrm{~S} / \mathrm{m}, \mu_{s}=\mu_{0}, \mathcal{H}_{0}=\mu_{0}^{-1} \boldsymbol{e}_{z} \mathrm{~Wb}$ and angular frequencies $\omega=[5,50] 2 \pi \mathrm{rad} / \mathrm{s}$ is considered. To simulate this problem, the computational domain is chosen to be $\Omega^{m}=([0,4] \times[-4,4]) \mathrm{m}^{2}$ and a suitably simplified version of Algorithm 1 is solved. For this problem there is no static component of the magnetic field and as such $\left.\delta_{\hat{\mathcal{A}}_{\phi}}\right|_{\partial \Omega_{n}}=\left.\hat{\mathcal{A}}_{\phi}\right|_{\partial \Omega_{n}}$ and $\hat{\mathcal{A}}_{\phi}:=\delta_{\hat{\mathcal{A}}_{\phi}}$.

To test the convergence of the $h p$-finite element solution a series of meshes of $[330,598,1248,4952,13882,31117]$ unstructured quasi-uniform triangular elements of maximum size $h=[0.5,0.375,0.25,0.125,0.075,0.05] \mathrm{m}$, respectively, are generated. The convergence is measured using the relative error measure $\| \mathcal{A}_{\phi}-$ $\mathcal{A}_{\phi}\left\|_{L^{2}\left(\Omega^{m}\right)} /\right\| \mathcal{A}_{\phi} \|_{L^{2}\left(\Omega^{m}\right)}$ and $\left\|\mathcal{A}_{\phi}-\mathcal{A}_{\phi}\right\|_{H^{1}\left(\Omega^{m}\right)} /\left\|\mathcal{A}_{\phi}\right\|_{H^{1}\left(\Omega^{m}\right)}$.

A blending function approach, discussed in Section 4.4.2, is considered to represent the exact geometry of the sphere's surface [244]. This function avoids any geometrical error in the solution due to coarse approximation of the boundary.

\section{$h$-refinement}

Figures 5.2 and 5.3 plot the relative error measures $\left\|\mathcal{A}_{\phi}-\mathcal{A}_{\phi}\right\|_{L^{2}\left(\Omega^{m}\right)} /\left\|\mathcal{A}_{\phi}\right\|_{L^{2}\left(\Omega^{m}\right)}$ and $\left\|\mathcal{A}_{\phi}-\mathcal{A}_{\phi}\right\|_{H^{1}\left(\Omega^{m}\right)} /\left\|\mathcal{A}_{\phi}\right\|_{H^{1}\left(\Omega^{m}\right)}$ against the global characteristic length of the elements for varying degrees of element order of $p=[1,2,3]$ under $h$-refinement for different excitation frequencies of $f=[5,50] \mathrm{Hz}$.

Figure 5.2 shows the convergence of the $L^{2}(\Omega)$ error against the maximum element edge length $h$ on a logarithmic scale, where each point on the curve represents a mesh refinement and each curve uses a different element order for $p=1,2,3$. After an initial region of pre-asymptotic behaviour, each curve indicates a straight line suggesting that the convergence is algebraic, as predicted in [244, 127, 23, 102, 22]. The slope triangles highlighted in the plots suggest that the correct $p+1$ rate of 
convergence for smooth problems ${ }^{1}$ [244], is achieved. Similar convergence rates are also obtained for $p>3$, as shown in [254, 222].

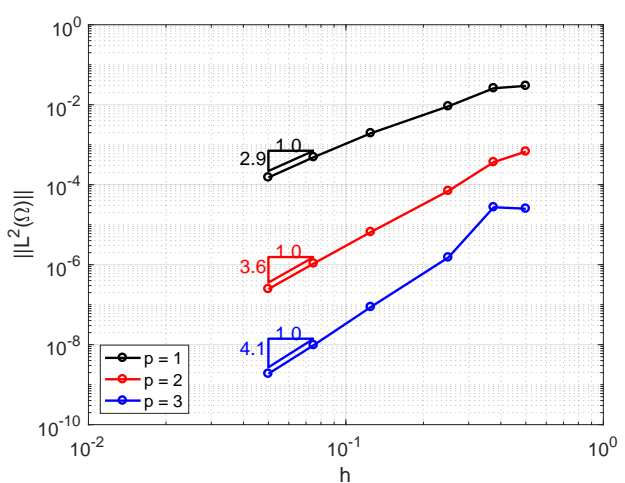

(a) $f=5 H z$

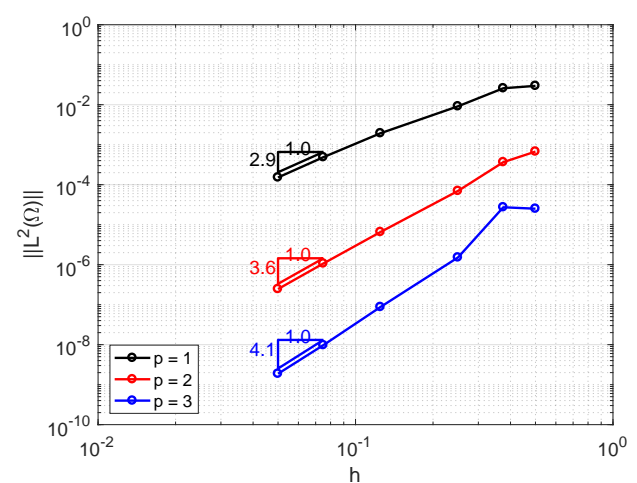

(b) $f=50 H z$

Figure 5.2: Conducting sphere in a uniform alternating magnetic field: convergence of $\| \mathcal{A}_{\phi}-$ $\mathcal{A}_{\phi}\left\|_{L^{2}\left(\Omega^{m}\right)} /\right\| \mathcal{A}_{\phi} \|_{L^{2}\left(\Omega^{m}\right)}$ under $h$-refinement for elements of $p=[1,2,3]$ for frequencies of $f=$ $[5,50] \mathrm{Hz}$, where the $L^{2}(\Omega)$ norm of the error is plotted against the maximum element edge length $h$.

Figure 5.3 shows the convergence of the $H^{1}(\Omega)$ error against the maximum element edge length $h$ on a logarithmic scale, where each point on the curve represents a mesh refinement and each curve uses a different element order for $p=1,2,3$. After initial pre-asymptotic behaviour, the curves show the expected algebraic $p$ rate of convergence for smooth problems ${ }^{2}$ as suggested in [244]. Similar convergence rates are, again, also obtained for $p>3$.

In the case of the lower frequencies, the rates of convergence are better than those predicted, such as in Figure 5.2a, where the rate of 2.1 for $p=1$ is greater than predicted etc. However, as the frequency increases, the theoretical rates of convergence of the error norms are no longer recovered for coarse discretisations, such as in Figure 5.2b, where only the last few fine meshes recover the correct convergence rate. The pre-asymptotic region is also more pronounced for higher frequencies, suggesting the importance of refinement for higher frequency. This effect is also apparent when looking at the $H^{1}(\Omega)$ error norm in Figure 5.3b. This effect is due to the decrease in the skin depth (see Appendix A for details) and thus larger gradients in the solution close to the surface of the conductor.

\footnotetext{
${ }^{1}$ The $p+1$ convergence rate of the $L^{2}(\Omega)$ error norm under $h$-refinement is given by the a priori error estimate $\|e\|_{L^{2}(\Omega)} \leq C h^{-(p+1)}$. Taking the $\log$ yields $\log \|e\|_{L^{2}(\Omega)} \leq \log C-(p+1) \log h$.

${ }^{2}$ The $p$ convergence rate of the $H^{1}(\Omega)$ error norm under $h$-refinement is given by the a priori error estimate $\|e\|_{H^{1}(\Omega)} \leq C h^{-p}$. Taking the $\log$ yields $\log \|e\|_{H^{1}(\Omega)} \leq \log C-p \log h$.
} 


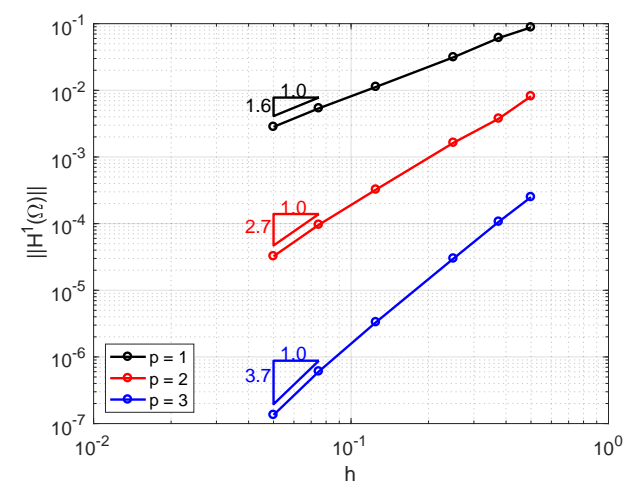

(a) $f=5 H z$

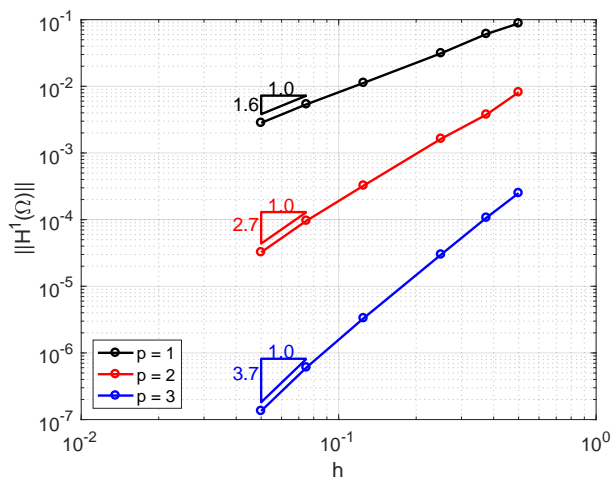

(b) $f=50 H z$

Figure 5.3: Conducting sphere in a uniform alternating magnetic field: convergence of $\| \mathcal{A}_{\phi}-$ $\mathcal{A}_{\phi}\left\|_{H^{1}\left(\Omega^{m}\right)} /\right\| \mathcal{A}_{\phi} \|_{H^{1}\left(\Omega^{m}\right)}$ under $h$-refinement for elements of $p=[1,2,3]$ for frequencies of $f=$ $[5,50] H z$, where the $H^{1}(\Omega)$ norm of the error is plotted against the maximum element edge length $h$.

Figure 5.4 plots the $L^{2}(\Omega)$ and $H^{1}(\Omega)$ error norms against the number of degrees of freedom for various element order $p=1,2,3$ under $h$-refinement. The slope triangles highlighted in the Figures illustrate that the correct convergence rates of $(p+1) / 2$ and $p / 2$ are achieved [244]. These plots are used to illustrate the comparison between the speed of the (slower) algebraic and exponential rates of convergence obtained by the $h$ - and $p$-refinement schemes, respectively.

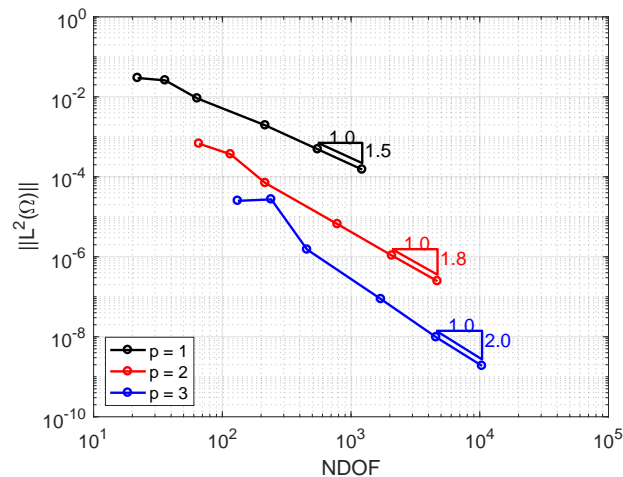

(a) $\left\|\mathcal{A}_{\phi}-\mathcal{A}_{\phi}\right\|_{L^{2}\left(\Omega^{m}\right)} /\left\|\mathcal{A}_{\phi}\right\|_{L^{2}\left(\Omega^{m}\right)}$

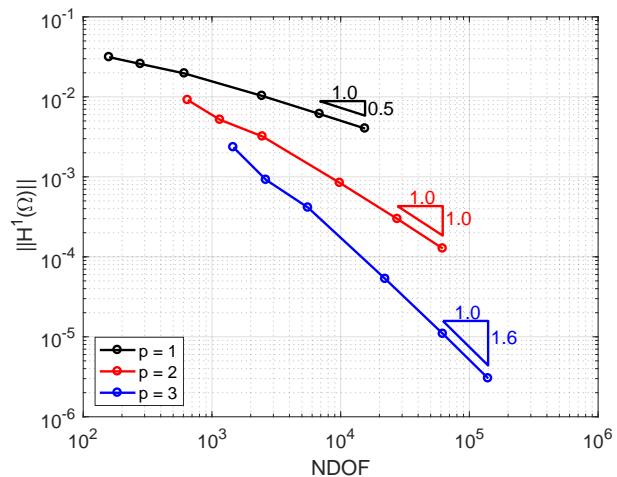

(b) $\left\|\mathcal{A}_{\phi}-\mathcal{A}_{\phi}\right\|_{H^{1}\left(\Omega^{m}\right)} /\left\|\mathcal{A}_{\phi}\right\|_{H^{1}\left(\Omega^{m}\right)}$

Figure 5.4: Conducting sphere in a uniform alternating magnetic field: convergence of $\| \mathcal{A}_{\phi}-$ $\mathcal{A}_{\phi}\left\|_{L^{2}\left(\Omega^{m}\right)} /\right\| \mathcal{A}_{\phi} \|_{L^{2}\left(\Omega^{m}\right)}$ and $\left\|\mathcal{A}_{\phi}-\mathcal{A}_{\phi}\right\|_{H^{1}\left(\Omega^{m}\right)} /\left\|\mathcal{A}_{\phi}\right\|_{H^{1}\left(\Omega^{m}\right)}$ at a frequency $f=5 H z$ for elements of $p=[1,2,3]$ against the number of degrees of freedom NDOF under $h$-refinement. 


\section{$p$-refinement}

Uniform polynomial enrichment corresponding to $p=[1,2, \cdots, 10]$ is now considered for a mesh of 1248 unstructured quasi-uniform triangular elements of maximum size $h=0.25 \mathrm{~m}$. Figure 5.5 plots the relative error measures $\left\|\mathcal{A}_{\phi}-\mathcal{A}_{\phi}\right\|_{L^{2}\left(\Omega^{m}\right)} /\left\|\mathcal{A}_{\phi}\right\|_{L^{2}\left(\Omega^{m}\right)}$ and $\left\|\mathcal{A}_{\phi}-\mathcal{A}_{\phi}\right\|_{H^{1}\left(\Omega^{m}\right)} /\left\|\mathcal{A}_{\phi}\right\|_{H^{1}\left(\Omega^{m}\right)}$ against the number of degrees of freedom NDOF for varying frequencies of the alternating magnetic field under $p$-refinement.

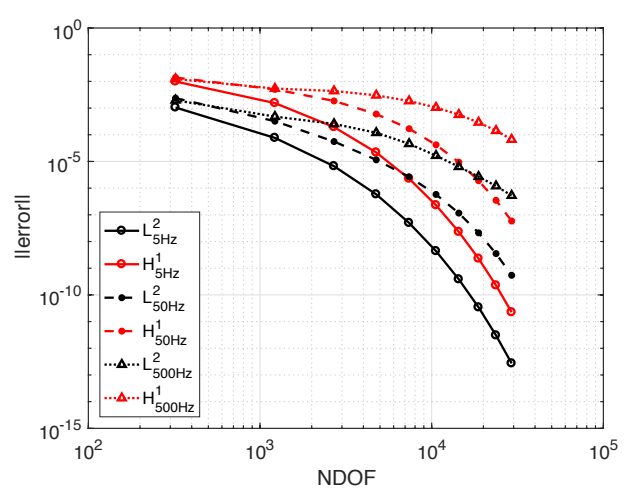

(a) $\log (\mathrm{NDOF})$

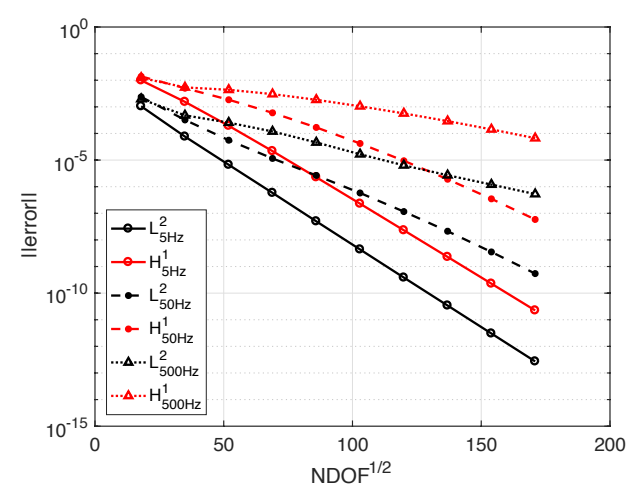

(b) $\mathrm{NDOF}^{1 / 2}$

Figure 5.5: Conducting sphere in a uniform alternating magnetic field: convergence of $\| \mathcal{A}_{\phi}-$ $\mathcal{A}_{\phi}\left\|_{L^{2}\left(\Omega^{m}\right)} /\right\| \mathcal{A}_{\phi} \|_{L^{2}\left(\Omega^{m}\right)}$ and $\left\|\mathcal{A}_{\phi}-\mathcal{A}_{\phi}\right\|_{H^{1}\left(\Omega^{m}\right)} /\left\|\mathcal{A}_{\phi}\right\|_{H^{1}\left(\Omega^{m}\right)}$ under $p$-refinement.

Figure 5.5a shows the convergence of the error against the number of degrees of freedom on a logarithmic scale, where each point represents a polynomial refinement and the different curves correspond to different frequencies and error measures. Each line indicates a downward sloping curve suggesting that the convergence is exponential. This is confirmed by plotting the error on a logarithmic scale against the number of degrees of freedom raised to the power $1 / 2$ on an algebraic scale in Figure 5.5b, which agrees with the a-priori error estimates in $[23,22]$. After a preasymptotic region, each curve becomes a straight line indicating that convergence of the numerical to the analytical solution is exponential with respect to the square root number of degrees of freedom for $p$-refinement of this problem ${ }^{3}$. This corresponds to the expected rate for smooth solutions, as reported in [244].

As the frequency of the alternating magnetic field increases, the gradient of the lines in Figure 5.5b reduces indicating that, although still exponential, the rate of convergence is lower. Physically, this is due to the smaller skin depths $s=\sqrt{2 /(\omega \gamma \mu)}$

\footnotetext{
${ }^{3}$ The convergence rate under $p$-refinement is given by the a priori error estimate $\|e\|_{L^{2}(\Omega)} \leq$ $C e^{-\left(\beta N_{d o f}^{1 / 2}\right)}$. Taking the $\log$ yields $\log \|e\|_{L^{2}(\Omega)} \leq \log C-\beta N_{d o f}^{1 / 2}$, where $\beta$ is a positive constant.
} 
[234] (see Appendix A.2), which characterises the depth to which the eddy currents $\boldsymbol{g}^{o}=\gamma \mathcal{E} \approx \mathrm{i} \omega \gamma \mathcal{A}_{\phi} \boldsymbol{e}_{\phi}$ decay to $1 / e$ of their surface value, associated with higher $\omega$, where $e \approx 2.72$ represents the natural number. It is possible to improve the gradient in the plots in Figure 5.5b by using a graded mesh towards the sphere's surface [244].

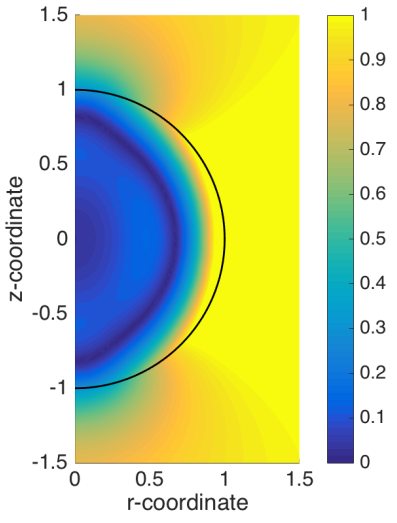

(a) $\omega=5 \mathrm{rad} / \mathrm{s}$

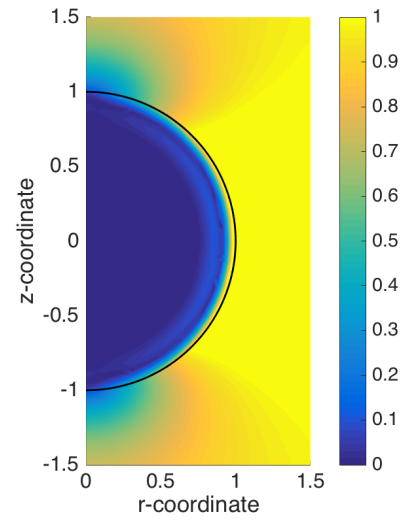

(b) $\omega=50 \mathrm{rad} / \mathrm{s}$

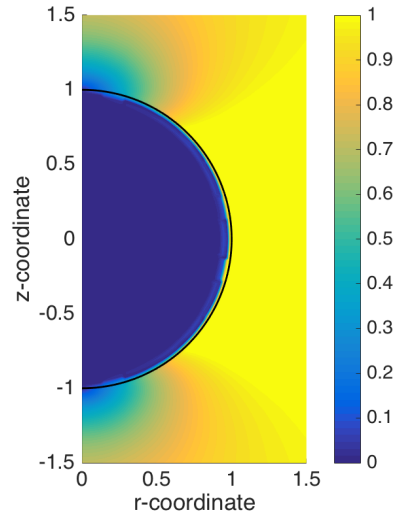

(c) $\omega=500 \mathrm{rad} / \mathrm{s}$

Figure 5.6: Conducting sphere in a uniform alternating magnetic field: contours of $|\boldsymbol{B}| \approx \mid \nabla \times$ $\left(\mathcal{A}_{\phi} \boldsymbol{e}_{\phi}\right) \mid$ around the conducting sphere at different frequencies.

To illustrate the different fields and skin depth effects for different frequencies, Figure 5.6 shows the contours of $|\boldsymbol{B}| \approx\left|\nabla \times\left(\mathcal{A}_{\phi} \boldsymbol{e}_{\phi}\right)\right|$ for the various frequencies of the converged solutions. This Figure illustrates the smaller $s$ for higher $\omega$ and the need to use higher fidelity discretisations to capture the solution with the same degree of accuracy.

\section{Infinite Elements}

If the analytical solution to the above problem was not known then a value of the field at the outer boundary would be required in order to solve the problem. In the case of an external uniform field the value of the solution at the farfield would correspond to this external field. Applying this as an approximate Dirichlet boundary condition would result in an error associated with the distance of the outer truncated boundary from the interior sphere.

In order to improve the accuracy of the far field boundary condition, without having to use large domains, the infinite element approach described in Section 4.4.4 can be applied. To apply the infinite elements the solution needs to be adjusted such that $\mathcal{H}=\mathcal{H}_{0}+\mathcal{H}_{*}$ and thus $\boldsymbol{A}=\boldsymbol{A}_{0}+\boldsymbol{A}_{*}$, where $\nabla \times \boldsymbol{A}_{0}=\mu_{0} \boldsymbol{H}_{0}$ and $\mathcal{H}_{0}$ corresponds to the applied exterior magnetic field. Rather than solving for $\mathcal{A}$, 
as was the case in Section 5.3.1, the problem may now be described in terms of solving for $\mathcal{A}_{*}$, where the decay condition holds for infinite elements (see Appendix $\mathrm{F}$ for details). However, in the case of the MRI magnet problem then the field decay naturally tends to 0 and no perturbation in the fields is required.

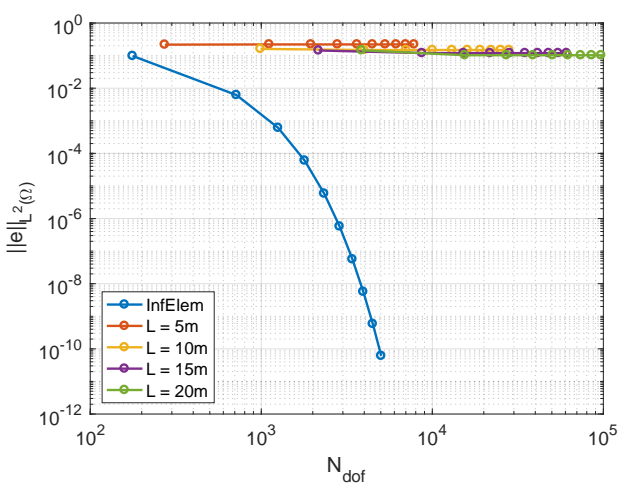

(a) $L^{2}(\Omega)$ error

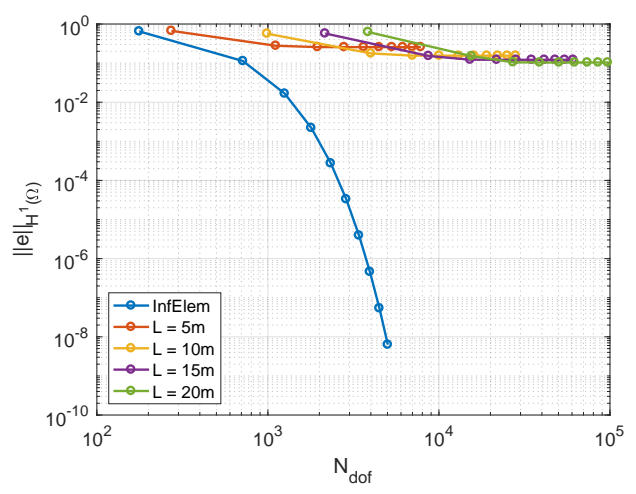

(b) $H^{1}(\Omega)$ error

Figure 5.7: Conducting sphere in a uniform alternating magnetic field: convergence of $\left\|\mathcal{A}_{\phi}-\mathcal{A}_{\phi}\right\|_{L^{2}\left(\Omega^{m}\right)} /\left\|\mathcal{A}_{\phi}\right\|_{L^{2}\left(\Omega^{m}\right)}$ and $\left\|\mathcal{A}_{\phi}-\mathcal{A}_{\phi}\right\|_{H^{1}\left(\Omega^{m}\right)} /\left\|\mathcal{A}_{\phi}\right\|_{H^{1}\left(\Omega^{m}\right)}$ for the perturbed eddy current problem using both infinite elements and an approximate boundary condition at $L=[5,10,15,20] \mathrm{m}$ under $p$-refinement.

Figure 5.7 plots the relative error measures $\left\|\mathcal{A}_{\phi}-\mathcal{A}_{\phi}\right\|_{L^{2}\left(\Omega^{m}\right)} /\left\|\mathcal{A}_{\phi}\right\|_{L^{2}\left(\Omega^{m}\right)}$ and $\left\|\mathcal{A}_{\phi}-\mathcal{A}_{\phi}\right\|_{H^{1}\left(\Omega^{m}\right)} /\left\|\mathcal{A}_{\phi}\right\|_{H^{1}\left(\Omega^{m}\right)}$ against the number of degrees of freedom NDOF for a frequency of the alternating magnetic field of $f=5 \mathrm{~Hz}$ for both infinite elements and approximate boundary conditions applied to domains of varying outer boundary location $L=[5,10,15,20] m$ under $p$-refinement.

Figure 5.7a and 5.7b show the convergence of the solution in the $L^{2}(\Omega)$ and $H^{1}(\Omega)$ norms on a logarithmic scale, respectively, where each point represents a polynomial refinement. Each of the curves (red, yellow, purple, green) represents a truncated domain of different size $\Omega^{m}=([0, L] \times[-L, L])$, where the outer boundary is located at $L=[5,10,15,20] \mathrm{m}$, respectively. The blue line corresponds to the original domain of size $L=4 m$ with a layer of infinite elements on the outer boundary. In this case the boundary condition is approximated to the decay condition such that $\boldsymbol{A}_{*}=\mathbf{0}$ on $\partial \Omega^{m}$. The curves corresponding to the truncated domains all show that the error is dominated primarily by the approximate boundary condition, which results in relatively large errors compared with the spatial discretisation, highlighted by the plateau of the curves. In this case, even larger domains would be required in order to obtain a reasonable accuracy, which would result in a significant increase in the number of degrees of freedom. On the other hand, applying a layer of 32 infinite 
elements on the outer boundary allows for this truncation error to be overcome, thus recovering the optimal rates of convergence [244], shown on the blue curve.

\subsubsection{Mechanical Shell Subject to a Pressure Field}

To verify the treatment of the elasticity system, the solution of Equation (2.23) is considered for $\boldsymbol{\sigma}^{e}=\mathbf{0}$. The static displacements $\boldsymbol{u} \neq \boldsymbol{u}(t)$ are computed for the case of a spherical mechanical shell $\Omega=\Omega_{c}=\left\{\boldsymbol{x}: r_{i}^{2} \leq|\boldsymbol{x}|^{2} \leq r_{o}^{2}\right\}$ of inner and outer radii $r_{i}, r_{o}$, respectively. The inner and outer surfaces of the shell are subject to traction conditions $-P_{i} \boldsymbol{n}$ and $-P_{o} \boldsymbol{n}$ resulting from internal and external pressures $P_{i}, P_{o}$ respectively, on different parts of $\partial \Omega^{m N}$, as illustrated in Figure 5.8. This problem is axisymmetric and has an analytical solution [41], which can be expressed in terms of the cylindrical displacement components $\left(u_{r}, u_{z}\right)$.

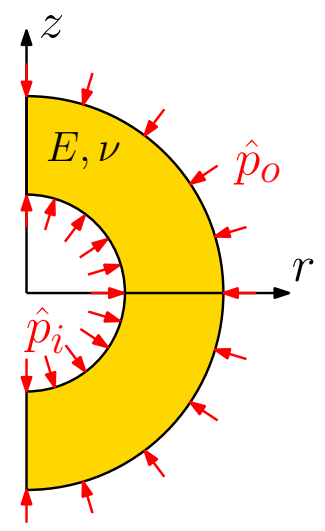

Figure 5.8: Mechanical shell subject to a pressure field: problem setup.

Specifically, the problem is solved for $r_{i}=0.5 \mathrm{~m}, r_{o}=1 \mathrm{~m}, E=210 \times 10^{9} \mathrm{~Pa}$, $P_{i}=P_{o}=10^{4} \mathrm{~Pa}$ and $\nu=0.49$ so that the shell is nearly incompressible. As described in Section 2.3, part of the boundary of the shell must be fixed in order to ensure a unique solution of the field. A suitably simplified version of Algorithm 1 for a single iteration is solved, where the displacements are chosen to be fixed $\boldsymbol{\delta}_{\mathrm{u}}^{D C[0]}=\mathrm{u}_{r}^{D C} \boldsymbol{e}_{r}+\mathrm{u}_{z}^{D C} \boldsymbol{e}_{z}$ on a small boundary segment $\partial \Omega^{m D}$ according to the analytical solution. The region of computation corresponds to $\Omega^{m}=\Omega_{c}^{m}=\{(r, z)$ : $\left.r_{i}^{2} \leq\left(r^{2}+z^{2}\right) \leq r_{o}^{2}\right\}$.

Like the conducting sphere problem, a series of meshes of $[11,22,44,177,489,1126]$ unstructured quasi-uniform triangular elements of maximum element edge size $h=$ $[0.5,0.375,0.25,0.125,0.075,0.05] \mathrm{m}$, respectively, are generated to test the convergence of the $h p$-finite element solution. The convergence is measured using the relative error measure $\|\boldsymbol{U}-\mathcal{U}\|_{L^{2}} /\|\boldsymbol{U}\|_{L^{2}}$ and $\|\boldsymbol{U}-\mathcal{U}\|_{S N S} /\|\boldsymbol{U}\|_{S N S}$ where $\mathcal{U}:=$ 
$r \delta_{\hat{\mathcal{U}}_{\mathrm{r}}} \boldsymbol{e}_{r}+\delta_{\mathcal{U}_{\mathrm{z}}} \boldsymbol{e}_{z}$. Again, a blending function approach is used to represent curved boundaries (for details see the previous problem).

\section{$h$-refinement}

Figures 5.2 and 5.3 plot the relative error measures $\|\boldsymbol{U}-\boldsymbol{U}\|_{L^{2}} /\|\boldsymbol{U}\|_{L^{2}}$ and $\| \boldsymbol{U}-$ $\mathcal{U}\left\|_{S N S} /\right\| \boldsymbol{U} \|_{S N S}$ against the global characteristic length of the elements for varying degrees of element order of $p=[1,2,3]$ under $h$-refinement.

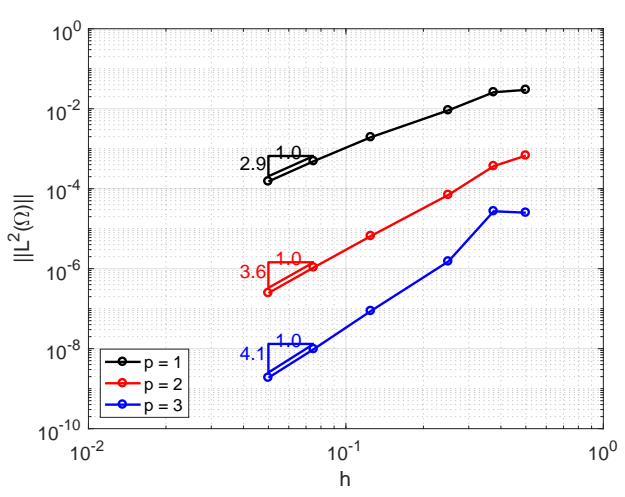

(a) $\|\mathcal{U}-\mathcal{U}\|_{L^{2}} /\|\boldsymbol{U}\|_{L^{2}}$

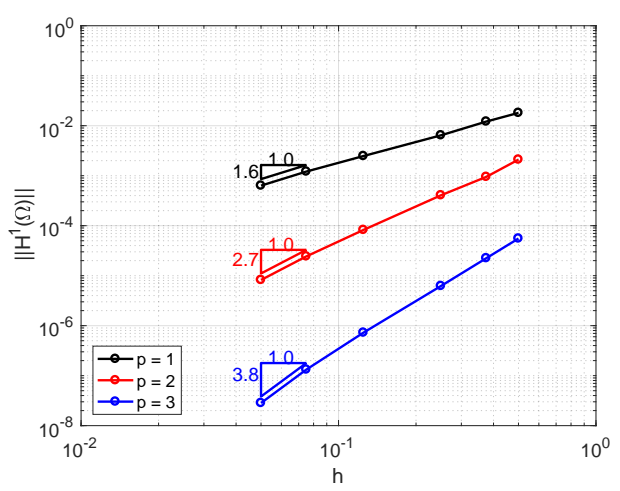

(b) $\|\boldsymbol{U}-\mathcal{U}\|_{S N S} /\|\mathcal{U}\|_{S N S}$

Figure 5.9: Mechanical shell subject to a pressure field: convergence of $\|\boldsymbol{U}-\mathcal{U}\|_{L^{2}} /\|\boldsymbol{U}\|_{L^{2}}$ and $\|\boldsymbol{U}-\mathcal{U}\|_{S N S} /\|\mathcal{U}\|_{S N S}$ under $h$-refinement for elements of $p=[1,2,3]$, where the errors are plotted against the maximum edge length $h$.

Figures 5.2 and 5.3 show the convergence of the $L^{2}(\Omega)$ and $H^{1}(\Omega)$ error against the maximum element edge length $h$ on a logarithmic scale, where each point on the curve represents a mesh refinement stage and each curve uses a different element order for $p=1,2,3$. After an initial region of pre-asymptotic behaviour, each curve indicates a straight line suggesting that the convergence is algebraic, as in the previous problem. The slope triangles highlighted in the plots suggest that the correct rate of convergence of at least $p+1$ for $L^{2}(\Omega)$ and $p$ for $H^{1}(\Omega)$ for smooth problems [244], is achieved. Similar convergence rates are also obtained for $p>3$, as shown in $[254,222]$.

\section{$p$-refinement}

Uniform polynomial enrichment corresponding to $p=[1,2, \cdots, 10]$ is now considered for a mesh of 44 unstructured quasi-uniform triangular elements of maximum size $h=0.25 \mathrm{~m}$. Figure 5.5 plots the relative error measures $\|\boldsymbol{U}-\boldsymbol{U}\|_{L^{2}} /\|\boldsymbol{U}\|_{L^{2}}$ and 
$\|\boldsymbol{U}-\mathcal{U}\|_{S N S} /\|\boldsymbol{U}\|_{S N S}$ against the number of degrees of freedom NDOF for varying frequencies of the alternating magnetic field under $p$-refinement.

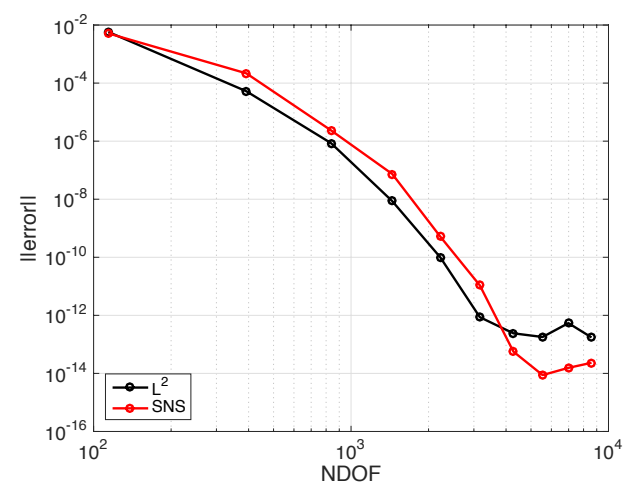

(a) $\log (\mathrm{NDOF})$

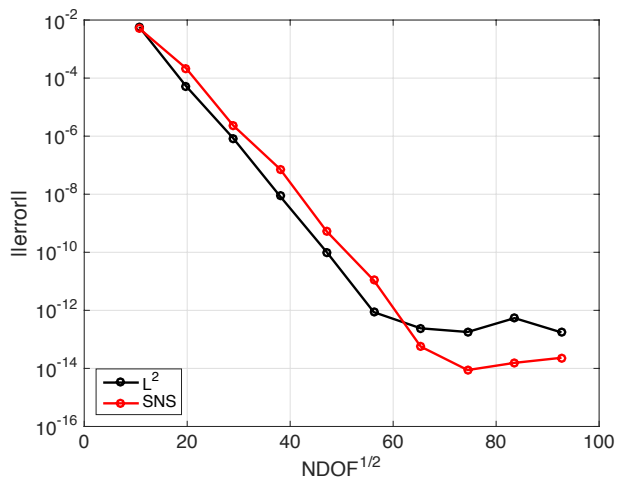

(b) $\mathrm{NDOF}^{1 / 2}$

Figure 5.10: Mechanical shell subject to a pressure field: convergence of $\|\boldsymbol{U}-\mathcal{U}\|_{L^{2}} /\|\boldsymbol{U}\|_{L^{2}}$, $\|\boldsymbol{U}-\mathcal{U}\|_{S N S} /\|\boldsymbol{U}\|_{S N S}$ under $p$-refinement.

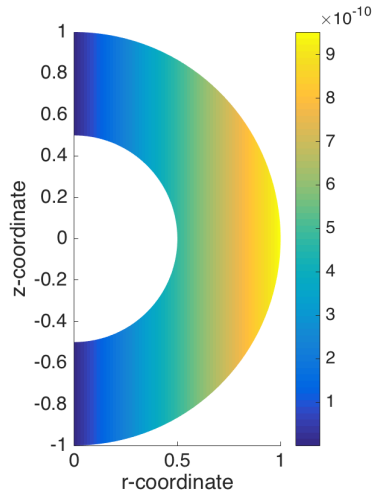

(a) Radial displacement $\mathcal{U}_{r}$

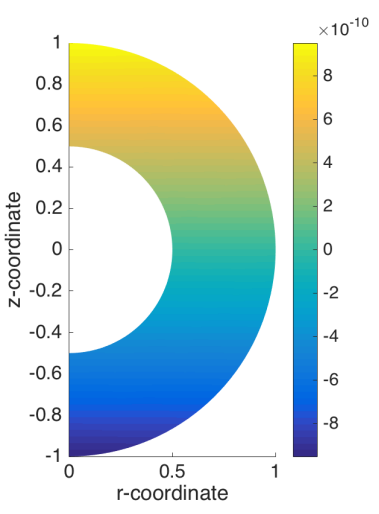

(b) Axial displacement $\mathcal{U}_{z}$

Figure 5.11: Mechanical shell subject to a pressure field: contours of $\mathcal{U}_{r}$ and $\mathcal{U}_{z}$.

The results shown in Figures 5.10a and 5.10b illustrate similar trends to those shown previously, indicating that exponential convergence with respect to the number of degrees of freedom raised to the power $1 / 2$ is also achieved through $p$ refinement for the mechanical problem. In particular, $p$-refinement serves as a method for overcoming volumetric locking that is known to be associated with the displacement formulation of elasticity for nearly incompressible material $[243,52$, 255 , and leads to exponential rates of convergence of the error measured in the 
$S N S(\Omega)$ norm. Although, there is stagnation of convergence when the norms of the error reach $10^{-13}$, which coincides with the numerical precision of the computation. The displacements in the radial and axial directions of the shell, obtained using $p=10$, are illustrated in Figure 5.11.

\subsubsection{Sound-Hard Sphere Subject to an Incident Acoustic Pressure Field}

Finally, to verify the acoustic system $(2.31,2.33)$, the problem of a sound hard sphere of radius $R$ in considered, illustrated in Figure 5.12. The sphere is illuminated by a harmonic incident wave $\boldsymbol{P}^{i n}=\mathcal{P}_{0} e^{\mathrm{i} k z}$ of amplitude $\mathcal{P}_{0}$ and wavenumber $k:=\omega / c$. The complete solution is of the form $\mathcal{P}=\mathcal{P}^{i n}+\mathcal{P}^{s c}$, subject to the boundary condition $\boldsymbol{n} \cdot \nabla \mathscr{P}=0$ on $\partial \Omega_{c}$ and admits an axisymmetric analytical solution for $\mathcal{P}^{s c}[49]$.

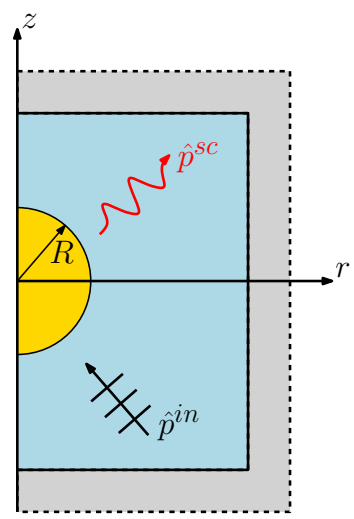

Figure 5.12: Sound-hard sphere subject to an incident acoustic pressure field: problem setup.

Specifically, the problem is solved for $R=1 \mathrm{~m}, \mathcal{P}_{0}=1 \mathrm{~Pa}, k=[4 \pi / 3,10,30] \mathrm{m}^{-1}$. A suitably simplified version of Algorithm 1 is solved on the computational domain $\Omega^{m}=([0,5.6] \times[-5.6,5.6]) \backslash\left\{(r, z): r^{2}+z^{2} \leq R^{2}\right\} \mathrm{m}^{2}$ of which $\Omega_{p m l}^{m}=$ $\Omega^{m} \backslash([0,4] \times[-4,4]) \mathrm{m}^{2}$, with thickness parameters $\left[t_{r}, t_{z}\right]=[1.6,1.6] \mathrm{m}$, distance parameters $\left[d_{r}, d_{z}\right]=[4,4] \mathrm{m}$ and $\delta_{\mathcal{P}}=0$ on $\partial \Omega^{m}$.

Like the two previous benchmark problems, a series of meshes consisting of [315, 569, 1 191, 4 716, 13 228, 29 597] unstructured quasi-uniform triangular elements of maximum size $h=[0.5,0.375,0.25,0.125,0.075,0.05] \mathrm{m}$, respectively, are generated to test the convergence of the $h p$-finite element solution. The convergence is measured using the relative error measure $\left\|\mathcal{P}^{s c}-\mathcal{P}^{s c}\right\|_{L^{2}} /\left\|\mathcal{P}^{s c}\right\|_{L^{2}}$ and $\| \mathcal{P}^{s c}-$ $\mathcal{P}^{s c}\left\|_{H^{1}} /\right\| \mathcal{P}^{s c} \|_{H^{1}}$, where $\mathcal{P}^{s c}:=\delta_{\mathcal{P}}$. Again, a blending function approach is used to represent curved boundaries (for details see the previous problems). 


\section{$h$-refinement}

In terms of uniform mesh refinement for the acoustic problem, particularly in cases of low $p$, the computational error will be dominated by the element size in the PML region, which will result in artificially polluted solutions [175]. Therefore, to provide an accurate error analysis the refinement of the PML has been chosen such that the error of the PML is sufficiently resolved such that the error is all within the mesh of the solution. This is done by setting the number of quadrilateral elements in the PML to correspond to the width of the outer layer of elements in the non-conducting domain $\Omega_{n}$ and the number of layers of elements in the layer is then defined as $n_{l}$ ayer $=20 t_{P M L} k / p$, where $t_{P M L}$ is the thickness of the PML in a specified direction and the coefficient of 20 is used to imply the general rule-ofthumb of number of points per wavelength required to be sufficiently resolved [27]. This results in $[4320,5760,8640,17280,28800,43$ 200] quadrilateral PML elements for each of the specified meshes for $p=1$, approximately $1 / 2$ that for $p=2$ and approximately $1 / 3$ that for $p=3$.

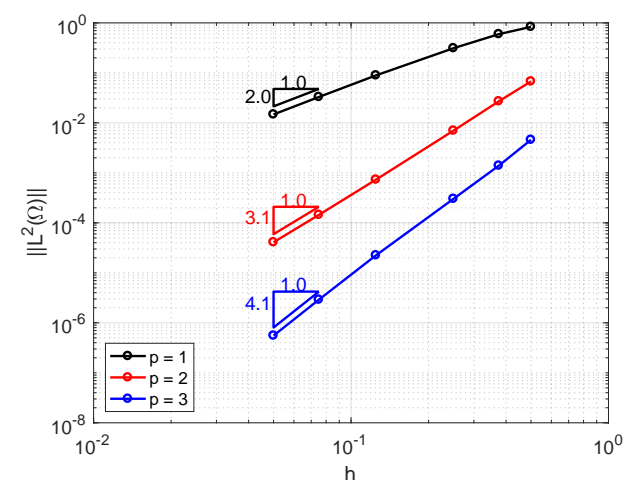

(a) $k=4 \pi / 3 m^{-1}$

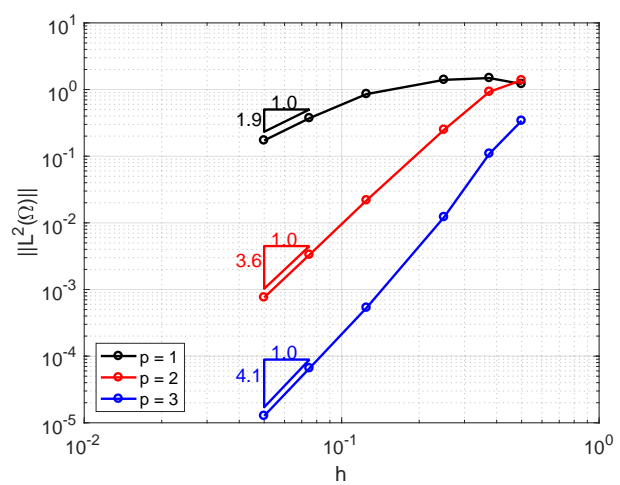

(b) $k=10 m^{-1}$

Figure 5.13: Sound-hard sphere subject to an incident acoustic pressure field: convergence of $\left\|\mathcal{P}^{s c}-\mathcal{P}^{s c}\right\|_{L^{2}} /\left\|\mathcal{P}^{s c}\right\|_{L^{2}}$, under $h$-refinement for elements of $p=[1,2,3]$ for wave numbers of $k=[4 \pi / 3,10] m^{-1}$, where the $L^{2}(\Omega)$ norm of the error is plotted against the maximum element edge length $h$.

Figures 5.13 and 5.14 show the convergence of the $L^{2}(\Omega)$ and $H^{1}(\Omega)$ error against the maximum element edge length $h$ on a logarithmic scale, where each point on the curve represents a mesh refinement stage and each curve uses a different element order for $p=1,2,3$. After an initial region of pre-asymptotic behaviour, each curve indicates a straight line suggesting that the convergence is algebraic, as in the previous problem. In the case of the acoustic problem, for the higher wave numbers the mesh requires further refinement, beyond that seen in the previous 
examples, in order to for the convergence rate to stabilise and this is due to numerical dispersion effects ${ }^{4}$ observed in wave propagation problems. However, once the effects of dispersion [17] are overcome further $h$-refinement results in the same rates of convergence as shown for the eddy current problem. For further details of this effect refer to the $p$-refinement study in the next section. The slope triangles highlighted in the plots suggest that the correct rates of convergence of at least $p+1$ for $L^{2}(\Omega)$ and $p$ for $H^{1}(\Omega)$ for smooth problems [244], are achieved. Similar convergence rates can also obtained for $p>3$.

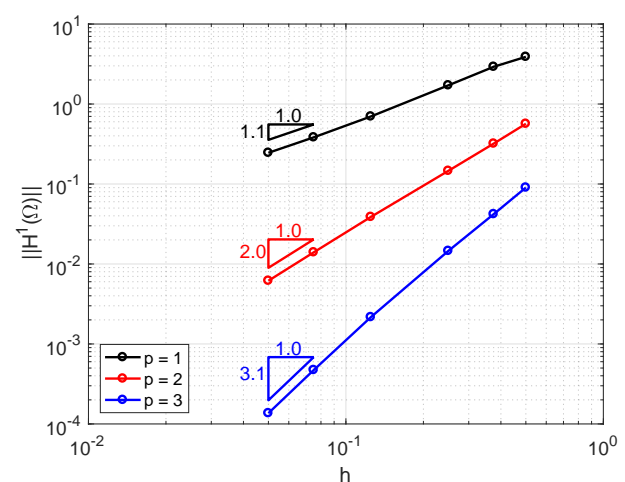

(a) $k=4 \pi / 3 m^{-1}$

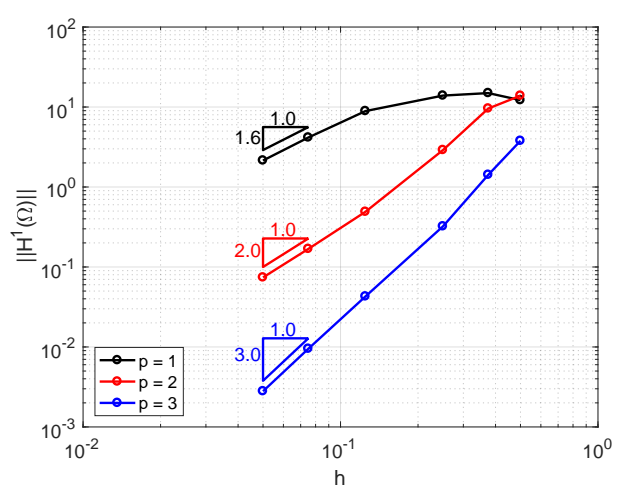

(b) $k=10 m^{-1}$

Figure 5.14: Sound-hard sphere subject to an incident acoustic pressure field: convergence of $\left\|\mathcal{P}^{s c}-\mathcal{P}^{s c}\right\|_{H^{1}} /\left\|\mathcal{P}^{s c}\right\|_{H^{1}}$, under $h$-refinement for elements of $p=[1,2,3]$ for wave numbers of $k=[4 \pi / 3,10] \mathrm{m}^{-1}$, where the $H^{1}(\Omega)$ norm of the error is plotted against the maximum element edge length $h$.

\section{$p$-refinement}

For this problem, a fixed quasi-uniform mesh of 1191 unstructured triangular elements of maximum size $h=0.25 \mathrm{~m}$ is considered, where the same refinement study of $p=1,2 \cdots, 10$ as the previous problems is performed. The convergence is measured using $\left\|\mathcal{P}^{s c}-\mathcal{P}^{s c}\right\|_{L^{2}} /\left\|\mathcal{P}^{s c}\right\|_{L^{2}},\left\|\mathcal{P}^{s c}-\mathcal{P}^{s c}\right\|_{H^{1}} /\left\|\mathcal{P}^{s c}\right\|_{H^{1}}$, where $\mathcal{P}^{s c}:=\delta_{\mathcal{P}}$.

Figures $5.15 \mathrm{a}$ and $5.15 \mathrm{~b}$ illustrate similar downward sloping trends, as shown in the previous examples. However, in this case the pre-asymptotic region is now affected by the increase in wave number. For higher wave numbers there is an initial stage of increase in error, which results from wave dispersion effects4, discussed in [16] and [116]. This effect is overcome by further increasing $p$ and eventually results in the same expected exponential rates of convergence as before, confirmed

\footnotetext{
${ }^{4}$ Numerical dispersion is the effect of waves behaving differently under poor numerical discretisation [17]. The under resolution of waves result in waves that do not propagate correctly.
} 


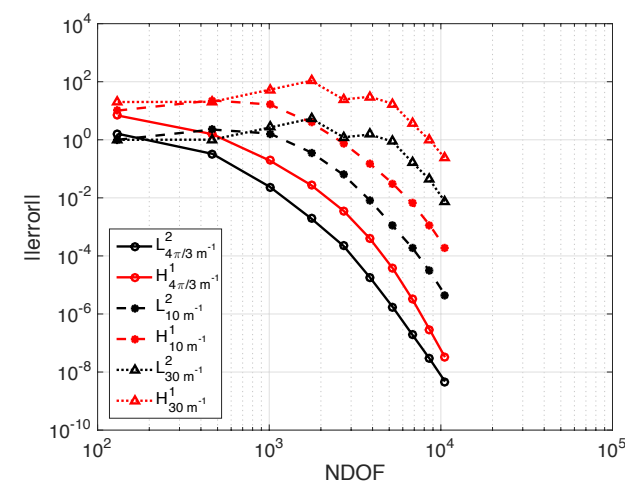

(a) $\log (\mathrm{NDOF})$

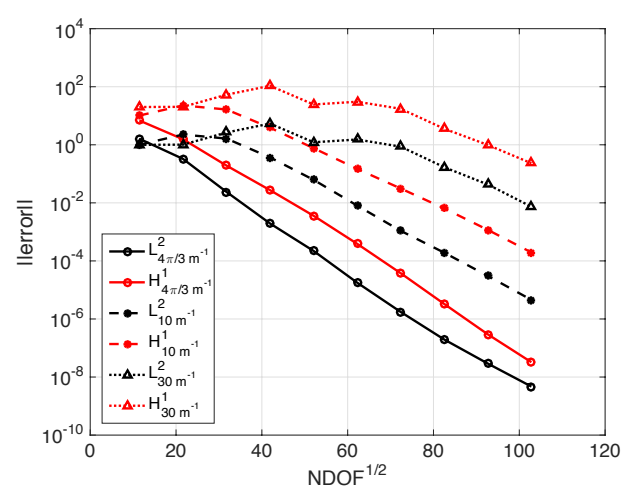

(b) $\mathrm{NDOF}^{1 / 2}$

Figure 5.15: Sound-hard sphere subject to an incident acoustic pressure field: convergence of $\left\|\mathcal{P}^{s c}-\mathcal{P}^{s c}\right\|_{L^{2}} /\left\|\mathcal{P}^{s c}\right\|_{L^{2}},\left\|\mathcal{P}^{s c}-\mathcal{P}^{s c}\right\|_{H^{1}} /\left\|\mathcal{P}^{s c}\right\|_{H^{1}}$ under $p$-refinement for wave numbers $k=[4 \pi / 3,10,30]$.

in Figure 5.15b. This again, indicates that exponential convergence, with respect to the NDOF raised to the power $1 / 2$, is also achieved through $p$-refinement for the acoustic problem, provided sufficiently high refinement is used to eliminate numerical dispersion. For the case of $k=4 \pi / 3$ for $p>7$ the convergence behaviour is suboptimal due to the effect of the PML, which is an approximate absorbing boundary condition, but nevertheless, accurate solutions are still obtained. The finest solution, using $p=10$, of the scattered pressure field arising from the incident pressure field for wave numbers $k=[4 \pi / 3,10,30]$ are illustrated in Figure 5.16.

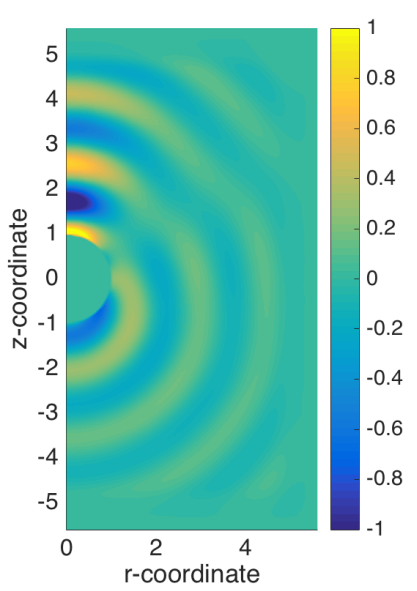

(a) $k=4 \pi / 3 \mathrm{~m}^{-1}$

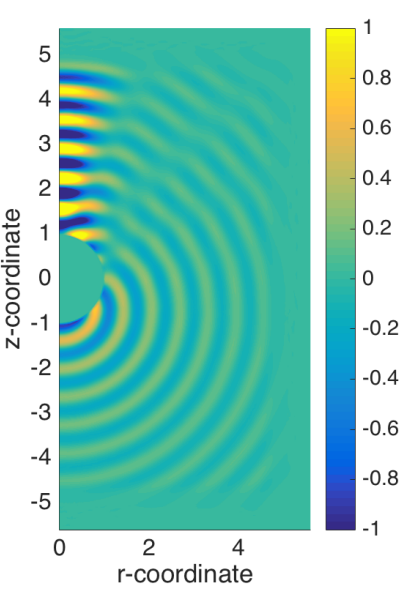

(b) $k=10 \mathrm{~m}^{-1}$

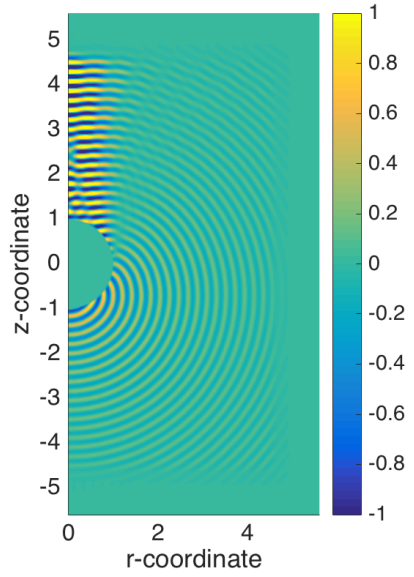

(c) $k=30 \mathrm{~m}^{-1}$

Figure 5.16: Sound-hard sphere subject to an incident acoustic pressure field: contours of $\operatorname{Re}\left(\mathcal{P}^{s c}\right)$ for differing wave numbers $k$. 


\subsection{Coupled Multi-Physics Problems}

\subsubsection{Acoustic Wave Scattering of Thin Elastic Shell}

A coupled acousto-mechanical problem is now considered. This problem consists of a thin elastic shell of thickness $t$, mid surface radius $R$ and material parameters $\rho_{s}, \nu$ and $E$. The shell is placed in a background medium, described by $\rho_{f}$ and $c_{f}$, and is illuminated by a harmonic incident pressure field $\mathcal{P}^{i n}$. The configuration is illustrated in Figure 5.17. This problem requires the solution of Equation (2.36) in absence of electromagnetic coupling and naturally lends itself to a harmonic treatment. For thin shells, the solution to this problem can be approximated by Kirchoff shell theory $[49,190]$ and the total exterior pressure becomes $\mathcal{P}=\mathcal{P}^{i n}+$ $\mathcal{P}^{s c}+\mathcal{P}^{r}$. For the incident field $\mathcal{P}^{i n}=P_{0} e^{\mathrm{i} k z}$, the component $\mathcal{P}^{s c}$ corresponds to the hard scattering by the sphere and $\mathcal{P}^{r}$ to the radiated pressure $[49,190]$.

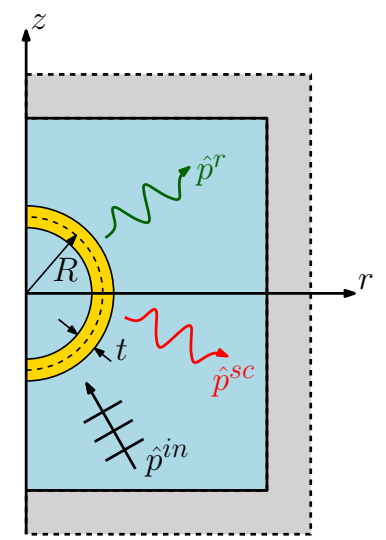

Figure 5.17: Elastic shell subject to an incident acoustic pressure field: problem setup.

The problem is solved for $d_{t}=0.05 \mathrm{~m}, R=1 \mathrm{~m}, P_{0}=1 \mathrm{~Pa}, k=\omega / c_{f}=$ $[4 \pi / 3,10,30] \mathrm{m}^{-1}, \rho_{f}=1000 \mathrm{~kg} / \mathrm{m}^{3}, c_{f}=1460 \mathrm{~m} / \mathrm{s}, \rho_{s}=7800 \mathrm{~kg} / \mathrm{m}^{3}, \nu=0.3$, $E=210 \mathrm{GPa}$. The problem is treated computationally by applying a suitably simplified version of Algorithm 1 with $\delta_{\mathcal{P}}:=\mathcal{P}^{s c}+\mathcal{P}^{r}$ on the computational domain $\Omega^{m}=([0,5.6] \times[-5.6,5.6]) \backslash\left\{(r, z): r^{2}+z^{2} \leq(R-t / 2)^{2}\right\} \mathrm{m}^{2}$ with the same PML settings as in Section 5.3.3. As in Section 5.3.2, to avoid the shell from floating away, a small boundary segment $\partial \Omega^{m D}$ is fixed to have displacements $\boldsymbol{U}$ according to the analytical solution. The problem is driven by the incident pressure field in the form of a Neumann condition set on the external boundary of the shell $\left\{(r, z): r^{2}+z^{2}=(R+t / 2)^{2}\right\}$ as $\boldsymbol{n} \cdot \nabla \delta_{\mathcal{P}}=-\boldsymbol{n} \cdot \nabla \mathcal{P}^{i n}$ and the coupling according to the interface conditions in Equation (2.36). The inside boundary of the shell is left free and the acoustic effects inside of the shell are ignored. 


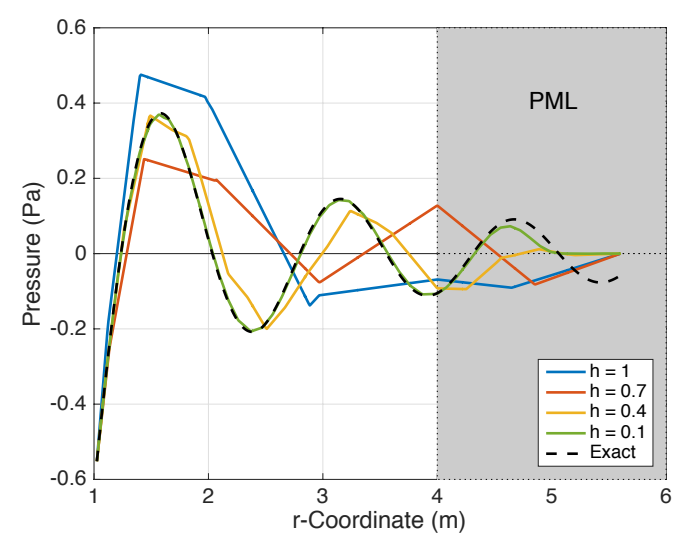

(a) $h$-enriched solutions

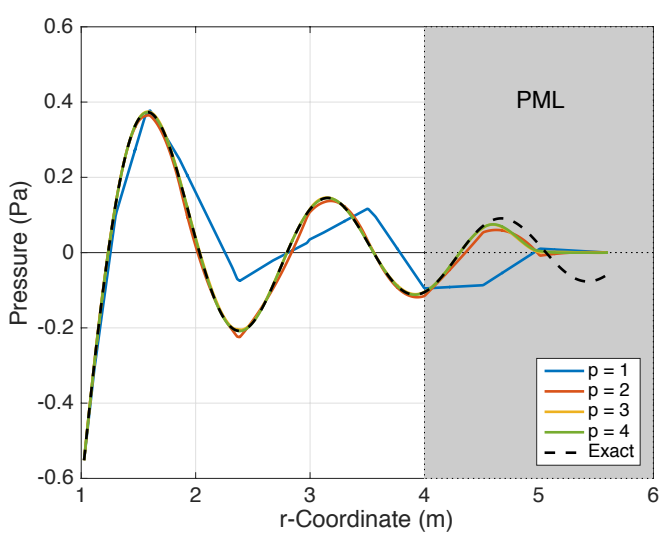

(b) $p$-enriched solutions

Figure 5.18: Elastic shell subject to an incident acoustic pressure field: effects of $h-$ and $p$ refinement on the acoustic pressure field for $k=4 \pi / 3 \mathrm{~m}^{-1}$, plotted along a line along the $z=0$ axis from $r=1$ to $r=5.6$.

Given that the convergence rates of the mechanical and acoustic fields have already been verified in Sections 5.3.2 and 5.3.3, the effectiveness of both $h-$ and $p$ refinement are now compared directly with the analytical solution for $k=4 \pi / 3 \mathrm{~m}^{-1}$. In Figure 5.18, various $h p$ enriched solutions are plotted for the line segment $1.025 \mathrm{~m} \leq$ $r \leq 5.6 \mathrm{~m}, z=0$, taken from the outer surface of the shell to the truncated boundary. In the case of Figure 5.18a a series of meshes consisting of [867, 922, 1 198, 8134] elements corresponding to $h=[1,0.7,0.4,0.1]$ for $p=1$ are used. In contrast, in the case of Figure 5.18b the same mesh, consisting of $h=0.4$ and 1198 elements, and $p=[1,2,3,4]$ are used. For both $h$ - and $p$-refinement the computed solution tends to the analytical for $r \leq 4 \mathrm{~m}$. However, for $h$-refinement a mesh of $h \approx 0.1 \mathrm{~m}$ with 8134 elements, with 7572 unknowns, is required to obtain good agreement with the analytical solution, with a level of accuracy of $O\left(10^{-2}\right)$. On the other hand, using $p=2$ on a mesh with 867 elements requires only 1021 unknowns for comparable accuracy in the solution. If $p$ is further refined to $p=4$ then the number of unknowns increases to 4163 , but with improvement in the relative accuracy by two orders of magnitude to $O\left(10^{-4}\right)$. The PML is defined by the grey area, in which the computed solution is non physical and absorbed.

Figure 5.19 shows comparisons in computed and analytical solutions for higher wave numbers of $k=[10,30] \mathrm{m}^{-1}$ and a fixed mesh of $h=0.5$ corresponding to 1658 elements. For both cases the order of $p=4$, used to obtain the finest solution in Figure 5.18, offers reasonable agreement with the analytical solution and is able to capture the higher frequencies of the waves. However, for increasing wave numbers 


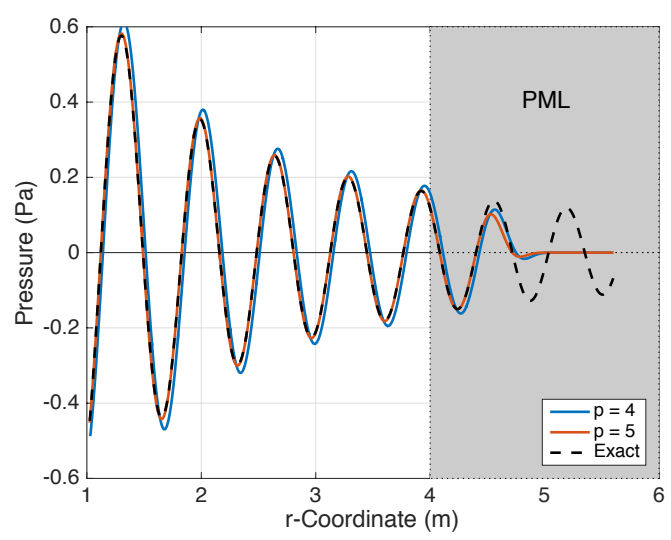

(a) $k=10 \mathrm{~m}^{-1}$

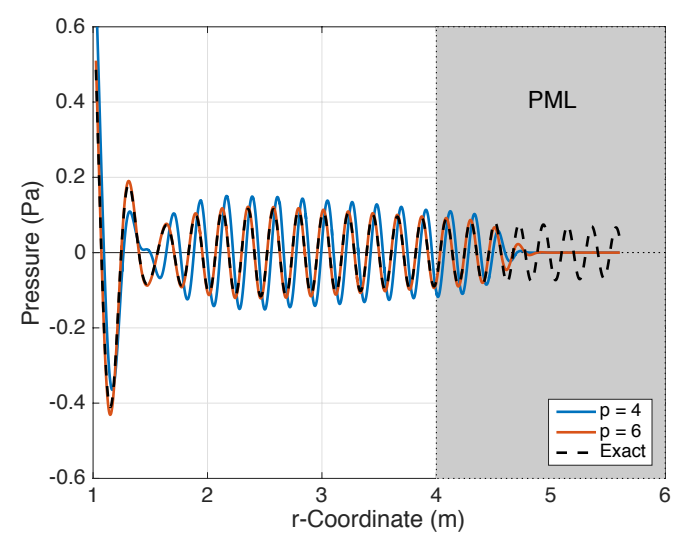

(b) $k=30 \mathrm{~m}^{-1}$

Figure 5.19: Elastic shell subject to an incident acoustic pressure field: effects of $p$-refinement for high wave number $k$ on the acoustic pressure field.

the computed solution requires even further $p$-refinement in order to accurately capture the solution in these regions due to the aforementioned dispersion effects. The solution case for $p=4$ and a suitably refined solution of each case is plotted against the analytical solution in Figures 5.19a and 5.19b. The mesh density required for $p=1$ elements to capture the high frequency wave effects at $k=30 \mathrm{~m}^{-1}$ results in a significantly greater number of degrees of freedom for such geometries.

The interaction between the pressure field and the displacements of the shell is illustrated in Figure 5.20, where the computed deformed shape of the shell is plotted in the surrounding acoustic field.

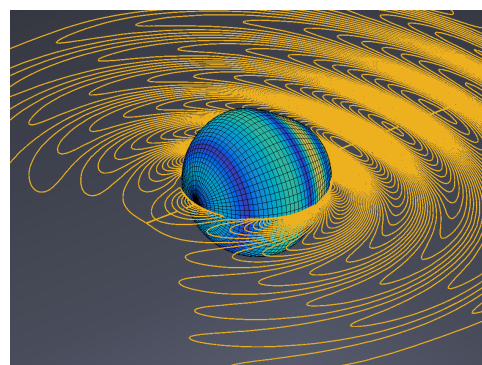

(a) $k=4 \pi / 3 \mathrm{~m}^{-1}$

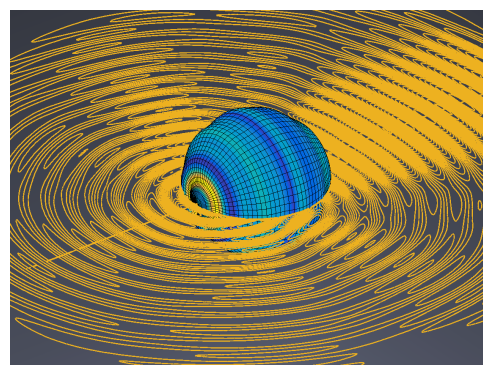

(b) $k=10 \mathrm{~m}^{-1}$

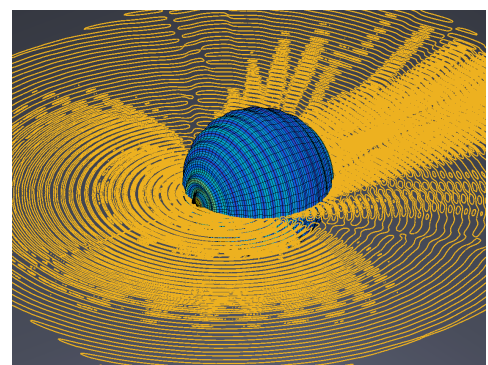

(c) $k=30 \mathrm{~m}^{-1}$

Figure 5.20: Elastic shell subject to an incident acoustic pressure field: deformed shell interacting with surrounding acoustic pressure field.

The success of high order $h p$ discretisations for the preceding case studies motivates the strategy for the following industrial example, which does not have an 
analytical solution.

\subsubsection{Test Magnet Problem}

An industrially relevant benchmark problem, proposed by Siemens Magnet Technology, is now considered. This problem involves a simplified quarter-size representation of an MRI scanner and is also modelled and presented in $[150,26]$. The problem setup comprises of the same main components illustrated in Figure 1.2, with a reduced complexity in the coil configuration. The setup comprises of three metallic shields known as the Outer Vacuum Chamber (OVC) $\Omega_{c}^{O V C}, 77 \mathrm{~K}$ radiation shield $\Omega_{c}^{77 K}$ and $4 K$ helium vessel $\Omega_{c}^{4 K}$, which make up $\Omega_{c}$ and each with different material parameters $(\gamma, \mu, \nu, E, \rho)$. A pair of main coils, with static current source $\boldsymbol{J}^{D C}$, are located on the outside of the three shields and a pair of gradient coils, with alternating current source $\boldsymbol{J}^{A C}(t)$, are located within the imaging bore. Both are assumed as Biot-Savart coils (see Section 2.5.2) and are illustrated in Figure 5.21.

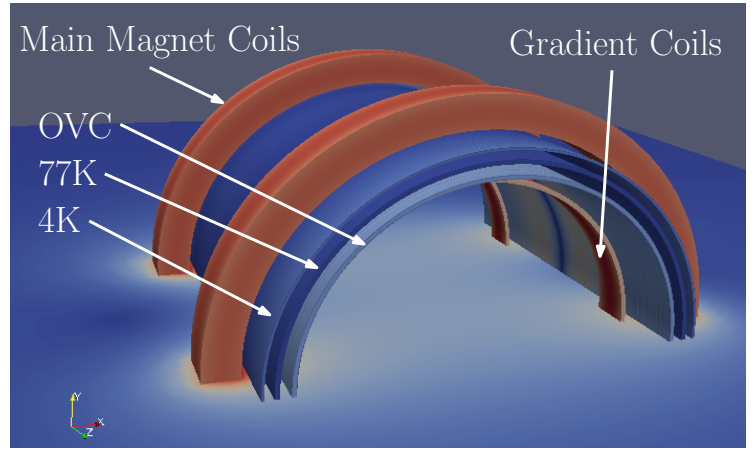

(a) 3D geometry

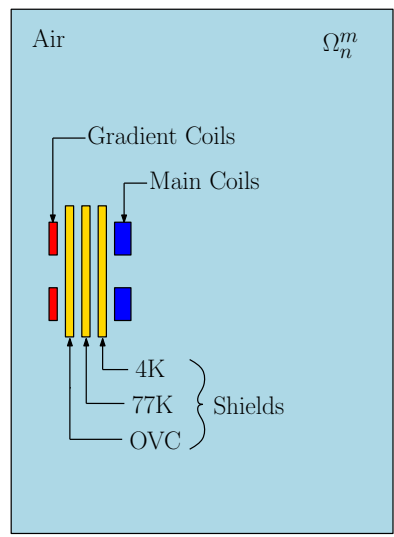

(b) Axisymmetric plane

Figure 5.21: Simplified MRI scanner subject to alternating and static current driven coils: problem setup.

This problem is treated computationally for two cases; in the first case, a suitably simplified version of Algorithm 1 is applied, in which the acoustic effect is neglected and the focus is purely on the magneto-mechanical coupling mechanisms, as in [150]. In the second case, the fully coupled acousto-magneto-mechanical systems in Algorithm 1 is considered. The non-conducting region, comprised of air, is truncated to create the domain $\Omega^{m}=([0,1.26] \times[-1.68,1.68]) \mathrm{m}^{2}$, with the PML $\Omega_{p m l}^{m}=$ $\Omega^{m} \backslash([0,0.9] \times[-1.2,1.2]) \mathrm{m}^{2}$. As in Section 5.3.2, to provide unique solutions, a small boundary segment $\partial \Omega_{c}^{D}$ of the conductors is fixed to have displacements $\boldsymbol{U}=\mathbf{0}$, 
which represents the physical fixings on actual MRI systems. The exact geometries and material parameters of the conducting components are confidential and so are not displayed in this Thesis. The solution is analysed for an unstructured mesh of 8464 triangles of maximum size $h=0.25 \mathrm{~m}$, but with substantial refinement in $\Omega_{c}^{m}$. Of these elements, $\sim 1700$ are located within $\Omega_{c}^{m}$. This example serves to show the predictive capability of the linearised approach and is validated with comparison of the results with industrial data supplied by Siemens Magnet Technology (SMT).

\section{Dissipated Power and Eddy Currents}

A quantity of industrial interest is the power dissipated in $\Omega_{c}^{O V C}, \Omega_{c}^{77 K}$ and $\Omega_{c}^{4 K}$. This measure is used to quantify the resonance behaviour of the MRI system and to determine the frequencies at which operation is undesirable. This measure is given in terms of the eddy (Ohmic) currents, and for the harmonic component of the magnetic vector potential $\boldsymbol{\delta}_{\mathcal{A}}$ (see Remark 5.1), becomes

$$
P_{\Omega}^{o}\left(\omega, \boldsymbol{\delta}_{\mathcal{A}}\right)=\frac{1}{2} \int_{\Omega} \frac{1}{\gamma}\left|\boldsymbol{g}^{e}\right|^{2} \mathrm{~d} \Omega=\frac{1}{2} \int_{\Omega} \gamma|\boldsymbol{E}|^{2} \mathrm{~d} \Omega \approx \pi \omega^{2} \int_{\Omega_{m}} \gamma\left|\boldsymbol{\delta}_{\mathcal{A}}\right|^{2} r \mathrm{~d} \Omega^{m}
$$

Remark 5.1 The time averaged output power over a time period $T$ is given as

$$
P_{\Omega}^{o}\left(\omega, \boldsymbol{\delta}_{A}\right)=\frac{1}{T} \int_{\Omega} \int_{t}^{t+T} \frac{1}{\gamma}\left|\boldsymbol{J}^{e}(t)\right|^{2} \mathrm{~d} t \mathrm{~d} \Omega
$$

where for a linear problem the time period associated with a single frequency is $T=2 \pi / \omega$. With the complex time harmonic representation the eddy currents become

$$
\boldsymbol{J}^{e}(t)=\operatorname{Re}\left(\boldsymbol{g}^{e} e^{\mathrm{i} \omega t}\right)=\operatorname{Re}\left(\boldsymbol{g}^{e}\right) \cos (\omega t)-\operatorname{Im}\left(\boldsymbol{g}^{e}\right) \sin (\omega t)
$$

and thus the output power dissipation becomes

$$
\begin{aligned}
P_{\Omega}^{o}\left(\omega, \boldsymbol{\delta}_{A}\right)= & \frac{\omega}{2 \pi} \int_{\Omega} \int_{t}^{t+T} \frac{1}{\gamma}\left|\boldsymbol{J}^{e}(t)\right|^{2} \mathrm{~d} t \mathrm{~d} \Omega \\
=\frac{\omega}{2 \pi} \int_{\Omega} \int_{t}^{t+T} \frac{\omega}{2 \pi}( & \operatorname{Re}\left(\boldsymbol{g}^{e}\right)^{2} \cos ^{2}(\omega t)+\operatorname{Im}\left(\boldsymbol{g}^{e}\right)^{2} \sin ^{2}(\omega t) \\
& \left.\quad-2 \operatorname{Re}\left(\boldsymbol{g}^{e}\right) \operatorname{Im}\left(\boldsymbol{J}^{e}\right) \cos (\omega t) \sin (\omega t)\right) \mathrm{d} t \mathrm{~d} \Omega .
\end{aligned}
$$

By using the definitions of the integrals of trigonometric function [241], the time 
harmonic definition of the output power becomes

$$
P_{\Omega}^{o}\left(\omega, \boldsymbol{\delta}_{A}\right)=\frac{1}{2} \int_{\Omega} \frac{1}{\gamma}\left|\boldsymbol{g}^{e}\right|^{2} \mathrm{~d} \Omega
$$

In [150], the output power $P_{\Omega_{c}^{O V C}}^{o}\left(\omega, \boldsymbol{A}^{A C}\right), P_{\Omega_{c}^{77 K}}^{o}\left(\omega, \boldsymbol{A}^{A C}\right)$ and $P_{\Omega_{c}^{4 K}}^{o}\left(\omega, \boldsymbol{A}^{A C}\right)$, where $\mathcal{A}^{A C}$ is the equivalent to $\boldsymbol{\delta}_{\mathcal{A}}$ but obtained by the fixed point scheme, was compared to a set of industrial results using the NACS software [8]. This is revisited by including the results of the new monolithic formulation, both with and without acoustic effects. The converged results are plotted in Figure 5.22, where a sweep over the alternating current driving frequencies is performed in the range $\omega:=2 \pi f=$ $2 \pi(10 \leq f \leq 5000) \mathrm{rad} / \mathrm{s}$ for a frequency step $\Delta f=10 \mathrm{~Hz}$. In the Figure; the black line represents the results obtained by Siemens using NACS, the red line the results of the previous fixed point scheme, the blue line the monolithic magneto-mechanical formulation and the yellow line the fully coupled monolithic system with acoustic effects.

In absence of acoustic effects, and for $f \leq 3000 \mathrm{~Hz}$, the fixed point, monolithic and NACS results for $P_{\Omega_{c}^{O V C}}^{o}\left(\omega, \boldsymbol{A}_{A C}\right)$ and $P_{\Omega_{c}^{77 K}}^{o}\left(\omega, \boldsymbol{A}_{A C}\right)$ are in close agreement with each other since in this case the problem is well approximated by the pure eddy current model. The fixed point and monolithic results for $P_{\Omega_{c}^{4 K}}^{o}\left(\omega, \boldsymbol{A}_{A C}\right)$ also give good agreement in this region, however, the results obtained by NACS offer very small differences of $O\left(10^{-1}\right)$ for $f>1000 \mathrm{~Hz}$. It can be conjectured that this is due to the limitations of the low order elements in accurately resolving the skin depth effects with increasing frequency, illustrated in Figures 5.23 and 5.24. In this problem the $4 K$ shield is the thinnest and so the eddy currents will penetrate this shield more quickly than the others, thus requiring a better resolution, than that of the low order elements used. These methodologies also give a similar prediction of the resonance region occupied by $3500 \mathrm{~Hz} \leq f \leq 4500 \mathrm{~Hz}$ with the NACS model being damped due to the artificial Rayleigh damping [114]. The effect of this damping on the response of the system results in a change in the amplitude and frequency range of the resonance region $[114,181]$.

The newly proposed monolithic framework of the linearised approach offers computational advantages over the fixed point strategy, in [150], since the solution is obtained in a single iteration, as opposed to multiple iterations, which grows in the 
resonance region. This enables rapid and robust solutions at each frequency to be performed, which offer trivial parallelism and greater resolution in the resonance region for the same computational cost. Notably, the resonant frequencies computed using the fixed point scheme exactly match those obtained by the monolithic scheme, albeit with differing magnitude of the peaks due to the effects of matrix equation conditioning and the fact that for undamped systems the solution tends to infinity at resonance.

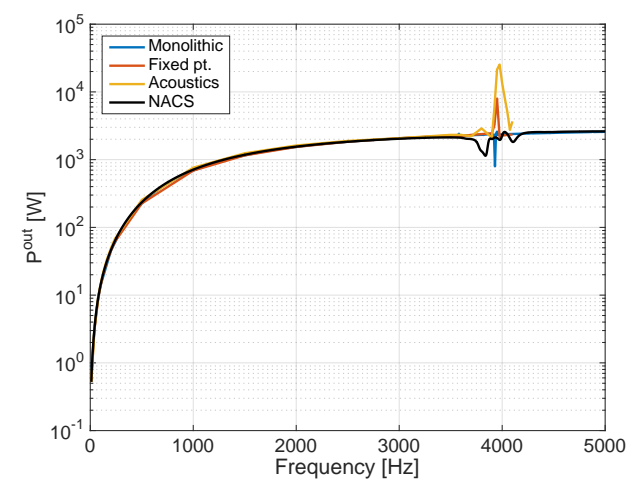

(a) $P_{\Omega_{c}^{O V V}}^{o}\left(\omega, \boldsymbol{A}_{A C}\right)$

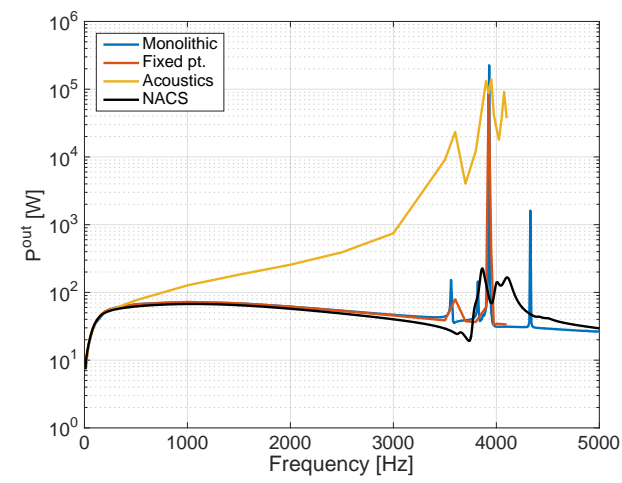

(b) $P_{\Omega_{c}^{77 K}}^{o}\left(\omega, \boldsymbol{A}_{A C}\right)$

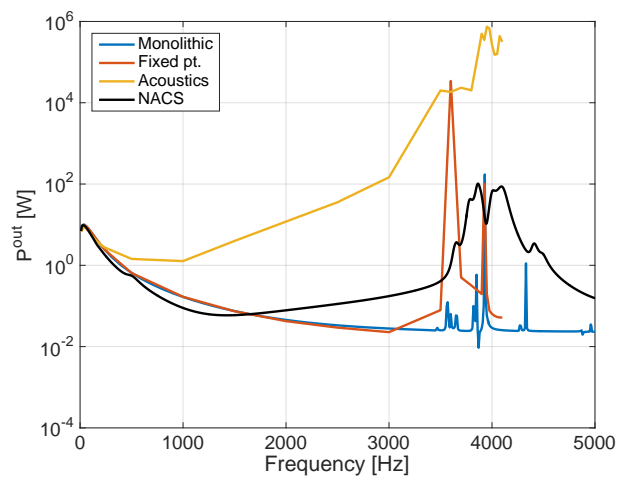

(c) $P_{\Omega_{c}^{4 K}}^{o}\left(\omega, \boldsymbol{A}_{A C}\right)$

Figure 5.22: Simplified MRI scanner subject to alternating and static current driven coils: ohmic power dissipation as a function of alternating current frequency.

The predicative capability of the linearised approach is further demonstrated by the inclusion of acoustic effects, which has substantial effects in $P_{\Omega_{c}^{77 K}}^{o}\left(\omega, \boldsymbol{A}_{A C}\right)$ and $P_{\Omega_{c}^{4 K}}^{o}\left(\omega, \boldsymbol{A}_{A C}\right)$ for $f \geq 500 \mathrm{~Hz}$, not included in the NACS software or the fixed point scheme in [150]. Negligible effects for $P_{\Omega_{c}^{O V C}}^{o}\left(\omega, \boldsymbol{A}_{A C}\right)$ are obtained since the OVC is located closest to the inner bore tube and, therefore, the gradient coils, and dissipated power is dominated by the electromagnetic effects. In contrast, the other two shields are located to the outside of the OVC and hence acoustic propagation effects 
cause these shields to further perturb the output power. Note that repeating the results with coils treated as deformable conductors leads to only negligible changes in the output power and, therefore, these results are not shown.

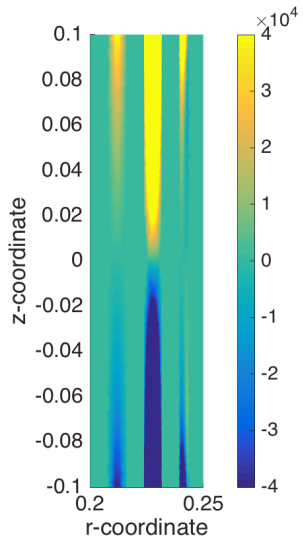

(a) $f=160 \mathrm{~Hz}, p=1$

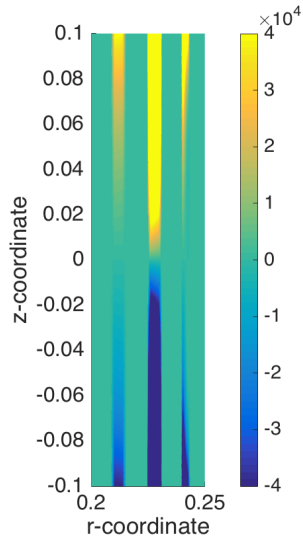

(b) $f=160 \mathrm{~Hz}, p=5$

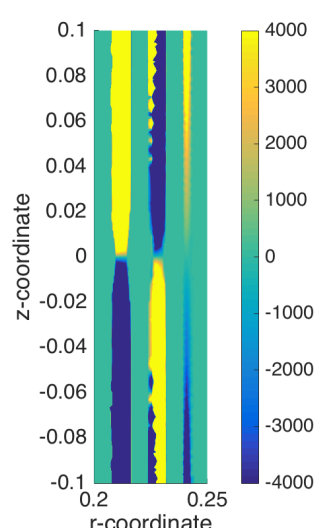

(c) $f=4100 \mathrm{~Hz}, p=1$

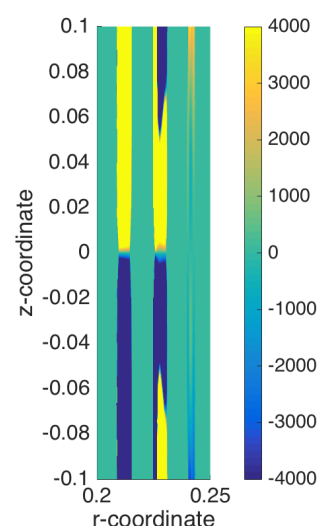

(d) $f=4100 \mathrm{~Hz}, p=5$

Figure 5.23: Simplified MRI scanner subject to alternating and static current driven coils: contours of the eddy currents $\operatorname{Re}\left(\boldsymbol{g}_{\phi}^{e}\right)$ for $p=1,5$ and $f=[160,4100] \mathrm{Hz}$.

To illustrate the skin effect at different frequencies, Figure 5.23 shows the eddy current distributions at $f=160 \mathrm{~Hz}$ and $f=4100 \mathrm{~Hz}$ for both $p=1$ and $p=5$. At the lower frequency the skin effects are already well resolved by $p=1$ elements, but the higher frequency $p \geq 4$ elements are required to resolve the small skin depth. This is further illustrated in Figure 5.24, which shows the convergence of $\boldsymbol{g}^{e}$ in $\Omega_{c}^{4 K}$ along the line $z=0.04 \mathrm{~m}$ for $p=1,2, \cdots, 5$.

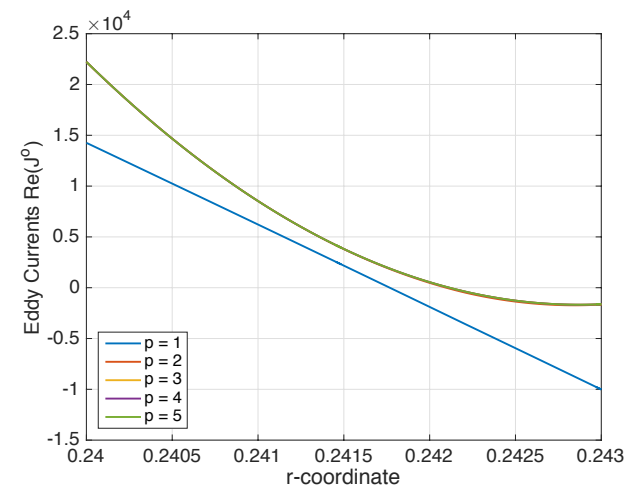

(a) $f=160 \mathrm{~Hz}$

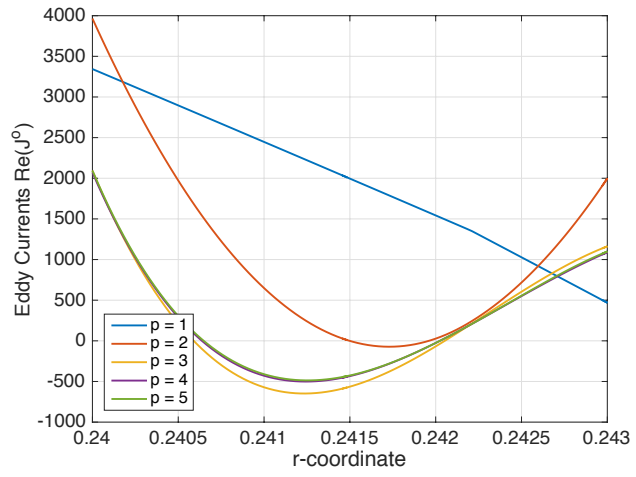

(b) $f=4100 \mathrm{~Hz}$

Figure 5.24: Simplified MRI scanner subject to alternating and static current driven coils: effects of $p$ - enrichment on the eddy current resolution $\operatorname{Re}\left(J_{\phi}^{o}\right)$ in $\Omega_{c}^{4 K}$ for $f=[160,4100] \mathrm{Hz}$. 


\section{Kinetic Energy and Mode Shapes}

The kinetic energy of $\Omega_{c}^{O V C}, \Omega_{c}^{77 K}$ and $\Omega_{c}^{4 K}$ is of industrial interest for understanding the motion of the conductors, highlighting the resonance frequencies and the corresponding mode shapes of the scanner's structure. In terms of the computed displacements $\boldsymbol{\delta}_{\mathcal{U}}$ this is ${ }^{5}$

$$
E_{\Omega}^{k}\left(\omega, \boldsymbol{\delta}_{u}\right)=\frac{1}{4} \int_{\Omega} m|\boldsymbol{v}|^{2} \mathrm{~d} \Omega=\frac{1}{4} \int_{\Omega} \rho \omega^{2}\left|\boldsymbol{\delta}_{u}\right|^{2} \mathrm{~d} \Omega \approx \frac{\pi}{2} \int_{\Omega^{m}} \rho \omega^{2}\left|\boldsymbol{\delta}_{\mathrm{u}}\right|^{2} r \mathrm{~d} \Omega^{m} .
$$

Figures 5.25, 5.26 and 5.27 illustrate the kinetic energy as a function of the driving current frequency in the gradient coils in $\Omega_{c}^{O V C}, \Omega_{c}^{77 K}$ and $\Omega_{c}^{4 K}$ respectively. For each spike in the kinetic energy, or resonant frequency, a corresponding 3D axisymmetric mode shape of the shield is included. Higher resonant frequencies excite higher order sinusoidal modes as the Figures illustrate. The resonant frequencies of the kinetic energy in the shields coincide with the frequencies experienced in the output power, which suggests that the primary source of excitation in the conductors is that of the eddy currents dissipated in the shields.

The computed modes of the OVC, illustrated in Figure 5.25 correspond to the Eigen-modes of the mechanical system. Table 5.1 shows the modes computed by the linearised approach and the corresponding Eigen-modes, obtained from an Eigenvalue analysis of the mechanical system $[81,114]$. As highlighted in Table 5.1, only the odd numbered modes appear to be activated in the coupled system. This is due to the forcing of the system through the placement of the gradient coils. The mechanical system is excited through magnetic stresses, which for the transient case are generated through the transient magnetic field. Due to the location of the coils, inside of the bore tube, the primary direction of the magnetic gradient field is in the radial direction and so only the radial modes would become excited.

\section{Fully Coupled Response}

Finally, the complex behaviour of the magnetic flux lines, acoustic contour lines and deformed structure are illustrated in Figure 5.28 for a range of frequencies in both the eddy current dominant (low frequency) and the resonance (high frequency) regions. This Figure illustrates that in the low frequency region $(120 \mathrm{~Hz} \leq f \leq 1500 \mathrm{~Hz})$ a patient in the bore can become exposed to sufficiently higher noise levels than that of the exterior region of the scanner, with the sound radiating and decaying outwards in space. This is due to the dominance of the harmonic magnetic field

\footnotetext{
${ }^{5}$ By applying a similar analysis to that in Remark 5.1.
} 


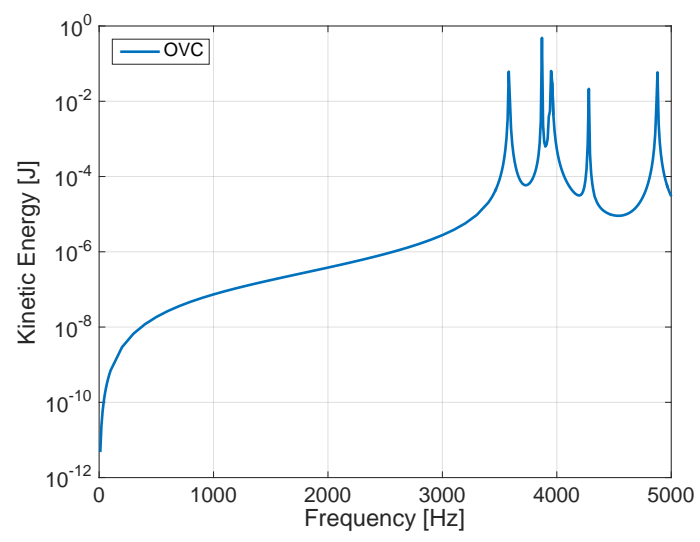

(a) $\left.E_{\Omega_{c}^{O V C}}^{k}\left(\omega, \boldsymbol{\delta}_{\mathfrak{U}}\right)\right)$

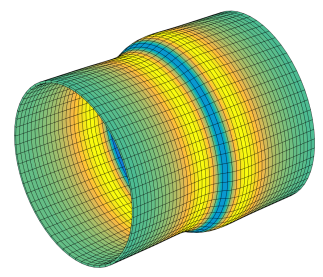

(b) $3580 \mathrm{~Hz}$

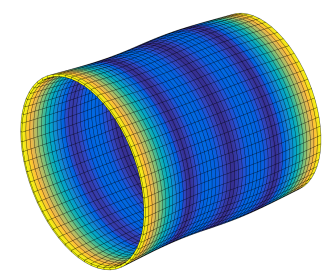

(c) $3870 \mathrm{~Hz}$

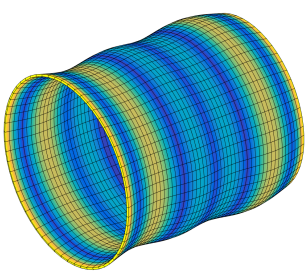

(d) $3950 \mathrm{~Hz}$

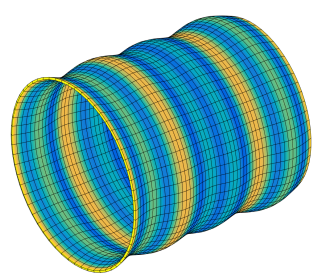

(e) $4280 \mathrm{~Hz}$

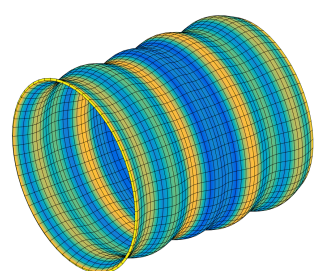

(f) $4880 \mathrm{~Hz}$

Figure 5.25: Simplified MRI scanner subject to alternating and static current driven coils: $E_{\Omega_{c}^{o V C}}^{k}\left(\omega, \boldsymbol{\delta}_{\mathcal{U}}\right)$ and resonant mode shapes.

\begin{tabular}{||ccc||}
\hline OVC Mode & Mag-mech $(\mathrm{Hz})$ & Eigenvalues $(\mathrm{Hz})$ \\
\hline \hline 1 & 3580 & 3584.52 \\
\hline 2 & & 3772.69 \\
\hline 3 & 3870 & 3869.44 \\
\hline 4 & & 3875.44 \\
\hline 5 & 3950 & 3954.48 \\
\hline 6 & & 4030.39 \\
\hline 7 & 4280 & 4279.24 \\
\hline 8 & & 4511.34 \\
\hline 9 & 4880 & 4878.37 \\
\hline
\end{tabular}

Table 5.1: Simplified MRI scanner subject to alternating and static current driven coils: Computed modal frequencies and corresponding Eigen-frequencies for $\Omega_{c}^{O V C}$. 


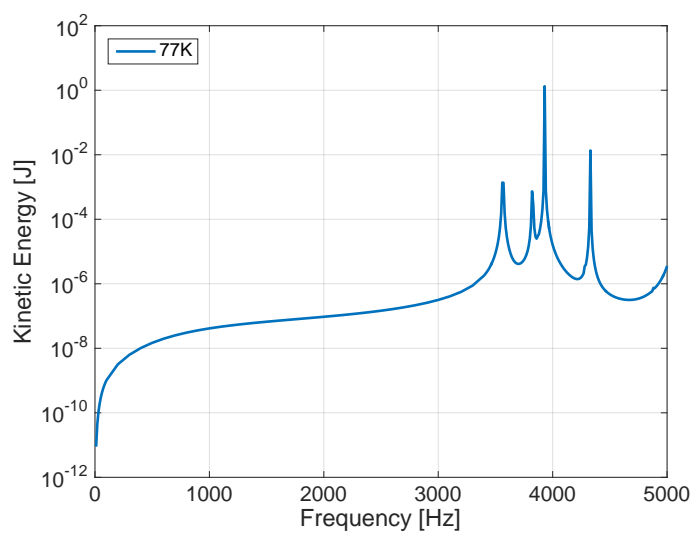

(a) $E_{\Omega_{c}^{77 K}}^{k}\left(\omega, \boldsymbol{\delta}_{\mathcal{U}}\right)$

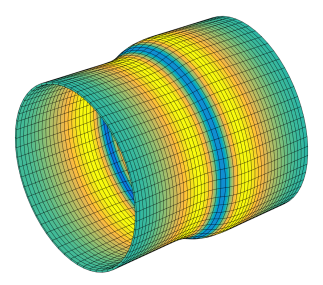

(b) $3560 \mathrm{~Hz}$

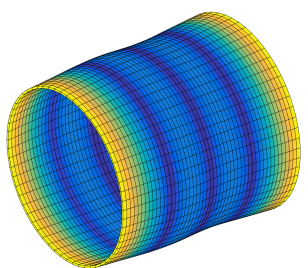

(c) $3820 \mathrm{~Hz}$

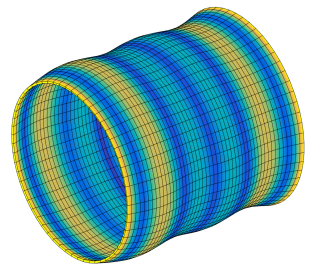

(d) $3930 \mathrm{~Hz}$

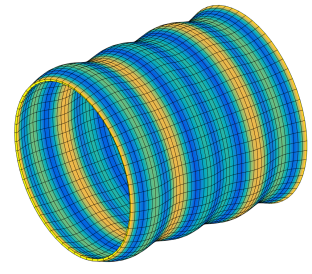

(e) $4330 \mathrm{~Hz}$

Figure 5.26: Simplified MRI scanner subject to alternating and static current driven coils: $E_{\Omega_{c}^{77 K}}^{k}\left(\omega, \boldsymbol{\delta}_{\mathcal{U}}\right)$ and resonant mode shapes. 


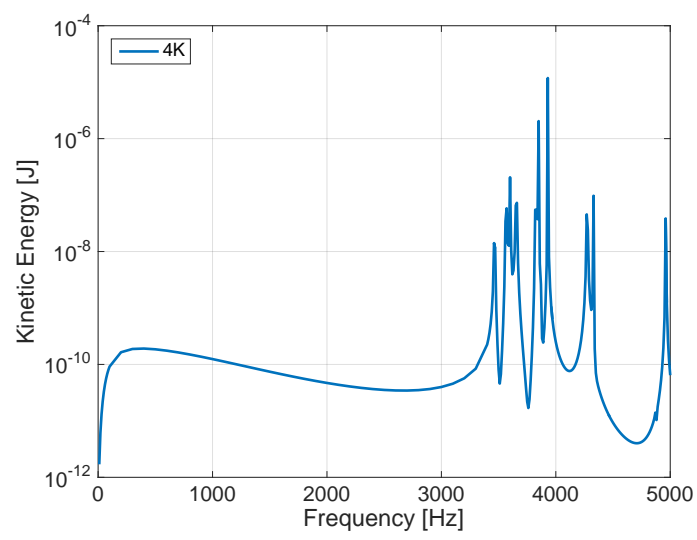

(a) $\left.E_{\Omega_{c}^{4 K}}^{k}\left(\omega, \boldsymbol{\delta}_{\mathcal{U}}\right)\right)$

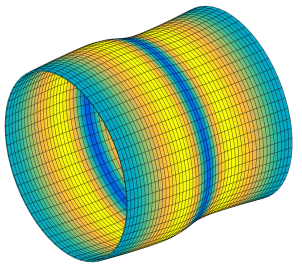

(b) $3460 \mathrm{~Hz}$

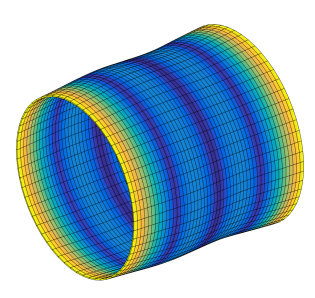

(c) $3600 \mathrm{~Hz}$

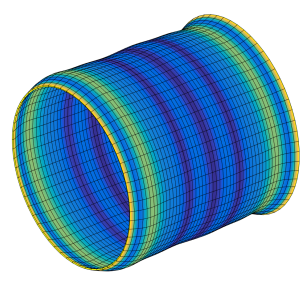

(d) $3930 \mathrm{~Hz}$

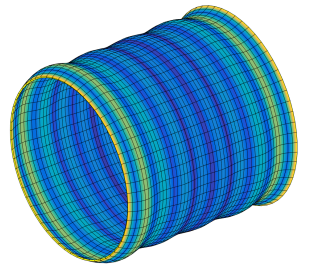

(e) $4330 \mathrm{~Hz}$

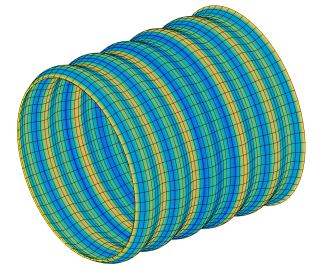

(f) $4960 \mathrm{~Hz}$

Figure 5.27: Simplified MRI scanner subject to alternating and static current driven coils: $E_{\Omega_{c}^{4 K}}^{k}\left(\omega, \boldsymbol{\delta}_{\mathcal{U}}\right)$ and resonant mode shapes. 
arising from the gradient coils, located inside the imaging volume, which gives rise to the source term in the acoustic Helmholtz system in (2.31). As the frequency increases $(1500 \mathrm{~Hz} \leq f \leq 3700 \mathrm{~Hz})$, the effect of the mechanical resonance begins to dominate and the acoustic field is further excited by the displacement of the shields and the sound intensity outside of the scanner increases. The case of $f=4075 \mathrm{~Hz}$ illustrates a higher frequency mode shape and the effect of the displacement on the acoustic field. In this case the magnetic field is further perturbed due to the increase in Lorentz currents resulting from the acoustic excitation. Notably, in each case, the greatest sound intensity is that inside the bore tube, suggesting that the highest noise levels are experienced by the patient.

\subsection{Chapter Summary}

This Chapter has presented a series of industrial and academic benchmark problems to test the capability of the novel linearised approach of the acousto-magnetomechanical problem derived in Chapter 3 and using the discrete approach outlined in Chapter 4. Each individual physical field; electromagnetic, mechanical and acoustic, has been rigorously benchmarked by performing convergence studies and measuring the error norms and shown to give accurate results compared to the analytical solutions with the correct theoretical rates of convergence [244, 127]. The coupled system has been benchmarked through comparisons with both analytical solutions and comparisons with commercial codes, as well as the fixed point magneto-mechanical scheme presented in [150].

The results obtained, for the range of examples considered, show that the linearised approach offers accurate solutions for a range of different problems analysed and thus aligns with the objective of "To determine the accuracy of the linearised approach across the full operating range of MRI scanners" .

The next step is to compare the performance of this newly proposed linearised approach in accurately predicting the behaviour of the fully coupled non-linear system. The next three Chapters of this Thesis present an approach to the solution of the full non-linear coupled problem through the formulation, numerical implementation and a series of numerical examples. This approach, named the non-linear approach is used to compare against the linearised approach to determine the accuracy of the linearised approach in computing the solution to the fully coupled system. 


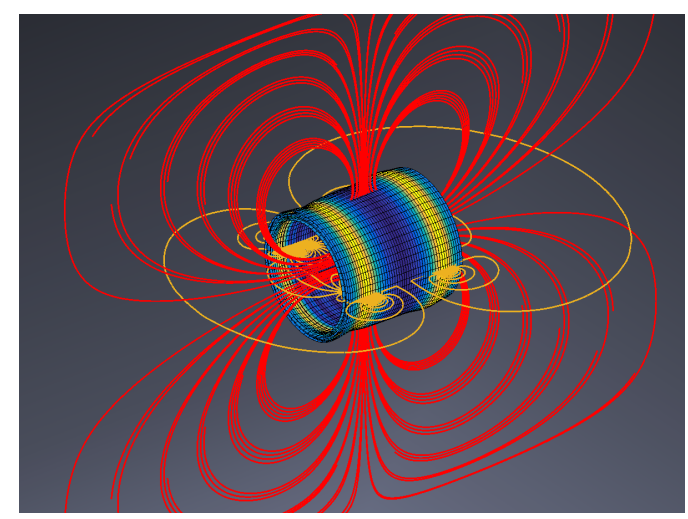

(a) $10 \mathrm{~Hz}$

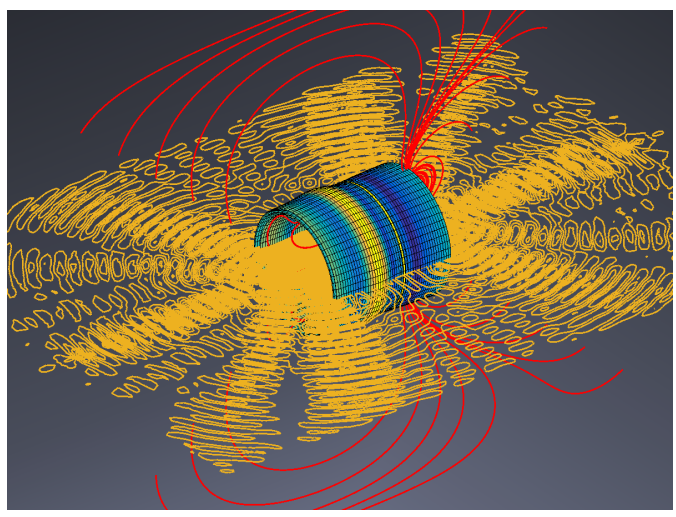

(c) $3600 \mathrm{~Hz}$

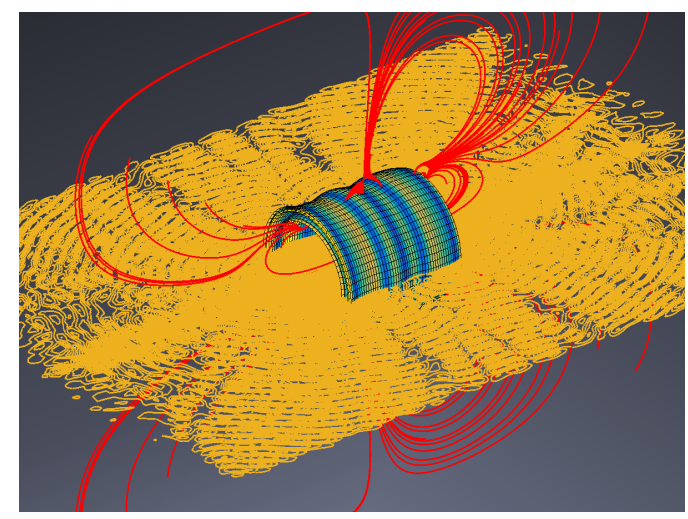

(e) $3950 \mathrm{~Hz}$

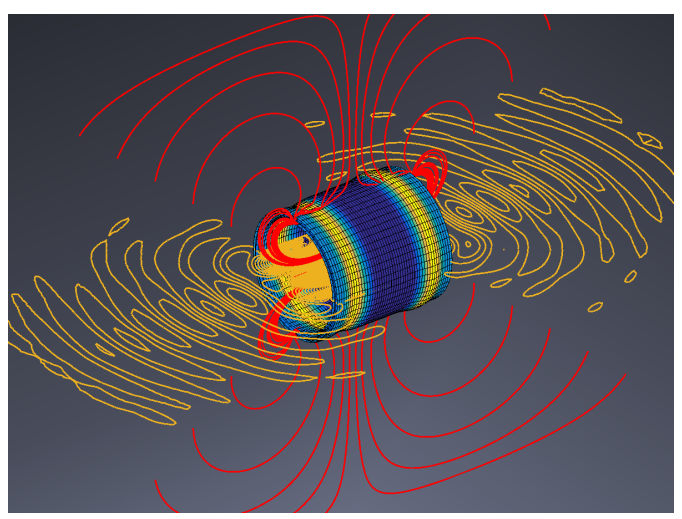

(b) $1500 \mathrm{~Hz}$

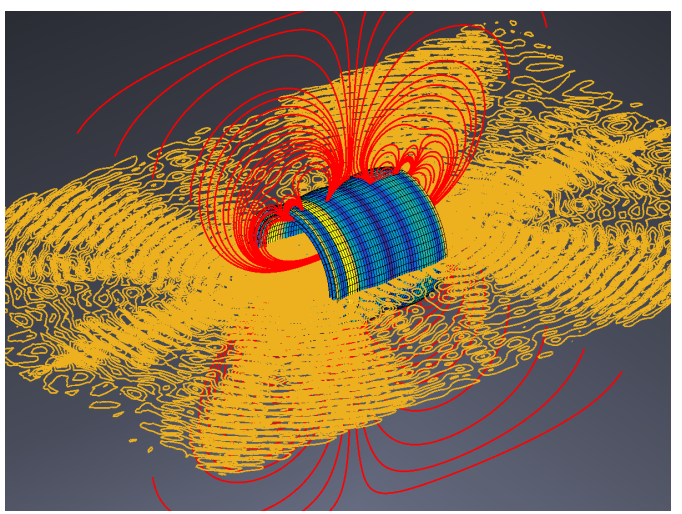

(d) $3800 \mathrm{~Hz}$

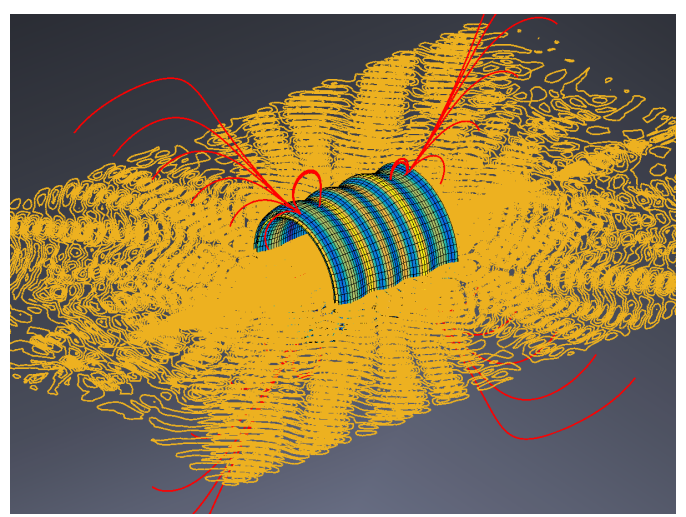

(f) $4075 \mathrm{~Hz}$

Figure 5.28: Simplified MRI scanner subject to alternating and static current driven coils: magnetic flux lines (red), acoustic contour lines (yellow) and displaced shields $\Omega_{c}$. 


\section{Part IV}

\section{Non Linear Approach}





\section{Chapter 6}

\section{Non-Linear Approach}

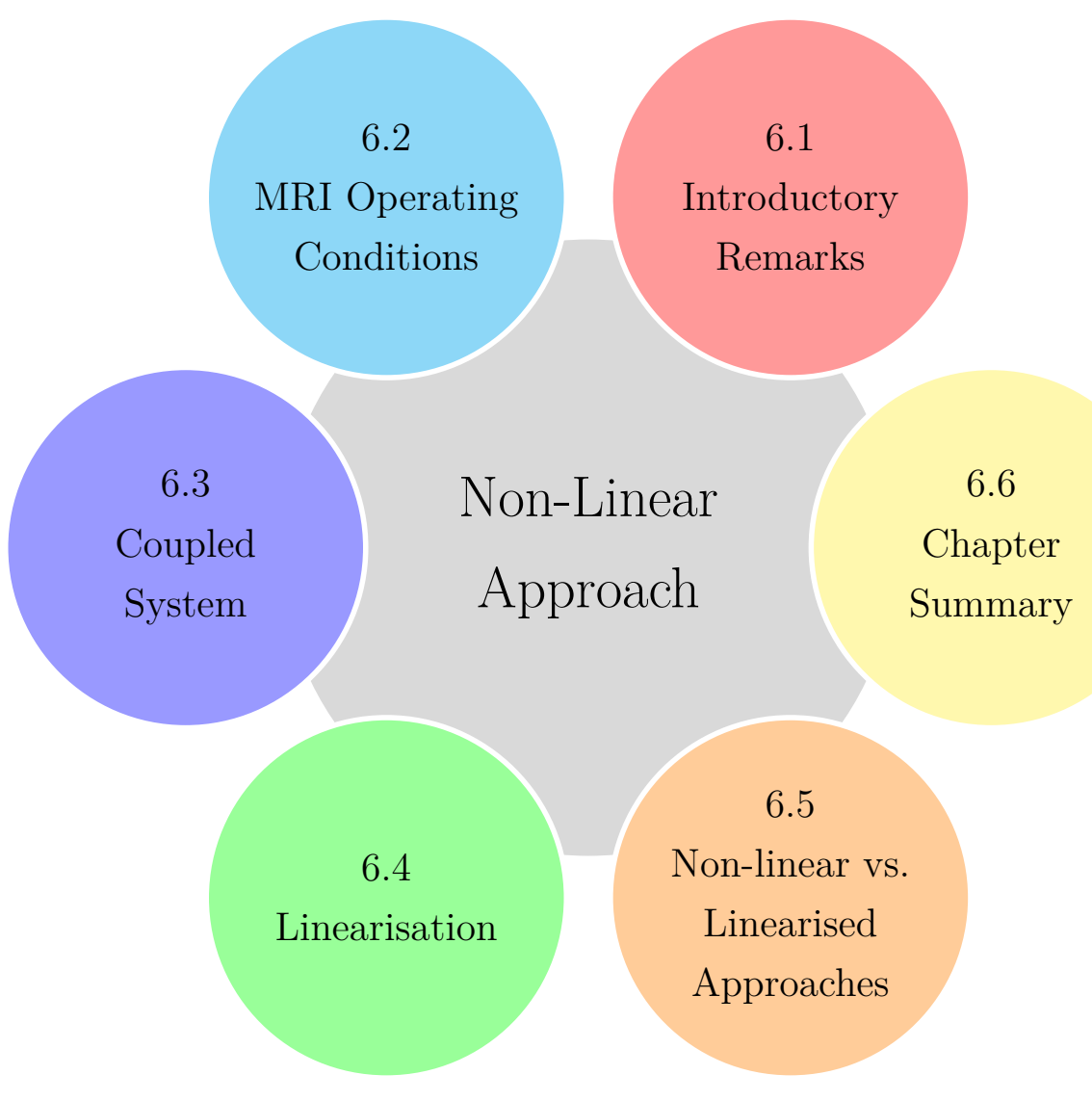

"If at first you don't succeed; call it version 1.0." 


\subsection{Introductory Remarks}

This Chapter describes the treatment of the non-linearity of the novel non-linear coupled acousto-magneto-mechanical equation through the linearisation of the system. In Chapter 3, a linearisation about the static solution fields was formulated which allows for the generation of a discrete single step monolithic solution strategy, described in Chapter 4. This Chapter builds on the work presented in Part III of this Thesis by revisiting the linearisation of the non-linear equations in Chapter 3, and provides an alternative formulation, suitable for transient simulations of the full non-linear problem. A set of rigorously derived bounds on the relative contributions of the non-linear terms in the non-linear approach that are not present in the linearised approach are also presented.

The relative importance of the non-linear terms will depend on the strength of the coupling between the acoustic, mechanical and electromagnetic fields. In the context of MRI scanners, it is thus imperative to answer two important questions: 1) How good is the agreement between the computationally efficient linearised approach compared with the intensive treatment of the fully non-linear system, denoted as the non-linear approach?; and 2) Over what range of MRI operating conditions can the linearised approach be expected to provide acceptable results for MRI scanner design?

From the objectives of this Thesis, discussed in section 1.4, the complete nonlinear approach can be used to compare against the computationally efficient linearised approach and this comparison aligns with the objectives; "To develop the complete non-linear approach to solving the coupled system of equations" and "To determine a series of theoretical measures that can be used to measure the nonlinearity of the coupled problem in MRI scanners." The contents of this Chapter builds on the work carried out in the submitted paper [25], through an extension of the discussion of the technical details.

The Chapter starts with a brief recollection of the coupled transmission problems, previously presented in Chapter 2, with a further discussion on the initial conditions of the system in Section 6.3. Section 6.4 then briefly summarises the NewtonRaphson procedure of the linearised system of non-linear equations and presents it in the form of a dynamic second order Equation of Motion (EOM), where the mass, stiffness and damping terms are defined. In Section 6.5, a comparison of the energy associated with a fully non-linear solution and that of the linearised approach is made resulting in a series of rigorous bounds. In doing so, a series of measures 
for the non-linearity of the acousto-magneto-mechanical coupling in MRI scanners can be developed which can be used to determine the accuracy of the linearised approach. The contents of this Chapter are then summarised in Section 6.6.

Remark 6.1 Part IV of this Thesis works directly with the transient transmission problem in Equation (2.36), Chapter 2 in the time domain. As such, the time harmonic representation of the variables $(\mathcal{A}, \mathcal{U}, \mathcal{P})$, used in Part III, are no longer required and the time dependent solution variables $(\boldsymbol{A}, \boldsymbol{u}, P)$ will be treated directly.

\subsection{MRI Operating Conditions}

MRI scanner resolution is determined by the strength of the static magnetic field, $\boldsymbol{H}^{D C}$, produced by the main magnet. The magnitude of magnetic flux density of such fields, $\left|\boldsymbol{B}^{D C}\right|$, is typically in the region of 1.5 -3T (approximately 30,000 to 70,000 times the strength of that of the Earth) for clinical operation [196, 229, 228], with some $7 \mathrm{~T}$ units in use for medical research applications [249, 129]. Recently, the Siemens 7T magnet, the MAGNETOM Terra [227], has also been cleared for clinical use as well as use in research. The magnitude of the magnetic flux density, quoted by manufacturers, is defined as the maximum value of the flux density magnitude on the imaging bore axis ${ }^{1}$, in the centre where the patient lies, shown in Figure 6.1. Recent advances in MRI design have resulted in magnets of flux densities of up to $12 \mathrm{~T}$ coming into production, which will allow for very high resolution images to be obtained, compared with the current systems [211]. These scanners all typically utilise superconducting magnets consisting of wound conducting wires, resembling solenoids, that are supercooled by being immersed in liquid helium (to temperatures of approximately 4K). Some open C-shaped MRI scanners, which utilise permanent magnets to generate the static field, are still available, but these are less common in current imaging units due to their relatively low flux densities of approximately $0.3 \mathrm{~T}$.

In addition to the static field generated by the main magnet, MRI scanners use pulsed time varying magnetic field gradients, generated through sets of resistive coils, which excite the tissues and generate images of the patient. The gradient in

\footnotetext{
${ }^{1}$ The imaging bore is located in free space and as such $\boldsymbol{B}^{D C}=\mu_{0} \boldsymbol{H}^{D C}$, where $\mu_{0}=4 \pi \times$ $10^{-7} \mathrm{H} / \mathrm{m}$ is the permeability of free space.
} 


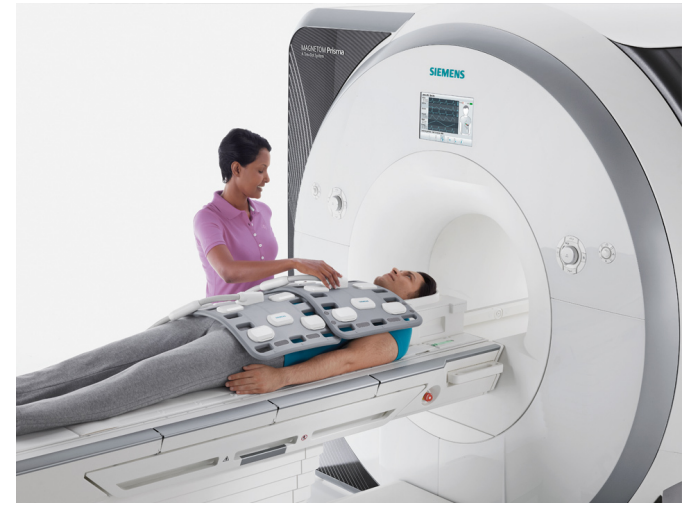

Figure 6.1: Patient on MRI scanner bed with localised torso receiver coils, courtesy of Siemens.

the magnetic flux density of these fields is much smaller than the flux density of the main static field, typically with amplitudes in the region of $30-80 \times 10^{-3} \mathrm{~T} / \mathrm{m}$ $[196,229,228]^{2}$.

\subsection{Coupled System}

The fully coupled system of non-linear equations describing the magnetic, mechanic and acoustic behaviours of an MRI scanner are presented in Chapter 2. The transmission conditions, present at the interface between the conducting and non-conducting regions as well as initial and far field conditions of the system are also described. From this coupled transmission problem, the linearised approach was introduced by a suitable additive split of the exciting current source $\boldsymbol{J}^{s}(t)$, described in Chapters 3, 4 and 5. This Section briefly recalls the fully coupled transmission problem derived in Chapter 2 and focusses on the treatment of the transient nonlinear problem as a means of verifying the linearised approach.

\subsubsection{Transient Non-Linear System}

The fully coupled transient acousto-magneto-mechanical system is described through the transmission problem defined in (2.36) Chapter 2. This system describes the interaction between the magnetic, mechanical and acoustic fields, $\boldsymbol{A}, \boldsymbol{u}, P$ respectively, through a series of coupled body terms and interface conditions. The magnetomechanical coupling arises due to Maxwell stresses and Lorentz currents that arise in the conductor (see Appendix A for further details). The acousto-mechanical cou-

\footnotetext{
${ }^{2}$ The gradient field is typically measured in terms of spatial rate of change in the magnetic flux density along the imaging bore axis, in Teslas per metre $(\mathrm{T} / \mathrm{m})$. The magnitude of the magnetic flux density arising from these coils is orders of magnitude smaller than that of the main coils.
} 
pling arises due to continuity conditions in the traction forces and accelerations at the conductor-non-conductor interface (see Appendix B for details). In the case of Biot-Savart coils (see Section 2.5.2), a one-way acousto-magnetic coupling term arises inside the coil regions, which are located in free space. These coupling phenomena appear in the linear system and the terms highlighted in Chapter 4 Equation (4.45).

The non-linear approach to solving this system of equations is obtained by treating this system directly using the time dependent variables $(\boldsymbol{A}, \boldsymbol{u}, P)$.

\subsubsection{Static Problem}

MRI scanners are driven by time varying current sources through a series of conducting coils and thus the system, in (2.36), is excited through the current source $\boldsymbol{J}^{s}(t)$. During operation, however, MRI scanners typically remain at static field and only apply time varying currents during imaging sequences [238]. This allows for the decomposition of the current source $\boldsymbol{J}^{s}(t)=\boldsymbol{J}^{D C}+\boldsymbol{J}^{A C}(t)$, where $\boldsymbol{J}^{D C}$ corresponds to the static current source of the main magnetic coils and $\boldsymbol{J}^{A C}(t)$ the transient current source of the gradient coils. The solution to the static problem $\left(\boldsymbol{A}^{D C}, \boldsymbol{u}^{D C}, P^{D C}\right)$ is achieved by setting $\boldsymbol{J}^{s}=\boldsymbol{J}^{D C}$ and assuming the fields are not time dependent in (2.36) resulting in the system for $\left(\boldsymbol{A}^{D C}, \boldsymbol{u}^{D C}, P^{D C}\right)$, in Equation (2.38). After linearising the system and applying a Newton-Raphson scheme the solution to the linear system is obtained by solving (3.26) and (3.27).

\subsubsection{Initial Conditions}

In the treatment of the linearised approach, in Chapter 3, the initial conditions in (2.36) were set to be zero, such that they represented a system at rest. This corresponds to a MRI scanner with the magnetic field completely switched off. In practice, however, MRI scanners are typically maintained at full field strength, whilst in clinical use, due to the time required for the static magnetic field to reach the desired field strength. The gradient (time varying) fields are then only applied during imaging sequences of the patient. This offers physical motivation to set the initial conditions for (2.36) to the solution of the static field components and so become

$$
\begin{aligned}
& \boldsymbol{A}(t=0)=\boldsymbol{A}^{D C} \quad \text { in } \mathbb{R}^{3}, \\
& \boldsymbol{u}(t=0)=\boldsymbol{u}^{D C}, \frac{\partial \boldsymbol{u}}{\partial t}(t=0)=\mathbf{0} \quad \text { in } \Omega_{c},
\end{aligned}
$$




$$
P(t=0)=P^{D C}, \frac{\partial P}{\partial t}(t=0)=\mathbf{0} \quad \text { in } \mathbb{R}^{3} \backslash \Omega_{c} .
$$

Setting these initial conditions for the system in (2.36) permits the solution of the much larger static component independently of time. The transient simulations can then be started from this point. With the knowledge of the static field, only solutions of the transient components of the fields at each time instance are required, resulting in shorter simulation times. This choice also more accurately represents an MRI scanner during actual clinical use [238].

\subsection{Linearisation}

The transient problem in (2.36) presents a non-linear system of coupled equations describing electromagnetic, mechanic and acoustic behaviours in an MRI environment. In this section the linearisation of (2.36) is briefly recalled and the linearised and non-linear approaches are presented for the solution of (2.36). The focus is then placed on the construction of the linearised dynamic system of equations by separating out the terms involving the first and second temporal derivatives. This representation, after applying spatial discretisation, will allow for the transient system to be presented in terms of a dynamic system involving mass, damping and stiffness matrices.

\subsubsection{Non-Linear Approach}

In order to solve the fully non-linear system of equations the linearised weighted residual form of the coupled equation set in (3.28) and (3.29) must be solved directly. In this case, the solution fields are time dependent and as such will require further treatment in order to solve.

\section{Coupled Weak Form}

The solution to the linearised system of coupled non-linear equations, presented in weighted residual form in Equations (3.28) and (3.29), may be expressed, in a continuous sense, in terms of the following iterative NR procedure: Find $\left(\boldsymbol{\delta}_{A}^{[\mathrm{k}]}, \boldsymbol{\delta}_{u}^{[\mathrm{k}]}, \delta_{P}^{[\mathrm{k}]}\right)(t) \in$ $(X \times Y(\mathbf{0}) \times Z)[0, T]$ such that

$$
D R_{A}\left(\boldsymbol{A}^{\delta} ; \boldsymbol{A}^{[\mathrm{k}]}, \boldsymbol{u}^{[\mathrm{k}]}\right)\left[\boldsymbol{\delta}_{A}^{[\mathrm{k}]}\right]+D R_{A}\left(\boldsymbol{A}^{\delta} ; \boldsymbol{A}^{[\mathrm{k}]}, \boldsymbol{u}^{[\mathrm{k}]}\right)\left[\boldsymbol{\delta}_{u}^{[\mathrm{k}]}\right]=-R_{A}\left(\boldsymbol{A}^{\delta} ; \boldsymbol{A}^{[\mathrm{k}]}, \boldsymbol{u}^{[\mathrm{k}]}\right),
$$




$$
\begin{aligned}
D R_{u}\left(\boldsymbol{u}^{\delta} ; \boldsymbol{A}^{[\mathrm{k}]}, \boldsymbol{u}^{[\mathrm{k}]}, P^{[\mathrm{k}]}\right)\left[\boldsymbol{\delta}_{A}^{[\mathrm{k}]}\right] & +D R_{u}\left(\boldsymbol{u}^{\delta} ; \boldsymbol{A}^{[\mathrm{k}]}, \boldsymbol{u}^{[\mathrm{k}]}, P^{[\mathrm{k}]}\right)\left[\boldsymbol{\delta}_{u}^{[\mathrm{k}]}\right] \\
& +D R_{u}\left(\boldsymbol{u}^{\delta} ; \boldsymbol{A}^{[\mathrm{k}]}, \boldsymbol{u}^{[\mathrm{k}]}, P^{[\mathrm{k}]}\right)\left[\delta_{P}^{[\mathrm{k}]}\right]=-R_{u}\left(\boldsymbol{u}^{\delta} ; \boldsymbol{A}^{[\mathrm{k}]}, \boldsymbol{u}^{[\mathrm{k}]}, P^{[\mathrm{k}]}\right), \\
D R_{P}\left(P^{\delta} ; \boldsymbol{A}^{[\mathrm{k}]}, \boldsymbol{u}^{[\mathrm{k}]}, P^{[\mathrm{k}]}\right)\left[\boldsymbol{\delta}_{A}^{[\mathrm{k}]}\right] & +D R_{P}\left(P^{\delta} ; \boldsymbol{A}^{[\mathrm{k}]}, \boldsymbol{u}^{[\mathrm{k}]}, P^{[\mathrm{k}]}\right)\left[\boldsymbol{\delta}_{u}^{[\mathrm{k}]}\right] \\
+ & D R_{P}\left(P^{\delta} ; \boldsymbol{A}^{[\mathrm{k}]}, \boldsymbol{u}^{[\mathrm{k}]}, P^{[\mathrm{k}]}\right)\left[\delta_{P}^{[\mathrm{k}]}\right]=-R_{P}\left(P^{\delta} ; \boldsymbol{A}^{[\mathrm{k}]}, \boldsymbol{u}^{[\mathrm{k}]}, P^{[\mathrm{k}]}\right),
\end{aligned}
$$

for all $\left(\boldsymbol{A}^{\delta}, \boldsymbol{u}^{\delta}, P^{\delta}\right) \in X \times Y(\mathbf{0}) \times Z$ for a particular iteration $[\mathrm{k}]$, where the system of weak variational residuals and their directional derivatives are defined in (3.28) and (3.29) respectively and obtained by adopting the approach in [38]. The definitions $X, Y, Z$ were previously introduced in Chapter 3 Equation (3.5).

The solution fields are obtained by first defining an initial guess of the three fields $\left(\boldsymbol{A}^{[0]}, \boldsymbol{u}^{[0]}, P^{[0]}\right)$ and then solving (6.2) to obtain the solution updates $\left(\boldsymbol{\delta}_{A}^{[\mathrm{k}]}, \boldsymbol{\delta}_{u}^{[\mathrm{k}]}, \delta_{P}{ }^{[\mathrm{k}]}\right)$. The solution fields are then updated, for a given iteration, by summing the solution field of the previous iteration and the solution updates

$$
\begin{aligned}
& \boldsymbol{A}^{[\mathrm{k}+1]}=\boldsymbol{A}^{[\mathrm{k}]}+\boldsymbol{\delta}_{A}^{[\mathrm{k}]}, \\
& \boldsymbol{u}^{[\mathrm{k}+1]}=\boldsymbol{u}^{[\mathrm{k}]}+\boldsymbol{\delta}_{u}^{[\mathrm{k}]}, \\
& P^{[\mathrm{k}+1]}=P^{[\mathrm{k}]}+\delta_{P}^{[\mathrm{k}]},
\end{aligned}
$$

until $\left|R_{A}, R_{u}, R_{\hat{p}}\right|<$ TOL where TOL is a user supplied tolerance. The resulting solutions are $\boldsymbol{A}(t), \boldsymbol{u}(t)$ and $P(t)$. Equation (6.2) is similar to the static NR system presented in Equation (3.26), Chapter 3. However, given that it is a transient system, will require more than a single iteration to converge, due to the non-linearities present in the transient terms that result in two-way coupling mechanisms. But the residuals $\left(R_{A}, R_{u}, R_{P}\right)$ will converge quadratically to 0 .

\section{Reformulation of the Newton-Raphson Procedure}

With a view to introducing an alternative formulation of (6.2), in which the terms associated with the zeroth-, first- and second- temporal derivative are isolated, the problem is recast in terms of a vector of unknowns containing the three physical fields $\boldsymbol{q}:=\left\{\boldsymbol{q}_{A} \boldsymbol{q}_{u} q_{P}\right\}^{T}=\{\boldsymbol{A} \boldsymbol{u} P\}^{T}$. The discretised bilinear forms associated with these temporal derivatives will later form the mass, damping and stiffness terms in the fully discrete approach, presented later in Chapter 7. This separation permits Equation (6.2) to be recast to introduce the following Newton-Raphson 
iteration: Find $\boldsymbol{\delta}_{q}^{[\mathrm{k}]} \in W$ at each time $t \in(0, T]$, such that

$$
\begin{aligned}
M\left({\frac{\partial^{2} \boldsymbol{\delta}_{q}}{\partial t^{2}}}^{[\mathrm{k}]}, \boldsymbol{q}^{\delta}\right)+C\left({\frac{\partial \boldsymbol{\delta}_{q}}{\partial t}}^{[\mathrm{k}]}, \boldsymbol{q}^{\delta} ; \boldsymbol{q}^{[\mathrm{k}]}\right) & +K\left(\boldsymbol{\delta}_{q}^{[\mathrm{k}]}, \boldsymbol{q}^{\delta} ; \boldsymbol{q}^{[\mathrm{k}]},{\left.\frac{\partial \boldsymbol{q}^{[\mathrm{k}]}}{\partial t}\right)=}-R_{q}\left(\boldsymbol{q}^{\delta} ; \boldsymbol{q}^{[\mathrm{k}]}, \frac{\partial \boldsymbol{q}^{[\mathrm{k}]}}{\partial t}, \frac{\partial^{2} \boldsymbol{q}^{[\mathrm{k}]}}{\partial t^{2}}\right),\right.
\end{aligned}
$$

for all $\boldsymbol{q}^{\delta} \in W$, where the updated solution vector is

$$
\boldsymbol{q}^{[\mathrm{k}+1]}=\boldsymbol{q}^{[\mathrm{k}]}+\boldsymbol{\delta}_{q}^{[\mathrm{k}]}
$$

and $W:=X \times Y(\mathbf{0}) \times Z$. The system is iterated until $\left|R_{q}\right|<$ TOL and the resulting solution is $\boldsymbol{q}(t)$. The forms of $M, C$ and $K$, which are associated with the mass, damping and stiffness contributions to the system, respectively, arise from this splitting of the directional derivatives and are given as

$$
\begin{aligned}
& M\left({\frac{\partial^{2} \boldsymbol{\delta}_{q}}{\partial t^{2}}}^{[\mathrm{k}]}, \boldsymbol{q}^{\delta}\right):=\int_{\Omega_{c}} \rho{\frac{\partial^{2} \boldsymbol{\delta}_{u}}{\partial t^{2}}}^{[\mathrm{k}]} \cdot \boldsymbol{q}_{u}^{\delta} \mathrm{d} \Omega-\left.\int_{\partial \Omega_{c}^{N}} \rho^{+} \frac{\partial^{2} \boldsymbol{\delta}_{u}}{\partial t^{2}}\right|^{-} \cdot \boldsymbol{n}^{+} q_{P}^{\delta} \mathrm{d} S \\
& +\int_{\mathbb{R}^{3} \backslash \Omega_{c}} \frac{1}{c^{2}}{\frac{\partial^{2} \delta_{P}}{\partial t^{2}}}^{[\mathrm{k}]} \cdot q_{P}^{\delta} \mathrm{d} \Omega \\
& C\left({\frac{\partial \boldsymbol{\delta}_{q}}{\partial t}}^{[\mathrm{k}]}, \boldsymbol{q}^{\delta} ; \boldsymbol{q}^{[\mathrm{k}]}\right):=\int_{\mathbb{R}^{3}} \gamma{\frac{\partial \boldsymbol{\delta}_{A}}{\partial t}}^{[\mathrm{k}]} \cdot \boldsymbol{q}_{A}^{\delta} \mathrm{d} \Omega-\int_{\Omega_{c}} \gamma{\frac{\partial \boldsymbol{\delta}_{u}}{\partial t}}^{[\mathrm{k}]} \times\left(\nabla \times \boldsymbol{q}_{A}^{[\mathrm{k}]}\right) \cdot \boldsymbol{q}_{A}^{\delta} \mathrm{d} \Omega, \\
& K\left(\boldsymbol{\delta}_{q}^{[\mathrm{k}]}, \boldsymbol{q}^{\delta} ; \boldsymbol{q}^{[\mathrm{k}]}, \frac{\partial \boldsymbol{q}^{[\mathrm{k}]}}{\partial t}\right):=\int_{\mathbb{R}^{3}} \mu^{-1}\left(\nabla \times \boldsymbol{\delta}_{A}^{[\mathrm{k}]}\right) \cdot\left(\nabla \times \boldsymbol{q}_{A}^{\delta}\right) \mathrm{d} \Omega \\
& -\int_{\Omega_{c}} \gamma{\frac{\partial \boldsymbol{q}_{u}}{\partial t}}^{[\mathrm{k}]} \times\left(\nabla \times \boldsymbol{\delta}_{A}^{[\mathrm{k}]}\right) \cdot \boldsymbol{q}_{A}^{\delta} \mathrm{d} \Omega \\
& +\int_{\Omega_{c}} \mu^{-1} \mathcal{S}\left(\boldsymbol{q}_{A}^{[\mathrm{k}]}, \boldsymbol{\delta}_{A}^{[\mathrm{k}]}\right): \nabla \boldsymbol{q}_{u}^{\delta} \mathrm{d} \Omega \\
& -\left.\int_{\partial \Omega_{c}^{N}} \mu_{0}^{-1} \mathcal{S}\left(\boldsymbol{q}_{A}^{[\mathrm{k}]}, \boldsymbol{\delta}_{A}^{[\mathrm{k}]}\right)\right|^{+} \boldsymbol{n}^{-} \cdot \boldsymbol{q}_{u}^{\delta} \mathrm{d} S \\
& +\int_{\Omega_{c}} \boldsymbol{\sigma}^{m}\left(\boldsymbol{\delta}_{u}^{[\mathrm{k}]}\right): \nabla \boldsymbol{q}_{u}^{\delta} \mathrm{d} \Omega-\left.\int_{\partial \Omega_{c}^{N}} \delta_{P}^{[\mathrm{k}]}\right|^{+} \boldsymbol{n}^{-} \cdot \boldsymbol{q}_{u}^{\delta} \mathrm{d} S \\
& -\int_{\operatorname{supp}\left(\boldsymbol{J}^{s}\right)}\left(\nabla \times \boldsymbol{\delta}_{A}^{[\mathrm{k}]} \times\left(\nabla \times\left(\mu_{0}^{-1} \nabla \times \boldsymbol{q}_{A}^{[\mathrm{k}]}\right)\right)\right. \\
& \left.+\nabla \times \boldsymbol{q}_{A}^{[\mathrm{k}]} \times\left(\nabla \times\left(\mu_{0}^{-1} \nabla \times \boldsymbol{\delta}_{A}^{[\mathrm{k}]}\right)\right)\right) \cdot \nabla q_{P}^{\delta} \mathrm{d} \Omega \\
& +\int_{\mathbb{R}^{3} \backslash \Omega_{c}} \nabla \delta_{P}^{[\mathrm{k}]} \cdot \nabla q_{P}^{\delta} \mathrm{d} \Omega
\end{aligned}
$$


where the corresponding matrices will follow from their discrete counterparts, which will be discussed in Chapter 7 . The system residual vector $R_{q}$ is defined as

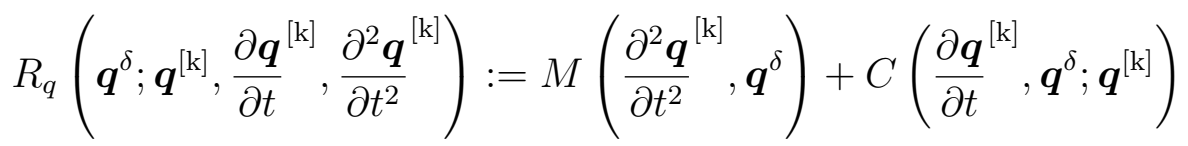

$$
\begin{aligned}
& +\int_{\mathbb{R}^{3}} \mu^{-1}\left(\nabla \times \boldsymbol{q}_{A}^{[\mathrm{k}]}\right) \cdot\left(\nabla \times \boldsymbol{q}_{A}^{\delta}\right) \mathrm{d} \Omega+\int_{\Omega_{c}}\left(\boldsymbol{\sigma}^{m}\left(\boldsymbol{q}_{u}^{[\mathrm{k}]}\right)+\boldsymbol{\sigma}^{e}\left(\boldsymbol{q}_{A}^{[\mathrm{k}]}\right)\right): \nabla \boldsymbol{q}_{u}^{\delta} \mathrm{d} \Omega \\
& -\left.\int_{\partial \Omega_{C}^{N}}\left(q_{P}^{[\mathrm{k}]} \boldsymbol{I}+\boldsymbol{\sigma}^{e}\left(\boldsymbol{q}_{A}^{[\mathrm{k}]}\right)\right)\right|^{+} \boldsymbol{n}^{-} \cdot \boldsymbol{q}_{u}^{\delta} \mathrm{d} S \\
& +\int_{\mathbb{R}^{3} \backslash \Omega_{c}}\left(\nabla q_{P}^{[\mathrm{k}]} \cdot \nabla q_{P}^{\delta}+\left(\nabla \cdot \boldsymbol{\sigma}^{e}\left(\boldsymbol{q}_{A}^{[\mathrm{k}]}\right)\right) \cdot \nabla q_{P}^{\delta}\right) \mathrm{d} \Omega-\int_{\mathbb{R}^{3}} \boldsymbol{J}^{s}(t) \cdot \boldsymbol{q}_{A}^{\delta} \mathrm{d} \Omega .
\end{aligned}
$$

The linearised electromagnetic stress tensor $\mathcal{S}$ was previously introduced in Chapter 3 Equation (3.25). The initial guesses of the system in (6.3) and (6.4) are such that $\left(\boldsymbol{q}^{[0]}, \frac{\partial \boldsymbol{q}^{[0]}}{\partial t},{\frac{\partial^{2} \boldsymbol{q}^{[0]}}{\partial t^{2}}}^{[0}\right) \in\left(X \times Y\left(\boldsymbol{u}^{D}\right) \times Z\right) \times\left(X \times Y\left(\partial \boldsymbol{u}^{D} / \partial t\right) \times Z\right) \times(X \times$ $\left.Y\left(\partial^{2} \boldsymbol{u}^{D} / \partial t^{2}\right) \times Z\right)$.

\subsubsection{Linearised Approach}

The transient case of the linearised approach is derived in (3.31) and (3.32), where the solutions fields are time dependent. In order to perform a comparison with the non-linear approach this case must be treated in the transient form, as opposed to using a time harmonic description, as was the case in Part III. As with the nonlinear approach in Section 6.4.1, the linearised approach can be recast in a similar vectorial form. First, $\boldsymbol{q}^{D C}:=\left\{\boldsymbol{A}^{D C} \boldsymbol{u}^{D C} P^{D C}\right\}^{T}$ is set as the static solution, which can be obtained from solving (3.26) or similarly from (6.3) by looking for a time invariant solution and replacing $\boldsymbol{J}^{A C}$ with $\boldsymbol{J}^{D C}$. Then, in light of the fact that the linearisation of the coupled non-linear system about $\boldsymbol{q}^{D C}$ results in a system that is linear in the solution variables, it can be recast in a similar manner to (6.2), where the system is independent of iterations, as: Find $\boldsymbol{\delta}_{q} \in W$, such that

$$
\tilde{M}\left(\frac{\partial^{2} \boldsymbol{q}}{\partial t^{2}}, \boldsymbol{q}^{\delta}\right)+\tilde{C}\left(\frac{\partial \boldsymbol{q}}{\partial t}, \boldsymbol{q}^{\delta}\right)+\tilde{K}\left(\boldsymbol{q}, \boldsymbol{q}^{\delta}\right)=-\tilde{R}_{q}\left(\boldsymbol{q}^{\delta}\right)
$$

for all $\boldsymbol{q}^{\delta} \in W$ at each time $t \in[0, T]$. The bilinear forms $\tilde{M}, \tilde{C}$ and $\tilde{K}$ will, once discretised, form the separated mass, damping and stiffness matrices in the linearised approach, and can be expressed in terms of the definitions in (6.5) as

$$
\tilde{M}\left(\frac{\partial^{2} \boldsymbol{\delta}_{q}}{\partial t^{2}}, \boldsymbol{q}^{\delta}\right):=M\left(\frac{\partial^{2} \boldsymbol{\delta}_{q}}{\partial t^{2}}, \boldsymbol{q}^{\delta}\right)
$$




$$
\begin{aligned}
\tilde{C}\left(\frac{\partial \boldsymbol{\delta}_{q}}{\partial t}, \boldsymbol{q}^{\delta}\right) & :=C\left(\frac{\partial \boldsymbol{\delta}_{q}}{\partial t}, \boldsymbol{q}^{\delta} ; \boldsymbol{q}^{D C}\right), \\
\tilde{K}\left(\boldsymbol{\delta}_{q}, \boldsymbol{q}^{\delta}\right) & :=K\left(\boldsymbol{\delta}_{q}, \boldsymbol{q}^{\delta} ; \boldsymbol{q}^{D C}, \mathbf{0}\right), \\
\tilde{R}_{q}\left(\boldsymbol{q}^{\delta}\right) & :=R_{q}\left(\boldsymbol{q}^{\delta} ; \boldsymbol{q}^{D C}, \mathbf{0}, \mathbf{0}\right),
\end{aligned}
$$

where the temporal derivatives of the static solution vanish, due to the temporal independence of the static fields.

This system in (6.7) is independent of iterations and so can be solved in a single step, hence named the linearised approach, described in Chapter 3. In this case the solution obtained from (6.7) is $\boldsymbol{\delta}_{q}(t)$, which contains only the transient component of the fields, and thus the complete fields are described as $\boldsymbol{q}(t)=\boldsymbol{q}^{D C}+\boldsymbol{\delta}_{q}(t)$, where $\boldsymbol{q}^{D C}$ is given from the initial conditions. The linear nature of this system also allows for a time harmonic description of the transient components of the fields to be adopted, which was the approach followed in Part III of this Thesis.

\subsection{Non-Linear vs. Linearised Approaches}

The linearised approach, described in Part III, provides an efficient way of solving the fully coupled acousto-magneto-mechanical problem as the discretisation of (6.7) results in system matrices that are independent of time, or frequency (if a time harmonic description is used). In particular, by performing the linearisation about the static solution, the non-linear terms in (6.3) disappear. The relative importance of these terms will depend on a comparison of the energy associated with a fully non-linear solution and that of the linearised approach. In this Section, the residual energy in the non-linear approach, not described by the linearised approach, will be bounded in terms of practical quantities of interest. A summary of these measures of interest is then presented. Finally, a simple analytical model is presented that can relate these measures to manufacturing data in order to analyse the operating range of magnetic field strengths used in actual MRI scanners.

\subsubsection{Comparison of Energies in Non-Linear and Linearised Approaches}

In order to determine if the linearised approach, presented in a time dependent sense in Section 6.4.2, accurately approximates the solution to the non-linear approach (6.2), at a continuous level, the magnitude of the energy associated with the non- 
linear approach must be compared with the corresponding energy of the linearised approach.

Recalling the complete weak form of the system in (3.21), it is assumed there exists a fixed point weak solution of the form $(\boldsymbol{A}, \boldsymbol{u}, P)(t) \in\left(X \times Y\left(\boldsymbol{u}_{D}\right) \times Z\right)[0, T]$ that satisfies the dynamic problem

$$
\begin{gathered}
\int_{\mathbb{R}^{3}} \mu^{-1} \nabla \times \boldsymbol{A} \cdot \nabla \times \boldsymbol{A}^{\delta} \mathrm{d} \Omega+\int_{\Omega_{c}} \gamma \frac{\partial \boldsymbol{A}}{\partial t} \cdot \boldsymbol{A}^{\delta} \mathrm{d} \Omega \\
-\int_{\Omega_{c}} \gamma \frac{\partial \boldsymbol{u}}{\partial t} \times(\nabla \times \boldsymbol{A}) \cdot \boldsymbol{A}^{\delta} \mathrm{d} \Omega-\int_{\operatorname{supp}\left(\boldsymbol{J}^{s}\right)} \boldsymbol{J}^{s} \cdot \boldsymbol{A}^{\delta} \mathrm{d} \Omega=0, \\
\int_{\Omega_{c}}\left(\boldsymbol{\sigma}^{m}(\boldsymbol{u})+\boldsymbol{\sigma}^{e}(\boldsymbol{A})\right): \nabla \boldsymbol{u}^{\delta} \mathrm{d} \Omega+\int_{\Omega_{c}} \rho \frac{\partial^{2} \boldsymbol{u}}{\partial t^{2}} \cdot \boldsymbol{u}^{\delta} \mathrm{d} \Omega \\
-\left.\int_{\partial \Omega_{c}^{N}}\left(P \boldsymbol{I}+\boldsymbol{\sigma}^{e}(\boldsymbol{A})\right)\right|_{\partial \Omega_{c}} ^{+} \boldsymbol{n} \cdot \boldsymbol{u}^{\delta} \mathrm{d} S=0, \\
\int_{\mathbb{R}^{3} \backslash \Omega_{c}}\left(\nabla P+\nabla \cdot \boldsymbol{\sigma}^{e}(\boldsymbol{A})\right) \cdot \nabla P^{\delta} \mathrm{d} \Omega+\int_{\mathbb{R}^{3} \backslash \Omega_{c}} \frac{1}{c^{2}} \frac{\partial^{2} P}{\partial t^{2}} P^{\delta} \mathrm{d} \Omega \\
-\left.\int_{\partial \Omega_{c}^{N}} \rho^{+} \boldsymbol{n} \cdot \frac{\partial^{2} \boldsymbol{u}}{\partial t^{2}}\right|_{\partial \Omega_{c}^{N}} ^{-} P^{\delta} \mathrm{d} S=0,
\end{gathered}
$$

for all $\left(\boldsymbol{A}^{\delta}, \boldsymbol{u}^{\delta}, P^{\delta}\right) \in\left(X \times Y\left(\boldsymbol{u}_{D}\right) \times Z\right)$, where $(X, Y(\boldsymbol{g}), Z)$ have their usual definitions as in (3.5).

The linearised approximation of the system (previously shown in Chapter 3 to obtain the linearised approach) can now be introduced, where the solutions take the form $\left(\boldsymbol{A}^{D C}+\boldsymbol{A}^{A C}\right),\left(\boldsymbol{u}^{D C}+\boldsymbol{u}^{A C}\right),\left(P^{D C}+P^{A C}\right)$, with static weak solutions $\left(\boldsymbol{A}^{D C}, \boldsymbol{u}^{D C}, P^{D C}\right) \in\left(X \times Y\left(\boldsymbol{u}_{D}\right) \times Z\right)$ being the fixed point solution of ${ }^{3}$

$$
\begin{array}{r}
\int_{\mathbb{R}^{3}} \mu^{-1} \nabla \times \boldsymbol{A}^{D C} \cdot \nabla \times \boldsymbol{A}^{\delta} \mathrm{d} \Omega-\int_{\operatorname{supp}\left(\boldsymbol{J}^{s}\right)} \boldsymbol{J}^{D C} \cdot \boldsymbol{A}^{\delta} \mathrm{d} \Omega=0 \\
\int_{\Omega_{c}}\left(\boldsymbol{\sigma}^{m}\left(\boldsymbol{u}^{D C}\right)+\boldsymbol{\sigma}^{e}\left(\boldsymbol{A}^{D C}\right)\right): \nabla \boldsymbol{u}^{\delta} \mathrm{d} \Omega \\
-\left.\int_{\partial \Omega_{c}^{N}}\left(P^{D C} \boldsymbol{I}+\boldsymbol{\sigma}^{e}\left(\boldsymbol{A}^{D C}\right)\right)\right|_{\partial \Omega_{c}} ^{+} \boldsymbol{n} \cdot \boldsymbol{u}^{\delta} \mathrm{d} S=0, \\
\int_{\mathbb{R}^{3} \backslash \Omega_{c}}\left(\nabla P^{D C}+\nabla \cdot \boldsymbol{\sigma}^{e}\left(\boldsymbol{A}^{D C}\right)\right) \cdot \nabla P^{\delta} \mathrm{d} \Omega=0
\end{array}
$$

for all $\left(\boldsymbol{A}^{\delta}, \boldsymbol{u}^{\delta}, P^{\delta}\right) \in(X \times Y(\mathbf{0}) \times Z)$ and "linearised" weak solutions to the transient problem $\left(\boldsymbol{A}^{A C}, \boldsymbol{u}^{A C}, P^{A C}\right) \in\left(X \times Y\left(\boldsymbol{u}_{D}^{A C}\right) \times Z\right)$ being the solution of

$$
\int_{\mathbb{R}^{3}} \mu^{-1} \nabla \times \boldsymbol{A}^{A C} \cdot \nabla \times \boldsymbol{A}^{\delta} \mathrm{d} \Omega+\int_{\Omega_{c}} \gamma \frac{\partial \boldsymbol{A}^{A C}}{\partial t} \cdot \boldsymbol{A}^{\delta} \mathrm{d} \Omega
$$

\footnotetext{
${ }^{3}$ The temporal derivatives of the static fields disappear as they are not dependant on time.
} 


$$
\begin{array}{r}
-\int_{\Omega_{c}} \gamma \frac{\partial \boldsymbol{u}^{A C}}{\partial t} \times\left(\nabla \times \boldsymbol{A}^{D C}\right) \cdot \boldsymbol{A}^{\delta} \mathrm{d} \Omega-\int_{\operatorname{supp}\left(\boldsymbol{J}^{s}\right)} \boldsymbol{J}^{A C} \cdot \boldsymbol{A}^{\delta} \mathrm{d} \Omega=0 \\
\int_{\Omega_{c}}\left(\boldsymbol{\sigma}^{m}\left(\boldsymbol{u}^{A C}\right)+\mu^{-1} \mathcal{S}\left(\boldsymbol{A}^{D C}, \boldsymbol{A}^{A C}\right)\right): \nabla \boldsymbol{u}^{\delta} \mathrm{d} \Omega+\int_{\Omega_{c}} \rho \frac{\partial^{2} \boldsymbol{u}^{A C}}{\partial t^{2}} \cdot \boldsymbol{u}^{\delta} \mathrm{d} \Omega \\
-\left.\int_{\partial \Omega_{c}^{N}}\left(P^{A C} \boldsymbol{I}+\mu^{-1} \mathcal{S}\left(\boldsymbol{A}^{D C}, \boldsymbol{A}^{A C}\right)\right)\right|_{\partial \Omega_{c}} ^{+} \boldsymbol{n} \cdot \boldsymbol{u}^{\delta} \mathrm{d} S=0 \\
\int_{\mathbb{R}^{3} \backslash \Omega_{c}}\left(\nabla P^{A C}+\nabla \cdot \boldsymbol{\sigma}^{e}\left(\boldsymbol{A}^{A C}\right)\right) \cdot \nabla P^{\delta} \mathrm{d} \Omega+\int_{\mathbb{R}^{3} \backslash \Omega_{c}} \frac{1}{c^{2}} \frac{\partial^{2} P^{A C}}{\partial t^{2}} P^{\delta} \mathrm{d} \Omega \\
-\left.\int_{\partial \Omega_{c}^{N}} \rho^{+} \boldsymbol{n} \cdot \frac{\partial^{2} \boldsymbol{u}^{A C}}{\partial t^{2}}\right|_{\partial \Omega_{c}^{N}} P^{\delta} \mathrm{d} S=0
\end{array}
$$

for all $\left(\boldsymbol{A}^{\delta}, \boldsymbol{u}^{\delta}, P^{\delta}\right) \in(X \times Y(\mathbf{0}) \times Z)$, where the definitions of $\boldsymbol{\sigma}^{e}(\boldsymbol{A})$ and $\mathcal{S}\left(\boldsymbol{A}^{D C}, \boldsymbol{A}^{A C}\right)$ are given in (2.24) and (3.25) respectively.

In order to determine how well the solution to the full non-linear system $(\boldsymbol{A}, \boldsymbol{u}, P)$ from (6.9) is approximated by the solution of the "linearised" system $\left(\left(\boldsymbol{A}^{D C}+\right.\right.$ $\left.\left.\boldsymbol{A}^{A C}\right),\left(\boldsymbol{u}^{D C}+\boldsymbol{u}^{A C}\right),\left(P^{D C}+P^{A C}\right)\right)$ from (6.10) and (6.11), the energy associated with that of the non-linear problem is compared with the linearised approach. To do this, the test functions are first set to $\left(\boldsymbol{A}^{\delta}, \boldsymbol{u}^{\delta}, P^{\delta}\right)=(\boldsymbol{A}, \boldsymbol{u}, P)$ in (6.9) to construct the energy functional associated with the non-linear problem. Here, it is assumed that $\boldsymbol{u}_{D}=\mathbf{0}$. Next, the ansatz $(\boldsymbol{A}, \boldsymbol{u}, P)=\left(\left(\boldsymbol{A}^{D C}+\boldsymbol{A}^{A C}\right),\left(\boldsymbol{u}^{D C}+\boldsymbol{u}^{A C}\right),\left(P^{D C}+P^{A C}\right)\right)$, previously seen in Chapter 3 , is used. In doing so, the resulting system will no longer be guaranteed to be equal to 0 and instead the residuals $R_{A}, R_{u}, R_{P}$ are introduced corresponding to the remainder, leading to ${ }^{4}$

$$
\begin{gathered}
\int_{\mathbb{R}^{3}} \mu^{-1} \nabla \times\left(\boldsymbol{A}^{D C}+\boldsymbol{A}^{A C}\right) \cdot \nabla \times\left(\boldsymbol{A}^{D C}+\boldsymbol{A}^{A C}\right) \mathrm{d} \Omega \\
-\int_{\Omega_{c}} \gamma \frac{\partial \boldsymbol{u}^{A C}}{\partial t} \times\left(\nabla \times\left(\boldsymbol{A}^{D C}+\boldsymbol{A}^{A C}\right)\right) \cdot\left(\boldsymbol{A}^{D C}+\boldsymbol{A}^{A C}\right) \mathrm{d} \Omega \\
+\int_{\Omega_{c}} \gamma \frac{\partial\left(\boldsymbol{A}^{A C}\right)}{\partial t} \cdot\left(\boldsymbol{A}^{D C}+\boldsymbol{A}^{A C}\right) \mathrm{d} \Omega-\int_{\operatorname{supp}\left(\boldsymbol{J}^{s}\right)} \boldsymbol{J}^{s} \cdot\left(\boldsymbol{A}^{D C}+\boldsymbol{A}^{A C}\right) \mathrm{d} \Omega=R_{A} \\
-\left.\int_{\partial \Omega_{c}^{N}}\left(\left(P^{D C}+P^{A C}\right) \boldsymbol{I}+\boldsymbol{\sigma}^{e}\left(\left(\boldsymbol{A}^{D C}+\boldsymbol{A}^{A C}\right)\right)\right)\right|_{\partial \Omega_{c}} ^{+} \boldsymbol{n} \cdot\left(\boldsymbol{u}^{D C}+\boldsymbol{u}^{A C}\right) \mathrm{d} S
\end{gathered}
$$

\footnotetext{
${ }^{4}$ Note that $\boldsymbol{\sigma}^{e}\left(\boldsymbol{A}^{D C}+\boldsymbol{A}^{A C}\right)=\mu^{-1}\left(\nabla \times \boldsymbol{A}^{D C} \otimes \nabla \times \boldsymbol{A}^{D C}+\nabla \times \boldsymbol{A}^{D C} \otimes \nabla \times \boldsymbol{A}^{A C}+\nabla \times \boldsymbol{A}^{A C}\right.$ $\left.\otimes \nabla \times \boldsymbol{A}^{D C}+\nabla \times \boldsymbol{A}^{A C} \otimes \nabla \times \boldsymbol{A}^{A C}-\frac{1}{2}\left(\left|\nabla \times \boldsymbol{A}^{D C}\right|^{2}+\left|\nabla \times \boldsymbol{A}^{A C}\right|^{2}+2 \nabla \times \boldsymbol{A}^{D C} \cdot \nabla \times \boldsymbol{A}^{D C}\right) \boldsymbol{I}\right)$ which reduces to $\boldsymbol{\sigma}^{e}\left(\boldsymbol{A}^{D C}+\boldsymbol{A}^{A C}\right)=\boldsymbol{\sigma}^{e}\left(\boldsymbol{A}^{D C}\right)+\boldsymbol{\sigma}^{e}\left(\boldsymbol{A}^{A C}\right)+\mu^{-1} \mathcal{S}\left(\boldsymbol{A}^{D C}, \boldsymbol{A}^{A C}\right)$.

${ }^{5}$ Note that $\boldsymbol{\sigma}^{m}\left(\boldsymbol{u}^{D C}+\boldsymbol{u}^{A C}\right)=\boldsymbol{\sigma}^{m}\left(\boldsymbol{u}^{D C}\right)+\boldsymbol{\sigma}^{m}\left(\boldsymbol{u}^{A C}\right)$.
} 


$$
+\int_{\Omega_{c}} \rho \frac{\partial^{2} \boldsymbol{u}^{A C}}{\partial t^{2}} \cdot\left(\boldsymbol{u}^{D C}+\boldsymbol{u}^{A C}\right) \mathrm{d} \Omega=R_{u}
$$

$$
\begin{gathered}
\int_{\mathbb{R}^{3} \backslash \Omega_{c}}\left(\nabla\left(P^{D C}+P^{A C}\right)+\nabla \cdot \boldsymbol{\sigma}^{e}\left(\left(\boldsymbol{A}^{D C}+\boldsymbol{A}^{A C}\right)\right)\right) \cdot \nabla\left(P^{D C}+P^{A C}\right) \mathrm{d} \Omega \\
+\int_{\mathbb{R}^{3} \backslash \Omega_{c}} \frac{1}{c^{2}} \frac{\partial^{2} P^{A C}}{\partial t^{2}}\left(P^{D C}+P^{A C}\right) \mathrm{d} \Omega-\left.\int_{\partial \Omega_{c}^{N}} \rho^{+} \boldsymbol{n} \cdot \frac{\partial^{2} \boldsymbol{u}^{A C}}{\partial t^{2}}\right|_{\partial \Omega_{c}^{N}} ^{-}\left(P^{D C}+P^{A C}\right) \mathrm{d} S=R_{P} .
\end{gathered}
$$

For the static system in (6.10), substituting $\boldsymbol{A}^{\delta}=\left(\boldsymbol{A}^{D C}+\boldsymbol{A}^{A C}\right), \boldsymbol{u}^{\delta}=\left(\boldsymbol{u}^{D C}+\right.$ $\left.\boldsymbol{u}^{A C}\right), P^{\delta}=\left(P^{D C}+P^{A C}\right)$, the following is obtained

$$
\begin{array}{r}
\int_{\mathbb{R}^{3}} \mu^{-1} \nabla \times \boldsymbol{A}^{D C} \cdot \nabla \times\left(\boldsymbol{A}^{D C}+\boldsymbol{A}^{A C}\right) \mathrm{d} \Omega-\int_{\operatorname{supp}\left(\boldsymbol{J}^{s}\right)} \boldsymbol{J}^{D C} \cdot\left(\boldsymbol{A}^{D C}+\boldsymbol{A}^{A C}\right) \mathrm{d} \Omega=0 \\
\int_{\Omega_{c}}\left(\boldsymbol{\sigma}^{m}\left(\boldsymbol{u}^{D C}\right)+\boldsymbol{\sigma}^{e}\left(\boldsymbol{A}^{D C}\right)\right): \nabla\left(\boldsymbol{u}^{D C}+\boldsymbol{u}^{A C}\right) \mathrm{d} \Omega \\
-\left.\int_{\partial \Omega_{c}^{N}}\left(P^{D C} \boldsymbol{I}+\boldsymbol{\sigma}^{e}\left(\boldsymbol{A}^{D C}\right)\right)\right|_{\partial \Omega_{c}} ^{+} \boldsymbol{n} \cdot\left(\boldsymbol{u}^{D C}+\boldsymbol{u}^{A C}\right) \mathrm{d} S=0, \\
\int_{\mathbb{R}^{3} \backslash \Omega_{c}}\left(\nabla P^{D C}+\nabla \cdot \boldsymbol{\sigma}^{e}\left(\boldsymbol{A}^{D C}\right)\right) \cdot \nabla\left(P^{D C}+P^{A C}\right) \mathrm{d} \Omega=0,
\end{array}
$$

and for the "linearised" transient system in (6.10), again substituting $\boldsymbol{A}^{\delta}=\left(\boldsymbol{A}^{D C}+\right.$ $\left.\boldsymbol{A}^{A C}\right), \boldsymbol{u}^{\delta}=\left(\boldsymbol{u}^{D C}+\boldsymbol{u}^{A C}\right), P^{\delta}=\left(P^{D C}+P^{A C}\right)$, it follows that

$$
\begin{gathered}
\int_{\mathbb{R}^{3}} \mu^{-1} \nabla \times \boldsymbol{A}^{A C} \cdot \nabla \times\left(\boldsymbol{A}^{D C}+\boldsymbol{A}^{A C}\right) \mathrm{d} \Omega \\
-\int_{\Omega_{c}} \gamma \frac{\partial \boldsymbol{u}^{A C}}{\partial t} \times\left(\nabla \times \boldsymbol{A}^{D C}\right) \cdot\left(\boldsymbol{A}^{D C}+\boldsymbol{A}^{A C}\right) \mathrm{d} \Omega \\
+\int_{\Omega_{c}} \gamma \frac{\partial \boldsymbol{A}^{A C}}{\partial t} \cdot \boldsymbol{A}^{\delta} \mathrm{d} \Omega-\int_{\operatorname{supp}\left(\boldsymbol{J}^{s}\right)} \boldsymbol{J}^{A C} \cdot\left(\boldsymbol{A}^{D C}+\boldsymbol{A}^{A C}\right) \mathrm{d} \Omega=0 \\
\int_{\Omega_{c}}\left(\boldsymbol{\sigma}^{m}\left(\boldsymbol{u}^{A C}\right)+\mu^{-1} \mathcal{S}\left(\boldsymbol{A}^{D C}, \boldsymbol{A}^{A C}\right)\right): \nabla\left(\boldsymbol{u}^{D C}+\boldsymbol{u}^{A C}\right) \mathrm{d} \Omega \\
-\left.\int_{\partial \Omega_{c}^{N}}\left(P^{A C} \boldsymbol{I}+\mu^{-1} \mathcal{S}\left(\boldsymbol{A}^{D C}, \boldsymbol{A}^{A C}\right)\right)\right|_{\partial \Omega_{c}} ^{+} \boldsymbol{n} \cdot\left(\boldsymbol{u}^{D C}+\boldsymbol{u}^{A C}\right) \mathrm{d} S \\
+\int_{\Omega_{c}} \rho \frac{\partial^{2} \boldsymbol{u}^{A C}}{\partial t^{2}} \cdot\left(\boldsymbol{u}^{D C}+\boldsymbol{u}^{A C}\right) \mathrm{d} \Omega=0,
\end{gathered}
$$


$\int_{\mathbb{R}^{3} \backslash \Omega_{c}}\left(\nabla P^{A C}+\nabla \cdot \boldsymbol{\sigma}^{e}\left(\boldsymbol{A}^{A C}\right)\right) \cdot \nabla\left(P^{D C}+P^{A C}\right) \mathrm{d} \Omega$
$+\int_{\mathbb{R}^{3} \backslash \Omega_{c}} \frac{1}{c^{2}} \frac{\partial^{2} P^{A C}}{\partial t^{2}}\left(P^{D C}+P^{A C}\right) \mathrm{d} \Omega-\left.\int_{\partial \Omega_{c}^{N}} \rho^{+} \boldsymbol{n} \cdot \frac{\partial^{2} \boldsymbol{u}^{A C}}{\partial t^{2}}\right|_{\partial \Omega_{c}^{N}} ^{-}\left(P^{D C}+P^{A C}\right) \mathrm{d} S=0$.

Now, by using (6.13) and (6.14) in (6.12), it follows that

$$
\begin{aligned}
& R_{A}=-\int_{\Omega_{c}} \gamma \frac{\partial \boldsymbol{u}^{A C}}{\partial t} \times\left(\nabla \times\left(\boldsymbol{A}^{A C}\right)\right) \cdot\left(\boldsymbol{A}^{D C}+\boldsymbol{A}^{A C}\right) \mathrm{d} \Omega, \\
& R_{u}=\int_{\Omega_{c}} \boldsymbol{\sigma}^{e}\left(\left(\boldsymbol{A}^{A C}\right)\right): \nabla\left(\boldsymbol{u}^{D C}+\boldsymbol{u}^{A C}\right) \mathrm{d} \Omega-\left.\int_{\partial \Omega_{c}^{N}} \boldsymbol{\sigma}^{e}\left(\boldsymbol{A}^{A C}\right)\right|_{\partial \Omega_{c}} ^{+} \boldsymbol{n} \cdot\left(\boldsymbol{u}^{D C}+\boldsymbol{u}^{A C}\right) \mathrm{d} S, \\
& R_{P}=-\int_{\operatorname{supp}\left(\boldsymbol{J}^{A C}\right)} \nabla \times \boldsymbol{A}^{A C} \times \boldsymbol{J}^{A C} \cdot \nabla\left(P^{D C}+P^{A C}\right) .
\end{aligned}
$$

For the linearised approach to accurately describe the solution of the non-linear problem, the residuals $R_{A}, R_{u}$ and $R_{P}$ need to be small. A series of bounds are now derived to relate these residuals to practical quantities of interest.

\section{$R_{A}$ Term}

Looking first at the residual of the magnetic problem in (6.15a), taking the magnitude of this term and applying the Cauchy-Schwartz inequality results in the following bound

$$
\begin{aligned}
\left|R_{A}\right| & :=\left|\int_{\Omega_{c}} \gamma \frac{\partial \boldsymbol{u}^{A C}}{\partial t} \times\left(\nabla \times\left(\boldsymbol{A}^{A C}\right)\right) \cdot\left(\boldsymbol{A}^{D C}+\boldsymbol{A}^{A C}\right) \mathrm{d} \Omega\right| \\
& \leq\left(\int_{\Omega_{c}}\left|\gamma \frac{\partial \boldsymbol{u}^{A C}}{\partial t}\right|^{2} \mathrm{~d} \Omega\right)^{\frac{1}{2}}\left(\int_{\Omega_{c}}\left|\nabla \times \boldsymbol{A}^{A C}\right|^{2} \mathrm{~d} \Omega\right)^{\frac{1}{2}}\left(\int_{\Omega_{c}}\left|\boldsymbol{A}^{D C}+\boldsymbol{A}^{A C}\right|^{2} \mathrm{~d} \Omega\right)^{\frac{1}{2}} \\
& \leq\left\|\gamma \frac{\partial \boldsymbol{u}^{A C}}{\partial t}\right\|_{L^{2}\left(\Omega_{c}\right)}\left\|\nabla \times \boldsymbol{A}^{A C}\right\|_{L^{2}\left(\Omega_{c}\right)}\left(\left\|\boldsymbol{A}^{D C}\right\|_{L^{2}\left(\Omega_{c}\right)}+\left\|\boldsymbol{A}^{A C}\right\|_{L^{2}\left(\Omega_{c}\right)}\right)
\end{aligned}
$$

In the above, the first step was to apply the magnitude to the residual term. From this, the second step was to bound the magnitude by the $L^{2}\left(\Omega_{c}\right)$ norm and the third step applied the triangle inequality. From [178], it can be shown that the $L^{2}$-norm of the magnetic vector potential is bounded by its curl as

$$
\left\|\boldsymbol{A}^{A C}\right\|_{L^{2}\left(\Omega_{c}\right)} \leq C\left\|\nabla \times \boldsymbol{A}^{A C}\right\|_{L^{2}\left(\Omega_{c}\right)}
$$


The $L^{2}$-norm can be bounded by the $\|\cdot\|_{L^{2}\left(\Omega_{c}\right)} \leq\left|\Omega_{c}\right|\|\cdot\|_{L^{\infty}\left(\Omega_{c}\right)}$, where $\left|\Omega_{c}\right|$ defines the size of the domain $\Omega_{c}$. Combining this with (6.16) and (6.17) results in

$$
\begin{aligned}
\left|R_{A}\right| \leq C\left\|\gamma \frac{\partial \boldsymbol{u}^{A C}}{\partial t}\right\|_{L^{\infty}\left(\Omega_{c}\right)}\left\|\nabla \times \boldsymbol{A}^{A C}\right\|_{L^{\infty}\left(\Omega_{c}\right)}\left(\left\|\nabla \times \boldsymbol{A}^{D C}\right\|_{L^{\infty}\left(\Omega_{c}\right)}+\right. \\
\left.\left\|\nabla \times \boldsymbol{A}^{A C}\right\|_{L^{\infty}\left(\Omega_{c}\right)}\right) .
\end{aligned}
$$

Provided that $\left\|\nabla \times \boldsymbol{A}^{A C}\right\|_{L^{\infty}\left(\Omega_{c}\right)} \ll\left\|\nabla \times \boldsymbol{A}^{D C}\right\|_{L^{\infty}\left(\Omega_{c}\right)}$, or in other terms $\left\|\boldsymbol{B}^{A C}\right\|_{L^{\infty}\left(\Omega_{c}\right)} \ll\left\|\boldsymbol{B}^{D C}\right\|_{L^{\infty}\left(\Omega_{c}\right)}$, the magnetic residual may be bounded by

$$
\left|R_{A}\right| \leq C\left\|\gamma \frac{\partial \boldsymbol{u}^{A C}}{\partial t}\right\|_{L^{\infty}\left(\Omega_{c}\right)}\left\|\boldsymbol{B}^{D C}\right\|_{L^{\infty}\left(\Omega_{c}\right)}^{2}
$$

where $C$ depends on the size of the domain $\Omega_{c}$.

\section{$R_{u}$ Term}

Looking now at the residual of the mechanical problem in (6.15b), taking the magnitude of this term and applying the Cauchy-Schwartz inequality results in the following bound

$$
\begin{aligned}
\left|R_{u}\right|:= & \left|\int_{\Omega_{c}} \boldsymbol{\sigma}^{e}\left(\left(\boldsymbol{A}^{A C}\right)\right): \nabla\left(\boldsymbol{u}^{D C}+\boldsymbol{u}^{A C}\right) \mathrm{d} \Omega-\int_{\partial \Omega_{c}^{N}} \boldsymbol{\sigma}^{e}\left(\boldsymbol{A}^{A C}\right)\right|_{\partial \Omega_{c}}^{+} \boldsymbol{n} \cdot\left(\boldsymbol{u}^{D C}+\boldsymbol{u}^{A C}\right) \mathrm{d} S \mid \\
\leq & \left\|\nabla \times \boldsymbol{A}^{A C}\right\|_{L^{2}\left(\Omega_{c}\right)}^{2}\left(\left\|\nabla \boldsymbol{u}^{D C}\right\|_{L^{2}\left(\Omega_{c}\right)}+\left\|\nabla \boldsymbol{u}^{A C}\right\|_{L^{2}\left(\Omega_{c}\right)}\right) \\
& +\left\|\nabla \times \boldsymbol{A}^{A C}\right\|_{L^{2}\left(\partial \Omega_{c}^{N}\right)}^{2}\left(\left\|\boldsymbol{u}^{D C}\right\|_{L^{2}\left(\partial \Omega_{c}^{N}\right)}+\left\|\boldsymbol{u}^{A C}\right\|_{L^{2}\left(\partial \Omega_{c}^{N}\right)}\right) \\
\leq & C\left\|\nabla \times \boldsymbol{A}^{A C}\right\|_{L^{\infty}\left(\Omega_{c}\right)}^{2}\left(\left\|\nabla \boldsymbol{u}^{D C}\right\|_{L^{\infty}\left(\Omega_{c}\right)}+\left\|\nabla \boldsymbol{u}^{A C}\right\|_{L^{\infty}\left(\Omega_{c}\right)}\right) .
\end{aligned}
$$

In the above, the first step was to apply the magnitude to the residual term. From this, the second step was to bound the magnitude by the $L^{2}\left(\Omega_{c}\right)$ norm and apply the triangle inequality. Again, bounding the $L^{2}$-norm by the $L^{\infty}$-norm, the residual term in (6.20) can be bounded as

$$
\begin{aligned}
\left|R_{u}\right| \leq & C\left\|\boldsymbol{B}^{A C}\right\|_{L^{\infty}\left(\Omega_{c}\right)}^{2}\left(\left\|\nabla \boldsymbol{u}^{D C}\right\|_{L^{\infty}\left(\Omega_{c}\right)}+\left\|\nabla \boldsymbol{u}^{A C}\right\|_{L^{\infty}\left(\Omega_{c}\right)}\right) \\
& +C\left\|\boldsymbol{B}^{A C}\right\|_{L^{\infty}\left(\partial \Omega_{c}^{N}\right)}^{2}\left(\left\|\boldsymbol{u}^{D C}\right\|_{L^{\infty}\left(\partial \Omega_{c}^{N}\right)}+\left\|\boldsymbol{u}^{A C}\right\|_{L^{\infty}\left(\partial \Omega_{c}^{N}\right)}\right)
\end{aligned}
$$


where $C$ depends on the size of $\Omega_{c}$ and $\partial \Omega_{c}^{N}$.

\section{$R_{P}$ Term}

Finally, looking at the residual of the acoustic problem in $(6.15 \mathrm{c})$, taking the magnitude of this term and applying the Cauchy-Schwartz inequality results in the following bound

$$
\begin{aligned}
\left|R_{P}\right| & :=\left|\int_{\operatorname{supp}\left(\boldsymbol{J}^{A C}\right)} \nabla \times \boldsymbol{A}^{A C} \times \boldsymbol{J}^{A C} \cdot \nabla\left(P^{D C}+P^{A C}\right)\right| \\
& \leq\left\|\nabla \times \boldsymbol{A}^{A C}\right\|_{L^{2}\left(\operatorname{supp}\left(\boldsymbol{J}^{A C}\right)\right)}\left\|\boldsymbol{J}^{A C}\right\|_{L^{2}\left(\operatorname{supp}\left(\boldsymbol{J}^{A C}\right)\right)}\left(\left\|\nabla P^{D C}\right\|_{L^{2}\left(\operatorname{supp}\left(\boldsymbol{J}^{A C}\right)\right)}+\right. \\
& \left.\left\|\nabla P^{A C}\right\|_{L^{2}\left(\operatorname{supp}\left(\boldsymbol{J}^{A C}\right)\right)}\right) .
\end{aligned}
$$

In the above, the first step was to apply the magnitude to the residual term. From this, the second step was to bound the magnitude by the $L^{2}\left(\Omega_{c}\right)$ norm and apply the triangle inequality. From the above, the curl of the vector potential can be expressed as $\left\|\nabla \times \boldsymbol{A}^{A C}\right\|_{L^{2}\left(\operatorname{supp}\left(\boldsymbol{J}^{A C}\right)\right)}=\mu_{0}\left\|\boldsymbol{H}^{A C}\right\|_{L^{2}\left(\operatorname{supp}\left(\boldsymbol{J}^{A C}\right)\right)}$ and the bound in (6.22) becomes

$$
\begin{array}{r}
\left|R_{P}\right| \leq \mu_{0}\left\|\boldsymbol{H}^{A C}\right\|_{L^{2}\left(\operatorname{supp}\left(\boldsymbol{J}^{A C}\right)\right)}\left\|\boldsymbol{J}^{A C}\right\|_{L^{2}\left(\operatorname{supp}\left(\boldsymbol{J}^{A C}\right)\right)}\left(\left\|\nabla P^{D C}\right\|_{L^{2}\left(\operatorname{supp}\left(\boldsymbol{J}^{A C}\right)\right)}+\right. \\
\left.\left\|\nabla P^{A C}\right\|_{L^{2}\left(\operatorname{supp}\left(\boldsymbol{J}^{A C}\right)\right)}\right) .
\end{array}
$$

Now expressing the $L^{2}$-norm in terms of the $L^{\infty}$-norm the pressure residual is bounded by

$$
\begin{array}{r}
\left|R_{P}\right| \leq C \mu_{0}\left\|\boldsymbol{H}^{A C}\right\|_{L^{\infty}\left(\operatorname{supp}\left(\boldsymbol{J}^{A C}\right)\right)}\left\|\boldsymbol{J}^{A C}\right\|_{L^{\infty}\left(\operatorname{supp}\left(\boldsymbol{J}^{A C}\right)\right)}\left(\left\|\nabla P^{D C}\right\|_{L^{\infty}\left(\operatorname{supp}\left(\boldsymbol{J}^{A C}\right)\right)}+\right. \\
\left.\left\|\nabla P^{A C}\right\|_{L^{\infty}\left(\operatorname{supp}\left(\boldsymbol{J}^{A C}\right)\right)}\right)
\end{array}
$$

where $C$ depends on the size of the gradient coils supp $\left(\boldsymbol{J}^{A C}\right)$. 


\subsubsection{Ratio of the Magnetic Field Strengths}

In summary, it becomes clear that the linearised approach is expected to offer good agreement with the non-linear approach provided that the following criteria are met:

1) $\left\|\gamma \partial \boldsymbol{u}^{A C} / \partial t\right\|_{L^{\infty}\left(\Omega_{c}\right)}\left\|\boldsymbol{B}^{D C}\right\|_{L^{\infty}\left(\Omega_{c}\right)}^{2} \ll 1$,

2) $\left\|\boldsymbol{B}^{A C}\right\|_{L^{\infty}\left(\Omega_{c}\right)}^{2}\|\nabla \boldsymbol{u}\|_{L^{\infty}\left(\Omega_{c}\right)} \ll 1,\left\|\boldsymbol{B}^{A C}\right\|_{L^{\infty}\left(\Omega_{c}\right)}^{2}\|\boldsymbol{u}\|_{L^{\infty}\left(\Omega_{c}\right)} \ll 1$,

3) $\mu_{0}\left\|\boldsymbol{H}^{A C}\right\|_{L^{\infty}\left(\operatorname{supp}\left(\boldsymbol{J}^{A C}\right)\right)}\left\|\boldsymbol{J}^{A C}\right\|_{L^{\infty}\left(\operatorname{supp}\left(\boldsymbol{J}^{A C}\right)\right)}\|\nabla P\|_{L^{\infty}\left(\operatorname{supp}\left(\boldsymbol{J}^{A C}\right)\right)} \ll 1$,

4) $\left\|\boldsymbol{B}^{A C}\right\|_{L^{\infty}\left(\Omega_{c}\right)} /\left\|\boldsymbol{B}^{D C}\right\|_{L^{\infty}\left(\Omega_{c}\right)} \ll 1$,

In the case of 1) small velocities in comparison to $\gamma$ and $\left\|\boldsymbol{B}^{D C}\right\|_{L^{\infty}\left(\Omega_{c}\right)}^{2}$ are required for the linearised approach to be applicable, which is typically the case for MRI scanners. For 2) small displacements and strains (displacement gradients) in comparison to $\left\|\boldsymbol{B}^{A C}\right\|_{L^{\infty}\left(\Omega_{c}\right)}^{2}$ and $\left\|\boldsymbol{B}^{A C}\right\|_{L^{\infty}\left(\partial \Omega_{c}^{N}\right)}^{2}$ are required for the linearised approach to be applicable, which is typically the case for MRI scanners. For 3) small pressure gradients in the coils are required for the linearised approach to be applicable, which is typically the case for MRI scanners under the Biot-Savart coil assumption. When modelling the coils as conductors this term drops from the weak form all together and so does not appear. For 4) the $\boldsymbol{B}^{D C}$ field is driven by the strong main magnet and consequently $\left\|\boldsymbol{B}^{D C}\right\|_{L^{\infty}(\Omega)}$ is orders of magnitude larger than $\left\|\boldsymbol{B}^{A C}\right\|_{L^{\infty}(\Omega)}$, which results from the AC coils and field perturbations caused by eddy and Lorentz currents in the conductors.

It is clear that the ratio $\left\|\boldsymbol{J}^{A C}\right\|_{L^{\infty}\left(\Omega_{c}\right)} /\left\|\boldsymbol{J}^{D C}\right\|_{L^{\infty}\left(\Omega_{c}\right)} \ll 1$ is a direct consequence of condition 4 ), and vice versa, and so must also hold. MRI coils are designed such that the current strengths are fairly uniform over the main magnet and gradient coils [140]. Thus $\left\|\boldsymbol{J}^{D C}\right\|_{L^{\infty}\left(\operatorname{supp}\left(\boldsymbol{J}^{s}\right)\right)}=\left|\boldsymbol{J}^{D C}\right|,\left\|\boldsymbol{J}^{A C}\right\|_{L^{\infty}\left(\operatorname{supp}\left(\boldsymbol{J}^{s}\right)\right)}=\left|\boldsymbol{J}^{A C}\right|$ and, in current MRI applications $\left|\boldsymbol{J}^{A C}\right|<\left|\boldsymbol{J}^{D C}\right|$. Thus, provided the velocities of the conductors are small the linearised approach is expected to give good agreement with the non-linear approach.

\subsubsection{Simple Model Relating Field and Current Strengths}

Whilst the true magnetic field strength $\boldsymbol{B}=\mu \boldsymbol{H}$ can only be found after solving (2.36) by means of the non-linear or linearised approaches, described in this Chapter, there is merit in also considering a simple model to relate $\boldsymbol{B}$ to the applied current density in the coils. This is because manufacturer's data, available for MRI scanners, is typically only quoted in terms of the maximum capable main and gradient field 
strengths at the central axis of the imaging bore [196, 229, 228]. Therefore, in order to determine the operating ranges of the current densities on in-use scanners, the relationships given by this simplified model offer useful insight.

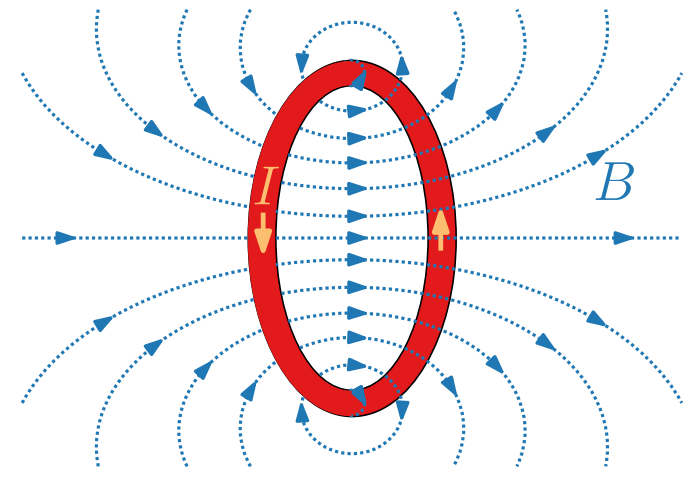

(a) Magnetic Field around single current loop

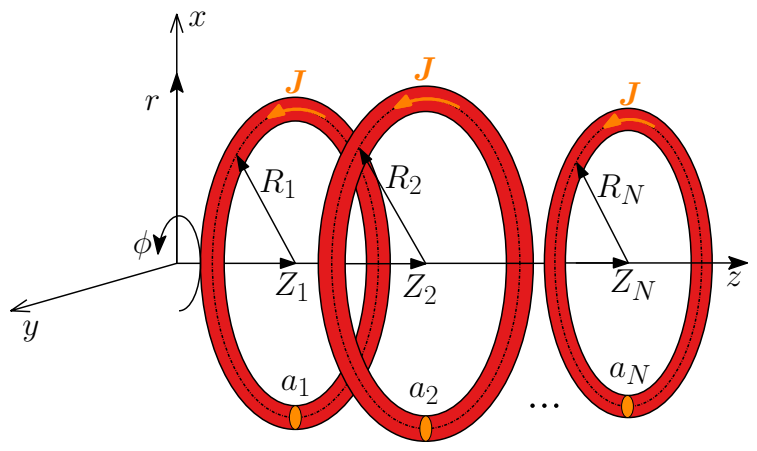

(b) Multiple coil configuration

Figure 6.2: Single current loop, representing a lumped mass of coils.

In the simplified model, shown in Figure 6.2, all transient, eddy current and coupling effects are neglected, as is the mutual inductance between coils. Coil $i$ has a constant cross sectional area $a_{i}$, is circular and hence rotationally symmetric and so is best expressed in terms of cylindrical coordinates $(r, \phi, z)$ and carries a uniform current density $\boldsymbol{J}^{s}=J_{\phi}^{s} e_{\phi}$. Then, by an additive application of the Biot-Savart law for a single coil [100], the following relationship can be derived for the field strength along the axis $r=0$ for $N$ coils placed at different locations $\left(R_{i}, Z_{i}\right)$

$$
\left|\boldsymbol{J}^{s}\right|=\sum_{i}^{N} \frac{\left(\left(z-Z_{i}\right)^{2}+R_{i}^{2}\right)^{3 / 2}}{R_{i} a_{i}}|\boldsymbol{B}(0, \phi, z)|
$$

where $\boldsymbol{B}(0, \phi, z)=B_{z}(r=0, z) \boldsymbol{e}_{z}$ along this axis, $R_{i}$ is the radius and $Z_{i}$ the axial position of the $i^{t h}$ current source relative to the scanner's central axes. Provided the aforementioned assumptions are enforced, this relationship can be applied to obtain the current densities that are associated only with the main magnet $\left(\boldsymbol{J}^{s}=\boldsymbol{J}^{D C}\right)$ or with the gradient coil $\left(\boldsymbol{J}^{S}=\boldsymbol{J}^{A C}\right)$ from their produced field strengths. In the context of Section 6.5.2, it also provides a guide as to the ranges of ratios of $\left|\boldsymbol{J}^{A C}\right| /\left|\boldsymbol{J}^{D C}\right|$ and $\left\|\boldsymbol{B}^{A C}\right\|_{L^{\infty}(\Omega)} /\left\|\boldsymbol{B}^{D C}\right\|_{L^{\infty}(\Omega)}$ over which the linearised approach is applicable.

In Chapter 8, this simplified model is revisited for each of the industrially relevant numerical examples, where the results of this simplified model to the corresponding problems are presented and the ranges of the ratios 2) and 3) that MRI scanners operate within are shown. 


\subsection{Chapter Summary}

This Chapter has presented the treatment of the novel transient non-linear coupled acousto-magneto-mechanical system, presented in Chapter 2 through a complete linearisation, named the non-linear approach. The linearised and non-linear approaches have been recast in terms of the associated mass, damping and stiffness contributions to the system to allow for the natural treatment of the system through temporal integration, discussed in Chapter 7.

A novel comparison between the energy associated with both the linearised and non-linear approaches has been performed, where a set of bounds have been rigorously derived to obtain measures for determining the non-linearity of the problem. A simple analytical model has also been presented to relate these measures to industrial data, where these measures can be used to determine the non-linearity of the coupled MRI problem across the operable conditions of MRI scanners. Ultimately, these measures will be used to determine the applicability of the linearised approach across the operating conditions of MRI scanners.

From the objectives of this Thesis, discussed in section 1.4, the topics discussed in this Chapter align with the objectives: "To develop the complete non-linear approach to solving the coupled system of equations" and "To determine a series of theoretical measures that can be used to measure the non-linearity of the coupled problem in MRI scanners."

In the next Chapter, the numerical discretisation of the transient coupled system of equations for both the linearised and non-linear approaches is discussed. The spatial discretisation of the system, previously carried out in Chapter 4, is briefly revisited before the temporal discretisation of the system is introduced. 



\section{Chapter 7}

\section{Computational Treatment of the Non Linear Approach}

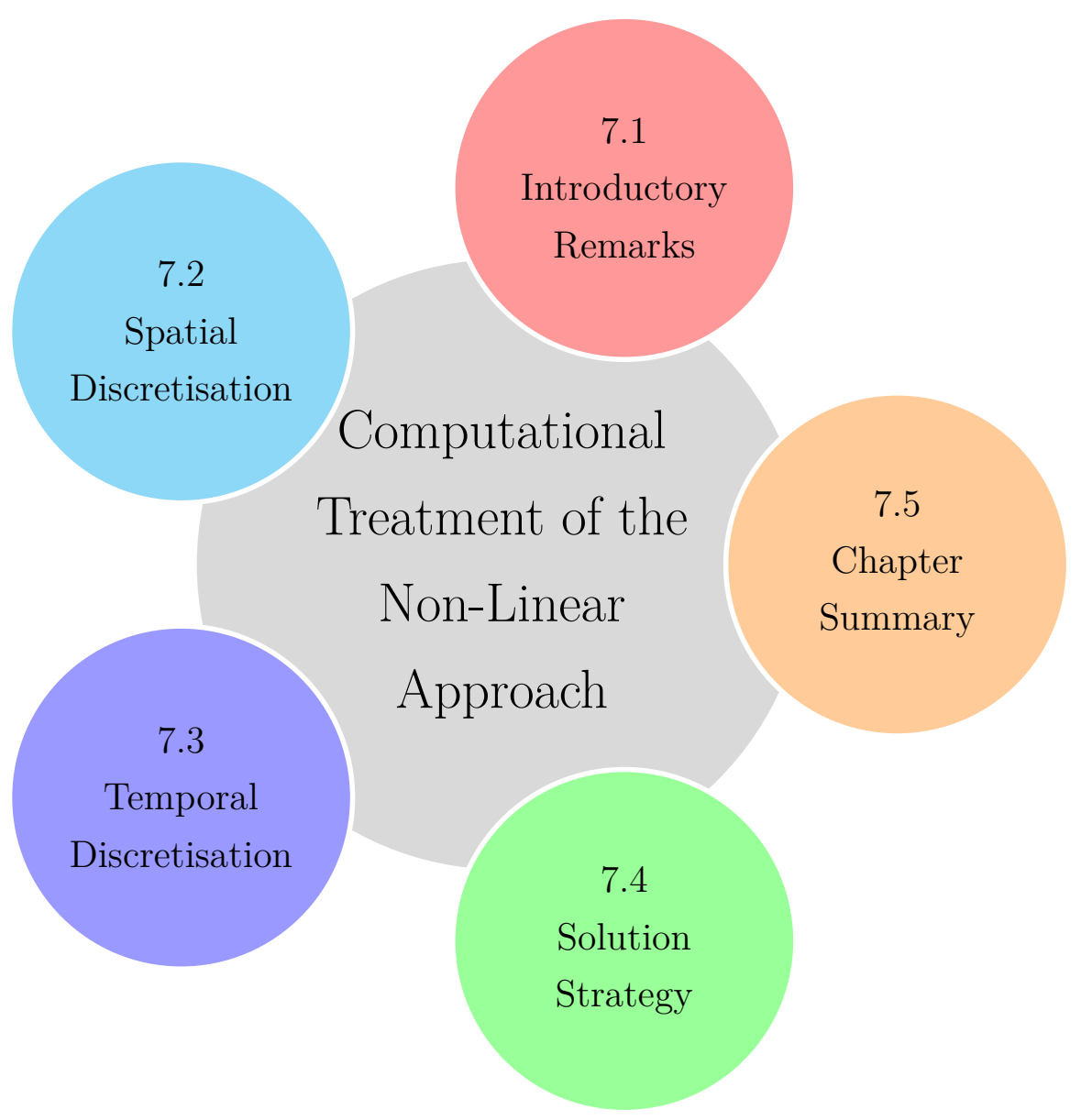

"Aim for the sky to reach the tree tops" 


\subsection{Introductory Remarks}

This Chapter describes the temporal discretisation of the novel acousto-magnetomechanical coupled system for both the linearised and non-linear approaches. In Part III of this Thesis, the linearised approach was presented in a time harmonic representation. In order to solve the fully non-linear system the temporal dependence of the fields must be fully resolved, which requires the use of a temporal discretisation scheme. Both approaches are fully discretised through a $h p$-finite element spatial discretisation, as discussed in Chapter 4, and an $\alpha$-type time integration scheme is used to provide a temporal discretisation. In doing so, the two approaches can be compared directly in the time domain to determine the linearity of the MRI problem and test the ratios within MRI operating conditions, presented in Chapter 6.

From the objectives of this Thesis, discussed in section 1.4, the temporally discrete description of the linearised approach can be used to compare against the non-linear approach to determine the validity of the system and aligns with the objective; "To provide an efficient computational methodology for handling the solution to the coupled problem of MRI scanners using both approaches." The contents of this Chapter builds on the work carried out in the submitted paper [25], where the discussion of the technical details has been extended.

This Chapter begins with a brief recollection of the spatial discretisation in a $h p$-finite element sense and presents both the linearised and non-linear approaches as coupled discrete second order ODEs in Section 7.2. Section 7.3 then presents the temporal discretisation of both approaches that permit solutions in the time domain. The solution strategy for the temporal non-linear approach is then summarised in Section 7.4. The contents of this Chapter are then summarised in Section 7.5.

\subsection{Spatial Discretisation}

The spatial discretisation of the linearised system of coupled acousto-magnetomechanical equations in (6.3) is extensively covered in Chapter 4 of this Thesis, where the treatment of the axisymmetric system and far field conditions are also presented. This approach permits refinement of both the mesh spacing and the order of the elements. This has been shown to be an effective approach, which leads to accurate solutions, for benchmark eddy current, mechanical and acoustic problems as well as fully coupled MRI configurations, as shown previously in Chapter 5. 
In this Chapter, the same spatial discretisation as in Chapter 4 is applied, where the spatially discrete system of second order ODEs obtained for the non-linear approach can be expressed as the Newton-Raphson iteration: Find $\boldsymbol{\delta}_{q}^{[\mathrm{k}]} \in \mathbb{R}^{4 N}$ at each time $t \in(0, T]$ such that ${ }^{1}$

$$
\begin{aligned}
\mathbf{M} \ddot{\boldsymbol{\delta}}_{\mathrm{q}}{ }^{[\mathrm{k}]}+\mathbf{C}^{[\mathrm{k}]} \dot{\boldsymbol{\delta}}_{\mathrm{q}}{ }^{[\mathrm{k}]}+\mathbf{K}^{[\mathrm{k}]} \boldsymbol{\delta}_{\mathrm{q}}^{[\mathrm{k}]} & =-\mathbf{R}_{\mathrm{q}}^{[\mathrm{k}]}, \\
\mathbf{q}^{[\mathrm{k}+1]} & =\mathbf{q}^{[\mathrm{k}]}+\boldsymbol{\delta}_{\mathrm{q}}^{[\mathrm{k}]},
\end{aligned}
$$

where $\boldsymbol{\delta}_{\mathrm{q}}^{[\mathrm{k}]}$ and $\mathbf{q}^{[\mathrm{k}]}$ are spatially discrete forms of the continuous variables $\boldsymbol{\delta}_{q}^{[\mathrm{k}]}$ and $\boldsymbol{q}^{[\mathrm{k}]}$ and $\mathbf{M}, \mathbf{C}^{[\mathrm{k}]}, \mathbf{K}^{[\mathrm{k}]}$ and $\mathbf{R}_{\mathrm{q}}^{[\mathrm{k}]}$ are the discrete linearised mass, damping and stiffness matrices and residual vector, respectively. These are obtained by discretising the terms in (6.5) and depend, with the exception of the mass matrix, on the solution at iteration $[\mathrm{k}]$. The partial time derivatives of the solution vectors become ordinary time derivatives due to their independence of spatial coordinates.

In a similar manner, the linearised approach takes the form: Find $\boldsymbol{\delta}_{q}^{[\mathrm{k}]} \in \mathbb{R}^{4 N}$ at each time $t \in(0, T]$ such that

$$
\tilde{\mathbf{M}} \ddot{\boldsymbol{\delta}}_{\mathrm{q}}+\tilde{\mathbf{C}} \dot{\boldsymbol{\delta}}_{\mathrm{q}}+\tilde{\mathbf{K}} \boldsymbol{\delta}_{\mathrm{q}}=-\tilde{\mathbf{R}}_{\mathrm{q}}
$$

where $\mathbf{q}^{D C}$ is the vector of discrete solutions to the static problem and $\tilde{\mathbf{M}}, \tilde{\mathbf{C}}, \tilde{\mathbf{K}}$ and $\tilde{\mathbf{R}}_{\mathrm{q}}$ are the discrete mass, damping and stiffness matrices and the residual vector, respectively, obtained by discretising the terms in (6.8). In this case, the NewtonRaphson procedure would converge in a single iteration and hence, in principle, no further iteration is required.

\subsection{Temporal Discretisation}

In order to solve the spatially discrete system for both the linearised and non-linear approaches in time domain, presented in (7.1) and (7.2), the fields must be solved at each time instance. In order to resolve the transient components of the fields a time integration scheme can be used. This Section discusses a series of possible time integration schemes, before presenting the fully discrete linearised and non-linear approaches for the chosen scheme.

\footnotetext{
${ }^{1}$ Here the spatial discretisation consists of $\mathrm{N}$ basis functions with same order elements employed for each field. There are $4 \mathrm{~N}$ unknowns since, associated with basis functions, the degrees of freedom for $A_{\phi}, \tilde{u}=\left\{\tilde{u}_{r} u_{z}\right\}^{T}$ and $P$, in the axisymmetric case, see [26] for details.
} 


\subsubsection{Time Integration Schemes}

There are a considerable number of alternative time integration techniques that could be employed for the temporal integration of (7.1) and (7.2), such as Euler schemes [161], trapezoidal schemes [205], and the Newmark method [20]. In this Thesis, a second order generalised- $\alpha$ scheme will be adopted, see [57], to discretise the system of equations. This scheme is designed to allow for tailor-made numerical dissipation in the solution and is of sufficient degree, given the second order nature of (7.1) and (7.2). Given the complexity of the coupled system, it is difficult to know the true initial conditions for the full transient problem, when applying a forced excitation. The numerical dissipation of the generalised- $\alpha$ scheme is therefore beneficial as it can be used to damp out any artificial frequencies that pollute the solution.

Transient eddy current problems often require solutions to configurations consisting of high conductivity and relatively small frequencies, as discussed in Chapter 2. This typically results in very small time steps to provide stable solutions for explicit solvers $[79,71,186]$, which has resulted in much research utilising implicit approaches [202, 58, 73, 214, 185], as the solutions are unconditionally stable. Furthermore, in the context of this Thesis, the aim is to create a solver for non-linear coupled problems, where explicit solvers cannot guarantee stability. Therefore, in the interest of maintaining stable and accurate solutions, an implicit time integration scheme is adopted as these are typically more robust than explicit time integrators. This is due to less restrictive time stability constraints.

There exists also a number of high order integration schemes, such as backward differentiation formulas [42], explicit Runge-Kutta and leap-frog type methods $[250,237]$, that allow for higher order accuracies in time and larger time steps, although with increased temporal accuracy comes a significant increase in computational cost. However, for the coupled problems presented in this Thesis, the required timestep size is dictated by the need to capture the excitation frequency and resonant frequencies of the system (typically in the region of $5000 \mathrm{~Hz}$ ). For this reason, the need for higher order accuracy is outweighed by the requirement of smaller timestep sizes and thus the second order accurate generalised- $\alpha$ scheme appears a sensible choice.

Whilst this work could look into motivating a high order time integration scheme to further increase the accuracy of the temporal solutions, the main purpose of implementing a transient solver in this Thesis is to provide sufficiently accurate 
solutions to validate the reliability of the linearised approach ${ }^{2}$. This validation is performed on a series of industrially relevant examples in Chapter 8 by comparing the transient response of the fields using the linearised approach versus the non-linear approach, as the non linear system can only be solved in the time domain.

\subsubsection{Generalised- $\alpha$ Time Integration Scheme ( $2^{\text {nd }}$ Order)}

The focus of this Section lies in the development of the temporal discretisation of (7.1), which can similarly be developed for (7.2). The implicit form of this scheme evaluates the system of non-linear equations in (7.1) at an intermediate time step $t_{n+1-\alpha_{f}}$ as

$$
\left.\left.\mathbf{M} \ddot{\boldsymbol{\delta}}_{\mathrm{q}_{n+1-\alpha_{m}}^{[\mathrm{k}]}}^{[\mathrm{C}}\right|_{t_{n+1-\alpha_{f}}} ^{[\mathrm{k}]} \dot{\boldsymbol{\delta}}_{\mathrm{q}_{n+1-\alpha_{f}}^{[\mathrm{k}]}}^{[\mathbf{K}}\right|_{t_{n+1-\alpha_{f}}} ^{[\mathrm{k}]} \boldsymbol{\delta}_{\mathrm{q}_{n+1-\alpha_{f}}^{[\mathrm{k}]}}^{\left[{ }^{2}\right.}=-\left.\mathbf{R}_{\mathrm{q}}\right|_{t_{n+1-\alpha_{f}}} ^{[\mathrm{k}]},
$$

where $\left.\mathbf{C}\right|_{t_{n+1-\alpha_{f}}} ^{[\mathrm{k}]},\left.\mathbf{K}\right|_{t_{n+1-\alpha_{f}}} ^{[\mathrm{k}]}$ and $\left.\mathbf{R}_{\mathrm{q}}\right|_{t_{n+1-\alpha_{f}}} ^{[\mathrm{k}]}$ are evaluated at the intermediate timestep $t_{n+1-\alpha_{f}}$ and the values of the fields at this timestep are given by

$$
\begin{aligned}
\ddot{\mathbf{q}}_{n+1-\alpha_{m}}^{[\mathrm{k}]} & =\left(1-\alpha_{m}\right) \ddot{\mathbf{q}}_{n+1}^{[\mathrm{k}]}+\alpha_{m} \ddot{\mathbf{q}}_{n}, \\
\dot{\mathbf{q}}_{n+1-\alpha_{f}}^{[\mathrm{k}]} & =\left(1-\alpha_{f}\right) \dot{\mathbf{q}}_{n+1}^{[\mathrm{k}]}+\alpha_{f} \dot{\mathbf{q}}_{n}, \\
\mathbf{q}_{n+1-\alpha_{f}}^{[\mathrm{k}]} & =\left(1-\alpha_{f}\right) \mathbf{q}_{n+1}^{[\mathrm{k}]}+\alpha_{f} \mathbf{q}_{n}, \\
t_{n+1-\alpha_{f}} & =\left(1-\alpha_{f}\right) t_{n+1}+\alpha_{f} t_{n} .
\end{aligned}
$$

The generalised- $\alpha$ scheme, described in [57], expresses $\mathbf{q}_{n+1}^{[\mathrm{k}]}$ and $\dot{\mathbf{q}}_{n+1}^{[\mathrm{k}]}$ in terms of $\ddot{\mathbf{q}}_{n+1}^{[\mathrm{k}]}$, known as an acceleration based formulation. This can be manipulated into a displacement based formulation, where $\dot{\mathbf{q}}_{n+1}^{[\mathrm{k}]}$ and $\ddot{\mathbf{q}}_{n+1}^{[\mathrm{k}]}$ are expressed in terms of $\mathbf{q}_{n+1}^{[\mathrm{k}]}$, as

$$
\begin{aligned}
& \ddot{\mathbf{q}}_{n+1}^{[\mathrm{k}]}=\frac{1}{\beta}\left(\frac{\mathbf{q}_{n+1}^{[\mathrm{k}]}-\mathbf{q}_{n}}{\Delta t^{2}}-\frac{\dot{\mathbf{q}}_{n}}{\Delta t}-\left(\frac{1}{2}-\beta\right) \ddot{\mathbf{q}}_{n}\right), \\
& \dot{\mathbf{q}}_{n+1}^{[\mathrm{k}]}=\frac{\varsigma}{\beta}\left(\frac{\mathbf{q}_{n+1}^{[\mathrm{k}]}-\mathbf{q}_{n}}{\Delta t}+\left(\frac{\beta}{\varsigma}-1\right) \dot{\mathbf{q}}_{n}+\left(\frac{\beta}{\varsigma}-\frac{1}{2}\right) \Delta t \ddot{\mathbf{q}}_{n}\right),
\end{aligned}
$$

where $\varsigma:=\frac{1}{2}-\alpha_{m}+\alpha_{f}, \beta:=\frac{1}{4}\left(1-\alpha_{m}+\alpha_{f}\right)^{2}, \alpha_{m}:=\frac{2 \rho_{\infty}-1}{\rho_{\infty}+1}, \alpha_{f}:=\frac{\rho_{\infty}}{\rho_{\infty}+1}, \Delta t$

\footnotetext{
${ }^{2}$ The linearised approach permits solutions in the frequency domain (as seen in part III) and as such only requires a spatial discretisation as the temporal independence is removed by transformation to the frequency domain. As a result the high order spatial discretisation is favoured over high order time integration schemes to provide an accurate linearised frequency domain solver.
} 
is the timestep size and $\rho_{\infty}$ denotes the user-specified value of the spectral radius in the high frequency limit ${ }^{3}$.

\subsubsection{Predictor Multi-Corrector Step}

The eventual aim of this formulation is to handle fully coupled non-linear problems and employ an iterative procedure at each timestep to fully resolve the solution. Iterative procedures require a prediction of the solution field to compute a converged solution. Therefore, to allow for the robust treatment of the solution at each timestep the introduction of a predictor-corrector algorithm, similar to those proposed in [43], is required. The prediction step $[\mathrm{k}=0]$, based on an initial guess of the displacement field $\mathbf{q}_{n+1}^{[0]}$, is defined as

$$
\begin{aligned}
& \ddot{\mathbf{q}}_{n+1}^{[0]}=\frac{1}{\beta}\left(\frac{\mathbf{q}_{n+1}^{[0]}-\mathbf{q}_{n}}{\Delta t^{2}}-\frac{\dot{\mathbf{q}}_{n}}{\Delta t}-\left(\frac{1}{2}-\beta\right) \ddot{\mathbf{q}}_{n}\right) \\
& \dot{\mathbf{q}}_{n+1}^{[0]}=\frac{\varsigma}{\beta}\left(\frac{\mathbf{q}_{n+1}^{[0]}-\mathbf{q}_{n}}{\Delta t}+\left(\frac{\beta}{\varsigma}-1\right) \dot{\mathbf{q}}_{n}+\left(\frac{\beta}{\varsigma}-\frac{1}{2}\right) \Delta t \ddot{\mathbf{q}}_{n}\right),
\end{aligned}
$$

where (7.6a) and (7.6b) are consistent with (7.5a) and (7.5b) respectively. In other words, both the velocity and accelerations predictors preserve second-order accuracy, as discussed in [118]. Directing the reader's attention to (7.5a) and (7.5b) and substituting the fields at iteration step $[\mathrm{k}+1]$, the update variables of the first and second order fields are obtained as

$$
\begin{gathered}
\ddot{\boldsymbol{\delta}}_{\mathrm{q} n+1}^{[\mathrm{k}]}=\frac{1}{\beta \Delta t^{2}} \boldsymbol{\delta}_{\mathrm{q}}{ }_{\mathrm{q}+1}^{[\mathrm{k}]}, \\
\dot{\boldsymbol{\delta}}_{\mathrm{q} n+1}^{[\mathrm{k}]}=\frac{\varsigma}{\beta \Delta t} \boldsymbol{\delta}_{\mathrm{q}}{ }^{[\mathrm{k}]}{ }_{n+1} .
\end{gathered}
$$

\subsubsection{Fully Discrete Non-Linear Approach}

Using the relations between the updates, in (7.7), the discrete system, in (7.3), can be expressed in terms of the update in the zeroth order solution vector $\boldsymbol{\delta}_{\mathrm{q}}{ }_{n+1}^{[\mathrm{k}]}$ at time $t_{n+1}$ as the following Newton-Raphson procedure: Find $\boldsymbol{\delta}_{\mathrm{q}_{n+1}}^{[\mathrm{k}]} \in \mathbb{R}^{4 N}$ at each

\footnotetext{
${ }^{3}$ Traditionally with the generalised $\alpha$ method, $\gamma$ is used to refer to the first stability parameter, however, given that this Thesis reserves $\gamma$ for the material conductivity, instead, the alternative symbol $\varsigma$ is used.
} 
time $t \in(0, T]$ such that

$$
\begin{aligned}
&\left(\frac{\left(1-\alpha_{m}\right)}{\beta \Delta t^{2}} \mathbf{M}+\left.\frac{\varsigma\left(1-\alpha_{f}\right)}{\beta \Delta t} \mathbf{C}\right|_{t_{n+1} \alpha_{f}} ^{[\mathrm{k}]}+\left.\left(1-\alpha_{f}\right) \mathbf{K}\right|_{t_{n+1-\alpha_{f}}} ^{[\mathrm{k}]}\right) \boldsymbol{\delta}_{\mathrm{q}}^{[\mathrm{k}]}{ }_{n+1}=-\left.\mathbf{R}_{\mathrm{q}}\right|_{t_{n+1-\alpha_{f}}} ^{[\mathrm{k}]}, \\
& \mathbf{q}_{n+1}^{[\mathrm{k}+1]}=\mathbf{q}_{n+1}^{[\mathrm{k}]}+\boldsymbol{\delta}_{\mathrm{q}_{n+1}}^{[\mathrm{k}]},
\end{aligned}
$$

which is iterated over $[\mathrm{k}]$ until $\left|\mathbf{R}_{\mathrm{q}}^{[\mathrm{k}]}\right|<$ TOL.

\subsubsection{Fully Discrete Linearised Approach}

The fully discrete version of (7.3) for the linearised approach is similarly expressed as: Find $\boldsymbol{\delta}_{\mathrm{q}_{n+1}} \in \mathbb{R}^{4 N}$ at each time $t \in(0, T]$ such that

$$
\left(\frac{\left(1-\alpha_{m}\right)}{\beta \Delta t^{2}} \tilde{\mathbf{M}}+\frac{\varsigma\left(1-\alpha_{f}\right)}{\beta \Delta t} \tilde{\mathbf{C}}+\left(1-\alpha_{f}\right) \tilde{\mathbf{K}}\right) \boldsymbol{\delta}_{\mathrm{q}_{n+1}}=-\left.\tilde{\mathbf{R}}_{\mathrm{q}}\right|_{t_{n+1-\alpha_{f}}}
$$

where the system matrices $\tilde{\mathbf{M}}, \tilde{\mathbf{C}}$ and $\tilde{\mathbf{K}}$ are independent of the previous temporal solutions and $\tilde{\mathbf{R}}_{\mathrm{q}}$ depends only on the evaluation of $\boldsymbol{J}^{A C}$ at time level $t_{n+1-\alpha_{f}}$ and so can be solved in a single step, which is a simplified version of (7.8).

\subsection{Solution Strategy}

A general algorithm for computing the transient variation in the fields for both the non-linear and linearised approaches, proposed above, under a generalised- $\alpha$ scheme is summarised in the following algorithm, where for the latter approach no iterations are required and so $N_{\mathrm{k}}=0$, thus rendering the predictor-multi-corrector algorithm to a predictor-corrector.

Figure 7.1 summarises the steps required to construct the discretised NewtonRapshon iterative scheme in (7.8) from the transient non-linear transmission problem $(2.36)$.

\subsection{Chapter Summary}

This Chapter has presented a temporal discretisation of both the novel linearised and non-linear approaches. The temporally discrete systems are presented as second order ODEs - the EOMs, which express the mass, stiffness and damping matrices. 
$\overline{\text { Algorithm } 2 \text { Algorithm to compute the time variation of the coupled transient }}$ system.

1: Define the initial conditions of the fields $\mathbf{q}_{0}, \dot{\mathbf{q}}_{0}, \ddot{\mathbf{q}}_{0}$ from (6.1)

2: for $n=0,1,2 \ldots N_{\Delta t}$ do

$\triangleright$ Number of timesteps $N_{\Delta t}$

3: $\quad$ Predict the solution at the current timestep $\mathbf{q}_{n+1}^{[0]}, \dot{\mathbf{q}}_{n+1}^{[0]}, \ddot{\mathbf{q}}_{n+1}^{[0]}$ from (7.6)

4: $\quad$ while $\left|\mathbf{R}_{\mathrm{q}}\right|<\mathrm{TOL} ; k=0,1,2 \ldots N_{\mathrm{k}}$ do $\quad \triangleright$ Number of iterations $N_{\mathrm{k}}$

5: $\quad$ Compute the fields $\mathbf{q}_{n+1-\alpha_{f}}^{[\mathrm{k}]}, \dot{\mathbf{q}}_{n+1-\alpha_{f}}^{[\mathrm{k}]}$ and $\ddot{\mathbf{q}}_{n+1-\alpha_{m}}^{[\mathrm{k}]}$ at $t_{n+1-\alpha_{f}}$ from (7.4)

6: $\quad$ Solve the linear system (7.8) or (7.9) for the solution update

$$
\mathbf{K}_{T S M}^{[\mathrm{k}]} \boldsymbol{\delta}_{\mathrm{q} n+1}^{[\mathrm{k}]}=-\left.\mathbf{R}_{\mathrm{q}}\right|_{t_{n+1-\alpha_{f}}} ^{[\mathrm{k}]},
$$

where $\mathbf{K}_{T S M}^{[\mathrm{k}]}=\left(\frac{\left(1-\alpha_{m}\right)}{\beta \Delta t^{2}} \mathbf{M}+\left.\frac{\varsigma\left(1-\alpha_{f}\right)}{\beta \Delta t} \mathbf{C}\right|_{t_{n+1-\alpha_{f}}} ^{[\mathrm{k}]}+\left.\left(1-\alpha_{f}\right) \mathbf{K}\right|_{t_{n+1-\alpha_{f}}} ^{[\mathrm{k}]}\right)$ for the non-linear approach and $\mathbf{K}_{T S M}=$ $\left(\frac{\left(1-\alpha_{m}\right)}{\beta \Delta t^{2}} \tilde{\mathbf{M}}+\frac{\varsigma\left(1-\alpha_{f}\right)}{\beta \Delta t} \tilde{\mathbf{C}}+\left(1-\alpha_{f}\right) \tilde{\mathbf{K}}\right)$ and $\quad \mathbf{R}_{\mathrm{q}}$ is replaced by $\tilde{\mathbf{R}}_{\mathrm{q}}$ for the linearised approach

7: $\quad$ Update the solution fields

$$
\begin{aligned}
& \mathbf{q}_{n+1}^{[\mathrm{k}+1]}=\mathbf{q}_{n+1}^{[\mathrm{k}]}+\boldsymbol{\delta}_{\mathrm{q}}{ }^{[\mathrm{k}]}{ }_{n+1} \\
& \dot{\mathbf{q}}_{n+1}^{[\mathrm{k}+1]}=\dot{\mathbf{q}}_{n+1}^{[\mathrm{k}]}+\frac{\varsigma}{\beta \Delta t} \boldsymbol{\delta}_{\mathrm{q}}{ }^{[\mathrm{k}]} \\
& \ddot{\mathbf{q}}_{n+1}^{[\mathrm{k}+1]}=\ddot{\mathbf{q}}_{n+1}^{[\mathrm{k}]}+\frac{1}{\beta \Delta t^{2}} \boldsymbol{\delta}_{\mathrm{q} n+1}^{[\mathrm{k}]}
\end{aligned}
$$

\section{8: $\quad$ end while}

9: end for
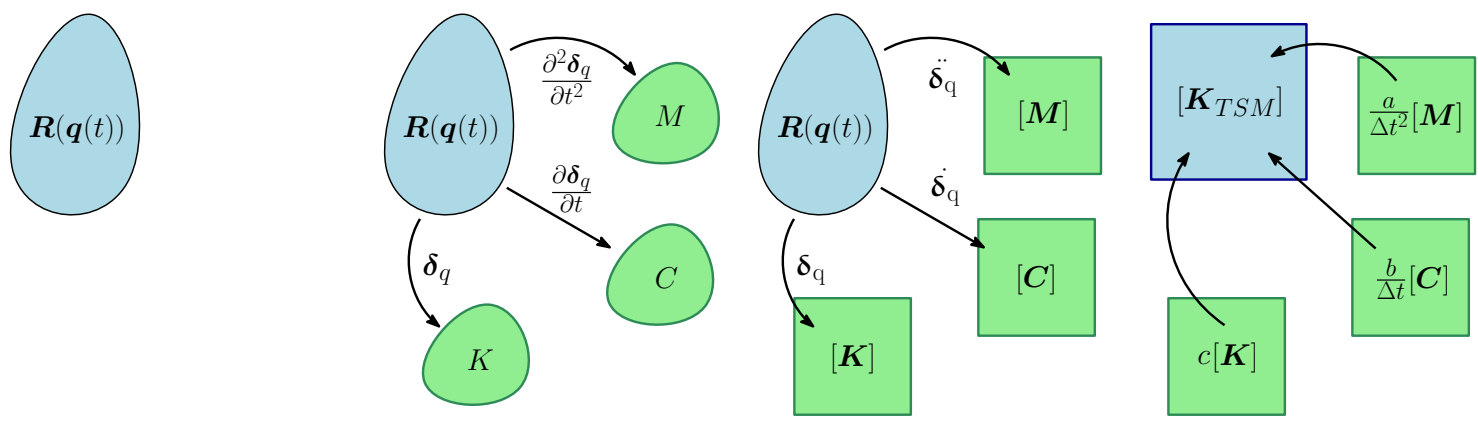

(a) Residual weak form (b) Linearisation of the of coupled non-linear non-linear system system

(c) Discretisation of (d) Temporal discretithe directional deriva- sation and building systives tem matrix

Figure 7.1: Summary of steps to solving transient non-linear system. 
This description permits the easy application of the time integration scheme and allows for the individual matrices to be computed separately. The temporally discrete linearised approach results in a system where the matrices are independent of the fields and so can be solved in a single iteration independent of time, or frequency, as in Chapter 4. This allows for a computationally efficient implementation where only the residual must be recalculated, where a method for its efficient calculation is summarised in Appendix G.

From the objectives of this thesis, discussed in Section 1.4, the topics discussed in this Chapter align with the objective: "To provide an efficient computational methodology for handling the solution to the coupled problem of MRI scanners using both approaches."

In the next Chapter, temporal solutions to a series of industrially relevant MRI scanner type problems will be computed. The temporal solutions will be computed using both the linearised and non-linear approaches and compared also with the results from the time harmonic system of the linearised approach, presented in Part III. The different approaches will be compared using a range of different industrial measures of interest across the full operating range of MRI scanners to determine the validity of the computationally efficient linearised approach. 



\section{Chapter 8}

\section{Numerical Examples of the Non-Linear Approach}

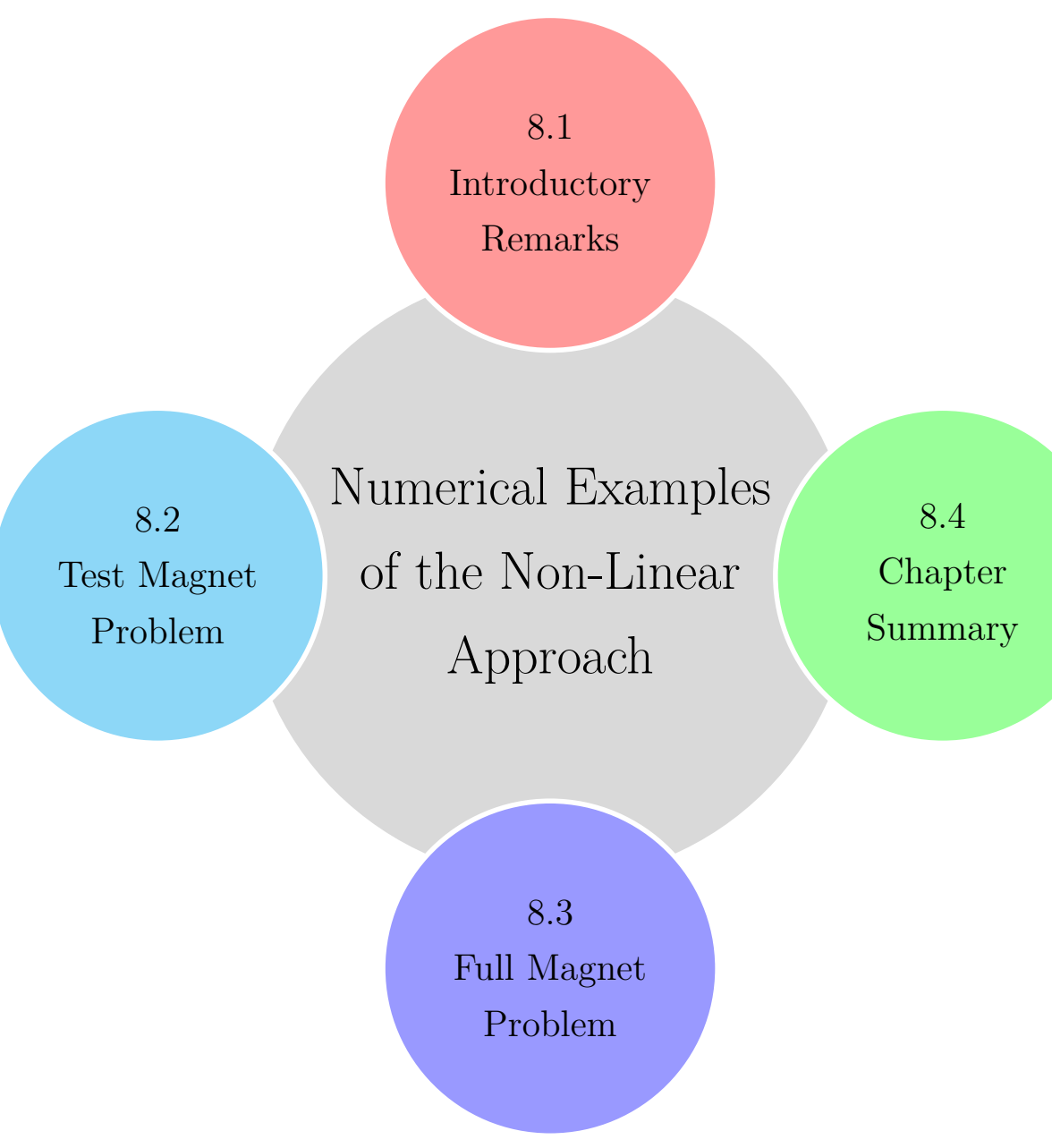

"Not all those who wander are lost." 


\subsection{Introductory Remarks}

This Chapter presents two industrially relevant benchmark MRI scanner problems: the first problem is the same test magnet presented in Chapter 5, and the other problem comprises of a more realistic MRI scanner geometry that accurately represents current scanners in operation, to compare the linearised and non-linear approaches in the time domain. A series of studies are performed across a range of measures of interest in order to determine the accuracy of the linearised approach in capturing the full non-linear behaviour of MRI scanners. Solutions to each problem are computed for the linearised approach both in the time harmonic and time dependent formulations and the non-linear approach across a range of MRI scanner operating conditions, in order to prove the conjectures presented in Chapter 6.

From the objectives of this Thesis, discussed in Section 1.4, this Chapter addresses the objective: "To determine the accuracy of the linearised approach across the full operating range of MRI scanners", by comparing the two approaches across the full range of operating conditions, and beyond, of current clinical MRI scanners. The operating conditions for each problem are derived from the MRI scanner manufacturer's data and a simplified model for relating the field strength of the magnet to the current density in the coils, summarised in Chapter 6.

This Chapter begins with the analysis of the test magnet problem in Section 8.2, where a full analysis across a series of measures of interest are performed, before moving on to the analysis of the full magnet problem in Section 8.3. Finally, the contents of this Chapter are summarised in Section 8.4.

\subsection{Test Magnet Problem}

First, solutions to the industrially relevant test magnet problem are considered, which was previously presented in Chapter 5 . The conducting region $\Omega_{c}$ of the test magnet consists of three metallic shields, known as the Outer Vacuum Chamber (OVC) $\Omega_{c}^{O V C}, 77 K$ radiation shield $\Omega_{c}^{77 K}$ and $4 K$ helium vessel $\Omega_{c}^{4 K}$, each with different material parameters $(\gamma, \mu, \lambda, G, \rho)$. The exact geometries and material parameters of the conducting components are commercially sensitive and as such are not displayed in this Thesis. By choosing to study only the Z-gradient coils and noting that the currents in the main coils and geometry of the conducting components are rotationally symmetric, the problem may be reduced to an axisymmetric description and solved in cylindrical coordinates $(r, \phi, z)$ on the meridian plane $\Omega^{m}$. 
The full 3D representation of this simplified MRI scanner is depicted in Figure 8.1.

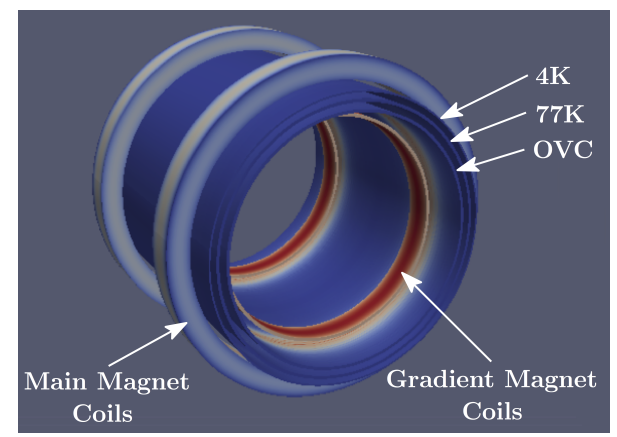

Figure 8.1: Test magnet problem: components of the simplified geometry.

A pair of main magnet coils, each with a static current source $\boldsymbol{J}^{D C}=J_{\phi}^{D C}(r, z) \boldsymbol{e}_{\phi}$, are located on the outside of the three shields and a pair of Z-gradient magnet coils, each with alternating current source $\boldsymbol{J}^{A C}(t)=J_{\phi}^{A C}(t, r, z) \boldsymbol{e}_{\phi}$, are located within the imaging bore, both of which are assumed as Biot-Savart conductors and are located in free space. Realistic excitations of the gradient coil are non-sinusoidal in nature, however for the purposes of comparison between the linearised and nonlinear approaches only sinusoidal excitations will be considered, where the current density is described by $J_{\phi}^{A C}(t)=\operatorname{Re}\left(\left|J_{\phi}^{A C}\right| e^{\mathrm{i} \omega t}\right)$. A range of excitation frequencies of $\omega=2 \pi[1000,1500,2000] \mathrm{rad} / \mathrm{s}$ are considered for this problem, which lie outside of the resonance region of $\omega \geq 2 \pi$ [3500], predicted in Chapter 5. The magnitude of the static $\left|J_{\phi}^{D C}\right|$ and gradient current sources $\left|J_{\phi}^{A C}\right|$ to be considered are obtained from manufacturer's data $[196,229,228]$. This data quotes the maximum capable flux density on the central axis of the imaging bore $(r=0)$ and in order to obtain the corresponding current densities the model described in Section 6.5.3 is used, the results of which are summarised, for key clinical field strengths, in Table 8.1.

\begin{tabular}{|c|c||c|c|}
\hline \multicolumn{2}{|c||}{ Main Coil } & \multicolumn{2}{c|}{ Gradient Coil } \\
\hline \hline $\max \left|\boldsymbol{B}_{z=0}^{D C}\right|[\mathrm{T}]$ & $\left|J_{\phi}^{D C}\right|\left[\times 10^{-6} \mathrm{~A} / \mathrm{m}^{2}\right]$ & $\max \left|\boldsymbol{B}_{z=0}^{A C}\right|\left[\times 10^{-3} \mathrm{~T} / \mathrm{m}\right]$ & $\left|J_{\phi}^{A C}\right|\left[\times 10^{-6} \mathrm{~A} / \mathrm{m}^{2}\right]$ \\
\hline 1.5 & 218.35 & 30 & 4.70 \\
3 & 436.70 & 80 & 12.53 \\
11.7 & 1703.10 & 100 & 15.66 \\
\hline
\end{tabular}

Table 8.1: Test magnet problem: typical values of the current densities in static and gradient coils and ranges of static and gradient field strengths from manufacturers data [196, 229, 228].

Given criteria 2) and 3) in Section 6.5.2, the greatest non-linearity should appear for a magnet with weakest static field of $1.5 \mathrm{~T}$ and strongest gradient field of 
$100 \times 10^{-3} \mathrm{~T} / \mathrm{m}$. This would result in a ratio between the static and gradient current density values of $\left|J_{\phi}^{A C}\right| /\left|J_{\phi}^{D C}\right| \approx 7.2 \%$ for this problem. The two approaches are, therefore, studied across a range of current density ratios of $\left|J_{\phi}^{A C}\right| /\left|J_{\phi}^{D C}\right|=$ $[5,10,15,20] \%$ to provide a rigorous test of the linearised approach for applications of higher levels of non-linearity than current MRI scanners are capable of. The problem is subject to the following boundary conditions: the Dirichlet boundaries of the conductors are set to $\boldsymbol{u}^{D}=\mathbf{0}$ to fix the conductors in space and the value of the magnetic vector potential on the outer boundary is set to $A_{\phi}=0$ due to the eddy current decay. The initial conditions of the problem are defined by those described in Section 6.3.3.

This problem is treated computationally for both the linearised and non-linear approaches. The non-conducting free space region, comprised of air, is truncated to create the domain $\Omega^{m}$, which is the same as in Chapter 5. In terms of spatial discretisation, the solution is analysed for a single unstructured mesh of 2,842 triangles of maximum size $h=0.25 \mathrm{~m}$, but with substantial refinement in the conductors $\Omega_{c}^{m}$, resulting in 570 elements in $\Omega_{c}^{m}$, with $p$-refinement for elements of order $p=[1,2,3,4,5]$. The mesh parameters used are the same as in [26], however, due to improvements in the mesh generator used [78] results in a better distribution of elements and hence fewer elements. A single layer of 18 infinite elements is applied to the outer boundary of $\Omega^{m}$ to resolve the static decay of the magnetic field, so that the boundary condition $A_{\phi}=0$ is effectively imposed at infinity. The temporal discretisation used to resolve these waves are studied for a timestep size of $\Delta t=2 \pi /\left(\omega N_{\Delta t}\right)$, where the number of points per wavelength of the excitation frequency is varied for $N_{\Delta t}=[10,20,30,40]$. The spectral radius of the $\alpha$-scheme time integrator $\rho_{\infty}$ allows for damping of certain frequency regimes. For $\rho_{\infty}=1$ the amplitude of the wave is fully preserved and no damping of any high frequencies is introduced. For $\rho_{\infty}=0$ the scheme is fully dissipative and higher frequency waves are completely damped, however, the damping of physical modes also occurs. Given that the recovery of only physical modes is desirable in the problem, a value of $\rho_{\infty}=0.8$ is chosen, to allow for numerical damping of any non-physical high frequencies induced through the forcing, whilst still preserving the physical lower frequency waves.

Solutions to this problem are obtained by applying Algorithm 2 for both the nonlinear and linearised approaches across the range of current densities and excitation frequencies discussed. 


\subsubsection{Temporal convergence of the physical fields}

In order to determine the correct implementation of the generalised $\alpha$ temporal integration scheme, the Test Magnet problem was run for a series of different temporal disretisations consisting of number of timesteps per wavelength equal to $N_{\Delta t}=[2,4,8,16,24,32,48,56,64]$ for $p=4$ elements, for an excitation frequency of $f=1000 \mathrm{~Hz}$. The reference solution is computed as the steady state time response generated from the computed frequency domain solutions for the uncoupled case of each field, where the magnetics is excited by the current source and the mechanics is excited by a time varying external pressure to each of the shields. Figure 8.2 plots the relative error of the solution fields against the number of time steps per wavelength for the zeroth and first order time derivatives of both the time dependent electromagnetic and mechanical fields. The relative error is measured as the difference in computed and reference solutions at some time $t$, which is taken at a time after a steady state response has been achieved. From the figure it is clear that the rate of convergence of the temporal scheme is second order accurate, which is in agreement with the fact that the generalised $\alpha$ scheme is a second order accurate scheme [57].

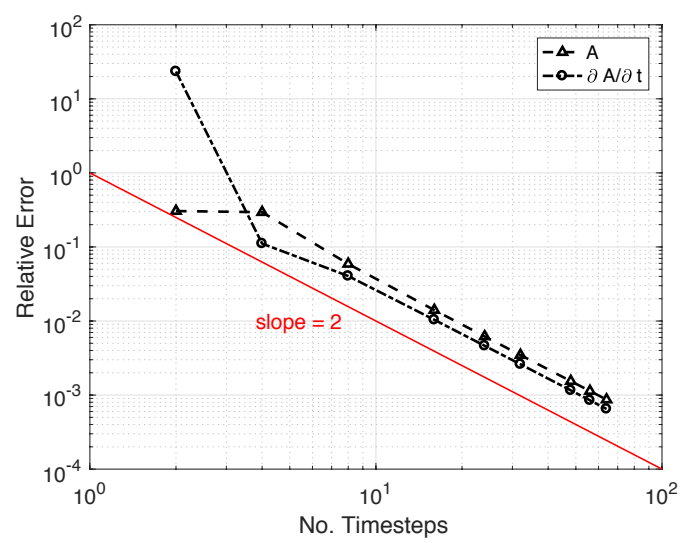

(a) $\left\|\boldsymbol{A}(t)-\boldsymbol{A}_{r e f}(t)\right\|_{L^{2}} /\left\|\boldsymbol{A}_{r e f}(t)\right\|_{L^{2}}$

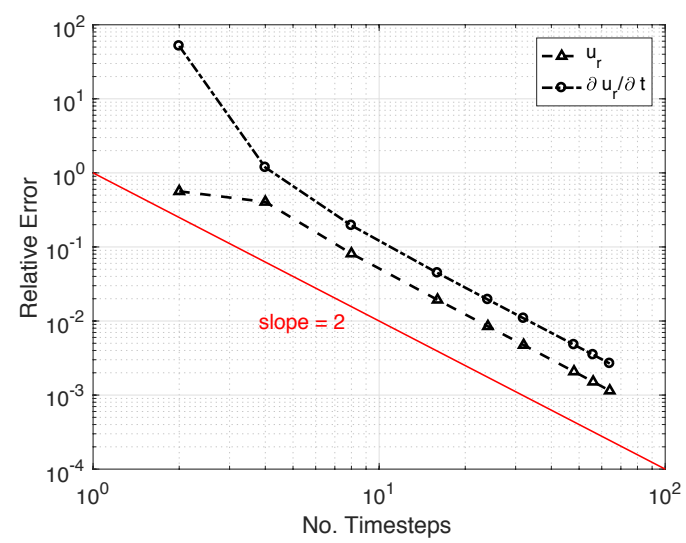

(b) $\left\|\boldsymbol{u}(t)-\boldsymbol{u}_{r e f}(t)\right\|_{L^{2}} /\left\|\boldsymbol{u}_{r e f}(t)\right\|_{L^{2}}$

Figure 8.2: Test magnet problem: temporal convergence of the generalised $\alpha$ scheme ( $2^{\text {nd }}$ order). The error is taken as the relative error between the computed and reference solutions at some time $t$, which is taken as some time after the steady state response is achieved. 


\subsubsection{Spatial Discretisation Study in the Outputs of Interest}

Quantities of industrial interest for both the mechanical and electromagnetic fields are now considered. For the electromagnetic field the output power dissipation in the conducting components $P_{\Omega_{c}}^{o}$ is considered and for the mechanical field the kinetic energy of the conducting components $E_{\Omega_{c}}^{k}$. The formal definition of these quantities in a full time domain description, where the quantities are averaged over the time period $T$ of excitation frequency, are

$P_{\Omega_{c}}^{o}(t, \boldsymbol{A})=\frac{1}{T} \int_{t}^{t+T} \int_{\Omega_{c}} \gamma\left|\frac{\partial \boldsymbol{A}}{\partial t}\right|^{2} \mathrm{~d} \Omega \mathrm{d} t, \quad E_{\Omega_{c}}^{k}(t, \boldsymbol{u})=\frac{1}{T} \int_{t}^{t+T} \frac{1}{2} \int_{\Omega_{c}} \rho\left|\frac{\partial \boldsymbol{u}}{\partial t}\right|^{2} \mathrm{~d} \Omega \mathrm{d} t$,

which reduce, in a time harmonic representation, to

$$
P_{\Omega_{c}}^{o}(\omega, \boldsymbol{A})=\frac{1}{2} \int_{\Omega} \gamma \omega^{2}|\boldsymbol{A}|^{2} \mathrm{~d} \Omega, \quad E_{\Omega_{c}}^{k}(\omega, \boldsymbol{U})=\frac{1}{4} \int_{\Omega} \rho \omega^{2}|\boldsymbol{u}|^{2} \mathrm{~d} \Omega
$$

as seen in Chapter 5. In the above, $\mathcal{A}$ and $\boldsymbol{U}$ are the complex amplitudes of their respective fields as $\boldsymbol{A}(t)=\operatorname{Re}\left(\mathcal{A} \mathrm{e}^{\mathrm{i} \omega t}\right)$ and $\boldsymbol{u}(t)=\operatorname{Re}\left(\boldsymbol{U} \mathrm{e}^{\mathrm{i} \omega t}\right)$, as described in Chapter 3. To compute these quantities for the test magnet problem, using either the transient solutions obtained by the linearised or non-linear approaches, Algorithm 2 is run at specific $N_{\Delta t}$ (which defines $\Delta t$ ) and $\rho_{\infty}$ for a sufficiently long time until the field responses reach steady state. Equation 8.1 is then applied over the time period of excitation $T$.

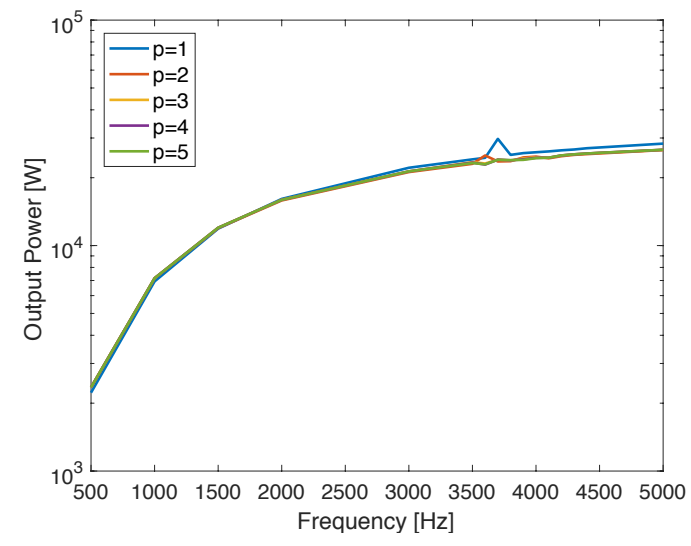

(a) $P_{\Omega_{c}^{O V C}}^{o}\left(\omega, \boldsymbol{A}_{A C}\right)$

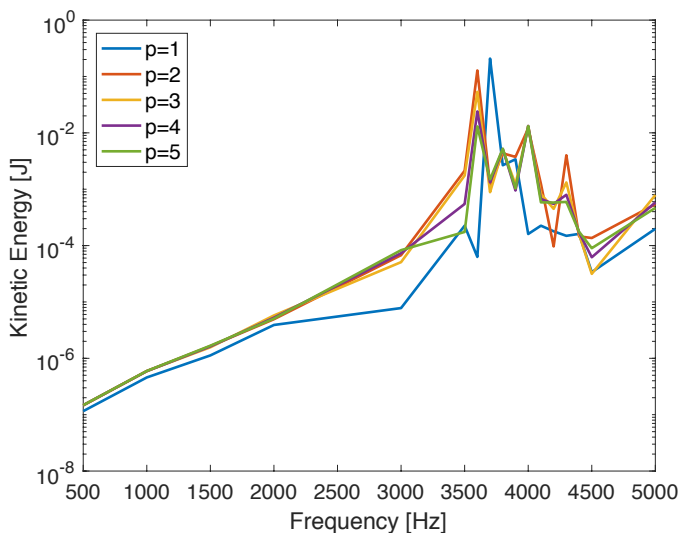

(b) $E_{\Omega_{C}^{O V C}}^{k}\left(\omega, \boldsymbol{u}_{A C}\right)$

Figure 8.3: Test magnet problem: output power (left) and kinetic energy (right) of the OVC $\Omega_{c}^{O V C}$ for element order $p=[1,2,3,4,5]$. 
In order to determine suitable spatial resolution in the solution, a $p$-refinement study is performed. Algorithm 2 is then applied for $N_{\Delta t}=30, \rho_{\infty}=0.8$ using the linearised approach to compute the output power and kinetic energy across the frequency spectrum for $\omega \leq 2 \pi$ [5000], the results of which are illustrated for the OVC, $77 K$ and $4 K$ radiation shields in Figures 8.3, 8.4 and 8.5, respectively ${ }^{1}$. For $p \leq 3$ the computed curves, illustrated in Figures 8.3, 8.4 and 8.5 do not match one another and so the results have not yet reached convergence. The curves for $p=4$ and $p=5$, however, are practically indistinguishable for both the output power and kinetic energy and suggests that for elements of order $p=4$ the results are sufficiently converged. Hence, elements of $p=4$ are adopted for all subsequent computations of this problem on the mesh specified previously.

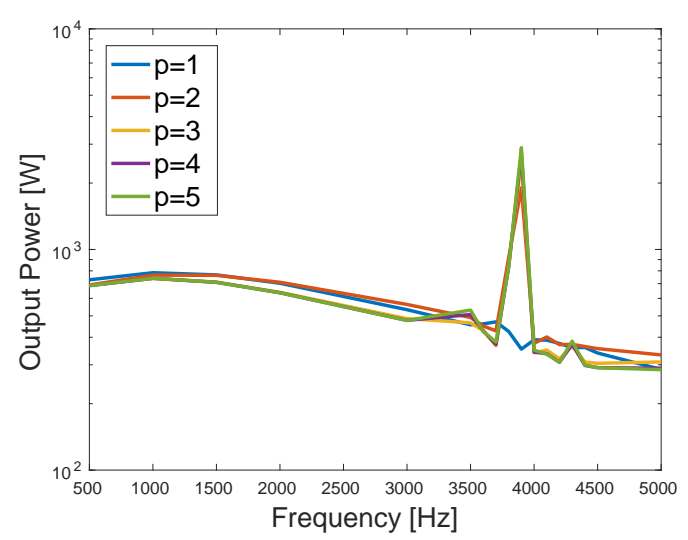

(a) $P_{\Omega_{c}^{O} O C}^{o}\left(\omega, \boldsymbol{A}_{A C}\right)$

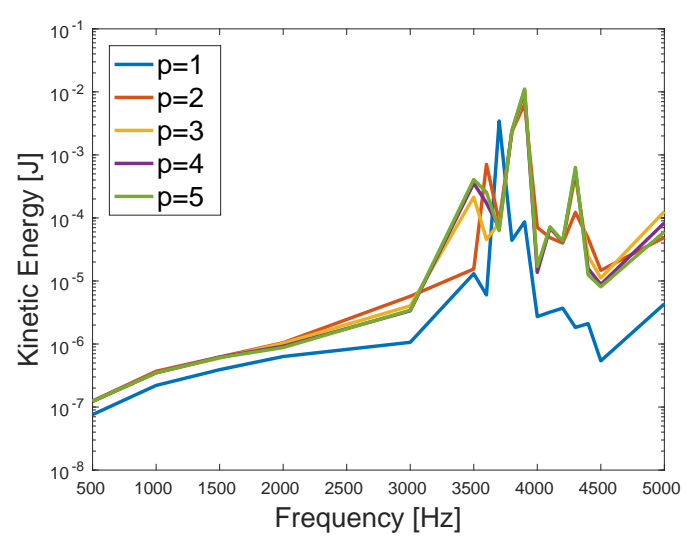

(b) $E_{\Omega_{c} O V C}^{k}\left(\omega, \boldsymbol{u}_{A C}\right)$

Figure 8.4: Test magnet problem: output power (left) and kinetic energy (right) of the $77 \mathrm{~K}$ radiation shield $\Omega_{c}^{77 K}$ for element order $p=[1,2,3,4,5]$.

A similar study to determine the required timestep size was also carried out, where a range of number of time steps per wavelength $N_{\Delta t}=[10,20,30,40]$ for $p=4$ elements were studied. The results suggest that $N_{\Delta t}=30$ offers sufficient temporal resolution to capture the amplitudes of the dominant frequency as well as frequencies twice the dominant frequency $2 \omega$. The importance of this frequency doubling will be explained later in Section 8.2.2. Thus for an excitation frequency of $\omega=2 \pi[1000]$ the timestep size $\Delta t=3.333 \times 10^{-5} s$, for $\omega=2 \pi[1500]$ the $\Delta t=2.222 \times 10^{-5} s$ and for $\omega=2 \pi[2000]$ the $\Delta t=1.667 \times 10^{-5} s$.

\footnotetext{
${ }^{1}$ When using an explicit time integration scheme the stability of the algorithm will depend on the restrictions of the time step size. To determine a sufficiently small time step one could perform stability analysis as in $[233,111]$. However, this is only valid for simple linear uncoupled problems and certainly not guaranteed for the coupled problems considered in this Thesis. Hence, for accuracy an implicit scheme is chosen.
} 


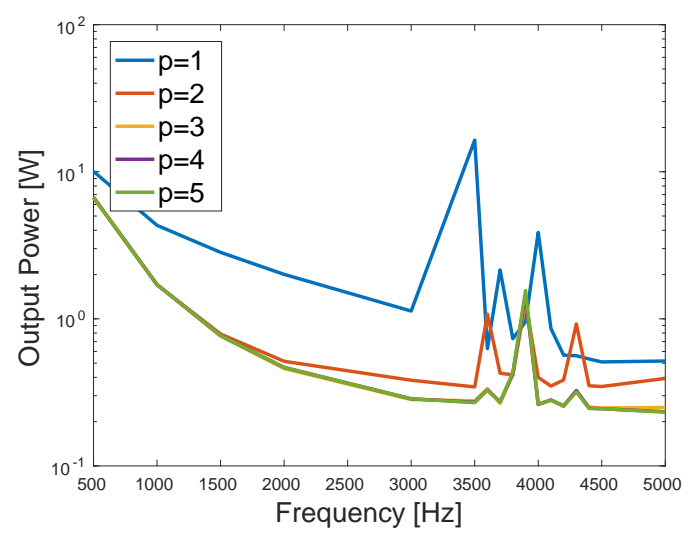

(a) $P_{\Omega_{c}^{O V C}}^{o}\left(\omega, \boldsymbol{A}_{A C}\right)$

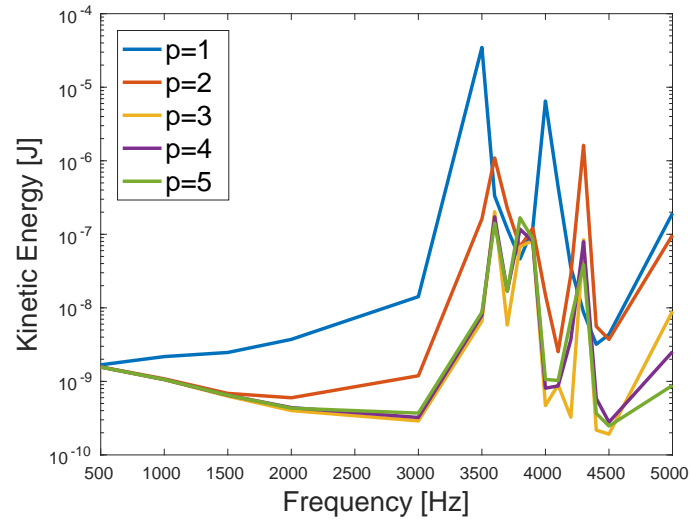

(b) $E_{\Omega_{c}^{O V C}}^{k}\left(\omega, \boldsymbol{u}_{A C}\right)$

Figure 8.5: Test magnet problem: output power (left) and kinetic energy (right) of the $4 K$ radiation shield $\Omega_{c}^{4 K}$ for element order $p=[1,2,3,4,5]$.

Using these parameters, in Section 8.2.2, the quantities in (8.1) are computed by applying Algorithm 2 for the linearised and non-linear approaches in the time domain and compared with the frequency domain solver [26], discussed in Part III. However, first the results of the linearised and non-linear approaches for transient electromagnetic and mechanical fields are considered.

\section{Electromagnetic Field}

Firstly, in order to perform comparisons between the linearised and non-linear approaches, the transient response of the magnetic field for both approaches is compared. Given that the output power of the conductors $P_{\Omega_{c}}^{o}$, described above, is driven by the temporal derivative of the magnetic vector potential $\partial \boldsymbol{A} / \partial t$, the response of this field for both approaches is measured and compared.

Figure 8.6 summarises the transient results of both approaches for the $\omega=$ $2 \pi[2000] \mathrm{rad} / \mathrm{s}$ sinusoidal excitation. The graphs in the left hand column plot the time signal obtained from both the linearised approach (in red) and non-linear approach (in black). The right hand column plots the corresponding frequency spectrum of the signal, obtained by performing Fast Fourier Transforms (FFTs) ${ }^{2}$ It is clear from this figure that the linearised approach provides an accurate approximation of the magnetic vector potential across the full range of current density ratios. The magnitude of the frequencies across the spectrum are almost identical and the

\footnotetext{
${ }^{2}$ The resolution of an FFT is determined by the time step size $\Delta t$ and the accuracy of the amplitude by length of the time signal. The examples presented here have all been run for sufficiently long with a sufficient time step size to capture the important frequency range.
} 


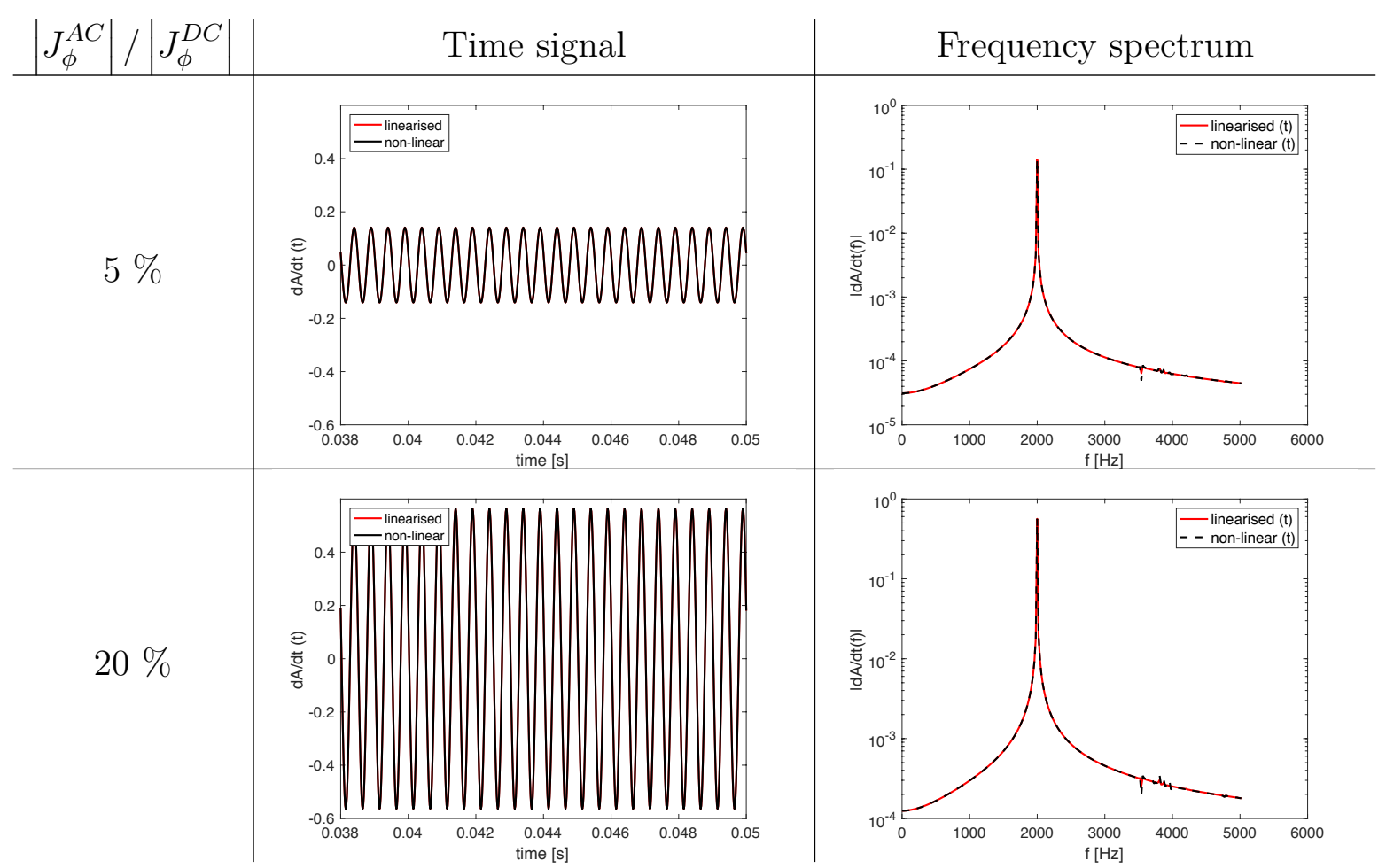

Figure 8.6: Test magnet problem: time signals and corresponding FFTs of $\partial \boldsymbol{A} / \partial t$ for both the linearised (red line) and non-linear approaches (black line), for various values of $\left|J_{\phi}^{A C}\right| /\left|J_{\phi}^{D C}\right|$ subject to a $\omega=2 \pi[2000] \mathrm{rad} / \mathrm{s}$ sinusoidal excitation.

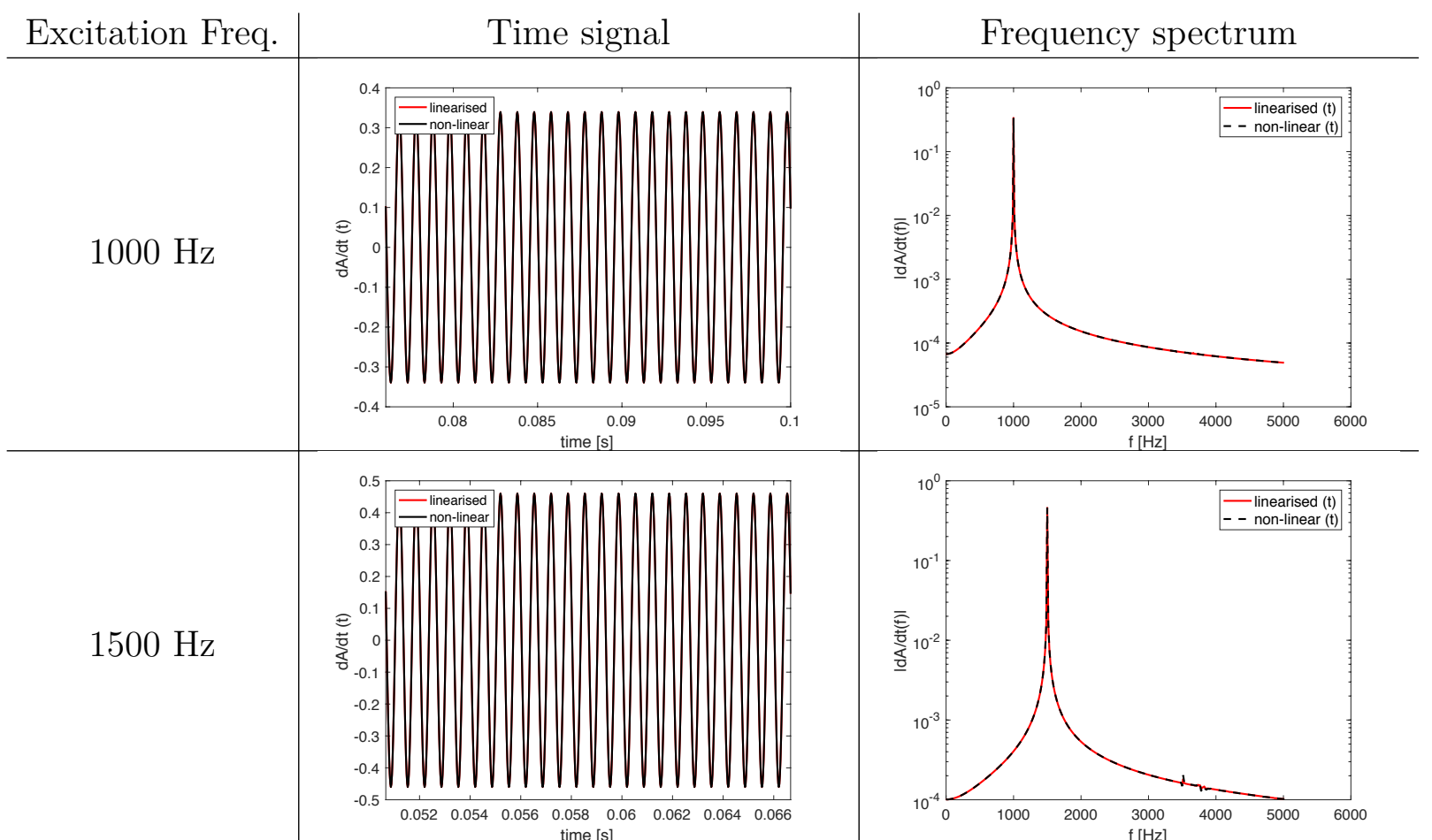

Figure 8.7: Test magnet problem: time signals and corresponding FFTs of $\partial \boldsymbol{A} / \partial t$ for both the linearised and non-linear approaches, for $\left|J_{\phi}^{A C}\right| /\left|J_{\phi}^{D C}\right|=20 \%$ subject to various frequencies of excitation. 
linearised approach is even capable of capturing all the fundamental frequencies, around $\omega=2 \pi[3500-4000] \mathrm{rad} / \mathrm{s}$.

Figure 8.7 plots the transient response and corresponding frequency spectrum for a current density ratio of $\left|J_{\phi}^{A C}\right| /\left|J_{\phi}^{D C}\right|=20 \%$ and a range of excitation frequencies. These plots, again, illustrate the agreement between the linearised and non-linear approaches for different excitation frequencies.

\section{Mechanical Field}

A comparison of the mechanical field response between the linearised and non-linear approaches is now considered. Given that the kinetic energy of the conductors $E_{\Omega_{c}}^{k}$, described in Section 8.2.2, is driven by their mechanical velocity $\partial \boldsymbol{u} / \partial t$, the response of this field for both approaches is measured and compared.

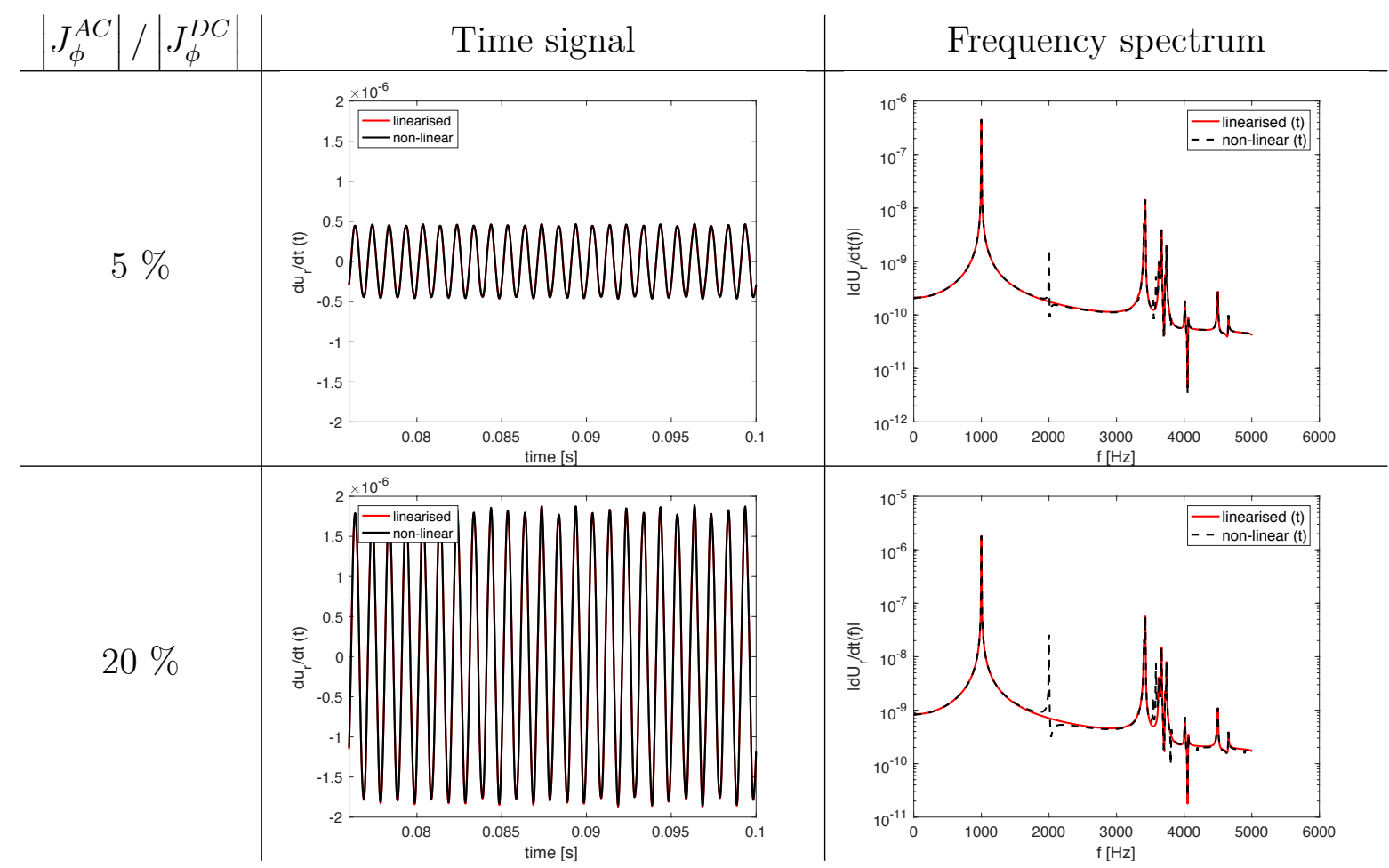

Figure 8.8: Test magnet problem: time signals and corresponding FFTs of $\partial u_{r} / \partial t$ for both the linearised and non-linear approaches, for various values of $\left|J_{\phi}^{A C}\right| /\left|J_{\phi}^{D C}\right|$ subject to a $\omega=$ $2 \pi[1000] \mathrm{rad} / \mathrm{s}$ sinusoidal excitation.

Figure 8.8 summarises the transient results of both approaches for the $\omega=$ $2 \pi[1000] \mathrm{rad} / \mathrm{s}$ sinusoidal excitation. The graphs in the left hand column plot the time signal obtained from both the linearised approach (in red) and non-linear approach (in black). From the plots it appears as though the two approaches offer good agreement, especially when looking at the time signals. However, in the fre- 
quency spectrum there appears to be an extra frequency at $\omega=2 \pi[2000] \mathrm{rad} / \mathrm{s}$ that is picked up in the non-linear approach, but not in the linearised approach. This term appears due to the non-linearity in the Maxwell stress tensor (2.24), which is observed to be quadratic in the magnetic field. From a decomposition of the magnetic field into static and dynamic components, as shown in Section 6.3.2, it can be shown that this non-linear term comprises of a product of the dynamic component of the field with itself, which disappears in the linearised approach. This term causes a frequency doubling effect, in other words it results in a component of excitation of the mechanical field that is double the frequency of the $\mathrm{AC}$ currents. So for a $\omega=2 \pi[1000] \mathrm{rad} / \mathrm{s}$ wave, as in Figure 8.9, an excitation at $\omega=2 \pi[2000] \mathrm{rad} / \mathrm{s}$ would also appear, which matches exactly with the results obtained. However, from Figure 8.9, it is clear that the magnitude of this term is far smaller than that of the amplitude associated with the exciting frequency and thus has little effect on the solution. As the ratio of the current densities increases so too does the magnitude of this term. However, even for a ratio of $20 \%$ the magnitude is still several orders smaller than the main excitation and smaller also than the most dominant resonant frequencies. Thus the magnitude of this doubled frequency component provides a useful measure in determining the non-linearity of the problem.

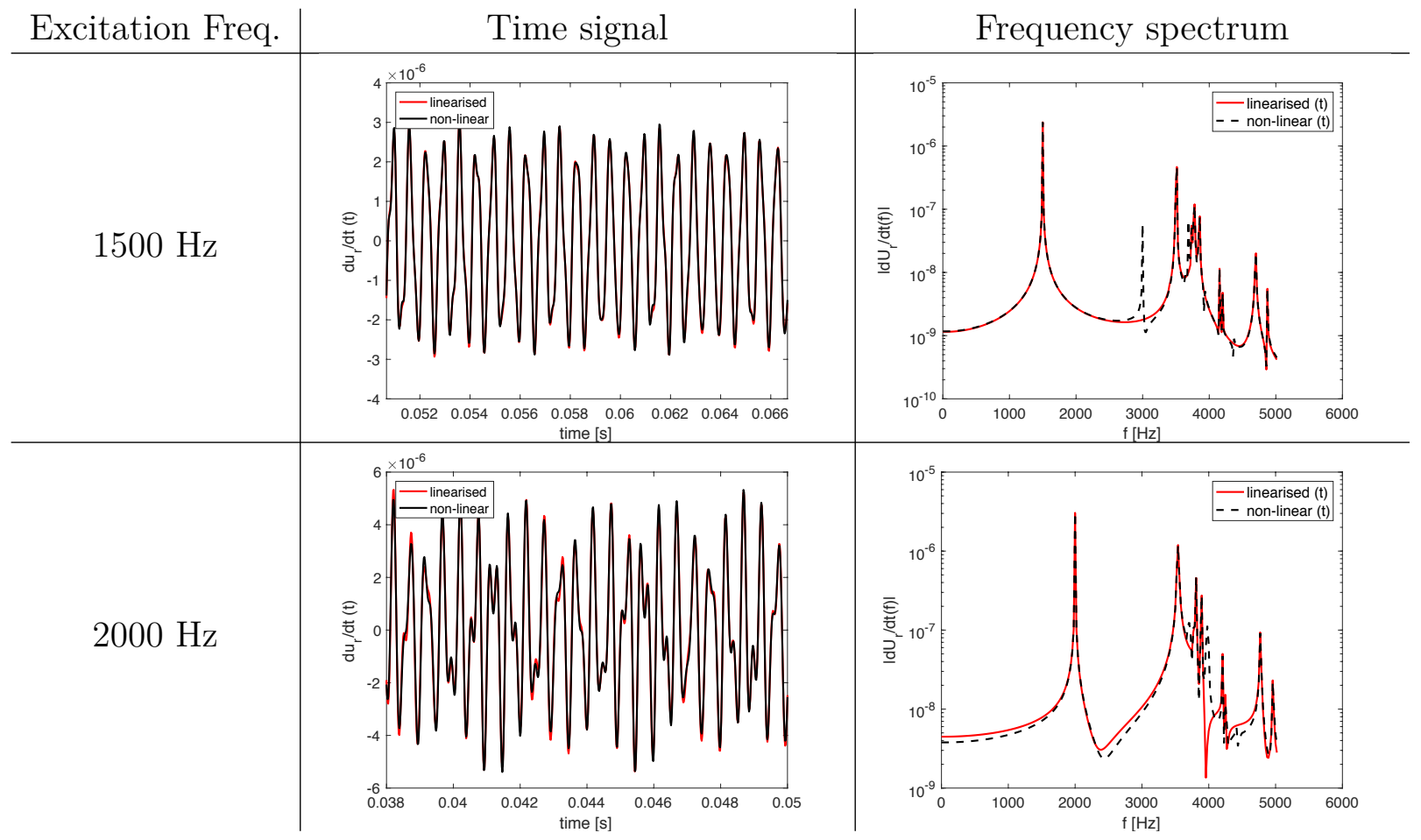

Figure 8.9: Test magnet problem: time signals and corresponding FFTs of $\partial u_{r} / \partial t$ for both the linearised and non-linear approaches, for $\left|J_{\phi}^{A C}\right| /\left|J_{\phi}^{D C}\right|=20 \%$ subject to various frequencies of excitation. 
Figure 8.9 plots the transient response and corresponding frequency spectrum for a current density ratio of $\left|J_{\phi}^{A C}\right| /\left|J_{\phi}^{D C}\right|=20 \%$ and a range of excitation frequencies. It is seen from these plots that for the AC current frequencies of $1500 \mathrm{~Hz}$ the agreement between the two approaches results in almost indistinguishable time signals. However, for the $\omega=2 \pi[2000] \mathrm{rad} / \mathrm{s}$ AC currents differences in the time signal become more visible. This is because the doubled frequency excitation component of $4000 \mathrm{~Hz}$ lies within the resonance region. When exciting close to the resonance region the problem results in matrices of high condition numbers, which are close to singular. Consequently, the tangent stiffness matrix inversion becomes more challenging and less reliable and, as a result, can lead to differences in the amplitudes across the frequency spectrum. Despite this effect however, the differences in the time signal are still very small and the characteristics of the system and prediction of the resonance region remain well captured by the linearised approach.

\section{Comparison of the Linearised and Non-Linear Approaches}

To benchmark the accuracy of the solution from the linearised approach with the non-linear approach, a comparison in the computation of the outputs of interest between two approaches, presented in Section 8.2.2, is performed across a range of frequencies $\omega \leq 2 \pi[5000] \mathrm{rad} / \mathrm{s}$. Figures $8.10,8.11$ and 8.12 illustrate the outputs of interest computed by the linearised approach in both frequency and time domain and the non-linear approach in time domain, using the definitions in (8.1) and (8.2) for a current density ratio of $\left|J_{\phi}^{A C}\right| /\left|J_{\phi}^{D C}\right|=10 \%$.

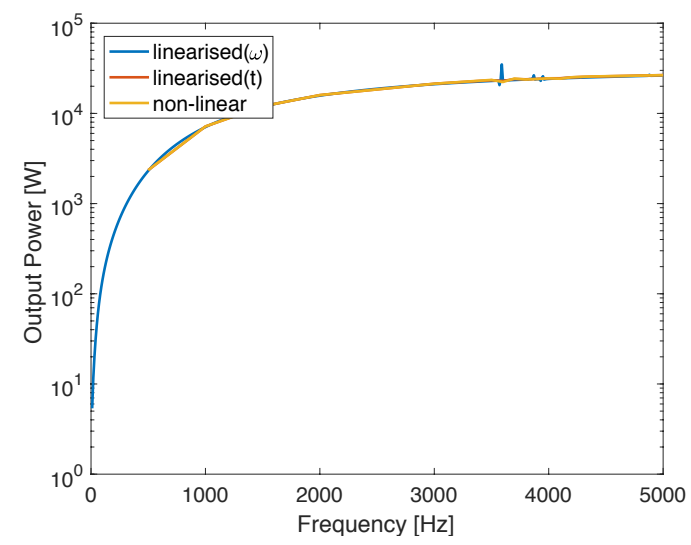

(a) $P_{\Omega_{c}^{O V V}}^{o}\left(\omega, \boldsymbol{A}^{A C}\right)$

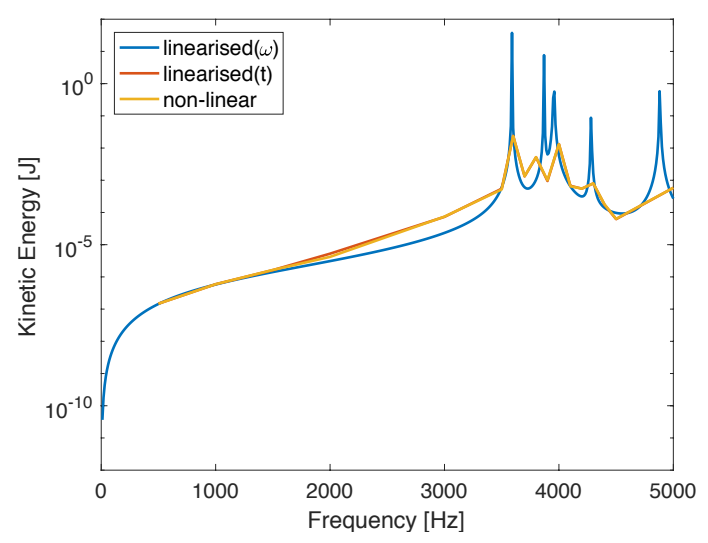

(b) $E_{\Omega_{c}^{O V C}}^{k}\left(\omega, \boldsymbol{u}^{A C}\right)$

Figure 8.10: Test magnet problem: computation of the Output Power (left) and Kinetic Energy (right) of the OVC $\Omega_{c}^{O V C}$ for both the linearised approach in time and frequency domain as well as the non-linear approach. 


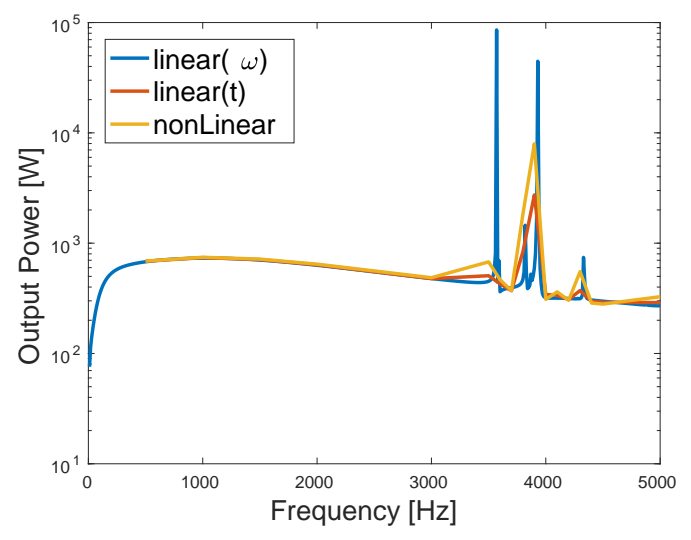

(a) $P_{\Omega_{c}^{77 K}}^{o}\left(\omega, A^{A C}\right)$

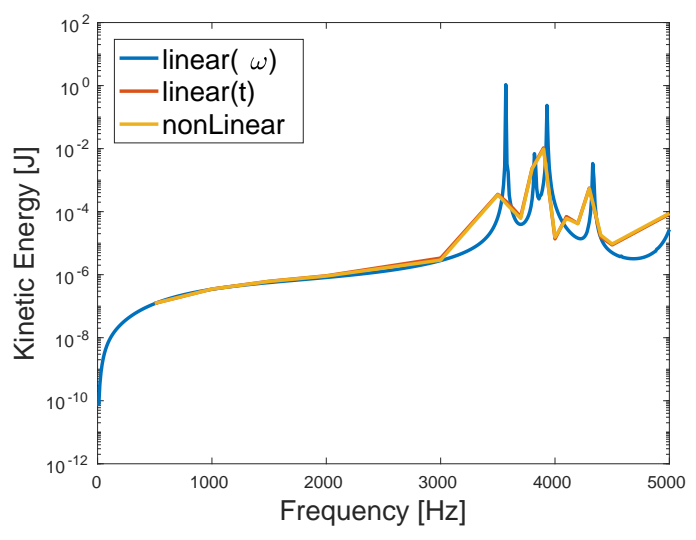

(b) $E_{\Omega_{c}^{77 K}}^{k}\left(\omega, \boldsymbol{u}^{A C}\right)$

Figure 8.11: Test magnet problem: computation of the Output Power (left) and Kinetic Energy (right) of the $77 \mathrm{~K}$ radiation shield $\Omega_{c}^{77 K}$ for both the linearised approach in time and frequency domain as well as the non-linear approach.

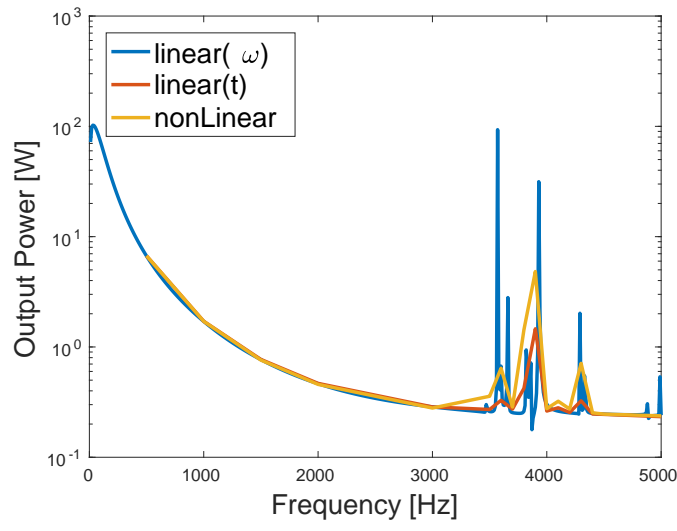

(a) $P_{\Omega_{c}^{4 K}}^{o}\left(\omega, \boldsymbol{A}^{A C}\right)$

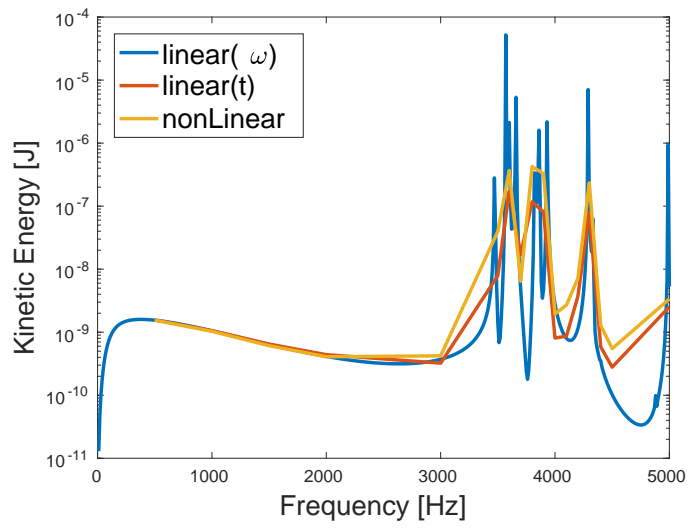

(b) $E_{\Omega_{c}^{4 K}}^{k}\left(\omega, \boldsymbol{u}^{A C}\right)$

Figure 8.12: Test magnet problem: computation of the Output Power (left) and Kinetic Energy (right) of the $4 K$ radiation shield $\Omega_{c}^{4 K}$ for both the linearised approach in time and frequency domain as well as the non-linear approach. 
Using the frequency domain approach, described in Part III and [26], the outputs of interest $P_{\Omega_{c}}^{o}$ and $E_{\Omega_{c}}^{k}$ can be directly computed for a given excitation frequency. Whereas, in the time domain the time solver must be run until steady state is obtained and then apply the definition in (8.1) across a time period. Due to the increased computational cost of computing the outputs of interest for transient solutions, a coarser frequency sweep is performed compared to the frequency domain results. Figures 8.10a, 8.11a and 8.12a plot the output power in the OVC $P_{\Omega_{c}^{O V V C}}^{o}\left(\omega, \boldsymbol{A}^{A C}\right)$, the $77 K$ radiation shield $P_{\Omega_{c}^{77 K}}^{o}\left(\omega, \boldsymbol{A}^{A C}\right)$ and the $4 K$ radiation shield $P_{\Omega_{c}^{4 K}}^{o}\left(\omega, \boldsymbol{A}^{A C}\right)$, respectively. Figures $8.10 \mathrm{~b}, 8.11 \mathrm{~b}$ and $8.12 \mathrm{~b}$ plot the kinetic energy in the OVC $E_{\Omega_{c}^{O V C}}^{k}\left(\omega, \boldsymbol{A}^{A C}\right)$, the $77 K$ radiation shield $E_{\Omega_{c}^{77 K}}^{k}\left(\omega, \boldsymbol{A}^{A C}\right)$ and the $4 K$ radiation shield OVC $E_{\Omega_{c}^{4 K}}^{k}\left(\omega, \boldsymbol{A}^{A C}\right)$, respectively. From the plots, the curves produced by the linearised and non-linear approaches are in close agreement across the frequency spectrum which suggests that the linearised approach provides a very accurate approximation to the full non-linear approach across the full spectrum for $\left|J_{\phi}^{A C}\right| /\left|J_{\phi}^{D C}\right|=10 \%$ for the two outputs of interest. In fact, given that the individual fields, analysed in Sections 8.2.2 and 8.2.2, also show very good agreement for $\left|J_{\phi}^{A C}\right| /\left|J_{\phi}^{D C}\right|=20 \%$, this can be hypothesised to be the case also for the outputs of interest as they are directly related.

Now, a comparison in the displacements of the mechanical shields is performed by plotting the displacements of the OVC, in three dimensions, at interesting instances of the time signal for the radial velocity $\partial u_{r} / \partial t$ computed for the case of $\left|J_{\phi}^{A C}\right| /\left|J_{\phi}^{D C}\right|=20 \%$ and $\omega=2 \pi(2000) \mathrm{rad} / \mathrm{s}$ in Figure 8.13. The chosen time instances across the time signal are plotted, where the difference in the mechanical velocities between the linearised and non-linear approaches is noticeable. The contour plots of the displacement fields are all scaled such that the colour maps between the linearised (in time) and non-linear approaches are the same. The displacements in Figure 8.13 are scaled by several orders of magnitude to show visually the displacement shapes of the shield. It is clear that for all snapshots the differences between the two approaches are almost indistinguishable. This suggests that even for $\left|J_{\phi}^{A C}\right| /\left|J_{\phi}^{D C}\right|=20 \%$ and an excitation frequency of $\omega=2 \pi(2000) \mathrm{rad} / \mathrm{s}$, where the doubled frequency component of $\omega=2 \pi(4000) \mathrm{rad} / \mathrm{s}$ resides in the resonance region, see Section 8.2.2, that the linearised approach provides accurate and comparable results to the full non-linear approach.

The average computational timings, per timestep, for the two approaches across a range of different element orders $p$ are summarised in Table 8.2. The comparison between the computational timings of the two approaches suggests that the linearised 


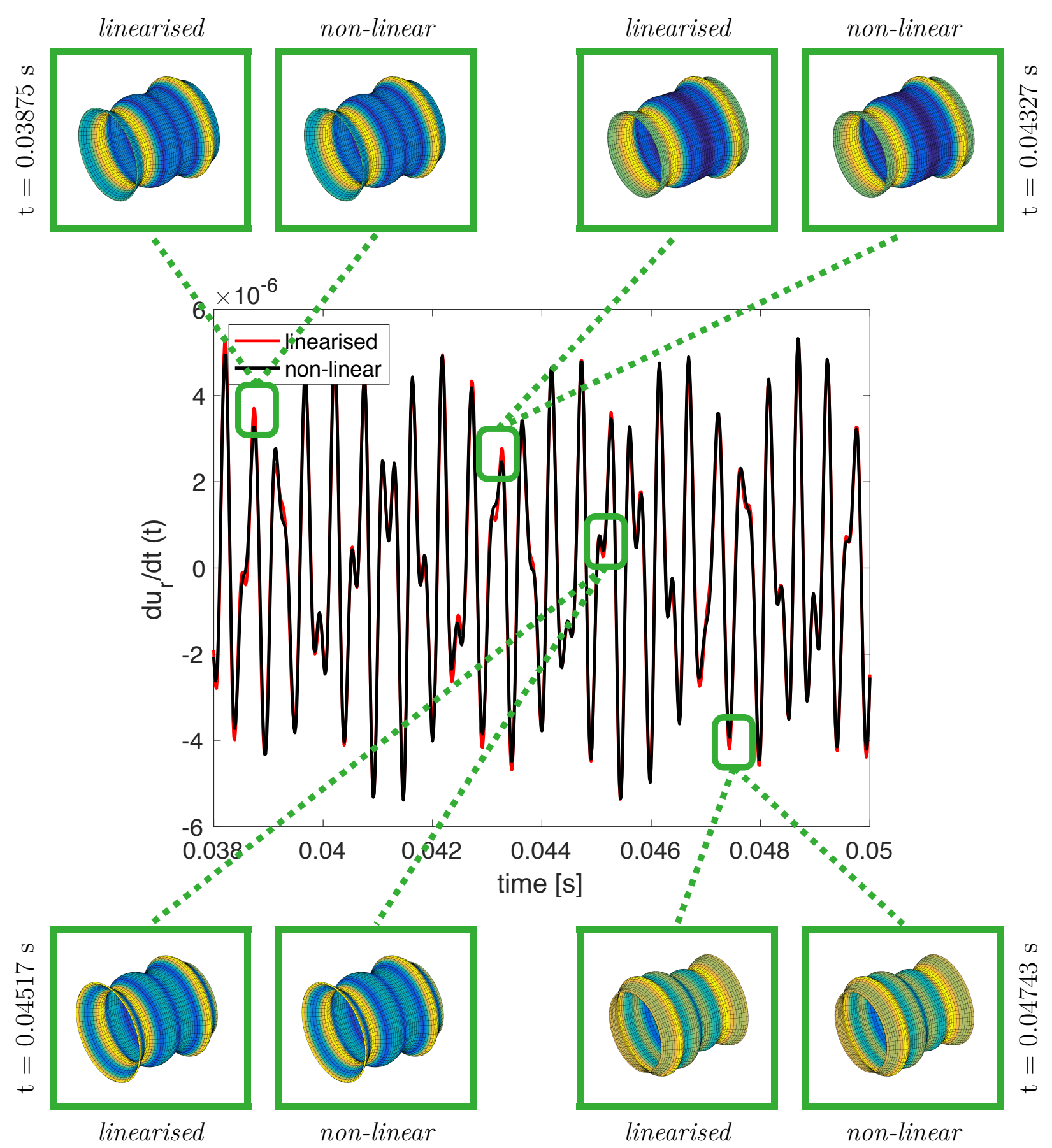

Figure 8.13: Test magnet problem: comparison between the time dependant linearised and nonlinear approaches for displacements of the OVC at different times for $\left|J_{\phi}^{A C}\right| /\left|J_{\phi}^{D C}\right|=20 \%$ and $\omega=2 \pi(2000) \mathrm{rad} / \mathrm{s}$. 
approach is orders of magnitude more efficient in terms of computational cost than the non-linear approach. For low order $p=1$ elements the linearised approach (in time) requires 383 times less computational effort than the non-linear approach. Whereas, for higher order $p=5$ elements the linearised approach (in time) requires 164 times less computational effort than the non-linear approach. This speed-up factor appears to offer an inverse exponential behaviour with $p$, which is due to the higher requirement on the solver for higher order elements. Nevertheless, the computational timings displayed in the table suggest that the linearised approach offers orders of magnitude increase in computational efficiency over the non-linear approach.

\begin{tabular}{|c|c||c|c||c|}
\hline \multicolumn{2}{|c||}{ linearised approach } & \multicolumn{2}{c|}{ non-linear approach } & \\
\hline \hline$p$ & Computational time $[\mathrm{s}]$ & $p$ & Computational time $[\mathrm{s}]$ & Speed-up \\
\hline 1 & 0.037 & 1 & 14.175 & 383 \\
2 & 0.181 & 2 & 49.327 & 273 \\
3 & 0.489 & 3 & 116.830 & 239 \\
4 & 1.150 & 4 & 206.424 & 179 \\
5 & 2.187 & 5 & 358.914 & 164 \\
\hline
\end{tabular}

Table 8.2: Test magnet problem: average computational times per timestep of the time dependant linearised and non-linear approaches in the time domain for specific element order $p=[1,2,3,4,5]$.

\subsection{Realistic Magnet Problem}

A more realistic problem is now considered that represents, very accurately, the sorts of MRI scanner designs currently used in clinical operation. The geometry is illustrated in Figure 8.14a. This problem consists of a similar construction to the previous problem, where the conducting region is comprised of the three radiation shields $\Omega_{c}=\Omega_{c}^{O V C} \cup \Omega_{c}^{77 K} \cup \Omega_{c}^{4 K}$, each with different material parameters $(\gamma, \mu, \lambda$, $G, \rho)$. The geometry of the radiation shields, however, is more complex and their topology represents that of closed cylindrical shells of trapezoidal cross section, with curved face end sections. However, despite the increased complexity in topology, the geometry is still cylindrical and can be treated as axisymmetric. Again, the exact geometries and material parameters of the conducting components are commercially sensitive and as such are not displayed in this Thesis. The configuration of the static main coil consists of the same block cross section as the test magnet problem, but contains more sets of coils including also a set of secondary coils, which act to minimise the magnetic stray field by reversing the polarity of the magnetic field. 
The gradient coils of this problem represent a far more realistic Z-gradient coil structure, which also contains a set of primary and secondary coils for shielding. The coils are sourced in the same way as the test magnet problem. The cross section of this problem, projected onto the positive half axisymmetric meridian domain $\Omega^{m}(r, z \geq 0)$, is illustrated in Figure 8.14b.

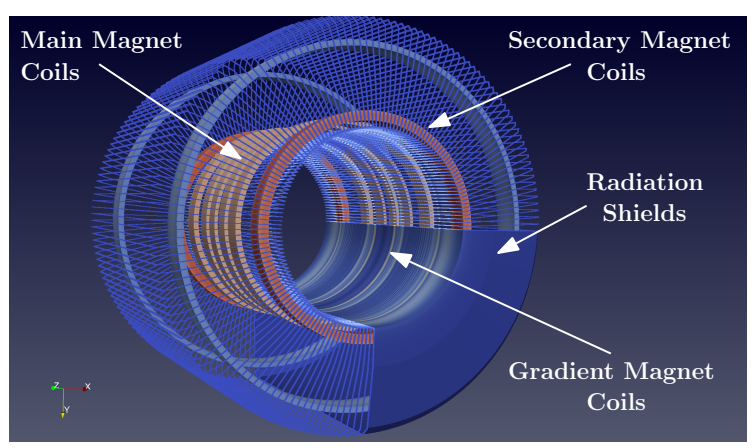

(a) $\mathbb{R}^{3}$ domain, $\Omega$

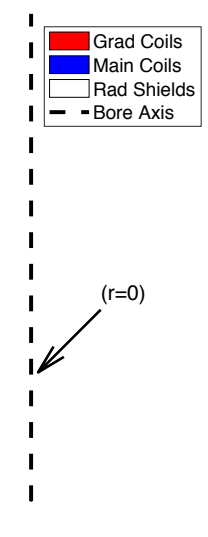

(b) Positive Half Meridian domain, $\Omega^{m}$

Figure 8.14: Realistic magnet problem: components of the simplified geometry.

Given criteria 2) and 3) in Section 6.5.2, the greatest non-linearity is expected to appear for a magnet with weakest static field of $1.5 \mathrm{~T}$ and strongest gradient field of $100 \times 10^{-3} \mathrm{~T} / \mathrm{m}$. This would result in a ratio between the static and gradient current density values of $\left|J_{\phi}^{A C}\right| /\left|J_{\phi}^{D C}\right| \approx 12.3 \%$ for this problem. The two approaches are therefore studied for an extreme case of a current density ratio of $\left|J_{\phi}^{A C}\right| /\left|J_{\phi}^{D C}\right|=$ $15 \%$ to rigorously test the linearised approach. The form of the excitation of $J_{\phi}^{A C}$ is as described in Section 8.2.

The non-conducting free space region, comprised of air, is truncated to create the domain $\Omega^{m}$ and boundary conditions similar to the test magnet problem are applied, whereby the Dirichlet boundaries of the conductors are set to $\boldsymbol{u}^{D}=\mathbf{0}$ to fix the conductors in space. The magnetic vector potential is set to $A_{\phi}=0$ on the outer boundary.

In terms of spatial discretisation, the solution is analysed for an unstructured mesh of 19,218 triangles of maximum size $h=0.25 \mathrm{~m}$, but with substantial refinement in the conductors $\Omega_{c}^{m}$, resulting in 4,085 elements in $\Omega_{c}^{m}$. A single layer of 40 infinite elements are applied on the outer boundary of $\Omega^{m}$ to resolve the static decay of the magnetic field. Having carried out similar convergence studies to those presented for the test magnet problem, $p=4$ elements have been chosen for the 
spatial discretisation of the problem, $N_{\Delta t}=30$ timesteps per excitation frequency $\omega$ for the temporal discretisation and set $\rho_{\infty}=0.8$ for the solutions to this problem.

Comparisons between the displacements of the mechanical shields are now preformed by plotting the velocity magnitude of the system across the time signal using both approaches for $\left|J_{\phi}^{A C}\right| /\left|J_{\phi}^{D C}\right|=15 \%$ and $\omega=2 \pi(1500) \mathrm{rad} / \mathrm{s}$ in Figure 8.15. Given that the differences in both the time signal and the displaced shapes of the OVC between the two approaches are in very close agreement, instead snapshots of the mechanical displacement in the inner OVC shell are highlighted at various time instances across the time signal for the linearised approach. The displaced OVC and static magnetic field for the corresponding gradient fields, at various time instances, are plotted in Figure 8.16. The displacements in Figures 8.15 and 8.16 are scaled by several orders of magnitude to show visually the displacement shapes of the OVC.

\subsection{Chapter Summary}

This Chapter has presented two industrially relevant benchmark MRI scanner problems to test the validity of the novel linearised approach across a range of MRI operating conditions that heavily exceed current MRI scanners. A series of tests using a range of measures of interest have been performed in order to test the linearity of the MRI problem and hence the accuracy of th linearised approach compared with the non-linear approach. Furthermore, the computational efficiency that the linearised approach provides over the non-linear approach has been measured for a range of spatial discretisations.

The results obtained, for the range of examples and operating conditions considered, show that the linearised approach offers very good agreement with the nonlinear approach and thus aligns with the objective "To determine the accuracy of the linearised approach across the full operating range of MRI scanners."

The next Chapter summarises the contents of the Thesis and provides a series of conclusions and outcomes of the work performed. The Chapter then goes on to present a series of recommendations for future work that will build on the underlying concepts provided within this Thesis and further enhance the created computational model. 


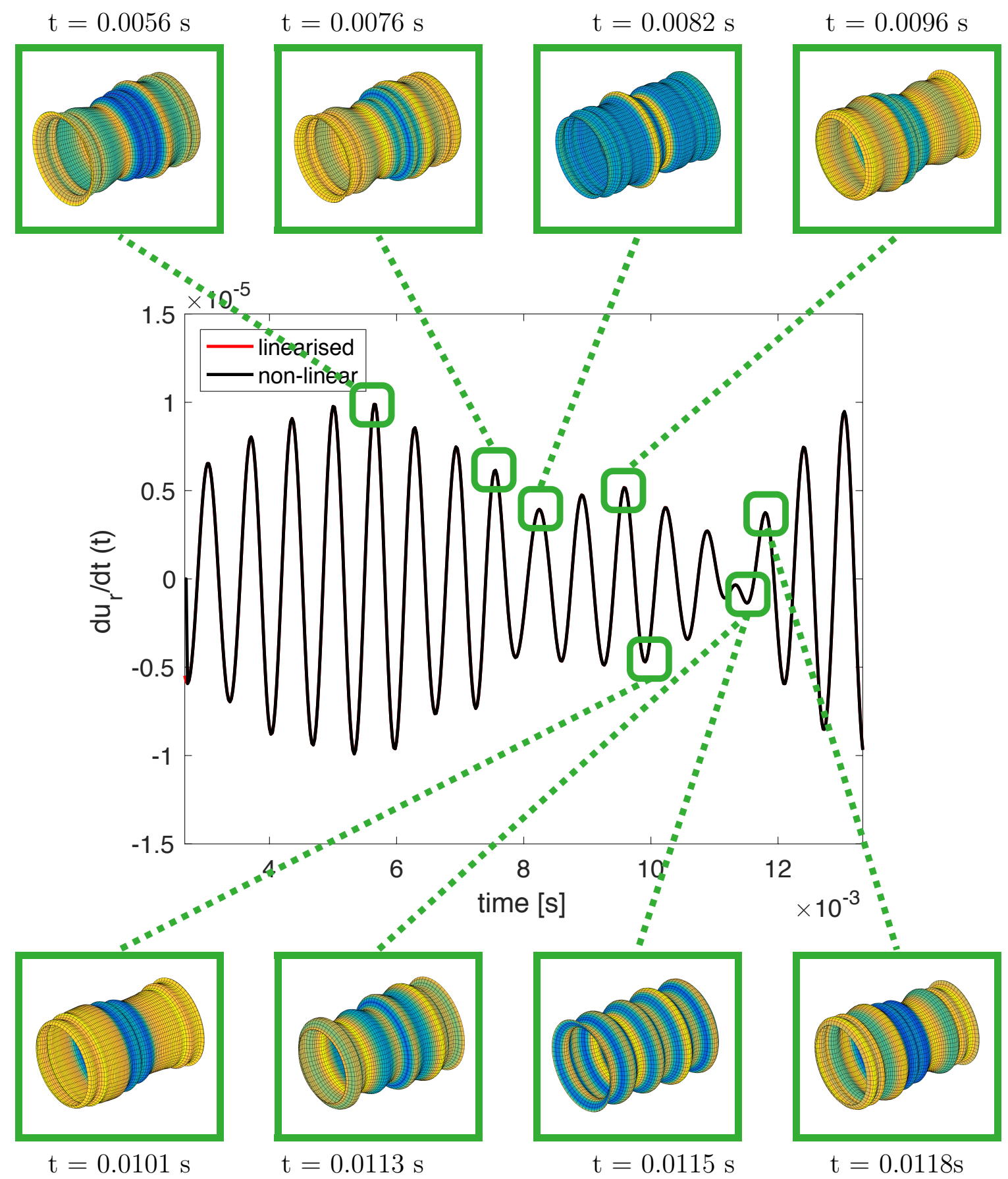

Figure 8.15: Full magnet problem: displacements of the OVC at different times for $\left|J_{\phi}^{A C}\right| /\left|J_{\phi}^{D C}\right|=15 \%$ and $\omega=2 \pi(1500) \mathrm{rad} / \mathrm{s}$ for both the time dependant linearised and nonlinear approaches. 


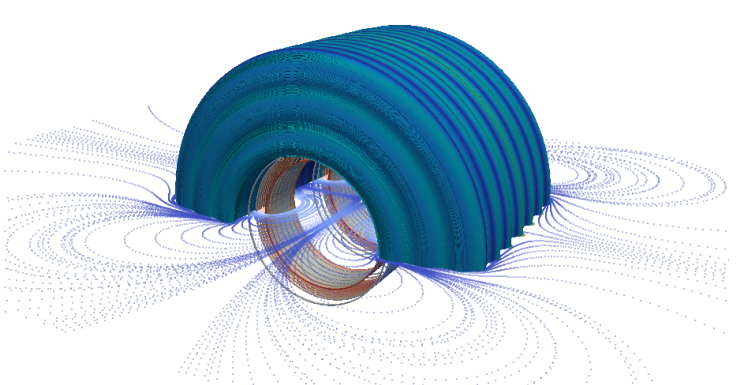

(a) $\mathrm{t}=0.0056 \mathrm{~s}$

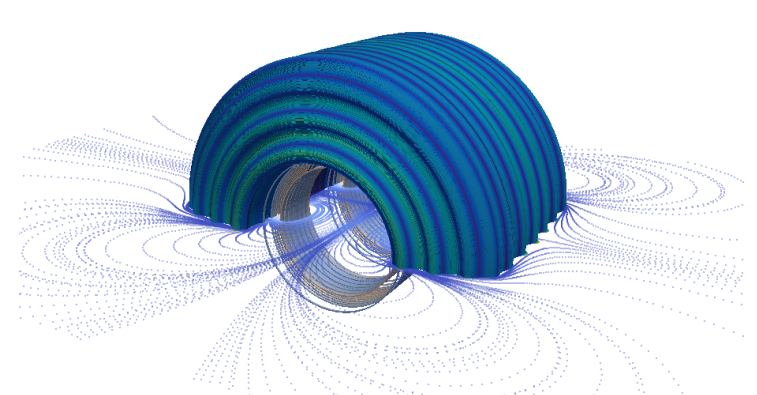

(c) $\mathrm{t}=0.0082 \mathrm{~s}$

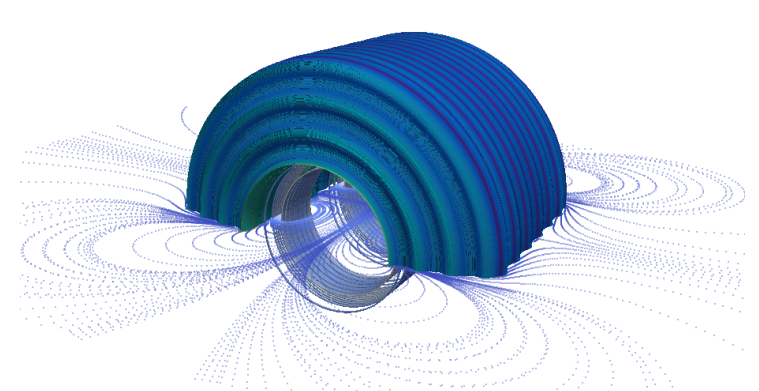

(e) $\mathrm{t}=0.0101 \mathrm{~s}$

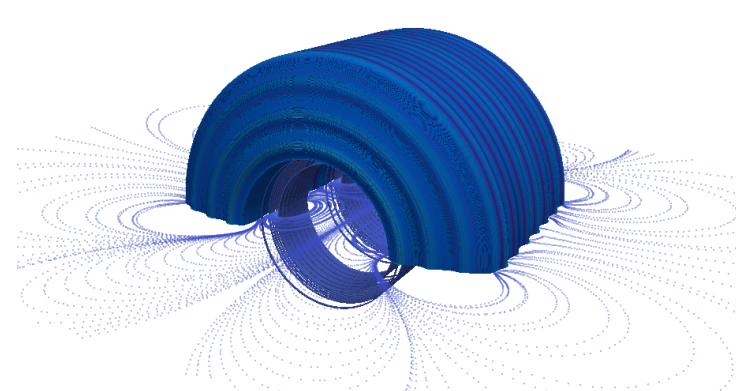

(g) $\mathrm{t}=0.0115 \mathrm{~s}$

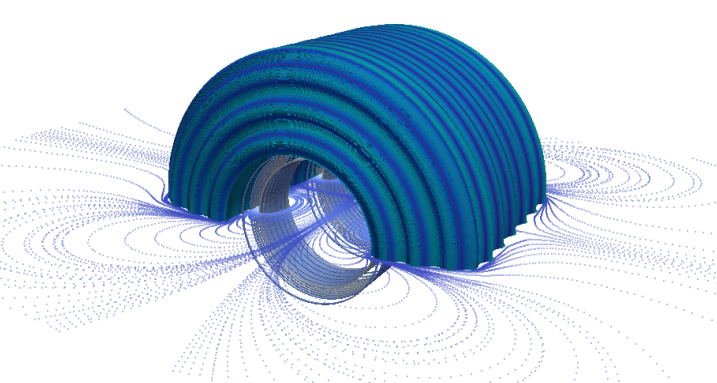

(b) $\mathrm{t}=0.0076 \mathrm{~s}$

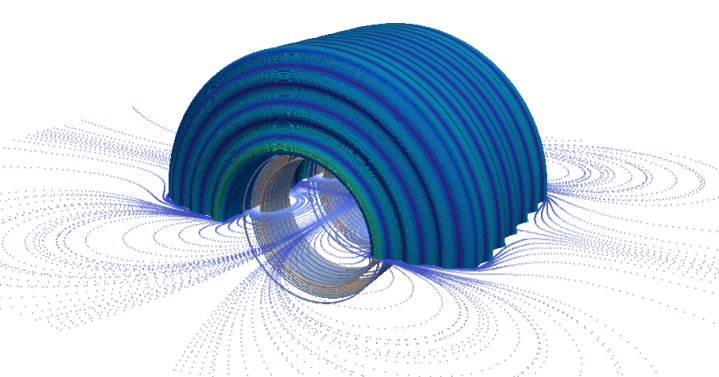

(d) $\mathrm{t}=0.0096 \mathrm{~s}$

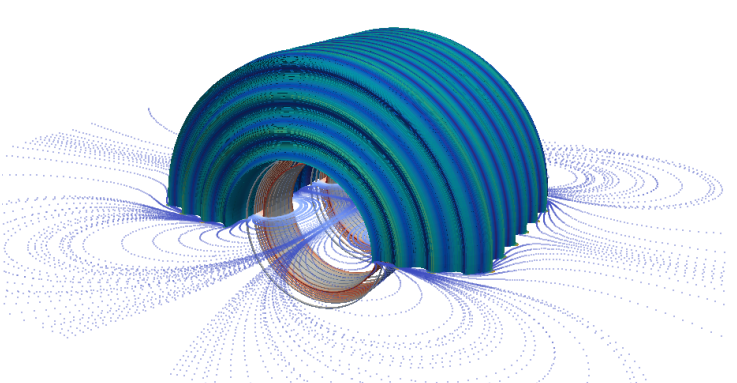

(f) $\mathrm{t}=0.0113 \mathrm{~s}$

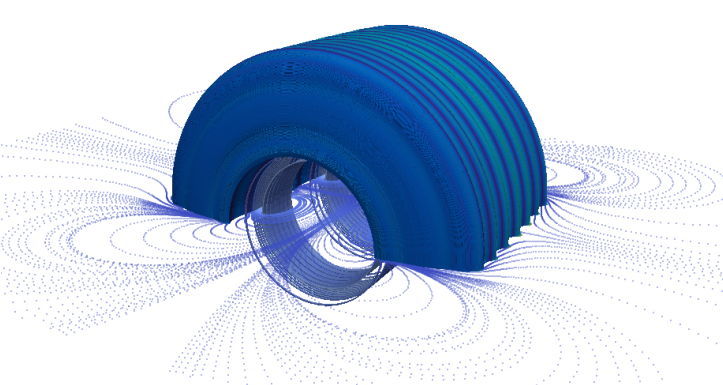

(h) $\mathrm{t}=0.0118 \mathrm{~s}$

Figure 8.16: Full magnet problem: snapshots of the distorted OVC, gradient coils and corresponding gradient magnetic field lines at various time intervals for $\left|J_{\phi}^{A C}\right| /\left|J_{\phi}^{D C}\right|=15 \%$ and $\omega=2 \pi(1500) \mathrm{rad} / \mathrm{s}$. 


\section{Part V}

Conclusions and Further Work 



\section{Chapter 9}

\section{Conclusions and Future Development}

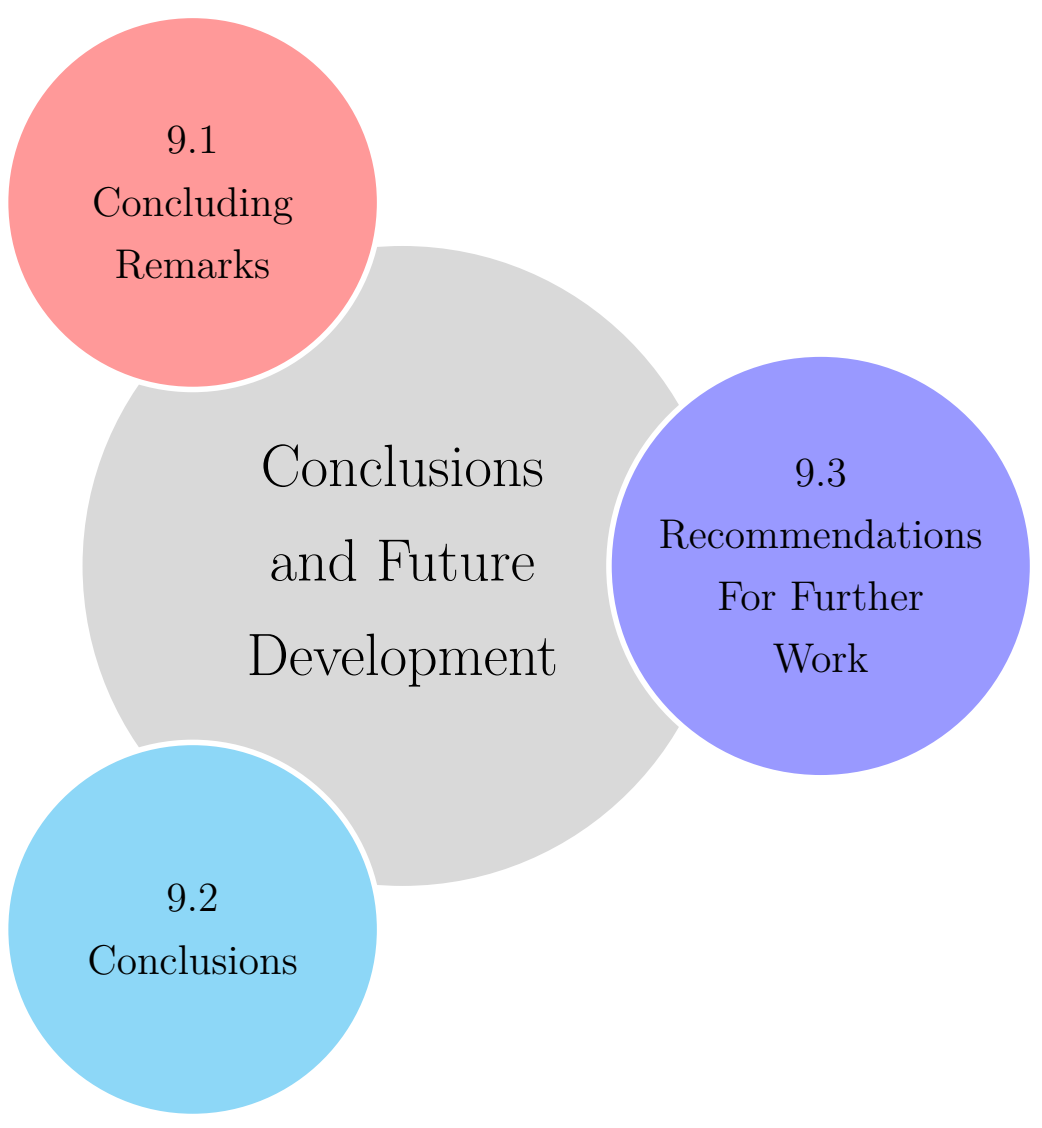

"Don't cry because it's over, smile because it happened." 


\subsection{Concluding Remarks}

This Thesis has presented the formulation and efficient computational implementation of both a novel linearised and non-linear approach, using an efficient $h p$-finite element framework, for the solution to coupled acousto-magneto-mechanical problems for MRI scanner design. The novel linearised approach has been rigorously compared against the full non-linear approach to determine its validity in solving the full MRI scanner problem for a range of operating conditions, which both mimic and exceed the current operable limits of MRI scanners in clinical operation. This comparison has been performed both through a novel mathematical comparison of the two approaches and through a series of industrially applicable examples. The aims and objectives outlined in Chapter 1 have been fully achieved. In the following sections, several conclusions are made and some recommendations for further work are given.

\subsection{Conclusions}

The governing coupled acousto-magneto-mechanical transmission problems, for both the static and transient fields, and the coupling mechanisms between the individual physical fields have been successfully derived in the full 3D representation.

- In Chapter 2, the mathematical description of the individual physical fields has been presented and the coupling mechanisms identified and derived. By means of a physically motivated additive split approach of the current sources of an MRI scanner two fully coupled transmission problems were derived: static and transient. These two problems have been used to form the novel linearised approach. This Chapter successfully addresses the objective "To accurately describe the individual physical fields and the coupling mechanisms associated with MRI scanners."

The computationally efficient linearised approach has been successfully established in both a time dependent and efficient time harmonic representation. The full 3D description of the formulation and the rigorous linearisation has been developed where the computational $h p$-finite element approach has been reduced to an axisymmetric representation to provide an efficient reduced order computational model. 
- In Chapter 3, the weighted residual forms of both the static and transient coupled transmission problems have been derived. A rigorous linearisation of the weighted residuals has also been established to represent the coupled problem in terms of an iterative monolithic Newton-Raphson procedure. The previous additive splitting of the current sources, presented in Chapter 2, has been used to derive the novel linearised approach, where the resulting linearised terms are independent of time and permit the use of a single step monolithic solution strategy [26]. Based on this fact, the linearised approach has been successfully presented in terms of a time harmonic formulation to allow for rapid sweeps over excitation frequencies to be performed. This approach provides a far more computationally efficient algorithm over solving the full non-linear problem. This Chapter addresses the objective "To develop an efficient novel solution procedure, named the linearised approach, through a rigorous linearisation of the non-linear system of equations."

- In Chapter 4, the axisymmetric representation of the fields has been presented. Instead of adjusting the finite element basis functions to satisfy the axisymmetric weighted spaces, a suitable scaling of the variables has been performed to permit the use of standard $H^{1}(\Omega)$ conforming basis functions. The treatment of the radiation conditions, for both the electromagnetic and acoustic fields, in unbounded domains are then suitably approximated for artificially truncated finite domains. The topic of $h p$-finite elements is then briefly discussed, where the high order shape functions are introduced and the suitable mapping functions to the reference finite elements are applied. The spatially discrete axisymmetric linearised approach to the coupled acousto-magneto-mechanical problem to both the static and transient fields are then presented. This Chapter addresses the objective "To provide an efficient computational methodology for handling the solution to the coupled problem of MRI scanners using both approaches."

- In Chapter 5, a series of academic and industrial benchmark numerical examples are then presented. First, a series of single physics problems are considered to validate the implementation, through the evaluation of the performance of the numerical approximation against analytical solutions. The correct optimal convergence behaviours for both the $h$ - and $p$-refinement are illustrated. The successful resolution of the small skin depths and wavelengths using high order elements are shown. A series of coupled multiphysics benchmark examples are then analysed that demonstrate the rapid and accurate predictive performance 
of the fully coupled linearised approach. This Chapter addresses the objective "To determine the accuracy of the linearised approach across the full operating range of MRI scanners."

The non-linear approach has been successfully established using an iterative monolithic Newton-Raphson procedure and a time integration scheme to evaluate the transient response of the fields. The time dependent formulation of the linearised approach has also been successfully established to allow for direct comparison between the two approaches in the time domain.

- In Chapter 6, the treatment of the non-linear approach has been established. The resulting linearised system for both approaches has been recast in the form of a second order dynamic system, where the terms represent the mass, stiffness and damping contributions. This form allows for the natural application of time integration schemes. A comparison of the energy associated with the two approaches has been performed to derive a set of rigorous bounds used to define a series of theoretical measures that determine the non-linearity of the problem. These measures can later be used to determine the validity of the linearised approach. A simplified model which relates these measures to industrial data has also been presented, which is useful to determine the range of operating conditions over which to compare the two approaches. This Chapter addresses both the objectives "To develop the complete non-linear approach to solving the coupled system of equations" and "To determine a series of theoretical measures that can be used to measure the non-linearity of the coupled problem in MRI scanners."

- In Chapter 7, the computational treatment of the transient fields for both approaches in a time domain formulation using a generalised $\alpha$ time integration scheme has been presented. A generalised solution strategy described through a computational algorithm is presented. This Chapter addresses the objective "To provide an efficient computational methodology for handling the solution to the coupled problem of MRI scanners using both approaches."

- In Chapter 8, a series of numerical examples of a simplified and a realistic MRI scanner geometry have been studied. The theoretical measures and simplified model, derived in Chapter 6, have been successfully applied to each example problem to determine the range of operating conditions that current MRI scanners operate within. A series of numerical studies have been performed both within and exceeding current operable limits and the non-linearity of 
the problem has been successfully quantified. These numerical studies have successfully shown the validity of the linearised approach in accurately approximating the full non-linear problem for current and future MRI scanner designs. A summary of the computational saving of the linearised approach has also been provided, where significant computational saving is achieved. This Chapter addresses the objective "To determine the accuracy of the linearised approach across the full operating range of MRI scanners."

In summary, the $h p$ finite element framework for coupled acousto-magnetomechanical coupling in MRI scanners has been successfully applied to a range of numerical problems and accurate results have been obtained. The small scale phenomena of the physical fields have been accurately resolved using the high order finite elements and accurate approximations of the farfield boundary conditions have been achieved. The novel computationally efficient linearised approach and the non-linear approach have been benchmarked and applied to full MRI scanner geometries and a comparison over a range of operating conditions have shown that: 1) there is accurate agreement between the two approaches and that, not only does the linearised approach provide accurate approximations to the transient response of the fields, but it also accurately predicts the quantities of interest and the resonance behaviour of MRI scanners; and 2) the linearised approach provides accurate results across the full region of interest in the frequency spectrum for a range of current density ratios $J_{\phi}^{A C} / J_{\phi}^{D C} \leq 20 \%$. In terms of current clinical MRI scanner applications, this ratio is restricted to around $4-12 \%$ and thus the analysis validates the use of the linearised approach in providing accurate solutions in current and future MRI scanner design, with orders of magnitude saving in the computational cost over the non-linear approach.

\subsection{Recommendations For Further Work}

Given the achievements of the research performed in this Thesis, there are many potential avenues for further work to be performed in order to further enhance the capabilities of the current methodology presented.

- One avenue for further research is the enhancement of the material model in the coupled formulation. Currently, the materials considered are based on homogeneous and isotropic definitions. However, with the advancement in materials science, new materials are being developed that could significantly 
impact the design of current MRI scanners and further enhance their performance. Reductions in cost, increases in material performance and vacuum integrity are all factors that will impact the future of magnet design for MRI scanners. The inclusion of inhomogeneous and anisotropic [232, 130] material models $[96,188,88]$ will allow for a greater range of materials to be implemented into the code and further improve the capabilities.

- Another area of research where significant enhancements in the model can be made is in the area of reduced order modelling, specifically in the application of Proper Generalised Decomposition (PGD) [53, 54]. The PGD allows the full problem to be decomposed into functions of the spatial domain and the parametric domain, which could include time/frequency, material parameters or a combination of these. The method computes a series of "off-line" solutions, or modes, in order to obtain sufficient data for interpolation of the solution to compute the "on-line" solutions. Given that the novel linearised approach poses a new linear system for the coupled system of non-linear equations, the system matrices (mass, stiffness and damping) are independent of time/frequency and NR iteration, and so they can be used to obtain the spatial discretisation and the PGD applied to solve for the parameter. This method will allow for almost real-time interpolation to perform rapid parametric studies of a range of scanner configurations.

- Another possible avenue of research is the inclusion of thermal effects, through an additional field in the system. Inside the Helium vessel (or $4 K$ shield), the main magnet coils are immersed in liquid helium to supercool them. Having already computed the energy transfer to the coils from the magnetic field in this work, the next step would be to compute any thermal energy transfer within the vessel due to heat radiation etc.

- A further topic of research would be to allow for the simulation of the nonaxisymmetric current sources in the $x$ and $y$ gradient coils. The current axisymmetric formulation can be further exploited through the decomposition of the current source into a series of Fourier coefficients in the azimuthal direction. The full problem could then be reduced to solving for a series of axisymmetric problems related to each Fourier coefficient, where the solution fields would have all three vector components, see [112] for details on similar methods. 
- Finally, the generalisation of the current model to solving the full threedimensional problem will provide a further possible avenue for research. This Thesis presents the fully 3D formulation of the problem, to which an axisymmetric representation is applied to develop an efficient reduced order axisymmetric model. Solving directly for the full 3D problem will provide a highfidelity computational tool, where similar high order finite element discretisations can be used, to determine the behaviour of non-axisymmetric geometries and complete MRI scanner models. 



\section{Part VI}

Appendices 



\section{Appendix A}

\section{Explanation of Key Physical Phenomena}

When describing the complex physical behaviour of the transient coupled system of acousto-magneto-mechanical equations several known physical phenomena become active. These phenomena provide mechanisms for a two-way coupling between the magnetic and mechanical fields, resulting in the magneto-mechanical coupled system, with the acoustics being excited as a result of these phenomena. In order to fully understand the complex behaviour of the multi-field system in an MRI scanner, the effects that moving conductors have on the magnetic field and varying magnetic fields have on conductors must be understood. This Appendix describes the key physical phenomena and coupling mechanisms between the magnetic and mechanical fields and enriches the topics discussed in Chapters 1 and 2.

\section{A.1 Eddy Currents}

Eddy (or Ohmic) Currents are electrical current loops that occur in conducting bodies when time varying magnetic fields cause closed current loops inside conductors to form. The eddy currents flow in closed loops inside of conductors in planes perpendicular to the magnetic field. Eddy currents themselves also generate magnetic fields that oppose the time varying magnetic field that generated them. The eddy current density can thus be expressed as

$$
\boldsymbol{J}^{e}(t)=\gamma \boldsymbol{E}(t)
$$

where $\gamma$ is the conductivity of the medium and $\boldsymbol{E}$ is the electric field. The electric field can then be expressed in terms of the magnetic flux density field $\boldsymbol{B}$ by Faraday's law of induction as

$$
\nabla \times \boldsymbol{E}=-\frac{\partial \boldsymbol{B}}{\partial t} .
$$

Figure A.1 illustrates eddy currents forming inside of a conductor when subject to a time varying magnetic field $\boldsymbol{H}(t)=\mu^{-1} \boldsymbol{B}(t)$ that is excited by an alternating current $\boldsymbol{J}^{A C}$, passed through a set of coils, where $\mu$ is the magnetic permeability 


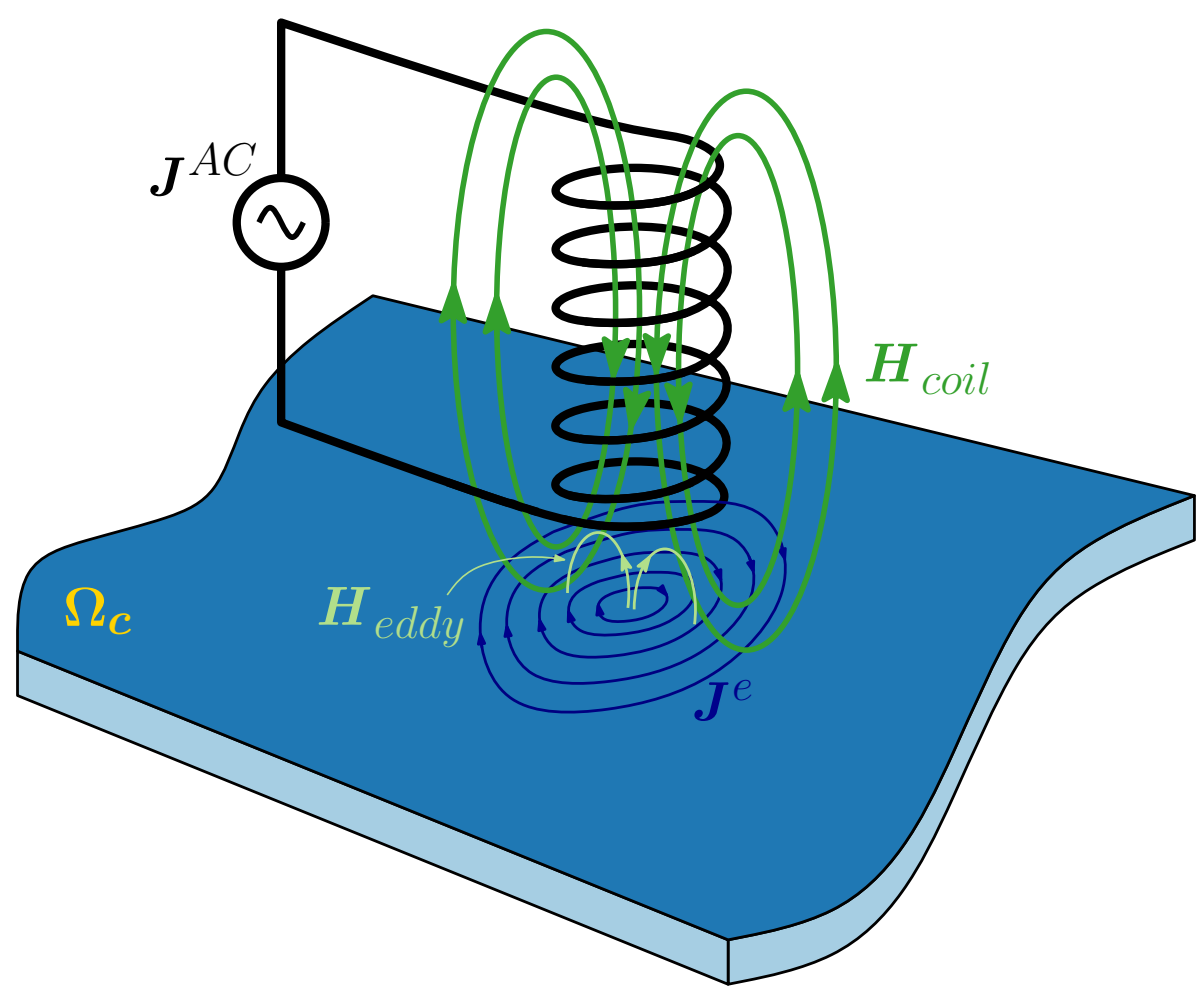

Figure A.1: Eddy currents $\boldsymbol{J}^{e}$ arising from time varying magnetic field.

of the medium. This represents a similar setup to that of an MRI scanner, where conductors (metallic radiation shields) are subject to an alternating magnetic field generated through AC current sources in the gradient coils. The eddy currents themselves generate their own magnetic field $\boldsymbol{H}_{e d d y}$ which perturbs the field generated by the coils $\boldsymbol{H}_{\text {coil }}$.

\section{A.2 Skin Depth Effect}

The skin effect is an effect whereby the electrical current, inside of a conductor, tends to become distributed such that the current density is largest at the surface and decreases with depth in the conductor. This distribution of the currents is due to the generation of eddy currents inside of conductors. The eddy currents are generated by time varying magnetic fields arising in the conductor and act to oppose the currents inside the body of the conductor and complement the currents towards the surface, illustrated in Figure A.2. As a result of the eddy currents the time varying magnetic field does not completely permeate the conductor.

The electric current flows primarily close to the surface of the conductor, in a layer between the surface and a depth called the skin depth. An alternating current density $\boldsymbol{J}$ inside a conductor decreases exponentially from its value at the surface $\boldsymbol{J}_{S}$ according to the depth from the surface $d$ as

$$
\boldsymbol{J}=\boldsymbol{J}_{S} e^{-(1+\mathrm{i}) d / s},
$$




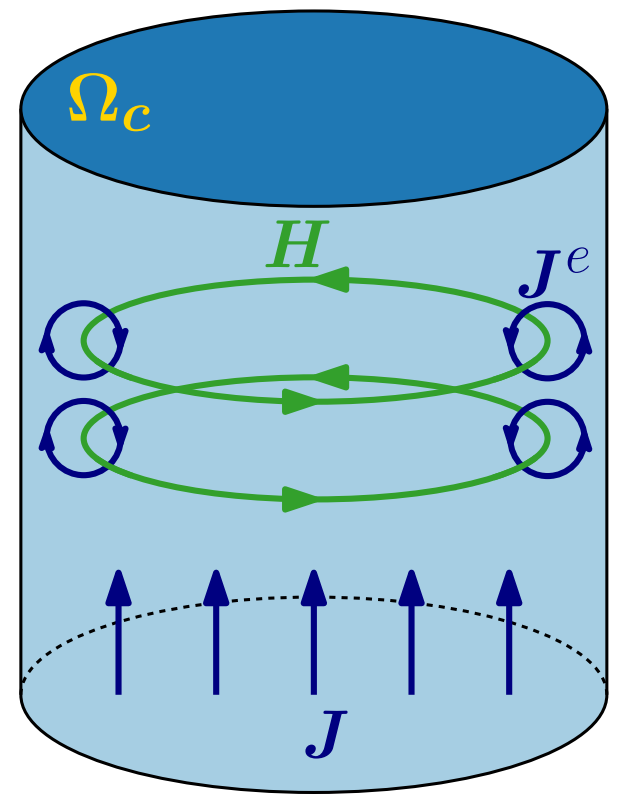

Figure A.2: Circulating eddy currents induced by a changing $\boldsymbol{H}$ field inside a conductor. The eddy currents $\boldsymbol{J}^{e}$ reinforce the currents at the surface and oppose the currents towards the centre of the conductor.

where $s$ denotes the skin depth ${ }^{1}$.

The skin depth is thus defined as the depth below the surface of the conductor at which the current density has fallen to $1 / e$ (about 0.37 ) of $\boldsymbol{J}_{S}$. The relation for the skin depth of a conductor is thus given by

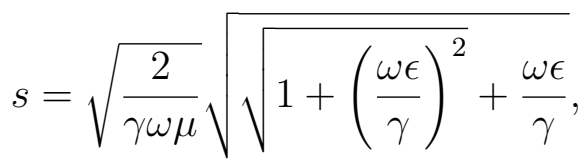

where $\omega$ is the angular frequency of the current, measured in $\mathrm{rad} / \mathrm{s}, \mu$ the permeability, $\gamma$ the conductivity and $\epsilon$ the permittivity of the conductor. At frequencies much below $\gamma / \epsilon$ the square root term is close to unity and thus the skin depth is approximated as

$$
\delta=\sqrt{\frac{2}{\gamma \omega \mu}} .
$$

The current distribution inside of a conductor $\Omega_{c}$, with thickness thick, is illustrated in Figure A.3.

\section{A.3 Lorentz Currents}

Lorentz currents (sometimes also referred to as induced eddy currents) are closed electrical current loops that form inside of a conductor when moving through a mag-

\footnotetext{
${ }^{1}$ The skin depth is often referred to in the literature as $\delta$, however, given that $\delta$ is reserved for the incremental update field variables the skin depth is denoted $s$.
} 


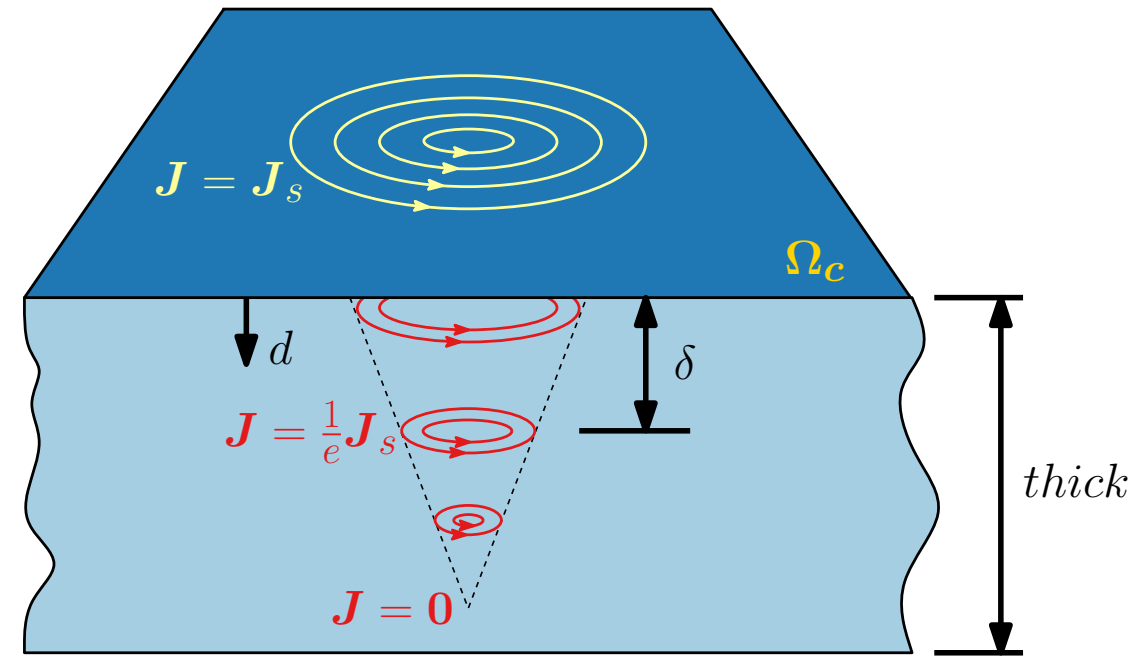

Figure A.3: Current distribution through the cross section of a conductor $\Omega_{c}$ of thickness thick. The current strength decays asymptotically to 0 from the surface.

netic field. The movement of the conductor causes a magnetic field that effectively varies in time and thus produces a current loop in the conductor. This current loop also results in a magnetic field which opposes the main field. The Lorentz current is quantified as

$$
\boldsymbol{J}^{l}(t)=\gamma \boldsymbol{v}(t) \times \boldsymbol{B}
$$

where $\gamma$ is the electrical conductivity of the medium. Figure A.4, below, illustrates a conducting plate $\Omega_{c}$ moving with velocity $\boldsymbol{v}$ through a static magnetic field with flux density $\boldsymbol{B}$. The dark navy lines illustrate the Lorentz currents inside the conductor.

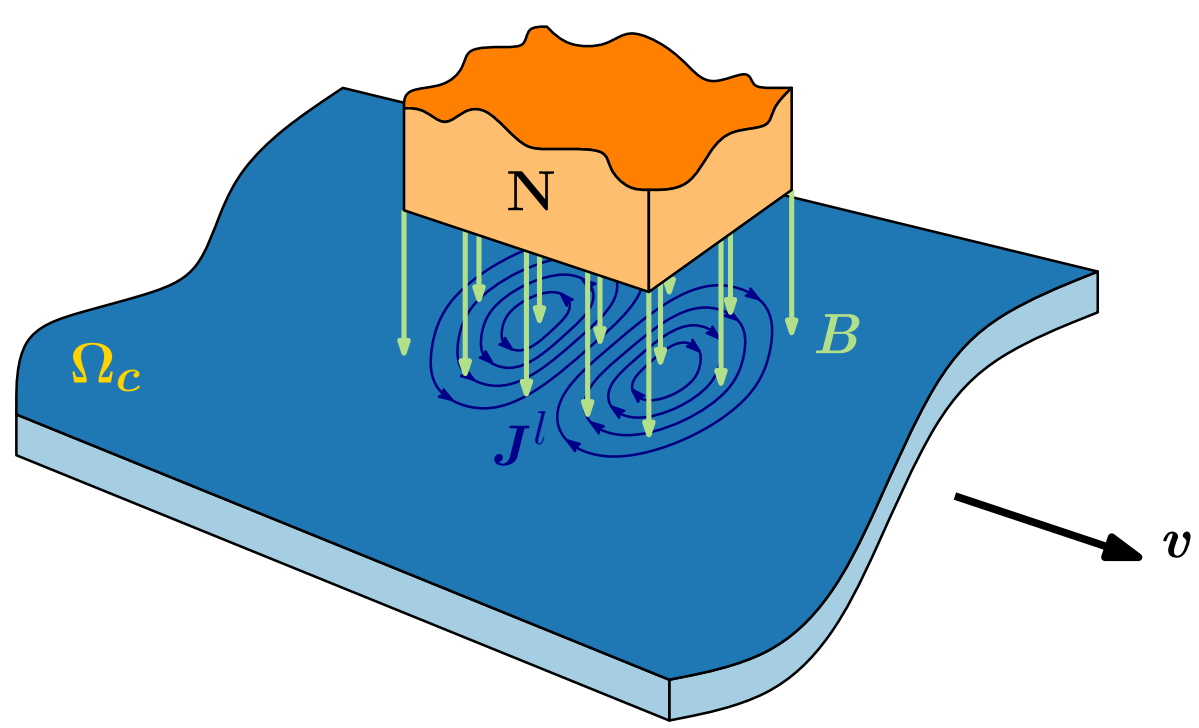

Figure A.4: Lorentz currents $\boldsymbol{J}^{l}$ induced by moving a conductor $\Omega_{c}$ through a magnetic field, with flux density $\boldsymbol{B}$, at a velocity $\boldsymbol{v}$. 


\section{A.4 Electromotive Force}

The electromotive force is the force exerted on a charge, or more generally a volume of charge such as a conductor, moving through a magnetic field. From the Lorentz force law [100], the electromotive force on a unit of charge is given by

$$
\boldsymbol{f}=q(\boldsymbol{E}+\boldsymbol{v} \times \boldsymbol{B}),
$$

where $q$ denotes a unit of charge, $\boldsymbol{E}$ is the electric field, $\boldsymbol{B}$ the magnetic flux density field and $\boldsymbol{v}$ the velocity of the charge. The electromotive force per unit volume on a distribution of charge is given by

$$
\boldsymbol{f}=\rho_{V}(\boldsymbol{E}+\boldsymbol{v} \times \boldsymbol{B}),
$$

where $\rho_{V}$ is the volume charge density and $\boldsymbol{J}=\rho_{V} \boldsymbol{v}$ the current density.

The electromotive force, which is perpendicular to $\boldsymbol{B}$ and $\boldsymbol{v}$, acting on a conductor moving through a magnetic flux density field is illustrated in Figure A.5.

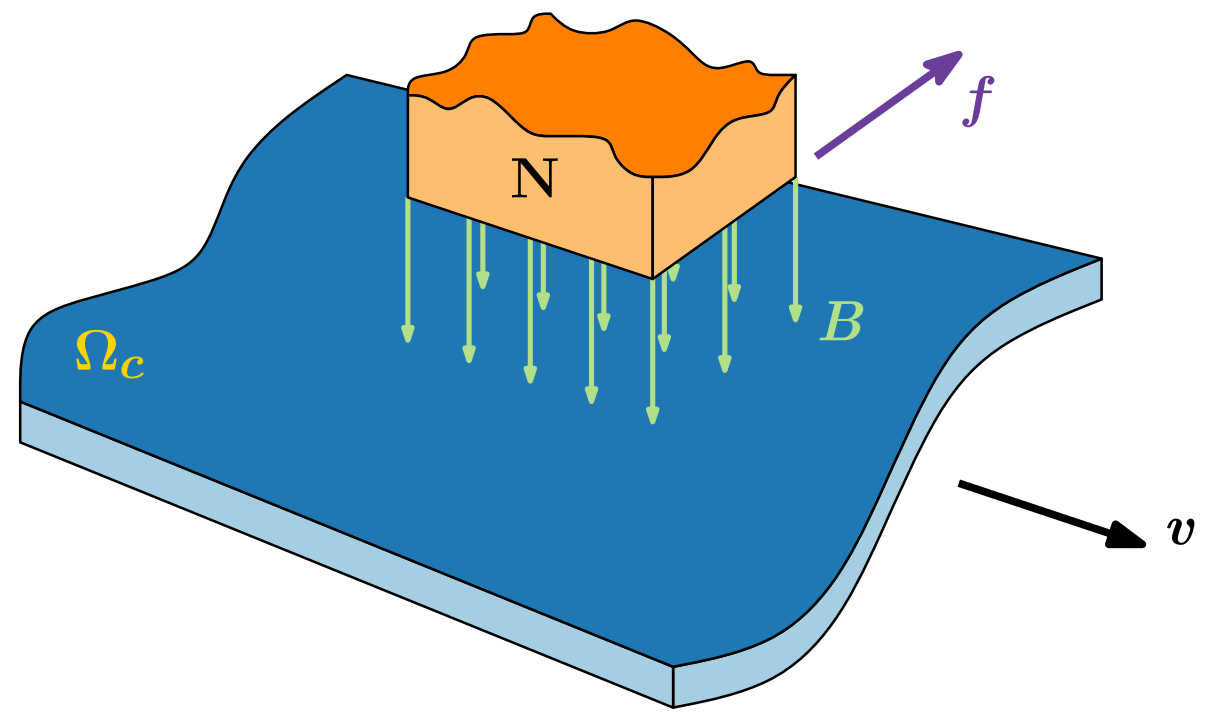

Figure A.5: Electromotive (Lorentz) forces $\boldsymbol{f}^{e}$ induced by moving a conductor $\Omega_{c}$ through a magnetic field, with flux density $\boldsymbol{B}$, at a velocity $\boldsymbol{v}$.

\section{A.5 Electromagnetic (Maxwell) Stress}

The electromagnetic stress, described through the Maxwell stress tensor, is the stress that a magnetic field imparts on a conductor. The electromotive force in (A.8), can be re-expressed only in terms of the field variables by replacing $\rho_{V}$ and $\boldsymbol{J}$ by the fields $\boldsymbol{E}$ and $\boldsymbol{B}$, using Gauss' and Ampére's law, in (2.1d) and (2.1a) to give

$$
\boldsymbol{f}=\rho_{V} \boldsymbol{E}+\boldsymbol{J} \times \boldsymbol{B}
$$




$$
=\epsilon(\nabla \cdot \boldsymbol{E}) \boldsymbol{E}+\mu^{-1}(\nabla \times \boldsymbol{B}) \times \boldsymbol{B}-\epsilon \frac{\partial \boldsymbol{E}}{\partial t} \times \boldsymbol{B} .
$$

In the above and throughout, the materials will be assumed as isotropic and homogeneous and as such all electro- and magneto-strictive effects will be neglected.

Applying now the product rule and Faraday's law, in $(2.1 \mathrm{~b})$, to the last term in (A.9) yields

$$
\boldsymbol{f}=\epsilon(\nabla \cdot \boldsymbol{E}) \boldsymbol{E}+\mu^{-1}(\nabla \times \boldsymbol{B}) \times \boldsymbol{B}-\epsilon\left(\frac{\partial}{\partial t}(\boldsymbol{E} \times \boldsymbol{B})+\boldsymbol{E} \times(\nabla \times \boldsymbol{E})\right) .
$$

Adding the term $(\nabla \cdot \boldsymbol{B}) \boldsymbol{B}=0$, due to Gauss' law of magnetism in (2.1c), and gathering the terms involving $\boldsymbol{E}$ and $\boldsymbol{B}$ gives

$$
\begin{array}{r}
\boldsymbol{f}=\epsilon((\nabla \cdot \boldsymbol{E}) \boldsymbol{E}-\boldsymbol{E} \times(\nabla \times \boldsymbol{E}))+\mu^{-1}((\nabla \cdot \boldsymbol{B}) \boldsymbol{B}-\boldsymbol{B} \times(\nabla \times \boldsymbol{B})) \\
-\epsilon \frac{\partial}{\partial t}(\boldsymbol{E} \times \boldsymbol{B}),
\end{array}
$$

where the curls can be eliminated ${ }^{2}$ to yield

$$
\begin{array}{r}
\boldsymbol{f}=\epsilon((\nabla \cdot \boldsymbol{E}) \boldsymbol{E}-\boldsymbol{E} \cdot \nabla \boldsymbol{E})+\mu^{-1}((\nabla \cdot \boldsymbol{B}) \boldsymbol{B}-\boldsymbol{B} \cdot \nabla \boldsymbol{B}) \\
-\frac{1}{2} \nabla\left(\epsilon(\boldsymbol{E} \cdot \boldsymbol{E})+\mu^{-1}(\boldsymbol{B} \cdot \boldsymbol{B})\right)-\epsilon \frac{\partial}{\partial t}(\boldsymbol{E} \times \boldsymbol{B}) .
\end{array}
$$

Then electromagnetic body force can then be expressed in terms of the divergence of an electromagnetic (Maxwell) stress tensor ${ }^{3}$ as

$$
\boldsymbol{f}=\nabla \cdot \boldsymbol{\sigma}^{e}(\boldsymbol{E}, \boldsymbol{B})+\epsilon \frac{\partial}{\partial t}(\boldsymbol{B} \times \boldsymbol{E}),
$$

which is similar to the result obtained in [80], but for homogeneous isotropic materials. In the above, the Maxwell stress tensor can be split into an electric and magnetic part as

$$
\begin{aligned}
\boldsymbol{\sigma}^{e}(\boldsymbol{E}, \boldsymbol{B}) & =\boldsymbol{\sigma}_{E}^{e}(\boldsymbol{E})+\boldsymbol{\sigma}_{M}^{e}(\boldsymbol{B}) \\
& =\epsilon\left(\boldsymbol{E} \otimes \boldsymbol{E}-\frac{1}{2}(\boldsymbol{E} \cdot \boldsymbol{E}) \boldsymbol{I}\right)+\mu^{-1}\left(\boldsymbol{B} \otimes \boldsymbol{B}-\frac{1}{2}(\boldsymbol{B} \cdot \boldsymbol{B}) \boldsymbol{I}\right),
\end{aligned}
$$

which depends on both the electric and magnetic flux density fields respectively.

\footnotetext{
${ }^{2}$ Recalling here the relation $\frac{1}{2} \nabla(\boldsymbol{A} \cdot \boldsymbol{A})=\boldsymbol{A} \times(\nabla \times \boldsymbol{A})+\boldsymbol{A} \cdot \nabla \boldsymbol{A}$.

${ }^{3}$ Recalling here the relation $\stackrel{2}{\nabla} \cdot(\boldsymbol{A} \otimes \boldsymbol{A})=(\nabla \cdot \boldsymbol{A}) \boldsymbol{A}+\boldsymbol{A} \cdot \nabla \boldsymbol{A}$.
} 


\section{Appendix B}

\section{Interface Conditions}

When describing a complex problem in its strong form a series of differential Equations governing the behaviour of systems are required. This strong form is derived from the governing set of integral Equations and must hold throughout the space in which the problem is valid. For complex domains consisting of multiple mediums with different material properties, the description of the physical behaviour of the fields at the interfaces are non-trivial. In order to fully represent these problems in their strong form one requires knowledge of the continuity in the fields across these interfaces, an important circumstance which is often overlooked. This Appendix describes the process of obtaining the interface conditions from the integral form of the governing Equations and enriches the topics discussed in Chapter 2.

\section{B.1 Electromagnetic Interface Conditions}

Determining the interface conditions in the electromagnetic fields requires the full set of transient Maxwell's Equations to be described, in terms of their integral forms. This system is defined as

$$
\begin{aligned}
\oint_{\partial \Sigma} \boldsymbol{E} \cdot \boldsymbol{t} \mathrm{d} l & =-\frac{\mathrm{d}}{\mathrm{d} t} \int_{\Sigma} \boldsymbol{B} \cdot \boldsymbol{n} \mathrm{d} S \\
\oint_{\partial \Sigma} \boldsymbol{H} \cdot \boldsymbol{t} \mathrm{d} l & =\int_{\Sigma} \boldsymbol{J} \cdot \boldsymbol{n} \mathrm{d} S+\frac{\mathrm{d}}{\mathrm{d} t} \int_{\Sigma} \boldsymbol{D} \cdot \boldsymbol{n} \mathrm{d} S \\
\oint_{\partial \Omega} \boldsymbol{D} \cdot \boldsymbol{n} \mathrm{d} S & =\int_{\Omega} \rho_{V} \mathrm{~d} \Omega \\
\oint_{\partial \Omega} \boldsymbol{B} \cdot \boldsymbol{n} \mathrm{d} S & =0
\end{aligned}
$$

where $\Omega$ denotes a volume, enclosed by a surface $\partial \Omega$ and $\Sigma$ denotes a surface enclosed by a contour $\partial \Sigma$. In the above, $\boldsymbol{E}, \boldsymbol{H}, \boldsymbol{D}$ and $\boldsymbol{B}$ are the electric, magnetic, electric flux and magnetic flux intensity field vectors, respectively, $\boldsymbol{J}$ the current density vector, $\rho_{V}$ the volume charge density and $\boldsymbol{n}$ and $\boldsymbol{t}$ denote the unit normal and tangential vectors, respectively.

The differential form of Maxwell's system of Equations, stated in Chapter 2, is obtained through manipulation of the integral form in (B.1). The Equations in- 
volving the curl of the vector fields, Ampére's and Faraday's law, are obtained by applying Stokes' theorem ${ }^{1}$ to the contour integral terms in Equation (B.1a) and (B.1b) and realising they must hold for every $\Sigma$. The Equations involving the divergence of the vector fields, Gauss' law and Gauss' law for magnetism, are obtained by applying the divergence theorem ${ }^{2}$ to the surface integral terms in Equation (B.1c) and (B.1d) and realising they must hold for every $\Omega$. [100].

In order to determine the continuity conditions of the electromagnetic fields at the interface $\partial \Omega^{i n t}$, between two mediums $\Omega_{1}$ and $\Omega_{2}$, a closed loop contour $\partial \Sigma$ enclosing a surface $\Sigma$ around $\partial \Omega^{i n t}$, as illustrated in Figure B.1, is considered.

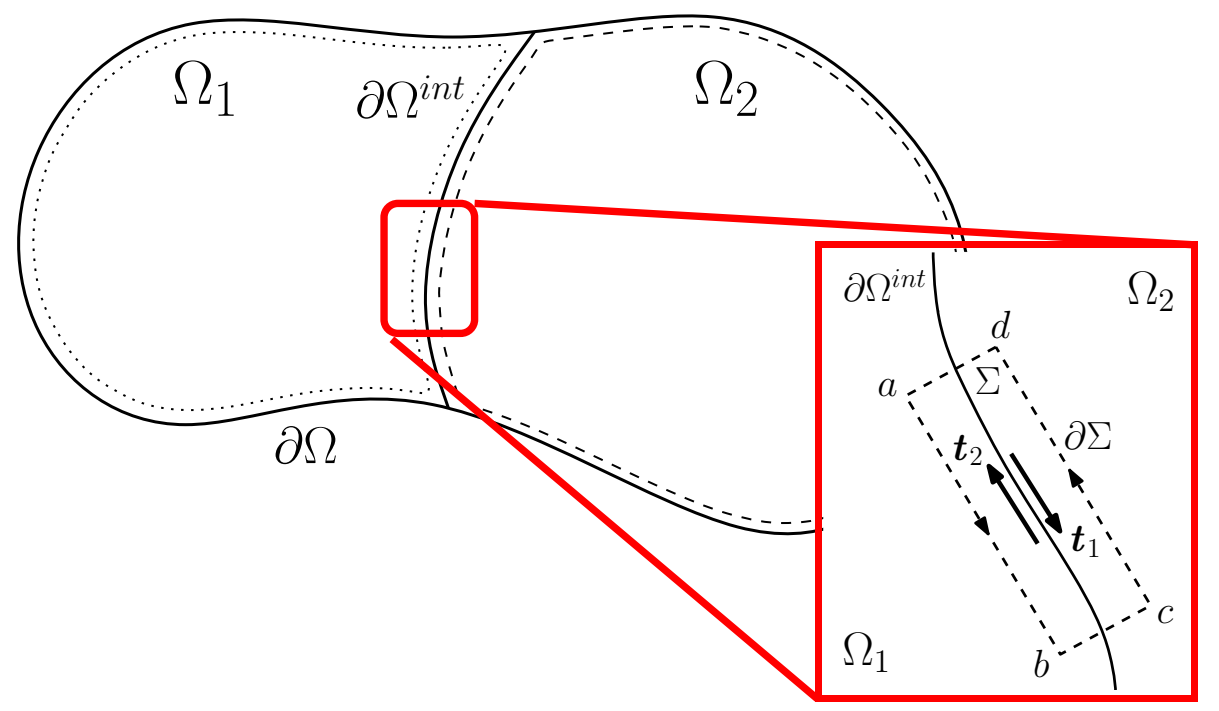

Figure B.1: A domain $\Omega=\Omega_{1} \cup \Omega_{2}$ consisting of multiple mediums $\Omega_{1}, \Omega_{2}$ with an outer boundary $\partial \Omega$ and an interface $\partial \Omega^{i n t}$. A closed loop contour $\partial \Sigma$ is applied at the interface of the two domains, where each side of the interface has an associated unit tangent vector.

\section{B.1.1 Electric Field Interface Condition}

The condition on the electric field $\boldsymbol{E}$ at the interface $\partial \Omega^{\text {int }}$ can be determined by considering Faraday's law (B.1a) on $\Sigma$, in Figure B.1, such that

$$
\int_{a}^{b} \boldsymbol{E} \cdot \boldsymbol{t} \mathrm{d} l+\int_{b}^{c} \boldsymbol{E} \cdot \boldsymbol{t} \mathrm{d} l+\int_{c}^{d} \boldsymbol{E} \cdot \boldsymbol{t} \mathrm{d} l+\int_{d}^{a} \boldsymbol{E} \cdot \boldsymbol{t} \mathrm{d} l=-\frac{\mathrm{d}}{\mathrm{d} t} \int_{\Sigma} \boldsymbol{B} \cdot \boldsymbol{n} \mathrm{d} S
$$

By allowing the surface $\Sigma$ to collapse to an infinitesimally thin area, with edges

\footnotetext{
${ }^{1}$ Stokes' theorem, correctly known the Kelvin-Stokes theorem, relates a contour integral to a surface integral by $\oint_{\partial \Sigma} \boldsymbol{A} \cdot \boldsymbol{t} \mathrm{d} l=\int_{\Sigma}(\nabla \times \boldsymbol{A}) \cdot \boldsymbol{n} \mathrm{d} S$.

${ }^{2}$ The divergence theorem, or Gauss' divergence theorem, relates the flux of a vector field through a surface to its behaviour inside the volume as $\int_{\Omega} \nabla \cdot \boldsymbol{A} \mathrm{d} \Omega=\oint_{\partial \Omega} \boldsymbol{A} \cdot \boldsymbol{n} \mathrm{d} S$.
} 
$l_{b c}$ and $l_{d a}$ collapsing to zero length, such that

$$
\int_{b}^{c} \boldsymbol{E} \cdot \boldsymbol{t} \mathrm{d} l+\int_{d}^{a} \boldsymbol{E} \cdot \boldsymbol{t} \mathrm{d} l=0, \quad \frac{\mathrm{d}}{\mathrm{d} t} \int_{\Sigma} \boldsymbol{B} \cdot \boldsymbol{n} \mathrm{d} S=0,
$$

the integral form of Faraday's law B.2, applied to $\Sigma$, therefore reduces to

$$
\int_{a}^{b} \boldsymbol{E}_{1} \cdot \boldsymbol{t}_{1} \mathrm{~d} l+\int_{c}^{d} \boldsymbol{E}_{2} \cdot \boldsymbol{t}_{2} \mathrm{~d} l=0
$$

Integrating out (B.3) results in

$$
\boldsymbol{E}_{1} \cdot \boldsymbol{t}_{1} l_{a b}+\boldsymbol{E}_{2} \cdot \boldsymbol{t}_{2} l_{c d}=0
$$

Given that the tangent vectors, of each domain, at the interface are related as $\boldsymbol{t}_{2}=-\boldsymbol{t}_{1}=\boldsymbol{t}$ and the edge lengths $l_{a b}=l_{c d}$, the continuity in the tangential component of the electric field across $\partial \Omega^{\text {int }}$ can be defined as

$$
\left(\boldsymbol{E}_{2}-\boldsymbol{E}_{1}\right) \cdot \boldsymbol{t}=0
$$

where this relation holds for any surface tangent vector on the interface. This condition of the tangential continuity of the electric field across $\partial \Omega^{i n t}$ may be rewritten in terms of the normal vector ${ }^{3}$ on the interface as

$$
\boldsymbol{n} \times[\boldsymbol{E}]_{\partial \Omega^{i n t}}=0 .
$$

In the above, $[\cdot]_{\partial \Omega^{i n t}}$ denotes a jump in the field across the interface $\partial \Omega^{\text {int }}$.

\section{B.1.2 Magnetic Field Interface Condition}

The condition on the magnetic field $\boldsymbol{H}$ at the interface $\partial \Omega^{\text {int }}$ can be determined by considering Ampére's law (B.1b) on $\Sigma$, in Figure B.1, such that

$$
\int_{a}^{b} \boldsymbol{H} \cdot \boldsymbol{t} \mathrm{d} l+\int_{b}^{c} \boldsymbol{H} \cdot \boldsymbol{t} \mathrm{d} l+\int_{c}^{d} \boldsymbol{H} \cdot \boldsymbol{t} \mathrm{d} l+\int_{d}^{a} \boldsymbol{H} \cdot \boldsymbol{t} \mathrm{d} l=\int_{S} \boldsymbol{J} \cdot \boldsymbol{n} \mathrm{d} S+\frac{\mathrm{d}}{\mathrm{d} t} \int_{\Sigma} \boldsymbol{D} \cdot \boldsymbol{n} \mathrm{d} S .
$$

Again, allowing $\Sigma$ to tend to an infinitesimally thin area, with edges $l_{b c}$ and $l_{d a}$ collapsing to zero length, such that

$$
\int_{b}^{c} \boldsymbol{H} \cdot \boldsymbol{t} \mathrm{d} l+\int_{d}^{a} \boldsymbol{H} \cdot \boldsymbol{t} \mathrm{d} l=0, \quad \frac{\mathrm{d}}{\mathrm{d} t} \int_{\Sigma} \boldsymbol{D} \cdot \boldsymbol{n} \mathrm{d} S=0,
$$

the integral form of Ampére's law B.5, applied to $\Sigma$, therefore reduces to

$$
\int_{a}^{b} \boldsymbol{H} \cdot \boldsymbol{t} \mathrm{d} l+\int_{c}^{d} \boldsymbol{H} \cdot \boldsymbol{t} \mathrm{d} l=\int_{S} \boldsymbol{J}^{s} \cdot \boldsymbol{n} \mathrm{d} S
$$

\footnotetext{
${ }^{3}$ The unit tangent and normal vectors are orthogonal and so a tangential component of a vector $\boldsymbol{E} \cdot \boldsymbol{t}$ must be orthogonal to its normal component, hence $\boldsymbol{E} \cdot \boldsymbol{t}=\boldsymbol{n} \times \boldsymbol{E}$.
} 
Note in this case, however, that as $\Sigma$ collapses to zero the curents on the surface can tend towards infinity and so the surface integral term of $\boldsymbol{J}^{s}$ remains. Integrating out (B.6) results in

$$
\boldsymbol{H}_{1} \cdot \boldsymbol{t}_{1} l_{a b}+\boldsymbol{H}_{2} \cdot \boldsymbol{t}_{2} l_{c d}=\boldsymbol{j}_{s} l,
$$

Given that $l_{a b}=l_{c d}=l$ and that the tangent vectors are related as $\boldsymbol{t}_{2}=-\boldsymbol{t}_{1}=\boldsymbol{t}$, the jump in the tangential component of the magnetic field across $\partial \Omega^{i n t}$ is given as

$$
\left(\boldsymbol{H}_{2}-\boldsymbol{H}_{1}\right) \cdot \boldsymbol{t}=\boldsymbol{j}_{s},
$$

where this relation holds for any surface tangent vector on the interface. This condition of the tangential jump of the magnetic field across $\partial \Omega^{i n t}$ may be rewritten in terms of the normal vector on the interface as

$$
\boldsymbol{n} \times[\boldsymbol{H}]_{\partial \Omega^{i n t}}=\boldsymbol{j}_{s}
$$

\section{B.2 Mechanical Interface Conditions}

Determining the interface conditions in the mechanical fields requires the transient Equations of equilibrium to be described, in their integral form as

$$
\int_{\partial \Omega} \boldsymbol{T} \mathrm{d} S+\int_{\Omega} \boldsymbol{f} \mathrm{d} \Omega=\int_{\Omega} \rho \boldsymbol{a} \mathrm{d} \Omega .
$$

where the sum of the internal (body) forces $\boldsymbol{f}$ and external (traction) $\boldsymbol{T}$ forces must equate to the transient force due to acceleration $\boldsymbol{a}$. In the above, the traction force

$$
\boldsymbol{T}=\boldsymbol{\sigma} \boldsymbol{n} \quad \text { on } \partial \Omega
$$

where $\boldsymbol{\sigma}$ is the stress tensor and $\rho$ is the density of the medium.

In order to determine the continuity conditions in the mechanical fields across the interface $\partial \Omega^{i n t}$, between two mediums $\Omega_{1}$ and $\Omega_{2}$, the setup in Figure B.2 is considered.

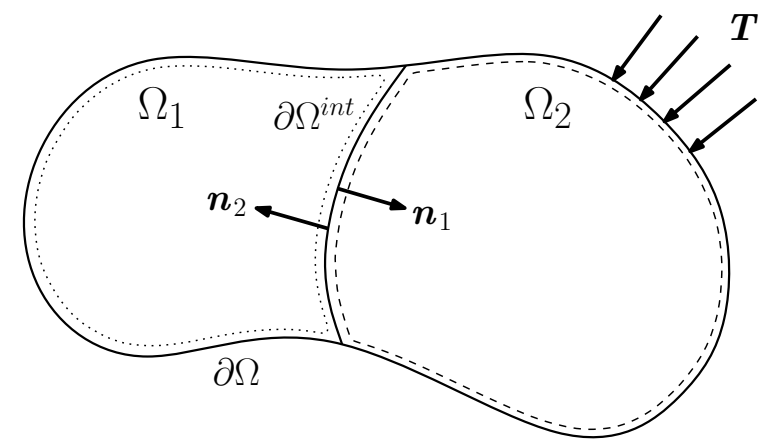

Figure B.2: A domain $\Omega=\Omega_{1} \cup \Omega_{2}$ consisting of two mediums $\Omega_{1}$ and $\Omega_{2}$ connected at an interface $\partial \Omega^{i n t}$, subject to a traction force $\boldsymbol{T}$. 


\section{B.2.1 Traction Interface Condition}

The condition on the traction forces $\boldsymbol{T}$ at the interface $\partial \Omega^{i n t}$ can be determined by considering (B.8) over the whole domain of multiple mediums. For the multi medium system illustrated in Figure B.2 the equilibrium Equation becomes

$$
\int_{\partial \Omega_{1}} \boldsymbol{T}_{1} \mathrm{~d} S+\int_{\Omega_{1}} \boldsymbol{f}_{1} \mathrm{~d} \Omega+\int_{\partial \Omega_{2}} \boldsymbol{T}_{2} \mathrm{~d} S+\int_{\Omega_{2}} \boldsymbol{f}_{2} \mathrm{~d} \Omega=\int_{\Omega_{1}} \rho_{1} \boldsymbol{a}_{1} \mathrm{~d} \Omega+\int_{\Omega_{2}} \rho_{2} \boldsymbol{a}_{2} \mathrm{~d} \Omega,
$$

This may be further split by considering the decomposition of the boundaries of each medium $\partial \Omega=\partial \Omega^{\text {int }} \cup \partial \Omega^{\text {ext }}$, where $\partial \Omega^{\text {ext }}$ is the exterior part and $\partial \Omega^{\text {int }}$ is the part on the interface. The equilibrium Equation for the full domain is described as

$$
\begin{aligned}
\int_{\partial \Omega_{1}^{\text {ext }}} \boldsymbol{T}_{1} \mathrm{~d} S+\int_{\partial \Omega_{1}^{\text {int }}} \boldsymbol{T}_{1} \mathrm{~d} S+\int_{\Omega_{1}} \boldsymbol{f}_{1} \mathrm{~d} \Omega+\int_{\partial \Omega_{2}^{\text {ext }}} \boldsymbol{T}_{2} \mathrm{~d} S & +\int_{\partial \Omega_{2}^{\text {int }}} \boldsymbol{T}_{2} \mathrm{~d} S+\int_{\Omega_{2}} \boldsymbol{f}_{2} \mathrm{~d} \Omega \\
& =\int_{\Omega_{1}} \rho_{1} \boldsymbol{a}_{1} \mathrm{~d} \Omega+\int_{\Omega_{2}} \rho_{2} \boldsymbol{a}_{2} \mathrm{~d} \Omega .
\end{aligned}
$$

The integrals over the individual external boundaries to the complete external boundary and the integrals over two mediums can be collapsed to the whole domain, which gives

$$
\int_{\partial \Omega_{1}^{i n t}} \boldsymbol{T}_{1} \mathrm{~d} S+\int_{\partial \Omega_{2}^{i n t}} \boldsymbol{T}_{2} \mathrm{~d} S+\int_{\partial \Omega} \boldsymbol{T} \mathrm{d} S+\int_{\Omega} \boldsymbol{f} \mathrm{d} \Omega=\int_{\Omega} \rho \boldsymbol{a} \mathrm{d} \Omega .
$$

From (B.8) it follows that (B.9) reduces to

$$
\int_{\partial \Omega_{1}^{i n t}} \boldsymbol{T}_{1} \mathrm{~d} S+\int_{\partial \Omega_{2}^{i n t}} \boldsymbol{T}_{2} \mathrm{~d} S=\mathbf{0}
$$

where the traction can related to the normal component of the stress to give

$$
\int_{\partial \Omega_{1}^{i n t}} \boldsymbol{\sigma}_{1} \boldsymbol{n}_{1} \mathrm{~d} S+\int_{\partial \Omega_{2}^{i n t}} \boldsymbol{\sigma}_{2} \boldsymbol{n}_{2} \mathrm{~d} S=\mathbf{0} .
$$

It holds across $\partial \Omega^{\text {int }}$ that $\boldsymbol{n}_{2}=-\boldsymbol{n}_{1}=\boldsymbol{n}$ and thus

$$
\int_{\partial \Omega^{i n t}}\left(\boldsymbol{\sigma}_{2}-\boldsymbol{\sigma}_{1}\right) \boldsymbol{n} \mathrm{d} S=\mathbf{0},
$$

where, in general,

$$
\left(\boldsymbol{\sigma}_{2}-\boldsymbol{\sigma}_{1}\right) \boldsymbol{n}=\mathbf{0},
$$

holds for any normal across $\partial \Omega^{\text {int }}$ and thus the continuity of stresses across interfaces between media in equilibrium becomes

$$
[\boldsymbol{\sigma}]_{\partial \Omega^{i n t}} \boldsymbol{n}=\mathbf{0} .
$$




\section{B.2.2 Acceleration Interface Condition}

The condition on the acceleration field $\boldsymbol{a}$ at the interface $\partial \Omega^{i n t}$ can be determined by starting with Euler's Equation in integral form. This Equation governs the behaviour of gases and is derived using the conservation of mass, which can be used also to describe the acoustic Helmholtz Equation [116]. Euler's Equation in integral form is given as

$$
\frac{\partial}{\partial t} \int_{\Omega} \rho \mathrm{d} \Omega=-\int_{\partial \Omega} \rho \boldsymbol{v} \cdot \boldsymbol{n} \mathrm{d} S
$$

where again $\rho$ is the density of the medium and $\boldsymbol{v}$ is the velocity. Applying Gauss' divergence theorem to the surface integral yields

$$
\frac{\partial}{\partial t} \int_{\Omega} \rho \mathrm{d} \Omega=-\int_{\Omega} \nabla \cdot(\rho \boldsymbol{v}) \mathrm{d} \Omega
$$

Since this has to be true for any volume $\Omega$ the following PDE is derived

$$
\frac{\partial \rho}{\partial t}=-\nabla \cdot(\rho \boldsymbol{v})
$$

Returning to the integral form of Euler's Equation, in (B.11), and applying it to the domain $\Omega=\Omega_{1} \cup \Omega_{2}$, in Figure B.2, yields

$$
\begin{aligned}
\frac{\partial}{\partial t} \int_{\Omega_{1}} \rho_{1} \mathrm{~d} \Omega+\frac{\partial}{\partial t} \int_{\Omega_{2}} \rho_{2} \mathrm{~d} \Omega= & -\int_{\partial \Omega_{1}^{\text {int }}} \rho_{1} \boldsymbol{v}_{1} \cdot \boldsymbol{n}_{1} \mathrm{~d} S-\int_{\partial \Omega_{2}^{\text {int }}} \rho_{2} \boldsymbol{v}_{2} \cdot \boldsymbol{n}_{2} \mathrm{~d} S \\
& -\int_{\partial \Omega_{1}^{\text {ext }}} \rho_{1} \boldsymbol{v}_{1} \cdot \boldsymbol{n}_{1} \mathrm{~d} S-\int_{\partial \Omega_{2}^{\text {ext }}} \rho_{2} \boldsymbol{v}_{2} \cdot \boldsymbol{n}_{2} \mathrm{~d} S
\end{aligned}
$$

The integrals over the individual domains and external boundaries can be reduced to integrals over the complete domain and boundary as

$$
\frac{\partial}{\partial t} \int_{\Omega} \rho \mathrm{d} \Omega=-\int_{\partial \Omega_{1}^{i n t}} \rho_{1} \boldsymbol{v}_{1} \cdot \boldsymbol{n}_{1} \mathrm{~d} S-\int_{\partial \Omega_{2}^{i n t}} \rho_{2} \boldsymbol{v}_{2} \cdot \boldsymbol{n}_{2} \mathrm{~d} S-\int_{\partial \Omega^{e x t}} \rho \boldsymbol{v} \cdot \boldsymbol{n} \mathrm{d} S .
$$

From (B.11) Equation (B.14) reduces to

$$
\int_{\partial \Omega_{1}^{i n t}} \rho_{1} \boldsymbol{v}_{1} \cdot \boldsymbol{n}_{1} \mathrm{~d} S+\int_{\partial \Omega_{2}^{\text {int }}} \rho_{2} \boldsymbol{v}_{2} \cdot \boldsymbol{n}_{2} \mathrm{~d} S=0
$$

It holds across $\partial \Omega^{\text {int }}$ that $\boldsymbol{n}_{2}=-\boldsymbol{n}_{1}=\boldsymbol{n}$ and thus

$$
\int_{\partial \Omega^{i n t}}\left(\rho_{2} \boldsymbol{v}_{2}-\rho_{1} \boldsymbol{v}_{1}\right) \cdot \boldsymbol{n} \mathrm{d} S=0
$$

where, in general,

$$
\left(\rho_{2} \boldsymbol{v}_{2}-\rho_{1} \boldsymbol{v}_{1}\right) \cdot \boldsymbol{n}=0
$$

holds for any normal across $\partial \Omega^{\text {int }}$ and thus the continuity of mass flux across inter- 
faces between media in equilibrium becomes

$$
[\rho \boldsymbol{v}]_{\partial \Omega^{i n t}} \cdot \boldsymbol{n}=0 .
$$

Taking the time derivative of the interface, where $\rho$ is constant in time for incompressible flows, the continuity condition becomes

$$
[\rho \boldsymbol{a}]_{\partial \Omega^{i n t}} \cdot \boldsymbol{n}=0 .
$$





\section{Appendix C}

\section{Low Frequency Electromagnetics}

For problems concerning low frequency electromagnetic fields, such as in MRI application, it becomes useful to examine the effects on the full set of Maxwell equations. In Chapter 2, the $\boldsymbol{A}$-based formulation of the Maxwell system is derived, by introducing the magnetic vector potential $\boldsymbol{A}$. For certain materials and frequencies of excitation the system can be reduced to the eddy current formulation through the eddy current approximation, where a series of conditions must hold (see Section 2.2.4 for details). This Appendix describes the process of obtaining the conditions which must be fulfilled in order to ensure the eddy current model remains valid and enriches the topics discussed in Chapter 2.

\section{C.1 Eddy Current Approximation}

In the absence of any current sources or Lorentz currents, the vector potential formulation of the full transient Maxwell system (from Section 2.2) results in Ampére's law being described as

$$
\nabla \times \mu^{-1} \nabla \times \boldsymbol{A}+\underbrace{\gamma \frac{\partial \boldsymbol{A}}{\partial t}}_{\boldsymbol{J}^{e}}-\underbrace{\epsilon \frac{\partial^{2} \boldsymbol{A}}{\partial t^{2}}}_{\frac{\partial \boldsymbol{D}}{\partial t}}=\mathbf{0} .
$$

The above is an un-gauged Equation and requires a series of gauge conditions for each domain, in order to define uniqueness in the solution of $\boldsymbol{A}$. See Chapter 2 for details on gauging the Equation.

For the transient eddy current approximation to hold, the magnitude of the displacement current term $\partial \boldsymbol{D} / \partial t$ must be negligible compared to the other terms in (C.1) in order to neglect it from the Equation. Therefore a dimensional analysis of the magnitude of each term in (C.1) must be performed, to determine under what conditions the magnitude of these terms remain dominant over the displacement currents. First, comparing the term associated with the curl-curl of the vector 
potential with the displacement currents deduces the following inequality

$$
\left|\epsilon \frac{\partial^{2} \boldsymbol{A}}{\partial t^{2}}\right|<<\left|\nabla \times \mu^{-1} \nabla \times \boldsymbol{A}\right|,
$$

which makes sense for low frequencies, since $\boldsymbol{A}$ will only propagate very slowly and so its acceleration will also be negligible. By performing a dimensional analysis, (C.2) reduces to

$$
\epsilon \tau^{-2}|\boldsymbol{A}|<<\alpha^{-2} \mu^{-1}|\boldsymbol{A}|
$$

where $\alpha$ is defined as the length scale and $\tau$ the time scale of the problem ${ }^{1}$.

Next, by comparing the magnitude of the term associated with the eddy currents $\boldsymbol{J}^{e}$ with the displacement current term, the inequality

$$
\left|\epsilon \frac{\partial^{2} \boldsymbol{A}}{\partial t^{2}}\right|<<\left|\gamma \frac{\partial \boldsymbol{A}}{\partial t}\right|,
$$

arises. This reduces, through a dimensional analysis, to

$$
\epsilon \tau^{-2}|\boldsymbol{A}|<<\gamma \tau^{-1}|\boldsymbol{A}|
$$

Thus, by eliminating the common terms in the two inequalities (C.3) and (C.4), the following conditions must be satisfied for the transient eddy current model to be a valid approximation

$$
\epsilon \mu \alpha^{2} \tau^{-2}<<1, \quad \frac{\epsilon}{\gamma \tau}<<1 .
$$

Following from this, the time scale $\tau$ can be related to the frequency $\omega$ of the problem by employing a time harmonic representation of the vector potential, through a Fourier decomposition, as

$$
\boldsymbol{A}(t)=\operatorname{Re}\left(\boldsymbol{A}(\omega) \mathrm{e}^{\mathrm{i} \omega t}\right)
$$

where $\mathcal{A}$ represents the complex amplitude of the field, which depends on the frequency. This is a valid representation of the field for problems concerning single frequency waves, but, in general this representation would require a sum over all frequencies present in the time signal. For a frequency domain problem the field associated with each frequency of the excitation signal, in Figure 1.4, could be solved individually by applying an FFT of the signals, as in Figure 2.2. The temporal derivative of the vector potential then becomes

$$
\frac{\partial \boldsymbol{A}}{\partial t}(t)=\operatorname{Re}\left(\mathrm{i} \omega \boldsymbol{A}(\omega) \mathrm{e}^{\mathrm{i} \omega t}\right)
$$

\footnotetext{
${ }^{1}$ The spatial derivatives $(\nabla \cdot, \nabla \times, \nabla)$ induce an inverse length scale $\alpha^{-1}$ and the temporal derivative $(\partial / \partial t)$ induces an inverse time scale $\tau^{-1}$.
} 
where the magnitude of this term is

$$
\left|\frac{\partial \boldsymbol{A}}{\partial t}\right|=\tau^{-1}|\boldsymbol{A}|=\omega|\boldsymbol{A}|
$$

It is clear from this that the time scale is equivalent to the inverse of the angular frequency $\tau=1 / \omega$ for temporally linear problems. For temporally non-linear problems this is not always the case, however, the magnitude of $\tau$ will remain of approximately the same order as the dominant excitation frequency $O(1 / \omega)[25]$.

\section{C.2 Electromagnetic Body Force}

The body force $\boldsymbol{b}$, present in the elasticity Equation, can be represented in terms of the divergence of the Maxwell stress tensor plus a momentum term [80] as

$$
\boldsymbol{b}(\boldsymbol{H}, \boldsymbol{D})=\nabla \cdot \boldsymbol{\sigma}^{e}(\boldsymbol{H}, \boldsymbol{D})-\frac{\partial}{\partial t}(\boldsymbol{D} \times \mu \boldsymbol{H}),
$$

where the Maxwell stress tensor can be further split into a magnetic and an electric component

$$
\boldsymbol{\sigma}^{e}(\boldsymbol{H}, \boldsymbol{D})=\boldsymbol{\sigma}_{M}^{e}(\boldsymbol{H})+\boldsymbol{\sigma}_{E}^{e}(\boldsymbol{D}) .
$$

The individual components of the Maxwell stress tensor can be expressed in terms of magnetic and electric fields as

$$
\begin{aligned}
\boldsymbol{\sigma}_{M}^{e}(\boldsymbol{H}) & =\mu\left(\boldsymbol{H} \otimes \boldsymbol{H}-\frac{1}{2}(\boldsymbol{H} \cdot \boldsymbol{H}) \boldsymbol{I}\right), \\
\boldsymbol{\sigma}_{E}^{e}(\boldsymbol{D}) & =\epsilon^{-1}\left(\boldsymbol{D} \otimes \boldsymbol{D}-\frac{1}{2}(\boldsymbol{D} \cdot \boldsymbol{D}) \boldsymbol{I}\right),
\end{aligned}
$$

which are valid for homogeneous isotropic materials, considered in this Thesis, in the absence of electro- and magneto- strictive effects, which are considered in [119].

Taking the divergence of the magnetic component of the stress tensor yields ${ }^{2} 3$

$$
\begin{aligned}
\nabla \cdot \boldsymbol{\sigma}_{M}^{e}(\boldsymbol{H}) & =\mu\left(\nabla \cdot(\boldsymbol{H} \otimes \boldsymbol{H})-\frac{1}{2} \nabla \cdot((\boldsymbol{H} \cdot \boldsymbol{H}) \boldsymbol{I})\right) \\
& =\mu\left(\boldsymbol{H} \nabla \cdot \boldsymbol{H}+(\nabla \boldsymbol{H}) \boldsymbol{H}-\frac{1}{2} \nabla(\boldsymbol{H} \cdot \boldsymbol{H})\right) \\
& =\mu(\nabla \times \boldsymbol{H}) \times \boldsymbol{H},
\end{aligned}
$$

and the divergence of the electric component of the stress tensor yields

$$
\nabla \cdot \boldsymbol{\sigma}_{E}^{e}(\boldsymbol{D})=\epsilon^{-1}(\nabla \times \boldsymbol{D}) \times \boldsymbol{D} .
$$

\footnotetext{
${ }^{2}$ Utilising the fact that the magnetic field is divergence free $\nabla \cdot \boldsymbol{H}=\mathbf{0}$.

${ }^{3}$ Recall here the relationship between the curl and gradient operators $(\nabla \boldsymbol{H}) \boldsymbol{H}-1 / 2 \nabla(\boldsymbol{H} \cdot \boldsymbol{H})=$ $(\nabla \times \boldsymbol{H}) \times \boldsymbol{H}$.
} 
The body force can therefore be expressed purely in terms of $\boldsymbol{D}$ and $\boldsymbol{H}$ as

$$
\boldsymbol{b}(\boldsymbol{H}, \boldsymbol{D})=\underbrace{\mu(\nabla \times \boldsymbol{H}) \times \boldsymbol{H}}_{\nabla \cdot \boldsymbol{\sigma}_{M}^{e}}+\underbrace{\epsilon^{-1}(\nabla \times \boldsymbol{D}) \times \boldsymbol{D}}_{\nabla \cdot \boldsymbol{\sigma}_{E}^{e}}-\underbrace{\frac{\partial \boldsymbol{D}}{\partial t} \times \mu \boldsymbol{H}-\boldsymbol{D} \times \mu \frac{\partial \boldsymbol{H}}{\partial t}}_{\text {momentum term }},
$$

which in an $\boldsymbol{A}$-based formulation yields

$$
\begin{aligned}
\boldsymbol{b}=\underbrace{\mu^{-1}(\nabla \times \nabla \times \boldsymbol{A}) \times \nabla \times \boldsymbol{A}}_{\nabla \cdot \boldsymbol{\sigma}_{M}^{e}} & +\underbrace{\epsilon\left(\nabla \times \frac{\partial \boldsymbol{A}}{\partial t}\right) \times \frac{\partial \boldsymbol{A}}{\partial t}}_{\nabla \cdot \boldsymbol{\sigma}_{E}^{e}} \\
& +\underbrace{\epsilon \frac{\partial^{2} \boldsymbol{A}}{\partial t^{2}} \times(\nabla \times \boldsymbol{A})+\epsilon \frac{\partial \boldsymbol{A}}{\partial t} \times\left(\nabla \times \frac{\partial \boldsymbol{A}}{\partial t}\right)}_{\text {momentum term }} .
\end{aligned}
$$

Combining like terms arising from the electric component of the stress and the momentum term, (C.8) reduces to ${ }^{4}$

$$
\boldsymbol{b}=\underbrace{\mu^{-1}(\nabla \times \nabla \times \boldsymbol{A}) \times \nabla \times \boldsymbol{A}}_{\nabla \cdot \boldsymbol{\sigma}_{M}^{e}}-\epsilon \frac{\partial^{2} \boldsymbol{A}}{\partial t^{2}} \times(\nabla \times \boldsymbol{A}) .
$$

In order to determine the conditions for which the momentum term and electric component of the Maxwell stress tensor can be neglected, a dimensional analysis of the individual terms is considered. Thus for the terms in (C.9) to be neglected the following condition must hold

$$
\left|\epsilon \frac{\partial^{2} \boldsymbol{A}}{\partial t^{2}} \times(\nabla \times \boldsymbol{A})\right|<<\left|\mu^{-1}(\nabla \times \nabla \times \boldsymbol{A}) \times \nabla \times \boldsymbol{A}\right| .
$$

Applying the length and time scaling, to the derivative components, yields

$$
\epsilon \tau^{-2} \alpha^{-1}|\boldsymbol{A}|^{2}<<\mu^{-1} \alpha^{-3}|\boldsymbol{A}|^{2}
$$

Eliminating the common terms results in the following condition, for eliminating the components of body force,

$$
\epsilon \mu \alpha^{2} \tau^{-2}<<1
$$

This is the same as one of the conditions derived for the transient eddy current approximation. Thus if the eddy current approximation remains valid for the application then the electric component of the Maxwell stress and the momentum term in the body force can also be neglected.

\footnotetext{
${ }^{4}$ Noting the relationship of the cross product $A \times B=-B \times A$.
} 


\section{Appendix D}

\section{Acoustic Far Field Treatment}

The radiation condition for the acoustic field must be satisfied in order to avoid artificial reflections of the acoustic waves back into the computational domain. This Appendix summarises the steps required to approximate the radiation of the acoustic field in truncated domains and enriches the topics discussed in Chapter 4.

\section{D.1 Approximation of the Sommerfeld Condition}

The Sommerfeld radiation condition ${ }^{1}$, which approximates the decaying behaviour of the field towards infinity, can be defined as

$$
\lim _{|\boldsymbol{x}| \rightarrow \infty}\left(\nabla P \cdot \boldsymbol{n}+\frac{\partial P}{\partial t}\right)=O\left(|\boldsymbol{x}|^{-1}\right) \quad \text { as }|\boldsymbol{x}| \rightarrow \infty
$$

For truncated computational domains then this condition may be used to approximate the behaviour of the field at the outer boundary. Whilst the boundary is truncated to lie a finite distance away from the radiation source, it can still allow for good approximations of the field if the boundary is located sufficiently far away. Figure D.1 illustrates the truncated computational domain, where the domain $\Omega=\Omega_{c} \cup \Omega_{n}$, the non-conducting boundary $\partial \Omega_{n}=\partial \Omega_{c} \cup \partial \Omega_{+}$and the outer boundary $\partial \Omega_{+}$are located a finite distance from the scatterer.

The Sommerfeld condition for the computational domain, is approximated by

$$
\nabla P \cdot \boldsymbol{n}=-\frac{\partial P}{\partial t} \quad \text { on } \partial \Omega_{+},
$$

where the normal gradient of the pressure is directly related to the first temporal derivative of the field. This condition will be used to relate the boundary integral arising from the acoustic Helmholtz equation.

\footnotetext{
${ }^{1}$ Here the Sommerfeld absorbing boundary condition is given as a first order boundary condition. Higher order absorbing boundary conditions can be obtained by using a Taylor series expansion.
} 


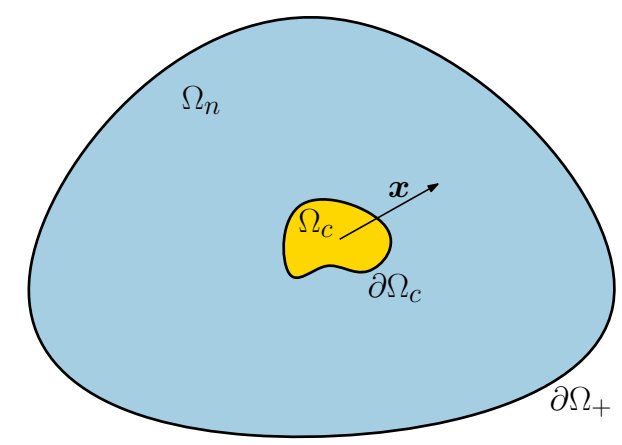

Figure D.1: Computational domain with truncated outer boundary.

The weak form of the Helmholtz acoustic wave equation in the non-conducting region, where terms involving the Maxwell stress $\boldsymbol{\sigma}^{e}$ are omitted for simplicity, is given by

$$
\int_{\Omega_{n}} \nabla P \cdot \nabla P^{\delta} \mathrm{d} \Omega+\int_{\Omega_{n}} \frac{1}{c^{2}} \frac{\partial^{2} P}{\partial t^{2}} P^{\delta} \mathrm{d} \Omega=\int_{\partial \Omega_{c}} \boldsymbol{n} \cdot \nabla P P^{\delta} \mathrm{d} S+\int_{\partial \Omega_{+}} \boldsymbol{n} \cdot \nabla P P^{\delta} \mathrm{d} S
$$

where the boundary term is obtained by applying the divergence theorem. From the approximate farfield condition in (D.2), the weak variational form of the acoustic Helmholtz equation in the non-conducting region becomes

$$
\int_{\Omega_{n}} \nabla P \cdot \nabla P^{\delta} \mathrm{d} \Omega+\int_{\Omega_{n}} \frac{1}{c^{2}} \frac{\partial^{2} P}{\partial t^{2}} P^{\delta} \mathrm{d} \Omega=\int_{\partial \Omega_{c}} \boldsymbol{n} \cdot \nabla P P^{\delta} \mathrm{d} S-\int_{\partial \Omega_{+}} \frac{\partial P}{\partial t} P^{\delta} \mathrm{d} S
$$

where the boundary term on $\partial \Omega_{+}$will form part of the damping matrix in the transient system.

\section{D.2 Perfectly Matched Layer Formulation}

In order to allow for the computational treatment of unbounded regions a truncation of the outer boundary is required. Boundary conditions must then be imposed on the artificial outer boundary. For problems with a known analytical solution this is trivial as one can simply define the analytical solution on the boundary. However, in general not all problems have known analytical solutions and so the boundary conditions are less trivial. For non wave type problems, such as the eddy current model, this poses less of an issue as one can simply impose the farfield solution at the boundary and increase the size of the computational domain to obtain more accurate results. However, for wave type problems, such as the acoustics, imposing a simple farfield condition will result in artificial reflections that pollute the solution. Therefore, for the acoustic problem further treatment at this outer boundary is required to obtain more accurate solutions.

The perfectly matched layer (PML) has been recently introduced by Bérenger [32] as a means of minimizing wave reflections into computational solutions. The PML is an absorbing layer that allows for the absorption of incoming waves into the 
layer by means of a complex coordinate stretching. The idea works to extend the solution onto the complex plane in order to allow the solution to decay to a prescribed farfield condition. Figure D.2 illustrates the effect of the PML by allowing incoming waves $P$ in $\Omega_{P M L}$ to decay, which results in a reduction in the reflected waves $P^{r e f}$. For sufficient absorption factors $\sigma$ the magnitude of these reflected waves tends to zero.

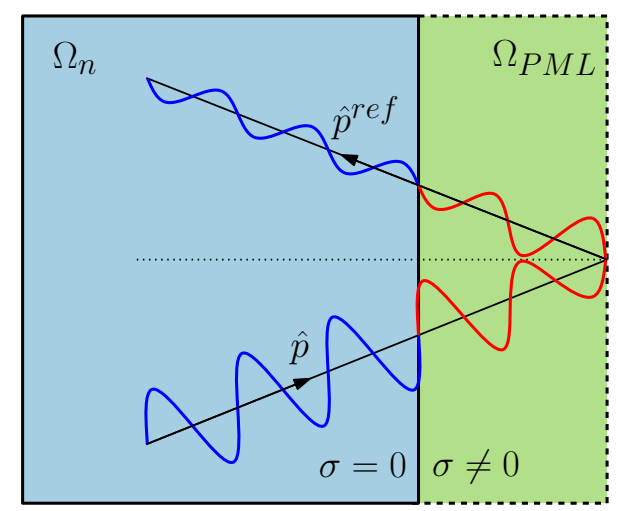

Figure D.2: Absorption of outgoing pressure wave through the PML.

Starting from the governing first order differential equations, when transformed from Equations (2.28) and (2.25), in terms of the velocity $\boldsymbol{v}$

$$
\begin{aligned}
& \frac{\partial P}{\partial t}=\kappa \nabla \cdot \boldsymbol{v} \\
& \frac{\partial \boldsymbol{v}}{\partial t}=\frac{1}{\rho} \nabla P .
\end{aligned}
$$

\section{D.2.1 Axisymmetric (2D Cylindrical) Split Field Form}

It is convenient to introduce a split-field formulation, first for equation (D.5)

$$
\frac{\partial P_{r}}{\partial t}=\kappa \frac{1}{r} \frac{\partial}{\partial r}\left(r v_{r}\right), \quad \frac{\partial P_{z}}{\partial t}=\kappa \frac{\partial v_{z}}{\partial z},
$$

where the pressure field is split as follows

$$
P=P_{r}+P_{z}
$$

Note here that $P_{r}, P_{z}$ are just convenient variables to introduce and have no physical meaning. Equation (D.6) then becomes

$$
\frac{\partial v_{r}}{\partial t}=\frac{1}{\rho} \frac{\partial P}{\partial r}, \quad \frac{\partial v_{z}}{\partial t}=\frac{1}{\rho} \frac{\partial P}{\partial z} .
$$

In order to damp the acoustic pressure in the PML and allow the absorption of outgoing waves and minimise artificial reflections at the boundary an absorption term is required. Reformulating the above split-field equations with the absorption 
terms ("artificial conductivities") $\sigma_{r}, \sigma_{z}$ yields

$$
\begin{aligned}
\frac{\partial v_{r}}{\partial t}+\sigma_{r}(r) v_{r} & =\frac{1}{\rho} \frac{\partial P}{\partial r}, & \frac{\partial v_{z}}{\partial t}+\sigma_{z}(z) v_{z} & =\frac{1}{\rho} \frac{\partial P}{\partial z} \\
\frac{\partial P_{r}}{\partial t}+\sigma_{r}(r) P_{r} & =\kappa \frac{1}{r} \frac{\partial}{\partial r}\left(r v_{r}\right), & \frac{\partial P_{z}}{\partial t}+\sigma_{z}(z) P_{z} & =\kappa \frac{\partial v_{z}}{\partial z}
\end{aligned}
$$

\section{D.2.2 Time Harmonic Description}

In a time harmonic representation of the fields, where the time dependent pressure and velocity can be described in terms of a complex phasor as $P(t)=\operatorname{Re}\left(\mathcal{P} e^{\mathrm{i} \omega t}\right)$ and $\boldsymbol{v}(t)=\operatorname{Re}\left(\boldsymbol{v} e^{\mathrm{i} \omega t}\right)$ respectively, equation (D.10) reduces to

$$
v_{x^{m}}=\frac{1}{i \omega+\sigma_{x^{m}}\left(x^{m}\right)} \frac{1}{\rho} \frac{\partial \mathcal{P}}{\partial x^{m}}, \quad P_{x^{m}}=\frac{1}{r} \frac{\kappa}{\mathrm{i} \omega+\sigma_{x^{m}}\left(x^{m}\right)} \frac{\partial}{\partial x^{m}}\left(r v_{x^{m}}\right),
$$

where the meridian coordinate system $x^{m}=r, z$ is used. Combining these equations and dropping the argument of $\sigma_{x^{m}}$ for simplicity, gives

$$
\mathcal{P}_{x^{m}}=\frac{1}{r} \frac{\kappa}{\rho} \frac{1}{\mathrm{i} \omega+\sigma_{x^{m}}} \frac{\partial}{\partial x^{m}}\left(\frac{r}{\mathrm{i} \omega+\sigma_{x^{m}}} \frac{\partial \mathcal{P}}{\partial x^{m}}\right) .
$$

Using the previous split-field analogy of the pressure in equation (D.8) results in

$$
\frac{1}{r} \frac{1}{\mathrm{i} \omega+\sigma_{r}} \frac{\partial}{\partial r}\left(\frac{r}{\mathrm{i} \omega+\sigma_{r}} \frac{\partial \mathcal{P}}{\partial r}\right)+\frac{1}{r} \frac{1}{\mathrm{i} \omega+\sigma_{z}} \frac{\partial}{\partial z}\left(\frac{r}{\mathrm{i} \omega+\sigma_{z}} \frac{\partial \mathcal{P}}{\partial z}\right)-\frac{1}{c^{2}} \mathcal{P}=0
$$

Multiplying through by $\left(\mathrm{i} \omega+\sigma_{r}\right)\left(\mathrm{i} \omega+\sigma_{z}\right)$ and noting that $\sigma_{r} \neq \sigma_{r}(z)$ and $\sigma_{z} \neq \sigma_{z}(r)$ yields

$$
\frac{1}{r} \frac{\partial}{\partial r}\left(r \frac{\mathrm{i} \omega+\sigma_{z}}{\mathrm{i} \omega+\sigma_{r}} \frac{\partial \mathcal{P}}{\partial r}\right)+\frac{1}{r} \frac{\partial}{\partial z}\left(r \frac{\mathrm{i} \omega+\sigma_{r}}{\mathrm{i} \omega+\sigma_{z}} \frac{\partial \mathcal{P}}{\partial z}\right)-\frac{\left(\mathrm{i} \omega+\sigma_{r}\right)\left(\mathrm{i} \omega+\sigma_{z}\right)}{c^{2}} \boldsymbol{P}=0
$$

Introducing a new coordinate system

$$
z_{x^{m}}\left(x^{m}\right)=x^{m}+\frac{1}{\mathrm{i} \omega} \int_{0}^{x} \sigma_{x^{m}}(\xi) \mathrm{d} \xi,
$$

such that its derivatives become

$$
\frac{\partial z_{x^{m}}\left(x^{m}\right)}{\partial x^{m}}=z_{x^{m}}^{\prime}\left(x^{m}\right)=\frac{\mathrm{i} \omega+\sigma_{x^{m}}}{\mathrm{i} \omega}=1-\mathrm{i} \frac{\sigma_{x^{m}}}{\omega},
$$

may now be substituted into (D.14), where $r \rightarrow z_{r}$ and multiplying through by $z_{r} / r$ yields

$$
\frac{1}{r} \frac{\partial}{\partial r}\left(\frac{z_{r} z_{z}^{\prime}}{z_{r}^{\prime}} \frac{\partial \mathcal{P}}{\partial r}\right)+\frac{1}{r} \frac{\partial}{\partial z}\left(\frac{z_{r} z_{r}^{\prime}}{z_{z}^{\prime}} \frac{\partial \mathcal{P}}{\partial z}\right)+\frac{z_{r} z_{r}^{\prime} z_{z}^{\prime}}{r} k^{2} \mathcal{P}=0,
$$

which is equivalent to the form of the PML adjusted equations derived in [175]. 
Equation (D.17) may be written, in vectorial form, in terms of the two PML tensors $\Lambda_{1}^{a x i}$ and $\Lambda_{2}^{a x i}$ as

$$
\frac{1}{r} \nabla^{m} \cdot\left(\Lambda_{1}^{a x i} \nabla^{m} \mathcal{P}\right)+\frac{1}{r} k^{2} \Lambda_{2}^{a x i} \mathcal{P}=0,
$$

which may be used to solve for the acoustic pressure in the PML region, where

$$
\Lambda_{1}^{a x i}(r, z)=\left[\begin{array}{cc}
\frac{z_{r} z_{z}^{\prime}}{z_{r}^{\prime}} & 0 \\
0 & \frac{z_{r} z_{r}^{\prime}}{z_{z}^{\prime}}
\end{array}\right] ; \quad \Lambda_{2}^{a x i}(r, z)=z_{r} z_{r}^{\prime} z_{z}^{\prime}
$$

\section{D.2.3 Time Dependent Description}

In order to allow for absorption of outgoing waves in the PML region in the time domain the time dependent split field system (D.10) must be solved. The two equations (D.10a) may be combined into a vector equation by realising that the artificial conductivity terms can be combined into a tensor as

$$
\boldsymbol{\sigma}(r, z)=\left[\begin{array}{cc}
\sigma_{r}(r) & 0 \\
0 & \sigma_{z}(z)
\end{array}\right] .
$$

Thus the weak form of the equation system becomes

$$
\begin{aligned}
\int_{\Omega_{p m l}} \frac{\partial P_{r}}{\partial t} q_{r}+\sigma_{r} P_{r} q_{r} \mathrm{~d} \Omega & =\int_{\Omega_{p m l}} \frac{\kappa}{r} \frac{\partial}{\partial r}\left(r v_{r}\right) q_{r} \mathrm{~d} \Omega \\
\int_{\Omega_{p m l}} \frac{\partial P_{z}}{\partial t} q_{z}+\sigma_{z} P_{z} q_{z} \mathrm{~d} \Omega & =\int_{\Omega_{p m l}} \kappa \frac{\partial v_{z}}{\partial z} q_{z} \mathrm{~d} \Omega \\
\int_{\Omega_{p m l}} \frac{\partial \boldsymbol{v}}{\partial t} \cdot \boldsymbol{w}+\boldsymbol{\sigma}(r, z) \boldsymbol{v} \cdot \boldsymbol{w} \mathrm{d} \Omega & =\int_{\Omega_{p m l}} \frac{1}{\rho} \nabla P \cdot \boldsymbol{w} \mathrm{d} \Omega .
\end{aligned}
$$

Applying integration by parts and the divergence theorem to (D.21c) yields

$$
\int_{\Omega_{p m l}} \frac{\partial \boldsymbol{v}}{\partial t} \cdot \boldsymbol{w}+\boldsymbol{\sigma}(r, z) \boldsymbol{v} \cdot \boldsymbol{w} \mathrm{d} \Omega+\int_{\Omega_{p m l}} \frac{1}{\rho} P \nabla \cdot \boldsymbol{w} \mathrm{d} \Omega=\int_{\partial \Omega_{p m l}} \frac{1}{\rho} P \boldsymbol{n} \cdot \boldsymbol{w} \mathrm{d} S .
$$

The boundary term that appears in the weak form needs further consideration due to the need to avoid any artificial reflections at the boundary. Figure D.3 shows the truncated computational domain with a PML on the outer boundary. The boundary of the PML region comprises of $\partial \Omega_{p m l}=\partial \Omega_{-} \cup \partial \Omega_{+}$and the boundary of the non conducting region of $\partial \Omega_{n}=\partial \Omega_{c} \cup \partial \Omega_{-}$.

The boundary term in (D.22) thus becomes

$$
\int_{\partial \Omega_{p m l}} \frac{1}{\rho} P \boldsymbol{n} \cdot \boldsymbol{w} \mathrm{d} S=\int_{\partial \Omega_{-}} \frac{1}{\rho} P \boldsymbol{n} \cdot \boldsymbol{w} \mathrm{d} S+\int_{\partial \Omega_{+}} \frac{1}{\rho} P \boldsymbol{n} \cdot \boldsymbol{w} \mathrm{d} S .
$$

For the PML formulation, the need to integrate over the outer boundary $\partial \Omega_{+}$ is eliminated by imposing this as a complete Dirichlet boundary and setting $P=0$ 


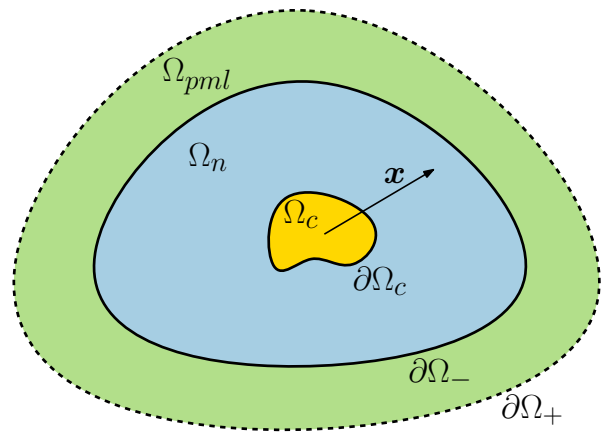

Figure D.3: Truncated computational domain including the PML region.

on $\partial \Omega_{+}$. The finite element shape functions have been constructed such that they vanish on Dirichlet boundaries and so (D.22) becomes

$$
\int_{\Omega_{p m l}} \frac{\partial \boldsymbol{v}}{\partial t} \cdot \boldsymbol{w}+\boldsymbol{\sigma}(r, z) \boldsymbol{v} \cdot \boldsymbol{w} \mathrm{d} \Omega+\int_{\Omega_{p m l}} \frac{1}{\rho} P \nabla \cdot \boldsymbol{w} \mathrm{d} \Omega=\int_{\partial \Omega_{-}} \frac{1}{\rho} P \boldsymbol{n} \cdot \boldsymbol{w} \mathrm{d} S,
$$

where the boundary term corresponds to the integration over the interface between $\Omega_{n}$ and $\Omega_{p m l}$.

Introducing the acoustic equation in the non conducting region results in

$$
\int_{\Omega_{n}} \nabla P \cdot \nabla P^{\delta} \mathrm{d} \Omega+\int_{\Omega_{n}} \frac{1}{c^{2}} \frac{\partial^{2} P}{\partial t^{2}} P^{\delta} \mathrm{d} \Omega=\int_{\partial \Omega_{c}} \boldsymbol{n} \cdot \nabla P P^{\delta} \mathrm{d} S+\int_{\partial \Omega_{-}} \boldsymbol{n} \cdot \nabla P P^{\delta} \mathrm{d} S
$$

where the $\boldsymbol{\sigma}^{e}$ terms have been omitted here for simplicity, as they only appear in $\operatorname{supp}\left(\boldsymbol{J}^{s}\right)$ and are therefore not required when talking about the farfield treatment. Now that the acoustic system for describing a PML in time domain have been represented in terms of integrals across the PML-non-conductor interface, some conditions across the interface are required to fully describe the problem. In order to avoid any reflections at $\partial \Omega_{-}$and allow the wave to fully propagate into $\Omega_{p m l}$ continuity in $P$ and $\nabla P$ must be maintained across $\partial \Omega_{-}$. Thus the following conditions

$$
\begin{aligned}
{[P]=0 } & & \text { on } \partial \Omega_{-}, \\
{[\nabla P] \cdot \boldsymbol{n}=0 } & & \text { on } \partial \Omega_{-},
\end{aligned}
$$

must hold. Given the earlier representation of $P=P_{r}+P_{z}$ in $\Omega_{p m l}$ then

$$
\begin{aligned}
P \boldsymbol{n} & =\left(P_{r}+P_{z}\right) \boldsymbol{n} & & \text { on } \partial \Omega_{-}, \\
\nabla P \cdot \boldsymbol{n} & =\left(\nabla P_{r}+\nabla P_{z}\right) \cdot \boldsymbol{n} & & \text { on } \partial \Omega_{-} .
\end{aligned}
$$

Thus the weak variational form of the equation system defining the acoustic pressure in the computational domain becomes

$$
\int_{\Omega_{p m l}} \frac{\partial P_{r}}{\partial t} q_{r}+\sigma_{r} P_{r} q_{r} \mathrm{~d} \Omega=\int_{\Omega_{p m l}} \frac{\kappa}{r} \frac{\partial}{\partial r}\left(r v_{r}\right) q_{r} \mathrm{~d} \Omega
$$




$$
\int_{\Omega_{p m l}} \frac{\partial P_{z}}{\partial t} q_{z}+\sigma_{z} P_{z} q_{z} \mathrm{~d} \Omega=\int_{\Omega_{p m l}} \kappa \frac{\partial v_{z}}{\partial z} q_{z} \mathrm{~d} \Omega,
$$

$$
\begin{aligned}
& \int_{\Omega_{p m l}}\left(\frac{\partial \boldsymbol{v}}{\partial t}+\boldsymbol{\sigma}(r, z) \boldsymbol{v}\right) \cdot \boldsymbol{w} \mathrm{d} \Omega+\int_{\Omega_{p m l}} \frac{1}{\rho}\left(P_{r}+P_{z}\right) \nabla \cdot \boldsymbol{w} \mathrm{d} \Omega=\int_{\partial \Omega_{-}} \frac{1}{\rho} P \boldsymbol{n} \cdot \boldsymbol{w} \mathrm{d} S, \\
& \int_{\Omega_{n}} \nabla P \cdot \nabla P^{\delta} \mathrm{d} \Omega+\int_{\Omega_{n}} \frac{1}{c^{2}} \frac{\partial^{2} P}{\partial t^{2}} P^{\delta} \mathrm{d} \Omega=\int_{\partial \Omega_{c}} \boldsymbol{n} \cdot \nabla P P^{\delta} \mathrm{d} S \\
& +\int_{\partial \Omega_{-}} \boldsymbol{n} \cdot\left(\nabla P_{r}+\nabla P_{z}\right) P^{\delta} \mathrm{d} S
\end{aligned}
$$





\section{Appendix E}

\section{Shape Functions on the Triangular Reference Element}

The vertex, edge and cell based $H^{1}(\Omega)$ conforming hierarchic (modal) shape functions on the reference elements in Chapter 4 must be defined. The reference triangular and quadrilateral elements used in this Thesis are illustrated in Figures 4.9 and 4.10, Chapter 4. This Appendix describes and illustrates the hierarchic shape functions generated on the reference elements and enriches the topics discussed in Chapter 4.

\section{E.1 Triangular Element}

The triangular elements are finite elements consisting of three vertices (or nodes) and three sides (or edges). The high order hierarchic shape functions are generated on this reference element and then mapped to the basis functions in the spatial domain. In general, the total number of shape functions $M_{\text {tria }}$ generated on the triangular elements for a given element order $p$ is given as $M_{\text {tria }}=M_{\text {tria }}^{V}+M_{\text {tria }}^{E}+M_{\text {tria }}^{I}=$ $(p+1)(p+2) / 2$.

\section{E.1.1 The $H^{1}(\Omega)$ Vertex Shape Functions}

The hierarchic vertex shape functions generated on the triangular reference element, in Figure 4.9, are illustrated in Figure E.1.

\begin{tabular}{|l||ccc|}
\hline & $V_{1}$ & $V_{2}$ & $V_{3}$ \\
\hline \hline \multirow{2}{*}{$p=1$} & & & \\
& & & \\
\hline
\end{tabular}

Figure E.1: Triangular finite element basis functions associated with the vertex degrees of freedom of the reference triangular element. 
The vertex shape functions, illustrated in Figure E.1, are generated by

$$
N_{i}^{V}=L_{i}
$$

where the subscripts $i$ of the vertex shape function denotes the vertices to which the shape functions are associated. These functions are equal to the barycentric/area coordinates of a triangle $L_{i}$, given mathematically as

$$
\begin{aligned}
& L_{1}=\frac{1}{2}\left(1+\xi-\frac{\eta}{\sqrt{3}}\right), \\
& L_{2}=\frac{\eta}{\sqrt{3}}, \\
& L_{3}=\frac{1}{2}\left(1-\xi-\frac{\eta}{\sqrt{3}}\right) .
\end{aligned}
$$

Note here that these shape functions are linear functions of the reference space $(\xi, \eta)$ and satisfy the partition of unity condition, that

$$
N_{1}^{V}+N_{2}^{V}+N_{3}^{V}=1
$$

such that the functions have unit value at the node to which they are associated and zero value at the other two nodes. These shape functions are the same as the standard nodal (or Lagrange-type) shape functions for a nodal finite element basis, implemented in most low order ( $h$-version) finite element codes. The vertex shape functions are continuous between neighbouring elements in the spatial domain.

For triangular elements the vertex shape functions are generated for all $p \geq 1$ and the number of vertex shape functions always corresponds to the number of vertices of the element, hence $M_{\text {tria }}^{V}=3$ in this case.

\section{E.1.2 The $H^{1}(\Omega)$ Edge Shape Functions}

The hierarchic edge shape functions generated on the reference element, in Figure 4.9, are illustrated in Figure E.2.

The hierarchical type (modal) edge shape functions, illustrated in Figure E.2, are typically generated through scaled Legendre polynomials as

$$
N_{i-1}^{E_{m}}=\varphi_{i}^{\mathcal{S}}\left(L_{e_{2}}-L_{e_{1}}, L_{e_{2}}+L_{e_{1}}\right)
$$

where the subscript $i=2, \ldots, p$ denotes the shape function number for a given edge $E_{m}=\left\{e_{1}, e_{2}\right\}$ consisting of the two nodes $e_{1}, e_{2}, m=1,2,3$ defines the edge number of the triangle ${ }^{1}$ and $\varphi_{n}^{\mathcal{S}}$ denotes the scaled integrated Legendre polynomials ${ }^{2}$.

\footnotetext{
${ }^{1}$ For the triangular reference element the relative nodes $e_{1}, e_{2}$ of the edges are: $E_{1}=\{1,2\}$, $E_{2}=\{2,3\}$ and $E_{3}=\{3,1\}$.

${ }^{2}$ The scaled integrated Legendre polynomials are given as $\varphi_{n}^{\mathcal{S}}(s, t)=\int t^{n} \ell_{n-1}\left(\frac{s}{t}\right) \mathrm{d} s$, where $\ell_{n-1}$ are the standard Legendre polynomials.
} 
Note here that these shape functions vanish at the nodes and thus the partition of unity condition is still satisfied at the nodes of the element. Associated with these shape functions are conformity problems across the interface between two neighbouring elements, where the orientation of the edges may differ. For 2D hierarchical edge shape functions, this causes a sign conflict between the edge shape functions of the two neighbouring elements and this must be resolved by incorporating a sign change in the referential to spatial mapping to enforce the $H^{1}(\Omega)$ conformity. This only effects odd-order finite elements, see [17] for further details. The hierarchic edge shape functions are associated with a specific edge of the element and are zero valued at all the nodes and other edges. They are continuous across all interfaces between elements containing the edge in the spatial domain.

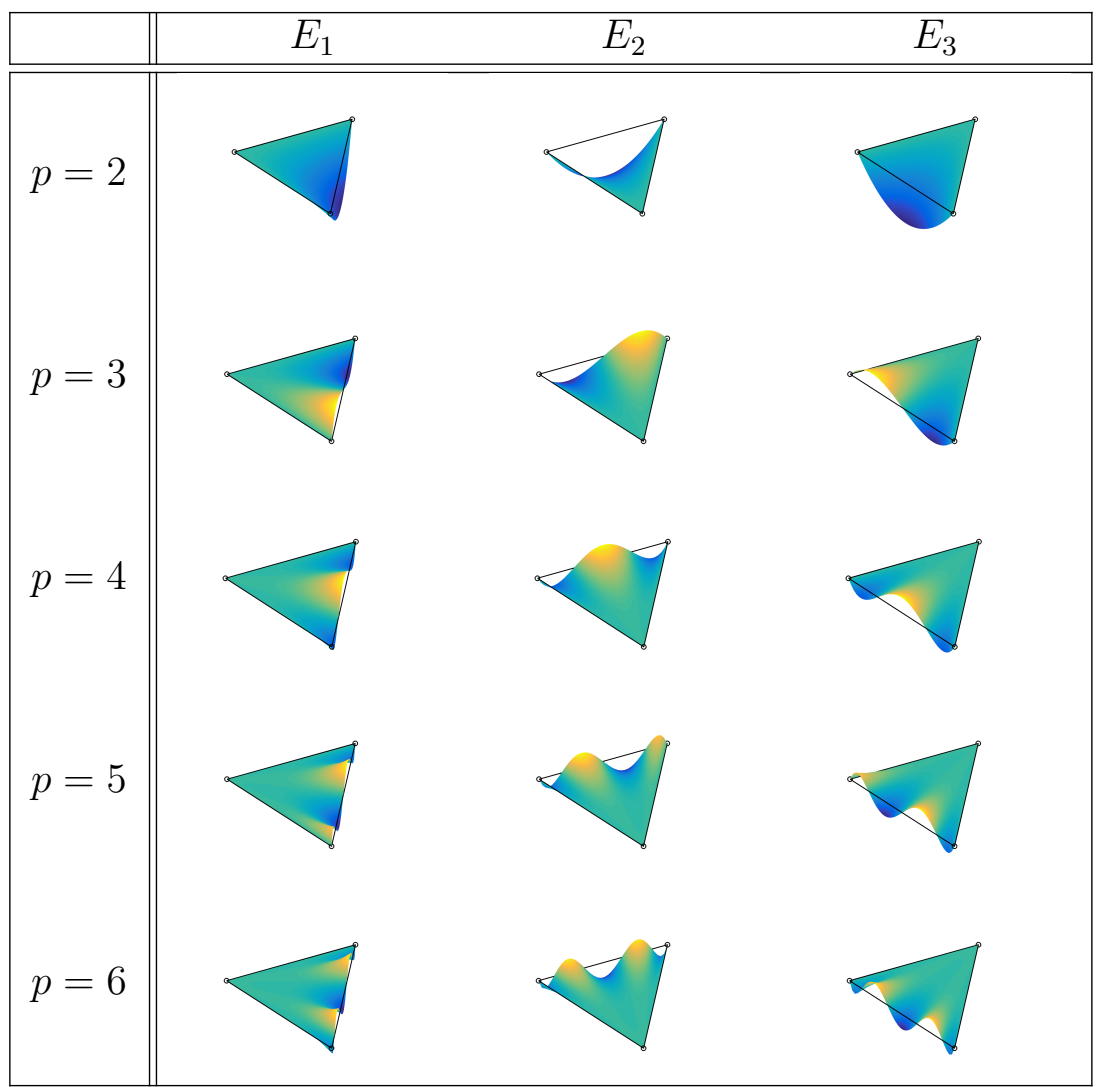

Figure E.2: Triangular finite element basis functions associated with the edge degrees of freedom of the reference triangular element.

The edge shape functions are present only for higher order elements of $p \geq 2$ and the number of edge shape functions for the reference triangular element is $M_{\text {tria }}^{E}=3(p-1)$.

\section{E.1.3 The $H^{1}(\Omega)$ Interior Shape Functions}

The hierarchic interior shape functions generated on the reference element, in Figure 4.9, are illustrated in Figure E.2. 


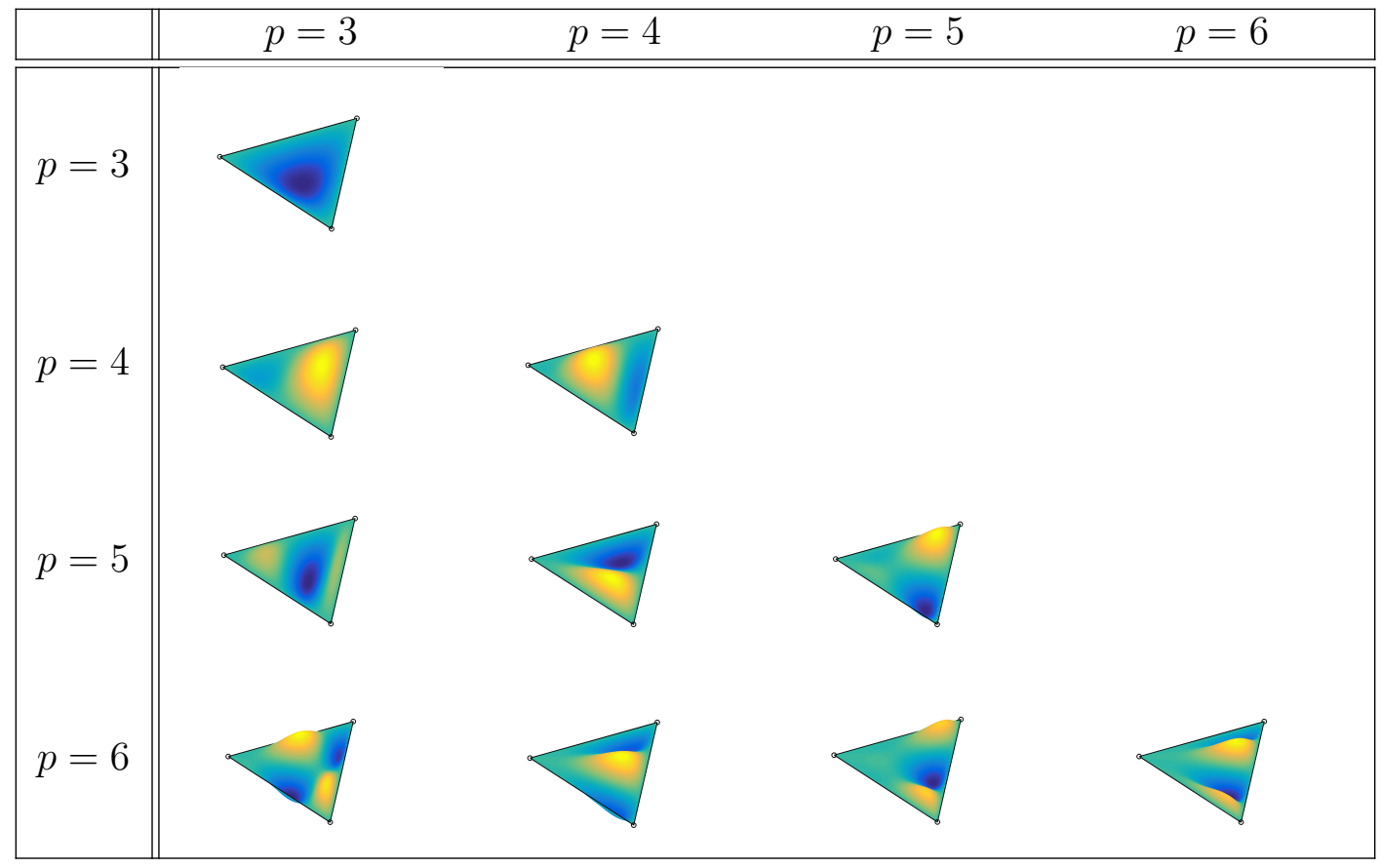

Figure E.3: Triangular finite element basis functions associated with the interior degrees of freedom of the reference triangular element.

The hierarchical type (modal) interior shape functions, illustrated in Figure E.3, are generated through multiplications of scaled integrated Legendre and scaled Legendre polynomials ${ }^{2}$ for fixed edges as

$$
N_{i-2, j-2}^{I}=L_{3} \varphi_{i-2}^{\mathcal{S}}\left(L_{2}-L_{1}, L_{2}+L_{1}\right) \ell_{j-2}\left(L_{3}-L_{1}-L_{2}\right),
$$

where the subscripts $i, j=3, \ldots, p$ and the shape function is only generated when $i \geq j$. To visualise how the shape functions stack up for each value of $p$, each subscript $i$ corresponds to a row and $j$ corresponds to a column in Figure E.3. So for $p=3$ only the top most shape function is generated, likewise for $p=4$ only the top three functions are generated and so on. The interior basis functions are zero valued at all nodes and edges and are thus trivially continuous between neighbouring elements in the spatial domain. They also satisfy the partition of unity condition at the nodes.

The interior shape functions are present only for higher order elements of $p \geq 3$ and the number of interior shape functions for the reference triangular element is $M_{\text {tria }}^{I}=(p-1)(p-2) / 2$.

\section{E.2 Quadrilateral Element}

The quadrilateral elements are finite elements consisting of four vertices (or nodes) and four sides (or edges). The high order hierarchic shape functions are generated on this reference element and then mapped to the basis functions in the spatial domain. In general, the total number of shape functions $M_{\text {quad }}$ generated on quadrilateral 
elements for a given element order $p$ is given as $M_{\text {quad }}=M_{\text {quad }}^{V}+M_{\text {quad }}^{E}+M_{\text {quad }}^{I}=$ $(p+1)^{2}$.

\section{E.2.1 The $H^{1}(\Omega)$ Vertex Shape Functions}

The hierarchic vertex shape functions generated on the quadrilateral reference element, in Figure 4.10, are illustrated in Figure E.4.

\begin{tabular}{|l||cccc|}
\hline & $V_{1}$ & $V_{2}$ & $V_{3}$ & $V_{4}$ \\
\hline \hline \multirow{2}{*}{$p=1$} & & - & -
\end{tabular}

Figure E.4: Quadrilateral finite element basis functions associated with the vertex degrees of freedom of the reference quadrilateral element.

The vertex shape functions, illustrated in Figure E.4, are generated by

$$
N_{i}^{V}=L_{i},
$$

where the subscripts $i$ of the vertex shape function denotes the vertices to which the shape functions are associated. These functions are equal to the nodal coordinates of a quadrilateral $L_{i}$, given mathematically as

$$
\begin{aligned}
& L_{1}=(1-\xi)(1-\eta), \\
& L_{2}=\xi(1-\eta), \\
& L_{3}=\xi \eta, \\
& L_{4}=(1-\xi) \eta .
\end{aligned}
$$

Note here that these shape functions are linear functions of the reference space $(\xi, \eta)$ and satisfy the partition of unity condition, that

$$
N_{1}^{V}+N_{2}^{V}+N_{3}^{V}+N_{4}^{V}=1,
$$

such that the functions have unit value at the node to which they are associated and zero value at the other two nodes. These shape functions are also the same as the standard nodal (or Lagrange-type) shape functions for a nodal finite element basis. The vertex shape functions are continuous between neighbouring elements in the spatial domain.

For quadrilateral elements the vertex shape functions are generated for all $p \geq 1$ and the number of vertex shape functions always corresponds to the number of vertices of the element, hence $M_{\text {quad }}^{V}=4$ in this case. 


\section{E.2.2 The $H^{1}(\Omega)$ Edge Shape Functions}

The hierarchic edge shape functions generated on the reference element, in Figure 4.10, are illustrated in Figure E.5.

\begin{tabular}{|c|c|c|c|c|}
\hline . & $E_{1}$ & $E_{2}$ & $E_{3}$ & $E_{4}$ \\
\hline$p=2$ & & & & \\
\hline$p=3$ & & & & \\
\hline$p=4$ & & & & \\
\hline$p=5$ & & & & \\
\hline$p=6$ & & & & \\
\hline
\end{tabular}

Figure E.5: Quadrilateral finite element basis functions associated with the edge degrees of freedom of the reference quadrilateral element.

The hierarchical type (modal) edge shape functions, illustrated in Figure E.5, are typically generated through scaled Legendre polynomials as

$$
N_{i-1}^{E_{m}}=\varphi_{i}^{\mathcal{S}}\left(L_{n_{2}}-L_{n_{1}}, L_{n_{2}}+L_{n_{1}}\right)
$$

where the subscript $i=2, \ldots, p$ denotes the shape function number for a given edge $E_{m}=\left\{n_{1}, n_{2}\right\}$ consisting of the two vertices $n_{1}, n_{2}, m=1,2,3,4$ defines the edge number of the quadrilateral ${ }^{3}$ and $\varphi_{n}^{\mathcal{S}}$ denotes the scaled integrated Legendre polynomials $^{4}$.

Note here that these shape functions vanish at the nodes and thus the partition of unity condition is still satisfied at the nodes of the element. Same as with the triangular element, these shape functions pose conformity issues between neighbouring elements in the spatial domain, where the orientation of the edges may differ, due to potential sign conflicts in the functions. This must be resolved in the same way as the triangular element by incorporating a sign change in the referential to spatial mapping to enforce the $H^{1}(\Omega)$ conformity. This only effects odd-order finite

\footnotetext{
${ }^{3}$ For the quadrilateral reference element the relative vertices $n_{1}, n_{2}$ of the edges are: $E_{1}=\{1,2\}$, $E_{2}=\{3,4\}, E_{3}=\{2,3\}$ and $E_{4}=\{4,1\}$.

${ }^{4}$ The scaled integrated Legendre polynomials are given as $\varphi_{n}^{\mathcal{S}}(s, t)=\int t^{n} \ell_{n-1}\left(\frac{s}{t}\right) \mathrm{d} s$, where $\ell_{n-1}$ is the standard Legendre polynomials.
} 
elements, see [17] for further details. This change restores the continuity across all interfaces between elements containing the edge in the spatial domain.

The edge shape functions are present only for higher order elements of $p \geq 2$ and the number of edge shape functions for the reference quadrilateral element is $M_{\text {quad }}^{E}=4(p-1)$.

\section{E.2.3 The $H^{1}(\Omega)$ Interior Shape Functions}

The hierarchic interior shape functions generated on the reference element, in Figure 4.10, are illustrated in Figure E.5.

\begin{tabular}{|c|c|c|c|c|c|}
\hline & $p=2$ & $p=3$ & $p=4$ & $p=5$ & $p=6$ \\
\hline$p=2$ & & & & & \\
\hline$p=3$ & & & & & \\
\hline$p=4$ & & & & & \\
\hline$p=5$ & & & & & \\
\hline$p=6$ & & & & & \\
\hline
\end{tabular}

Figure E.6: Quadrilateral finite element basis functions associated with the interior degrees of freedom of the reference quadrilateral element.

The hierarchical type (modal) interior shape functions, illustrated in Figure E.6, are generated through multiplications of scaled integrated Legendre polynomials for fixed edges as

$$
N_{i-1, j-1}^{I}=\varphi_{i-1}^{\mathcal{S}}\left(L_{2}-L_{1}, L_{2}+L_{1}\right) \varphi_{j-1}^{\mathcal{S}}\left(L_{4}-L_{1}, L_{4}+L_{1}\right),
$$

where the subscripts $i, j=2, \ldots, p$. To visualise how the shape functions stack up for each value of $p$, each subscript $i$ corresponds to a row and $j$ corresponds to a column in Figure E.6. So for $p=2$ only the top-left most shape function is generated, likewise for $p=3$ only the four top-left most functions are generated and so on. The interior basis functions are zero valued at all nodes and edges and are thus trivially continuous between neighbouring elements in the spatial domain.

The interior shape functions for quadrilateral elements are present only for higher order elements of $p \geq 2$ and the number of interior shape functions for the reference quadrilateral element is $M_{\text {quad }}^{I}=(p-1)^{2}$. 



\section{Appendix F}

\section{Perturbed Eddy Current Problem}

Infinite elements allow for the accurate solution to unbounded problems subject to decay conditions applied at $\infty$, see Section 4.4.4 for details. Accurate solutions are obtained by applying the exact boundary condition at infinity and mapping the outer boundary to infinity. In order to allow for this application the terms at $\infty$ must vanish and so the decay condition of the field must tend to 0 to remove any integration at infinity. This is naturally the case for the MRI scanner problem, however, in order to employ infinite elements on the Eddy current sphere problem, described in Section 5.3.1, the problem must be recast in terms of solving for a perturbed field variable such that the decay condition becomes 0. This Appendix describes the eddy current problem recast in the perturbed field case and enriches the topics discussed in Chapter 5.

\section{F.1 Traditional Eddy Current Problem}

The traditional eddy current problem in a time harmonic description, as described in Chapter 2 or [100], is defined as: Solve for the magnetic vector potential $\mathcal{A}$ such that

$$
\begin{aligned}
\nabla \times \mu^{-1} \nabla \times \mathcal{A}+\mathrm{i} \omega \gamma \mathcal{A} & =\mathbf{0} & & \text { in } \Omega_{c}, \\
\nabla \times \mu_{0}^{-1} \nabla \times \mathcal{A} & =\mathbf{0} & & \text { in } \mathbb{R}^{3} \backslash \Omega_{c}, \\
\nabla \times \mathcal{A} & =\mu_{0} \mathcal{H}_{0} & & \text { on } \partial \Omega \text { as }|\boldsymbol{x}| \rightarrow \infty, \\
{[\boldsymbol{n} \times \boldsymbol{A}]_{\partial \Omega_{c}} } & =\mathbf{0} & & \text { on } \partial \Omega_{c}, \\
{\left[\boldsymbol{n} \times \mu^{-1} \nabla \times \mathcal{A}\right]_{\partial \Omega_{c}} } & =\mathbf{0} & & \text { on } \partial \Omega_{c},
\end{aligned}
$$

where $\mu$ is the material permeability, $\gamma$ the conductivity, $\boldsymbol{n}$ the outward facing unit normal vector to the boundary, $\mathcal{H}_{0}$ is the applied external magnetic field. 


\section{F.2 Perturbed Field Problem}

In order to allow for the decay condition in (F.1c) to become 0 at $\infty$ the field must be composed into

$$
\mathcal{A}=\mathcal{A}_{0}+\mathcal{A}_{*} \quad \mathcal{H}=\mathcal{H}_{0}+\mathcal{H}_{*},
$$

where $\mathcal{H}_{*}$ is the perturbed magnetic field, $\mathcal{H}_{0}$ is the constant external applied magnetic field, $\boldsymbol{A}_{*}$ is the perturbed magnetic vector potential and the background vector potential $\boldsymbol{A}_{0}$ is expressed as

$$
\nabla \times \mathcal{A}_{0}=\mu_{0} \mathcal{H}_{0}
$$

This transformation of the magnetic field results in the decay condition of the vector potential in (F.1c) becoming

$$
\begin{aligned}
\nabla \times \mathcal{A} & =\mu_{0} \mathcal{H}_{0} & & \text { on } \partial \Omega \text { as }|\boldsymbol{x}| \rightarrow \infty \\
\nabla \times \boldsymbol{A}_{*}+\nabla \times \boldsymbol{A}_{0} & =\mu_{0} \boldsymbol{H}_{0} & & \text { on } \partial \Omega \text { as }|\boldsymbol{x}| \rightarrow \infty \\
\nabla \times \boldsymbol{A}_{*} & =\mathbf{0} & & \text { on } \partial \Omega \text { as }|\boldsymbol{x}| \rightarrow \infty,
\end{aligned}
$$

which allows for the infinite elements to be employed. The system in (F.1) under the transformation of the magnetic field now becomes: Solve for the perturbed magnetic vector potential $\boldsymbol{A}_{*}$ such that

$$
\begin{aligned}
\nabla \times \mu^{-1} \nabla \times \boldsymbol{A}_{*}+\mathrm{i} \omega \gamma \boldsymbol{A}_{*} & =-\mathrm{i} \omega \gamma \boldsymbol{A}_{0} & & \text { in } \Omega_{c} \\
\nabla \times \mu_{0}^{-1} \nabla \times \boldsymbol{A}_{*} & =\mathbf{0} & & \text { in } \mathbb{R}^{3} \backslash \Omega \\
\nabla \times \boldsymbol{A}_{*} & =\mathbf{0} & & \text { on } \partial \Omega \text { a } \\
{\left[\boldsymbol{n} \times \boldsymbol{A}_{*}\right]_{\partial \Omega_{c}} } & =-\left[\boldsymbol{n} \times \boldsymbol{A}_{0}\right]_{\partial \Omega_{c}} & & \text { on } \partial \Omega_{c} \\
{\left[\boldsymbol{n} \times \mu^{-1} \nabla \times \boldsymbol{A}_{*}\right]_{\partial \Omega_{c}} } & =-\left[\boldsymbol{n} \times \mu^{-1} \nabla \times \mathcal{A}_{0}\right]_{\partial \Omega_{c}} & & \text { on } \partial \Omega_{c} .
\end{aligned}
$$




\section{Appendix G}

\section{Computational Improvements}

The main topic throughout this Thesis has been the development of a computationally efficient method for solving coupled acousto-magneto-mechanical systems in MRI scanners. This work has resulted in a novel linearised approach, which provides a computationally efficient algorithm for solving these coupled systems. However, with the development of this approach, a series of aspects emerge which, if not suitably exploited, will result in suboptimal efficiency gains. This Appendix describes some of the key computer implementation aspects used to enhance the efficiency of the linearised approach and enriches the topics discussed in Chapters 4 and 7.

\section{G.1 Global System Assembly of Complex Fields}

\section{Sparse System Assembly}

In the context of a finite element code, the construction of the global system matrices (be it mass, damping and/or stiffness), typically results in sparse matrices. The sparsity patterns of these matrices can be exploited to reduce the memory required to store a complete matrix. A method for achieving this is to store the entries of the matrix into three separate column vectors denoted by $I_{Q \times 1}, J_{Q \times 1}$ and $V_{Q \times 1}$, which contain the row, column and value of the global system matrix entries, respectively. The size of the vectors $Q$ can be approximated a priori to assembly as

$$
Q=N_{d o f}^{2} N_{\text {elem }}
$$

where $N_{\text {dof }}$ defines the number of degrees of freedom per element ${ }^{1}$ and $N_{\text {elem }}$ defines the number of elements in the mesh. In the case of a multiphysics framework (as in this Thesis), then the number of elements and number of degrees of freedom per element will also depend on the physical fields. In the case of the electromagnetic field then (G.1) holds as the EM field appears in all elements, but in the mechanical

\footnotetext{
${ }^{1}$ The number of degrees of freedom per element $N_{\text {dof }}$ depends on the order and type of element. As an example, for a $p=1$ triangular element and 16 for a $p=3$ quadrilateral element for a scalar field (see Appendix E for further details.
} 
field then only the mechanical elements will be required and the number of degrees of freedom per element will double as $\boldsymbol{u}$ is a 2-component vector field. The assembly procedure of the $I, J$ and $V$ column vectors is summarised in algorithm 3.

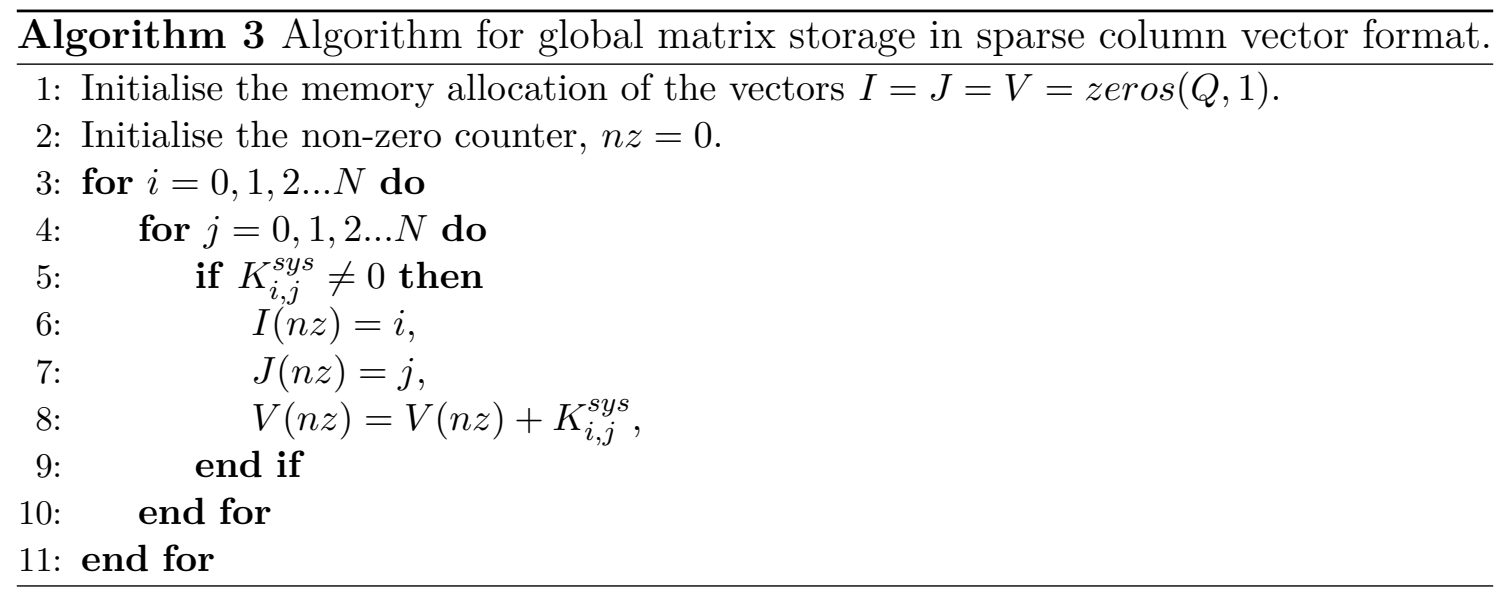

The typical sparsity pattern of an $N \times N$ system matrix, with the definition of the $I$ and $J$ directions and the annotation of the physical fields, as well as a depiction of the assembly into the $V$ column vector is illustrated in Figure G.1.

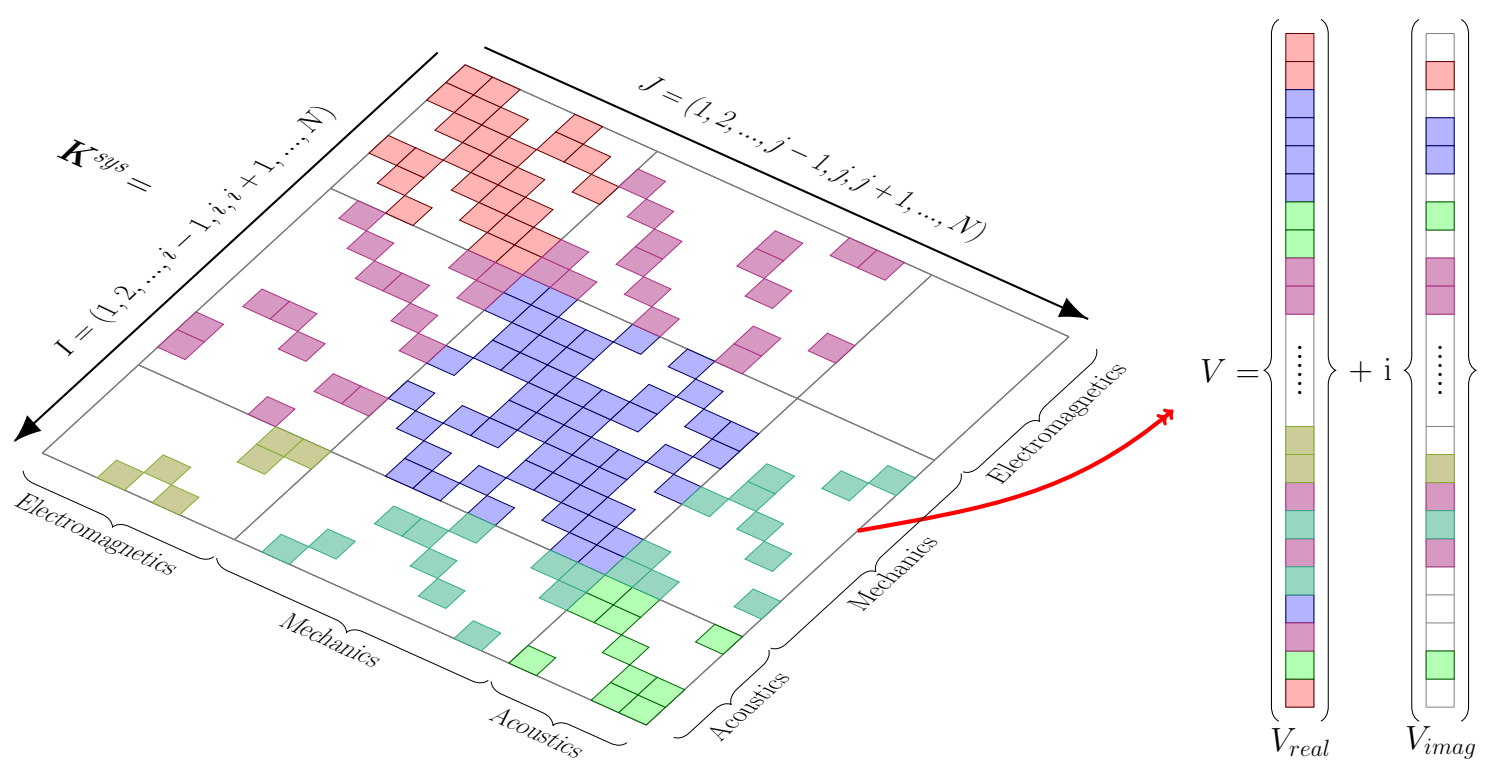

Figure G.1: Example of a fully coupled sparse system matrix with the definition of the rows and columns. The red, blue and green blocks are associated with the electromagnetic, mechanical and acoustic DOFs, respectively, where the mixed colours indicate coupled two-field DOFs.

\section{Problems with Complex Variables}

In the case of the time harmonic solver, however, the solution variables and hence some entries in $\mathbf{K}^{\text {sys }}$ are complex, which can cause significant increase in the computational time if not correctly handled in MATLAB. MATLAB inherently has a 
complex variable defined, which stores both a real and imaginary component to the variable. So, in the case of purely complex matrices, one could simply initialise the $V$ vector as a complex array and then proceed to store the variables to it.

However, in general, this is not the case for $\mathbf{K}^{\text {sys }}$, due to the complex nature of the coupling, which contains both real and imaginary valued components. In this case, if one was to initialise $V$ as a complex array, then in the case of storing a real variable to it, the real variable would first need to be converted to imaginary, with a 0 complex component, before being stored. Whilst this process is not particularly costly in isolation, doing this for large system matrices ,with thousands of DOFs will cause the code to bottleneck. Hence, the best way to treat this is to create two separate real valued $V$ vectors, one for the real and one for the complex component of a variable, and store the components individually inside them. Once the storage of the system matrix is complete, the two vectors can then be added together as

$$
V=V_{\text {real }}+\mathrm{i} V_{\text {imag }}
$$

to recover the complete system matrix in vector form, as illustrated in Figure G.1.

\section{G.2 Vectorisation}

MATLAB [1] is a compile-time scientific computing language that allows for rapid development of code with relative ease. The nature of this language is based around being able to handle matrix operations. With this in mind, compared with most other scientific computing languages, such as C, FORTRAN, Java etc., which utilise loops, a new programming concept has emerged, known as "vectorisation" [103]. This concept uses the idea of constructing matrices to then perform vector and matrix based operations, such as matrix multiplication, rather than performing loops, to significantly reduce the computation time.

An example, for the vectorisation of the multiplication of two matrices, would be to convert the following loop based code in algorithm 4 to the vectorised code in algorithm 5 as
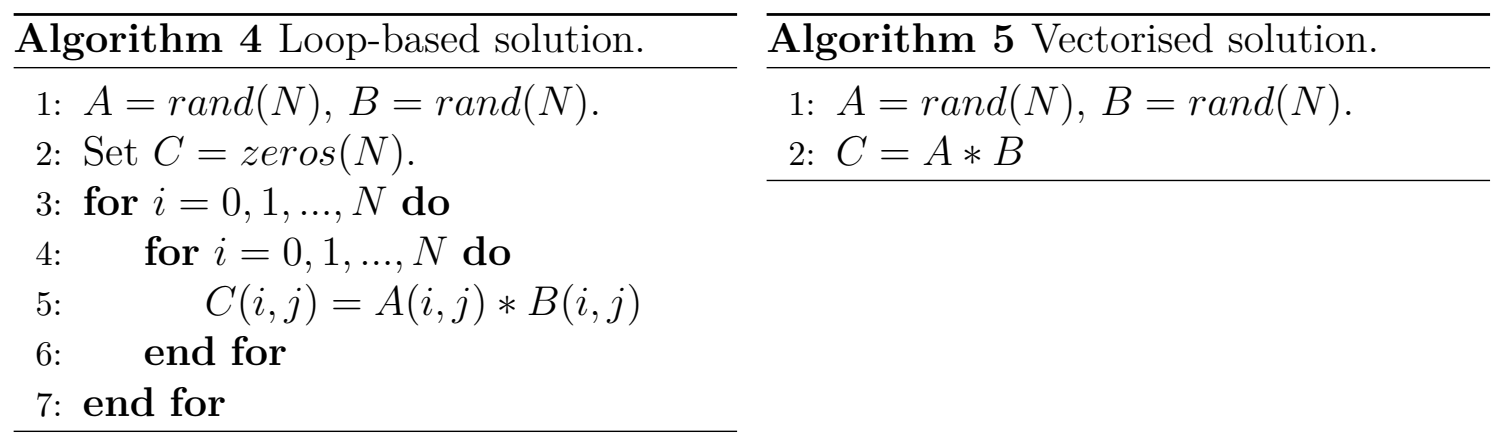

For more detailed studies of vectorisation of code see [103] and more specifically when dealing with $h p$-finite elements in MATLAB see [119]. 


\section{G.3 Residual Scaling}

In the linearised approach, the resulting residual vector $\tilde{\mathbf{R}}$ of the system contains purely forcing terms that depend on time, such as the current density in the coils and the applied mechanical body force. For this reason, the residual vector is independent of iteration and depends only on the sourcing function at the current timestep. In order to avoid reconstructing the residual vector at each timestep, a method for constructing a scaled residual and then post multiplying by the sourcing function is presented here.

First, the complete linear system matrices $\tilde{\mathbf{M}}, \tilde{\mathbf{C}}$ and $\tilde{\mathbf{K}}$ are constructed as per the description in Chapter 7. The residual vector is then constructed using a sourcing (current density, body force and acoustic sourcing (incident field), if they are present) vector with a magnitude of unity $\boldsymbol{J}_{\delta}, \boldsymbol{f}_{\delta}$. This concept also incorporates Dirichlet conditions and so these are also added with a magnitude of unity.

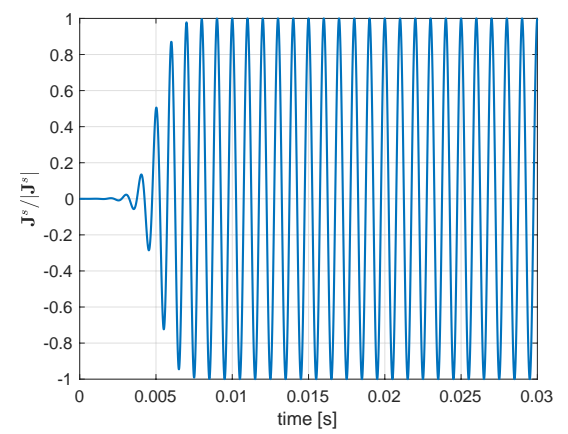

Figure G.2: Plot of the relative sourcing current density $\boldsymbol{J}^{s}(t) /\left|\boldsymbol{J}^{s}\right|$ as a function of time for a simple single frequency sinusoidal excitation .

With a priori knowledge of the sourcing functions in time, an example of which is illustrated in Figure G.2, the magnitude of the sourcing at a specific timestep can be determined. This value is then used to multiply with the unitised residual vector and the linear system solved to obtain the solution at each timestep. This method allows for a single construction of the linear system which can then be used at each timestep to determine the solution and will result in significant time savings.

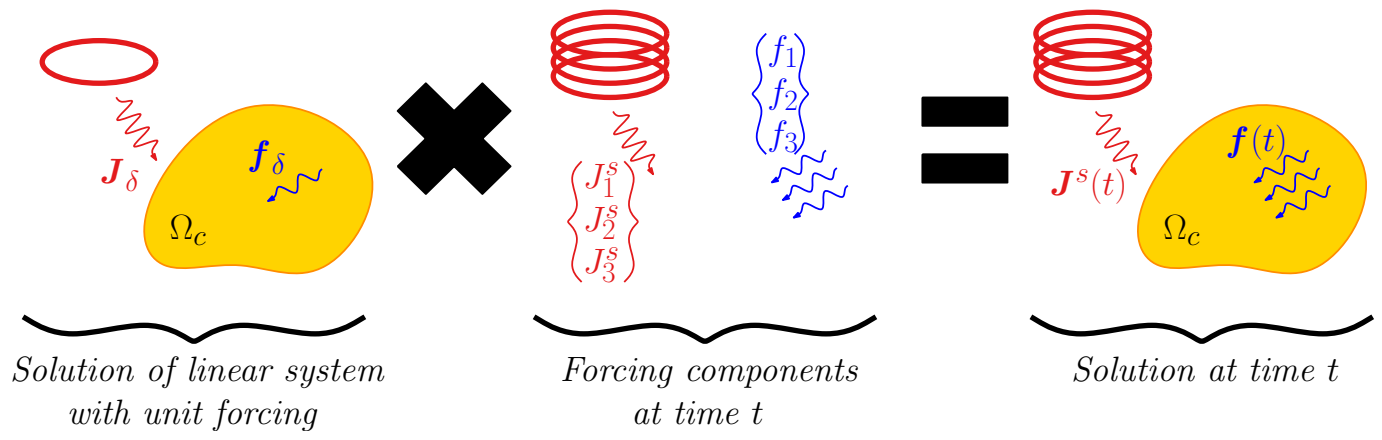

Figure G.3: Graphical representation of the solution to the system of equations using the scaled residual method. 


\section{Part VII}

\section{References}





\section{Bibliography}

[1] Mathworks: MATLAB. https://uk. mathworks.com/products/matlab. html, Date accessed: $11^{\text {th }}$ January 2018.

[2] AISI type 304 stainless steel. http://asm.matweb.com/search/ SpecificMaterial.asp?bassnum=mq304a, Date accessed $21^{\text {st }}$ January 2018.

[3] Properties of aluminium. http://www.aluminiumdesign.net/ why-aluminium/properties-of-aluminium/, Date accessed $21^{\text {st }}$ January 2018.

[4] NHS choices: CT scan. http://www.nhs.uk/conditions/CT-scan/Pages/ Introduction.aspx, Date accessed 25 ${ }^{\text {th }}$ August 2017.

[5] NHS choices: MRI scan. http://www.nhs.uk/conditions/mri-scan/ Pages/Introduction. aspx, Date accessed $25^{\text {th }}$ August 2017.

[6] Ansys software. http://www.ansys.com, Date accessed 25 th July 2016.

[7] COMSOL multiphysics software. https://www.comsol.com, Date accessed $25^{\text {th }}$ July 2016.

[8] NACS finite element analysis software. http://www.simetris.de/en/ Table/Products/NACS-Finite-Element-Simulation/, Date accessed $25^{\text {th }}$ July 2016.

[9] OECD health statistics. http://dx.doi.org/10.1787/data-00541-en, Date accessed $26^{\text {th }}$ August 2017.

[10] OPERA finite element analysis software. http://operafea.com/, Date accessed $29^{\text {th }}$ August 2017.

[11] Electromagnetic fields and public health: Static electric and magnetic fields. http://www.who.int/peh-emf/publications/facts/fs299/en/, Date accessed $3^{\text {rd }}$ September 2017.

[12] J. Esch J. Ekman A. Mayo A. Orlandi A. E. Ruehli, G. Antonini. Nonorthogonal PEEC formulation for time- and frequency-domain em and circuit modelling. IEEE Transactions on Electromagnetic Compatibility, 45(2):167-176, 2003. 
[13] V. Shankar A. H. Mohammadian and W. F. Hall. Computation of electromagnetic scattering and radiation using a time-domain finite-volume discretization procedure. Computer Physics Communications, 68(1):175-196, 1991.

[14] R. Aarnink and J. Overweg. Magnetic resonance imaging: a success story for superconductivity. Europhysics News, 43(4):26-29, 2012.

[15] H. U. Ahmed, A. Kirkham, M. Arya, R. Illing, A. Freeman, C. Allen, and M. Emberton. Is it time to consider a role for MRI before prostate biopsy? Nature Reviews Clinical Oncology, 6(4):197-206, 2009.

[16] M. Ainsworth. Discrete dispersion relation for $h p$-version finite element approximation at high wave number. SIAM Journal on Numerical Analysis, 42(2):553-575, 2004.

[17] M. Ainsworth and J. Coyle. Hierarchic finite element bases on unstructured tetrahedral meshes. International Journal For Numerical Methods In Engineering, 58(14):2103-2130, 2003.

[18] H. Ammari, A. Buffa, and J. C. Nedéléc. A justification of eddy currents model for the Maxwell equations. SIAM Journal on Applied Mathematics, 60:1805-1823, 2000.

[19] I. K. Argyros. Convergence and Applications of Newton-type Iterations. Springer, 2008.

[20] W. A. Artuzi. Improving the newmark time integration scheme in finite element time domain methods. IEEE Microwave and Wireless Components Letters, 15(12):898-900, 2005.

[21] R. J. Astley. Infinite elements for wave problems: a review of current formulations and an assessment of accuracy. International Journal for Numerical Methods in Engineering, 49(7):951-976, 2000.

[22] I. Babuška and B. Q. Guo. The $h, p$ and $h-p$ version of the finite element method: Basis theory and applications. Advances in Engineering Software, 15:159-174, 1992.

[23] I. Babuška and M. Suri. The $p$ - and $h-p$ versions of the finite element method, an overview. Computer Methods in Applied Mechanics and Engineering, 80(1):5-26, 1990.

[24] S. Baek, R. L. Gleason, K. R. Rajagopal, and J. D. Humphrey. Theory of small on large: Potential utility in computations of fluid-solid interactions in arteries. Computer Methods in Applied Mechanics and Engineering, 196:30703078, 2007. 
[25] S. Bagwell, P. D. Ledger, A. J. Gil, and M. Mallett. Transient solutions to nonlinear acousto-magneto-mechanical coupling for axisymmetric MRI scanner design. International Journal for Numerical Methods in Engineering. DOI: 10.1002/nme.5802, 2018.

[26] S. Bagwell, P. D. Ledger, A. J. Gil, M. Mallett, and M. Kruip. A linearised $h p$-finite element framework for acousto- magneto-mechanical coupling in axisymmetric MRI scanners. International Journal for Numerical Methods in Engineering, 112(10):1323-1352, 2017.

[27] C. A. Balanis. Modern Antenna Handbook. John Wiley \& Sons, 2011.

[28] G. Beer and J. O. Watson. Infinite boundary elements. International Journal for Numerical Methods in Engineering, 28(6):1233-1247, 1989.

[29] R. G. H. Beets-Tan, G. L. Beets, A. C. W. Borstlap, T. K. Oei, T. M. Teune, M. F. von Meyenfeldt, and M. A. van Engelshoven. Preoperative assessment of local tumor extent in advanced rectal cancer: CT or high-resolution MRI? Abdominal Imaging, 25(5):533-541, 2000.

[30] J. W. Belliveau, D. N. Kennedy, R. C. McKinstry, B. R. Buchbinder, R. M. Weisskoff, M. S. Cohen, J. M. Vevea, T. J. Brady, and B. R. Rosen. Functional mapping of the human visual cortex by magnetic resonance imaging. Science, 254(5032):716-719, 1991.

[31] J. P. Berenger. A perfectly matched layer for the absorption of electromagnetic waves. Journal of Computational Physics, 114(2):185-200, 1994.

[32] J. P. Berenger. Perfectly matched layer for the FDTD solution of wavestructure interaction problems. IEEE Transactions on Antennas and Propagation, 44(1):110-117, 1996.

[33] M. Besbes, Z. Ren, and A. Razek. Finite element analysis of magnetomechanical coupled phenomena in magnetostrictive materials. IEEE Transactions on Magnetics, 32(3):1058-1061, 1996.

[34] P. Bettess. Infinite Elements. Penshaw Press, 1992.

[35] P. Bettess and J. A. Bettess. Infinite elements for static problems. Engineering Computations, 1(1):4-16, 1984.

[36] J. Biermann, O. von Estorff, S. Petersen, and C. Wenterodt. Higher order finite and infinite elements for the solution of helmholtz problems. Computer Methods in Applied Mechanics and Engineering, 198(13):1171-1188, 2009.

[37] O. Biro. Edge element formulations of eddy current problems. Computer Methods in Applied Mechanics and Engineering, 169(3-4):391-405, 1999.

[38] J. Bonet, A. J. Gil, and R. D. Wood. Nonlinear Solid Mechanics for Finite Element Analysis. Cambridge University Press, 2017. 
[39] A. Bossavit. Whitney forms: a class of finite elements for three-dimensional computations in electromagnetism. IEE Proceedings A (Physical Science, Measurement and Instrumentation, Management and Education, Reviews), 135(8):493-500, 1988.

[40] F. Bouillaulta, A. Buffab, Y. Madayc, and F. Rapetti. Simulation of a magneto-mechanical damping machine: analysis, discretization, results. Computer Methods in Applied Mechanics and Engineering, 191:2587-ĂŞ2610, 2002.

[41] A. F. Bower. Applied Mechanics of Solids. CRC Press, Florida, 2009.

[42] R. K. Brayton, F. G. Gustavson, and G. D. Hachtel. A new efficient algorithm for solving differential-algebraic systems using implicit backward differentiation formulas. Proceedings of the IEEE, 60(1):98-108, 1972.

[43] A. N. Brooks and T. J. R. Hughes. Streamline upwind/Petrov-Galerkin formulations for convection dominated flows with particular emphasis on the incompressible Navier-Stokes equations. Computer Methods in Applied Mechanics and Engineering, 32(1-3):199-259, 1982.

[44] R. W Brown, Y. C. N. Cheng, E. M. Haacke, M. R. Thompson, and $\mathrm{R}$. Venkatesan. Magnetic resonance imaging: physical principles and sequence design. John Wiley \& Sons, 2014.

[45] A. Brydie and N. Raby. Early MRI in the management of clinical scaphoid fracture. The British Journal of Radiology, 76(905):296-300, 2003.

[46] X. Qiu L. Brooks D. Moreau C. Hansen, S. Snyder. Active Control of Noise and Vibration. CRC Press, 2012.

[47] H. Carr. Free precession techniques in nuclear magnetic resonance. $\mathrm{PhD}$ Thesis, Harvard University. 1952.

[48] J. A. Chalela, C. S. Kidwell, L. M. Nentwich, M. Luby, J. A. Butman, A. M. Demchuk, M. D. Hill, N. Patronas, L. Latour, and S. Warach. Magnetic resonance imaging and computed tomography in emergency assessment of patients with suspected acute stroke: a prospective comparison. The Lancet, 369(9558):293-298, 2007.

[49] Y. C. Chang and L. Demkowicz. Scattering on a spherical shell. comparison of 3-D elasticity and Kirchhoff shell theory results. Computer Assisted Mechanics and Engineering Sciences, 2:207-229, 1995.

[50] G. Chen and J. Zhou. Boundary Element Methods with Applications to Nonlinear Problems. Springer Science \& Business Media, Paris, 2010.

[51] J. Chen and X. Jiang. Stress analysis of a 7T actively shielded superconducting magnet for animal MRI. IEEE Transactions on Applied Superconductivity, 22(3):4903104-4903104, 2012. 
[52] L. Chilton and M. Suri. On the selection of a locking-free $h p$ element for elasticity problems. International Journal for Numerical Methods in Engineering, 40:2045-2062, 1997.

[53] F. Chinesta, A. Ammar, and E. Cueto. Recent advances and new challenges in the use of the proper generalized decomposition for solving multidimensional models. Archives of Computational Methods in Engineering, 17(4):327-350, 2010 .

[54] F. Chinesta, R. Keunings, and A. Leygue. The Proper Generalized Decomposition for Advanced Numerical Simulations: A Primer. Springer Science \& Business Media, 2013.

[55] M. Chiumenti, Q. Valverde, C. Agelet de Saracibar, and M. Cervera. A stabilized formulation for incompressible elasticity using linear displacement and pressure interpolations. Computer Methods in Applied Mechanics and Engineering, 191(46):5253 - 5264, 2002.

[56] Z. H. Cho, S. H. Park, l. H. Kim, S. C. Chung, S. T. Chung, I. Y. Chung, C. W. Moon, I. H. Yi, C. H. Sin, and E. K. Wong. Analysis of acoustic noise in MRI. Magnetic Resonance Imaging, 15:815-822, 1997.

[57] J. Chung and G. M. Hulbert. A Time Integration Algorithm for Structural Dynamics With Improved Numerical Dissipation: The Generalized- $\alpha$ Method. Journal of Applied Mechanics, 60:371-375, 1993.

[58] M. Clemens, M. Wilke, and T. Weiland. Linear-implicit time integration schemes for error-controlled transient nonlinear magnetic field simulations. IEEE Transactions on Magnetics, 39(3):1175-1178, 2003.

[59] R. Coifman, V. Rokhlin, and S. Wandzura. The fast multipole method for the wave equation: a pedestrian prescription. IEEE Antennas and Propagation Magazine, 35(3):7-12, 1993.

[60] C. M. Collins and Z. Wang. Calculation of radiofrequency electromagnetic fields and their effects in MRI of human subjects. Magnetic Resonance in Medicine, 65(5):1470-1482, 2011.

[61] L. Corradi. A displacement formulation for the finite element elastic-plastic problem. Meccanica, 18(2):77-91, 1983.

[62] T. C. Cosmus and M. Parizh. Advances in whole-body MRI magnets. IEEE Transactions on Applied Superconductivity, 21(3):2104-2109, 2011.

[63] S. C. Crozier and F. Liu. Numerical evaluation of the fields induced by body motion in or near high-field MRI scanners. Progress in Biophysics and Molecular Biology, 87(2-3):267-278, 2005. 
[64] Y. Dai, Q. Wang, C. Wang, L. Li, H. Wang, Z. Ni, S. Song, S. Chen, B. Zhao, H. Wang, Y. Li, X. Hu, C. Cui, J. Cheng, Y. Lei, and L. Yan. Structural design of a $9.4 \mathrm{~T}$ whole-body MRI superconducting magnet. IEEE Transactions on Applied Superconductivity, 22(3):4900404-4900404, 2012.

[65] R. Damadian. Tumor detection by nuclear magnetic resonance. Science, 171(3976):1151-1153, 1971.

[66] R. Damadian. Apparatus and method for detecting cancer in tissue, 1974. patent number: US3789832 A.

[67] R. Damadian, L. Minkoff, M. Goldsmith, M. Stanford, and J. Koutcher. Field focusing nuclear magnetic resonance (fonar): visualization of a tumor in a live animal. Science, 194(4272):1430-1432, 1976.

[68] P. J. Davis and P. Rabinowitz. Methods of Numerical Integration. 1984, Academic Press inc.

[69] F. de Vocht, T. Stevens, P. Glover, A. Sunderland, P. Gowland, and H. Kromhout. Cognitive effects of head-movements in stray fields generated by a 7 Tesla whole-body MRI magnet. Bioelectromagnetics, 28(4):247-255, 2007.

[70] A. Deraemaeker, I. Babuška, and P. Bouillard. Dispersion and pollution of the FEM solution for the helmholtz equation in one, two and three dimensions. International Journal for Numerical Methods in Engineering, 46(4):471-499, 1999.

[71] M. A. Dokainish and K. Subbaraj. A survey of direct time-integration methods in computational structural dynamics: Explicit methods. Computers 86 Structures, 32(6):1371 - 1386, 1989.

[72] S. Doll, K. Schweizerhof, R. Hauptmann, and C. Freischläger. On volumetric locking of low-order solid and solid-shell elements for finite elastoviscoplastic deformations and selective reduced integration. Engineering Computations, 17(7):874-902, 2000.

[73] J. Dutiné, M. Clemens, S. Schöps, and G. Wimmer. Explicit time integration of transient eddy current problems. International Journal of Numerical Modelling, e2227:9, 2017.

[74] W. A. Edelstein, R. A. Hedeen, R. P. Mallozzi, S. A. El-Hamamsy, R. A. Ackermann, and T. J. J. Havens Havens. Making MRI quieter.

[75] W. A. Edelstein, T. K. Kidane, V. Taracila, T. N. Baig, T. P. Eagan, Y. C. N. Cheng, R. W. Brown, and J. A. Mallick. Active-passive gradient shielding for MRI acoustic noise reduction. Magnetic Resonance in Medicine, 53(5):10131017, 2005. 
[76] A. El Kacimi and O. Laghrouche. Numerical modelling of elastic wave scattering in frequency domain by the partition of unity finite element method. International Journal for Numerical Methods in Engineering, 77(12):1646-1669, 2009 .

[77] H. C. Elman, D. J. Silvester, and A. J. Wathen. Finite Elements and Fast Iterative Solvers: With Applications in incompressible fluid dynamics. Oxford Science Publications, 2014.

[78] D. Engwirda. MESH2D - Delaunay-based unstructured meshgeneration. https://uk.mathworks.com/matlabcentral/fileexchange/ 25555-mesh2d-delaunay-based-unstructured-mesh-generation. Accessed 18/09/2017.

[79] K. Eriksson, C. Johnson, and A. Logg. Explicit time-stepping for stiff odes. SIAM Journal of Scientific Computing, 25(4):1142-?1157, 2012.

[80] A. Eringen and G. Maugin. Electrodynamics of Continua I: Foundations and Solid Media. Springer, Heidelberg, 2011.

[81] G. J. Fix. Eigenvalue approximation by the finite element method. Advances in Mathematics, 10(2):300-316, 1973.

[82] B. U. Foerster, D. Tomasi, and E. C. Caparelli. Magnetic field shift due to mechanical vibration in functional magnetic resonance imaging. Magnetic Resonance in Medicine, 54(5):1261-1267, 2005.

[83] W. Fong. Handbook of MRI Pulse Sequences. Academic Press, 2004.

[84] D. Gallichan, J. Scholz, A. Bartsch, T. E. Behrens, M. D. Robson, and K. L. Miller. Addressing a systematic vibration artifact in diffusion-weighted MRI. Human Brain Mapping, 31(2):193-202, 2010.

[85] N. Galopin, X. Mininger, F. Frederic, and L. Daniel. Finite element modeling of magnetoelectric sensors. IEEE Transactions on Magnetics, 44(6):834-837, 2008 .

[86] A. Gansen, M. El Hachemi, S. Belouettar, O. Hassan, and K. Morgan. An effective 3d leapfrog scheme for electromagnetic modelling of arbitrary shaped dielectric objects using unstructured meshes. Computational Mechanics, 56(6):1023-?1037, 2015.

[87] L. Gaul, M. Kögl, and M. Wagner. Boundary Element Methods for Engineers and Scientists. Springer, Berlin, 2003.

[88] T. D Gerhardt. A hybrid/finite element approach for stress analysis of notched anisotropic materials. Journal of Applied Mechanics, 51(4):804-810, 1984.

[89] W. C. Gibson. The Method of Moments in Electromagnetics. CRC Press, 2015. 
[90] A. J. Gil. Structural analysis of prestressed Saint Venant-Kirchhoff hyperelastic membranes subjected to moderate strains. Computers and Structures, 84(15-16):1012-1028, 2006.

[91] A. J. Gil and J. Bonet. Finite element analysis of prestressed structural membranes. Finite Elements in Analysis and Design, 42(8-9):683-697, 2006.

[92] A. J. Gil and J. Bonet. Finite element analysis of partly wrinkled reinforced prestressed membranes. Computational Mechanics, 40(3):595-615, 2007.

[93] A. J. Gil and P. D. Ledger. A coupled $h p$-finite element scheme for the solution of two-dimensional electrostrictive materials. International Journal for Numerical Methods in Engineering, 91:1158-1183, 2012.

[94] G. Giovannetti, V. Hartwig, V. Viti, P. Zadaricchio, L. Meini, L. Landini, and A. Benassi. Low field elliptical mr coil array designed by FDTD. Concepts in Magnetic Resonance Part B: Magnetic Resonance Engineering, 33B(1):32-38, 2008 .

[95] I. Gomez-Revuelto, L. E. Garcia-Castillo, and L. Demkowicz. A comparison between pml, infinite elements and an iterative bem as mesh truncation methods for $h p$ self-adaptive procedures in electromagnetics. Progress In Electromagnetics Research, 126:499-519, 2012.

[96] S. Gopalakrishnan, A. Chakraborty, and D. R. Mahapatra publisher=. Spectral Finite Element Method: Wave Propagation, Diagnostics and Control in Anisotropic and Inhomogeneous Structures.

[97] W. J. Gordon. Blending-function methods of bivariate and multivariate interpolation and approximation. SIAM Journal of Numerical Analysis, 8(1):158?177, 1971.

[98] D. Grainger. Safety Guidelines for Magnetic Resonance Imaging Equipment in Clinical Use. Medicines and Healthcare Products Regulatory Agency, 2015.

[99] M. J. Graves and D. G. Mitchell. Body MRI artifacts in clinical practice: a physicist's and radiologist's perspective. Journal of Magnetic Resonance Imaging, 38(2):269-287, 2013.

[100] D. J. Griffiths. Introduction to Electrodynamics. Pearson Education, 2014.

[101] M. Guan, X. Wang, L. Ma, Y. Zhou, and C. Xin. Magneto-mechanical coupling analysis of a superconducting solenoid magnet in self-magnetic field. IEEE Transactions on Applied Superconductivity, 24(3):1-4, 2014.

[102] W Gui and I Babuška. The $h, p$ and $h-p$ versions of the finite element method in 1 dimension. part 1 . the error analysis of the p-version. Numerische Mathematik, 49(6):577-612, 1986. 
[103] A. K. Gupta. Numerical Methods using MATLAB. Apress, Berkeley, CA, 2014.

[104] E. Haber and U. M. Ascher. Fast finite volume simulation of 3D electromagnetic problems with highly discontinuous coefficients. SIAM Journal on Scientific Computing, 22(6):1943-1961, 2006.

[105] E. Haber, U. M. Ascher, D. A. Aruliah, and D. W. Oldenburg. Fast simulation of 3D electromagnetic problems using potentials. Journal of Computational Physics, 163(1):150-171, 2000.

[106] R. F. Harrington. Field Computation by Moment Methods. IEEE Press, 1968.

[107] A. Hauck, T. Lahmer, and M. Kaltenbacher. Enhanced homogenization technique for magnetomechanical systems using the generalized finite element method. The International Journal for Computation and Mathematics in Electrical and Electronic Engineering (COMPEL), 28(4):935-947, 2009.

[108] D. Hawksworth, I. McDougall, J. Bird, and D. Black. Considerations in the design of MRI magnets with reduced stray fields. IEEE Transactions on Magnetics, 23(2):1309-1314, 1987.

[109] U. Heisserer, S. Hartmann, A. Düster, and Z. Yosibash. On volumetric lockingfree behaviour of $p$-version finite elements under finite deformations. Communications in Numerical Methods in Engineering, 24(11):1019-1032, 2008.

[110] F. Hennel, F. Girard, and T. Loenneker. "silent" MRI with soft gradient pulses. Magnetic Resonance in Medicine, 42:6-10.

[111] A. C. Hindmarsh, P. M. Gresho, and D. F. Griffiths. The stability of explicit euler time-integration for certain finite difference approximations of the multi-dimensional advection-diffusion equation. Numerical Methods in Fluids, 4(9):853-897, 1984.

[112] R. Hiptmair and P. D. Ledger. Computation of resonant modes for axisymmetric Maxwell cavities using $h p$-version edge finite elements. International Journal for Numerical Methods in Engineering, 62(12):1652-1676, 2005.

[113] R. Hiptmair and O. Sterz. Current and voltage excitations for the eddy current model. International Journal of Numerical Modelling: Electronic Networks, Devices and Fields, 18(1):1-21, 2005.

[114] T. J. R. Hughes. The Finite Element Method: Linear Static and Dynamic Finite Element Analysis. Prentice-Hall, Englewood Cliffs, 1987.

[115] N. X. Hung, S. P. A. Bordas, and N. D. Hung. Addressing volumetric locking and instabilities by selective integration in smoothed finite elements. Communications in Numerical Methods in Engineering, 25(1):19-34, 2009. 
[116] F. Ihlenburg. Finite Element Analysis of Acoustic Scattering. Springer Publishing Company, Incorporated, 2013.

[117] J.A. Jackson and W.H. Langham. Whole-body nmr spectrometer. Review of Scientific Instruments, 39(4):510-513, 1968.

[118] K. E Jansen, C. H. Whiting, and G. M. Hulbert. A generalized- $\alpha$ method for integrating the filtered navier-stokes equations with a stabilized finite element method. Computer Methods in Applied Mechanics and Engineering, 190(3):305-319, 2000.

[119] D. Jin. An $h p$-finite element computational framework for nonlinear magnetofluid problems including magnetostriction. PhD Thesis, Swansea University. 2016 .

[120] D. Jin, P. D. Ledger, and A. J. Gil. An $h p$-FEM framework for the simulation of electrostrictive and magnetostrictive materials. Computers and Structures, 133:131-148, 2014.

[121] D. Jin, P. D. Ledger, and A. J. Gil. hp-finite element solution of coupled stationary magnetohydrodynamics problems including magnetostrictive effects. Computers and Structures, 164:161-180, 2016.

[122] J. M. Jin. Electromagnetics in magnetic resonance imaging. IEEE Antennas and Propagation Magazine, 40(6):7-22, 1998.

[123] M. Kaltenbacher. Numerical Simulation of Mechatronic Sensors and Actuators. Springer Berlin Heidelberg, 2004.

[124] M. Kaltenbacher. Numerical Simulation of Mechatronic Sensors and Actuators. Springer Berlin Heidelberg, 2007.

[125] M. Kaltenbacher, H. Landes, and R. Lerch. An efficient calculation scheme for the numerical simulation of coupled magnetomechanical systems. IEEE Transactions on Magnetics, 33(2):1646-1649, 1997.

[126] M. Kaltenbacher, H. Landes, and R. Lerch. Software package for the numerical simulation of coupled field problems. Transactions on Engineering Sciences, 22:233-242, 1999.

[127] G. E. M. Karniadakis and S. Sherwin. Spectral/hp Element Methods for Computational Fluid Dynamics. Oxford University Press, 1999.

[128] C. T. Kelley. Iterative Methods for Linear and Nonlinear Equations. Society for Industrial and Applied Mathematics, 1995.

[129] Geoffrey A. Kerchner. Ultra-high field 7T MRI: A new tool for studying alzheimer's disease. Journal of Alzheimer's Disease, 26(3):91-95, 2011. 
[130] J.H. Keyak, J.M. Meagher, H.B. Skinner, and C.D. Mote. Automated threedimensional finite element modelling of bone: a new method. Journal of Biomedical Engineering, 12(5):389-397, 1990.

[131] C. S. Kidwell, J. A. Chalela, J. L. Saver, and et al. Comparison of MRI and ct for detection of acute intracerebral hemorrhage. Journal of the American Medical Association, 292(15):1823-1830, 2004.

[132] A. P. S. Kirkham, M. Emberton, and C. Allen. How good is MRI at detecting and characterising cancer within the prostate? European Urology, 50(6):1163$1175,2006$.

[133] M. Kirschneck, D. J. Rixen, H. Polinder, and R. van Ostayen. Effects of magneto-mechanical coupling on structural modal parameters. Topics in Modal Analysis II: Proceedings of the 32nd IMAC, A Conference and Exposition on Structural Dynamics, 8, 2014.

[134] K. Kose, Y. Matsuda, T. Kurimoto, S. Hashimoto, Y. Yamazaki, T. Haishi, S. Utsuzawa, H. Yoshioka, S. Okada, M. Aoki, and T. Tsuzaki. Development of a compact MRI system for trabecular bone volume fraction measurements.

[135] Narayan Kovvali. Theory and Applications of Gaussian Quadrature Methods. Morgan \& Claypool, 2011.

[136] M. Kozlov and R. Turner. Fast MRI coil analysis based on 3-D electromagnetic and rf circuit co-simulation. Journal of Magnetic Resonance, 200(1):147-152, 2009 .

[137] P. Kozlowski, S. D. Chang, E. C. Jones, K. W. Berean, H. Chen, and S.L. Goldenberg. Combined diffusion-weighted and dynamic contrast-enhanced MRI for prostate cancer diagnosis: Correlation with biopsy and histopathology. Journal of Magnetic Resonance Imaging, 24(1):108-113, 2006.

[138] J. M. B. Kroot. Analysis of eddy currents in a gradient coil. Scientific Computing in Electrical Engineering, 9:221-226, 2006.

[139] K. Krupa and M. Bekiesińska-Figatowska. Artifacts in magnetic resonance imaging. Polish Journal of Radiology, 80:93-106, 2015.

[140] T. Kupiszewski and O. R. Christianson. Conceptual design of superconducting magnet coils. http://aries . ucsd . edu/LIB/REPORT/SPPS/FINAL/chap4 . pdf, 1997.

[141] S. Kurz, J. Fetzer, G. Lehner, and W. M. Rucker. A novel formulation for 3D eddy current problems with moving bodies using a Lagrangian description and BEM-FEM coupling. IEEE Transactions on Magnetics, 34(5):3068-3073, 1998. 
[142] K.K. Kwong, J.W. Belliveau, D.A. Chesler, I.E. Goldberg, R.M. Weisskoff, B.P. Poncelet, D.N. Kennedy, B.E. Hoppel, M.S. Cohen, R. Turner, H. Cheng, T.J. Brady, and B.R. Rosen. Dynamic magnetic resonance imaging of human brain activity during primary sensory stimulation. Proceedings of the National Academy of Sciences, 89(12):5951-5955, 1992.

[143] P. Lacoste. Solution of Maxwell equation in axisymmetric geometry by fourier series decomposition and by use of $H(r o t)$ conforming finite element. $\mathrm{Nu}$ merische Mathematik, 84(4):577-609, 2000.

[144] R. Garcia Lage, C. M. Mota Soares, C. A. Mota Soares, and J. N. Reddy. Layerwise partial mixed finite element analysis of magneto-electro-elastic plates. Computers \& Structures, 82(17):1293-1301, 2004.

[145] O. Laghrouche and P. Bettess. Short wave modelling using special finite elements. Journal of Computational Acoustics, 08(01):189-210, 2000.

[146] O. Laghrouche and M. S. Mohamed. Locally enriched finite elements for the helmholtz equation in two dimensions. Computers \& Structures, 88(23):1469$1473,2010$.

[147] C. Lalanne. Mechanical Vibration and Shock, Fatigue Damage. John Wiley \& Sons, 2010.

[148] M. G. Larson and F. Bengzon. The Finite Element Method: Theory, Implementation, and Applications. Springer Science \& Business Media, 2013.

[149] P.C. Lauterbur. Image formation by induced local interactions: Examples of employing nuclear magnetic resonance. Nature, 242(5394):190-191, 1973.

[150] P. D. Ledger, A. J. Gil, R. Poya, M. Kruip, I. Wilkinson, and S. Bagwell. Solution of an industrially relevant coupled magneto-mechanical problem set on an axisymmetric domain. Applied Mathematical Modelling, 40:1959-1971, 2016 .

[151] P. D. Ledger, K. Morgan, J. Peraire, O. Hassan, and N. P. Weatherill. The development of an $h p$-adaptive finite element procedure for electromagnetic scattering problems. Finite Elements in Analysis and Design, 39(8):751-764, 2003.

[152] P. D. Ledger, J. Peraire, K. Morgan, O. Hassan, and N. P. Weatherill. Parameterised electromagnetic scattering solutions for a range of incident wave angles. Computer Methods in Applied Mechanics and Engineering, 193(33):3587-3605, 2004.

[153] P. D. Ledger and S. Zaglmayr. $h p$-finite element simulation of threedimensional eddy current problems on multiply connected domains. Computer Methods in Applied Mechanics and Engineering, 199:3386-3401, 2010. 
[154] L. Li, J. Cheng, C. Cui, Y. Li, Y. Dai, X. Hu, J. Liu, L. Wang, and Q. Wang. Numerical analysis of mechanical behavior for a 9.4-T whole-body MRI magnet. IEEE Transactions on Applied Superconductivity, 27(4):1-5, 2017.

[155] T. R. Ligon. Coil design for low field $\mathrm{nmr}$ and $\mathrm{nmr}$ measurements on the human arm. Masters Thesis, Oklahoma State University. 1967.

[156] J. C. Lin and Z. Wang. Acoustic pressure waves induced in human heads by rf pulses from high-field MRI scanners. Health Physics, 98:603-613, 2010.

[157] T. M. Link, S. Majumdar, J. C. Lin, D. Newitt, P. Augat, X. Ouyang, A. Mathur, and H. K. Genant. A comparative study of trabecular bone properties in the spine and femur using high resolution MRI and CT. Journal of Bone and Mineral Research, 13(1):122-132, 1998.

[158] F. Liu and S. Crozier. An FDTD model for calculation of gradient-induced eddy currents in MRI system. IEEE Transactions on Applied Superconductivity, 14(3):1983-1989, 2004.

[159] F. Liu, S. Crozier, H. Zhao, and B. Lawrence. Finite-difference time-domainbased studies of MRI pulsed field gradient-induced eddy currents inside the human body. Concepts in Magnetic Resonance, 15(1):26-36, 2002.

[160] F. Liu, H. Zhao, and S. Crozier. Calculation of electric fields induced by body and head motion in high-field MRI. Journal of Magnetic Resonance, 161(1):99-107, 2003.

[161] T. Liu, C. Zhao, Q. Li, and L. Zhang. An efficient backward euler timeintegration method for nonlinear dynamic analysis of structures. Computers ES Structures, 106:20-28, 2012.

[162] R. J. Luebbers, K. S. Kunz, M. Schneider, and F. Hunsberger. A finitedifference time-domain near zone to far zone transformation [electromagnetic scattering]. IEEE Transactions on Antennas and Propagation, 39(4):429-433, 1991.

[163] O. Steinbach M. Schanz. Boundary Element Analysis: Mathematical Aspects and Applications. Springer Science \& Business Media, 2007.

[164] R. L. Mackie, J. T. Smith, and T. R. Madden. Three-dimensional electromagnetic modelling using finite difference equations: The magnetotelluric example. Radio Science, 29(4):923-935, 1994.

[165] N. K. Madsen and R. W. Ziolkowski. A three-dimensional modified finite volume technique for Maxwell's equations. Electromagnetics, 10(1-2):147-161, 1990.

[166] P. Mansfield and P. Grannell. Diffraction and microscopy in solids and liquids by nmr. Physical Review B, 12(8):3618-3634, 1975. 
[167] S. S. Manson and G. R. Halford. Fatigue And Durability of Structural Materials. ASM International, 2006.

[168] D. W. Markiewicz, M. R. Vaghar, I. R. Dixon, and H. Garmestani. Generalized plane strain analysis of superconducting solenoids. Journal of Applied Physics, (12):7039-7051, 1999.

[169] P. C. Matthews. Vector Calculus. Springer, 1998.

[170] S. J. Matzat, J. van Tiel, G. E. Gold, and E. H. G. Oei. Quantitative MRI techniques of cartilage composition. Quantitative Imaging in Medicine and Surgery, 3(3):162-174, 2013.

[171] M. McJury and F. G. Shellock. Auditory noise associated with MR procedures:a review. Magnetic Resonance Imaging, 12:37-45, 2000.

[172] D. W. Mcrobbie. Occupational exposure in MRI. The British Journal of Radiology, 85(1012):293-312, 2012.

[173] C. K. Mechefske, Y. Wu, and B. K. Rutt. MRI gradient coil cylinder sound field simulation and measurement. Journal of Biomechanical Engineering, 124:450-455, 2002.

[174] L. R. Meneghelli and E. L. Cardoso. Design of compliant mechanisms with stress constraints using topology optimization. Optimization of Structures and Components, 43:35-48, 2013.

[175] C. Michler, L. Demkowicz, J. Kurtz, and D. Pardo. Improving the performance of perfectly matched layers by means of $h p$-adaptivity. Numerical Methods for Partial Differential Equations, 23:832-858, 2007.

[176] M. S. Mohamed, M. Seaid, J. Trevelyan, and O. Laghrouche. A partition of unity FEM for time-dependent diffusion problems using multiple enrichment functions. International Journal for Numerical Methods in Engineering, 93(3):245-265, 2013.

[177] A. Mollasadeghi, A. H. Mehrparvar, S. Atighechi, M. H. Davari, P. Shokouh, M. Mostaghaci, and M. Bahaloo. Sensorineural hearing loss after magnetic resonance imaging. Case Reports in Radiology, 2013, 2013.

[178] P. Monk. Finite Element Methods for Maxwell's Equations. Oxford University Press, 2003.

[179] F. Moukalled, L. Mangani, and M. Darwish. The Finite Volume Method in Computational Fluid Dynamics: An Advanced Introduction with OpenFOAM and Matlab. Springer International Publishing, 2015.

[180] V. Rokhlin N. Engheta, W. D. Murphy and M. Vassiliou. The fast multipole method for electromagnetic scattering computation. IEEE Transactions on Antennas and Propagation, 40:634-641, 1992. 
[181] A. D. Nashif, D. I. G. Jones, and J. P. Henderson. Vibration Damping. John Wiley \& Sons, 1985.

[182] S. Nath. Finite element and boundary element analysis of electromagnetic nde phenomena. PhD Thesis, Iowa State University. 1992.

[183] J. C. Nédélec. Mixed finite elements in $\mathbb{R}^{3}$. Numerische Mathematik, $35(3): 315-341,1980$.

[184] J. C. Nédélec. A new family of mixed finite elements in $\mathbb{R}^{3}$. Numerische Mathematik, 50(1):57-81, 1986.

[185] A. Nicolet and F. Delince. Implicit runge-kutta methods for transient magnetic field computation. IEEE Transactions on Magnetics, 32(3):1405-1408, 1996.

[186] G. Noh and K. J. Bathe. An explicit time integration scheme for the analysis of wave propagations. IEEE Transactions on Magnetics, 129:178-193, 2013.

[187] A. Oppelt, R. Graumann, H. Barfuss, H. Fischer, W. Hartl, and W. Schajor. Fisp - a new fast MRI sequence. Electromedica, 54(1).

[188] S. S. Pageau and S. B. Biggers. Finite element evaluation of free-edge singular stress fields in anisotropic materials. International Journal for Numerical Methods in Engineering, 38(13):2225-2239, 1995.

[189] P. Papadopoulos and R. L. Taylor. A mixed formulation for the finite element solution of contact problems. Computer Methods in Applied Mechanics and Engineering, 94(3):373-389, 1992.

[190] C. J. Partridge. Sound wave scattering from an elastic spherical shell. Technical report, 1993.

[191] M. Pastor, M. Quecedo, and O. C. Zienkiewicz. A mixed displacement-pressure formulation for numerical analysis of plastic failure. Computers 86 Structures, 62(1):13-23, 1997.

[192] D. W. Paty, J. J. F. Oger, L. F. Kastrukoff, S. A. Hashimoto, J. P. Hooge, A. A. Eisen, K. A. Eisen, S. J. Purves, M. D. Low, V. Brandejs, W. D. Robertson, and B. D. K. Li. MRI in the diagnosis of ms: A prospective study with comparison of clinical evaluation, evoked potentials, oligoclonal banding, and CT. Neurology, 38(2):180, 1988.

[193] P. Pierce. Benefits of using an open MRI vs. closed. https://www. providianmedical.com/blog/benefits-open-mri-vs-closed, 2016.

[194] R. Poya, A. J. Gil, and P. D. Ledger. A computational framework for the analysis of linear piezoelectric beams using $h p$-FEM. Computers 85 Structures, 152(Supplement C):155-172, 2015. 
[195] R. Poya, R. Sevilla, and A. J. Gil. A unified approach for a posteriori highorder curved mesh generation using solid mechanics. Computational Mechanics, 2016.

[196] D. Price, I. Delakis, C. Renaud, and R. Dickinson. MRI scanners: A buyer's guide. https://pdfs.semanticscholar.org/df6b/ bd32e99e1889bfcc61001285c6c96297e574.pdf, Date accessed $25^{\text {th }}$ June 2017 .

[197] D. L. Price, J. P. De Wilde, A. M. Papadaki, J. S. Curran, and R. I. Kitney. Investigation of acoustic noise on $15 \mathrm{MRI}$ scanners from $0.2 \mathrm{~T}$ to $3 \mathrm{~T}$. Magnetic Resonance Imaging, 13(2):288-93, 2001.

[198] C. Qian, I. S. Masad, J. T. Rosenberg, M. Elumalai, W. W. Brey, S. C. Grant, and P.L. Gor'kov. A volume birdcage coil with an adjustable sliding tuner ring for neuroimaging in high field vertical magnets: ex and in vivo applications at 21.1t. Journal of Magnetic Resonance, 221:110-116, 2012.

[199] C. H. Lee R. Ortigosa, A. J. Gil. A computational framework for large strain nearly and truly incompressible electromechanics based on convex multivariable strain energies. Computer Methods in Applied Mechanics and Engineering, 310:297-334, 2016.

[200] I.I. Rabi, J.R. Zacharias, S. Millman, and P. Kusch. A new method of measuring nuclear magnetic moment. Physical Review, 53(4):318-327, 1938.

[201] P. Radomskij, M. A. Schmidt, C. W. Heron, and D. Prasher. Effect of MRI noise on cochlear function. The Lancet, 359:1485-1486, 2002.

[202] M. Rausch, M. Gebhardt, M. Kaltenbacher, and H. Landes. Magnetomechanical field computations of a clinical magnetic resonance imaging (MRI) scanner. The International Journal for Computation and Mathematics in Electrical and Electronic Engineering (COMPEL), 22:576-588, 2003.

[203] M. Rausch, M. Gebhardt, M. Kaltenbacher, and H. Landes. Computeraided design of clinical magnetic resonance imaging scanners by coupled magnetomechanical-acoustic modelling. IEEE Transactions on Magnetics, 41:72-81, 2005.

[204] Z. Ren and A. Razek. A strong coupled model for analysing dynamic behaviours of non-linear electromechanical systems. IEEE Transactions on Magnetics, 30(5):3252-3255, 1994.

[205] P. J. Roache and D. E. Dietrich. Evaluation of the filtered leapfrog-trapezoidal time integration method. Numerical Heat Transfer, 14(2):149-164, 1988.

[206] A. A. Rodríguez and A. Valli. Eddy Current Approximation of Maxwell Equations: Theory, algorithms and applications. Springer, Milano, 2010. 
[207] T. Y. Rong and A. Q. Lu. Generalized mixed variational principles and solutions of ill-conditioned problems in computational mechanics: Part i. volumetric locking. Computer Methods in Applied Mechanics and Engineering, 191(3):407-422, 2001.

[208] N. B. Roozen, A. H. Koevoets, and A. J. den Hamer. Active vibration control of gradient coils to reduce acoustic noise of MRI systems. IEEE/ASME Transactions on Mechatronics, 13(3):325-334, 2008.

[209] J. Saitz. Newton-raphson method and fixed-point technique in finite element computation of magnetic field problems in media with hysteresis. IEEE Transactions on Magnetics, 35(3):1398-1401, 1999.

[210] C. C. Sánchez, H. Power, S. G. Garcia, and A. R. Bretones. Quasi-static multidomain inverse boundary element method for MRI coil design with minimum induced E-field. Engineering Analysis with Boundary Elements, 35(3):264-272, 2011.

[211] N. Savage. The world's most powerful MRI takes shape. http://spectrum.ieee.org/biomedical/imaging/ the-worlds-most-powerful-mri-takes-shape, Date accessed $30^{\text {th }}$ June 2017.

[212] K. Schaap, Y. C. de Vries, C. K. Mason, F. de Vocht, L. Portengen, and H. Kromhout. Occupational exposure of healthcare and research staff to static magnetic stray fields from 1.5-7 Tesla MRI scanners is associated with reporting of transient symptoms. Occupational and Environmental Medicine, 71(6):423-429, 2014.

[213] D. J. Schaefer, J. D. Bourland, and J. A. Nyenhuis. Review of patient safety in time-varying gradient fields. Journal of Magnetic Resonance Imaging, 12:20 29, 2000 .

[214] M. Schinnerl, M. Kaltenbacher, U. Langer, R. Lerch, and J. Schöberl. An efficient method for the numerical simulation of magneto-mechanical sensors and actuators. European Journal of Applied Mathematics, 18:233-271, 2007.

[215] M. Schinnerl, J. Schoberl, M. Kaltenbacher, and R. Lerch. Multigrid methods for the three-dimensional simulation of nonlinear magnetomechanical systems. IEEE Transactions on Magnetics, 38(3):1497-1511, 2002.

[216] K. Schmidt, O. Sterz, and R. Hiptmair. Estimating the eddy-current modeling error. IEEE Transactions on Magnetics, 44(6):686-689, 2008.

[217] J. Schöberl and S. Zaglmayr. High order Nédélec elements with local complete sequence properties. The International Journal for Computation and Mathematics in Electrical and Electronic Engineering (COMPEL), 24:374-384, 2005. 
[218] G. Seriani and E. Priolo. Spectral element method for acoustic wave simulation in heterogeneous media. Finite Elements in Analysis and Design, 16(3):337348, 1994.

[219] R. Sevilla, S. Fernández-Méndez, and A. Huerta. NURBS-enhanced finite element method (NEFEM). International Journal for Numerical Methods in Engineering, 76(1):56-83, 2008.

[220] R. Sevilla, S. Fernández-Méndez, and A. Huerta. 3D NURBS-enhanced finite element method (NEFEM). International Journal for Numerical Methods in Engineering, 88(2):103-125, 2011.

[221] R. Sevilla, S. Fernández-Méndez, and A. Huerta. Comparison of high-order curved finite elements. International Journal for Numerical Methods in Engineering, 87(8):719-734, 2011.

[222] R. Sevilla, O. Hassan, and K. Morgan. The use of hybrid meshes to improve the efficiency of a discontinuous Galerkin method for the solution of Maxwell's equations. Computers \& Structures, 137(Supplement C):2-13, 2014.

[223] V. Shankar, A. H. Mohammadian, and W. F. Hall. A time-domain, finitevolume treatment for the Maxwell equations. Electromagnetics, 10(1-2):127$145,1990$.

[224] W. Shao and C. K. Mechefske. Acoustic analysis of a gradient coil winding in an MRI scanner. Concepts in Magnetic Resonance Part B: Magnetic Resonance Engineering, 24B(1):15-27, 2005.

[225] F. G. Shellock and J. V. Crues. Mr procedures: Biologic effects, safety, and patient care. Radiology, 232(3):635-652, 2004.

[226] S. Shvartsman, M. Morich, G. DeMeester, and Z. Zhai. Ultrashort shielded gradient coil design with 3D geometry. Concepts in Magnetic Resonance, 26B(1):1-15, 2005.

[227] Siemens. MAGNETOM Terra: Translate 7T research power into clinical care. https://www.healthcare.siemens.co.uk/magnetic-resonance-imaging/ 7t-mri-scanner/magnetom-terra. Accessed: 17/11/2017.

[228] Siemens. Magnetic resonance imaging: The definitive portfolio for MRI. https://www .healthcare.siemens.com/magnetic-resonance-imaging? stc=wwhim800053, Date accessed $25^{\text {th }}$ July 2017.

[229] Siemens. MAGNETOM family of MRI systems. http: //www.deltamedicalsystems.com/DeltaMedicalSystems/media/ Product-Details/MAGNETOM-Family-Brochure.pdf, Date accessed $25^{\text {th }}$ June 2017. 
[230] J. C. Simo and J. W. Ju. Strain- and stress-based continuum damage models: Ii. computational aspects. International Journal of Solids and Structures, 23(7):841-869, 1987.

[231] R.J. Singer. Blood-flow rates by nmr measurements. Review of Scientific Instruments, 130:1652-1653, 1959.

[232] D. R. Smith, D. C. Vier, T. Koschny, and C. M. Soukoulis. Electromagnetic parameter retrieval from inhomogeneous metamaterials. Physical Reviews E., 71:36617-36628, 2005.

[233] P. Smolinski. Stability analysis of a multi-time step explicit integration method. Computer Methods in Applied Mechanics and Engineering, 95(3):291 - 300, 1992.

[234] W. R. Smythe. Static and Dynamic Electricity. McGraw-Hill, USA, 1950.

[235] W. T. Sobol. Recent advances in MRI technology: Implications for image quality and patient safety. Saudi Journal of Ophthalmology, 26(4):393-399, 2017.

[236] K. Somasundaram and P. Kalavathi. Analysis of imaging artifacts in mr brain images. Oriental Journal of Computer Science \& Technology, 5(1):135-141, 2012 .

[237] H. Spachmann, R. Schuhmann, and T. Weiland. Higher order explicit time integration schemes for Maxwell's equations. International Journal of $\mathrm{Nu}$ merical Modelling: Electronic Networks, Devices and Fields, 15(5-6):419-437, 2002.

[238] P. Sprawls. Magnetic Resonance Imaging: Principles, Methods, and Techniques. Medical Physics Publishing, 2000.

[239] A. Stadler, W. Schima, A. Ba'ssalamah, J. Kettenbach, and E. Eisenhuber. Artifacts in body mr imaging: Their appearance and how to eliminate them. European Radiology, 17(5):1242-1255, 2007.

[240] Diagnostic Imaging Staff. Patient movement during MRI expensive for radiology. http://www.diagnosticimaging.com/mri/ patient-movement-during-mri-expensive-radiology, 2015.

[241] G. Strang. Calculus. Wellesley-Cambridge Press, 1991.

[242] A. H. Stroud and D. Secrest. Gaussian quadrature formulas. Englewood Cliffs, N.J. : Prentice-Hall, 1966.

[243] M. Suri. Analytical and computational assessment of locking in the $h p$ finite element method. Computer Methods in Applied Mechanics and Engineering, 133:347-371, 1996. 
[244] B. Szabó and I. M. Babuška. Finite Element Analysis. New York: John Wiley and Sons, 1991.

[245] A. Taflove. Application of the finite-difference time-domain method to sinusoidal steady-state electromagnetic-penetration problems. IEEE Transactions on Electromagnetic Compatibility, 22(3):191-202, 1980.

[246] A. Taflove and S. C. Hagness. Computational Electrodynamics: The FiniteDifference Time-Domain Method. Artech House, 2005.

[247] L. L. Thompson. A review of finite-element methods for time-harmonic acoustics. The Journal of the Acoustical Society of America, 119(3):1315-1330, 2006.

[248] A. Trakic, H. Wang, F. Liu, H. S. Lopez, and S. Crozier. Analysis of transient eddy currents in MRI using a cylindrical FDTD method. IEEE Transactions on Applied Superconductivity, 16(3):1924-1936, 2006.

[249] A. G. van der Kolk, J. Hendrikse, J. J. M. Zwanenburg, F. Visser, and P. R. Luijten. Clinical applications of $7 \mathrm{~T}$ MRI in the brain. European Journal of Radiology, 82(5):708-718, 2013.

[250] A. H. van Zuijlen and H. Bijl. Implicit and explicit higher order time integration schemes for structural dynamics and fluid-structure interaction computations. Computers \& Structures, 83(2):93-105, 2005.

[251] T. Vaughan, L. DelaBarre, C. Snyder, J. Tian, C. Akgun, D. Shrivastava, W. Liu, C. Olson, G. Adriany, J. Strupp, P. Andersen, A. Gopinath, P.F. van de Moortele, M. Garwood, and K. Ugurbil. 9.4T human MRI: preliminary results. Magnetic Resonance in Medicine, 56(6):1274-1282, 2006.

[252] Y. Wang, F. Liu, and S. Crozier. Simulation study of noise reduction methods for a split MRI system using a finite element method. Medical Physics, 42(12):7122-7131, 2015.

[253] Y. Wang, F. Liu, E. Weber, F. Tang, J. Jin, Y. Tesiram, and S. Crozier. Acoustic analysis for a split MRI system using FE method. Concepts in Magnetic Resonance Part B: Magnetic Resonance Engineering, 45(2):85-96, 2015.

[254] M. Weberstadt. A high-fidelity stabilised finite element method for fluidstructure interaction problems. PhD Thesis, Swansea University. 2016.

[255] G. N. Wells, L. J. Sluys, and R. de Borst. A p-adaptive scheme for overcoming volumetric locking during plastic flow. Computer Methods in Applied Mechanics and Engineering, 191(29):3153-3164, 2002.

[256] S. Zaglmayr. High order finite element methods for electromagnetic field computation. PhD Thesis, Institut für Numerische Mathematik, Johannes Kepler Univerität Linz, Austria. 2006. 
[257] H. Zhao, S. Crozier, and F. Liu. Finite difference time domain (FDTD) method for modelling the effect of switched gradients on the human body in MRI. Magnetic Resonance in Medicine, 48(6):1037-1042, 2002.

[258] O. C. Zienkiewicz, C. Emson, and P. Bettess. A novel boundary infinite element. International Journal for Numerical Methods in Engineering, 19(3):393404, 1983.

[259] O. C. Zienkiewicz, R. L. Taylor, and J. Z. Zhu. The Finite Element Method: Its Basis $\&$ Fundamentals. Butterworth-Heinemann, Sixth Edition, 2007. 